\author{
UNIVERSITY OF SAO PAULO \\ ENGINEERING SCHOOL OF SÃO CARLOS \\ INSTITUTE OF PHYSICS OF SÃO CARLOS \\ INSTITUTE OF CHEMISTRY OF SÃO CARLOS \\ SUPERIOR NORMAL SCHOOL OF CACHAN \\ LABORATORY OF MECHANICS AND TECHNOLOGY
}

\title{
FATIGUE LIFE EVALUATION OF A356 ALUMINUM ALLOY USED FOR ENGINE CYLINDER HEAD
}




\section{MAURICIO ANGELONI}

\section{AVALIAÇÃO DA VIDA EM FADIGA DE LIGA DE ALUMÍNIO A356 UTILIZADA EM CABEÇOTE DE MOTOR}

Tese apresentada ao Programa de PósGraduação Interunidades em Ciência e Engenharia de Materiais da Universidade de São Paulo, para a obtenção do Título de Doutor em Ciência e Engenharia de Materiais.

Orientedores: Prof. Dr. Waldek W. Bose Filho Prof ${ }^{a}$. Dra. Sylvie Pommier

\section{São Carlos}



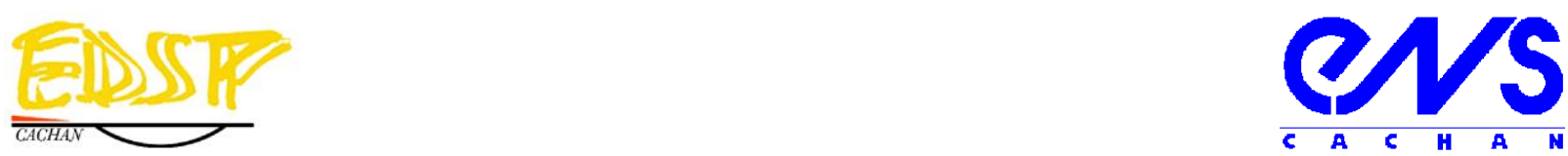

THÈSE DE DOCTORAT

DE L'ECOLE NORMALE SUPERIEURE DE CACHAN

\author{
PRESENTEE PAR \\ Monsieur Mauricio ANGELONI
}

POUR OBTENIR LE GRADE DE

DOCTEUR DE L'ECOLE NORMALE SUPERIEURE DE CACHAN

DOMAINE :

Mécanique- Génie mécanique - Génie civil

\title{
SUJET DE LA THESE: \\ Évaluation de la vie en fatigue en alliage d'aluminium A356 utilisé pour les culasses de moteurs
}

Thèse en cotutelle entre l'Universite de São Paulo, São Carlos (Brésil) et L'Ecole Normale Superieure de Cachan présentée et soutenue à l'Instituto de Física de São Carlos (Brésil) le 22 mars 2011 devant le jury composé de :

\begin{tabular}{|l|c|l|}
\hline M. Waldek W. Bose Filho & Professeur USP & Examinateur et directeur de thèse \\
\hline Mme. Sylvie Pommier & Professeur ENS-Cachan & Examinateur et directrice de thèse \\
\hline Renné Billardon & Professeur UPMC & Président et examinateur \\
\hline Yves Nadot & Professeur ENSMA & Rapporteur \\
\hline Sérgio P. B. Proença & Professeur USP & Examinateur \\
\hline José Alexander Araújo & Professeur UNB & Rapporteur et Examinateur \\
\hline Jonas de Carvalho & Professeur USP & Examinateur \\
\hline
\end{tabular}




\section{AUTORIZO A REPRODUÇÃO E DIVULGAÇÃO TOTAL OU PARCIAL DESTE TRABALHO, POR QUALQUER MEIO CONVENCIONAL OU ELETRÔNICO, PARA FINS DE ESTUDO E PESQUISA, DESDE QUE CITADA A FONTE.}

Ficha catalográfica preparada pela Seção de Tratamento da Informação do Serviço de Biblioteca - EECS/USP

\begin{tabular}{|c|c|}
\hline \multirow[t]{3}{*}{ A584a } & $\begin{array}{l}\text { Angeloni, Mauricio } \\
\text { Avaliação da Vida em Fadiga de Liga de Alumínio A356 Utilizada em } \\
\text { Cabeçote de Motor / Mauricio Angeloni; orientador Waldek Wladimir Bose } \\
\text { Filho e Sylvie Pommier. -- São Carlos, } 2011 .\end{array}$ \\
\hline & $\begin{array}{l}\text { Tese (Doutorado-Programa de Pós-Graduação e Área de Concentração } \\
\text { Interunidades em Ciência e Engenharia de Materiais) - Escola de Engenharia } \\
\text { de São Carlos, Instituto de Física de São Carlos, Instituto de Química de São } \\
\text { Carlos da Universidade de São Paulo, } 2011 \text {. }\end{array}$ \\
\hline & $\begin{array}{l}\text { 1. Ligas de Alumínio. 2. Cabeçote de Motor. 3. Fadiga Isotérmica. 4. Fadiga } \\
\text { Termomecânica. 5. Propagaçâo de Trinca por Fadiga. 6. Elementos Finitos. I. } \\
\text { Título. }\end{array}$ \\
\hline
\end{tabular}



I dedicate to my family, friends and everyone who helped and encouraged me in this study 


\section{ACKNOWLEDGEMENTS}

Professors Supervisors Dr. Waldek W. Bose Filho and Dra. Sylvie Pommier for his tireless and able guidance and also for the support, friendship and trust, without which this work would not have been accomplished.

To Professor. Dr. Spinelli Dirceu by trust and belief.

I thank my parents Gilberto Angeloni and Diva Maria Cordeiro Angeloni and my girlfriend Wuendy Fernanda Cardili for encouragement and support in difficult times.

The friends of the Department of Materials Engineering of Engineering School of São CarlosUSP and the friends of the Laboratory of Mechanics and Technology of ENS-Cachan for their immeasurable support in hard times.

AID AGENCIES search FAPESP and CAPES. 


\section{RESUMO}

ANGELONI, M. Avaliação da Vida em Fadiga de Liga de Alumínio A356 Utilizada em Cabeçote de Motor. 2011. 201p. Tese (Doutorado) - Escola de Engenharia de São Carlos, Instituto de Física de São Carlos, Instituto de Química de São Carlos, Universidade de São Paulo, São Carlos, 2011.

Para caracterizar componentes usados em aplicações de alta responsabilidade não basta apenas conhecer a composição química e os resultados de ensaios de tração, impacto e dureza, pois estes podem não fornecer os subsídios necessários que permitam prever, de maneira confiável, o comportamento dos componentes nas condições reais de trabalho. Exemplo disto são os cabeçotes de motor automotivos, submetidos a tensões térmicas e mecânicas relativamente altas durante seu uso normal e altíssimo em condições extremas. Durante longos tempos de funcionamento e eventuais falhas na refrigeração e ou lubrificação a temperatura pode chegar a valores próximos de $300^{\circ} \mathrm{C}$. Esta variação de temperaturas provoca choques térmicos que podem gerar trincas e/ou uma grande quantidade de deformação plástica em regiões próximas aos pistões. Desconsiderando a presença de choques térmicos provocados por falhas, ainda assim, uma pequena quantidade de ciclos de acionamento e parada do motor, é considerada como os principais causadores de pequenas trincas. Isso indica que o surgimento de trincas em cabeçotes de motor deve ser considerado um problema de fadiga termomecânica de baixo ciclo. Outro problema é a heterogeneidade microestrutural no componente devido ao processo de fundição, levando a propriedades mecânicas e físicas diferentes em uma mesma peça. Além da presença de porosidade gerada por bolhas de gás e vazios de solidificação, que podem adquirir tamanho tal que se aproximem de pequenas trincas, diminuindo a vida para a nucleação e assim mudando o foco do problema para o de propagação de trinca por fadiga. A proposta deste trabalho foi a de determinar as propriedades de fadiga isotérmica e termomecânica através de ensaios de fadiga de baixo ciclo, bem como as propriedades de propagação de trinca por fadiga, relaxação, caracterização microestrutural e modelagem do comportamento mecânico por elementos finitos para a liga de alumínio utilizada na fabricação de cabeçotes de motores automotivos pela indústria nacional. Todos os ensaios e análises computacionais foram realizados nos laboratórios da Universidade de São Paulo (EESC-USP) e da École Normale Supérieure de Cachan (ENS-LMT). Os ensaios de fadiga isotérmica foram realizados nas temperaturas de $120^{\circ} \mathrm{C}$ e $280^{\circ} \mathrm{C}$ e os ensaios termomecânicos foram realizados em fase entre as temperaturas de $120^{\circ} \mathrm{C}$ e $280^{\circ} \mathrm{C}$. Os ensaios de relaxação foram realizados em várias temperaturas com carregamento de onda trapezoidal, enquanto que os ensaios de propagação de trinca por fadiga foram realizados nas temperaturas de $120^{\circ} \mathrm{C}, 200^{\circ} \mathrm{C}$ e $280^{\circ} \mathrm{C}$ para carregamentos de onda senoidal e trapezoidal em controle de carga e de deslocamento. Foi feita também análise microestrutural nos corpos de prova, após os ensaios de fadiga, por microscopia óptica e por microscopia eletrônica de varredura MEV. Os resultados destes ensaios mostraram que os defeitos de fundição e a falta de homogeneidade no material, aliados a longos tempos de exposição a carregamentos e em altas temperaturas, constituem um fator crítico no desempenho do componente. Estes resultados ajudarão a estabelecer modelos precisos de previsão de vida para os cabeçotes de motor.

Palavras-chave: Ligas de Alumínios. Cabeçote de Motor. Fadiga Isotérmica. Fadiga Termomecânica. Propagação de Trinca por Fadiga. Elementos Finitos. 


\begin{abstract}
ANGELONI, M. Fatigue Life Evaluation of A356 Aluminum Alloy used for Engine Cylinder Head. 2011. 201p. Tese (Doutorado) - Escola de Engenharia de São Carlos, Instituto de Física de São Carlos, Instituto de Química de São Carlos, Universidade de São Paulo, São Carlos, 2011.

In order to characterize mechanical components used in high responsibility applications, the knowledge of chemical composition and results from regular experiments such as traction, impact and hardness tests is important, but not sufficient. They cannot supply the necessary information that permit anticipating, in a reliable way, the components' behavior in actual working conditions. As an example, there are engine's cylinder head submitted to mechanical and thermal tensions that are relatively high during the in service use, and very high in same very demanding condition. During long run times and any failure in the cooling and/or lubrication the temperature may reach $300^{\circ} \mathrm{C}$. This temperature variation causes thermal shocks which may generate cracks and/or a wide ranging of plastic deformation in regions close to the pistons. Even not considering the thermal shock effects caused by failure, even so, a short number of start-up and shutdown cycles of engine, are considered the main cause of small cracks. This indicates that the generation of cracks in cylinder head may be considered as low cycle thermomechanical fatigue problem. Another problem is the microstructure heterogeneity in the component due to the casting process, leading to different physical and mechanical properties in the same piece. Besides the presence of porosity generated by gas bubbles and voids of solidification, which may be as great as short crack, reducing the nucleation life and changing the problem's focus for the fatigue crack growth. The purpose of this study was to determine the isothermal and thermomechanical fatigue property through low cycle fatigue, as well as the fatigue crack growth, relaxation, microestrutural characterization and modeling of mechanical behavior by finite element for the aluminum alloy employed in the manufacture of engine cylinder head by the national automotive industry. All tests and computer analysis were performed in the laboratories of the University of São Paulo (EESC-USP) and the École Normale Supérieure de Cachan (ENS-LMT). Isothermal fatigue experiments were carried out at temperature of $120^{\circ} \mathrm{C}$ and $280^{\circ} \mathrm{C}$ and the thermomechanical performed in phase between the temperatures of $120^{\circ} \mathrm{C}$ and $280^{\circ} \mathrm{C}$. The relaxation experiments were performed at some temperatures with trapezoidal wave loading, whereas the experiments of fatigue crack growth carried out at temperatures of $120^{\circ} \mathrm{C}, 200^{\circ} \mathrm{C}$ and $280^{\circ} \mathrm{C}$ for sine and trapezoidal wave loading in displacement and load control. The microstructure analysis was also made in the specimens after the fatigue test by optical microscopy and scanning electron microscopy SEM. The results of these experiments showed that the casting defects and material's inhomogeneities, coupled long run times in high temperatures and loads, are a critical factor in the component performance. These results help us to establish accurate models for life prediction of the engine cylinder head.
\end{abstract}

Keywords: Aluminum Alloys. Cylinder head. Isothermal Fatigue. Thermomechanical Fatigue. Fatigue Crack Growth. Finite Element. 


\section{RÉSUMÉ}

ANGELONI, M. Évaluation de la Vie en Fatigue en Alliage d'Aluminium A356 utilisés pour la Culasse du Moteur. 2011. 201p. Tese (Doutorado) - Escola de Engenharia de São Carlos, Instituto de Física de São Carlos, Instituto de Química de São Carlos, Universidade de São Paulo, São Carlos, 2011.

Afin de caractériser les composants qui demande de haut-responsabilité ne suffit pas de connaître la composition chimique et les résultats des essais de traction, impact et dureté, car ils ne peuvent pas fournir des données nécessaire qui permettent de prédire avec certitude le comportement des composants dans les conditions réelles de travail. Des exemples sont des culasses du moteur, soumis à des contraintes thermiques et mécaniques relativement élevé en utilisation normale et très élevé dans des conditions extrêmes. Pour de longues périodes de fonctionnement et pour d'eventuels défauts de refroidissement ou de lubrification la température peut atteindre des valeurs proches de $300^{\circ} \mathrm{C}$. Cette variation de température provoque un choc thermique qui peut provoquer des fissures et/ou une grande quantité de déformation plastique dans les régions proches des pistons. Sans tenir compte la présence de chocs thermiques causés par des défaillance, encore, une petite quantité de cycles de démarrer et d'arrêt du moteur, est considérée comme la principale cause des fissures petites. Cela indique que l'apparition de fissures dans la culasses du moteur doivent être considérés comme un problème de fatigue thermomécanique oligocyclique. An autre problème est la hétérogénéité de la microstructure du composante en raison du processus de fonderie, conduisant à des différents propriétés physiques et mécaniques dans la même pièce. De plus, la présence de porosité généré par les bulles de gaz et les vides de la solidification, qui peut obtenir une telle taille qui viennent à proximité des petits fissures, réduisant la vie à la nucléation et donc changer le centre de l'attention pour la propagation des fissures par fatigue. Le but de cette étude était de déterminer les propriétés des essais de fatigue isotherme et thermomécanique utilisant de fatigue oligocyclique, ainsi que les propriétés de propagation des fissures de fatigue, la relaxation, la caractérisation et la modélisation du comportement mécanique par éléments finis pour une alliage d'aluminium utilisé dans la fabrication de culasse du moteur automobile par l'industrie nationale. Tous les essais et l'analyse numérique ont été effectuées dans les laboratoires de l'Université de São Paulo (USP-EESC) et l'École Normale Supérieure de Cachan (ENS-LMT). Les essais de fatigue isotherme ont été effectués à des températures de $120^{\circ} \mathrm{C}$ et $280^{\circ} \mathrm{C}$ et les thermomécanique ont été réalisées en phase entre les températures de $120^{\circ} \mathrm{C}$ et $280^{\circ} \mathrm{C}$. Les essais de relaxation ont été effectués à différentes températures avec des chargement d'ondes trapézoïdale, tandis que les essais de propagation des fissures de fatigue ont été réalisés à des températures de $120^{\circ} \mathrm{C}, 200^{\circ} \mathrm{C}$ et $280^{\circ} \mathrm{C}$ avec des chargement d'ondes trapézoïdale et sinusoïdales sûr le contrôle de charge et de déplacement. A été faite l'analyse des microstructures dans les échantillons, après les essais de fatigue, pour microscopie optique et microscopie électronique à balayage MEB. Les résultats de ces essais ont montré que les défauts de fonderie et le manque d'homogénéité dans le matériau, couplé avec des longue temps de exposition à des températures élevées et des charges, constituent un facteur critique dans l'exécution de la composante. Ces résultats aideront à établir des modèles précis de prédiction de la vie pour les culasses de moteur.

Mots clés: Les alliages d'aluminium. Culasse du Moteur. Fatigue isotherme. Fatigue thermomécanique. Fissuration. Éléments finis. 


\section{SUMÁRIO}

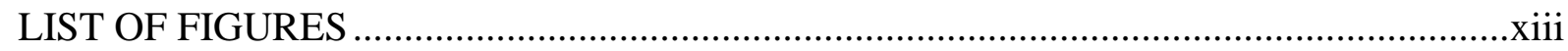

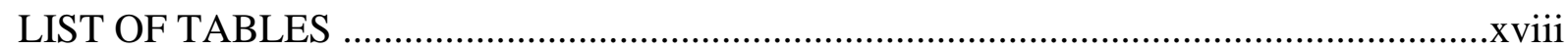

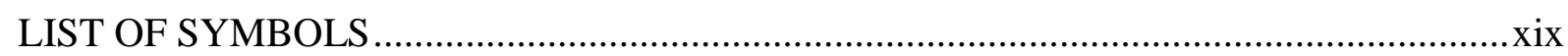

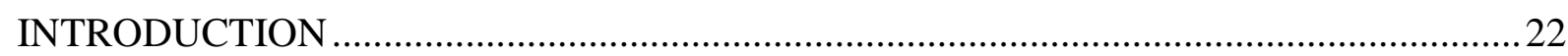

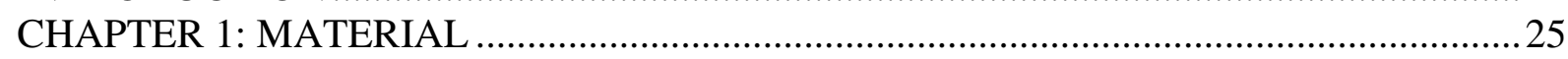

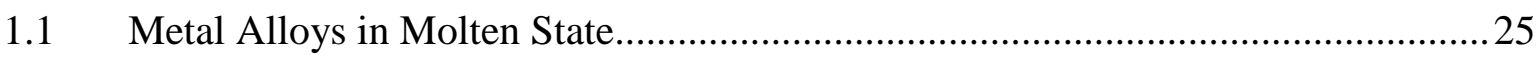

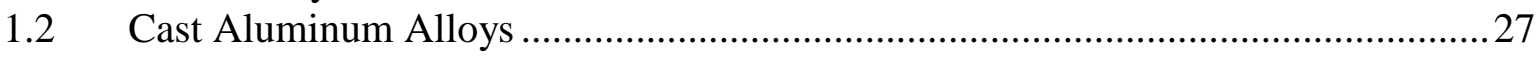

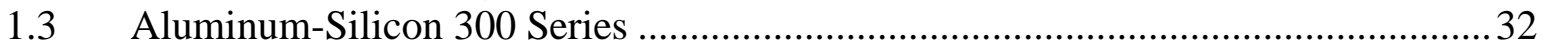

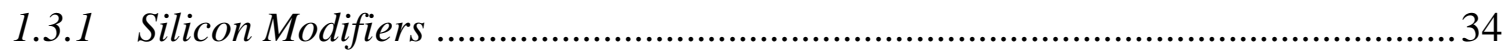

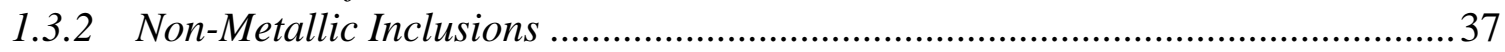

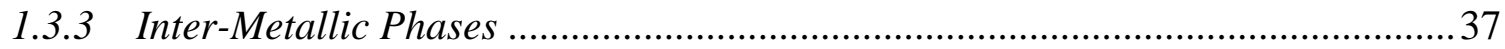

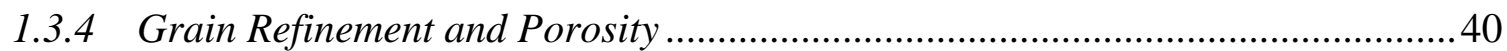

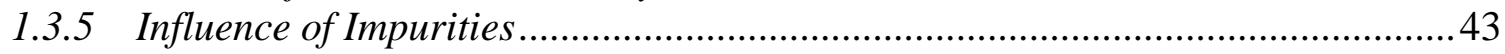

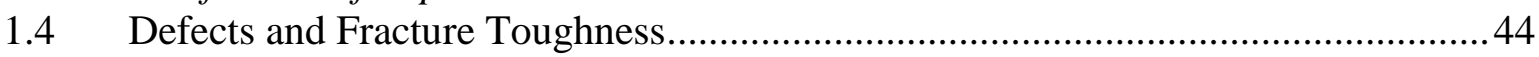

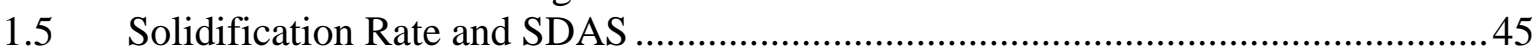

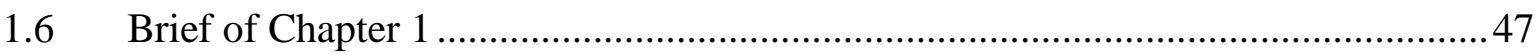

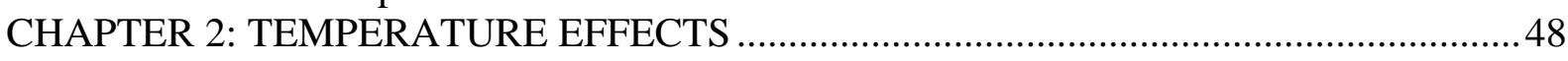

2.1 Effect of High Temperature on Materials............................................................. 48

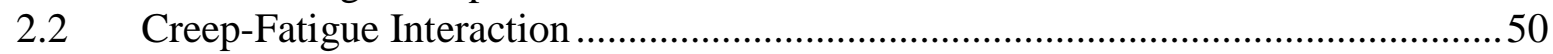

2.3 Damage and Crack Initiation at High Temperatures ............................................52

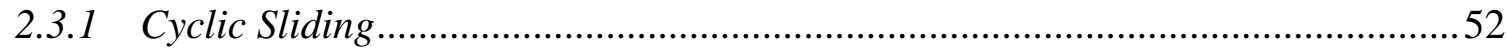

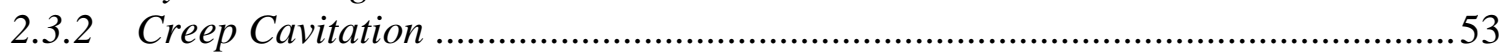

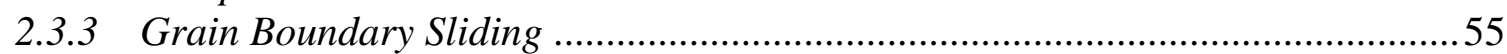

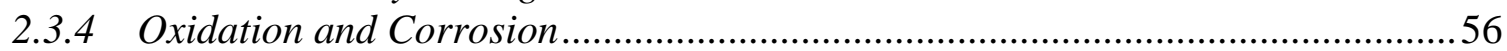

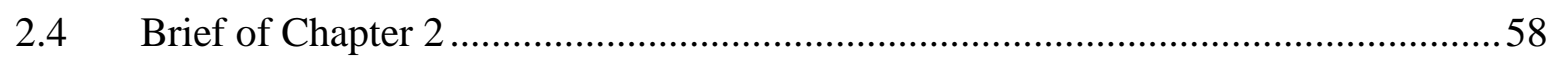

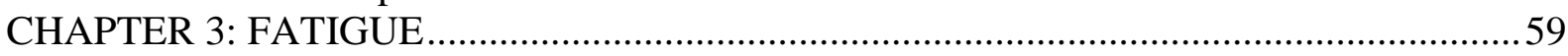

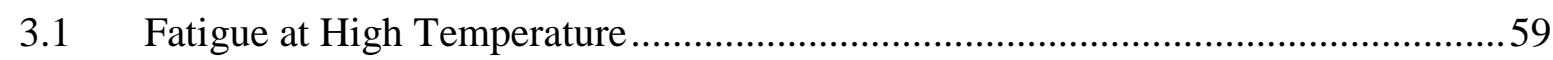

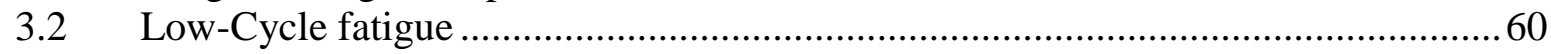

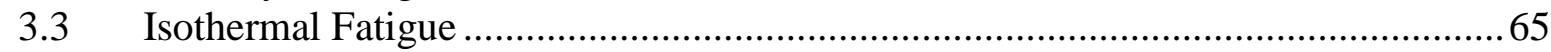

3.4 In-Phase and Out-of-Phase Thermomechanical Fatigue and Thermal Fatigue ........68

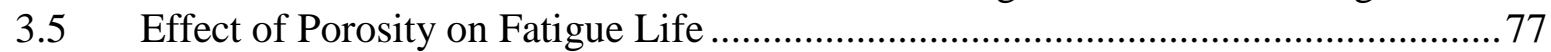

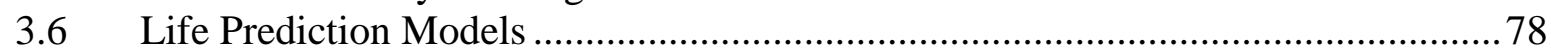

3.6.1 Damage Accumulation Models ........................................................................... 78

3.6.2 Modified-Frequency Coffin-Manson Equations ............................................... 79

3.6.3 Strain-Variation Partitioning Model ................................................................. 79

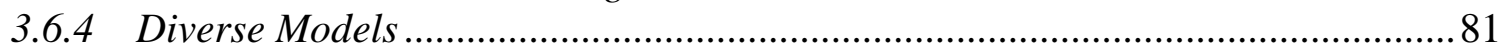

3.6.5 Modeling of Anisothermal Hysteresis as a Function of Isothermal Data ............ 83

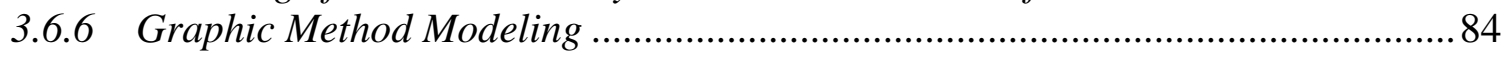

3.6.7 Thermo-Mechanical Fatigue Modeling for Nucleation of Cracks ........................86

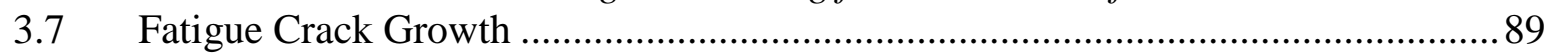

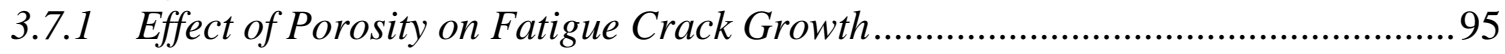

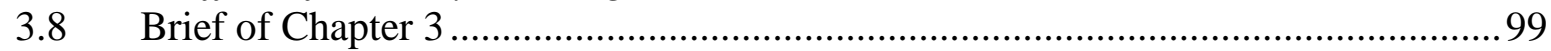

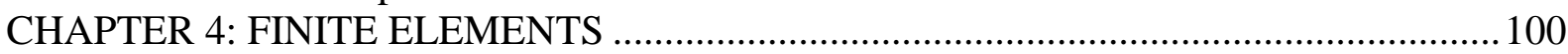

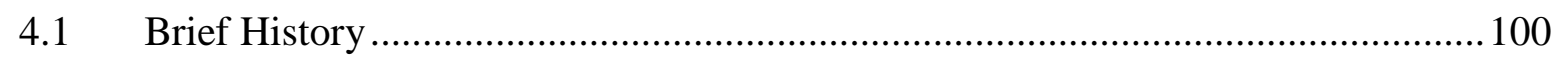

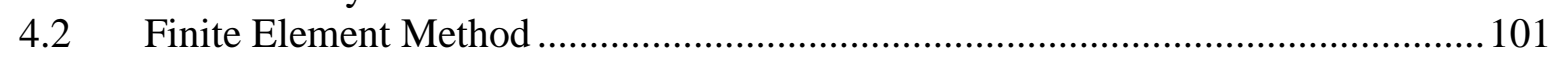

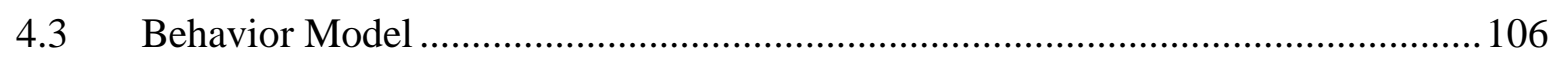

4.4 Presentation of Proposed Crack Growth Model .................................................. 107 


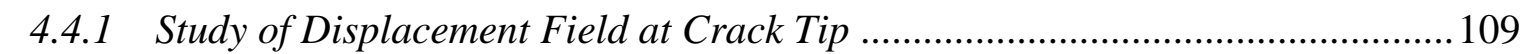

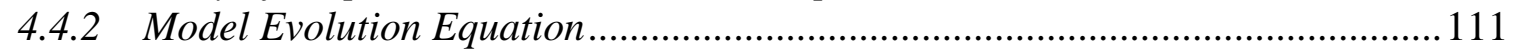

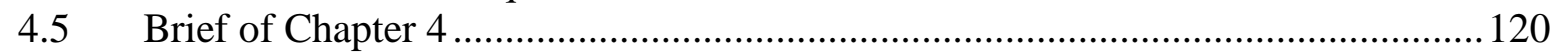

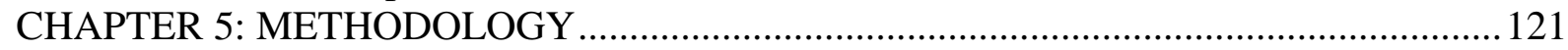

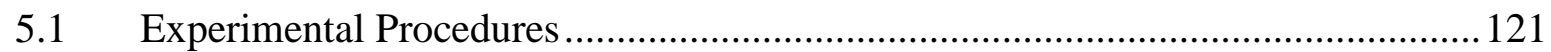

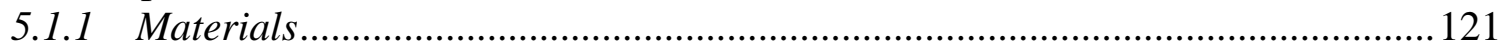

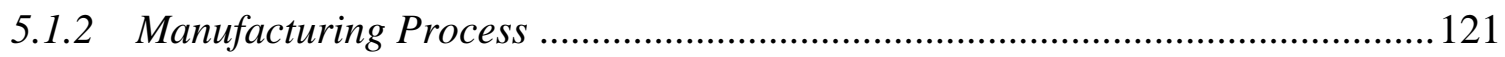

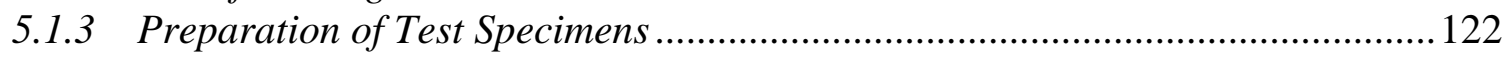

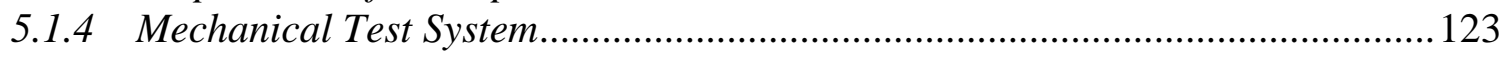

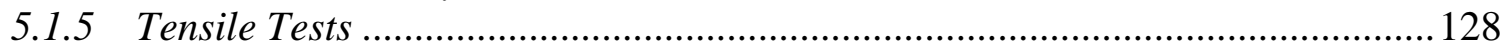

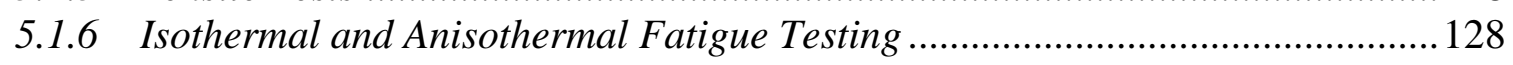

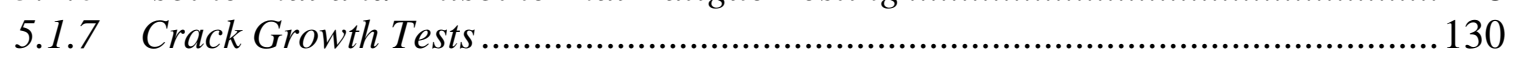

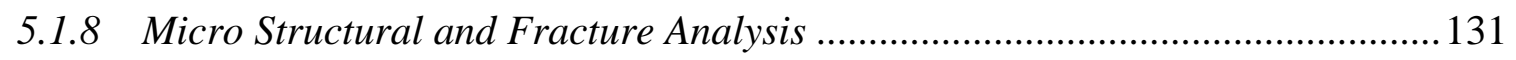

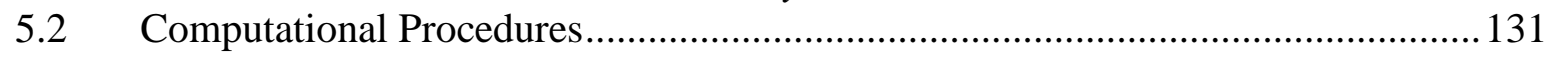

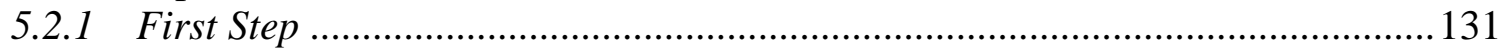

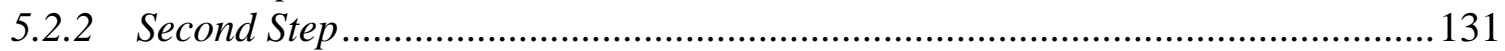

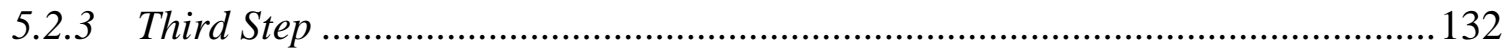

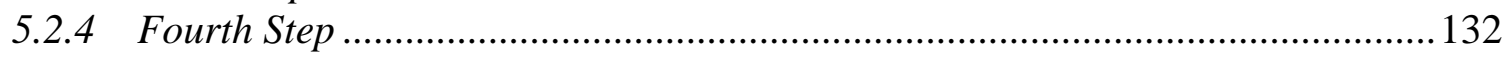

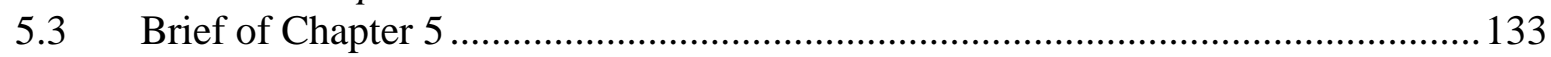

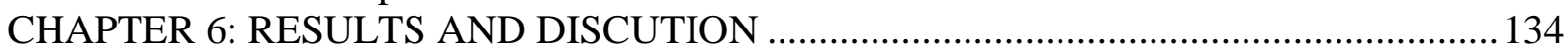

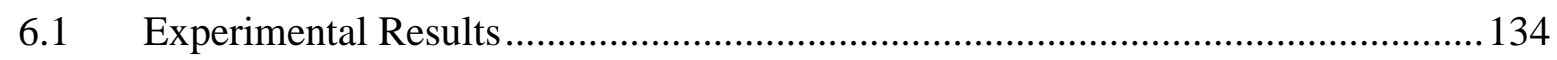

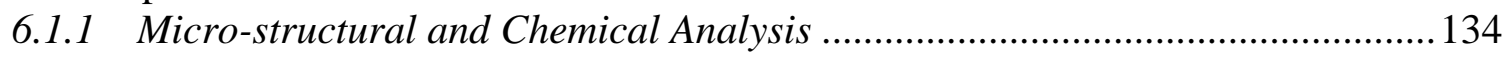

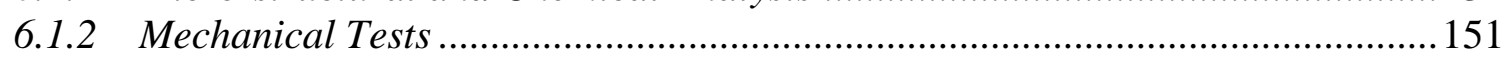

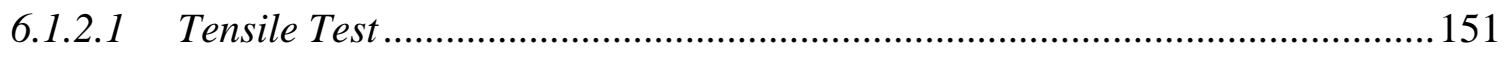

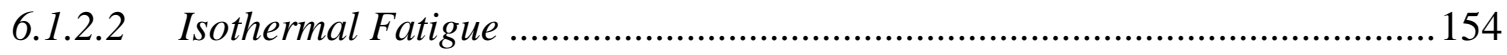

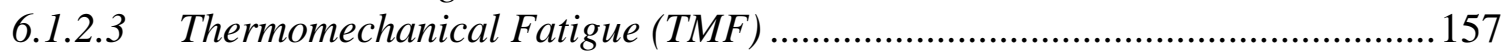

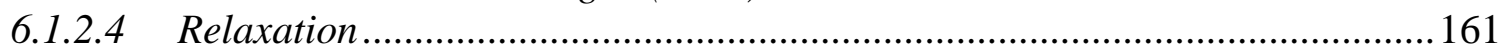

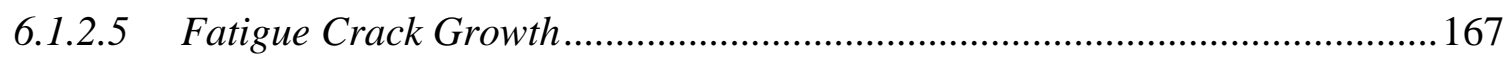

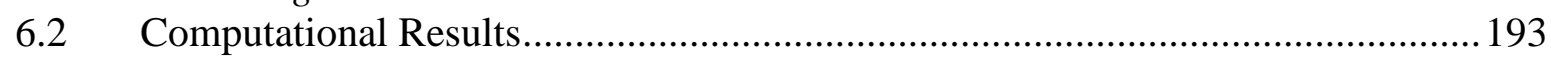

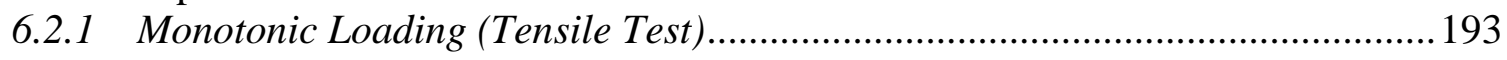

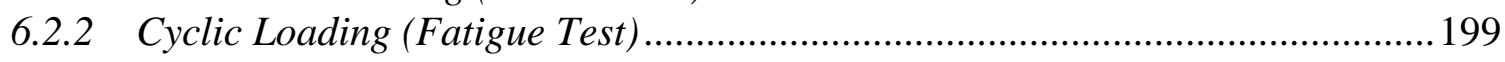

6.2.2.1 Identification of Material Behavior Law Parameters .................................... 199

6.2.2.2 Identification of Plastic Blunting Law Parameters .......................................203

6.2.2.3 Identification of Crack Propagation Law Parameter ......................................205

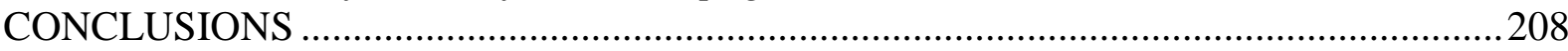

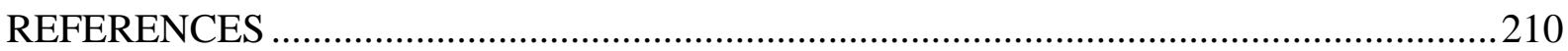




\section{LIST OF FIGURES}

Figure 1: Picture of a fatigue crack that caused the cylinder head to fail during operation.....22 Figure 2: (a) Homogeneous nucleation and (b) heterogeneous nucleation (Milan, 2004)...... 26 Figure 3: Schematic representation of (a) planar growth and (b) dendritic growth (Milan, 2004).

Figure 4: Solidification dendrites of an aluminum alloy with high silicon content (Milan, 2004).

Figure 5: Schematic representation of a dendrite (Massinon \& Constantin, 2002). ............... 28

Figure 6: (a) Principle of competitive growth leading to a lamellar eutectic, and (b) schematic representation of a lamellar eutectic (Barralis J., Maeder G., 1997).............................29

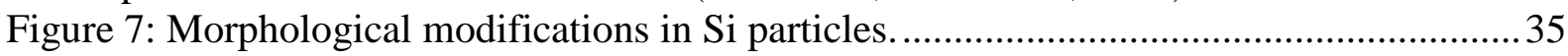

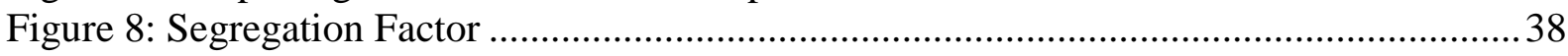

Figure 9: Influence of iron content, solidification rate, and SDAS on fracture toughness, ductility, and particle size in $\beta$ phase in an aluminum alloy (Al-7Si-0,4Mg) (Campbell, 1991).

Figure 10: (a) 3D schematic representation of a solidification void and its 2D projection; (b) and (c) optical micrograph of solidification voids (Anson \& Gruzleski, 1999)...............41

Figure 11: Schematic representation of void development by micro-contractions in the boundaries of grains and the influence of grain size (Hetke \& Gundlach, 1994)........... 42

Figure 12: Influence of solidification rate and SDAS on mechanical strength and ductility of an aluminum alloy casting (Al-7Si-0.4Mg) (Campbell, 1991)....................................... 43

Figure 13: Correlation between SDAS, grain size, and local solidification time for the Al-

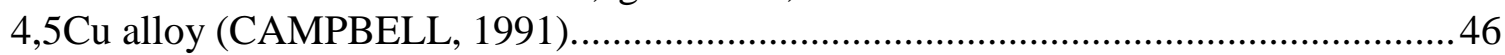

Figure 14: Influence of temperature on fatigue limit for different alloys (Fuchs, 2001)........ 49

Figure 15: (a) Balanced, slow-fast, and fast-slow fatigue loading; (b) map of mechanisms of life under fatigue in relation to temperature (for fixed values of non-linear variation of strain and frequency) for fast-slow loading in fatigue-creep (Reidel, 1987)...................54

Figure 16: Illustration of Fujita's mechanism for fatigue crack nucleation (Suresh, 1998).....57

Figure 17: Variation of plastic deformation in relation to life of AISI A289 alloys in air and vacuum with frequency and temperature variation (Coffin, 1973)...............................57

Figure 18: Plastic strain amplitude in relation to number of reverses, bi-logarithmic scale (Dieter, 1981).

Figure 19: Actual stress in relation to number of reverses, in bi-logarithmic scale (Dieter, 1981).

Figure 20: Total strain amplitude in relation to number of reverses to failure, obtained from the superposition of elastic and plastic deformation in relation to number of reverses to failure (Fuchs, 2001).

Figure 21: Ideal strain-stress hysteresis loop resulting from a low-cycle fatigue test under

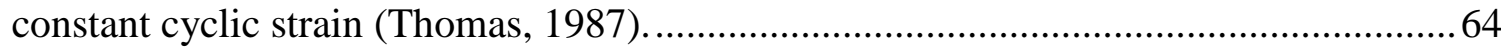

Figure 22: Isothermal and thermo-mechanical fatigue curves (Shi, 1998). ...........................65

Figure 23: Effect of temperature on fatigue life (Srinivasan, 2003) ....................................66

Figure 24: Cyclic stress response in relation to temperature (Srinivasan, 2003). ...................67

Figure 25: Comparison between thermal fatigue tests and thermo-mechanical tests (Engler-

Pinto Jr., 1996). Todos os parâmetros mecânicos medidos durante o ensaio..................... 70

Figure 26: Thermal and mechanical variation and total strain in relation to time for out-ofphase (a) and in-phase (b) thermo-mechanical fatigue.

Figure 27: Example of an out-of-phase anisothermal hysteresis loop (Engler-Pinto Jr., 1996). 
Figure 28: Stress-strain relationship for various loading histories and cycling types: (a) continuous strain cycling; (b) level of strain (strain hold); (c) continuous cyclic mean stress; (d) level of stress (stress hold); (e) stress-strain hold limit (CP); (e) thermomechanical status (mixed mechanical-thermal) (Fuchs, 1980). 75

Figure 29: Hysteresis curve under elastic thermo-mechanical strain conditions for phase

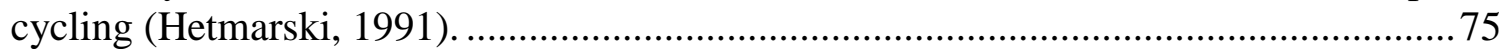

Figure 30: Hysteresis curve under plastic thermo-mechanical strain conditions for phase

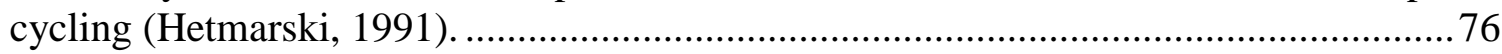

Figure 31: Hysteresis curve under creep thermo-mechanical strain conditions for phase

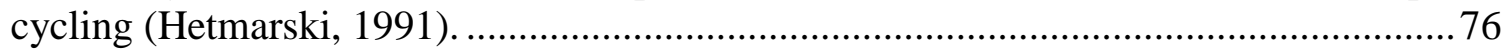

Figure 32: S-N curves for three aluminum alloys (A, B, and C), with $-0.131,-0.168$, and 0.158 slopes (Buffière et al., 2001).

Figure 33: Schematic representation of inelastic deformation variation partitioned into four components, E, P, and C representing elastic, plastic, and creep deformations, respectively (Suresh, 1998).

Figure 34: Schematic representation of fatigue-life relationships for the partitioning of

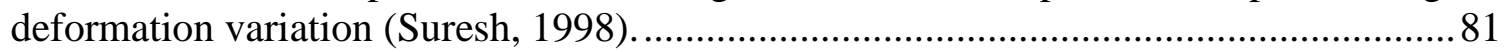

Figure 35: Schematic representation of difference between two inelastic equations (Engler \&

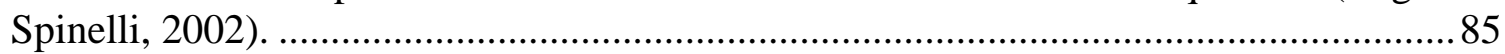

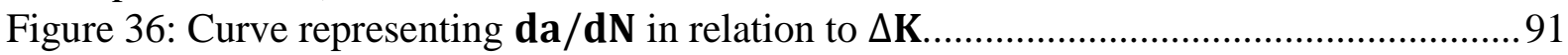

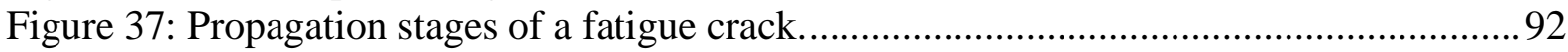

Figure 38: (a) Fatigue grooves observed on rupture surface and (b) mechanism of propagation

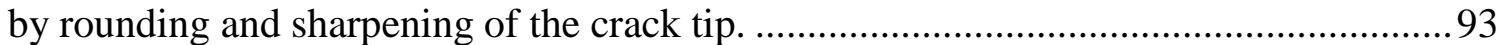

Figure 39: Relationship between groove paces and propagation rate. ................................... 94

Figure 40: (a) Classification of pores observed by tomography in a specimen after 233,000 cycles; for every pore, the effective stress level was measured in relation to the stress intensity factor, estimated according to pore dimensions $\mathrm{X}$ and $\mathrm{Y}$; (b) definition of $\mathrm{X}$ and $\mathrm{Y}$ on a pore $3 \mathrm{D}$ diagram.

Figure 41: (a) Classification of pore observed by tomography on the surface of a specimen after 233,000 cycles; for each pore, the measurement between the crack lateral length (Lc) on the surface (including pore) and the maximum lateral pore dimension below the surface (Lp) is indicated in relation to $\mathbf{L p}$; the different symbols indicate whether crack has not spread (x) or has spread ( $\boldsymbol{\Delta})$; (b) definition of Lc and Lp: in black, intersection of pore with specimen surface; in gray, 2D projection of 3D shape of pore below the

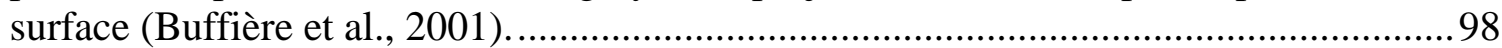

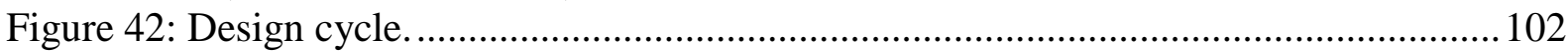

Figure 43: The exhaust fan (a) physical problem and (b) the continuum............................. 104

Figure 44: The continuum discretized by countless elements.............................................. 104

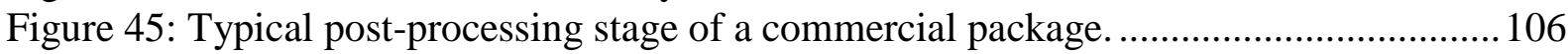

Figure 46: Coordinate system around the crack............................................................. 109

Figure 47. Evolution of plastic rounding in relation to applied stress intensity factor...........113

Figure 48: Algorithm of propagation with incremental law........................................... 116

Figure 49: Evolution of contact point in relation to the plastic rounding law at (a) negative contact level, and (b) positive contact level. ............................................................. 119

Figure 50: Permanent mold cast produced according to ASTM B108-08 (2008).................. 122

Figure 51: (a) 1.0 engine head supplied by General Motors Brazil and (b) cutting process

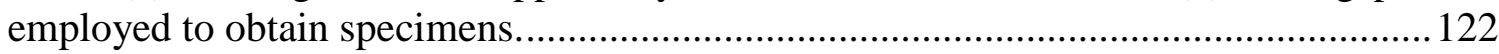

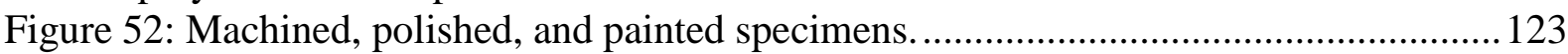

Figure 53: Testing system showing induction furnace and MTS 810 servo-hydraulic

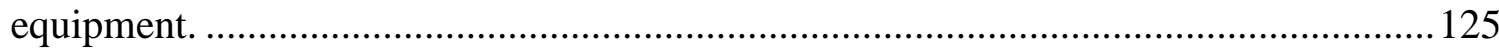

Figure 54: System of grips, infrared pyrometer, and extensometer for fatigue testing.......... 126

Figure 55: Details of specimen, induction coil, and auxiliary grip cooling system. ............. 126 


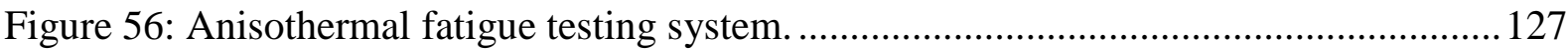

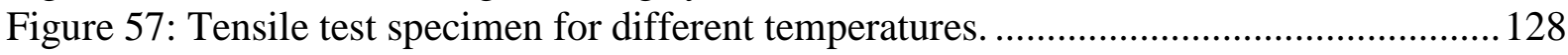

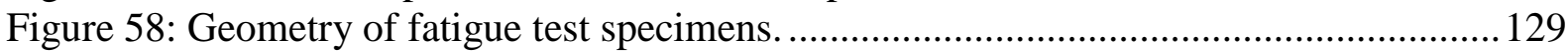

Figure 59: Anisothermal hystereses obtained between $600^{\circ} \mathrm{C}$ and $900^{\circ} \mathrm{C}$ for IN738LC alloy.

Figure 60: Cycle formats usually employed in thermo-mechanical fatigue tests. ................. 130

Figure 61: Scanning electron microscopy (SEM) image of fractured surface of a fatigue crack

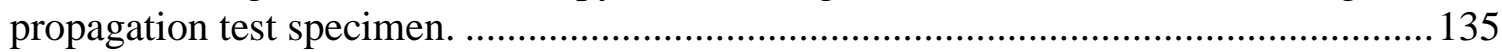

Figure 62: Analysis of chemical composition via EDX in specimen tested at $120^{\circ} \mathrm{C}$ for areas of (a) $25 \mathrm{~mm}^{2}$ and (b and c) $900 \mu \mathrm{m}^{2}$ in the regions of crack propagation and brittle

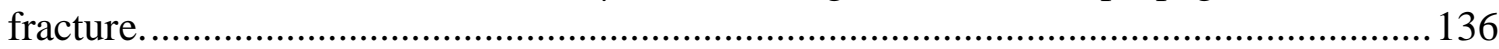

Figure 63: Analysis of chemical composition via EDX in specimen tested at $120^{\circ} \mathrm{C}$ for areas of (a) $25 \mathrm{~mm}^{2}$ and (b and c) $900 \mu^{2}$ in the regions of crack propagation and brittle fracture. 137

Figure 64: Analysis of chemical composition via EDX in specimen tested at $280^{\circ} \mathrm{C}$ for areas of (a) $25 \mathrm{~mm}^{2}$ and (b and c) $900 \mu \mathrm{m}^{2}$ in the regions of crack propagation and brittle fracture 138

Figure 65: Optical micrographs of the alloy showing (a) well refined dendrite microstructure and (b) coalesced silica particles that constitute the inter-dendrite eutectic (dark grey indicated by full arrow) and $\mathrm{Fe}_{2} \mathrm{Si}_{2} \mathrm{Al}_{9}$-type precipitates (dotted arrow)....

Figure 66: Metallographic analysis of specimens obtained from engine head transversally (a), (c), and (e) with corresponding images of casting voids (b), (d), and (f); and perpendicularly $(\mathrm{g}),(\mathrm{i})$, and $(\mathrm{k})$ with corresponding images of casting voids $(\mathrm{h}),(\mathrm{j})$, and

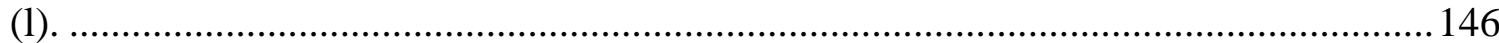

Figure 67: Detail of casting voids found in specimens obtained from the engine head,

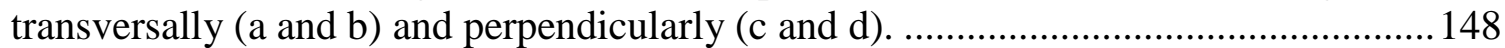

Figure 68: Fractographic analysis of the specimens submitted to (a) fatigue crack growth test at $120^{\circ} \mathrm{C}$ taken in the region of stable crack propagation and (b) ampliation of the same region, (c) thermomechanical fatigue test and (d) isothermal fatigue test at $280^{\circ} \mathrm{C} \ldots . . .150$

Figure 69: (a) Results of tensile tests conducted at $25^{\circ} \mathrm{C}, 120^{\circ} \mathrm{C}$, and $280^{\circ} \mathrm{C}$, (b) details of estimation of Young's modulus $(E)$, (c) yield stress at $0.2 \%(\sigma y 0.2 \%)$, and (d) rupture stress $(\sigma r)$. 153

Figure 70: Elasticity modulus vs. temperature for permanent mold cast A356 alloy............ 153

Figure 71: Curve of stress amplitude as a function of the number of reversals to fail for

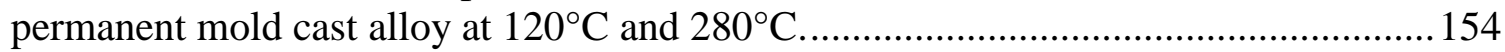

Figure 72: Curve of strain amplitude as a function of the number of reversals to fail for permanent mold cast alloy at $120^{\circ} \mathrm{C}$ and $280^{\circ} \mathrm{C}$

Figure 73: Hysteresis curve for isothermal fatigue tests at $120^{\circ} \mathrm{C}$ and total mechanical deformation of (a) $0.3 \%$ and (b) $0.5 \%$. 156

Figure 74: Hysteresis curve for isothermal fatigue tests at $280^{\circ} \mathrm{C}$ and $0.3 \%$ total mechanical deformation.

Figure 75: Curves of (a) mechanical deformation amplitude and (b) stress as a function of the number of reversals to failure.

Figure 76: Comparison between graphs of (a) mechanical deformation amplitude in relation to the number of reversals to fail, (b) stress amplitude as a function of the number of reversals to fail, and (c) hysteresis related to isothermal and anisothermal tests for $0.3 \%$ total mechanical deformation.

Figure 77: Chart of signal of perfectly adjusted command and response. .......................... 162

Figure 78: Relaxation test hystereses for total deformations of $0.1 \%, 0.3 \%, 0.5 \%, 0.7 \%$, and $0.9 \%$ at (a) $80^{\circ} \mathrm{C}$, (b) $100^{\circ} \mathrm{C}$, (c) $120^{\circ} \mathrm{C}$, (d) $140^{\circ} \mathrm{C}$, (e) $180^{\circ} \mathrm{C}$, and (f) $280^{\circ} \mathrm{C} \ldots \ldots \ldots \ldots . . .165$

Figure 79: Stress vs. time curves for a constant deformation level of $0.5 \%$ at $100^{\circ} \mathrm{C}, 180^{\circ} \mathrm{C}$, and $280^{\circ} \mathrm{C}$. 
Figure 80: Fracture surface of a specimen tested for fatigue crack propagation and (a) criteria employed to define the points $a 0$ and $a f$, and (b) and (c) calibration curves. 168

Figure 81: Graph of crack propagation $(\mathrm{dadN})$ as a function of stress intensity factor $(\Delta \mathrm{K})$ at $75^{\circ} \mathrm{C}$ under sinusoidal wave loading.

Figure 82: Graph of crack propagation ( dadN) as a function of stress intensity factor variation $(\Delta \mathbf{K})$ at $120^{\circ} \mathrm{C}$ and under sinusoidal and trapezoidal wave loadings. 170

Figure 83: Graph of crack propagation ( $\mathrm{dadN}$ ) as a function of stress intensity factor variation $(\Delta \mathrm{K})$ at $200^{\circ} \mathrm{C}$ and under sinusoidal and trapezoidal wave loadings. . 171

Figure 84: Graph of crack propagation (dadN) as a function of stress intensity factor variation $(\Delta \mathrm{K})$ at $280^{\circ} \mathrm{C}$ and under sinusoidal and trapezoidal wave loadings............................ 172

Figure 85: Comparative graph of displacement and strength in relation to time for a cycle, corresponding to crack growth tests under trapezoidal wave loading and (a) constant load and (b) constant displacement.

173

Figure 86: Graph of crack length as a function of the number of cycles for crack propagation tests under trapezoidal wave loading and (a) load control and (b) displacement control.

Figure 87: Comparative graph of crack propagation (dadN) as a function of stress intensity factor variation $(\Delta \mathrm{K})$ at $75^{\circ} \mathrm{C}, 120^{\circ} \mathrm{C}, 200^{\circ} \mathrm{C}$, and $280^{\circ} \mathrm{C}$ and under sinusoidal wave and dwell loadings.

Figure 88: Displacement vs. time graph for a crack propagation test at $120^{\circ} \mathrm{C}$ under dwell loading.

Figure 89: Shows the example of tow edge dislocations pinned at two obstacles. Dislocation 1 has to absorb vacancies to climb and dislocation 2 needs to emit them. Pile-up of dislocation at obstacles and vacancy diffusion. Dislocation 1 is a vacancy sink, dislocation 2 a vacancy source. (Roesler 2007) ....................................................... 179

Figure 90: Deformation mechanism maps. The grain size is $32 \mu \mathrm{m}$ in both cases. (Roesler 2007).

Figure 91: SEM analysis from a specimen tested by fatigue at $120^{\circ} \mathrm{C}$, dwell cycle in load control: (a) notch and stable crack growth region, (b) detail of the stable crack growth region at $120^{\circ} \mathrm{C}$ and (c) final fracture; (d) is SEM analysis from a specimen tested by fatigue at $120^{\circ} \mathrm{C}$, sinusoidal wave shape in load control $(10 \mathrm{~Hz}$ frequency) and (e) detail of (d).

Figure 92: SEM analysis from a specimen fatigue tested at $200^{\circ} \mathrm{C}$, sinusoidal wave shape, 10 $\mathrm{Hz}$ frequency): (a) region of stable crack growth, (b) detail of (a), (c) fatigue striations and (d) cast defect on fracture surface. 184

Figure 93: SEM analysis from a specimen fatigue tested at $280^{\circ} \mathrm{C}$, dwell cycle in load control: (a) notch and stable crack growth region, (b), (c), (d) and (e) detail of the stable crack growth region at $280^{\circ} \mathrm{C}$.

Figure 94: SEM analysis from a specimen fatigue tested at $280^{\circ} \mathrm{C}$, dwell cycle in displacement control: (a) notch and stable crack growth region, (b), (c), (d) and (e) detail of the stable crack growth region at $280^{\circ} \mathrm{C}$.

Figure 95: Photograph of specimen used for fatigue crack propagation testing showing (a) the region of the notch and pre-crack at room temperature and (b) tentative tests under anisothermal loading.

Figure 96: Modeling results of strain specimen at room temperature in terms of (a) displacement, (b) deformation, and (c) Von Mises stress.

Figure 97: Von Mises stress results of modeling on useful region of strain specimen at room temperature.

Figure 98: Tensile tests at $25^{\circ} \mathrm{C}$ : (a) loading as a function of total strain and 0.2 yield point;

(b) comparison between experimental and computational curves for elastic region. .... 196

Figure 99: Tensile tests at $120^{\circ} \mathrm{C}$ : (a) loading as a function of total strain and 0.2 yield point; (b) comparison between experimental and computational curves for elastic region. ....196 
Figure 100: Tensile tests at $280^{\circ} \mathrm{C}$ : (a) loading as a function of total strain and 0.2 yield point; (b) comparison between experimental and computational curves for elastic region. .... 196

Figure 101: Tensile tests at $25^{\circ} \mathrm{C}$ : (a) loading as a function of total strain and yield point taken at end of tangent to elastic region of curve; (b) comparison between experimental and computational curves for elastic region.

Figure 102: Tensile tests at $120^{\circ} \mathrm{C}$ : (a) loading as a function of total strain and yield point taken at end of tangent to elastic region of curve; (b) comparison between experimental and computational curves for elastic region.

Figure 103: Tensile tests at $280^{\circ} \mathrm{C}$ : (a) loading as a function of total strain and yield point taken at end of tangent to elastic region of curve; (b) comparison between experimental and computational curves for elastic region. 198

Figure 104: Comparison between computational and experimental tensile test curves......... 198

Figure 105: Axisymmetric model employed to identify the behavior law parameters of the material in question: (a) and (b) boundary conditions; and (c) Finite Element mesh ....200

Figure 106: Experimental hysteresis curves for isothermal fatigue tests at $120^{\circ} \mathrm{C}$ and total mechanical deformation of $0.3 \%$ and its corresponding computational hysteresis curve from ABAQUS.

Figure 107: Experimental hysteresis curves for isothermal fatigue tests at $280^{\circ} \mathrm{C}$ and total mechanical deformation of $0.3 \%$ and its corresponding computational hysteresis curve from ABAQUS. 202

Figure 108: Experimental hysteresis curves for anisothermal fatigue tests between 120 and $280^{\circ} \mathrm{C}$ and total mechanical deformation of $0.3 \%$ and its corresponding computational hysteresis curve from ABAQUS. 202

Figure 109: Relaxation test hystereses for total deformations of $0.1 \%, 0.3 \%, 0.5 \%$ and $0.7 \%$ at $180^{\circ} \mathrm{C}$ and its corresponding computational hysteresis curve from ABAQUS .............203

Figure 110: Finite Element calculation performed to identify plastic blunting law parameters. 204

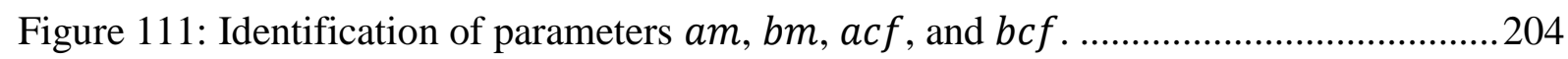

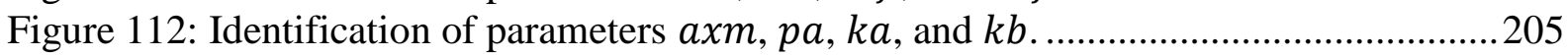

Figure 113: Graph of crack propagation (dadN) as a function of stress intensity factor variation $(\Delta \mathrm{K})$ at $120^{\circ} \mathrm{C}$ and under sinusoidal wave loadings at frequency of $10 \mathrm{~Hz} . . .206$ 


\section{LIST OF TABLES}

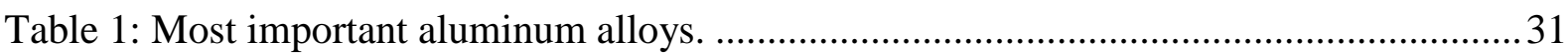

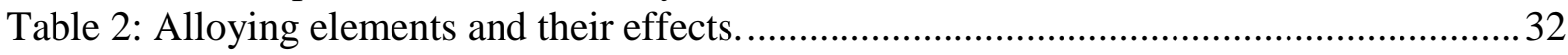

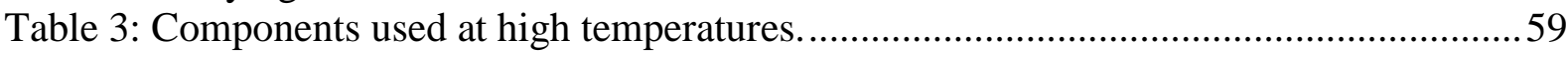

Table 4: Numerical parameter for Paris equation............................................................... 91

Table 5: Nominal chemical composition of the aluminum-silica alloy under study..............121

Table 6: Results of chemical analysis through spectrometry (\% in weight). .........................134

Table 7: Results of chemical analysis via EDX (\% in weight). .......................................... 138

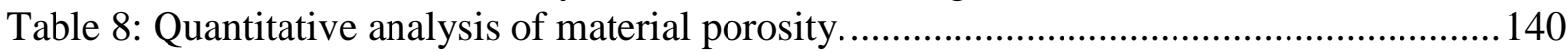

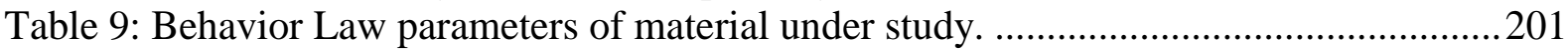

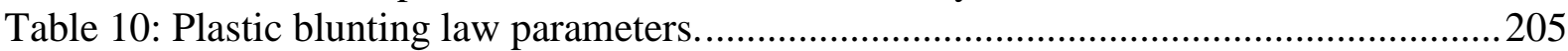




\section{LIST OF SYMBOLS}

$\zeta_{c}=$ fractions of creep damage.

$\zeta_{\mathrm{f}}=$ fractions of fatigue damage.

$\frac{\Delta \varepsilon_{e}}{2}=$ elastic deformation amplitude

$\frac{\Delta \varepsilon_{p}}{2}=$ plastic deformation amplitude

$\frac{\Delta \sigma}{2}=$ stress amplitude

$\frac{\Delta \varepsilon}{2}=$ total deformation amplitude

$2 N_{f}=$ number of reverse to failure

$\mathrm{d}_{\mathrm{g}}=$ grain size

$\mathrm{d}_{\mathrm{p}}=$ average particle size

$\mathrm{f}_{\mathrm{b}}=$ fraction by volume of cavity-nucleating particles at the grain boundary

$\mathfrak{J}_{2}=2$ nd stress tensor invariant

$\mathrm{K}_{\mathrm{p}}=$ elastic modulus tensor for plastic elements

$\mathrm{K}_{\mathrm{V}}=$ elastic modulus tensor for elastic elements

$\mathrm{T}_{\mathrm{S}}=$ solidification time

$\varepsilon_{\mathrm{e}}=$ elastic deformation

$\varepsilon_{\mathrm{el}}=$ elastic deformation tensor

$\varepsilon_{\mathrm{f}}^{\prime}=$ fatigue ductility coefficient

$\varepsilon_{\mathrm{fl}}=$ creep strain

$\varepsilon_{\text {mec }}=$ mechanical strain

$\varepsilon_{\mathrm{p}}=$ plastic deformation

$\varepsilon_{\text {term }}=$ thermal strain

$\varepsilon_{\mathrm{vp}}=$ visco-plastic deformation tensor

$\dot{\varepsilon}_{\text {cr }}=$ critical strain rate value 
$\sigma_{\mathrm{f}}^{\prime}=$ fatigue strength coefficient

$\sigma_{\mathrm{y}}=$ material yield stress

$\Delta \mathrm{w}=$ energy dissipated per cycle

$\epsilon=$ compliance tensor

$2 N=$ number of reverse

$\mathrm{c}=$ fatigue ductility exponent

$c=$ specific heat

DAS $=$ dendrite arm spacing

$\mathrm{E}=$ Young's modulus

$\mathrm{H}=$ strength modulus

$\mathrm{k}=$ Boltzmann's constant

$k=$ thermal conductivity

$\mathrm{m}=$ viscosity exponent

$\mathrm{S}=$ stress deviation tensor

$\mathrm{SF}=$ Segregation Factor

$\mathrm{T}=$ absolute temperature $(\mathrm{K})$

$\mathrm{X}=$ stress tensor (hardening)

$\gamma=$ plastic multiplier

$\varepsilon=$ total strain

$\eta=$ viscosity

$v=$ Poisson modulus

$\rho=$ density

$\sigma=$ stress

$\Omega=$ atomic volume

$b=$ fatigue strength exponent

$\mathrm{n}_{\mathrm{i}}=$ cycles under $\Delta \sigma_{\mathrm{i}}$ stress variation and the number of cycles to fail under this stress is $\mathrm{N}_{\mathrm{i}}$.

$\sum n_{i} / N_{i}=$ fraction of fatigue damage 
$t_{j}=$ average time for an applied average stress $\sigma_{j}$ and $t_{R j}$ is the time to fracture $\sum \mathrm{t}_{\mathrm{j}} / \mathrm{t}_{\mathrm{Rj}}=$ amount of accumulated creep damage

$\mathrm{d}=$ fraction of damage accumulated

$v_{c}=$ frequency into the Coffin-Manson relationship

$\Delta \varepsilon_{\mathrm{pp}}=$ plastic deformation in reverse strain-stress

$\Delta \varepsilon_{\mathrm{cp}}=$ creep deformation in tensile and plastic deformation in compression

$\Delta \varepsilon_{\mathrm{pc}}=$ plastic deformation in tension and creep in compression

$\Delta \varepsilon_{\mathrm{cc}}=$ creep deformation in reverse tension-compression

$D^{f}=$ fatigue accumulated damage

$D^{o x}=$ environmental attack (oxidation) accumulated damage

$D^{c}=$ creep accumulated damage

$D^{t}=$ Total accumulated damage

$\mathrm{E}_{\mathrm{pp}}=$ approximate slope of the hardening curve on a inelastic stress-strain graph

$\nabla T=$ Thermal gradient

$\mathrm{da} / \mathrm{dN}=$ crack growth rate

$\Delta \mathrm{K}=$ stress intensity factor

$\frac{\mathrm{d} \rho}{\mathrm{dt}}=$ plastic flow rate

$K_{\text {Max }}=$ maximum stress intensity factor

$K_{o p}=$ opening stress intensity factor

$\Delta K_{e f}=$ variation of effective stress intensity factor 


\section{INTRODUCTION}

Many machines have several types of components subjected to variations in temperature or load during operation. The induced thermal gradient inside these components can promote internal stresses and strains and repeated thermal cycles may lead to crack nucleation and propagation by a process known as thermo-mechanical fatigue (TMF).

This process can be clearly seen in internal combustion engines. When this type of engine is started, the temperature of the head and valves increases rapidly at the same time that the nearby material, which is water cooled, restrains the thermal expansion of the material. As a result, there is an increase in thermal stress and significant plastic deformation may occur at high temperatures if it exceeds the elastic limit under compression of the material.

Moreover, upon cooling to room temperature, tensile stresses are created and may promote cracks and be conducive to component failure (Figure 1). Repeated engine startupshutdown cycles can cause these components to undergo plastic deformation, leading to stress-strain hysteresis loops and, consequently, TMF. Therefore, the study of the material mechanical behavior and fracture mechanisms is vital to a proper engineering design of these components.

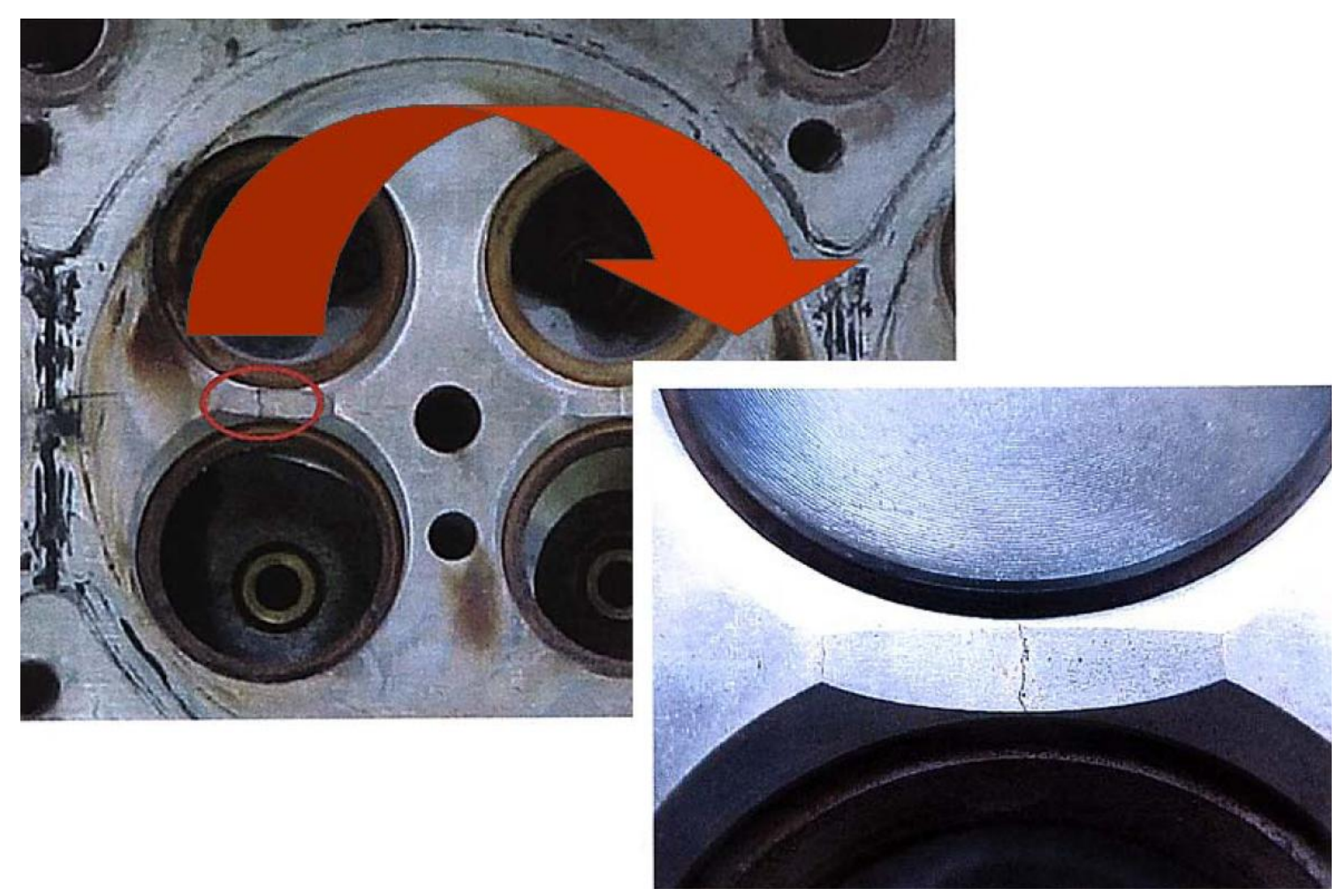

Figure 1: Picture of a fatigue crack that caused the cylinder head to fail during operation. 
Materials employed in the making of automotive engine cylinder heads are often submitted to critical thermo-mechanical efforts. The development of new materials for the automotive industry has been hitherto based almost exclusively on laboratorial specimen testing. The financial cost and time expenditure involved in the process, from selection of materials to approval for serial production, are high, demanding a wide array of mechanical tests. From project planning to production, there is a series of stages before the component is approved.

For example, in the case of an engine cylinder head, some of these steps comprise dynamometer tests, whose cost is around $\mathrm{R} \$ 140,000$, durability tests ( $\mathrm{R} \$ 150,000)$, piloting it in some 300 vehicles, and so forth. Therefore, minor project faults may cause huge financial losses and waste of time, which can be minimized by computational simulation. Nowadays, there are several models that enable the accurate modeling of the behavior of many materials submitted to isothermal loading. However, only recently has intensive development of behavior laws under anisothermal conditions started. In some cases, isothermal models can be extrapolated for anisothermal solicitation, but some materials display interaction effects during the thermal cycle, which can alter their mechanical behavior and lead to nonconservative predictions of residual lifetime. In this context, it is fundamental to conduct anisothermal testing.

Another problem is the casting-related microstructural heterogeneity, which can give rise to different mechanical and physical properties in the same component. Finite elements analysis have been used to model casting processes as well as components under real-life operational conditions so as to build satisfactory predictive mathematical models capable of determining fatigue lifetime of components, thereby diminishing the cost of material selection and/or improvement. This study deals with this problem by investigating its influence on the component under loading conditions closest to those observed in real life. Therefore, investigating the mechanical behavior and developing constitutive equations for these materials are vital to better determine their residual lifetime and implement projects of components more effectively.

In the industrial context of this study, i.e., production of cylinder heads of automotive engines, the choice of aluminum alloys is based on their good thermal conductivity (better engine cooling), good fluidity (implementation of part in casting), easy machining, and low weight. However, increased engine performance produces ever-higher operating 
temperatures - particularly with diesel engines — between intake valves, the warmest areas of the head.

Although there are studies on the morphology and development of cast aluminum alloys, the literature is deficient with regard to cyclic mechanical behavior of these alloys under varying temperature conditions, such as those found in transmission housings and cylinder heads of engines.

Numerical simulation is a useful tool for solving equations that predict material behavior. The finite element method (FEM) has been developed and applied very satisfactorily. Basically, micro-structural features and thermal, mechanical and geometrical aspects should be incorporated into TMF process modeling. There are two approaches to understanding the TMF process: (1) by modeling crack initiation and propagation at the microscopic level, and (2) by taking into account the material macroscopic behavior.

This study aimed to characterize the mechanical behavior of an aluminum alloy used in the production of automotive engine cylinder heads through mechanical testing laboratory conducted under conditions of loadings closer to reality of component operation and numerical simulation using a Finite Element Software. It is expected that the results presented in this study will provide support to product development areas in industry. 


\section{CHAPTER 1: MATERIAL}

\subsection{Metal Alloys in Molten State}

Casting is defined as the process of manufacturing metal parts from the liquid state. After melting, the metal is poured into molds to obtain components with complex geometries and/or close to final measures, in order to avoid waste of raw material and excessive machining costs. It is the oldest manufacturing process in recorded history, known for thousands of years. Even today it is one of the most widely used processes because it is relatively simple and comparatively inexpensive, though it may require the control of a large number of variables.

The production of most metal components involves solidification, a cooling phenomenon during which liquid metal is transformed into finished solid objects or ingots, which are then processed by other forming means. Basically, the solidification process can be divided into nucleation and growth stages.

During nucleation, liquids present a disordered structure, in which atoms and molecules are in constant motion, with no fixed positions. As temperature drops, the total energy of atoms decreases thereby increasing the force of attraction among them. At a given point, a set of atoms may arrange themselves according to metal crystallographic rules in a small area of the liquid, forming a solid nucleus. The development process of stable nuclei requires that the system temperature be below the metal solidification point for the energy balance to promote its stabilization.

If the super-cooling level is not sufficient, the nucleus can become unstable and melt again. The process may repeat until the temperature is low enough for the formed nuclei to display a minimum curvature radius, one that results in an energetically stable structure, as shown schematically in Figure 2a. This process is known as homogeneous nucleation. In pure metals, the super-cooling temperature needed for stable nucleation to occur may be in the order of hundreds of degrees Celsius, e.g., development of stable nuclei in iron only occurs at approximately $300^{\circ} \mathrm{C}$ below its solidification temperature.

Nevertheless, under real conditions, the presence of impurities and the mold walls containing the liquid advance nucleus development. In this case, nucleation is called heterogeneous. The presence of elements foreign to the liquid diminishes the amount of ordered atoms needed to develop into a solid particle with the minimum curvature radius 
required for stabilization (Figure 2b). Most cases of inoculation during casting are based on the principle of heterogeneous nucleation, in which particles are intentionally introduced into the liquid in order to act as nucleation substrates. The result is a structure with fine particle size and better mechanical properties.

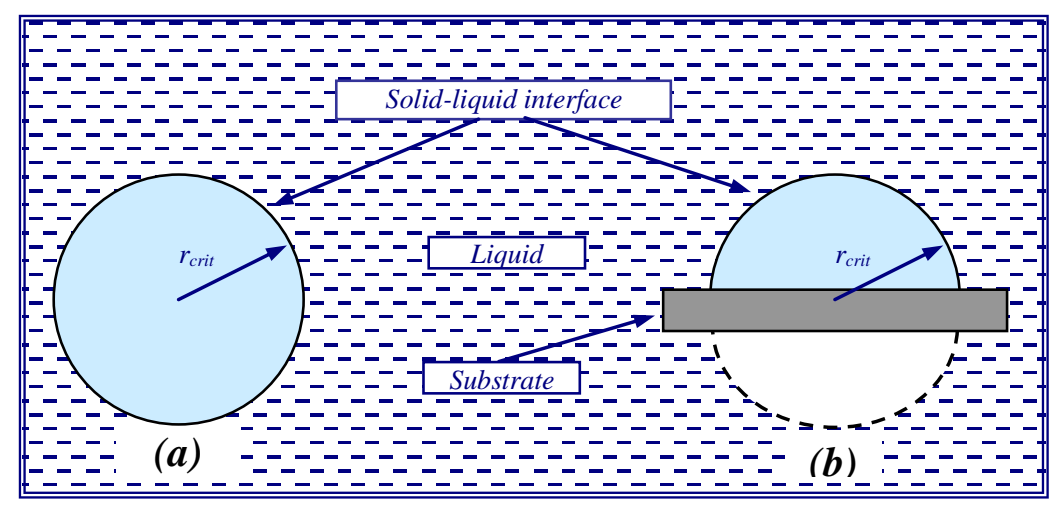

Figure 2: (a) Homogeneous nucleation and (b) heterogeneous nucleation (Milan, 2004).

After being formed, nuclei tend to grow by accumulation of more solid matter around them. This growth may be planar or dendritic.

If the liquid beyond the solid/liquid interface is at a temperature above fusion, the development of protuberances is inhibited and there occurs planar growth of the crystal solid, as shown in Figure 3a. Latent solidification heat is removed by thermal conduction through the mold walls and, consequently, through the solid formed.

Dendritic growth is favored when the liquid is in a super-cooled state, i.e., at a temperature $\left(\mathrm{T}_{1}\right)$ below its melting point $\left(\mathrm{T}_{\mathrm{f}}\right)$. In this case, the removal of latent heat needed for solidification of the liquid occurs by heat transfer, from the interface area to the supercooled liquid beyond. This mechanism favors the development of tree-shaped protuberances in the solid/liquid interface, called dendrites, as represented in Figure 3b. Development and growth of dendrites continue until super-cooling of the liquid continues or when they are blocked by the development/growth of other adjacent dendrites. Figure 4 shows the appearance of dendritic solidification on the fracture surface of an aluminum alloy with high silicon content. 


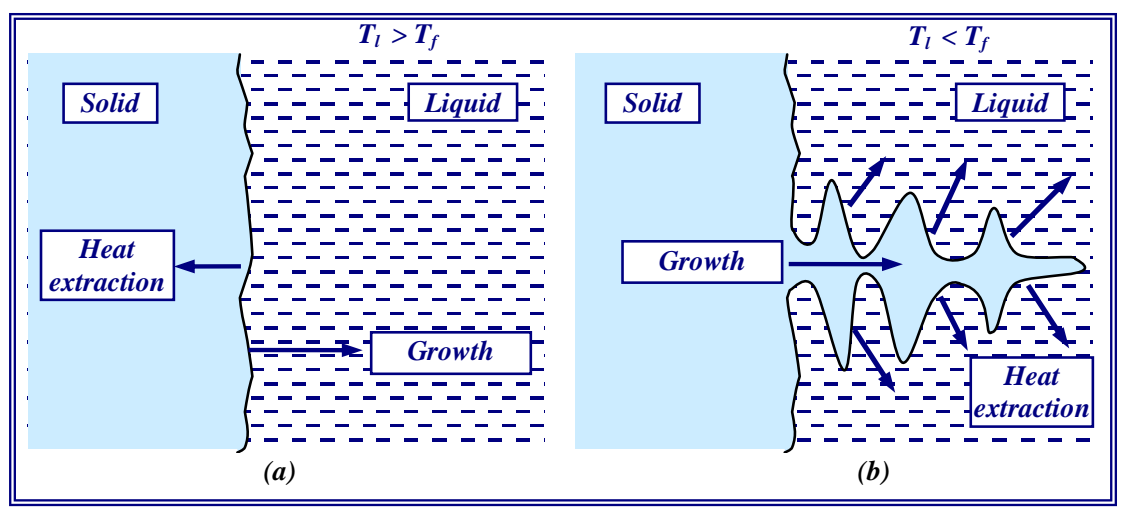

Figure 3: Schematic representation of (a) planar growth and (b) dendritic growth (Milan, 2004).

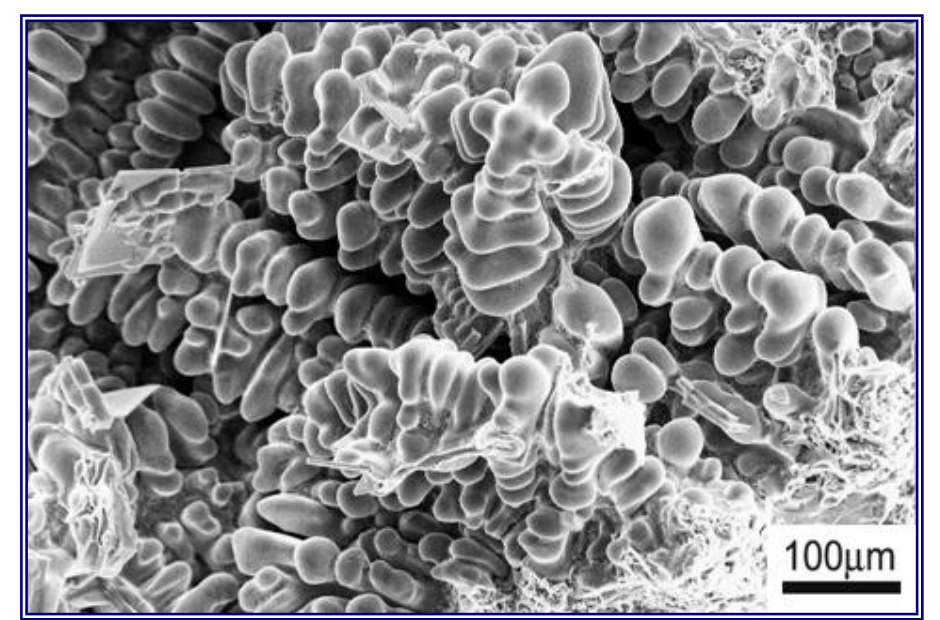

Figure 4: Solidification dendrites of an aluminum alloy with high silicon content (Milan, 2004).

\subsection{Cast Aluminum Alloys}

Pure aluminum is seldom used because its physic-chemical properties are poor. Nonetheless, its alloys are employed widely in automotive and aeronautical industry.

Aluminum alloys - due to their good mechanical properties and low specific weighthave been increasingly employed in the production of molded parts, especially in place of iron alloys. The specific weight of aluminum is approximately one third that of iron. Another important aspect favoring the use of aluminum is related to its recycling, which allows an overall assessment of costs compatible to iron components.

The structure of aluminum alloys comprises solid solutions in the form of more or less complex (binary and tertiary) dendrites (hypo-eutectic) and inter-metallic liquid components. 
In the Al-Si system, solid crystals of aluminum are formed on the metal mold walls and grow inwardly. Its $\mathrm{Si}$ composition is lower than that of the liquid that surrounds it, because $\mathrm{Si}$ solubility in $\mathrm{Al}$ decreases with temperature. Therefore, silicon is forced out to the interface between the liquid and the surface of growing crystals, thus lowering the solidification temperature of the liquid in the region through cryoscopy (liquid state below its normal solidification temperature).

This phenomenon slows solidification of this layer, since it requires that an above normal amount of heat be removed for it to occur. But if growing crystal germs find liquid at a below solidification temperature, thereby consisting of a silicon-poor medium thermodynamically favorable to solidification, they keep on growing as dendrites.

These initially unstable protuberances grow rapidly, which explains the fact that aluminum crystals develop into tree-like shapes (dendrites) instead of spheres or needles (Figure 5). Secondary arms are dozens of micrometers apart. This distance between dendrite arms is known as dendrite arm spacing (DAS) or secondary dendrite arm spacing (SDAS).

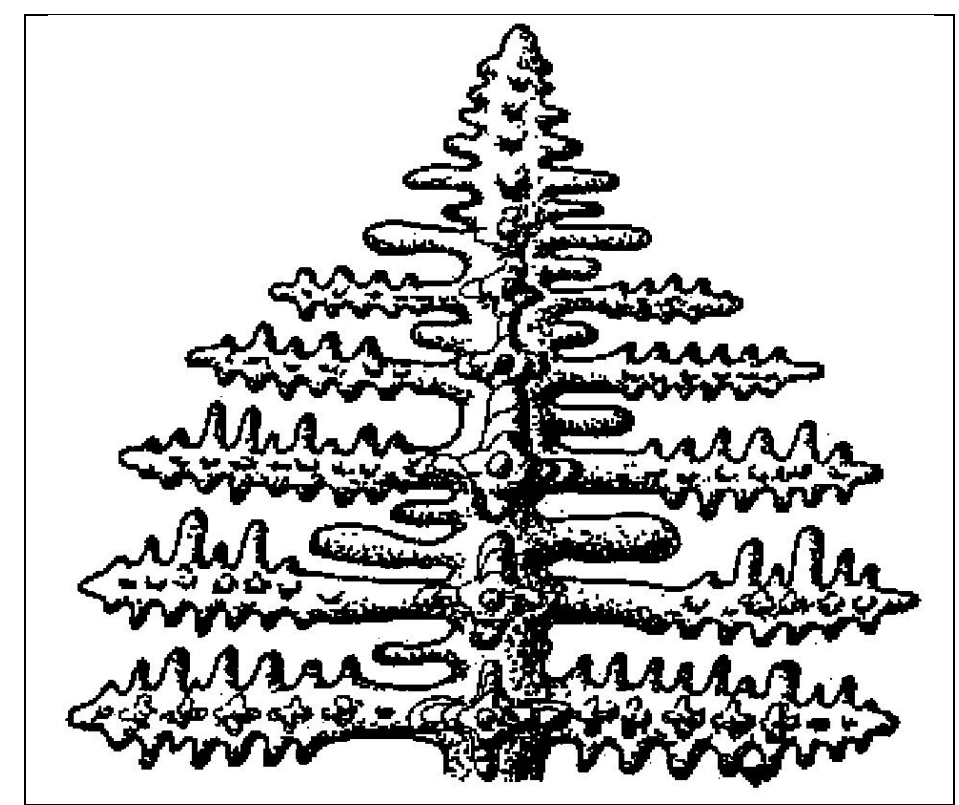

Figure 5: Schematic representation of a dendrite (Massinon \& Constantin, 2002).

Then, the eutectic solidifies amid dendrite arms, which are rich in silicon rejected from the solidification front (excess silicon in this region). This enriches the surrounding liquid metal with silicon, which, upon precipitating as platelets, drains silicon from the solution and creates an almost pure aluminum region in the vicinity. An aluminum lamella is, thus, formed. Little by little a lamellar eutectic is formed via competitive growth (Figure 6a). 


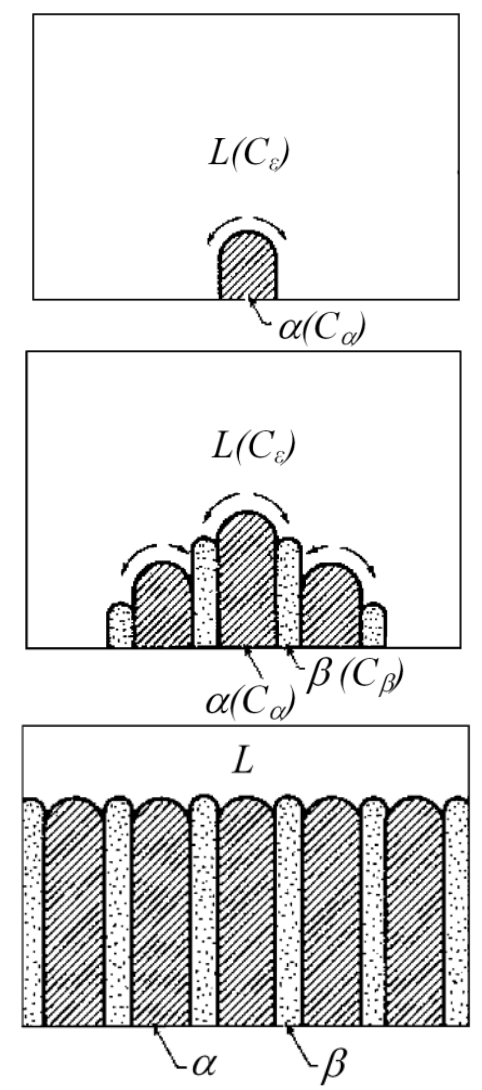

(a)

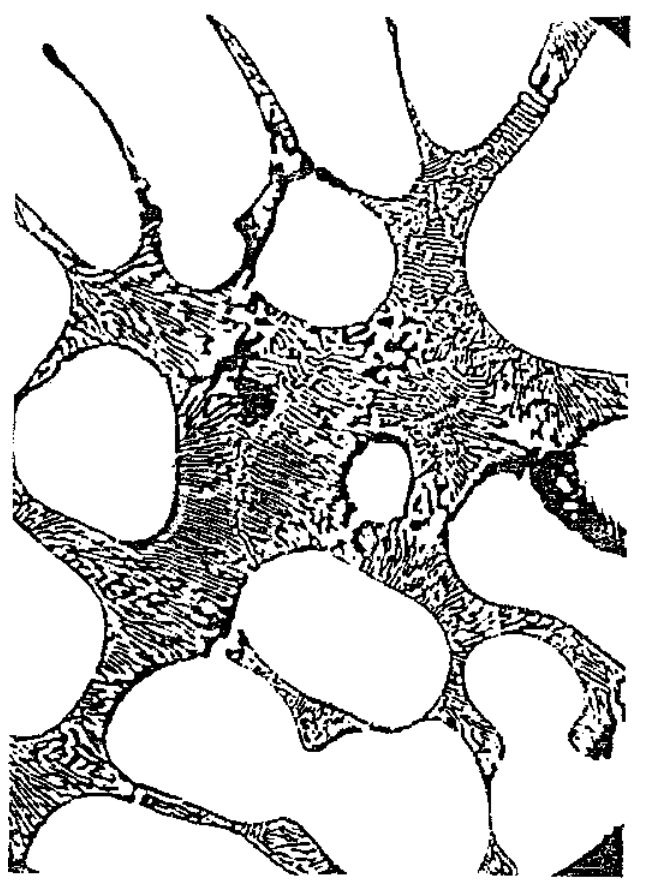

(b)

Figure 6: (a) Principle of competitive growth leading to a lamellar eutectic, and (b) schematic representation of a lamellar eutectic (Barralis J., Maeder G., 1997).

For instance, in heads of diesel engines, SDAS typically varies from $20 \mu \mathrm{m}$ inside the component to about $100 \mu \mathrm{m}$ in areas at lower temperatures. The grain size in cast aluminum alloys is generally in the order of millimeters and, therefore, well above the size of a dendrite. The mechanical properties of cast aluminum alloys strongly depend on micro-structural parameters; there are numerous studies that examine this dependence.

The acquisition of a given mechanical property also depends on the casting method employed (e.g., sand mold, permanent mold, lost-wax, and die casting). In fact, permanent mold casting promotes fast cooling, which provides the component with a thinner metallic structure (i.e., low SDAS) and higher tensile strength and elongation values as compared to those of the same alloy cast in sand, for instance. However, sand mold casting is easy to implement and prototypes can be quickly built in order to validate geometry, power, cooling, and so forth (except product life). It has been observed that low SDAS improves life under fatigue. According to empirical law, the shorter the solidification time $\left(T_{S}\right)$, the lower is SDAS (fast cooling):

$$
D A S(\mu m)=K . T_{s}^{1 / n}
$$

where $K$ and $n$ are constants. 
Solidification in the mold occurs on its walls first and progresses toward the center until the entire component is solid. This induces a gradient of microstructure and, consequently, properties. The literature also indicates the existence of a larger amount of pores and eutectic phase in the center, influenced by a lower cooling rate (Equation 1), which together with higher SDAS, leads to a shorter life under fatigue of specimens taken from this region as compared to those taken from outer regions, where cooling rates are higher.

Besides the influence of the casting method employed, it is also possible to alter the properties of alloys by adding different elements to them. Thus, cast aluminum alloys constitute several families of materials whose chemical composition depends on the relationship between casting requirements (e.g., flowability, machinability, shrinkage) and the way the component will be used (e.g., mechanical properties, surface appearance, and corrosion strength).

Aluminum alloys employed in casting are classified according to their main alloying elements. The most common of these classifications is that established by the Aluminum Association and adopted by other standards bodies (e.g., ASTM and SAE). According to this classification, the most important families are:

a) 1xx.X - pure aluminum alloys;

b) $2 \mathrm{xx} . \mathrm{x}$ - aluminum-copper alloys;

c) $3 x x . x$ - aluminum-silicon alloys (with addition of magnesium and/or copper);

d) 4xx.x - aluminum-silicon alloys;

e) 5xx.x - aluminum-magnesium alloys;

f) 7xx.x - aluminum-zinc alloys (with addition of magnesium, copper, chromium, manganese or combinations of the same);

g) $8 x x \cdot x$ - aluminum-tin alloys.

The last digit (after the dot) is employed to describe the nature of the alloy. Therefore, the digit " 0 " indicates the chemical composition limits; "1" and "2" represent cast components and ingots with pre-adjusted composition, respectively.

Variations in the original chemical composition — usually at impurity level—are indicated with a capital letter before the alloy number (e.g., 356, A356, A356). 
Alloys from 3xx.x and 4xx.x families are the most important, commercially, because of their mechanical, corrosion, and casting properties. Alloys belonging to families with silicon as their main alloying component represent over $90 \%$ of all cast products.

The other families of alloys display low castability levels, but with specific advantages such as: high mechanical properties $(2 \mathrm{xx})$, corrosion strength $(5 \mathrm{xx})$ or machinability $(5 \mathrm{xx}$, $7 \mathrm{xx}$, and 8xx).

Table 1 shows an overview of the series of most used aluminum alloys, along with their classification given by the AA (Aluminum Association), characteristics, and uses. Table 2 presents some alloying elements and their effects.

Table 1: Most important aluminum alloys.

\begin{tabular}{|c|c|c|c|}
\hline $\begin{array}{l}\text { Casting } \\
\text { Alloys }\end{array}$ & $\begin{array}{c}\text { AA } \\
\text { Classification }\end{array}$ & Characteristics & Uses \\
\hline $\mathrm{Al}>99.0 \%$ & $1 \mathrm{xx} . \mathrm{x}$ & $\begin{array}{ll}\text { - } & \text { Heat treatable } \\
\text { - } & \text { Excellent corrosion strength } \\
\text { - } & \text { Excellent weldability } \\
\text { - } & \text { Excellent formability }\end{array}$ & $\begin{array}{ll}\text { - } & \text { House ware } \\
\text { - } & \text { Accessories for Chemical } \\
& \text { Industry } \\
\text { - } & \text { Rotors for induction motors } \\
\text { - } & \text { Electrical Hardware }\end{array}$ \\
\hline $\mathrm{Al}-\mathrm{Cu}$ & $2 x x \cdot x$ & $\begin{array}{ll}\text { - } & \text { Heat treatable } \\
\text { - } & \text { Good mechanical strength } \\
\text { - } & \text { Good machinability } \\
\text { - } & \text { Fragility when hot }\end{array}$ & $\begin{array}{l}\text { Cast and/or machined } \\
\text { components subject to } \\
\text { stresses, operating in non- } \\
\text { corrosive environments }\end{array}$ \\
\hline $\mathrm{Al}-\mathrm{Si}-\mathrm{Cu} / \mathrm{Mg}$ & $3 x x \cdot x$ & $\begin{array}{ll}\text { - } & \text { Heat treatable } \\
\text { - } & \text { Easy production, including by } \\
& \text { pressure casting } \\
\text { - } & \text { Good strength } \\
\text { - } & \text { Good corrosion strength }\end{array}$ & $\begin{array}{ll} & \text { Automobiles } \\
\text { - } & \text { Ships } \\
\text { - } & \text { Frames of fans and pumps } \\
\text { - } & \text { Castings in general to be } \\
& \text { subject to loading }\end{array}$ \\
\hline $\mathrm{Al}-\mathrm{Si}$ & $4 x x \cdot x$ & $\begin{array}{ll}\text { - } & \text { Treatable by hardening } \\
\text { - } & \text { Excellent weldability } \\
\text { - } & \text { Excellent casting creep } \\
\text { - } & \text { Low machinability } \\
\text { - } & \text { Good corrosion strength }\end{array}$ & $\begin{array}{ll}\text { - } & \text { Castings with thin and } \\
\text { intricate walls }\end{array}$ \\
\hline $\mathrm{Al}-\mathrm{Mg}$ & $5 x x \cdot x$ & $\begin{array}{l}\text { Not heat treatable, except for } \\
\text { AA } 520.2(9.5 \% \mathrm{Mg}) \\
\text { - Best combination of } \\
\text { machinability, mechanical } \\
\text { properties, corrosion strength, } \\
\text { and finish }\end{array}$ & 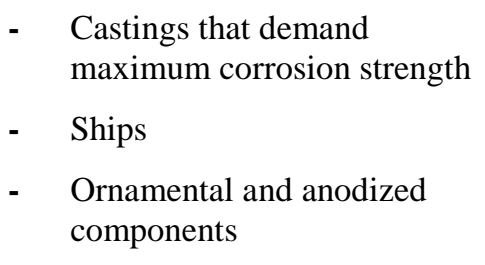 \\
\hline Al-Sn and & $8 \mathrm{xx} . \mathrm{x}$ & - $\quad$ Heat treatable & - $\quad$ Bearings and bushings for \\
\hline
\end{tabular}




\begin{tabular}{|c|l|ll|c|}
\hline $\begin{array}{c}\text { other } \\
\text { elements }\end{array}$ & $-\begin{array}{l}\text { Excellent resistance to } \\
\text { corrosion by lubricating oils }\end{array}$ & axes of trucks and laminators \\
& $-\quad$\begin{tabular}{l} 
Good resistance to fatigue \\
\hline
\end{tabular}
\end{tabular}

Table 2: Alloying elements and their effects.

\begin{tabular}{|c|c|c|c|}
\hline $\begin{array}{c}\text { Alloy } \\
\text { component }\end{array}$ & Usual Proportion & Advantage & Disadvantage \\
\hline $\mathrm{Cu}$ & 3 to $11 \%$ & $\begin{array}{ll}\text { - } & \text { Confers higher mechanical } \\
\text { strength } \\
\text { - } & \text { Improves machinability }\end{array}$ & $\begin{array}{l}\text { - } \begin{array}{l}\text { Lowers resistance to saline } \\
\text { corrosion }\end{array} \\
\text { - } \quad \text { Increases fragility in heat }\end{array}$ \\
\hline $\mathrm{Si}$ & 12 to $13 \%$ & $\begin{array}{ll}- & \text { Increases casting creep } \\
\text { - } & \text { Reduces expansion coefficient } \\
\text { - } & \text { Improves weldability }\end{array}$ & - $\quad$ Lowers machinability \\
\hline $\mathrm{Mg}$ & $>8 \%$ & $\begin{array}{ll}\text { - } & \text { Confers high weldability } \\
\text { - } & \text { Increases resistance to } \\
\text { corrosion in saline medium } \\
\text { - } \quad \text { Enables thermal treatment of } \\
\text { Al-Si alloys }\end{array}$ & $\begin{array}{l}\text { Hinders casting due to } \\
\text { oxidation (dreg) and } \\
\text { absorption of impurities (Fe } \\
\text { and other elements) }\end{array}$ \\
\hline $\mathrm{Zn}$ & 0.05 to $2.2 \%$ & $\begin{array}{l}\text { - } \quad \text { Always combined to } \mathrm{Mg} \\
\text { - } \quad \text { Confers higher mechanical } \\
\text { strength } \\
\text { - } \quad \text { Increases ductility }\end{array}$ & $\begin{array}{ll}\text { - } & \text { Decreases resistance to } \\
\text { saline corrosion }\end{array}$ \\
\hline $\mathrm{Mn}$ & 0.5 to $10.7 \%$ & $\begin{array}{ll}\text { - } & \text { Employed as corrector } \\
\text { - } & \text { Increases mechanical strength } \\
\text { in heat }\end{array}$ & - $\quad$ Low ductility \\
\hline
\end{tabular}

\subsection{Aluminum-Silicon 300 Series}

These are aluminum alloys that display the best casting characteristics, which is why about $90 \%$ of aluminum castings belong to the 300 series. Binary alloys have excellent ductility, corrosion strength, and good tensile strength. Si increases creep, reduces shrinkage, and improves weldability. However, high levels of Si hinder machining.

$\mathrm{Cu}$ addition to $\mathrm{Al}-\mathrm{Si}$ alloys improves machinability and increases mechanical strength, but reduces ductility. $\mathrm{Mg}$ addition allows alloys to be hardened by heat treatment thereby increasing their mechanical strength.

Alloys with lower Si concentrations (5 to $7 \%$ ) are usually employed in sand mold casting whereas alloys with high Si content (9 to 13\%) are commonly used in permanent mold or pressure casting. 
Hypereutectic alloys (up to $13 \%$ silicon) are known for their high wear strength, low thermal expansion coefficient, and high thermal conductivity.

Hetke and Gundlach (1994) sustain that the aluminum-silicon-magnesium alloy is among the easiest to cast irrespective of the casting technique employed. Its main features are good mechanical strength, high corrosion strength, heat treatability, and weldability, according to ASTM B108-97 (1997). This alloy is commonly used in the annealed condition and artificially aged, heat treatment T6. However, for casts with greater dimensional stability, it can be used in the solubilized and over-aged condition, T7, resulting in an increase in ductility with a slight reduction in strength as compared to T6, according to Byczynski et al. (1996).

During solubilization, magnesium is dissolved into the aluminum matrix after quenching and subsequent aging at intermediate temperatures (between $150^{\circ} \mathrm{C}$ and $200^{\circ} \mathrm{C}$ ); the $\mathrm{Mg}_{2} \mathrm{Si}$ phase precipitates in the aluminum matrix in the form of finely dispersed particles, which causes a substantial increase in strength, according to Wang and Cáceres (1997), Lee et al. (1998), and Zhang (1996).

Strength and fracture toughness of the metal matrix can be controlled by varying the temperature and aging time. In general, strength is inversely related to ductility and toughness. However, toughness can be increased through heat treatment, to the detriment of strength.

Hetke and Gundlach (1994) claim that when the metallurgical quality of castings lowers, both strength and toughness decrease. Whenever high toughness values are required, there are three ways of achieving these characteristics:

1. By performing the sub-aging treatment;

2. By softening via over-aging treatment;

3. By improving the quality of the cast.

The first two methods result in decreased mechanical strength and the last one increases toughness and strength. The quality of cast aluminum is influenced by various micro-structural factors, including the level of silicon modifiers, non-metallic inclusions, undesirable inter-metallic phases, micro-porosity, and grain refinement. These characteristics have a major impact on the cast fracture toughness and ductility. 
The main applications involve general-use components, intake manifolds, cylinder engine blocks and heads, automotive and wheel pistons, structural aerospace components, fuel and water pumps, and housing and suspension components.

\subsubsection{Silicon Modifiers}

The vast majority of cast components in aluminum alloys employ Al-Si alloys. It happens that the raw microstructure of these cast alloys features Si particles with acicular morphology, which is detrimental to ductility (Figure 7). Then, it is necessary to modify this morphology in order to obtain high strength and ductility.

The modification consists of an aluminum-silicon bath treatment by adding agents that modify Si-morphology. The most commonly used modifiers are: $\mathrm{Na}$ (sodium), $\mathrm{Sr}$ (strontium), $\mathrm{Ca}$ (calcium), and $\mathrm{Sb}$ (antimonium).

Additions of these elements (generally between $0.005 \%$ and $0.02 \%$ in weight) promote major changes in the growth of the $\beta$ phase (silicon) of the eutectic, which begins to display a more refined morphology, thus increasing the ductility of Al-Si alloy casts.

Irrespective of the modification treatment employed, high cooling rates, such as those obtained in metallic molds, contribute to refine grain size and the eutectic structure itself. They are also capable of changing the $\beta$ phase morphology in the same way that it occurs chemically, besides contributing to reducing micro-porosity. 


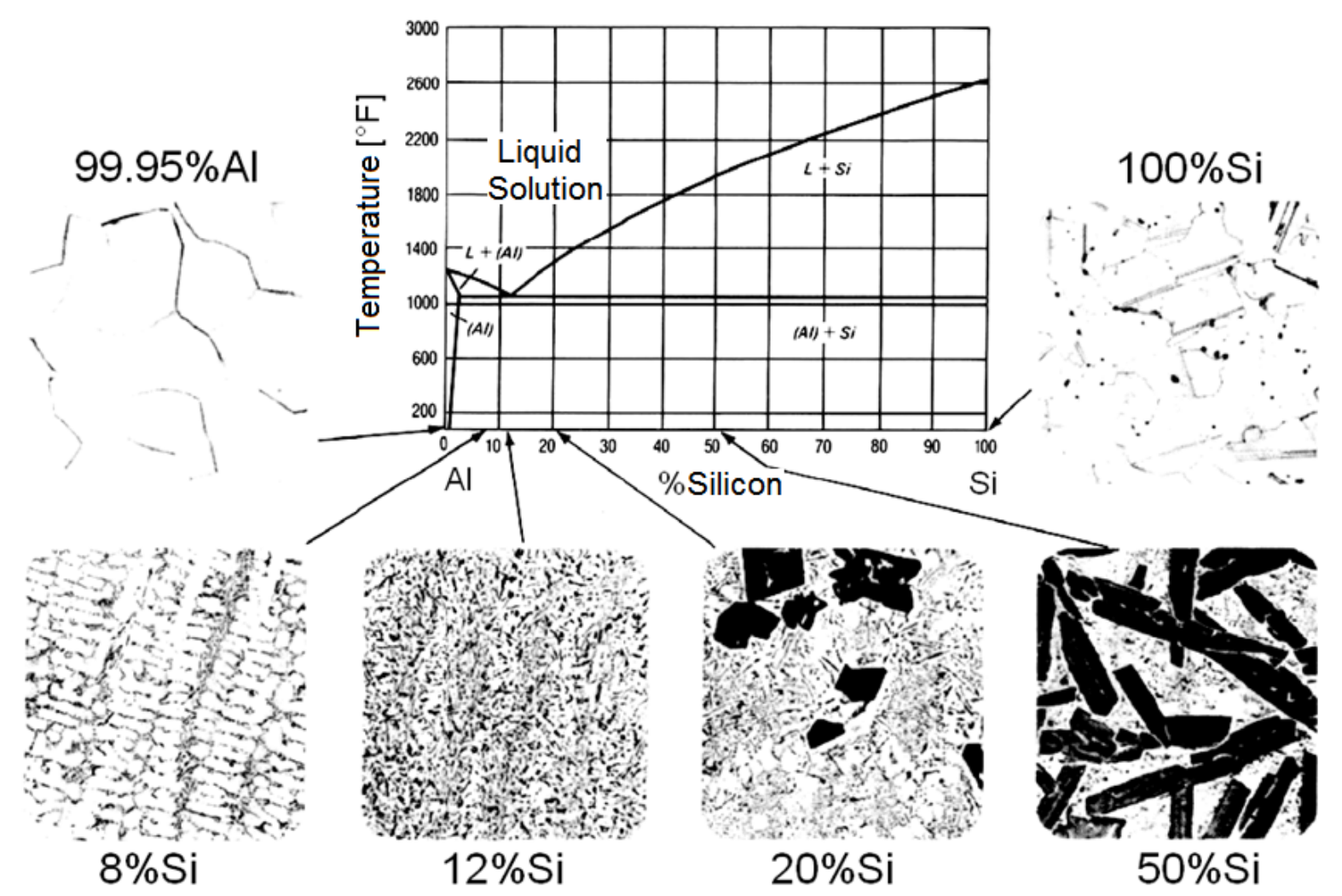

Figure 7: Morphological modifications in Si particles.

Sodium and strontium are employed commercially as effective eutectic modifiers. These elements, when added in appropriate amounts, refine and change the morphology of the $\beta$ phase of the eutectic from acicular or coarse lamellar to fibrous. This morphological change promotes substantial improvement in mechanical properties, especially ductility.

Modification with strontium is accomplished through the addition of strontium (Sr) in metal form of as Al-Sr pre-alloys. In general, additions are in the order of $0.02 \% \mathrm{Sr}$, which requires a 15-minute incubation time for bathing with little agitation.

Modification with sodium is done by adding metal sodium or fluxes containing this element. Sodium is a more efficient modifier than strontium, providing better modification degrees. However, due to a greater tendency to vaporize, its modifying effect is lost faster than that of strontium. In Na-modified baths, the modifying effect remains for about 30 minutes. In the case of $\mathrm{Sr}$, the effect lasts more than two hours. Another negative aspect of $\mathrm{Na}$ is the damage it causes to melting crucibles.

Ganiev and Vakhobov (1983) and Beumler and colleagues (1988) indicate that small additions of these elements change the morphology of the eutectic silicon phase from a coarse plate-shaped phase to a refined fibrous eutectic. The result is that fragile silicon particles become small finely-divided fibers, rather than broad and coarse plates that act as stress 
concentrators and weakness planes that promote crack propagation, according to Wang and Cáceres (1998) and Digho and Gokhale (1997).

The degree of silicon modification may vary from weak refinement, resulting in silicon platelets, to a thorough change, resulting in refined silicon fibers. According to Hetke and Gundlach (1994), the amount of Sr or Na required to promote thorough modification is strongly correlated with the solidification rate. The higher the cooling rate, the smaller is the required amount of modifiers. At low solidification rates, the required amount of modifiers is much greater. It may be very difficult to alter some larger sections of castings. However, high solidification rates promote high modification degrees and, consequently, improve mechanical properties.

$\mathrm{Na}$ and $\mathrm{Sr}$ are mutually compatible so it is possible to use both simultaneously, causing no problems due to scrap contamination.

As compared to non-modified alloys, the alloys treated with sodium and strontium show higher tendency to micro-porosity and lower propensity to macro-shrinkages in the thicker areas of the components.

Technologically speaking, this feature of modified alloys allows the production of parts with less need for hot top. At the same time, it is virtually impossible to eliminate microporosity formed in all component sections. Modified cast alloys with sodium or strontium should not be used when micro-porosity is not tolerated. In these applications, the use of unmodified alloys promotes to healthier parts. On the other hand, it requires feeding (hot top) in all thick areas where macro-shrinkages concentrate.

An alternative to modification with sodium or strontium is that with antimonium. When this treatment is employed, the $\beta$ phase morphology remains lamellar, undergoing just one refinement, which is enough to develop components with higher degree of reliability than that obtained in non-modified alloys.

Antimonium is widely used in Japan and parts of Europe. Strontium and, to a lesser extent, sodium are commonly used in the United States, Canada, parts of Europe, and Brazil. Calcium has, even today, little commercial application as a modifier.

Another kind of eutectic silicon modification takes place during solubilization treatment. Solubilization temperatures are high enough to allow silicon migration and later spheroidization. The eutectic silicon particles undergo dissolution and partial agglomeration. This type of silicon modification also promotes ductility. 


\subsubsection{Non-Metallic Inclusions}

Inclusions are foreign materials present in aluminum that can compromise its physical and chemical characteristics, such as reducing its mechanical and corrosion strength, as well as cause quality problems in the final product. There has been a constant search for understanding their origin and means to detect and control them.

Molten aluminum alloys contain highly reactive metals and oxidation of the molten metal is fast. Even in a protective atmosphere, a metal can initiate the process of oxidation. Very large oxide inclusions are not readily wet by the molten alloy and act as discontinuities, which drastically reduces mechanical strength and ductility. Furthermore, they can greatly reduce fatigue resistance, shortening the component life.

According to Crepeau (1998), various kinds of insoluble sludge can develop in molten aluminum alloys at high concentrations of certain impurities. This sludge can gather in the liquid pool and later contribute to defects arising in the cast. Over time, it attaches to the crucible bottom. Both oxides and sludge must be removed by having the liquid metal filtered before it enters the mold cavity.

\subsubsection{Inter-Metallic Phases}

In furnaces operating at low temperatures in which aluminum is not subjected to agitation, alloying elements, such as iron, chromium, manganese, and copper, precipitate and thus form a mud or sand at the bottom of the furnace or crucible. Under favorable conditions, these alloying elements, which by nature present high density, have their solubility limits diminished and thus accumulate at the bottom of the furnace, forming high-density/hardness compounds. Their development is predictable as it derives from the percentile participation of these alloying elements in aluminum, determined by a so-called Segregation Factor, which is calculated as follows:

$$
S F=\% F e+2 \cdot \% M n+3 \cdot \% C r
$$

The temperature required to dissolve inter-metallic compounds increases with iron, manganese, and chromium content, as shown in Figure 8. 


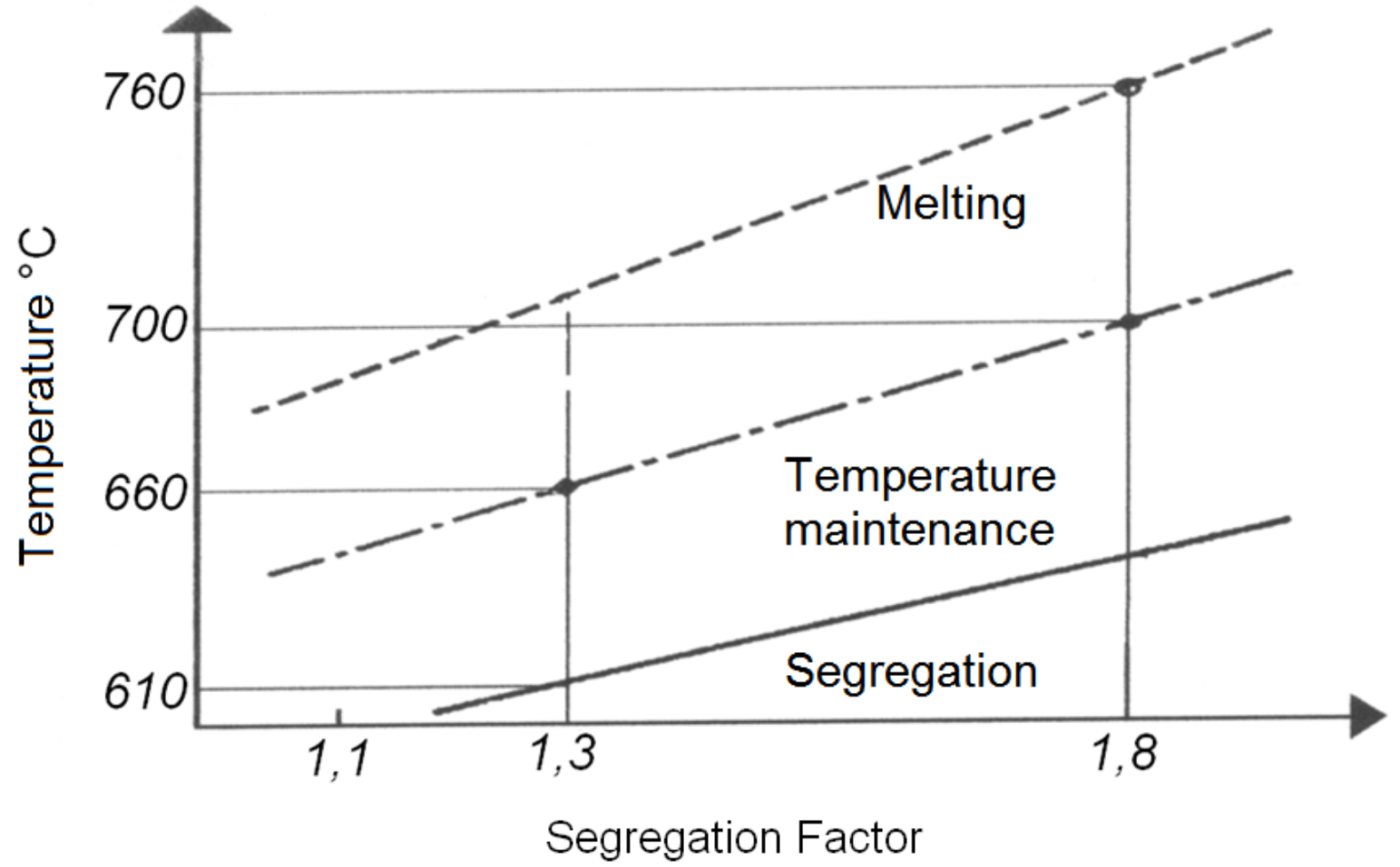

Figure 8: Segregation Factor

Without adequate control, the impurity content may rise to undesirable levels, resulting in the development of inter-metallic phases, which precipitate in inter-dendritic regions and around grains. These phases usually give rise to acicular platelets or interdendritic eutectic lattices, which can reduce matrix continuity. As a result, these hard particles act as stress raisers, propitiating crack development and decreasing fracture toughness, according to Verdu et al. (1996), and fatigue strength, according to Madelaine-Dupuich and Stolarz (1996).

Hetke and Gundlach (1994) sustain that iron and copper are major impurities in Al-Si alloys. Iron is particularly damaging because of development of acicular platelets, which significantly reduce ductility and fracture toughness. The size of acicular particles and, consequently, their influence on ductility are also affected by the solidification rate. When balanced with magnesium, iron forms a more compact eutectic phase, i.e., $\mathrm{Al}_{15}(\mathrm{Fe}, \mathrm{Mn}) 3 \mathrm{Si}_{2}$, shaped as "chinese writing", less detrimental to mechanical properties, as indicated by Crepeau (1998).

Crepeau (1998) conducted detailed studies on the effect of added iron on the mechanical properties of aluminum-silicon alloys. Vorren and colleagues (1984) observed that the effect of iron on ductility is highly dependent on the solidification rate. Kim and colleagues (1996) studied the effect of iron on mechanical properties (i.e., impact and fatigue) of aluminum-silicon alloys. Murali and colleagues (1996) investigated the addition of 
beryllium to aluminum-silicon alloys with the intention of nullifying the harmful effect of iron on mechanical properties. Figure 9 shows the influence of iron content, solidification rate, and SDAS (secondary dendrite arm spacing) on fracture toughness, ductility, and silicon particle size of the $\beta$ phase in a aluminum alloy (Al-7Si-0.4Mg).
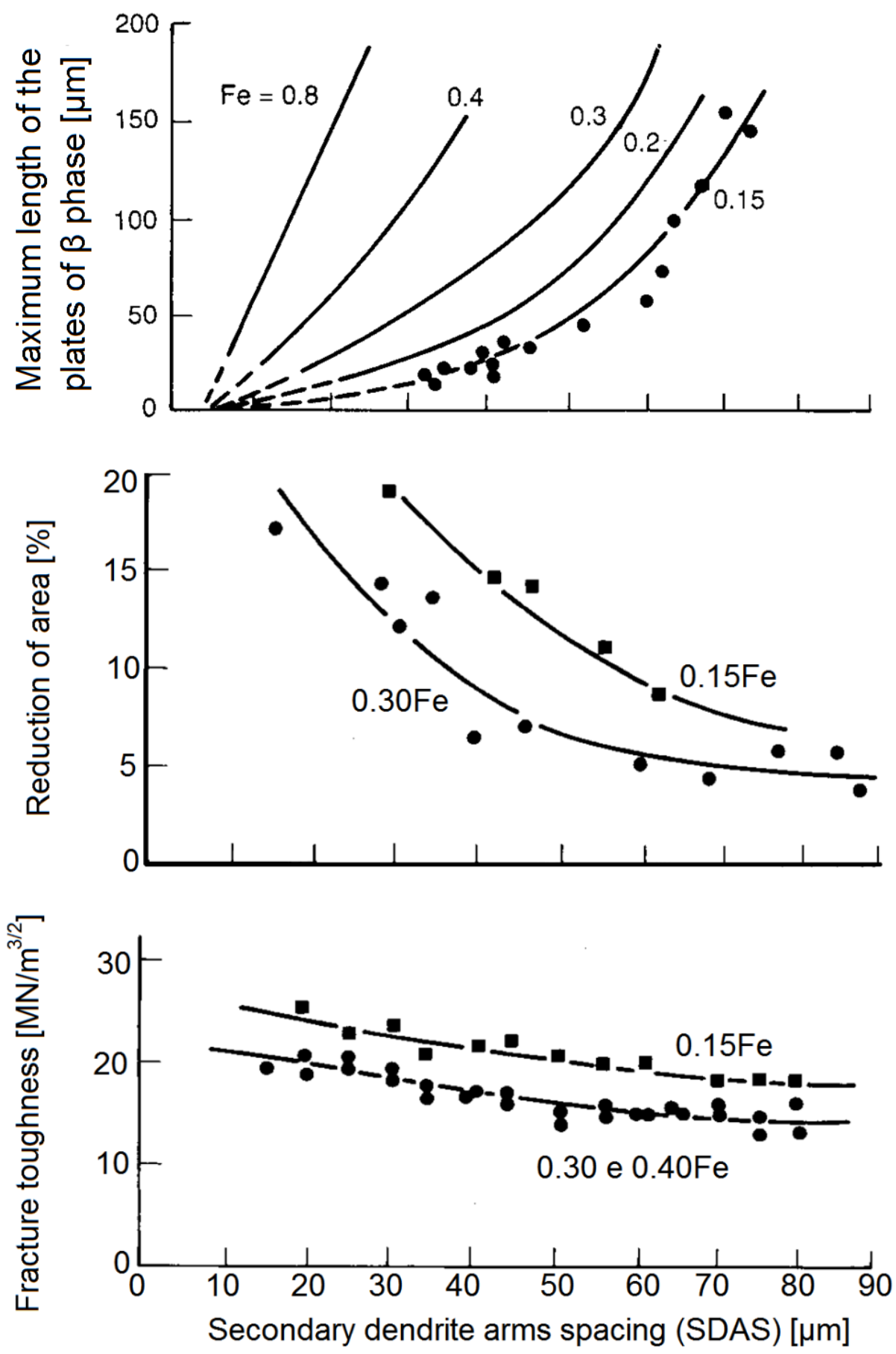

Figure 9: Influence of iron content, solidification rate, and SDAS on fracture toughness, ductility, and particle size in $\beta$ phase in an aluminum alloy (Al-7Si-0,4Mg) (Campbell, 1991). 


\subsubsection{Grain Refinement and Porosity}

Porosity in aluminum alloys is responsible for decrease in mechanical strength and occurrence of cracks and thus constitutes an important problem. According to Hetke and Gundlach (1994) and Anson and Gruzleski (1999), two varieties of micro-porosity can occur in aluminum castings: porosity resulting from gas (hydrogen) and porosity deriving from micro-contraction due to solidification.

Porosity caused by gas can be controlled through the level of hydrogen dissolved in the liquid metal. Hydrogen gas is highly soluble in liquid aluminum; the higher the temperature, the more hydrogen is dissolved in aluminum. During solidification, the amount of hydrogen dissolved in aluminum in equilibrium certainly diminishes; high cooling speed causes hydrogen to saturate in the metal, because the gas does not have enough time to leave and is thus trapped, giving rise to porosity especially in thicker areas where solidification last occurs. This porosity, usually oblong and bright, compromises the product mechanical properties and sealing, making it unfit for use.

Hydrogen contaminates liquid aluminum, especially by adding solid furnace loads contaminated with oil and residues from fusion furnaces. These residues, upon decomposition, release oxygen (which reacts with aluminum to form oxides), carbon-enrich gases or fumes, and the hydrogen is immediately incorporated into the liquid metal.

Relative humidity also contributes to aluminum contamination on the surface where oxidation and hydrogen absorption occur; liquid aluminum reacts with air moisture: $2 \mathrm{Al}+$ $3 \mathrm{H}_{2} \mathrm{O} \rightarrow 3 \mathrm{H}_{2}+\mathrm{Al}_{2} \mathrm{O}_{3}$. This gaseous hydrogen is partly dissolved into liquid aluminum in atomic form. During solidification, hydrogen becomes less soluble and recombines, is driven out of the solid phase to the liquid phase, which becomes rich in gas, creating bubbles that get trapped in the solid.

Most foundries degasify their baths with great care to minimize gas-related porosity. If control is not exercised, gas porosity can easily exceed $4 \%$ in volume, seriously degrading mechanical properties of castings. Another type of porosity caused by gas derives from turbulent feeding, resulting in elongated pores, as stated by Medeiros (1999).

Porosity caused by solidification micro-contractions occurs because aluminum contracts and silicon expands during solidification, which is conducive to a theoretical contraction of 0 to $2 \%$ (instead of $5.6 \%$ in the absence of silicon). This generates a lattice of 
cavities, which, when cut, form sets of non-spherical holes of variable sizes (Figure 10a, b e c).

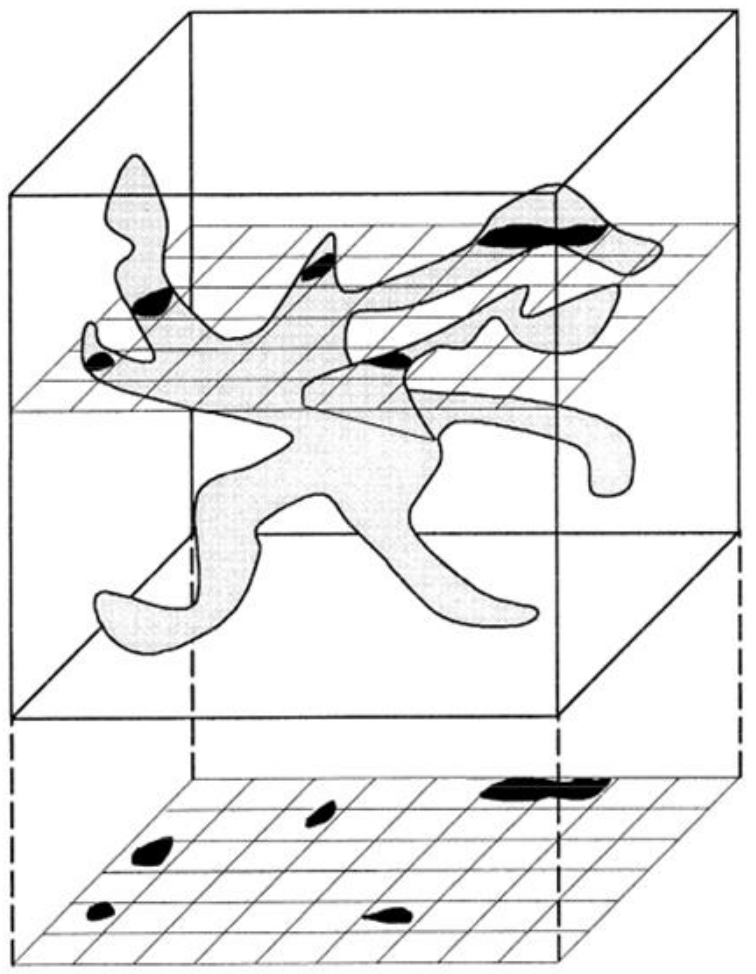

(a)

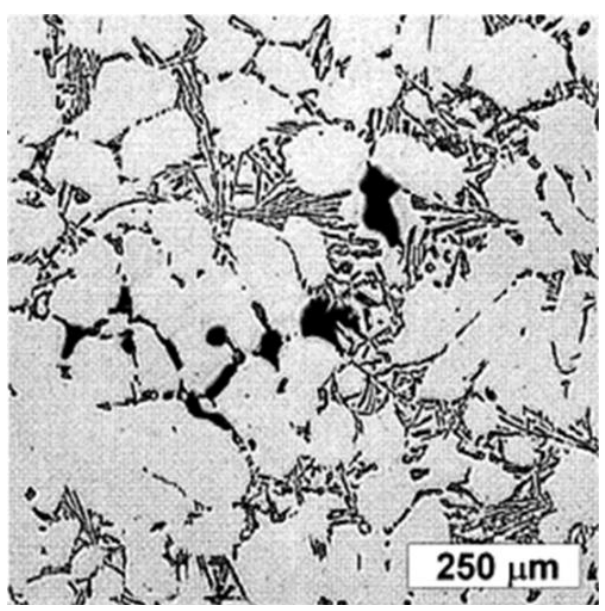

(b)

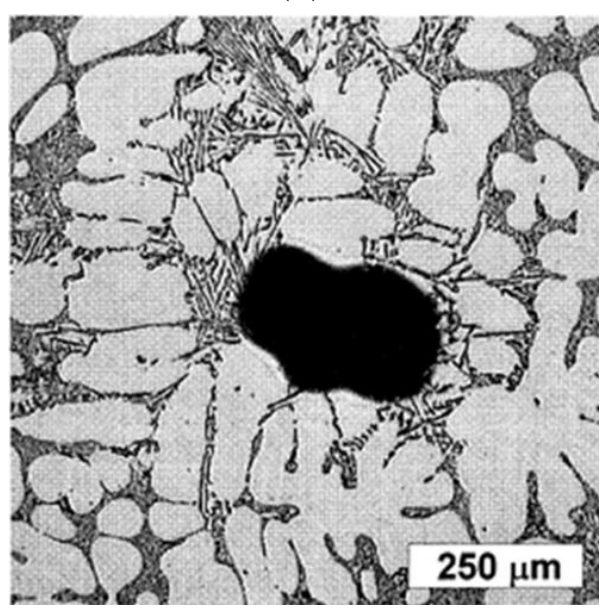

(c)

Figure 10: (a) 3D schematic representation of a solidification void and its 2D projection; (b) and (c) optical micrograph of solidification voids (Anson \& Gruzleski, 1999).

This type of porosity can be controlled through casting geometry, feeding, and design of feeding channels and hot tops. This defect is minimized when these factors are properly designed to promote directional solidification. Aluminum castings, when properly designed and processed, can exhibit low contraction porosity, $0.5 \%$ by volume or less. Hetke and Gundlach (1994) claim that pore size and porosity distribution are as important as volume fraction. Micro-contraction voids occur in the last solidification stage, i.e., at grain boundaries where adjacent grains grow and press against each other.

When two grains grow adjacent to one another, the feeding needed to make up for solid-liquid contraction is interrupted and micro-contraction voids appear. As a result, the size of contraction voids is partly determined by grain size; larger grains produce larger contraction voids whereas smaller grains produce smaller voids. Figure 11 shows a schematic 
representation of micro-contraction voids generated at grain boundaries and the influence of grain size.
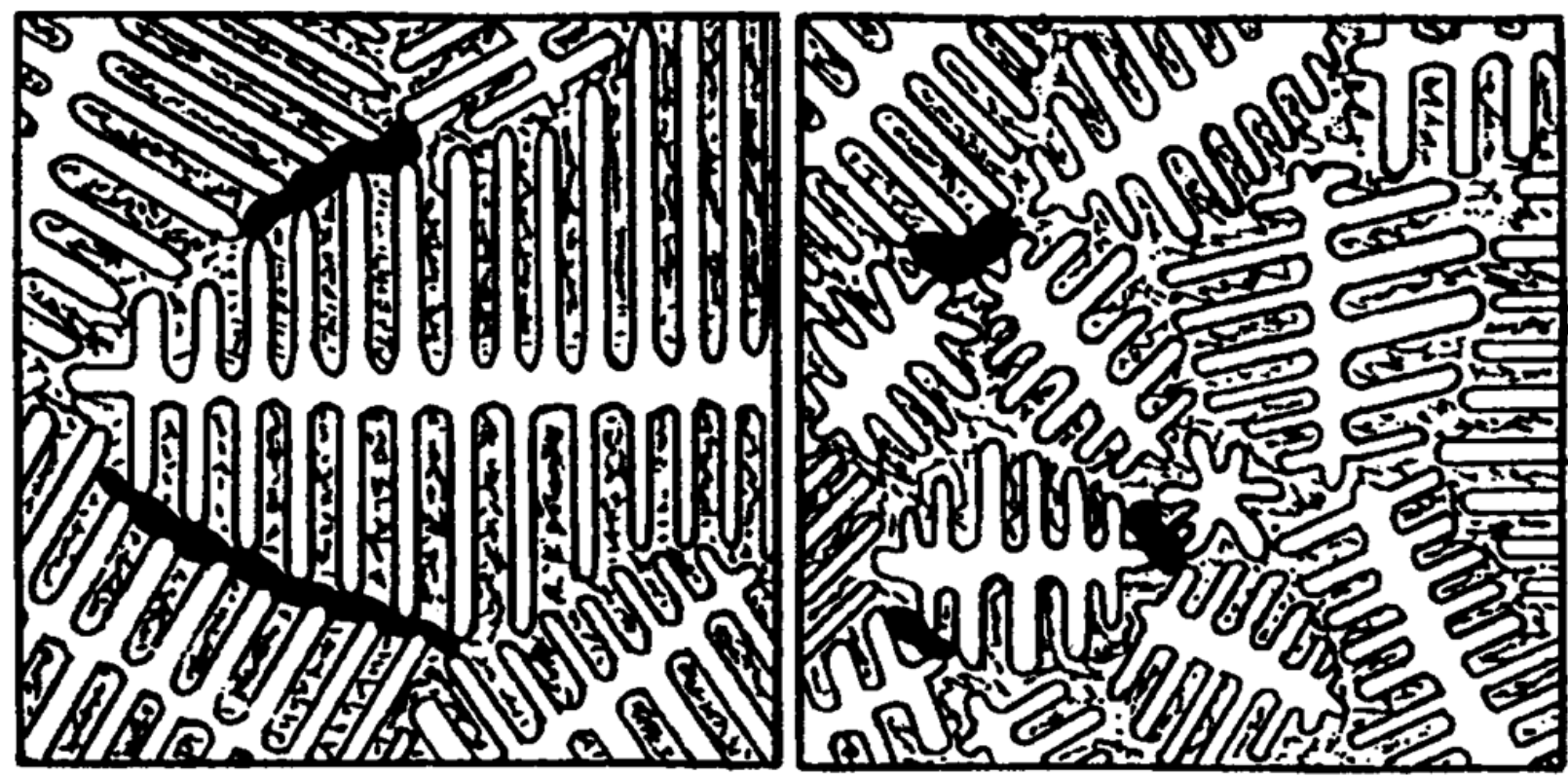

Figure 11: Schematic representation of void development by micro-contractions in the boundaries of grains and the influence of grain size (Hetke \& Gundlach, 1994).

High levels of impurity cause the production of inter-metallic phases, which precipitate at the grain boundaries, hindering the liquid metal flow and increasing the occurrence of micro-contractions. Since grain size is directly proportional to cooling rate, sections first to solidify display finer grain sizes and smaller and more dispersed microcontraction voids. The solidification rate and SDAS influence on strength and ductility of a cast aluminum alloy (Al-7Si-0.4Mg) is shown in Figure 12 (note that the solidification rate is inversely proportional to SDAS). These data show that strength and ductility increase significantly as solidification rate.

Grain size can also be reduced by adding inoculants, such as aluminum-titanium-boron alloys, which increase the number of grains forming during solidification. Grain refinement obtained through inoculation in aluminum bath is followed by contraction porosity dispersion, improving the mechanical properties of casts.

The main purpose of chemically refining the $\alpha$ phase is to reduce dendrite size, mainly by improving feeding conditions and cast mechanical properties and sealing and by reducing the tendency to develop hot cracks. In general, refiners hinder the development of columnar grains (elongated dendrites) and increase the development of equiaxed grains (less elongated dendrites). 


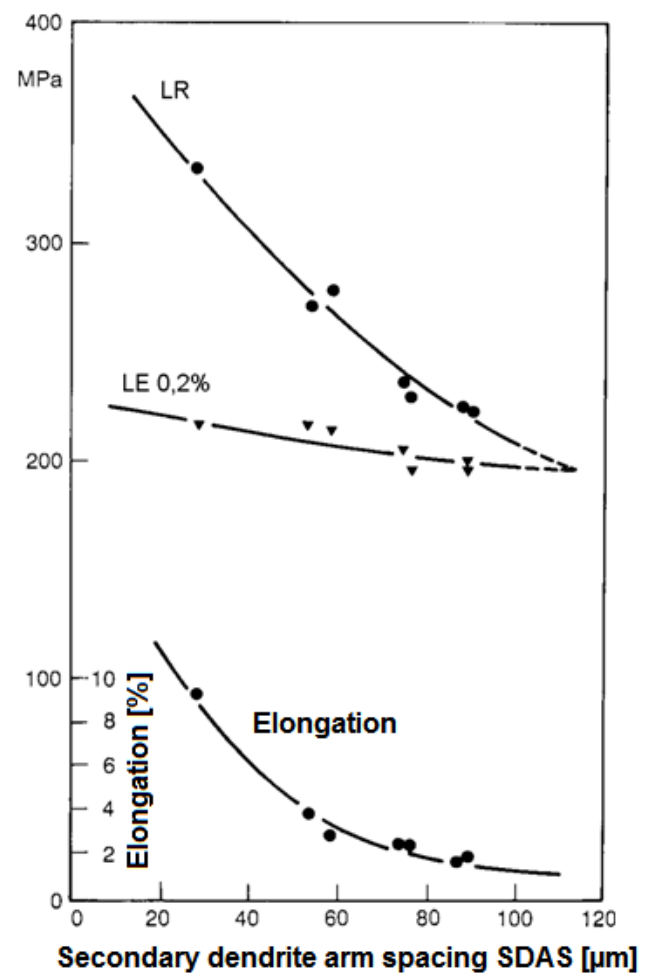

Figure 12: Influence of solidification rate and SDAS on mechanical strength and ductility of an aluminum alloy casting (Al-7Si-0.4Mg) (Campbell, 1991).

\subsubsection{Influence of Impurities}

Impurities contained in these alloys are important in that they modify, with or without intent, several of the material properties. The main impurities are:

Iron is a naturally-occurring impurity in silicon and aluminum. It weakens the component by promoting the development of hard and brittle inter-metallic compounds, which may under mechanical stress, facilitate crack nucleation by decohesion. In addition, it interferes with feeding of molten metal to parts of the mold by favoring the development of inter-metallic element platelets and diminishing sealing of supply channels. In order to display good mechanical properties, iron content must be less than $0.14 \%$ in first-melting alloys, said to display high purity; chromium and manganese have the same effect;

$>$ Zinc, together with manganese, promotes high impact and strain strength and ductility. A low content improves machinability and a high content promotes high contraction and hot brittleness;

Nickel slightly improves dimensional stability and mechanical properties at high temperatures, however at a high cost. Above 5\%, it favors high contraction; 
Lead content above $0.05 \%$ neutralizes modification by strontium and decreases mechanical properties of alloys;

Tin reduces mechanical properties and increases porosity;

$>$ Phosphor is detrimental to hypo-eutectic alloys, since it constitutes a silicon germination site; on the other hand, this element is a refiner for hyper-eutectic alloys (hyper-silica). In fact, in these alloys, the silicon platelets germinate first and, therefore, no aluminum dendrites develop;

Antimonium neutralizes phosphor, but is not compatible with strontium;

Lithium, easily oxidized, reacts significantly and quickly in alloys.

Additional elements that improve mechanical properties:

$>$ Titanium is a naturally-occurring impurity in aluminum, but it refines dendrites of the aluminum-rich solid solution; hence, it is regarded as a grain refiner; it increases tensile strength and ductility and decreases thermal conductivity;

$>$ Boron prolongs the effect of titanium in re-melting; like titanium, it acts as a grain refiner, increases tensile strength and ductility, and decreases thermal conductivity;

$>$ Sodium and strontium alter the eutectic structure from naturally lamellar to globular, which helps reduce risk of contraction, facilitates machining, and improves geometric properties;

Calcium prolongs modification by sodium.

Al-Si alloys are the most used in industry because of their melt properties, welding suitability, good dimensional stability and low melting point. And more, they present good resistance to wear, e.g., in periods of engine low lubrication.

\subsection{Defects and Fracture Toughness}

The quality of castings can be defined by the amount, type, and size of defects in their microstructure. Several papers present studies on the influence of defects on mechanical properties of aluminum alloys: Cáceres et al. (1999); Jiang et al. (1999); Dabayeh et al. 
(1998); Cáceres and Selling (1996); Dabayeh et al. (1996); Stanzl-Tschegg et al. (1995); Zieser and Sonsino (1993); and Wickberg et al. (1984). Since the matrix fracture toughness is fixed, the larger the defect, the smaller is the load required to initiate and propagate a crack.

A quality cast displays a structure with minor discontinuities and high fracture toughness. With regard to aluminum casts, as indicated by Todinov (1998), certain structural features, such as micro-contraction voids and non-metallic inclusions, may be regarded as discontinuities. The size and distribution of eutectic silicon phase, inter-metallic phases, and inclusions contribute to determining the value of fracture toughness. Therefore, higher strength and ductility values are obtained when:

1. Eutectic silicon modification is large;

2. Contraction voids are small and few;

3. Oxides are removed;

4. Impurities are minimized;

5. Microstructure exhibits minor dendrite arm spacing.

\subsection{Solidification Rate and SDAS}

High solidification rates produce important benefits that result in substantial improvement of mechanical properties of casts. Rapid solidification reduces grain size and refines and disperses porosity by contraction. It also improves eutectic silicon modification, resulting in more finely divided silicon particles. These refined silicon phases are more susceptible to further modification and agglomeration during heat treatment. Moreover, high solidification rates reduce the amount of segregation in the alloy. A decrease in solidification distances diminishes the degree of solute redistribution in dendrites and at grain boundaries. As a result, development of insoluble impurities is reduced and the size and volume of intermetallic phases are minimized, increasing toughness. The amount of Sr needed to modify eutectic silicon is also minimized.

Kuma (1996) sustains that processes with a high solidification rate result in casts with better mechanical properties and fatigue strength. Secondary dendrite arm spacing (SDAS) is the most direct measure of the solidification rate in a cast. Due to this factor, several studies have shown correlations between SDAS and mechanical properties of aluminum-silicon alloys, e.g., Doglione et al. (1996), Bamberg et al. (1986), and Oswalt and Misra (1981). 
Figure 13 shows a correlation between SDAS, grain size, and local solidification time for the Al-4,5Cu alloy. It has become usual to specify the SDAS in critical areas of aluminum casts to ensure that the mechanical properties specified in the project are achieved.

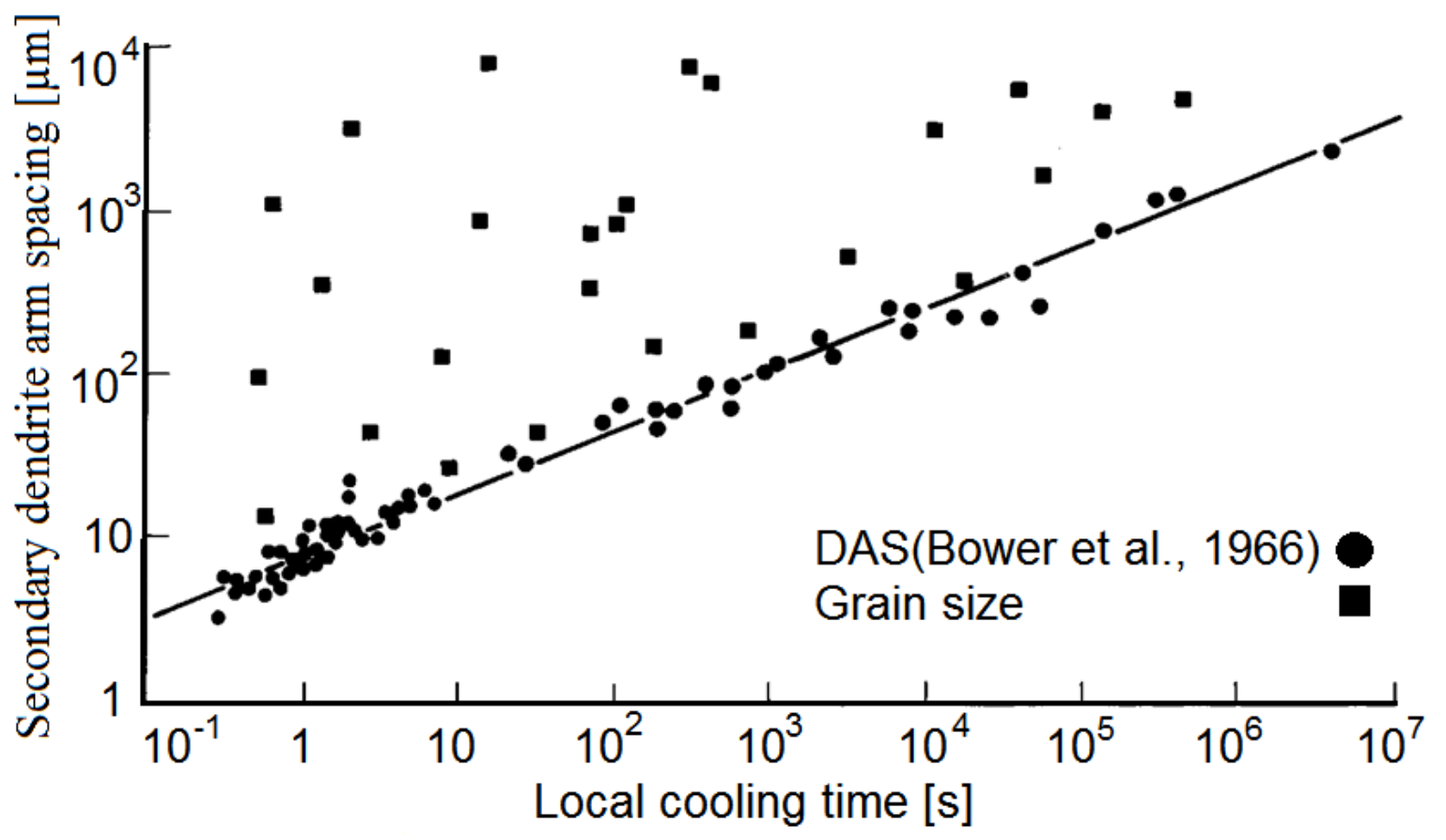

Figure 13: Correlation between SDAS, grain size, and local solidification time for the Al4,5Cu alloy (CAMPBELL, 1991). 


\subsection{Brief of Chapter 1}

- Al-Si alloys are the most used in industry because of their melt properties, welding suitability, good dimensional stability and low melting point.

- Aluminum-Silicon 300 Series are the aluminum alloys that display the best casting characteristics.

- The quality of castings can be defined by the amount, type, and size of defects in their microstructure.

- Porosity in aluminum alloys is responsible for decrease in mechanical strength and occurrence of cracks and thus constitutes an important problem. Two varieties of microporosity can occur in aluminum castings: porosity resulting from gas (hydrogen) and porosity deriving from micro-contraction due to solidification. 


\section{CHAPTER 2: TEMPERATURE EFFECTS}

\subsection{Effect of High Temperature on Materials}

Parts subjected to high temperatures display numerous problems, such as creep, oxidation (or corrosion), and fatigue. These damage mechanisms can act independently or in concert (which renders the study of damage at high temperatures more complex), according to material variables and operating conditions, such as maximum and minimum temperature, temperature range, mechanical strain range, strain rate (in-phase or out-of-phase) or environmental factors (Cai, 1999). Conform to Engler-Pinto Jr. (1995), the damage caused by these factors is in the inception of nucleation of cracks that propagate and cause fatigue failure. However, as temperature rises well above room temperature, creep becomes important and is the main cause of fracture at still higher temperatures (temperatures above half the melting point).

A high temperature for one material may not be considered high for another, then, it is usually expressed as homologous temperature, i.e., the ratio between the test temperature and melting temperature of the metal in question, both in absolute scale (Kelvin). According to Dieter (1981), as a rule, flow reaches significance in engineering at homologous temperatures higher than 0.5 .

In general, mechanical strength of metals decreases as temperature increases above room temperature (Figure 14). This is mainly due to increased mobility of atoms as temperature rises. 


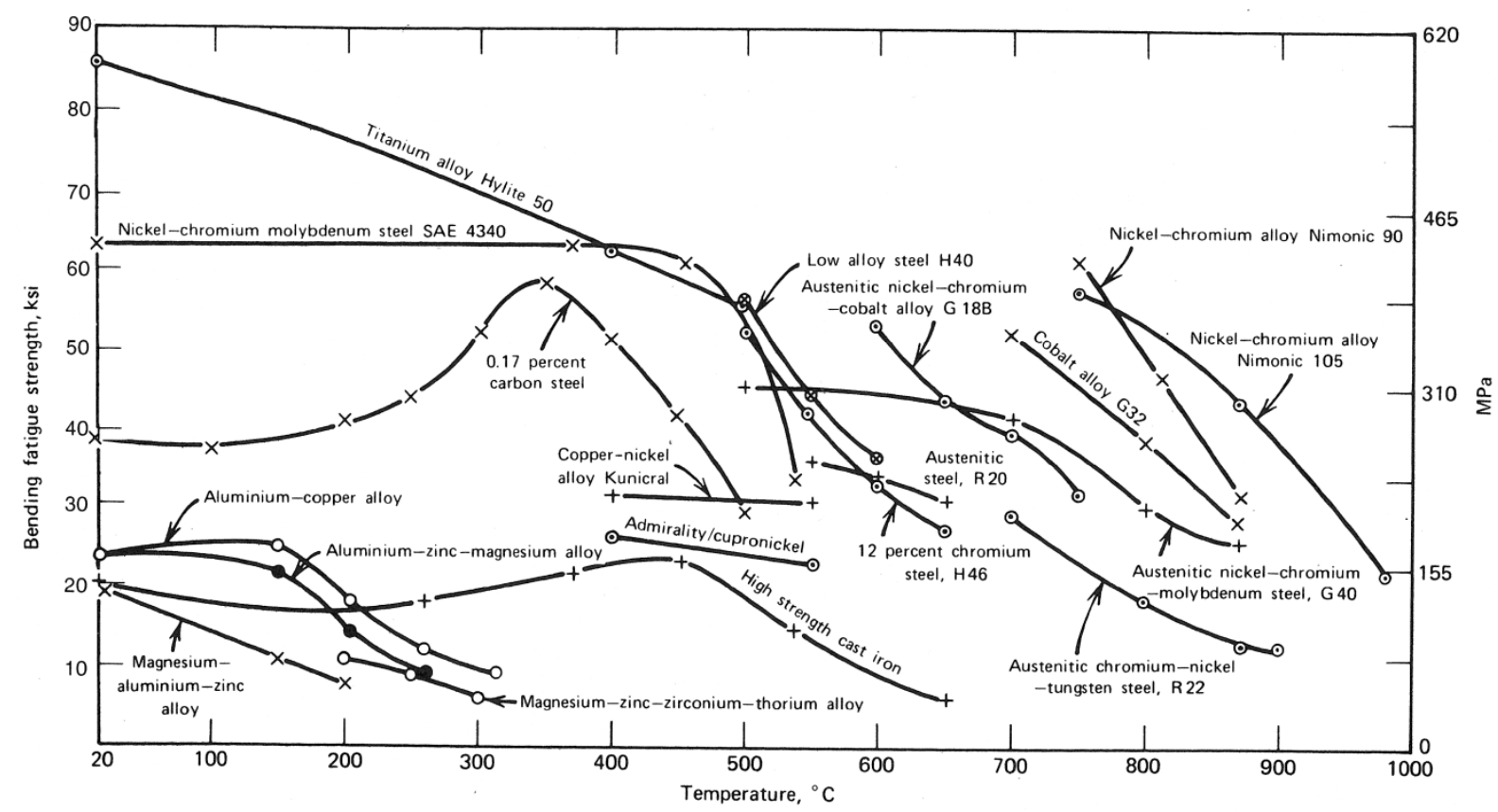

Figure 14: Influence of temperature on fatigue limit for different alloys (Fuchs, 2001).

Therefore, diffusion-controlled processes have a very significant impact on mechanical properties at high temperatures. High temperatures also result in greater mobility of dislocations due to the scaling mechanism, which becomes important because of higher dissemination and also because the concentration of gaps in equilibrium increases with increasing temperature. In addition, new strain mechanisms may become operative. In some metals, the sliding system changes or additional sliding systems are introduced.

Strain at grain boundaries becomes an additional possibility in strain of metals at high temperatures. Another important factor to consider is the effect of prolonged exposure of metals and alloys to high temperatures on their metallurgical stability. Cold-worked metals can re-crystallize and undergo grain growth whereas age-hardened alloys may undergo overaging and lose strength as second-phase particles grow. The metal-environment interaction, i.e., oxidation, is also an important factor to be taken into account (Dieter, 1981).

It is also important that mechanical properties at high temperatures should always be considered in relation to a time scale. The mechanical properties at room temperature of most engineering metals are not, for practical purposes, time-dependent. However, at high temperatures, strength becomes very dependent on both strain rate and exposure time. Under these conditions, many metals behave, in many respects, as visco-plastic materials.

Creep or crack growth can occur when materials are subjected to constant stress, which causes their strain response to change. A metal subjected to constant tractive loading and high temperatures flows and undergos an increase in length that depends on time. Under 
constant strain conditions, relaxation can occur, changing the strain exerted on the material. This time-dependence of mechanical properties at elevated temperatures differs for each material and is related to the temperature to which the material is exposed (Fuchs, 1980). Therefore, some important factors to be taken into account when materials are undergoing fatigue at high temperatures are discussed in the following section.

\subsection{Creep-Fatigue Interaction}

Components that are in a permanent state of cyclic loading at high temperatures may be subjected to creep-fatigue interaction, since they can be damaged by micro-structural creep. Therefore, resistance to low-cycle fatigue and mastery of the creep-fatigue interaction process are important criteria in the development of components that will operate under limit conditions. Therefore, it is necessary to understand cyclic strain and fracture behavior in materials at various loading rates and high temperatures (Srinivasan, 2003).

Creep is the strain occurring in solid materials over time when they are subjected to constant loading at high temperatures, which may eventually lead to fracture. Although this strain can occur at all temperatures, it is only significant when it occurs at temperatures corresponding to $30 \%$ of the melting point for pure metals and $40 \%$ for metal alloys (Dry, 2001; Es-Soun, 2001a, Langdon, 2002).

Transition from fatigue failure to creep failure with increasing temperature almost always changes fracture type from trans-granular, typical of fatigue failure, to inter-granular, characteristic of creep failure (Fuchs, 2001). Local oxidation of grain boundaries can significantly contribute to crack initiation. Dieter (1981) sustains that the amount of creep increases with average strain at all temperatures. Ferrous materials, which usually have a pronounced fatigue limit in tests at room temperature, display the same performance when tested at temperatures above about $420^{\circ} \mathrm{C}$. Fatigue tests at high temperatures also depend on how often strain is applied. It is common to refer to the total time required to fracture in the same way as to the number of cycles.

In general, the higher the material creep resistance, the greater is its fatigue strength at high temperatures. However, the metallurgical treatment that produces better fatigue properties at high temperatures does not necessarily result in better creep properties. This was shown by Toolin and Mochell in Dieter (1981) for several alloys in tests at high temperatures. At low temperatures, the smaller the metal grain size, the better is its performance under 
fatigue. As test temperature is increased, the difference between the performance of a finegrained material under fatigue and that of a coarse material decreases up to very high temperatures, at which flow dominates. Then, the material with larger grain size displays higher resistance. Typically, alloys mechanically worked beforehand have somewhat higher fatigue strength, while cast materials are often more resistant to creep. The solutions that succeed in reducing fatigue failure at room temperature may not be effective at high temperatures. Compressive residual stresses, for example, can be alleviated before the component operating temperature is reached.

Nucleation of fatigue cracks is basically a surface phenomenon. In contrast, creep damage is manifested both on the surface and inside the material. Thus, creep can influence both crack initiation and growth (Sarabanda, 1991). Wareing (1983) states that creep damage is manifested by cavity nucleation at grain boundaries due to cavity coalescence, dislocation stacking, and grain boundary sliding.

The same may happen to second-phase particles. The subsequent growth of these cavities leads to grain boundary cracking and inter-granular fracture. Under static loading, failure is established by catastrophic fracture; that is when cavities coalesce. In low-cycle fatigue with overlapping loading levels at high temperatures, fracture occurs when cavities achieve a critical geometric configuration.

Creep damage may derive from tensile residual stress caused by strain under compression followed by its reversion. In both cases, inelastic deformation takes place under zero total strain, though with differing signs of residual stress. Other loadings involving inelastic deformation, found in components subjected to thermal fatigue, may be simulated in mechanical tests (Wareing, 1983; Skelton, 1983; Hales, 1983). The dwell time at certain levels of stress or strain is another independent variable, ranging from seconds to hours.

Dwell under tensile load are generally more damaging than those under compression when applied separately. The application of retention under traction load and, shortly afterward, a dwell under compression load appears to produce less damage than applying the former in isolation. Wareing (1983) claims that this is due to a healing effect, that take place in the compressive part of loading cycle, on the cavities formed during the tensile part of the dwell cycle. This is a complex phenomenon and requires the knowledge of the mechanism of micro-structural creep damage. However, some side effects to elastic energy becoming inelastic deformation may be found even in the absence of micro-structural damage. The most important effect is the variation of the mean stress caused by the inelastic deformation, mainly for materials with high creep strength. For completely reverse cycles, a dwell in the tensile 
half-cycle leads to a compressive mean stress; in the case of a dwell in the compressive part of the cycle, the mean stress changes in the tensile direction. In the latter, life time under fatigue is reduced as a result of higher tensile stress and not quite because of inelastic deformation (ASM, 1985).

Another phenomenon occurring in materials is creep relaxation. Stress relaxation usually occurs in parts joined by screws (flanges) and hot-set assemblages. When these components are in operation at high temperatures, their joints can become loose and produce disconnection between their parts due to excessive screw stretching and/or flange rotation. Relaxation consists of reducing applied load (stress) over time at constant strain.

Strain constancy is obtained by reducing load or stress over time or by stopping the movement of the engine heads, considering load in relation to time at a fixed position of the heads. As temperature is usually high, these cases produce creep conditions. The total strain $(\varepsilon)$ is equal to the sum of elastic deformation $\left(\varepsilon_{e}\right)$ and plastic deformation or creep rate $\left(\varepsilon_{p}\right)$. For the strain $\varepsilon$ to remain constant, elastic deformation has to decrease with time and plastic deformation has to grow proportionately, and, as a result, the stress required to have a constant $\varepsilon$ should decrease with increasing creep. The increase in $\varepsilon_{e}$ is equal to or smaller than the increase in $\varepsilon_{p}$ (Souza, 1982).

\subsection{Damage and Crack Initiation at High Temperatures}

Nucleation of fatigue cracks in alloys at high temperatures is influenced by several temperature-dependent microscopic processes at both the strain level and the environment level. Mechanisms accountable for fatigue crack initiation may be usually grouped into the following categories: (a) cyclic sliding-induced cracking; (b) creep-related grain boundary cavitation; (c) grain boundary sliding and wedge crack development; (d) void nucleation and growth from inclusions and precipitates; and (e) oxidation and corrosion.

\subsubsection{Cyclic Sliding}

Cyclic sliding is affected by temperature. The following points illustrate the role played by temperature in this phenomenon. 
1. At temperatures higher than half the homologous temperature, most materials begin to exhibit alternated sliding characteristics. In alloys with solid solution, stacking failure energy generally increases with increasing temperature. Thermal activation also enables displacement movement of by cross slipping and climbing.

2. Kinematic irreversibility of cyclic sliding is increased by oxidation and/or gas diffusion at high temperatures.

3. Alternate sliding may be counteracted in some materials by dynamic strain aging that favors planar sliding.

4. Displacement movement by climbing is time-dependent, which makes strain at high temperatures sensitive to strain rate.

5. For most engineering materials, micro-structural stability decreases at higher temperatures. Thermally activated micro-structural changes may arise as a result of aging by strain, recovery, or precipitation. These micro-structural changes can be beneficial or harmful to fatigue strength; they can also modify significantly cyclic stress-strain characteristics.

\subsubsection{Creep Cavitation}

According to Suresh (1998), at temperatures between one and two thirds the melting point and at low stress, most metals, alloys, and ceramics exhibit creep by cavitation at grain boundaries. These cavities nucleate at triple points of grain boundaries, in grain boundary particles, or protrusions in intergranular facets. In many engineering alloys, creep cavities nucleate well below the $100 \mathrm{MPa}$ nominal stress, and their nucleation occurs continuously over a substantial fraction of creep life (Riedel, 1987). Shear slipping of grain boundary faces also favors cavity nucleation.

A particularly interesting aspect of creep cavitation under cyclic loading is that the degree of cavitation is strongly influenced by stress waveform. Slow creep rates under traction and fast rates under compression, i.e., waveforms with long periods of tensile stress, exhibit greater propensity for cavitation than balanced loading cycles in which the magnitude of part of the traction loading varies identically to the compression phase, as shown in Figure 15 (Majumdar, 1980; Baik, 1982). 
Reidel (1987) took into account different creep cavitation theories for slow and fast fatigue loading and presented a map of mechanisms for life under fatigue in relation to temperature for fixed values of deformation variation $(\Delta \varepsilon)$ and test frequency.

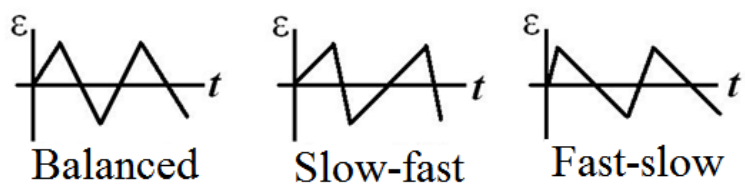

(a)

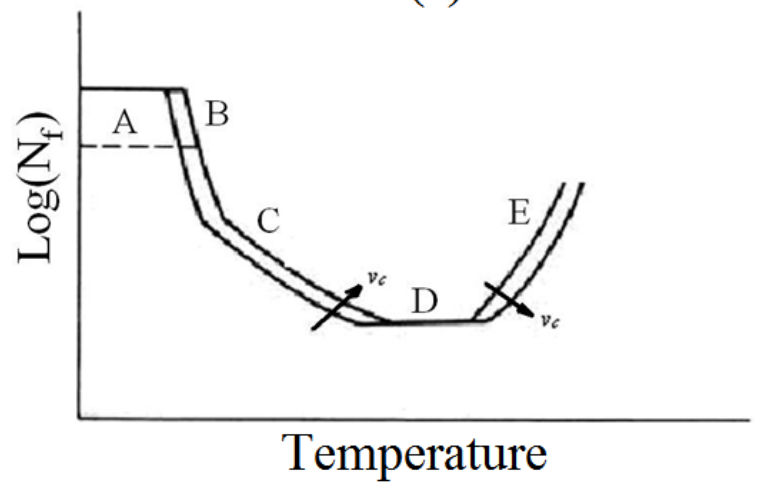

(b)

Figure 15: (a) Balanced, slow-fast, and fast-slow fatigue loading; (b) map of mechanisms of life under fatigue in relation to temperature (for fixed values of non-linear variation of strain and frequency) for fast-slow loading in fatigue-creep (Reidel, 1987).

At intermediate temperatures represented by region $\mathrm{C}$, cavity growth occurs by diffusion, in which cavities nucleate continuously throughout the creep-fatigue test. For low values of accumulated strain $\left(\mathbf{2} . \Delta \boldsymbol{\varepsilon} . \boldsymbol{N}_{\boldsymbol{f}}\right)$, the effect of frequency $\left(\boldsymbol{v}_{\boldsymbol{c}}\right)$ and temperature $(\boldsymbol{T})$ in region $C$ are described by the relationship $\left(\mathbf{N}_{\mathbf{f}} \cdot \boldsymbol{\alpha} \cdot\left(\boldsymbol{v}_{\mathbf{c}} \cdot \mathbf{T} / \boldsymbol{\delta}_{\mathbf{g}} \mathbf{D}_{\mathbf{b}}\right)^{2 / 5}\right)$, in which $\mathbf{D}_{\mathbf{b}}$ is the diffusion coefficient of the grain boundary. For larger values of $\mathbf{N}_{\mathbf{f}}$ (Regime B), the accumulated strain is so large that the density of cavities saturates early in fatigue life; in this case, $\mathbf{N}_{\mathbf{f}} \cdot \boldsymbol{\alpha} \cdot \boldsymbol{v}_{\mathbf{c}} \cdot \boldsymbol{T} / \boldsymbol{\delta}_{\mathbf{g}} \mathbf{D}_{\mathbf{b}}$. For low temperatures (Region A), the plastic growth of cavities is postulated as the dominant mechanism. This process does not depend on frequency if the development of surface cavities is irreversible.

For higher temperatures (Region D), cavities grow so fast that the applied strain rateassumed to be constant in Figure 15b-may not be capable of accommodating the cavity volume for a long time. As a result, cavity growth is hindered in the slow-fast loading mode. For even higher temperatures (Region E), the growth of restrained cavities takes place in the fast compression segment. Since diffusion rates are high at these temperatures, cavity growth is restrained under both traction and compression. This is conducive to zero cavity growth thereby improving life under fatigue. 


\subsubsection{Grain Boundary Sliding}

Many experimental studies (e.g., Driver, 1971; Min \& Raj, 1979) showed that grain boundary sliding plays an important role in nucleation of inter-granular cavities in creep fatigue. This process is also a mechanistic consideration in empirical partitioning of inelastic deformation variation when estimating creep-fatigue life. On the assumption that grain boundary sliding is the rate-limiting step in crack nucleation at triple junctions and that it does not occur above a critical strain rate value $\left(\dot{\varepsilon}_{c r}\right)$, Min and Raj (1979) developed the following model (Equation 3):

$$
\dot{\varepsilon}_{c r}=\frac{8 \cdot \sigma_{y} \cdot \Omega \cdot \delta_{g} \cdot D_{b}}{k \cdot T \cdot d_{g} \cdot f_{b} \cdot d_{p}^{2}}
$$

where $\sigma_{y}$ is the material yield stress, $\Omega$ is the atomic volume, $k$ is Boltzmann's constant, $T$ is absolute temperature $(\mathrm{K}), d_{g}$ is the grain size, $f_{b}$ is the fraction by volume of cavitynucleating particles at the grain boundary, and $d_{p}$ is the average particle size. The remaining terms are defined in the following section.

According to this theory, if the waveform is such that strain rates in stress and compression are below a critical value, no net harm is produced as long as grain boundary sliding occurs equally in the direction of advance and reverse loading. When fast and slow loading and strain rate, during compression, is greater than $\dot{\varepsilon}_{c r}$, maximum damage is produced. Although this model is a simplification of the grain sliding process, it provides understanding of how waveform affects fatigue at high temperatures in many alloys.

Grain boundary cavitation can be affected by relative temperature and stress variations with time. Experiments of Fujino and Taira (1980) on AISI 304 stainless steel showed that cyclic in-phase temperature and stress variations produce greater damage in fatigue than offphase thermo-mechanical loading. In addition, off-phase loading produces shorter fatigue life than isothermal mechanical fatigue. This variation of fatigue strength for different thermomechanical loading conditions seems to imply a correlation with amount of grain boundary sliding.

Sub-surface fatigue crack nucleation can occur at high temperatures by decohesion of inclusions and second-phase particles from the matrix. In some aluminum alloys, void nucleation around precipitates at the grain boundary causes cavitation even at low temperatures (Vasudevan, 1987). Furthermore, an increase in precipitation during the fatigue process at high temperatures produces a large number of void nucleation sites. 


\subsubsection{Oxidation and Corrosion}

The presence of an oxidizing or aggressive environment may have an influence on mechanisms of crack nucleation by fatigue at high temperatures as follows:

1. By preventing cavity closure or sintering at high temperatures;

2. Under the influence of applied stress, cavities develop around precipitates at grain boundaries (Wells, 1979). Moreover, precipitation of residual gases can provide nuclei for cavities, with internal gas pressure accelerating cavity growth. If grains near the surface are not protected by an oxide layer, oxygen or other embrittling element may spread at grain boundaries and react with existing precipitates.

3. The combination of surface diffusion and oxidation during sliding promotes kinematic increases in cyclic sliding, which results in nucleation of fatigue cracks (Figure 16). This process is known as Fujita's mechanism (Fujita, 1963).

4. Preferential oxidation at some micro-structural sites, e.g., the intersection of grain boundaries and free surfaces, gives rise to microscopic stress concentrators (notches). These micro-notches increase local stress and promote crack nucleation (Duquette, 1979).

5. Repeated loading breaks through the protecting oxide film on the material surface. The lack of this film provides an active path for chemical attack. Furthermore, cracking of the fragile oxide film per se can promote growth of catastrophic fatigue cracks (Wells, 1979).

For many materials, deterioration of fatigue strength at elevated temperatures is merely a result of interactions with the environment. The experimental basis for this inference may be found in Coffin (1973) for AISI A286 ferrous alloy (Figure 17).

Here the variation of plastic deformation $\left(\Delta \varepsilon_{p}\right)$ with the number of cycles to fail $\left(N_{f}\right)$ is not affected by temperature or frequency in vacuum. However, when testing frequency is lowered, low-cycle fatigue life is severely reduced at elevated temperatures in air. 

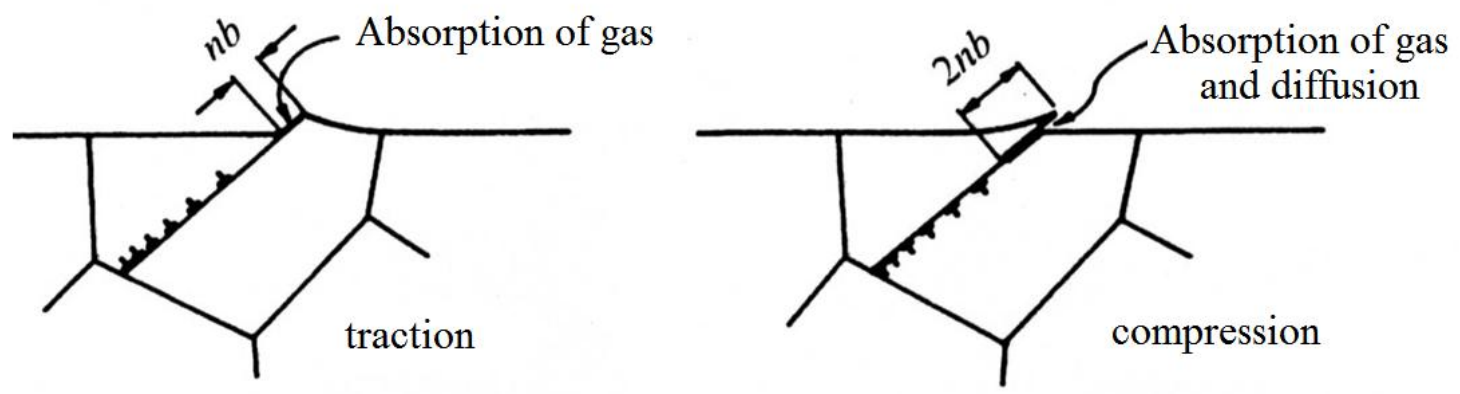

Figure 16: Illustration of Fujita's mechanism for fatigue crack nucleation (Suresh, 1998).

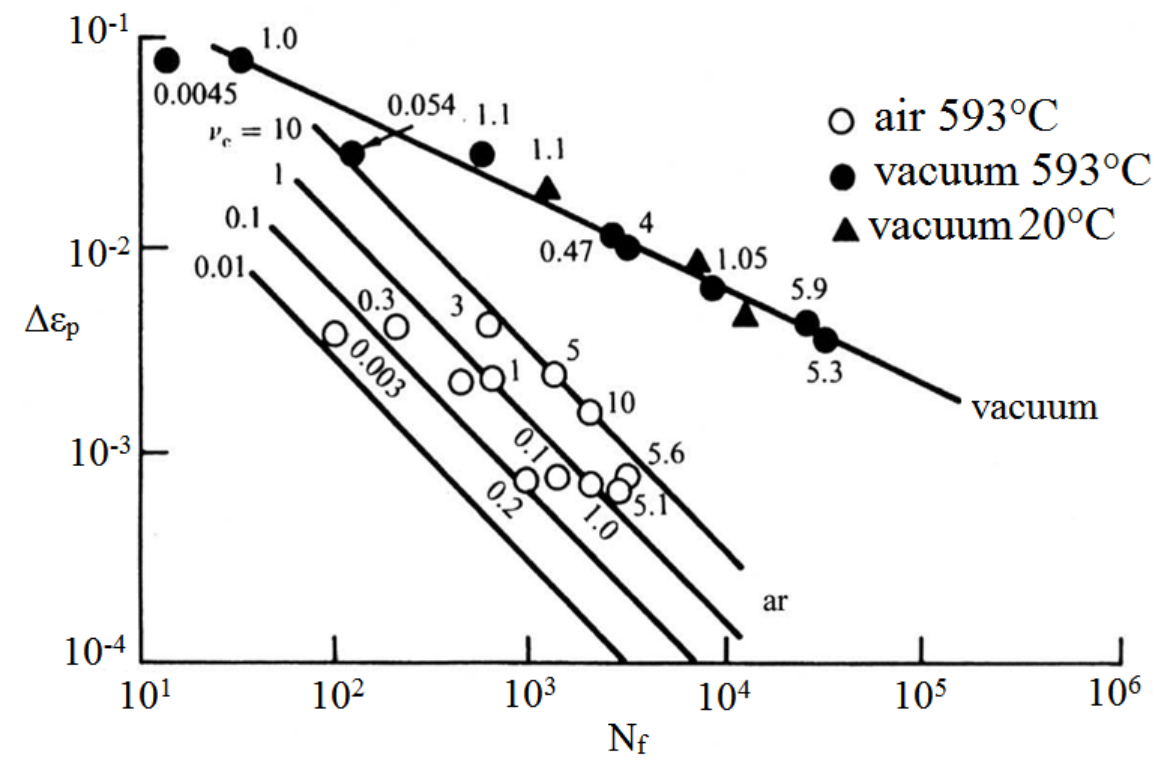

Figure 17: Variation of plastic deformation in relation to life of AISI A289 alloys in air and vacuum with frequency and temperature variation (Coffin, 1973). 


\subsection{Brief of Chapter 2}

- An important factor to consider is the effect of prolonged exposure of metals and alloys to high temperatures on their metallurgical stability.

- It is also important that mechanical properties at high temperatures should always be considered in relation to a time scale.

- A metal subjected to constant tractive loading and high temperatures flows and undergos an increase in length that depends on time. Under constant strain conditions, relaxation can occur, changing the strain exerted on the material.

- The important factor to be taken into account when materials are undergoing fatigue at high temperatures is Creep-Fatigue interaction. 


\section{CHAPTER 3: FATIGUE}

\subsection{Fatigue at High Temperature}

Several mechanical or structural components are subjected to high temperatures and/or thermal gradients during operation, as shown in Table 3. Temperature loops and external mechanical loadings may cause crack nucleation and propagation through processes known as fatigue, which may be of high or low cycle and occur isothermally or anisothermally (Sehitoglu, 1996).

Table 3: Components used at high temperatures.

\begin{tabular}{|c|c|c|}
\hline Component & Material & Maximum Temperature \\
\hline Ingot & Steel castings & $>800^{\circ} \mathrm{C}$ \\
\hline Rolling mill & Steel castings/forgings & $500-900^{\circ} \mathrm{C}$ \\
\hline Gas turbines & & $400^{\circ} \mathrm{C}$ \\
Compressor housing & Stainless steels & $400^{\circ} \mathrm{C}$ \\
Compressor drives & Ni Super-alloys & $>500^{\circ} \mathrm{C}$ \\
Combustion chamber & Ni Super-alloys & $<700^{\circ} \mathrm{C}$ \\
Turbine discs & Ni Super-alloys & $900-1100^{\circ} \mathrm{C}$ \\
Turbine blades & Ni Super-alloys & $<350^{\circ} \mathrm{C}$ \\
Automotive components & Al alloys & $<900^{\circ} \mathrm{C}$ \\
Cylinder head & Gray Cast Iron & $<350^{\circ} \mathrm{C}$ \\
Brake discs & Cast Iron & \\
Ventilation system & &
\end{tabular}

A fatigue test comprises the application of cyclic loading to an adequate and standardized specimen in relation to the type of test to be conducted. Metallic materials, when subjected to repetitive or fluctuating stress, i.e., when under cyclic stresses, break up at stress levels well below those predicted by stress and strain tests. Failure occurring under these dynamic conditions of stress application is known as fatigue failure. This phenomenon takes place within a considerable time after the material has been subjected to stresses. As technology develops, as required by industry (e.g., aviation and automotive), and incorporates new components and equipment that are continuously subjected to dynamic forces and vibration, fatigue has become the cause of over $90 \%$ of failure of metallic components in operation. Fatigue failure is rather unpredictable, because it happens without notice. 
Around 1871, German engineer A. Wöhler conducted the first systematic investigation of fatigue behavior of rail axes. He conducted fatigue tests with real-size models as well as small specimens subjected to cyclic bending, torsion and stress-compression, using various types of materials. Results were recorded in terms of conventional stress $(\sigma)$ in relation to the logarithm of the number of cycles $(N)$, which became known as $\sigma-\mathrm{N}$ curves or Wöhler curves. A $\sigma-N$ curve is usually determined using certain quantities of specimens according to recommendations found in ASTM E466 (1996), ASTM E468, and ASTM E739 (1991). It is recommended, among other things, a minimum quantity of specimens be used for each stress level tested, which is determined by the accuracy degree required of the test.

The stress-life $(\sigma-N)$ method was the first methodology employed in an attempt to understand and quantify fatigue of metals. This methodology is extensively used in design applications where applied stress is predominantly within the elastic regime of the material and the resulting life (cycles to fail) is long, stress and strain being linearly related, known as high-cycle fatigue. Therefore, in this period, the results of tests under controlled load or strain are equivalent, but $\sigma-N$ curves are generated by testing under controlled load. However, the stress-life method does not suit low-cycle applications, in which applied strains have a significant plastic component. In this case, a strain-based approach is more appropriate (Bannantine, 1990).

\subsection{Low-Cycle fatigue}

In the strain-based approach there is low-cycle fatigue related to operational conditions in which failures occur at relatively high stresses and low numbers of cycles to failure (less than $10^{5}$ ). The conditions for low-cycle fatigue to occur often arise when repeated stresses are of thermal origin. Because thermal stresses are due to the material thermal contraction or expansion, fatigue results from cyclic strain instead of cyclic stress.

Also, according to Suresh (1998), information from cyclic stress analyses based on continuum mechanics is primarily related to elastic deformation without mechanical restriction. In many practical applications, engineering components are often subjected to some degree of structural restriction and localized plastic deformation, particularly in areas of stress concentration. This type of problem should be taken into account when designing pressure vessels for nuclear industry, steam turbine components, aircraft engines, and many other types of equipment and machines, e.g., brake discs (Dieter, 1981). 
Low-cycle fatigue tests are carried out under controlled strain and results are plotted in graphs as strain logarithm in relation to the logarithm of number of reversals $\left(2 N_{f}\right)$.

Coffin and Manson, cited in Suresh (1998), worked independently on thermal fatigue problems and proposed the characterization of life under fatigue based on the extent of plastic deformation. They observed a linear relationship (Equation 4) between the logarithm of plastic deformation amplitude and the logarithm of the number of reverse to failure in metallic materials.

$$
\frac{\Delta \varepsilon_{p}}{2}=\varepsilon_{f}^{\prime}(2 N)^{c}
$$

where $\varepsilon_{f}^{\prime}$ is the fatigue ductility coefficient and $c$ the fatigue ductility exponent. In general $\varepsilon_{f}^{\prime}$ is approximately equal to the true ductility coefficient $\left(\varepsilon_{f}\right)$ in traction and $c$ ranges from -0.5 to -0.7 for most metals.

In bi-logarithmic scale, Equation 4 may be expressed as a straight line, as shown in Figure 18. The total strain amplitude of a test with constant strain amplitude may be described as the sum of elastic and plastic deformation amplitudes, as shown by Equation 5:

$$
\frac{\Delta \varepsilon}{2}=\frac{\Delta \varepsilon_{e}}{2}+\frac{\Delta \varepsilon_{p}}{2}
$$

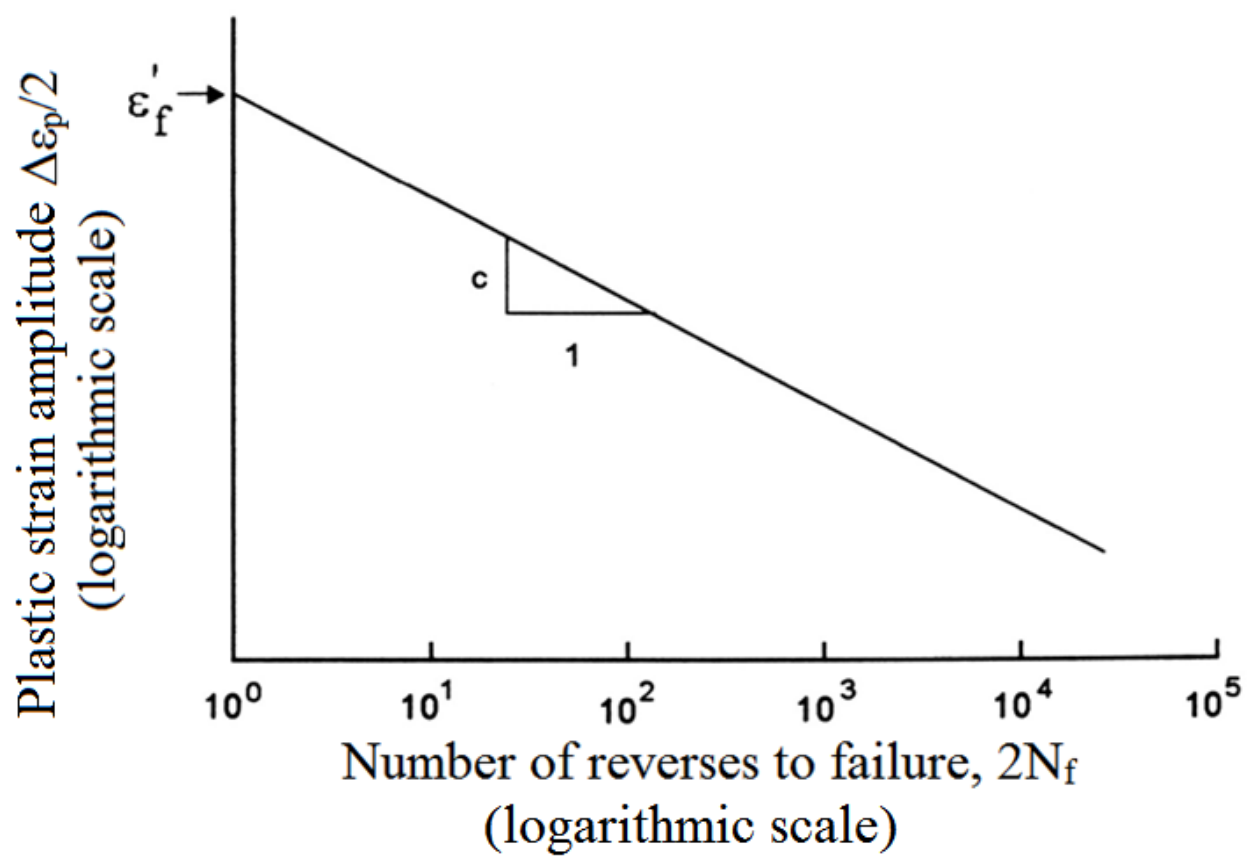

Figure 18: Plastic strain amplitude in relation to number of reverses, bi-logarithmic scale (Dieter, 1981).

By using Equation 6 of Basquin (cited in Suresh, 1998) to relate the stress amplitude (Figure 19): 


$$
\frac{\Delta \sigma}{2}=\sigma_{f}^{\prime}(2 N)^{b}
$$

where $\sigma_{f}^{\prime}$ is the fatigue strength coefficient and $\Delta \sigma / 2$ corresponds to stress amplitude $\left(\sigma_{a}\right)$. The fatigue strength coefficient $\left(\sigma_{f}^{\prime}\right)$ and the fatigue strength exponent (b) are fatigue properties of materials. Values of $b$ range from -0.05 to -0.12 , and for many metals $\sigma_{f}^{\prime}$ is approximately equal to the actual strain at fracture, determined by stress tests.

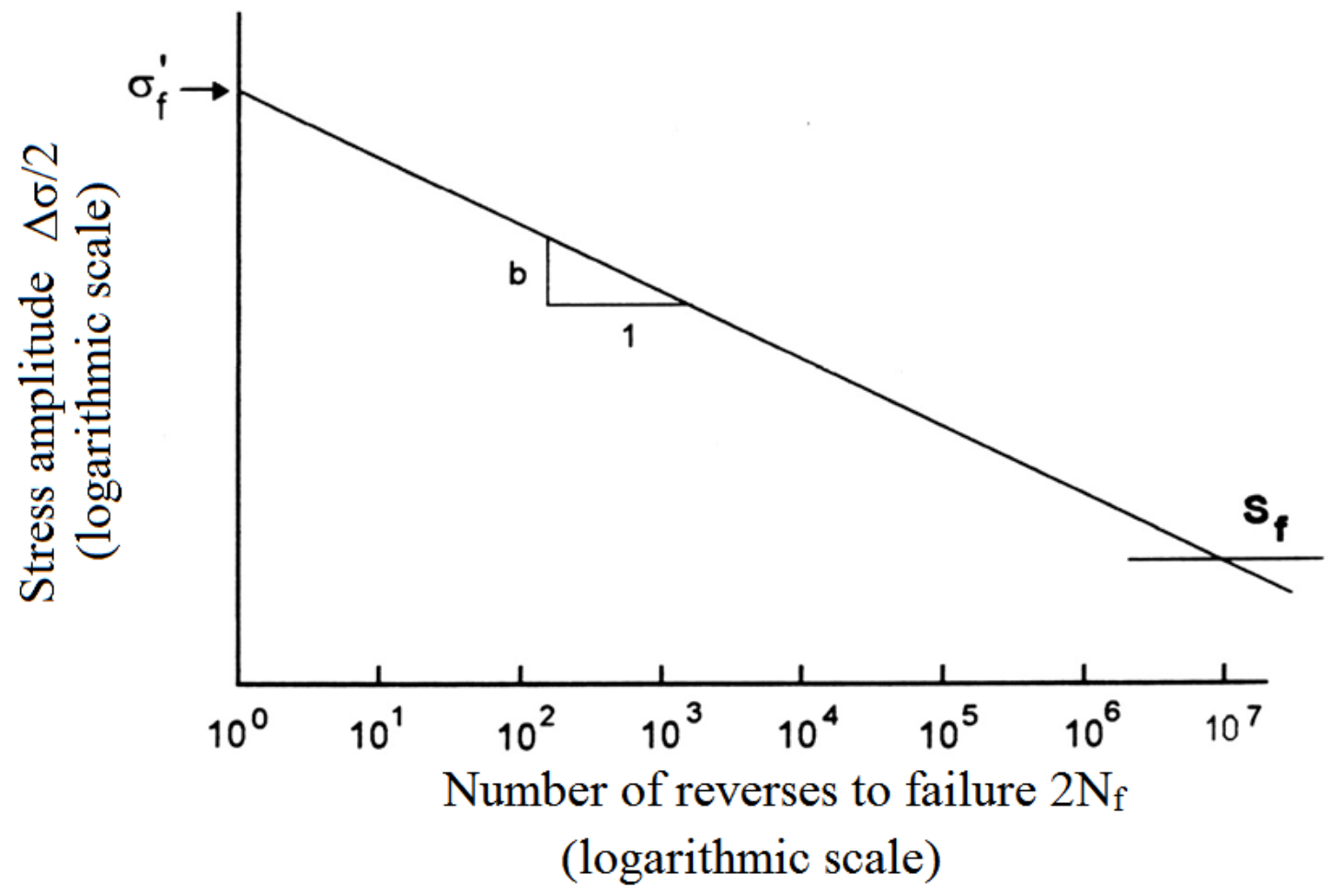

Figure 19: Actual stress in relation to number of reverses, in bi-logarithmic scale (Dieter, 1981).

\section{Noting Equation 7:}

$$
\frac{\Delta \varepsilon_{e}}{2}=\frac{\Delta \sigma}{2 E}=\frac{\sigma_{f}^{\prime}}{E}\left(2 N_{f}\right)^{b}
$$

where $E$ is Young's modulus.

It is possible to rewrite Equation 5 as indicated in Equation 8:

$$
\frac{\Delta \varepsilon}{2}=\frac{\sigma_{f}^{\prime}}{E}(2 N)^{b}+\varepsilon_{f}^{\prime}(2 N)^{c}
$$

The total, plastic and elastic strain amplitudes are shown in Figure 20 in relation to the number of reverses to failure $(2 N)$. It is possible to define a transition between low and highcycle fatigue at the point where elastic and plastic deformation amplitudes are the same $\left(2 N_{t}\right)$. According to Suresh (1998), plastic deformation predominates over elastic deformation and fatigue strength is controlled by the material ductility for low-cycle fatigue $\left(2 N<2 N_{t}\right)$. As for high-cycle fatigue $\left(2 N>2 N_{t}\right)$, elastic deformation is more significant than plastic 
deformation and life is controlled by the material strength. Optimization of fatigue properties is conducive to a balance between strength and ductility of the material.

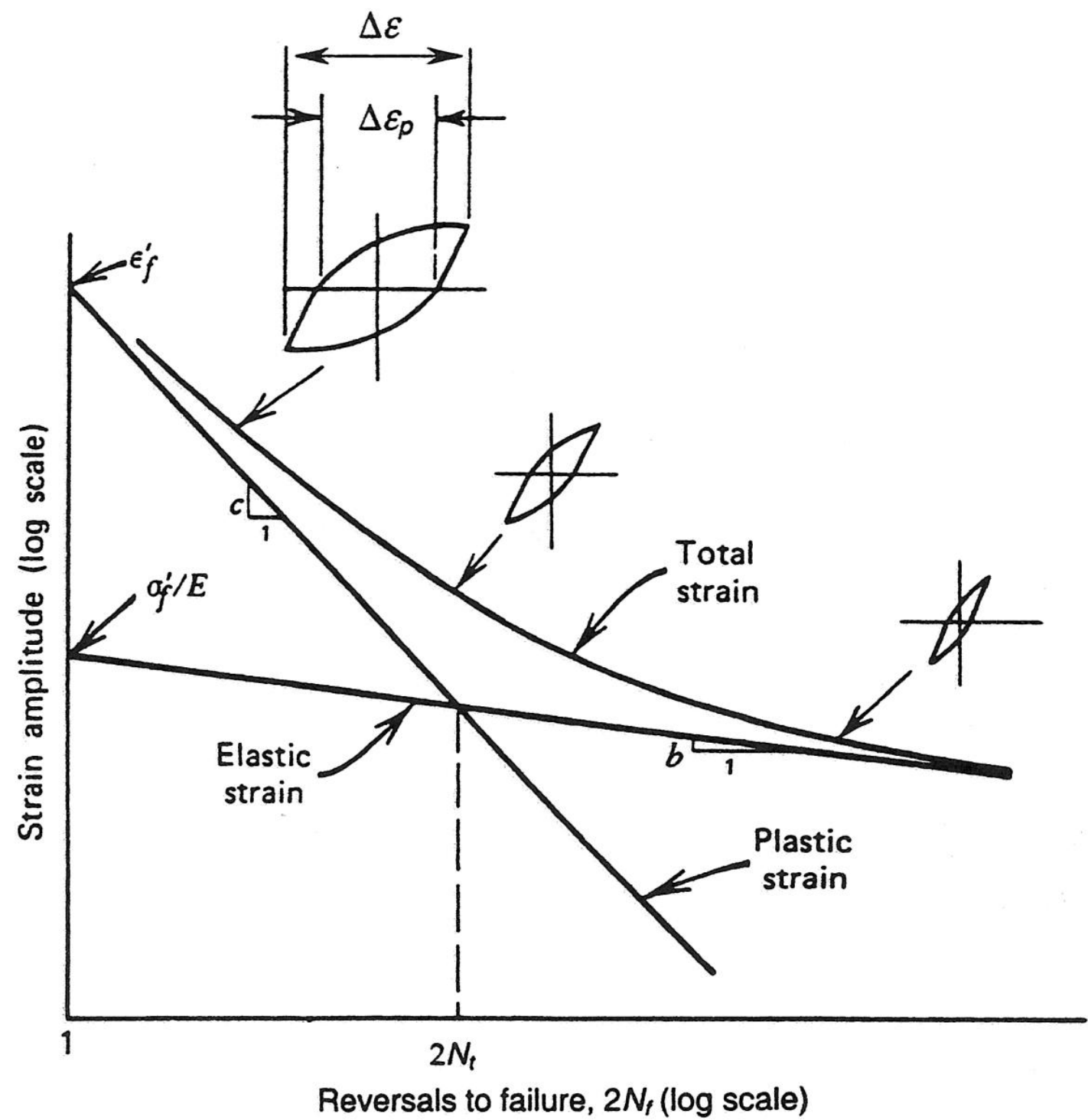

Figure 20: Total strain amplitude in relation to number of reverses to failure, obtained from the superposition of elastic and plastic deformation in relation to number of reverses to failure (Fuchs, 2001).

Low-cycle fatigue tests are usually conducted under controlled strain. Figure 21 show an ideal stress-strain loop resulting from a low-cycle fatigue test in which cyclic strain is kept constant. 


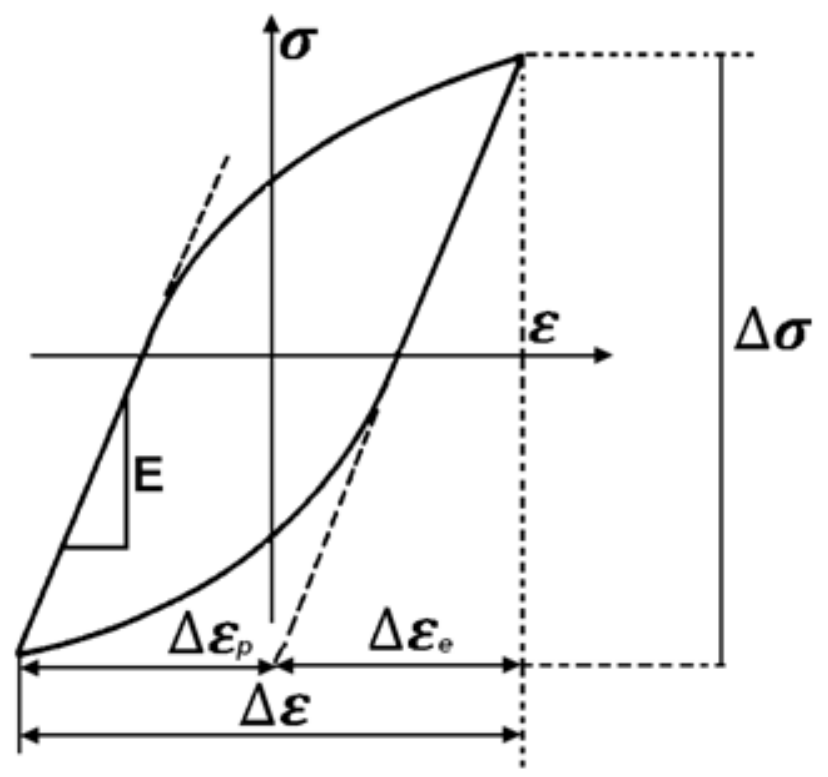

Figure 21: Ideal strain-stress hysteresis loop resulting from a low-cycle fatigue test under constant cyclic strain (Thomas, 1987).

During loading and unloading, a hysteresis loop originates, whose dimensions are described as width $(\Delta \varepsilon)$, total strain period, and height $(\Delta \sigma)$, which is the period between stresses. The total strain period $(\Delta \varepsilon)$ comprises an elastic deformation component $\left(\Delta \varepsilon_{e}=\right.$ $\Delta \sigma / E)$ and a plastic deformation component $\left(\Delta \varepsilon_{p}\right)$. The hysteresis loop width depends on the cyclic strain level; at low levels, hysteresis loops become very narrow.

For tests performed under constant $\Delta \varepsilon$, the stress period $(\Delta \sigma)$ usually varies with the increase in the number of cycles. Annealed materials undergo cyclic hardening, which means that $\Delta \sigma$ increases with the number of cycles until it saturates after about 100 strain cycles. The larger the value of $\Delta \varepsilon$, the greater is the increase in the stress period. Materials that have previously undergone cold work experience cyclic softening, i.e., $\Delta \sigma$ decreases with increasing number of strain cycles. Therefore, the usual way of presenting results of low-cycle fatigue tests is by plotting the strain amplitude $(\Delta \varepsilon / 2)$ as a function of the number of reversals to fail $\left(2 N_{f}\right)$.

Low-cycle fatigue is usually referred to as low-cycle isothermal fatigue, low-cycle thermal fatigue, or low-cycle thermo-mechanical fatigue. Low-cycle fatigue may be characterized as: (1) high-strain cycling when the range of inelastic deformation in the cycle exceeds that of elastic deformation; and (2) inelastic deformations of such magnitude that they spread through the microstructure evenly. Fatigue damage at high temperatures originates as a result of inelastic deformations when irrecoverable. In the case of low cycles, the material is damaged after a small number of cycles. Thermo-mechanical fatigue usually derives from low-cycle fatigue, e.g., in jet engines the highest thermal transients and gradients occur during 
engine startup/shutdown. The total number of take-offs and landings during an aircraft life is below 30,000 cycles. In this case, laboratory investigations are carried out under low-cycle conditions and may be completed in a short period of time.

\subsection{Isothermal Fatigue}

An isothermal fatigue test consists of imposing varying mechanical strains at constant temperature. This type of test has been widely used since the 1970s upon the advent of test equipment that operates in closed loop. Its main advantages are easy conduction and low cost as compared to an anisothermal test; results for several materials may be found in the literature (Coffin Jr., 1954).

Studies conducted by several researchers have shown that isothermal fatigue life is longer than that of anisothermal fatigue (Hertnarski, 1991; Shi, 1998). This may be attested by the study carried out by Shi et al. (cited in Shi, 1998), shown in Figure 22, with a molybdenum alloy containing $0.5 \% \mathrm{Ti}, 0.08 \% \mathrm{Zr}$, and $0.01-0.04 \%$ carbon.

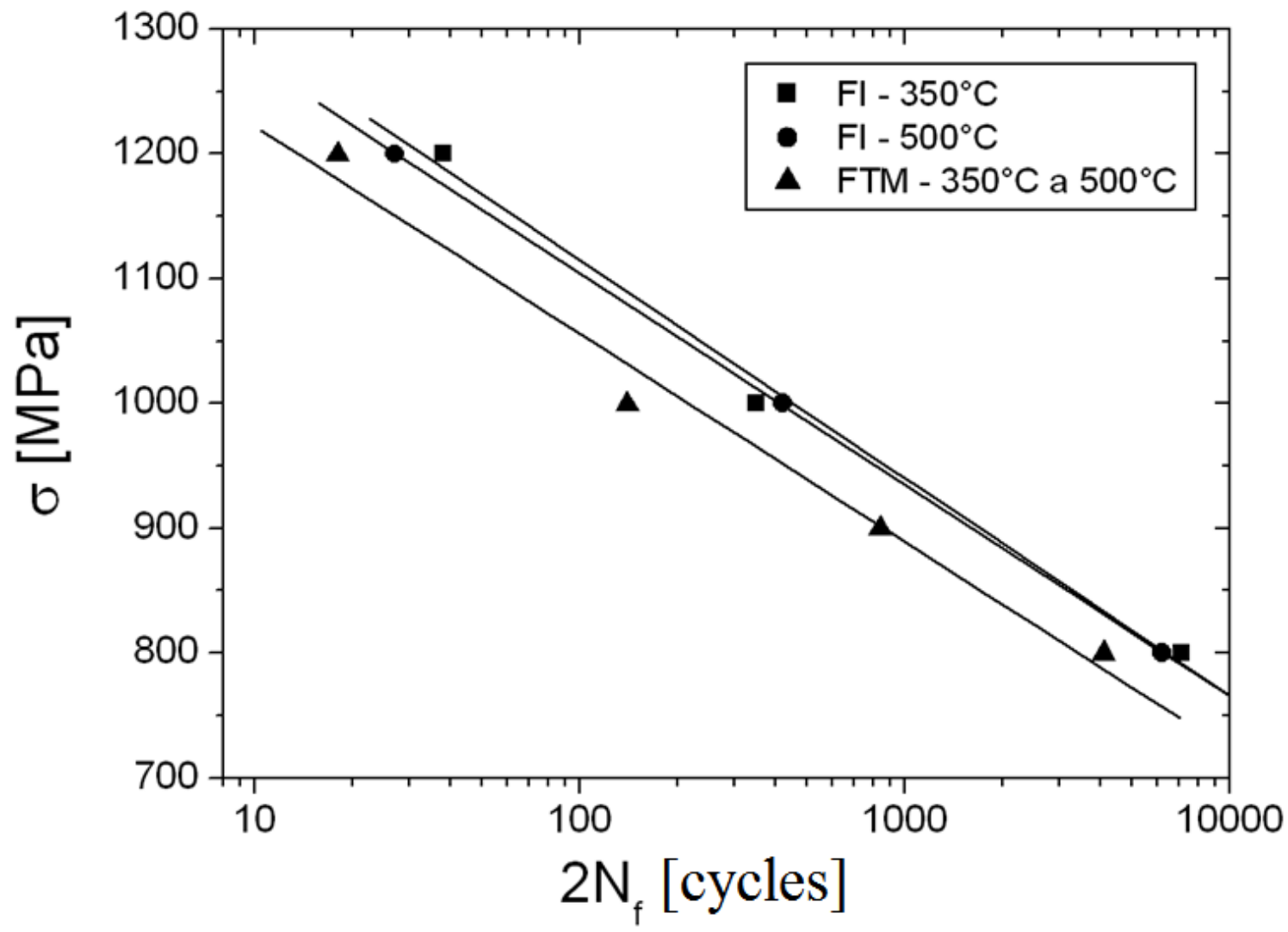

Figure 22: Isothermal and thermo-mechanical fatigue curves (Shi, 1998). 
Life obtained by isothermal fatigue tests for two temperature levels $\left(350^{\circ} \mathrm{C}\right.$ and $500^{\circ} \mathrm{C}$ ) was longer, in both cases, than that found in in-phase thermo-mechanical fatigue at temperatures ranging from $350^{\circ} \mathrm{C}$ to $500^{\circ} \mathrm{C}$, which points that temperature variation causes great damage to material. However, for the two types of isothermal tests under analysis, no clear difference was observed for number of cycles to specimen failure, which shows that the material displays good strength within this temperature range. Then, designs based solely on isothermal fatigue results of components that operate at high temperatures are not reliable. It is important to conduct an in-depth investigation into behavior of materials subjected to this phenomenon, including tests using different temperature ranges (anisothermal fatigue) and under varying stress and strain conditions.

Figure 23 shows that the longest life under isothermal fatigue occurs within an applied intermediate temperature range. Within this range the shortest life for $316 \mathrm{~L}(\mathrm{~N})$ austenitic stainless steel under isothermal fatigue occurred at room temperature $\left(25^{\circ} \mathrm{C}\right)$, where strain induced the development of martensite.

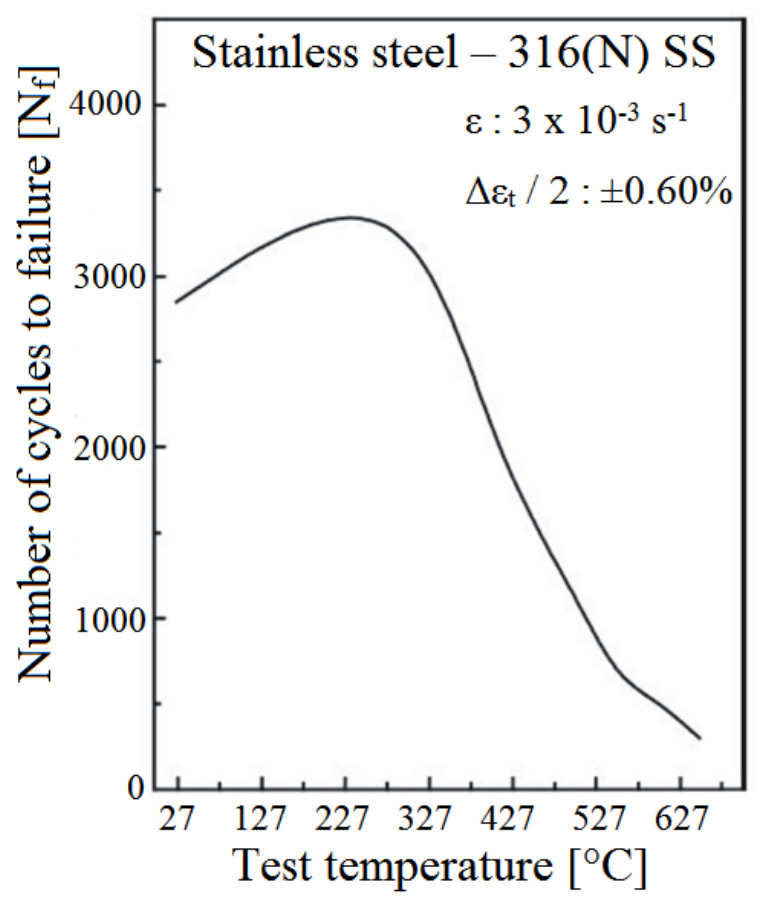

Figure 23: Effect of temperature on fatigue life (Srinivasan, 2003).

On the other hand, at $573 \mathrm{~K}\left(300^{\circ} \mathrm{C}\right)$ the material underwent sub-structural recovery, which was responsible for increased life.

However, a sub-structural recovery of the material occurred at $573 \mathrm{~K}\left(300^{\circ} \mathrm{C}\right)$, which was responsible for increase in life. The reduction in life as temperature continued to rise may be attributed to several effects of dynamic strain, e.g., stress concentration induced by pilling 
up of dislocations when the maximum stress of the cycle is reached, causing an increased crack growth rate. This may be clearly seen at temperatures higher than $873 \mathrm{~K}\left(600^{\circ} \mathrm{C}\right)$, at which life was significantly reduced by oxidation (Srinivasan, 2003).

Another aspect to be noted about isothermal fatigue with controlled strain is the behavior of cyclic stress in relation to life. For $316 \mathrm{~L}(\mathrm{~N})$ austenitic stainless steel, behavior was monitored in four stages, as shown in Figure 24 (Srinivasan, 2003).

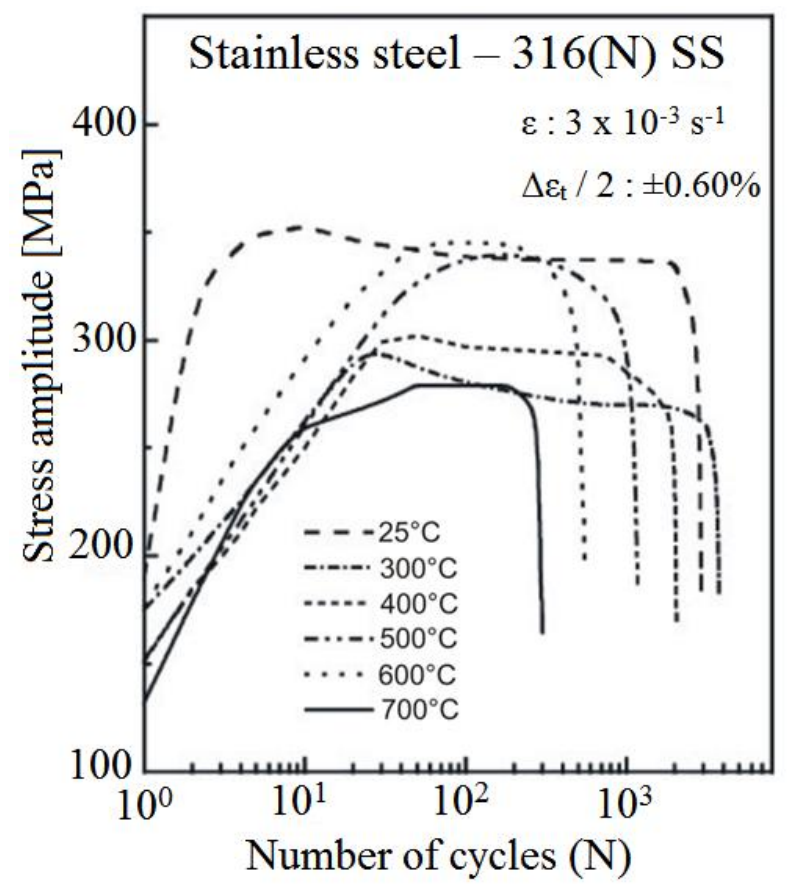

Figure 24: Cyclic stress response in relation to temperature (Srinivasan, 2003).

The alloy exhibited a short period of cyclic hardening, reaching the maximum stress in the first life stage. Then, it may be observed that cyclic softening takes place before stable regime is achieved. In the period prior to fracture, stress amplitude decreases rapidly, indicating the development of a macro-crack and its subsequent spread. The same figure indicates that the amplitude of the stress peak increases with increasing temperature between $573 \mathrm{~K}$ and $873 \mathrm{~K}$ (Srinivasan, 2003). Also, it shows that some factors contribute to the drop in material strength as temperature increases: (a) abnormal cyclic hardening rate and reduced plastic deformation amplitude in the intermediate life to fracture; and (b) increased maximum stress rate during the initial stages with increasing temperature due to inductive interaction developed between diffusion solutes and discrepancy mobility under strain. All these phenomena are considered to be a manifestation of dynamic strain processes.

A more detailed discussion comparing isothermal and anisothermal fatigue can be found below. 


\subsection{In-Phase and Out-of-Phase Thermomechanical Fatigue and Thermal Fatigue}

Several components are subjected to a variety of thermal and thermo-mechanical loads due to temperature variations during operation. The conditions of cyclic loading induced by temperature gradient are essentially strain-constrained loads. Then, laboratory studies on thermal fatigue are usually constrained by strain control in low-cycle fatigue tests (Hetnarski, 1991). Research on mechanical behavior and fracture mechanisms of these materials is important to improve design and use of these components.

If stress develops under thermal cycling without external loading in a component, the term thermal fatigue (TF) is employed. This process may result from steep temperature gradients in a component, or through a section, and may occur in a perfectly homogeneous and isotropic material. For example, when heated, a surface is compressed by the cooler material below the surface. Thus, the surface is subjected to compressive stresses. Under cooling, strain is in the opposite direction, and tensile stresses may develop. Under heating and cooling cycles, the surface can be damaged by TF. Classic TF examples may be found in train wheels subjected to the action of brake shoes, which generates temperature gradients and, consequently, internal stresses (Sehitoglu, 1996).

On the other hand, TF may develop in laboratory specimens, even under uniform temperature conditions, because of internal constraints, such as orientation of different grains at a microscopic level or anisotropy of the thermal expansion coefficient of some crystals (not cubic). Strains and internal stresses may be big enough to cause expansion, distortion and surface irregularities in the material. Consequently, thermal loops lead to micro-structural damage and deterioration. This behavior has been observed in pure metals such as uranium, tin, cadmium-based alloys, and duplex steels whose microstructure consists of ferrite and martensite.

The term thermo-mechanical fatigue (TMF) describes fatigue under variation of both temperature and mechanical strain. Mechanical strain is determined by subtracting thermal strain from total strain, which should be uniform throughout the specimen. Mechanical strain derives from external constraints or externally applied loads. For example, if a specimen is held between two rigid walls and subjected to thermal cycling (without allowing for expansion), it will undergo "external" compressive mechanical strain. TMF examples can be found in pressure vessels and pipelines in the power generation industry, where structures are subjected to pressure loads and thermal transients with temperature gradients in the thickness 
direction, and the aircraft industry, where turbine blades and disks are subjected to temperature gradients aggravated by rotation-related stress.

According to Sehitoglu (1996), thermo-mechanical fatigue may involve several mechanisms that add damage to that of "pure" fatigue, including creep and oxidation at elevated temperatures, which directly contribute to further damage. These mechanisms differ from each another, depending on strain and temperature history. They are different from those predicted by fluidity tests (no reversals) and by oxidation tests without stress (or with constant stress). Micro-structural degradation may occur under TMF as: (1) over-aging, such as coalescing of lamellae and precipitates; (2) strain aging for systems hardened by solid solution; (3) precipitation of second-phase particles; and (4) phase transformation at the cycle limit temperature. Variations in mechanical properties or in the thermal expansion coefficient between the matrix and precipitates - found in many alloys - also cause local stresses and cracks. These mechanisms affect the strain characteristics of the material, which are inexorably associated with damage processes.

For this reason, more advanced test techniques at varied temperatures were developed, such as the thermal fatigue (TF) test and thermo-mechanical fatigue (TMF) test, whose main differences are shown schematically in Figure 25 (Meyer-Olbersleben, 1995; Engler Pinto, Jr., 1994).

A TF test involves subjecting a specimen with geometry similar to that of the component under study to alternating heating and cooling cycles. Stresses of internal origin derive from the specimen geometry, warming and cooling rates, and material properties. It is a more technological test; its results enable immediate design application as it is capable of determining the life of a specimen with geometry similar to that of the component studied and can be used to reliably compare different types of materials. On the other hand, it has the disadvantage of not allowing direct strain and stress measurements, which have to be calculated, for instance, by means of the finite element method. To do so, according to Mallet et al. (1995), it is necessary to use a mathematical model to express the material mechanical behavior. 


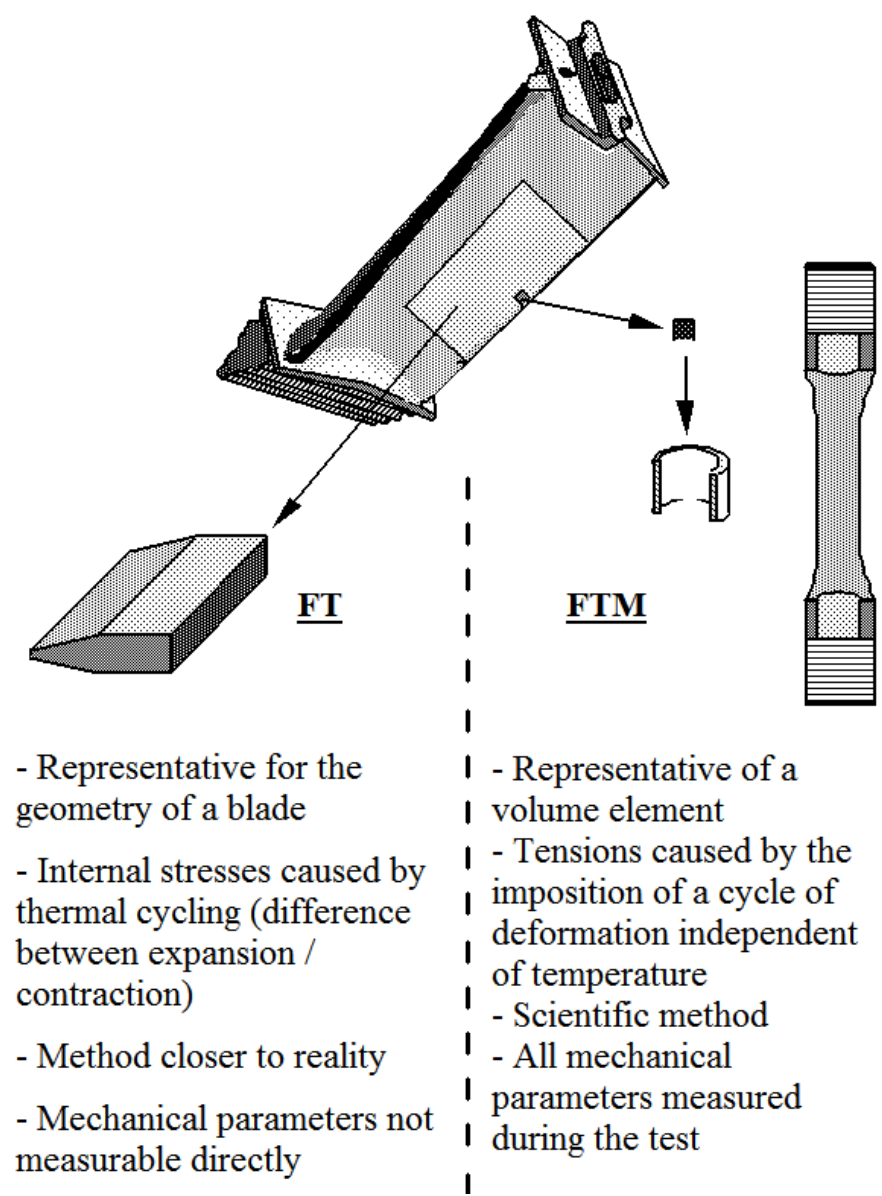

Figure 25: Comparison between thermal fatigue tests and thermo-mechanical tests (EnglerPinto Jr., 1996). Todos os parâmetros mecânicos medidos durante o ensaio

The TMF test, however, is characterized by independent and simultaneous overlapping of a strain cycle in relation to time $(\varepsilon(t))$ and a temperature vs. time cycle $(T(t))$ in accordance with a previously established thermo-mechanical history. The external strain imposed by an especially-adapted and computerized testing machine replaces the internal strain of the TF test. The test is uniaxial and should be performed at low frequencies in order to avoid temperature gradients throughout the specimen. Despite its complexity and high cost, this test is more scientific in that the researcher can control all the influential parameters. It is useful to determine the mechanical behavior of materials under anisothermal conditions (closer to reality). Hence, it assists in the development and allows the verification of mathematical models employed to calculate stresses and strains of real components or TF specimens (Mallet, 1995; Guedou, 1992).

Sehitoglu (1996) sustains that an important distinction must be made between TMF and thermal shock. Thermal shock involves a very rapid variation in temperature (due to surface heating or internal heat generation), and resulting strains are generally different from those produced under slow heating and cooling conditions (i.e., quasi-static). Physical properties such as conductivity and specific heat, which do not arise in cases of low strain 
rate, are clearly present in thermal shock cases. Strain rate affects the material response and should be taken into account when analyzing damage due to thermal shock or choosing materials with better thermal shock strength.

Sehitoglu (1996) and Sarabanda (1991) claim that if a body is subjected to thermal cycling conditions with overlaying loads in the section, the component will undergo cyclic thermal creep (thermal ratcheting), which is gradual accumulation of inelastic deformation cycles. This type of failure involves ductile rupture and fatigue mechanisms, which sometimes occur unintentionally during TMF tests when an area of the specimen is warmer than its surroundings, causing a lump to appear in the hot area.

Crack initiation under thermal fatigue is a decreasing function of distance from the surface of the solid due to the stress-strain-temperature field generated in superficial areas of structural components. Therefore, the driving force that propagates a thermal crack often decreases with crack growth, since the farther the crack is from the stress-strain-temperature field, the less it is influenced by thermal fatigue and the smaller is its propagation. However, in isothermal or thermo-mechanical fatigue testing, in which components are under uniform temperature conditions, the crack, when growing toward the interior of the material, reaches a more severe stress-strain field, since the cross-sectional area of the component is reduced by crack growth (Hetnarski, 1991).

The design procedure of real components subjected to anisothermal fatigue is quite complex and can be basically divided into three steps:

1. Determination of stress and strain distribution in these components during operation, which usually is accomplished with the aid of the finite element method.

2. Determination of constitutive equations that describe the material mechanical behavior for the in service conditions determined above. Whenever possible these equations should take into consideration possible changes in material properties during operation.

3. Development of life prediction models (number of cycles to failure), which enables the safe use of the component in question by establishing its inspection and replacement routines. These models should take into account factors other than fatigue, such as creep and oxidation interactions.

The total strain $\left(\varepsilon_{\text {tot }}\right)$ measured during an anisothermal fatigue test is the sum of the mechanical strain $\left(\varepsilon_{m e c}\right)$ imposed by the testing equipment and the thermal strain (thermal 
expansion or $\left.\left(\varepsilon_{\text {term }}\right)\right)$ resulting from the change in temperature: $\left(\varepsilon_{t o t}=\varepsilon_{m e c}+\varepsilon_{\text {term }}\right)$ for both in-phase and out-of-phase thermo-mechanical tests (Figure 26b and a, respectively).

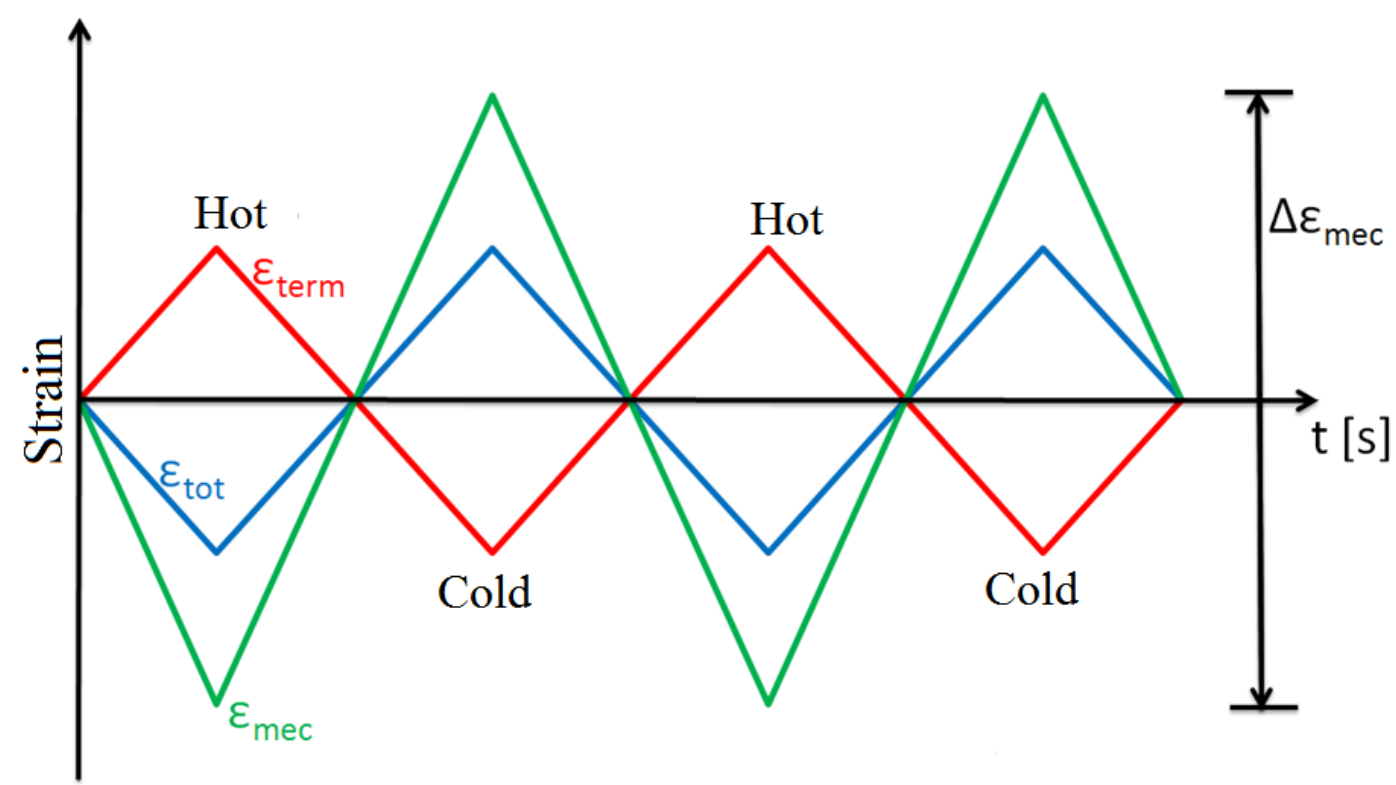

(a)

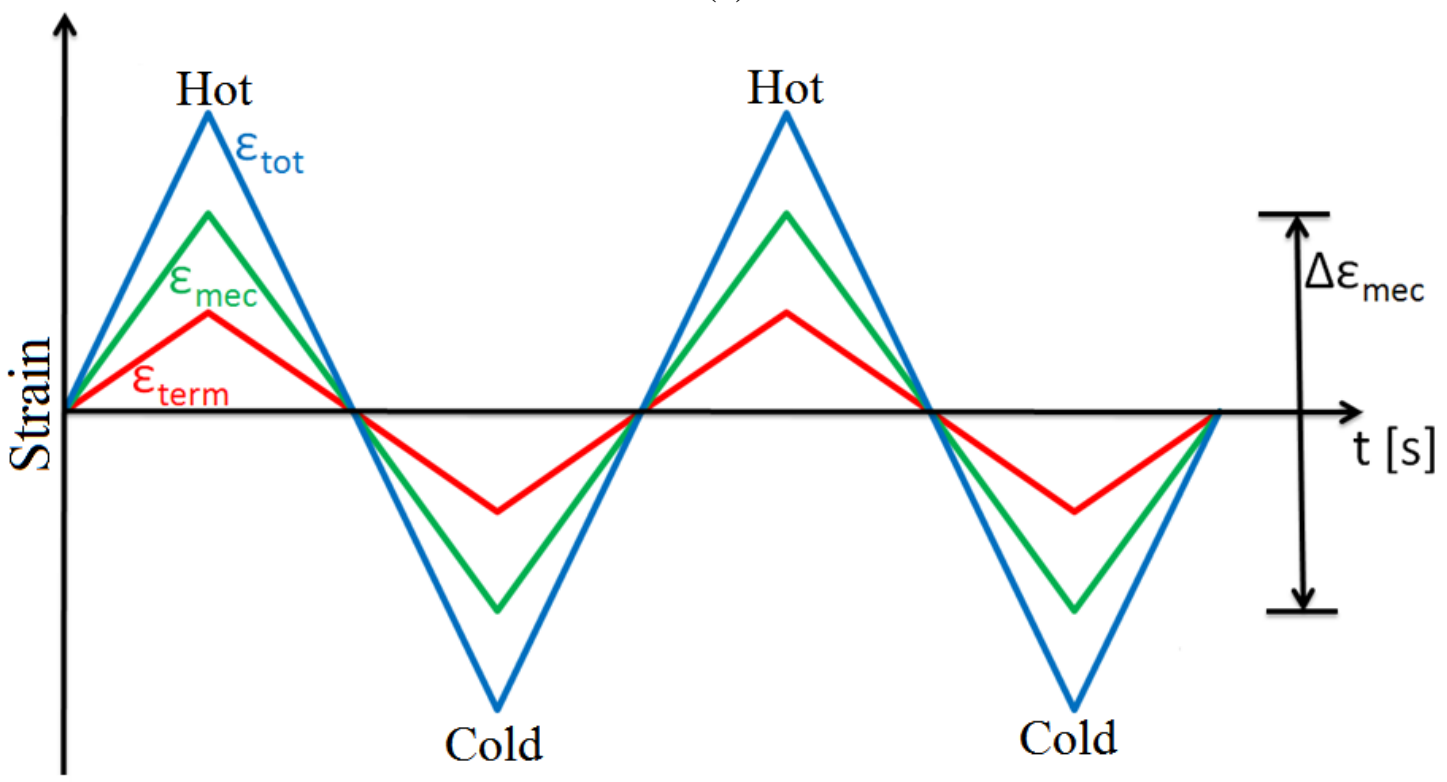

(b)

Figure 26: Thermal and mechanical variation and total strain in relation to time for out-ofphase (a) and in-phase (b) thermo-mechanical fatigue.

Thermal strain, obviously, does not cause stress and may be determined through the following equation:

$$
\varepsilon_{t h}=\alpha(T) .\left(T-T_{a m b}\right)
$$

where $\alpha(T)$ is the thermal expansion coefficient, $T$ is temperature and $T_{a m b}$ is room temperature. On the other hand, mechanical strain may be decomposed into elastic $\left(\varepsilon_{e}\right)$ and inelastic $\left(\varepsilon_{\text {in }}\right)$ deformations: 


$$
\varepsilon_{m e c}=\varepsilon_{e}+\varepsilon_{\text {in }}
$$

where $\varepsilon_{e}$ is obtained through Hooke's law:

$$
\varepsilon_{e}=\sigma / E(T)
$$

where $\sigma$ is the measured stress and $E(T)$ is the elastic modulus in relation to instantaneous temperature. In addition, $\varepsilon_{i n}$ consists of two distinct components at sufficiently high temperatures: plastic deformation $\left(\varepsilon_{p}\right)$, caused by stress higher than the material elasticity limit, and creep deformation $\left(\varepsilon_{f l}\right)$, due to thermal activation. These are some of the terms employed in an anisothermal hysteresis loop, as shown in Figure 27.

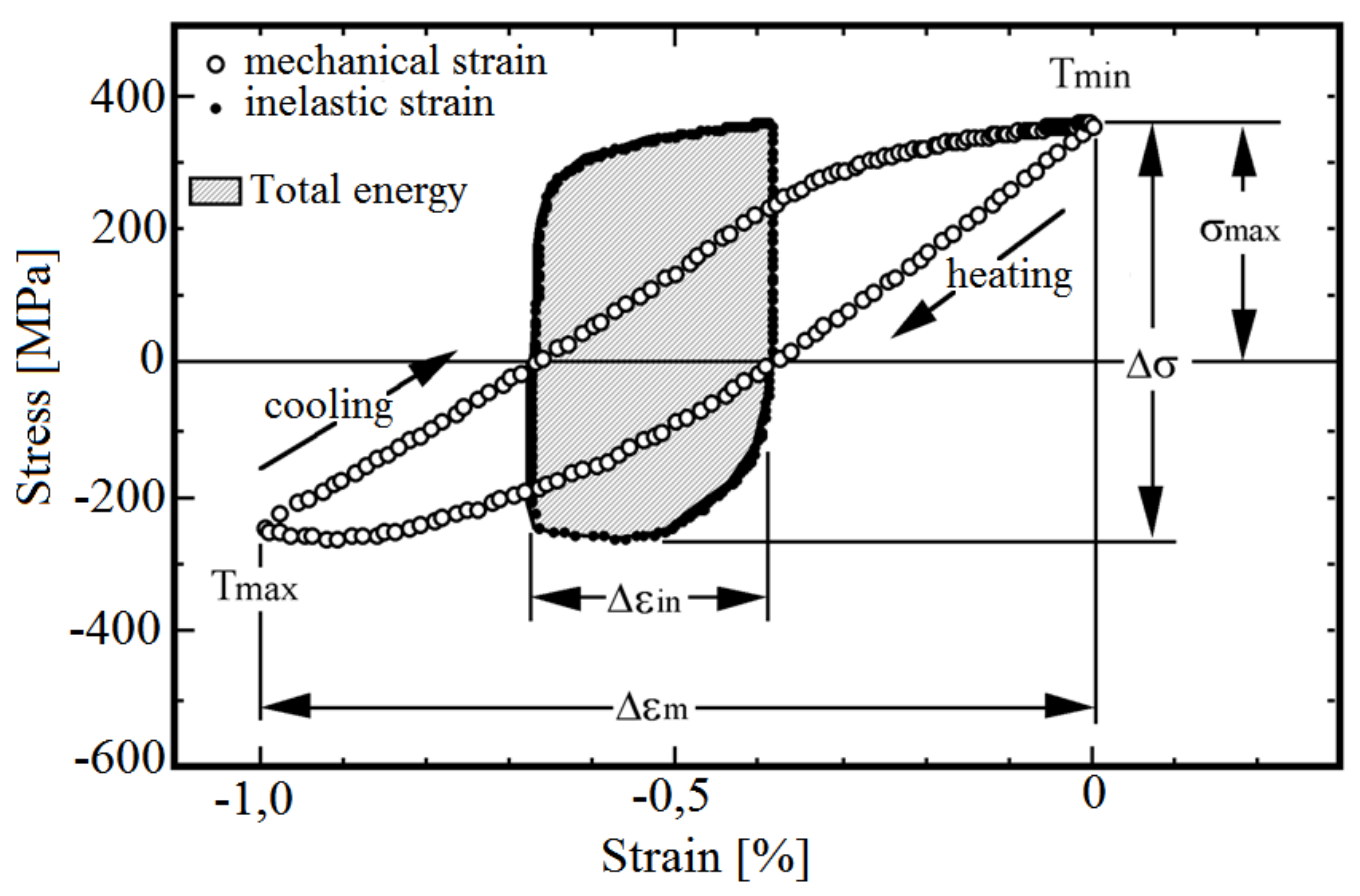

Figure 27: Example of an out-of-phase anisothermal hysteresis loop (Engler-Pinto Jr., 1996).

Coffin has summarized the basic stress-strain relationship for several heating histories and loops, as shown in Figure 28.

It is possible to see that the hysteresis curve is entirely complex and discontinuous, thereby explaining the great difficulty in predicting life under fatigue of components operating at high temperatures (Fuchs, 1980).

Thermal fatigue is seen as an unbalanced repetitive process, i.e., the response from the tensile part of the cycle differs from that of the other half (compressive region) and the response is a function of temperature. There are three basic aspects of stress-strain behavior that are important unbalanced cyclic mechanisms: elasticity, plasticity, and creep (Hetnarski, 1991). 
1. Elasticity - the elastic stress-strain response of a solid is a function of temperature, since the elasticity modulus invariably decreases with increasing temperature. Thus, an in-phase elastic thermo-mechanical cycle exhibits a nonlinear response since the solid is hardened at the lowest temperature of the cycle. The elastic cyclic stress-strain response, shown in Figure 29, displays a concave-downward curvature. The cycle shown is completely reversed in terms of strain, but not with respect to stress. It may be observed that tractive stress is lower than compressive stress, which corresponds to the difference between elasticity modulus values at high temperatures. Thus, the difference in unbalanced stress is a direct function of temperature range and level (Hetnarski, 1991).

2. Plasticity - if a thermo-mechanical strain is high enough, the material will undergo plastic deformation. This results in a tractive stress response in relation to non-linear and unbalanced compressive stress (Figure 30). Usually, the elasticity limit decreases as temperature increases thereby giving a lower stress response to higher temperatures. Plastic and elastic deformation changes in spite of strain being entirely reversed (Hetnarski, 1991).

3. Creep - the maximum stress peak is lower in thermo-mechanical tests with low strain rates, in which creep is favored, than in tests with higher strain rates because creep decreases material strength (Figure 31). The stress peak response at low temperatures is essentially independent from the strain rate. Therefore, half the cycle corresponding to the highest temperature becomes more unbalanced as regards half the cycle corresponding to the lowest temperature, with decreasing cycling rate and increasing maximum cycle temperature, which are factors that favor creep (Hetnarski, 1991). 


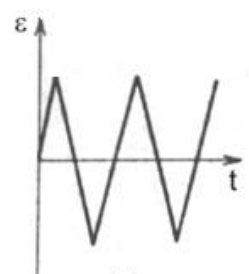

(a)

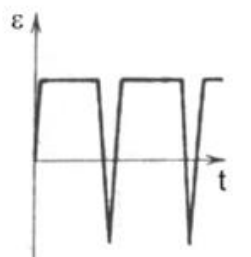

(b)

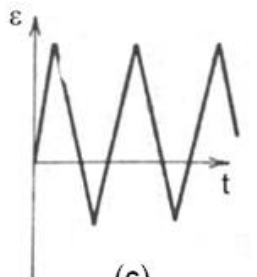

(c)
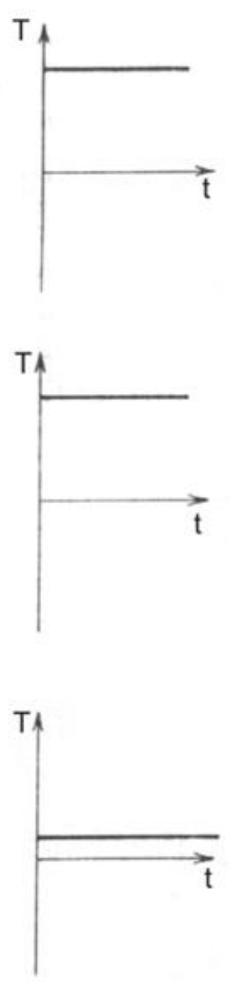
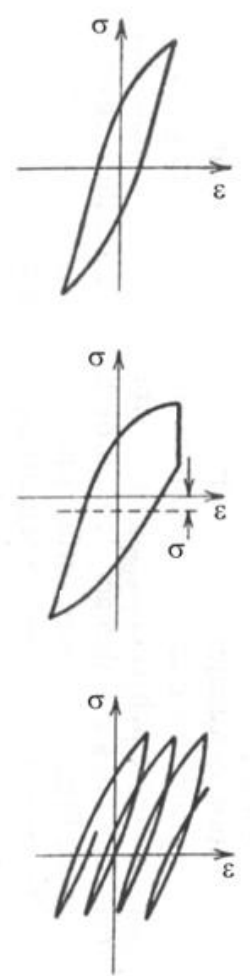
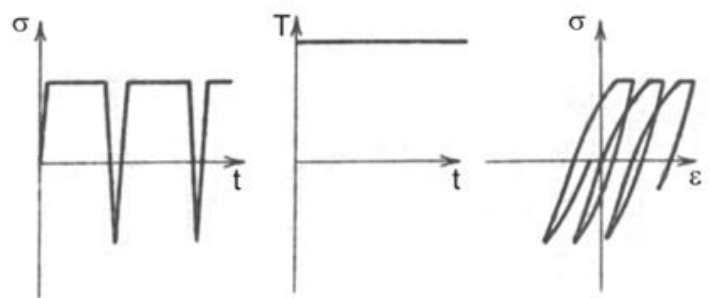

(d)
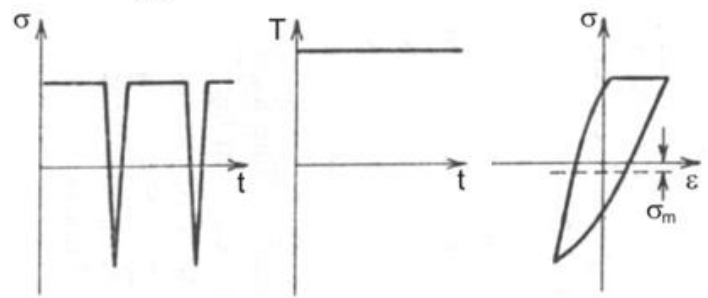

(e)

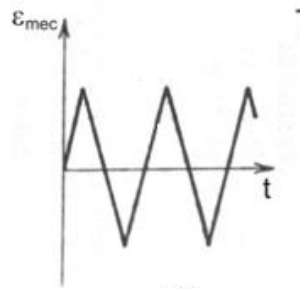

(f)

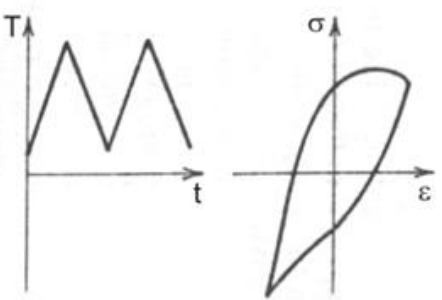

Figure 28: Stress-strain relationship for various loading histories and cycling types: (a) continuous strain cycling; (b) level of strain (strain hold); (c) continuous cyclic mean stress; (d) level of stress (stress hold); (e) stress-strain hold limit (CP); (e) thermo-mechanical status (mixed mechanical-thermal) (Fuchs, 1980).

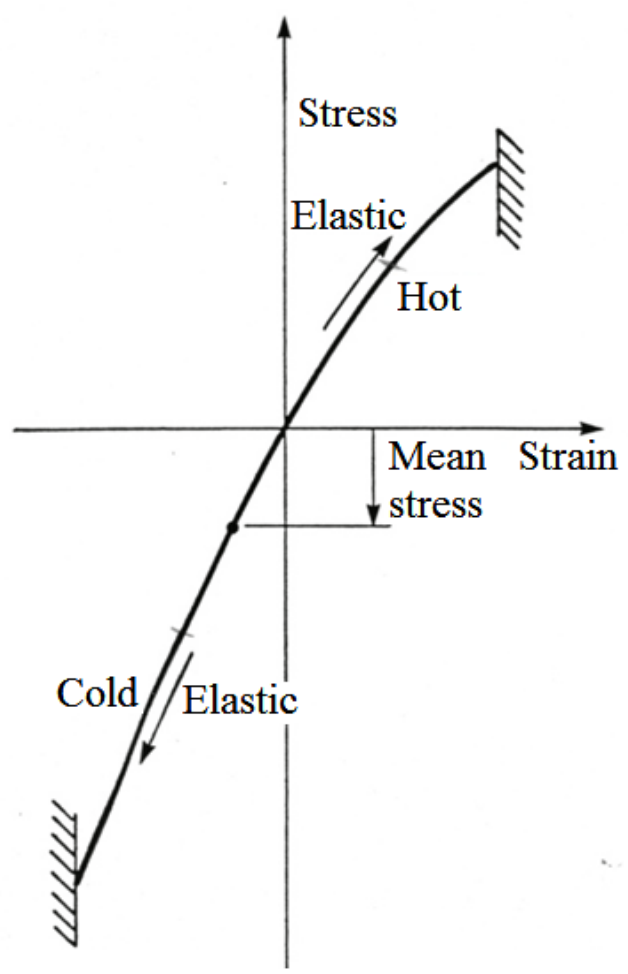

Figure 29: Hysteresis curve under elastic thermo-mechanical strain conditions for phase cycling (Hetmarski, 1991). 


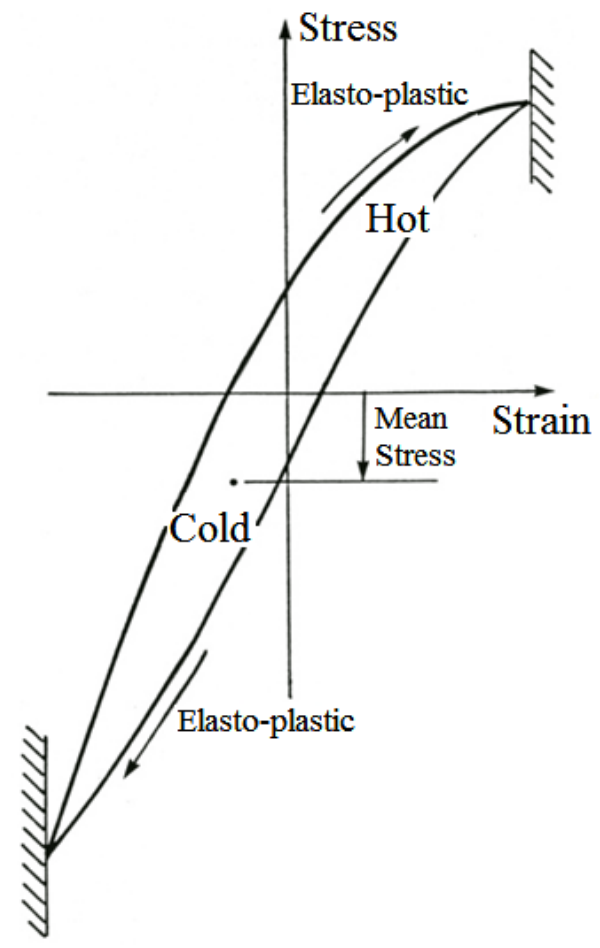

Figure 30: Hysteresis curve under plastic thermo-mechanical strain conditions for phase cycling (Hetmarski, 1991).

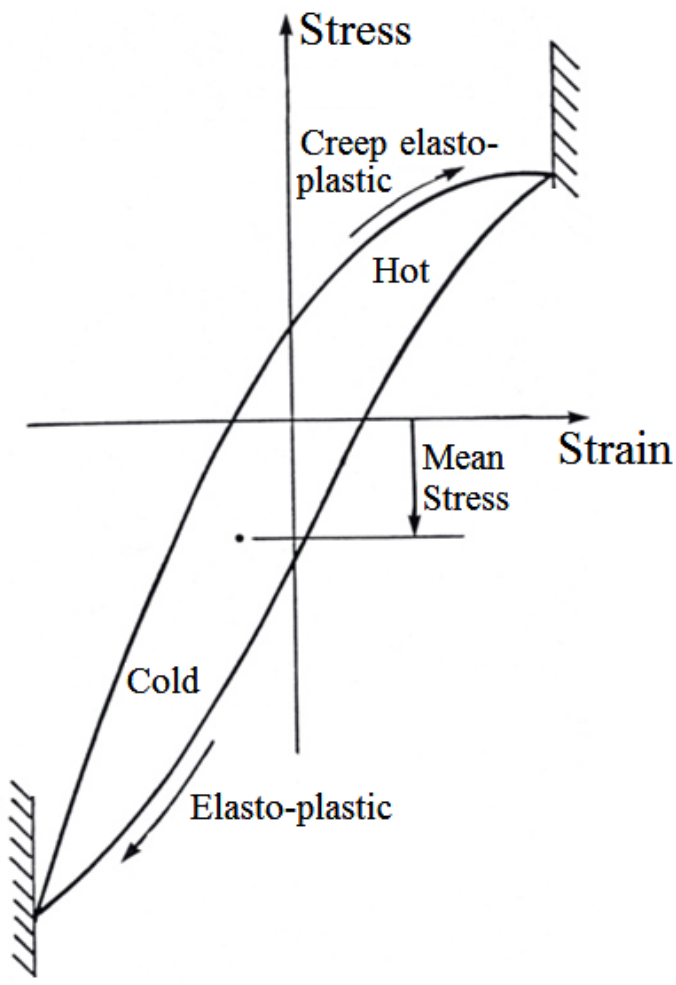

Figure 31: Hysteresis curve under creep thermo-mechanical strain conditions for phase cycling (Hetmarski, 1991). 


\subsection{Effect of Porosity on Fatigue Life}

Porosity has a catastrophic effect on mechanical properties of aluminum alloys. A $1 \%$ volumetric fraction of pores may reduce fatigue life by $50 \%$ and strength limit by $20 \%$ as compared to the same alloy without pores (Odegard \& Pedersen. 1994).

The number of cycles needed for crack nucleation depends on loading, ranging from $0 \%$ (at startup) to less than $10 \%$ of fatigue life (Slallerud \& Larsen, 1989), while the time needed for crack initiation decreases with pore size.

Woller curves (Figure 32) for aluminum alloys show that fatigue life increases when stress decreases and that for a given stress level the number of cycles to failure decreases when amount of gas introduced in the alloy increases (Buffière et al., 2001). The uncertainty about fatigue life increases when stress and/or amount of gas decreases. This shows the importance of pore quantity and distribution to fatigue life.

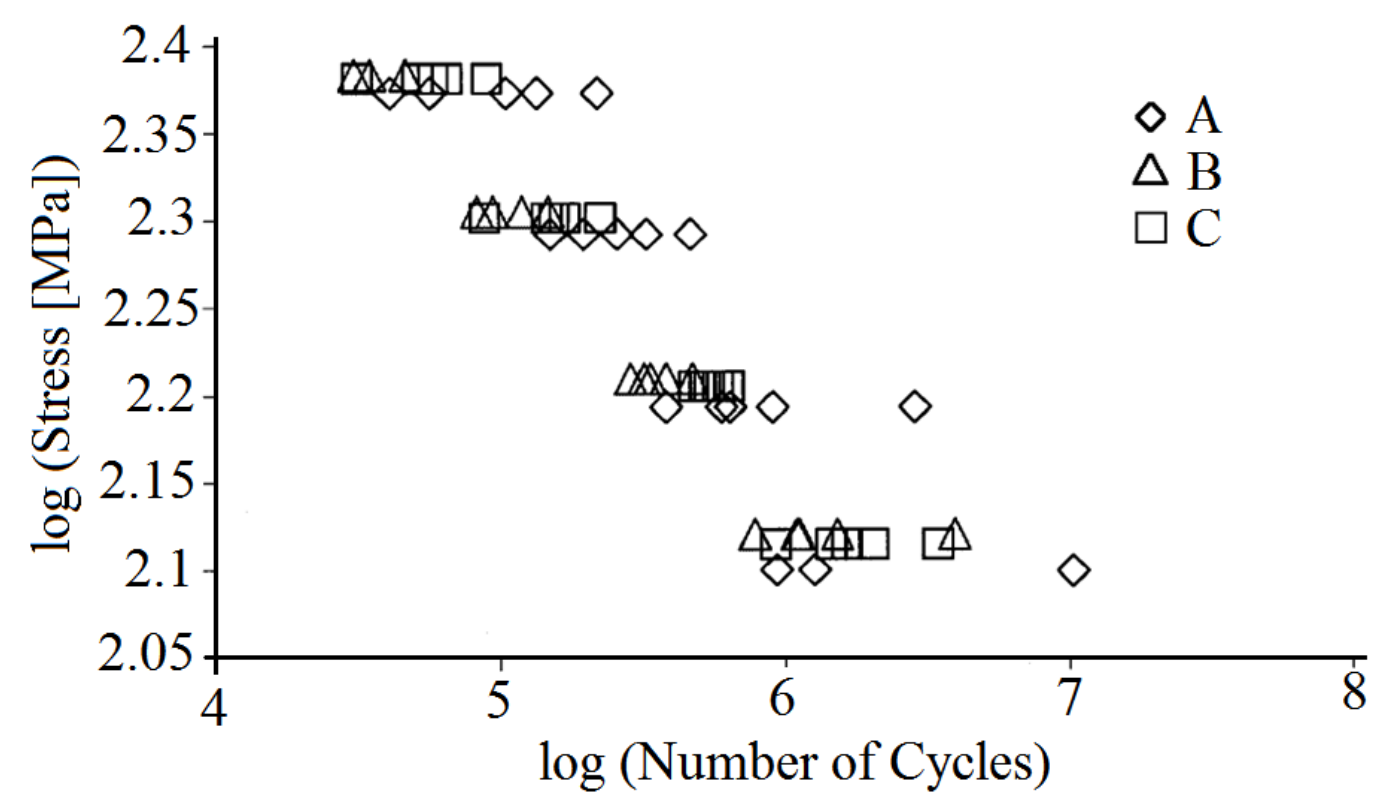

Figure 32: S-N curves for three aluminum alloys (A, B, and C), with $-0.131,-0.168$, and 0.158 slopes (Buffière et al., 2001).

Buffière and colleagues (2001) show that cracks responsible for ruptures nucleate on one or more pores located on the surface of specimens or just below it. Except for high stress levels, at which cracks nucleate by de-cohesion of internal silicon nodules (far from pores) probably located at grain boundaries. It was observed that in the pores responsible for ruptures crack nucleation occurs by de-cohesion of the silicon/matrix interface. In most cases this decohesion is produced on a silicon particle near a convex region (a pore) almost perpendicular 
to the applied load. It has been noted also that even at low stress levels the number of cycles required for crack nucleation is negligible as compared to that of total fatigue life $(<2 \%)$.

\subsection{Life Prediction Models}

Suresh (1998) states some approaches have been developed over the years in order to predict the total life of engineering materials under fatigue at elevated temperatures. Extensive reviews of life prediction models can be found in Coffin (1974), Tomkins and Wareing (1977), and Batte (1983). The most common life prediction methods may be classified into three groups according to Suresh (1998): (a) damage accumulation models, (b) modifiedfrequency Coffin-Manson equations and (c) deformation variation partitioning. A brief summary of each method is presented below.

\subsubsection{Damage Accumulation Models}

The simplest approach to fatigue-creep life prediction involves the linear accumulated damage model, as described by Suresh (1998). In this method, the accumulated damage by mechanical fatigue and creep are linearly overlaid by means of the following equation:

$$
\zeta_{f}+\zeta_{c}=1
$$

where $\zeta_{f}$ and $\zeta_{c}$ are fractions of damage fatigue and creep, respectively.

If the material is subjected to $n_{i}$ cycles under $\Delta \sigma_{i}$ stress variation and the number of cycles to fail under this stress is $N_{i}$, the fraction of fatigue damage is $\sum n_{i} / N_{i}$, where summation is performed for the total number of stress blocks with different amplitudes. Likewise, if $t_{j}$ is the average time for an applied average stress $\sigma_{j}$ and $t_{R j}$ is the time to fracture at that level of stress, then $\sum t_{j} / t_{R j}$ indicates the amount of accumulated creep damage. For variable amplitude loadings at high temperatures, the damage sum model is rewritten as follows:

$$
\sum \frac{n_{i}}{N_{f l}}+\sum \frac{t_{j}}{t_{R j}}=d
$$

where $d$ is the fraction of damage accumulated, which is usually equal to 1 .

This approach is widely used in low-cycle fatigue tests in dwell periods. However, Batte (1983) found at least three problems in implementing the concept of linear damage sum: 
i. In periods of permanence under constant strain, stresses relax continuously;

ii. Cyclic softening and hardening lead to different stress relaxation levels during fatigue strain; and

iii. Rupture stress results, employed in life prediction by means of Equation 12, are obtained through monotonic tests, which do not usually represent creep-fatigue behavior.

\subsubsection{Modified-Frequency Coffin-Manson Equations}

The strain-life relationship for modified frequency was proposed by Coffin (1973), considering the effect of frequency on cyclic response at high temperatures. This relationship incorporates phenomenologically the term frequency $\left(v_{c}\right)$ into the Coffin-Manson relationship given by Equation 4 as follows:

$$
\Delta \varepsilon_{p}=c_{f}\left(N \cdot v_{c}^{k-1}\right)^{-\beta}
$$

where the constant $c_{f}$ and the exponents $k$ and $\beta$ are specific to the temperature-materialenvironment system.

The strain-life behavior of many steels is satisfactorily described as an alternative to Equation 14, in which the deformation variation is separated into elastic and plastic components as in Equation 8:

$$
\Delta \varepsilon_{\mathrm{t}}=\Delta \varepsilon_{\mathrm{e}}+\Delta \varepsilon_{\mathrm{p}}=c_{f 1}\left(N \cdot v_{c}^{k-1}\right)^{-\beta 1}+c_{f 2}\left(N \cdot v_{c}^{k-1}\right)^{-\beta 2}
$$

The employment of Equations 14 and 15 to predict life under fatigue-creep requires knowledge of strain-stress loading hysteresis.

\subsubsection{Strain-Variation Partitioning Model}

The strain-variation partitioning methods proposed by Halford et al. (1973) divide the total damage caused by thermo-mechanical fatigue interactions into mechanical elements and those dependent on strain-variation time. Suresh (1998) claims that, on a microscopic scale, cyclic sliding is considered as the mechanism for time-independent mechanical fatigue, and cavitation and sliding of grain boundaries are considered as primary mechanisms affecting creep-fatigue damage. Methods of strain-variation partitioning also take into account the 
reversal of strain associated with fatigue and creep. In this method, any complete cycle of inelastic deformation variation is divided into four components:

i. $\quad \Delta \varepsilon_{\mathrm{pp}}$, plastic deformation in reverse strain-stress;

ii. $\Delta \varepsilon_{\mathrm{cp}}$, creep deformation in tensile and plastic deformation in compression;

iii. $\Delta \varepsilon_{\mathrm{pc}}$, plastic deformation in tension and creep in compression, and

iv. $\Delta \varepsilon_{\mathrm{cc}}$, creep deformation in reverse tension-compression. Figure 33 illustrates the method of strain-variation partitioning.
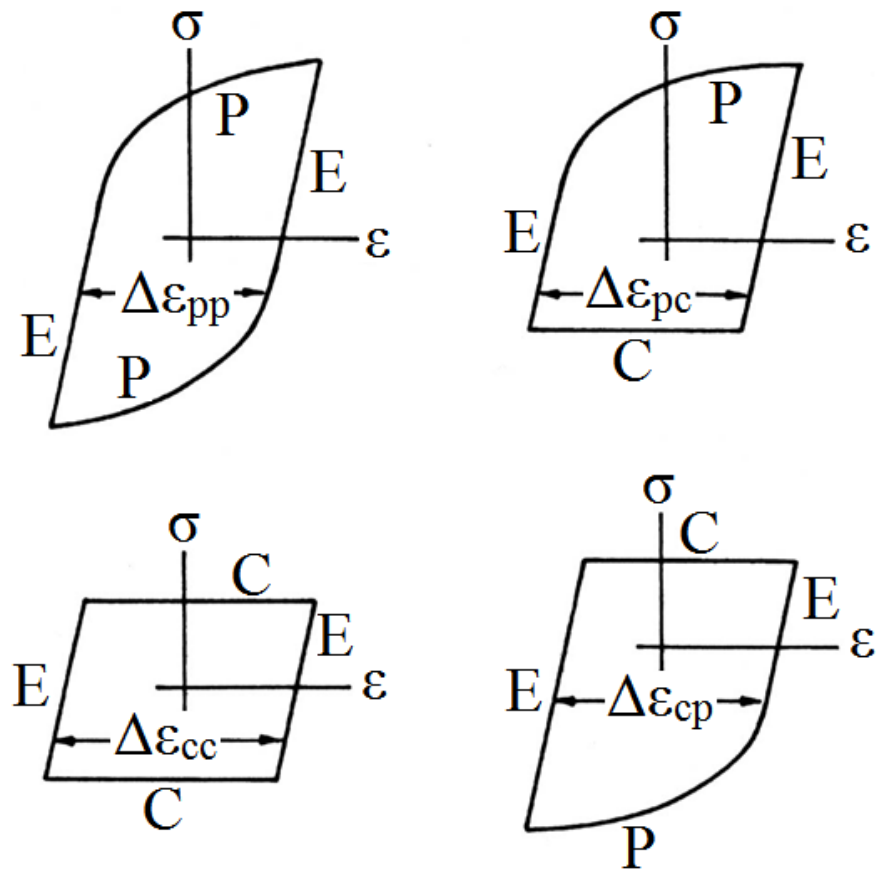

Figure 33: Schematic representation of inelastic deformation variation partitioned into four components, E, P, and C representing elastic, plastic, and creep deformations, respectively (Suresh, 1998).

Cyclic strength is associated with the four components of deformation variation by an equation of the type of Coffin-Manson potency law:

$$
\Delta \varepsilon_{p p}=c_{p p} \cdot N_{p p}^{-\beta 1}, \Delta \varepsilon_{c p}=c_{c p} \cdot N_{c p}^{-\beta 2}, \text { etc }
$$

Figure 34 shows the fatigue-life relationships for strain-variation partitioning.

The total life is thus determined by the sum of fractions of the four deformation modes, as follows:

$$
\frac{1}{N_{f}}=\frac{1}{N_{p p}}+\frac{1}{N_{c p}}+\frac{1}{N_{p c}}+\frac{1}{N_{c c}}
$$

where indices denote the same strain-variation modes. 
The damage fractions are sometimes measured in proportion to the deformationvariation magnitude of every mode. Several empirical modifications of this method are available in order to incorporate effects of slow-fast loading fatigue (including effects of different stress-strain paths in the cycle) and fatigue-creep dwell time in the estimation of fatigue life.

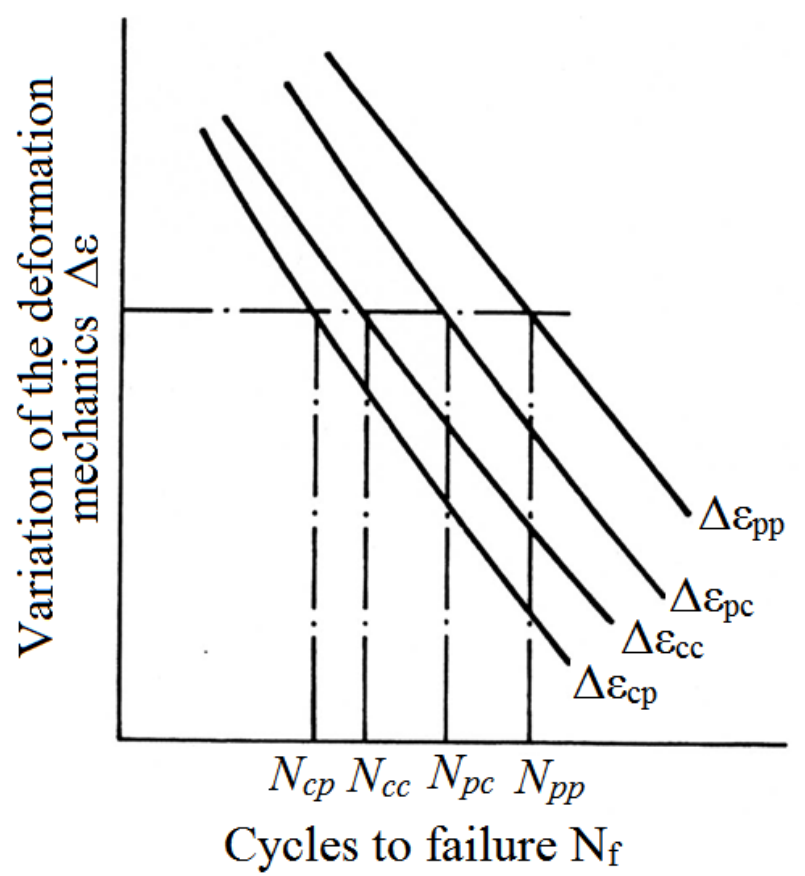

Figure 34: Schematic representation of fatigue-life relationships for the partitioning of deformation variation (Suresh, 1998).

\subsubsection{Diverse Models}

According to Shi et al. (1993), some life-prediction models based on low-cycle isothermal fatigue have been employed under thermo-mechanical conditions, such as the deformation variation partitioning model, the deformation energy parameter model, the constitutive model, and the thermal activation model. These models generally make use of the linear damage theory and estimate the damage per cycle in the stabilization period using the parameter of damage to plastic deformation or strain energy density. Shi and colleagues (1993) and Shi and Pluvinage (1993) investigated the TMF strength of 316L stainless steel and proposed firstly a model of nonlinear kinematic hardening using internal variables to describe cyclic stress-strain behavior. Then, they established a model to estimate the damage to the material from the characteristics of stabilized cycles. From isothermal fatigue data, they 
deduced a an equivalent model of variation of strain energy density as damage parameter with which TMF life was predicted satisfactory as compared to test results.

Goals and Ellyin (1988) presented a unified theory based on the criterion of cyclic strain energy density. It was shown that fatigue failure under high and low cycle, cumulative damage, and loading sequence can be expressed in terms of a single damage parameter. The damage criterion is based on the strain energy density per cycle (sum of energy of plastic and elastic traction deformation). The stages of initiation and propagation of fatigue cracks were incorporated into this model. The comparison to experimental data was satisfactory.

As indicated by Cai et al. (1999), the two most widely used models to predict life of various types of metallic materials subjected to TMF solicitation are models of damage accumulation and strain variation partitioning. However, these models must undergo some changes in order to satisfy the anisothermal solicitation condition.

The damage mechanisms present in TMF of various metals involve three main aspects involved in anisothermal solicitation: failure caused by fatigue, environment (oxidation), and creep. Depending on the temperature, deformation, and phase between the temperature and strain cycles, all three mechanisms can operate jointly, according Neu and Sehitoglu (1989b). The fatigue damage mechanism controls the life of metals under thermo-mechanical solicitation conditions when temperatures are low, so the contribution of oxidation mechanisms of creep on life may be neglected, according to Falcão (2002).

In an out-of-phase TMF test, in which the highest compressive stresses coincide with the highest temperatures during the cycle, oxidative damage may be significant whereas creep failure is negligible because growth of voids and inter-granular cracking is suppressed under compression. In this case, life is determined by oxidation.

Neu and Sehitoglu (1989a, 1989b) developed a general model for fatigue at elevated temperatures, including thermo-mechanical fatigue. This model incorporates accumulated damage due to fatigue, environmental attack (oxidation), and creep. The damage per cycle of these mechanisms are added up to obtain the total damage per cycle.

$$
D^{t}=D^{f}+D^{o x}+D^{c}
$$

Assuming that linear damage is equal to 1 in the failure, Equation 18 may be rewritten in terms of life $(\mathrm{N})$, in which damage is equal to $1 / \mathrm{N}$ :

$$
\frac{1}{N}=\frac{1}{N^{f}}+\frac{1}{N^{o x}}+\frac{1}{N^{c}}
$$


According to Cai et al. (1999), due to its complexity, a well-accepted framework for predicting TMF life has been rather vague. Several proposed models are but non-isothermal generalizations deriving from isothermal models. These models may be divided into two types:

1. General models for predicting life under TMF based on fundamental physical mechanisms of crack initiation and propagation under TMF. Such models may be used to capture and simulate damage mechanisms and their interactions under TMF loading conditions. These models should be based on data about several materials, including analyses and micro-structural observations, and generally associated with visco-plastic constitutive models. Nevertheless, the formulas or set of equations for these models are complex and not convenient to engineering application.

2. Empirical and engineering models have goals and direct applications aimed at engineering practice. They are intended for special materials to which the TMF life prediction model was established. The formulas of this model are simpler and more convenient for applications. However, they are not in common use among several materials.

\subsubsection{Modeling of Anisothermal Hysteresis as a Function of Isothermal Data}

Determining the behavior of anisothermal loading hysteresis using results from isothermal stress-strain curves is of great importance to life prediction models in TMF tests.

Cyclic behavior in low-cycle fatigue can be represented in three ways: (1) by analyzing cyclic hardening or softening; (2) through stress-strain hysteresis (mechanical or inelastic); or (3) by means of cyclic hardening curves. The difference between the latter two methods is that the cyclic hardening curve is represented by extreme stress and strain values obtained in several tests superimposed on a single graph. Stress-strain profiles thus obtained are not always individual hystereses, obtained during a test conducted within a fixed deformation amplitude.

Typically, cyclic strain hardening curves as well as stress-strain hystereses can be represented by a potency relationship, as suggested by Ramberg-Osgood, firstly for a monotonic solicitation: 


$$
\sigma=A_{m}\left(\varepsilon_{i n}\right)^{\beta}
$$

This equation may be adapted to cyclic loading:

$$
\frac{\Delta \sigma}{2}=A_{m}\left(\frac{\Delta \varepsilon_{i n}}{2}\right)^{\beta}
$$

where $A_{m}$ and $\beta$ are constants of the material, varying with temperature.

Through interpolation of results obtained isothermally it is possible to determine the values of these constants for the temperature range of the anisothermal cycle.

One of the problems of using an equation such as the Ramberg-Osgood equation to simulate an individual hysteresis is that, in the case of cyclic loading, the constants $A_{m}$ and $\beta$ tend to vary depending on the extent of plastic deformation imposed on the material. For this reason, it is unusual to find in the literature the employment of this equation as representing hysteresis itself, according Skelton (1994).

Another complication arising from the use of the Ramberg-Osgood equation is the absence of a purely elastic regime, since all applied stresses would result in deformation, however small. This gradual transition from elastic regime to plastic regime makes it particularly difficult to model the Bauschinger effect, whereby plastic deformation reduces the elastic limit in compression and vice-versa.

Skelton (1994) describes three methods employed to model anisothermal hysteresis: a graphical method, a tangent modulus method, and a secant modulus method. The graphical method is most used in the literature due to its better suitability.

\subsubsection{Graphic Method Modeling}

A simplified modeling of isothermal behavior is possible through approximation to cyclic behavior by a linear plastic-elastic model, whereby the relationship between strain and stress in the plastic regime is given by an equation of the following type:

$$
\sigma=\sigma_{e}+E_{p p} \cdot \varepsilon_{i n}, e m \text { tração }
$$

or else:

$$
\sigma=-\sigma_{e}+E_{p p} \cdot \varepsilon_{i n}, \text { em compressão }
$$

where $\sigma_{e}$ is the yield limit, $E_{p p}$ is the approximate slope of the hardening curve on a inelastic stress-strain graph, $\sigma$ is stress and $\varepsilon_{i n}$ is inelastic deformation (which is used instead of plastic deformation as it is a more suitable denomination for tests at high temperatures). 
These two equations define an upper boundary (traction) and a lower boundary (compression) of plastic deformation. The material behavior in the area between these two equations is purely elastic, governed by Hooke's law:

$$
\sigma=E\left(\varepsilon_{m e c}-\varepsilon_{i n}\right)
$$

where $E$ is the elasticity modulus and $\varepsilon$ is the total mechanical deformation.

In this case, the Baushinger effect is automatically taken into account, since the vertical distance between the two inelastic equations is fixed and equal to $2 \sigma_{\mathrm{e}}$ for any given temperature, as shown in Figure 35.

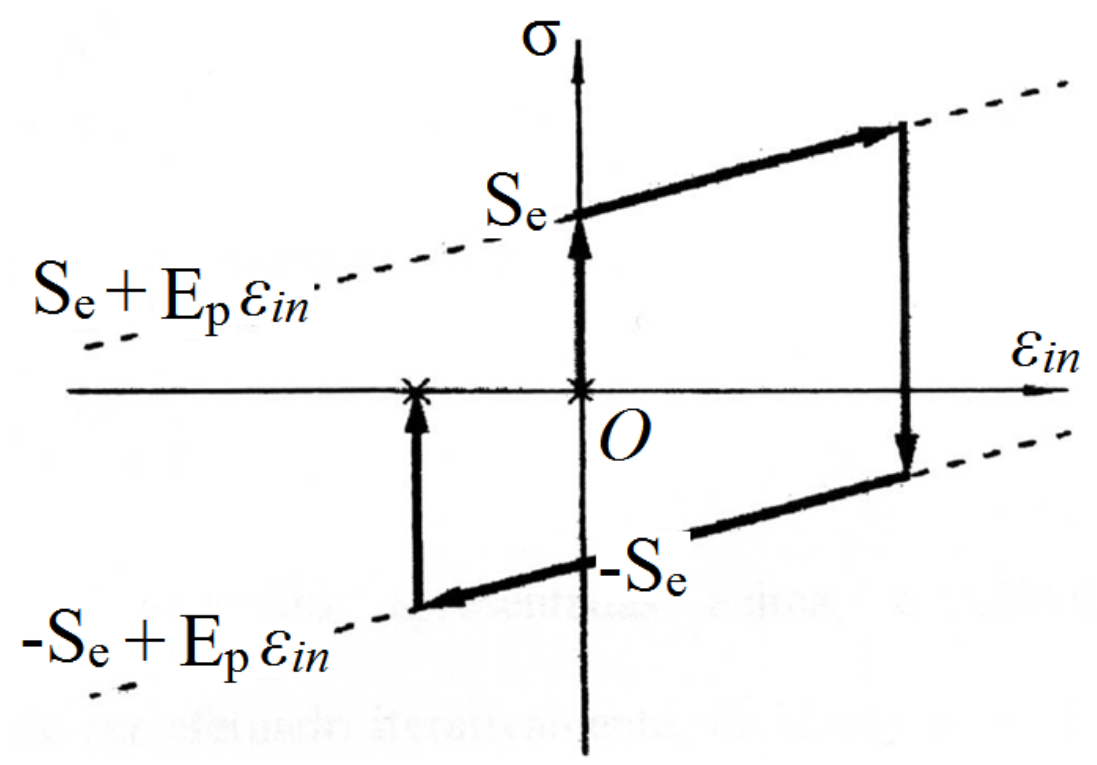

Figure 35: Schematic representation of difference between two inelastic equations (Engler \& Spinelli, 2002).

Equations for the plastic regime may also be represented in relation to total mechanical deformation $(\varepsilon)$ :

$$
\begin{gathered}
\sigma=S_{p}+E_{p} \cdot \varepsilon, \text { em tração } \\
\sigma=S_{p}-E_{p} \cdot \varepsilon, \text { em Compressão }
\end{gathered}
$$

where $E$ is the scaling constant for the plastic regime. $S_{p}$ and $E_{p}$ are related to values obtained with the aid of the inelastic stress-strain curve.

According to the model proposed by Engler and Spinelli (2002), $\sigma_{\mathrm{e}}$ and $\mathrm{E}_{\mathrm{pp}}$ are determined so as dissipated plastic energy (area under the curve) is the same as that in the model proposed by Ramberg-Osgood.

The elastic limit used in the model proposed by Engler and Spinelli (2002) is described by the following equation: 


$$
\sigma_{e}=A_{m}\left(\varepsilon_{\text {in }}\right)^{\beta-1}\left(\frac{1-\beta}{1+\beta}\right)
$$

and the scaling constant between $\sigma$ and $\varepsilon_{\text {in }}$ :

$$
E_{p p}=A_{m}\left(\varepsilon_{i n}\right)^{\beta-1}\left(\frac{2 \beta}{1+\beta}\right)
$$

According to the above equations, the estimation of anisothermal hystereses may be done iteratively by dividing the thermo-mechanical $(\varepsilon(\mathrm{T}))$ into $\mathrm{N}$ periods and estimating the inelastic strain and stress for each point.

The model proposed by Engler and Spinelli (2002) provides a satisfactory representation of loading hysteresis behavior for small inelastic deformations.

\subsubsection{Thermo-Mechanical Fatigue Modeling for Nucleation of Cracks}

The development of numerical simulations in the past 20 years has promoted the improvement of numerical tools and the development of new algorithms. It is possible today to estimate loads and displacement in structures rapidly and reliably. Nonetheless, studies on structures subjected to multi-axial thermo-mechanical loading are still rare.

Only a small number of automotive components undergo severe cyclic thermomechanical loading capable of producing low-cycle fatigue. Most of these components are found in engineering: engine head; engine exhaust; carter; brake disc, and so forth. It is important to note that problems caused by low-cycle fatigue to these components are related to the startup-shutdown-startup cycle, not to combustion cycles.

The process of thermo-mechanical fatigue (TMF) is quite complex, involving fracture mechanism due to creep and plastic deformation, which tend to be mainly influenced by the solicitation type and cyclic changes in the material microstructure of the material in operation, such as oxidation and phase transformation (Sermage, 2000; Neu, 1989). Models that attempt to predict the life of a component subjected to thermo-mechanical fatigue are scarce, derive mostly from isothermal models, are adapted to particular cases, and cannot be generalized to other situations (Shi, 1993). Basically, the TMF modeling process should incorporate microstructural features (which are responsible for the material mechanical strength within the applied temperature range), thermal aspects (analysis on the flow and thermal distribution present in the component), mechanical aspects (deformation and fracture mechanisms), and geometric aspects (knowledge about stress distribution in the component under solicitation). Two types of approach have been used to understand the TMF process: first, at the 
microscopic level, by modeling crack initiation and propagation, and secondly, by taking the material macroscopic behavior into account. The latter is more widely used and aimed at simulating the response given by a material under thermal and mechanical stresses. In this case, there have been employed continuum mechanics models as well as elasto-plastic and visco-plastic models (Oller, 2005; Constantinescu, 2004; Charkaluk, 2002).

Predicting the life of a given component subjected to thermo-mechanical loading requires the knowledge (mapping) of the distribution of temperatures and stresses in its volume (when in operation. In this context, one should begin by conducting a thermal analysis on the component, which should simulate the flow of fluids (if present), allowing for heat exchanges with the structure, and simulate the cyclic heating and cooling throughout the component. The temperature evolution may be determined by the following equation:

$$
\rho c\left(\frac{\partial T}{\partial t}\right)-\nabla \cdot k \nabla T=0
$$

where $\rho$ is density, $c$ is specific heat, and $k$ is thermal conductivity.

In order to solve this equation three boundary conditions are need: internal surface, external surface, and conduction on contact surface (adjacent components).

Thermal analysis is considered an important step in TMF modeling, because accuracy in temperature-related data requires accuracy when simulating the component mechanical behavior (Thomas, 2004).

The analysis of the behavior of a structure subjected to thermo-mechanical solicitation has been conducted, in this case, by means of an elastoviscoplastic model. This type of model has proven to be simpler in plotting of stress-strain curves during cycles, because hypotheses are assumed that eliminates many variables that would make the problem much more complex. Moreover, the elastoviscoplastic model is valid for the component entire operating temperature range. The main assumptions in this model are: (a) as the material cyclic behavior is stabilized, the initial transient, which can show material hardening or softening under solicitation, is disregarded; (b) the damage-related parameters may be overlooked in the constitutive law; therefore, there is decoupling in the approach to mechanical behavior with damage variables. Below are shown the equations of two elastoviscoplastic models proposed:

- Classic Visco-plastic Model combined with linear kinematic hardening, according to Chaboche (1997).

$$
\begin{gathered}
\varepsilon_{e l}=\frac{1+v}{E} \sigma-\frac{v}{E} \operatorname{tr}(\sigma) I \\
\sigma=\frac{3}{2} \frac{1}{H} X
\end{gathered}
$$




$$
\begin{gathered}
\dot{\varepsilon}_{v p}=\frac{3}{2}\left(\frac{\mathfrak{I}_{2}(\sigma-X)-\sigma_{y}}{\eta}\right)^{m} \frac{s-X}{\mathfrak{I}_{2}(s-X)} \\
\dot{\alpha}=\dot{\varepsilon}_{v p} \\
\sigma=\epsilon:\left(\varepsilon-\varepsilon_{v p}\right)
\end{gathered}
$$

where: $\varepsilon_{\mathrm{el}}=$ elastic deformation tensor, $v=$ Poisson modulus, $\mathrm{E}=$ elastic modulus, $\mathrm{X}=$ stress tensor (hardening), $\mathrm{H}=$ strength modulus, $\varepsilon_{v p}=$ visco-plastic deformation tensor, $\eta=$ viscosity, $\mathrm{s}=$ stress deviation tensor, $\sigma_{\mathrm{y}}=$ yield stress, $\sigma=$ deformation tensor (hardening), $\mathfrak{I}_{2}=2^{\text {nd }}$ stress tensor invariant, $\in=$ compliance tensor, and $\mathrm{m}=$ viscosity exponent.

- Two-Layered Visco-plastic Model, in which stress is divided into viscous and plastics elements, according to Kichenin (1992).

$$
\begin{gathered}
\varepsilon=\varepsilon_{v}^{e l}+\varepsilon_{v} \\
\varepsilon=\varepsilon_{p}^{e l}+\varepsilon_{p} \\
\alpha=\frac{3}{2} \frac{1}{H} X \\
\varepsilon_{v}^{e l}=\frac{1+v}{K_{v}} \sigma_{v}-\frac{v}{K_{v}} \operatorname{tr}\left(\sigma_{v}\right) I \\
\varepsilon_{p}^{e l}=\frac{1+v}{K_{p}} \sigma_{p}-\frac{v}{K_{p}} \operatorname{tr}\left(\sigma_{p}\right) I \\
\dot{\varepsilon}_{v}=\frac{3}{2}\left[\frac{\mathfrak{I}_{2}\left(\sigma_{v}\right)}{\eta}\right]^{m} \frac{S_{y}}{\mathfrak{I}_{2}\left(\sigma_{y}\right)} \\
\dot{\varepsilon}_{v}=\gamma \frac{S_{p}-X}{\mathfrak{I}_{2}\left(\sigma_{p}-X\right)} \\
\dot{\alpha}=\dot{\varepsilon}_{p} \\
\sigma_{p}=K_{p}:\left(\varepsilon-\varepsilon_{p}\right) \\
\sigma_{v}=K_{v}:\left(\varepsilon-\varepsilon_{v}\right) \\
\sigma_{v p}=\sigma_{p}+\sigma_{v}
\end{gathered}
$$

where: $\mathrm{K}_{\mathrm{p}}$ and $\mathrm{K}_{\mathrm{v}}=$ elastic modulus tensor for plastic and elastic elements, respectively, and $\gamma=$ plastic multiplier.

The material parameters, embedded in the elastoviscoplastic model (e.g., modulus, viscosity, and yield stress) may be obtained through mechanical tests of "tensioncompression-relaxation" performed isothermally at different temperatures. In these cases, the parameters are assumed to show linear variation with temperature.

Numerical simulation (EF) is a useful tool for solving equations specified in the aforementioned model. The "ABAQUS FINITE ELEMENT STANDARD CODE" program 
has been widely used in these cases. Three algorithm types have been tested: forward EULER, backward EULER, and backward EULER with a radial return (Simo, 1998), the latter producing the best results in less time under anisothermal loading (Charkaluk, 1999).

After conducting thermal and mechanical analyses, the third step in TMF modeling consists of selecting a fatigue criterion. This step has been considered challenging due to the anisothermal and multi-axial nature of the solicitations in this case. Two of the fatigue criteria are the relationship of a damage function (indicator) and the number of cycles to fail. The functions set for classic fatigue criteria (e.g., Manson-Coffin, Smith-Watson-Topper, deformation variation partitioning), which include parameters such as total deformation amplitude, maximum stress, Young's modulus, and material-dependent constants, have been proved inadequate for thermo-mechanical fatigue, mainly due to the difficulty in determining these parameters in case of multi-axial thermo-mechanical loading (Constantinescu, 2004; Charkaluk, 2002; Thomas, 2004). Therefore, other ways were sought to represent the damage function. In this context, energy dissipated per cycle $(\Delta \mathrm{w})$ has been chosen to make this representation. The expression that defines $\Delta \mathrm{w}$ is shown below, being applied (valid) when stress-strain cycles are stabilized.

$$
\Delta w(x)=\int_{\text {ciclo }} \sigma(x, t) \varepsilon(x, t) d t
$$

where $\sigma$ and $\varepsilon$ are stress and strain tensors, respectively, and $x$ indicates the point analyzed in the structure.

The energy dissipated per cycle may be considered a material parameter that expresses the energy required for crack propagation in the process zone. There are three advantages to using this parameter, according to Thomas (2004): (a) energy quantification can be generalized to multi-axial loading situations; (b) its determination is independent of temperature; and (c) $\Delta \mathrm{w}$ is representative of the material cyclic behavior. Regarding the number of cycles to fail $\left(\mathrm{N}_{\mathrm{f}}\right)$, it has been proposed that the number of cycles considered bordering $\left(\mathrm{N}_{\text {critical }}\right)$ is that at which the first (macroscopic) crack is seen in the material, or when there is a decrease of about $10 \%$ in load (Constantinescu, 2004). Finally, the fatigue criterion may be expressed as follows:

$$
\Delta w \cdot N_{\text {critical }}=C
$$

\subsection{Fatigue Crack Growth}


In the past, studies on fatigue focused on damage processes and crack development. From the 1960s onward interest shifted to crack growth processes (Forsyth, 1963), due to the fact that fatigue crack growth does not occur catastrophically (there is sub-critical crack growth, which may be halted under certain circumstances) and that crack nucleation occurs in a small part of the fatigue life. Cracks are usually formed very early in a material fatigue life (Fuchs \& Stephens, 1980).

Thus, the primary characteristic of fatigue failure is the gradual spread of a crack, caused primarily by oscillation of loads applied to the component. Geometric cracks are defects that resemble slots with very small tip radius, ideally zero. For this reason, it is not possible to apply the traditional stress analysis to cracks, because its stress concentration factor $(\mathrm{K})$ is very large.

However, propagation of fatigue cracks can be effectively dealt by traditional concepts of Fracture Mechanics, which show that the propagation rate da/dN or how much a crack grows per loading cycle depends primarily on the variation range of the stress intensity factor $\Delta \mathrm{K}$ applied. This became possible thanks to Paris and Erdogan (1963), who related the propagation rate of a fatigue crack to the stress intensity factor. By using mathematical tools of Fracture Mechanics to quantify the primary characteristic of fatigue failure, the slow growth of a crack in every loading cycle, Paris introduced the first truly innovative idea since Wöhler, representing an important milestone in the history of research on fatigue.

This method of analysis, called the Law of Paris, predicts a parabolic relationship between $\mathrm{da} / \mathrm{dN}$ and $\Delta \mathrm{K}$ (Equation 34) and is suitable to describe the region where crack growth rate is linear.

$$
\frac{d a}{d N}=C \cdot(\Delta K)^{m}
$$

where $\mathrm{C}$ are $\mathrm{m}$ constants dependent on the material, environment, frequency, temperature, and stress ratio.

Exponent $\mathrm{m}$ for metallic materials is approximately 4 (four), which may be rationalized as accumulated damage and associated with the plastic zone volume $\left(\mathrm{V}_{\mathrm{p}}\right)$, since volume $V_{p}$ is estimated with $r_{p}^{2}$ and that $r_{p} \propto K_{I}^{2}$, then $d a / d N \propto \Delta K^{4}$. Some $C$ and $m$ values for several alloys are presented in Table 4. 
Table 4: Numerical parameter for Paris equation.

\begin{tabular}{c|c|c}
\hline Metallic Alloy & $\boldsymbol{m}$ & $\boldsymbol{C}$ \\
\hline Steel & 3 & $10^{-11}$ \\
Aluminum & 3 & $10^{-12}$ \\
Nickel & 3.3 & $4 \times 10^{-12}$ \\
Titanium & 5 & $10^{-11}$ \\
\hline
\end{tabular}

The typical curves of fatigue crack propagation, when plotted on a graph (Figure 36) as $\log (\mathrm{da} / \mathrm{dN})$ in relation to $\log (\Delta \mathrm{K})$, show a characteristic sigmoidal shape with three distinct phases: (1) initial phase or Region I, with decreasing derivative; (2) intermediate phase or Region II, with constant derivative and linear behavior; and (3) final phase or Region III, with increasing derivative.

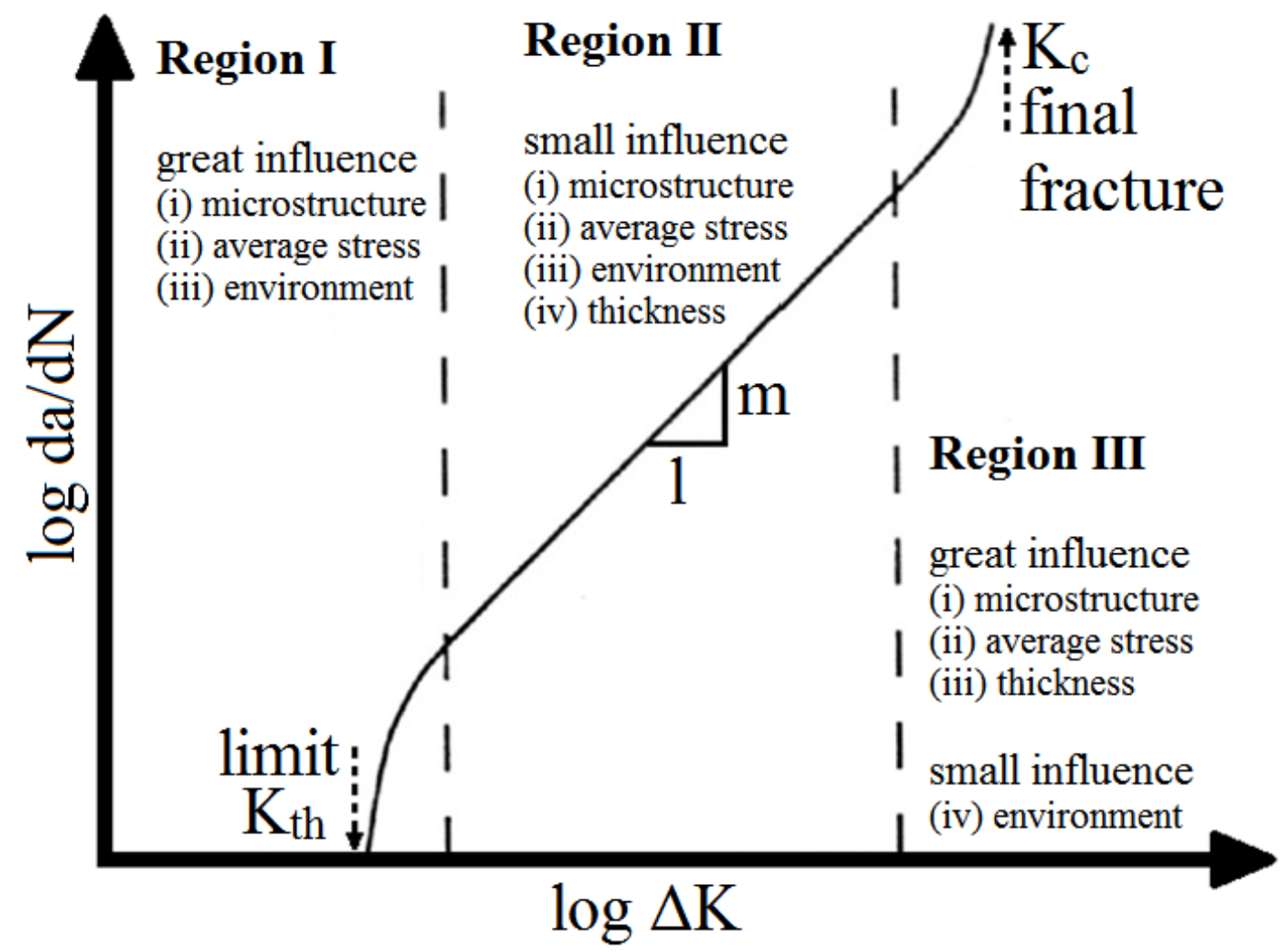

Figure 36: Curve representing $\mathbf{d a} / \mathbf{d} \mathbf{N}$ in relation to $\Delta \mathbf{K}$.

Therefore, there are three distinct areas or stages (Figure 37), in which the Law of Paris and Erdogan is only valid for the intermediate region of crack growth, i.e., Region II. 
Region I, with crack propagation rate underestimated by the Law of Paris and Erdogan, is characterized by the existence of an inferior limit $\left(\mathrm{K}_{\mathrm{th}}\right)$ below which there is no crack propagation or propagation occurs at an undetectable rate for practical purposes. This phase is very sensitive to average load, material microstructure, and environment.

Region III, of unstable crack propagation (typically between 10-8 and 10-6 m/cycle), is important to characterize the component final failure, but contributes little to its life under fatigue (expressed in number of cycles). This region tends to display static fracture mechanisms: ductile mechanisms (e.g., cavitation and void/micro-cavity coalescence) and fragile mechanisms (e.g., cleavage), the propagation rate being underestimated by the Law of Paris. This curve deviation occurs when $\mathrm{K}_{\max }$ reaches toughness to fracture as $\mathrm{K}_{\max }$ reaches values close to those of $\mathrm{K}_{\mathrm{C}}$ and $\mathrm{K}_{\mathrm{IC}}$ of the material.

Region II, or intermediate region of crack growth, the Paris-Erdogan equation describes well the growth of a crack with exponent $\mathrm{m}$ ranging typically between 2 and 5 . Crack growth is controlled by continuous mechanisms, insensitive to the microstructure, average load, environment, and thickness of components. From the fractographic standpoint, it is in this region that stretch marks are observed on the surface characteristic of fatigue.

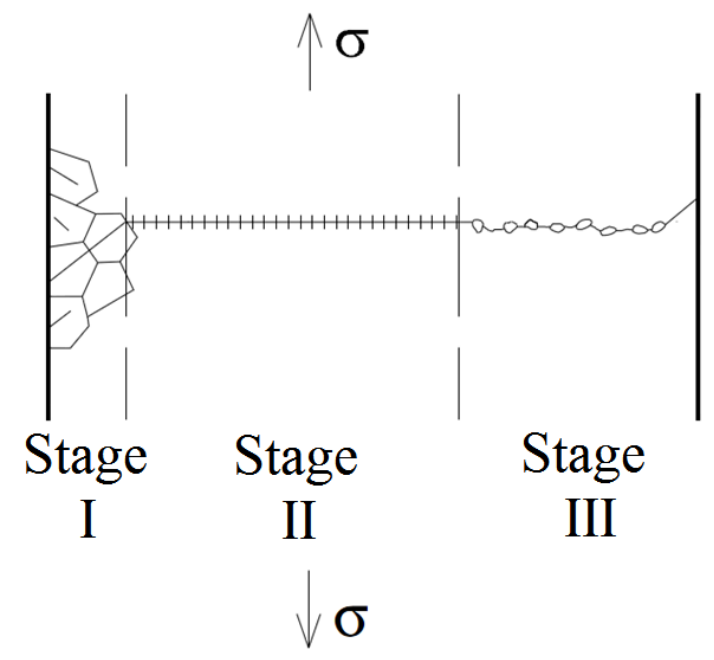

(a)

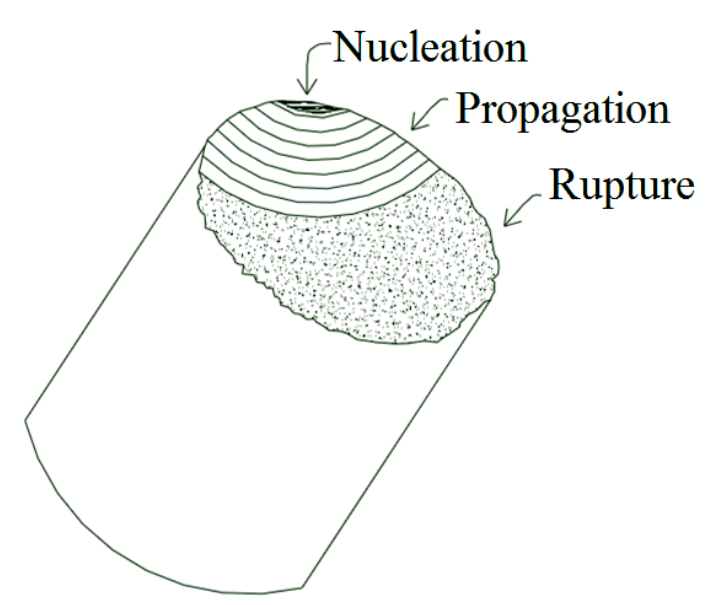

(b)

Figure 37: Propagation stages of a fatigue crack.

The observation of fatigue fracture surface of ductile materials often reveals the presence of grooves perpendicular to the direction of crack propagation (Figure 38a). Laird (1967) and Pelloux (1969) were the first to explain the occurrence of these grooves by successive crack blunting and sharpening due to plasticity. In order to understand the mechanism proposed by Laird one should follow the faces of a crack during a fatigue cycle (loading and unloading) (Figure 38b). At the beginning of loading, face displacement is done 
by a simple elastic opening. As loading increases, a plastic zone forms in the region ahead of the crack. It becomes less acute as the load increases and is accompanied by the creation of new surfaces of plasticity. At the beginning of unloading, the crack closes elastically and as the distant regions far from it are plastic, at zero load the deformation imposed on the plastic zone is zero. Therefore, the crack tip undergoes retained plastic deformation. This deformation occurs at the end of the cycle, when the crack is almost closed and cannot make up for the plastic deformation that occurred when it was opened. Moreover, due to chemical reactions with air, the newly-created free surfaces are irreversible. The crack propagates, thus, to a length approximately equal to the rounding radius. In this propagation mechanism, the length of a crack derives from a cyclic plastic deformation in the region ahead of it and, therefore, the material cyclic plastic behavior plays an important role in propagation.

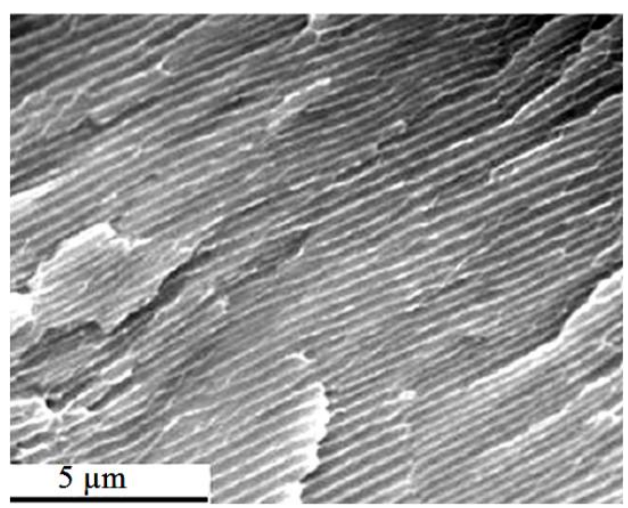

(a)

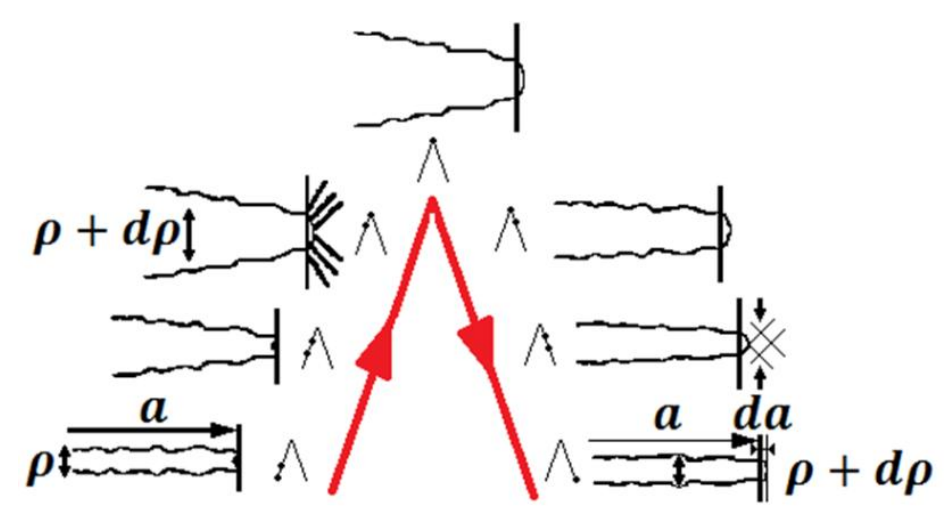

(b)

Figure 38: (a) Fatigue grooves observed on rupture surface and (b) mechanism of propagation by rounding and sharpening of the crack tip.

Plastic blunting $\rho$ measures deformation at the tip of a crack. Taking into account assumptions about displacement fields, $\rho$ may be understood as a permanent part of CTOD (Crack Tip Opening Displacement). It is generally accepted that CTOD is the parameter that governs fatigue crack propagation under constant and variable loading (Masahiro, 2004). The relationship between CTOD and crack propagation has been the subject of several studies that propose propagation scenarios from fractographic observations (Laird, 1967; Pelloux, 1969).

According to this scenario, it is possible to note that there is a strong link between crack length and surface opening. The law that equates these magnitudes is that of CTDO, proposed by McClintock (1967), which predicts crack propagation for loading of monotonic amplitude.

$$
\frac{d a}{d N}=\triangle C T O D
$$

For a material with perfect elasto-plastic behavior, $\triangle \mathrm{CTOD}$ is given by: 


$$
\triangle C T O D=\beta \cdot \frac{\Delta K^{2}}{2 R_{e} E}
$$

where $\beta$ is a scaling factor, $E$ is the elastic modulus, and $R_{e}$ is the yield stress of the material.

This last equation assumes a relationship between $\triangle$ CTOD and plastic zone size. In fact, the term $\Delta \mathrm{K}^{2}$ derives from the plastic zone size. Thus, in this case the Paris Law exponent is equal to 2 (two). Nebdal and colleagues (1989) proposed an empirical relationship between crack advancement and fatigue groove pitch, which is proportional to plastic rounding. This relationship was obtained by dividing the measured rate of macroscopic crack propagation during the test by the microscopic rate corresponding to the groove pace $(\mathrm{S})$ measured on the fracture surface (Figure 39).
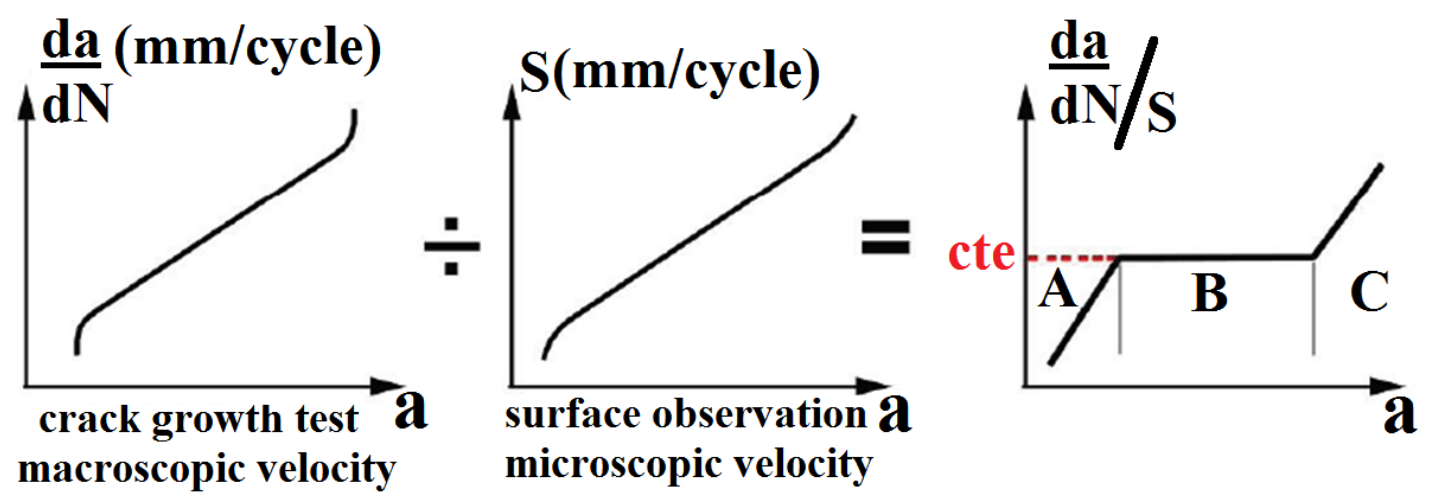

Figure 39: Relationship between groove paces and propagation rate.

The curve obtained comprises three regions corresponding to three Paris curve states. In Region A, macroscopic velocity is lower than microscopic velocity, because some cycles do not advance the crack (sterile cycles). The crack length in Region B corresponds to approximately one groove per cycle; this is the region in which the Law of Paris is valid. In Region $\mathrm{C}$, propagation rate is more than one groove per cycle, as other propagation mechanisms come into play such as ductile tearing. The proposed relationship (Equations 37 and 38) in this study for Region B is, thus, based on the relationship of Nebdal et al. (1989), but it is a function of time instead of cycle. This formalism allows the handling of cases with complex cycles at variable amplitude and can more easily take into account time and temperature-related effects on fatigue propagation.

$$
\begin{aligned}
& \begin{array}{lll}
\frac{d a}{d t}=\alpha \cdot \frac{d \rho}{d t} & \text { se } & \frac{d \rho}{d t}>0
\end{array} \\
& \begin{array}{lll}
\frac{d a}{d t}=-\alpha \cdot \frac{d \rho}{d t} & \text { se } & \frac{d \rho}{d t}>0
\end{array}
\end{aligned}
$$

where $\alpha$ is a parameter of the material identified with the assistance of a crack propagation test at constant amplitude. 
In order to establish the crack propagation rate at any given load, it suffices to determine the evolution of plastic rounding $(\rho)$ throughout loading.

Many empirical rules have been proposed to better describe the sigmoidal shape of the curve $\mathrm{da} / \mathrm{dN}$ in relation to $\Delta \mathrm{K}$ as well as the effects of some secondary variables, such as average load (usually quantified by $\mathrm{R}=\mathrm{K}_{\min } / \mathrm{K}_{\max }$ ) and crack opening load $\left(\mathrm{K}_{\mathrm{op}}\right)$. The best known rules are:

$$
\begin{array}{cc}
\frac{d a}{d N}=A \cdot\left(K_{\text {Max }}-K_{o p}\right)^{m}=A \cdot\left(\Delta K_{e f}\right)^{m} & \text { Elber } \\
\frac{d a}{d N}=A \cdot \Delta K^{m} \cdot K_{\text {max }}^{p} & \text { Walker } \\
\frac{d a}{d N}=A \cdot \Delta K^{m}\left(K_{\max }-\Delta K_{t h}\right)^{p} & \text { Hall } \\
\frac{d a}{d N}=\frac{A \cdot \Delta K^{m}\left(\Delta K-\Delta K_{t h}\right)^{p}}{(1-R) \cdot K_{C}-\Delta K} & \text { Forman (modificado) }
\end{array}
$$

All these rules require the determination of constants such as A, m, or p, which are obtained experimentally through fatigue crack propagation tests. Picking one of these rules depends on the particular set of data to which one wishes to apply it. In many instances, several of them may be used with satisfactory results (Chang \& Hudson, 1981).

\subsubsection{Effect of Porosity on Fatigue Crack Growth}

In most cases, fatigue cracks originate in pores irrespective of stress and deformation (Inguanti, 1985; Murali et al., 1997; Powell, 1994; Skallerud et al., 1993); most pores leading to cracks are located on the material surface or just below it (Couper et al., 1990; Seniw et al., 1997; Wigant \& Stephens, 1987). After crack nucleation, propagation chronology can be divided into four phases:

- Inter-dendritic crack propagation, alternatively in $\alpha$ matrix and along interfaces of silicon/matrix particles (Hoskin et al., 1988; Verdu et al., 1996);

- Intra-dendritic crack propagation (Powell, 1994)

- Rapid inter-dendritic crack propagation with damage to silicon particles ahead of crack (Crepeau et al., 1992; Plumtree \& Schafer, 1986);

- Final rupture. 
When the crack is still small and is in the vicinity of the pore, it is sometimes possible to note a decrease or even a halt in propagation with a special frequency of the order of SDAS (Evans et al., 1994).

The crack propagation mechanism may be divided into two distinct regimes corresponding to high and low stress levels. At high stress levels cracks propagate continuously in the material, i.e., they are not halted by micro-structural barriers and may be described by Paris law. At low stress levels, once nucleated, cracks propagate rapidly throughout the eutectic region until they reach a length of approximately $10-20 \mu \mathrm{m}$. Just a small amount of these cracks keep on propagating. When this happens, growth is discontinuous, with numerous halts and may last several thousand cycles. In the period between these halts, growth rate of surface cracks may be higher or lower than that observed in long cracks for the same stress intensity factor $\Delta \mathrm{K}$. These halts may be related to the presence of grain boundaries.

Topographic observations indicate that $85 \%$ of pores located on the material surface or right below it nucleate cracks. Figure 40 shows that the probability of crack nucleation from pores is neither necessarily related to the stress intensity factor nor to a maximum stress criterion.

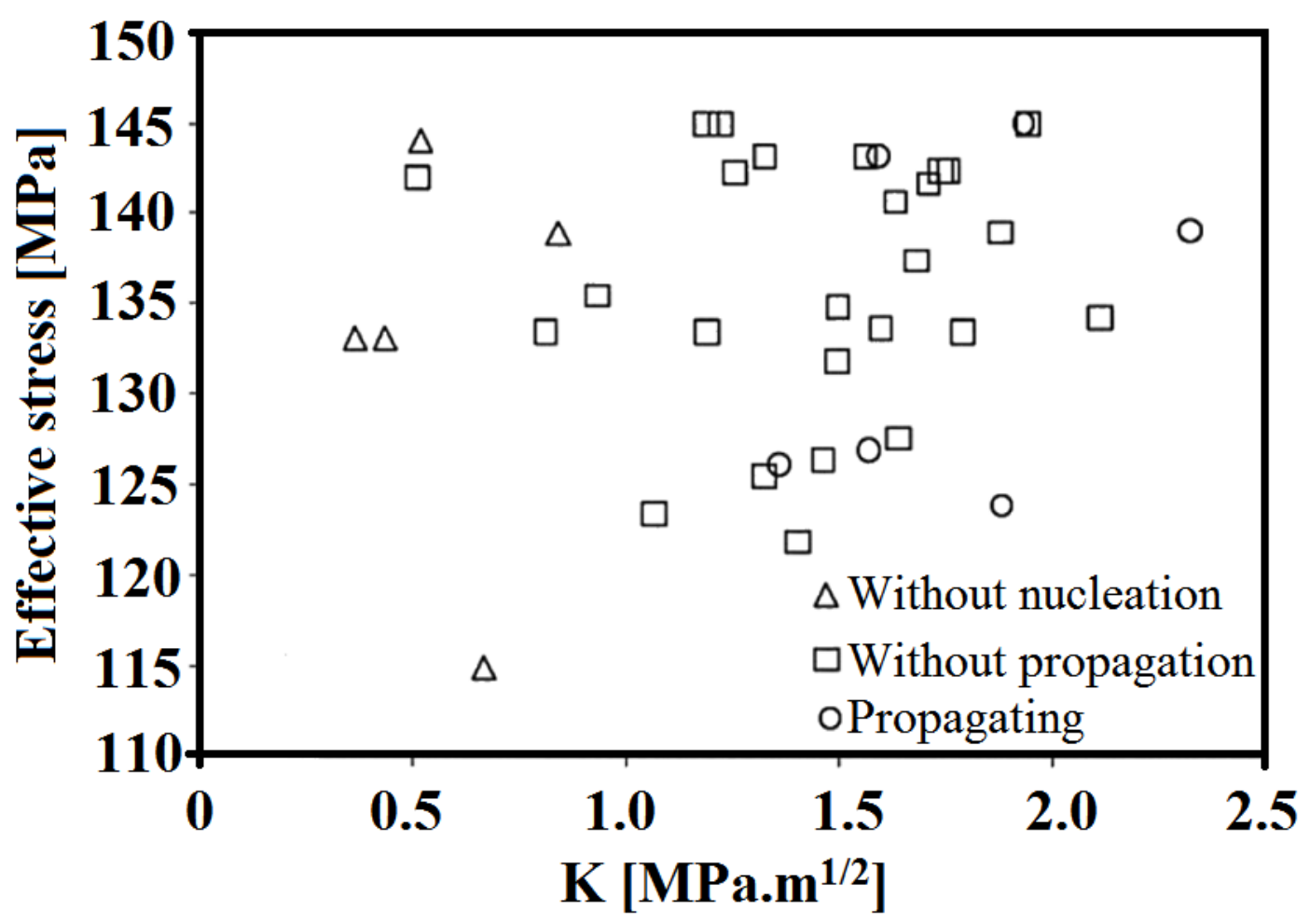

(a) 


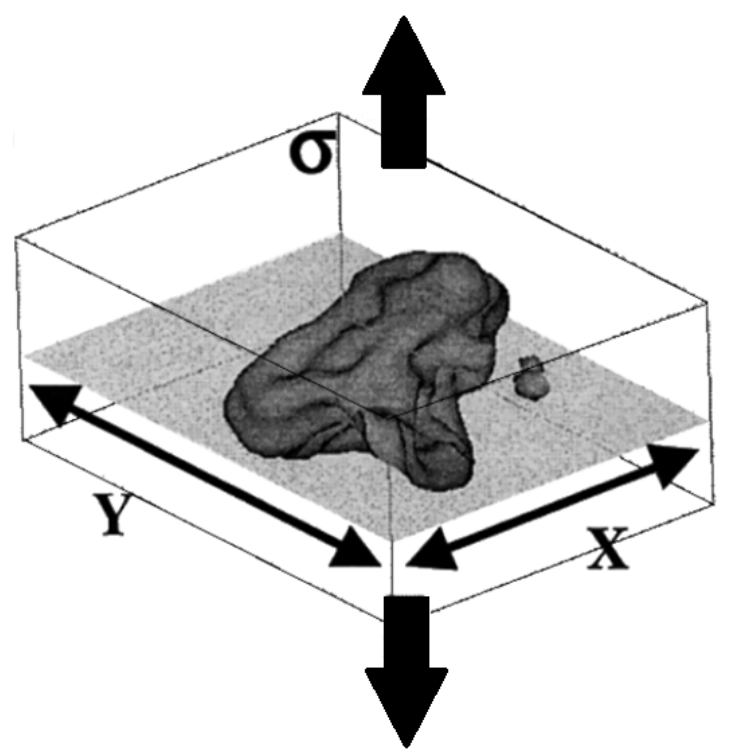

(b)

Figure 40: (a) Classification of pores observed by tomography in a specimen after 233,000 cycles; for every pore, the effective stress level was measured in relation to the stress intensity factor, estimated according to pore dimensions $\mathrm{X}$ and $\mathrm{Y}$; (b) definition of $\mathrm{X}$ and $\mathrm{Y}$ on a pore 3D diagram.

Finally, it seems that pores smaller than $50 \mu$ do not nucleate cracks (Savelli, 2001). 3D images show that cracks that do not propagate are smaller than the pores that nucleated them (Figure 41).

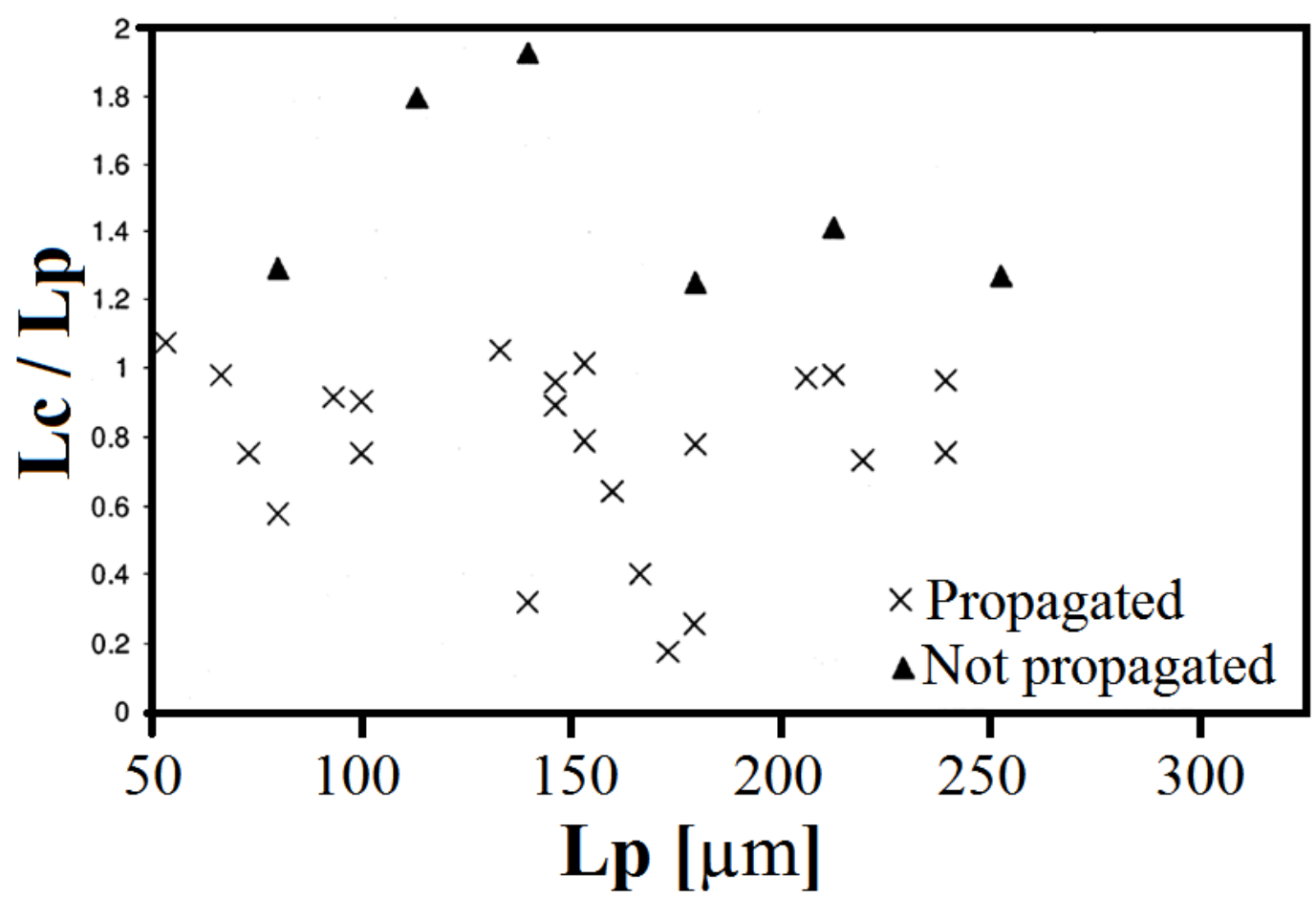

(a) 


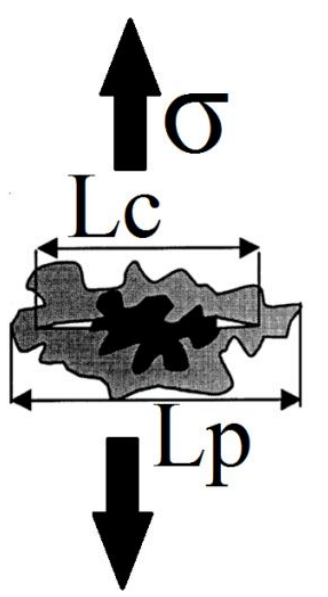

(b)

Figure 41: (a) Classification of pore observed by tomography on the surface of a specimen after 233,000 cycles; for each pore, the measurement between the crack lateral length $\left(\mathbf{L}_{\mathbf{c}}\right)$ on the surface (including pore) and the maximum lateral pore dimension below the surface $\left(\mathbf{L}_{\mathbf{p}}\right)$ is indicated in relation to $\mathbf{L}_{\mathbf{p}}$; the different symbols indicate whether crack has not spread (x) or has spread $(\boldsymbol{\Delta})$; (b) definition of $\mathbf{L}_{\mathbf{c}}$ and $\mathbf{L}_{\mathbf{p}}$ : in black, intersection of pore with specimen surface; in gray, 2D projection of 3D shape of pore below the surface (Buffière et al., 2001).

Crack propagation depends on the available distance between the pore border and the next favorably orientated grain boundary. If it is shorter than the corresponding pore diameter, the crack will not propagate.

Crack nucleation mechanisms are, therefore, the same for high and low stress levels. Applying a cyclic loading is conducive to de-cohesion between silicon particles and the matrix, which leads to the development of small cracks. Two factors affecting this decohesion are, on the one side, the plastic and elastic compatibility existing between hard and fragile silicon particles and the ductile matrix and, on the other hand, the location of silicon particles near convex regions of pores, which act as stress concentrators. Moreover, the grain boundaries play an important role due to supplementary plastic incompatibility, induced by it, because of its orientation. Crack nucleation at grain boundaries is favored because energy is minimized on the surface. Large and inter-granular pores also favor nucleation.

Indeed, the influence of grain boundaries on crack propagation has been reported in the literature for steels and aluminum. The physical processes that explain how a crack goes through a grain boundary are not yet clear. A probable mechanism is activation of sources of discrepancies in adjacent grains and creation of a new micro-crack by irreversible accumulation of discrepancies in the grain. As was observed by Buffière et al. (2001), the crack came to a halt in tests in which there was observed the development of plastic zones in the vicinity of the grain immediately ahead. 


\subsection{Brief of Chapter 3}

- In the strain-based approach there is low-cycle fatigue that may be characterized as: (1) high-strain cycling when the range of inelastic deformation in the cycle exceeds that of elastic deformation; and (2) inelastic deformations of such magnitude that they spread through the microstructure evenly.

- An isothermal fatigue test consists of imposing varying mechanical strains at constant temperature.

- Thermo-mechanical fatigue (TMF) describes fatigue under variation of both temperature and mechanical strain and may involve several mechanisms that add damage to that of "pure" fatigue, including creep and oxidation at elevated temperatures, which directly contribute to further damage. These mechanisms differ from each another, depending on strain and temperature history.

- There are three basic aspects of stress-strain behavior that are important unbalanced cyclic mechanisms: elasticity, plasticity, and creep.

- Porosity has a catastrophic effect on mechanical properties of aluminum alloys. A $1 \%$ volumetric fraction of pores may reduce fatigue life by $50 \%$ and strength limit by $20 \%$ as compared to the same alloy without pores and the time needed for crack initiation decreases with pore size.

- Some approaches have been developed over the years in order to predict the total life of engineering materials under fatigue at elevated temperatures.

- Propagation of fatigue cracks can be effectively dealt by traditional concepts of Fracture Mechanics. The typical curves of fatigue crack propagation, when plotted on a graph as $\log (\mathrm{da} / \mathrm{dN})$ in relation to $\log (\Delta \mathrm{K})$, show a characteristic sigmoidal shape with three distinct phases.

- In most cases, fatigue cracks originate in pores irrespective of stress and deformation, most pores leading to cracks are located on the material surface or just below it. After crack nucleation, propagation chronology can be divided into four phases: (1) interdendritic crack propagation, alternatively in $\alpha$ matrix and along interfaces of silicon/matrix particles; (2) intra-dendritic crack propagation; (3) rapid inter-dendritic crack propagation with damage to silicon particles ahead of crack; (4) final rupture. 


\section{CHAPTER 4: FINITE ELEMENTS}

\subsection{Brief History}

The idea behind the finite element method is not attributed to a single person or group of people from the same historical period. Over two thousand years, Greek philosophers developed theories which assumed that all things were composed of countless particles. So set Leucippus and Democritus that everything was made by an infinitely large number of particles, called atoms by the latter. Eudoxus, creator of the "exhaustion method", which consists of inscribing and circumscribing rectilinear figures into curvilinear figures, already proposed syncretizing a continuous figure to facilitate certain calculations. This method allowed the calculation of areas of curved figures and volumes of solids such as spheres and cones. It is equivalent to passing to the limit of integral and differential calculus.

More recently, in the 1930s, McHenry (1943) and Hrennikoff (1941) replaced a continuous element, e.g., a plate, for a structure formed by bars following the original geometry, maintaining the same binding conditions and loads.

Despite taking into consideration a continuous medium discretized by elements with known stiffness and elasticity, these methods, which have given rise to matrix analysis, do not present the conceptual aspect implicit in the finite element method.

Not only does it transform a continuous solid into a combination of discrete elements and write equations of compatibility and balance between them, but it also admits continuous functions that represent, for instance, the displacement field in the domain of an element and, subsequently, obtains the corresponding deformation condition, which, together with the material constitutive relationships, allows the definition of the stress condition in the entire element.

This stress condition is transformed into internal efforts, which have to be in balance with external actions.

This formulation derives from the Rayleigh-Ritz method, which is based on minimizing the total potential energy of the system, expressed in relation to the preestablished displacement field (displacement method).

A renowned mathematician, Courant (1943), applied this procedure to the study of Saint-Venant torsion of hollow sections.

Argyris and Kelsey (1964) published a series of papers in the mid-1950s, in which the matrix formulation of the Rayleigh-Ritz method was categorically determined and has been 
applied to analyze especially aircraft wings and fuselage, simulating them as consisting of bars and panels.

The finite element method had its formula established as it is presently known with the publication of the study of Turner and colleagues (1956). They showed that it was possible to replace the properties of the continuum in a more direct, not less intuitive way, assuming that small portions (elements) behave in a simplified fashion.

Clough (1980), author of the term (method of finite elements in opposition to infinitesimal elements of differential calculus), describes in details his involvement in the development of this method in a paper published in 1980 .

Despite its formulation having been known since the 1950s, the finite element method became disseminated and applied in various fields of engineering due to the rapid development and expansion of computers.

Today, there are hundreds of commercial computer programs currently used in various areas of knowledge, which employ this method in linear and nonlinear analysis.

The finite element method is now well-established in engineering so that its knowledge is essential to be able to intelligibly work with commercial software programs existing in almost all project management firms (Assan, 2003).

\subsection{Finite Element Method}

Numerical simulation is one of the stages to be developed in order to design a component (Figure 42). The component should be analyzed in light of strength criteria, the classic fatigue theory, and the finite element method.

In order to predict the performance of mechanical components, a designer has at hand today a series of mathematical tools that can be applied. Analytical solutions may be used in certain cases, but its application is limited to specific situations where a mathematical solution of the structure can be found. A more comprehensive way of dealing with structural problems is the use of numerical analysis methods. Although these methods provide approximate solutions, in many cases they are the only way designers have to find the answers they are seeking.

The Finite Element Method (FEM) takes into consideration the region of problem solution as consisting of small interconnected elements. The region under study is analytically modeled or estimated by a set of pre-defined discreet elements. Once these elements are placed together in a countless number of different configurations, it is possible to model very 
complex geometrical forms. Moreover, it provides designers with a great deal of flexibility in the application of loads and boundary conditions, which makes FEM the most widely employed method in structural analysis nowadays.

FEM is suitable to a wide range of problems of boundary values in engineering. In a boundary value problem, a solution is sought in the body region (domain) whereas in the boundaries of this region the values of dependent variables (or their derivatives) are known.

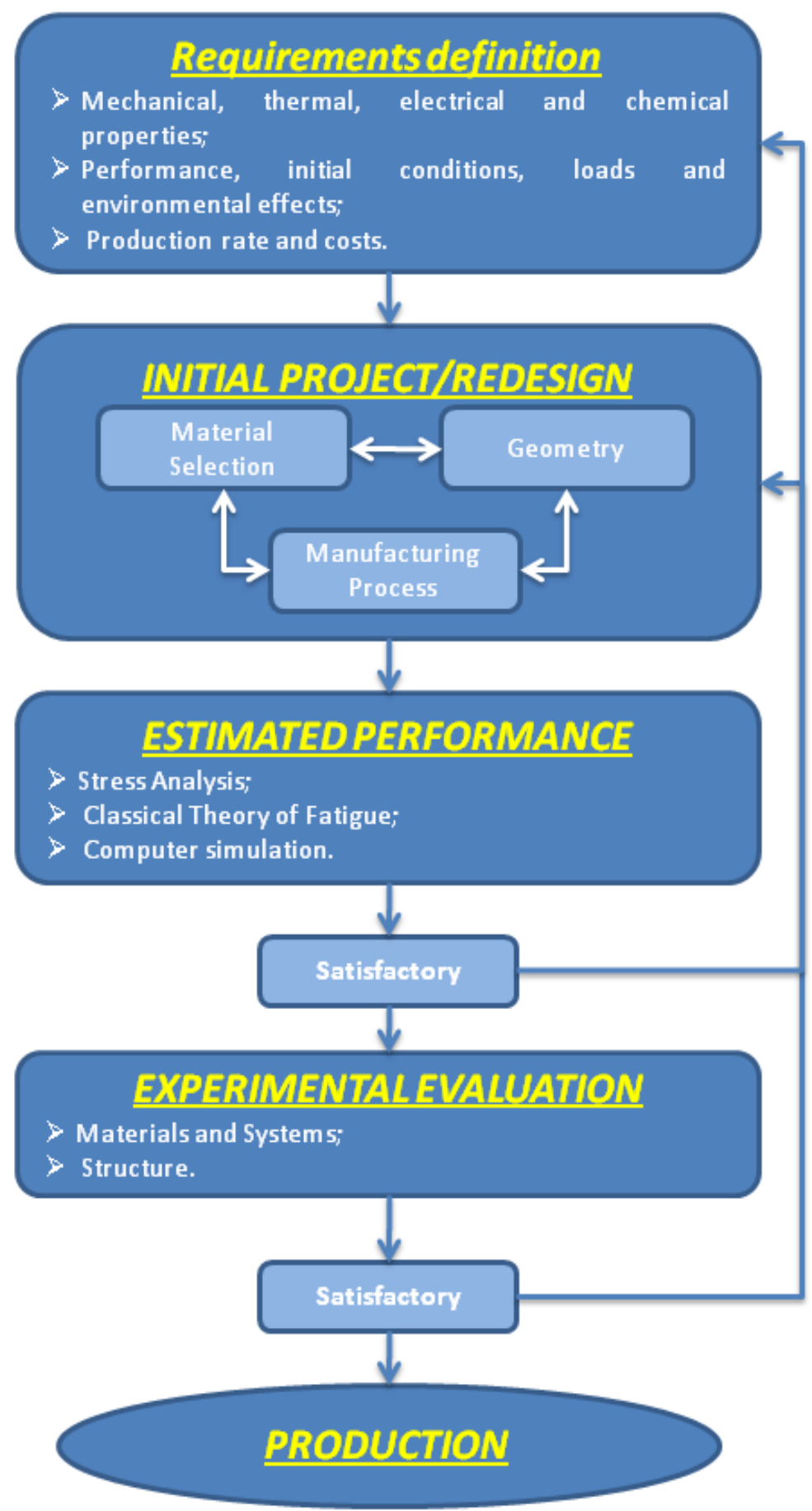

Figure 42: Design cycle. 
According to Huebner (1994), the method may be summarized into three stages:

1. Pre-processing: the preparation stage of the problem to be solved later. It is in this stage that the phenomenon is modeled, assuming hypotheses, initial conditions, boundary conditions, and loadings. It also includes selecting the element, material properties, and geometry that will represent the shape of the component in question. The following are two important preprocessing sub-stages:

- Continuum discretization: the continuum is the physical body shown in Figure 43, structure or solid under analysis. The discretization viewed in Figure 44 can be simply described as the process by which the domain is subdivided into an equivalent system of finite elements, which can be, for instance, triangles or quadrangles for $2 \mathrm{D}$ problems or tetrahedrons and hexahedrons for $3 \mathrm{D}$ problems. These elements have the so-called nodes, which can be internal or external, i.e., located in the interior of the element or at its edges. Although there have been attempts to automate the process of domain subdivision, it remains essentially dependent on engineers' discrimination and expertise;

- Selection of interpolation functions: the assumed displacement or interpolation functions represent only approximately the exact or actual distribution of displacements. Generally, the form adopted for the interpolation functions is the polynomial, due to its requiring simple mathematical manipulation. There are three interrelated factors influencing the selection of interpolation functions: choice of type and degree of function (as the type usually adopted is the polynomial, only the degree should be selected) and the type of field variables that describe the model (usually, displacements at the nodes or their derivatives). Finally, the model must meet certain requirements to ensure that numerical results come close to the correct solution; 


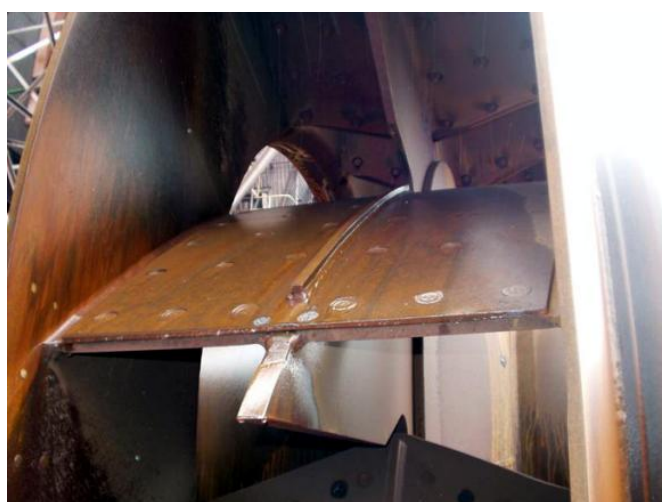

(a)

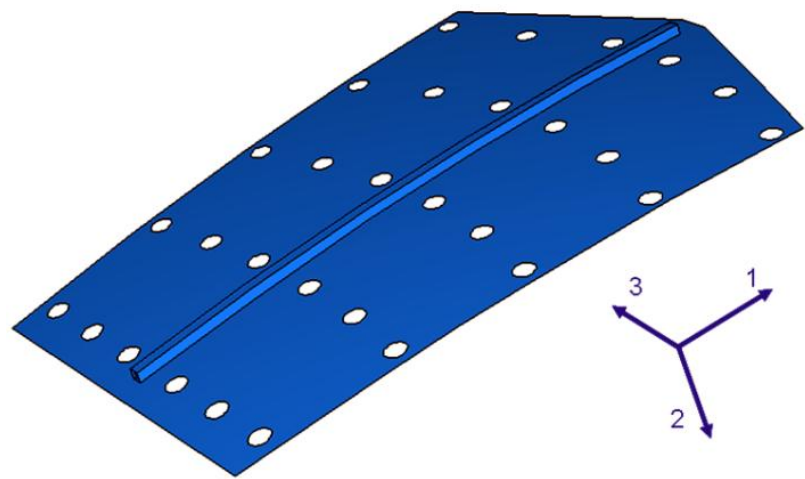

(b)

Figure 43: The exhaust fan (a) physical problem and (b) the continuum.

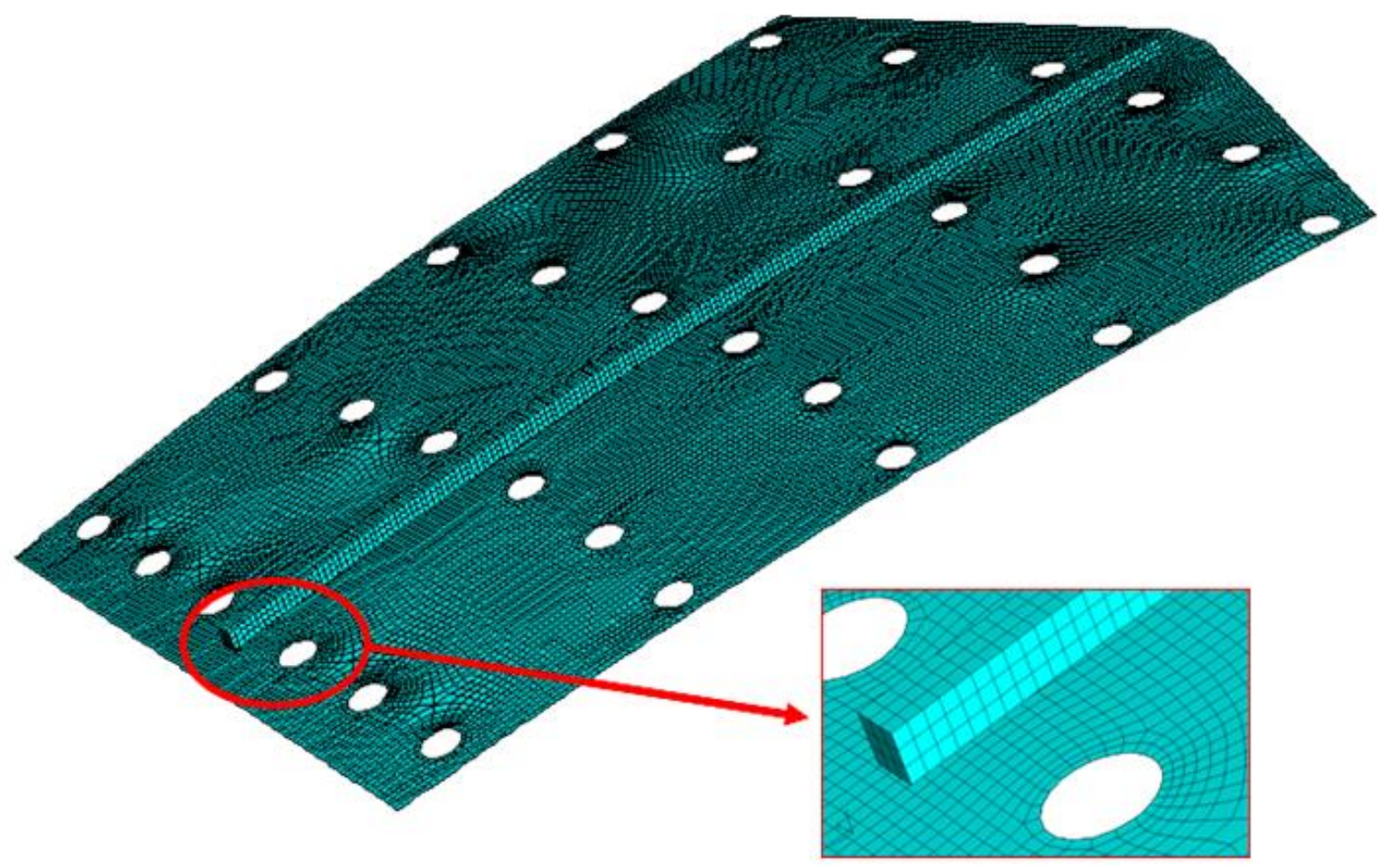

Figure 44: The continuum discretized by countless elements.

2. Solver: the solution of the problem has as its starting point the model set during the previous stage. Therefore, the accuracy of responses depends critically on the researcher's ability to take apart the phenomenon. The solution is based on a numerical algorithm aimed at solving as quickly and accurately as possible a differential equation with boundary conditions and/or initial conditions imposed by the model. Following are some sub-stages to solve the problem:

- Obtaining the elemental stiffness matrix: the stiffness matrix consists of coefficients of equilibrium equations derived from geometric and material properties of an element and can be obtained by using the principle of 
minimum potential energy. Stiffness relates nodal displacements to forces applied at the nodes. The equilibrium relationship between the stiffness matrix $[k]$, the nodal force vector $\{F\}$, and the nodal displacement vector $\{u\}$ is expressed as a set of simultaneous linear algebraic equations, $[k]\{u\}=\{F\}$. The stiffness matrix of an element depends on the interpolation function, element geometry, and local properties of the material;

- Assembly of algebraic equations for the entire domain: the process includes assembling the global stiffness matrix for the whole body from elementary stiffness matrixes and the global force vector from elemental force vectors. In general, the basis for an assembly method is the requirement of nodal interconnections. Displacements at a node must be the same for all adjacent elements;

- Solutions for unknown displacements: algebraic equations assembled in the previous step are solved for unknown displacements. In linear problems, this is a relatively straightforward application of matrix algebra techniques. However, for nonlinear problems, the desired solutions are obtained by a sequence of steps, each involving the modification of stiffness matrix and/or strength vector;

- Calculation of elemental strains and stresses from nodal displacements: in some cases, nodal displacements are the variables under study for the solution. Often, however, other quantities derived from unknown variables such as stresses and strains must be estimated. In general, stress and strain are proportional to the derivatives of displacements.

It is important to remark that these numerical algorithms may be implemented computationally to accelerate calculation. Nowadays, it is possible to find computational packages such as ANSYS, NASTRAN, ABAQUS and others, each program having advantages and disadvantages to problem modeling.

3. Post-processing: it is the last stage. It depends only on the researcher's modeling needs, i.e., it is the solution to the differential equation that describes the phenomenon under study as shown in Figure 45. It may be:

- Nodal displacements;

- Geometry deformations; 
- Stress gradients according to selected strength criterion;

- Temperature gradients;

- Nodal displacements over time;

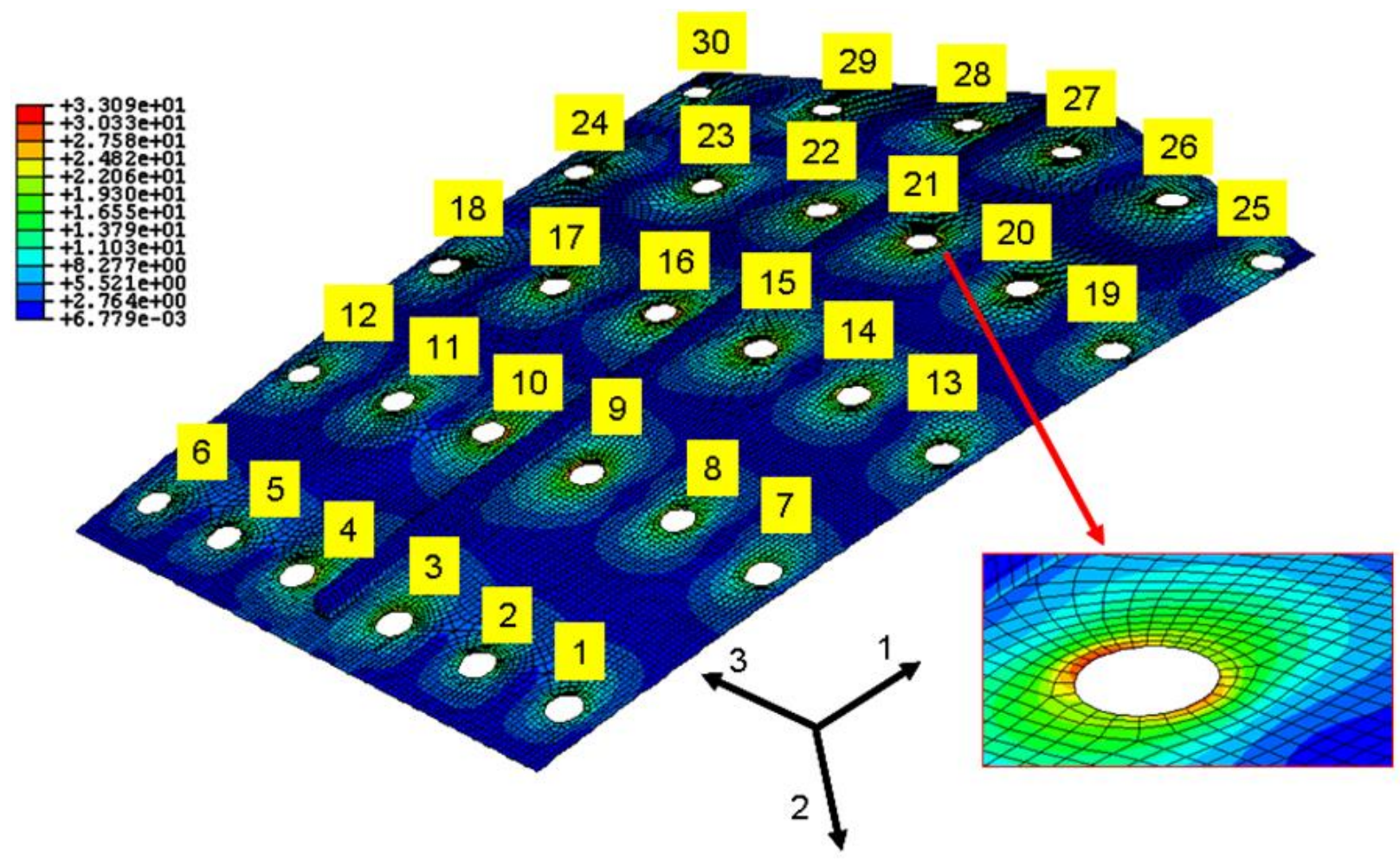

Figure 45: Typical post-processing stage of a commercial package.

These features, implemented computationally, allow the estimation of the solution to a complex problem in a relatively short time, optimizing the development time for projects of anisotropic or isotropic materials subjected to static, thermal, and dynamic loadings, and so forth.

\subsection{Behavior Model}

There are two classic models of material behavior:

- The first, purely phenomenological, is at a macroscopic scale, i.e., it operates through global variables (e.g., stress, strain, and internal variables). This approach allows the modeling of experimental phenomena with a limited number of variables, thus engendering a lack of physical basis. 
- The second, known as micro-macro, is at a microscopic scale and seeks to predict the macroscopic response by changing scales from physical parameters such as density and movement of discrepancies, nucleation and propagation phenomena, and the distance between precipitates. The problem with this type of application is that the number of steps between the microscopic mechanisms and macroscopic response is very large, which usually complicates the construction of complete models for industrial alloys, and that most authors use it only to predict yield stress (Gandin et al., 2002; Liu et al., 2003).

\subsection{Presentation of Proposed Crack Growth Model}

The first elements of this model were developed in a study on crack propagation in turbine disc carried out by Pommier and Risbet (2005). The model was subsequently identified, implemented, and validated for fatigue crack propagation in steel employed in train wheels at room temperature by Hamam and Pommer (2007). The incremental crack propagation model is composed of two parts:

- A propagation law that provides the instantaneous speed of creating the cracked area per length of the crack front in relation to the instantaneous speed of plastic rounding of the crack. This first part of the model is identified by using a test of fatigue crack propagation at constant amplitude.

- A plastic rounding law that allows the determination of the global speed of plastic deformation in the crack front region in relation to applied load and internal variables of the model. These internal variables define in a comprehensive way the condition of residual stresses and material hardening in the plastic zone. This second part of the model is identified by using finite element calculations and requires identification of the material cyclic elasticplastic behavior.

Thus, fatigue damage modeling is decoupled from plasticization modeling in the crack front region. This decoupling is possible due to the fact that the characteristic dimension of the damaged area is much inferior to that of the plasticized zone. The plasticized zone dimension is comparable to the size of fatigue grooves (in the order of micrometers) or to the size of grains. Hence, this hypothesis is valid as long as the plastic zone dimension is larger than $100 \mu \mathrm{m}$. 
Otherwise, FEM is only employed to establish the global cyclic plastic model of the crack front region. The approach used to establish this method - the plastic rounding law-is as follows:

Good finite element calculations enable to estimate the evolution of stresses and strains at each point in the crack front region. Taking the behavior peculiarities of the material into account by its behavior law, which comprises at least one isotropic hardening and one nonlinear kinematic hardening.

Next, a scale-changing method is applied to identify, from finite element simulation, a simplified model of cyclic elastic-plastic behavior in the region ahead of the crack on a global scale. The scale-change principle is simple, it is based on an approximation of the kinematics at the crack tip. In Mode I, the displacement field at the crack tip is assumed to decompose into an elastic part and a plastic part. It is assumed, for both parts, that they are the result of an intensity factor (a function of boundary conditions and crack geometry) and a reference field (a function of space coordinates only).

The study of plasticity at the global scale for the region ahead of the crack is done by investigating the intensity factor evolution of the plastic part of the displacement (plastic rounding) field, identified by $\rho_{\mathrm{I}}$.

The evolution of plastic rounding $\left(\rho_{\mathrm{I}}\right)$, estimated by means of finite elements, allows the establishment of a simplified model of global cyclic elastic-plastic behavior for the crack front region, i.e., a model that enables to calculate $d \rho_{\mathrm{I}} / \mathrm{dt}$ in relation to applied nominal loading $\mathrm{dK}_{\mathrm{I}} / \mathrm{dt}$ from a set of partial differential equations.

Otherwise, a crack propagation law is introduced to provide the instantaneous speed of creation of cracked area per unit of crack tip length da/dt in relation to the instantaneous speed of plastic rounding $\mathrm{d} \rho_{\mathrm{I}} / \mathrm{dt}$.

The set enables subsequently to calculate the propagation speed of fatigue cracks for complex loadings taking the effects of the loading history into account.

Several aspects of this approach are detailed below. In the context of this text, the sensitivity of this approach with respect to the material behavior law was investigated for a gravity-cast aluminum-silicon alloy, employed in the manufacture of automotive engine heads. 


\subsubsection{Study of Displacement Field at Crack Tip}

\section{Hypothesis of Displacement Field Projection}

Let $\overline{\mathrm{x}}=(\mathrm{x}, \mathrm{y})$ be a point in a local coordinate system fixed in a normal section in the region ahead of a crack (Figure 46). As the load applied to the crack is known, a simplified form of displacement of this point is then sought. The goal here is to reduce the number of freedom degrees of the problem so that simulation does not result in high calculation times, considering that industrial problems require quick and satisfactory responses.

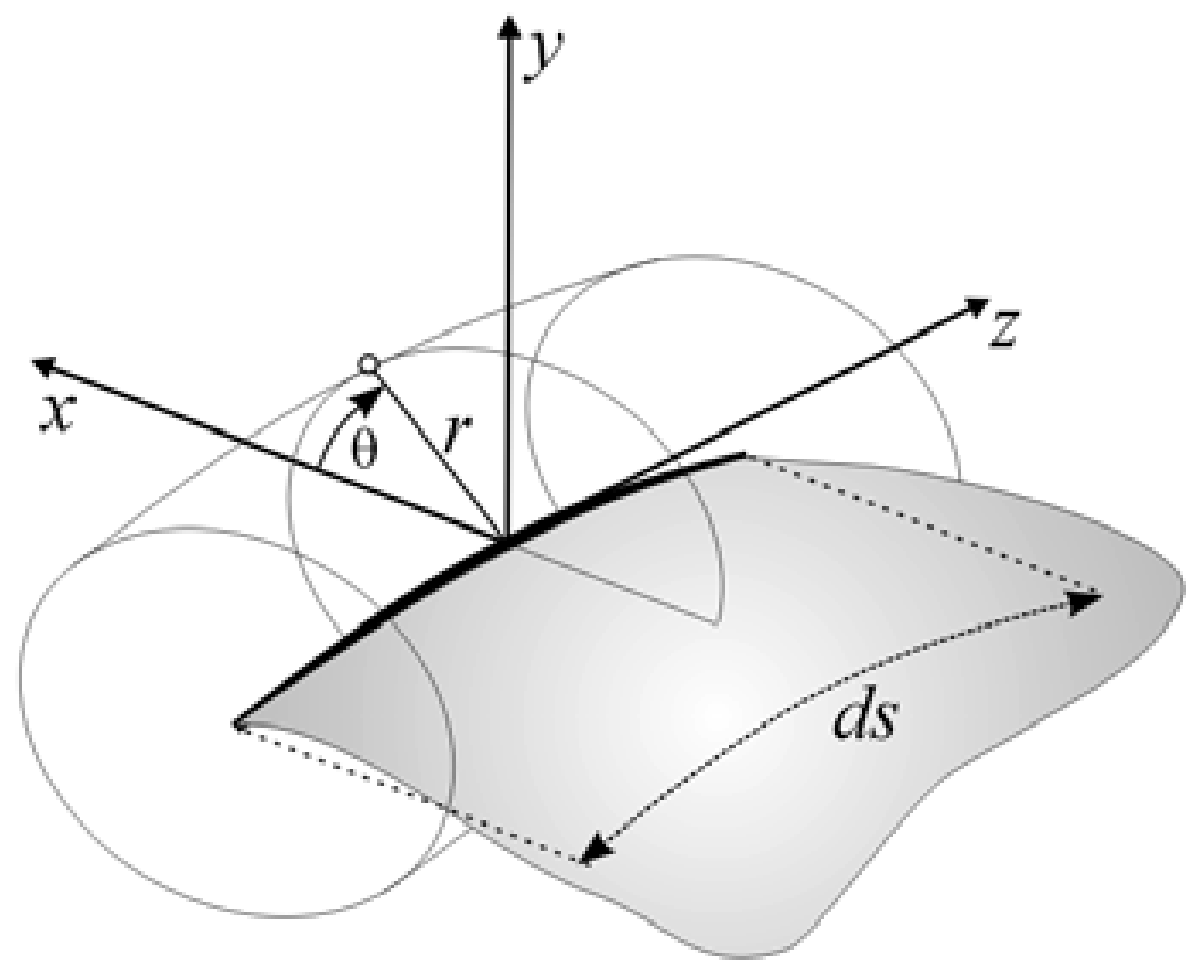

Figure 46: Coordinate system around the crack.

The rupture linear mechanics provides displacement fields in the crack tip region by asymptotic development in view of the distance $r$ to this tip. The first term of this development is expressed as the product of the stress intensity factor, varying over time, and a reference field solely dependent on the point coordinates with respect to the crack tip. Hence, at first just the stress intensity factor $\mathrm{K}_{\mathrm{I}}$ is useful to characterize loading at the crack tip. The crack propagation criteria are, thus, be described as a direct function of $\mathrm{K}_{\mathrm{I}}$, which becomes the only freedom degree of the problem. 
In the cyclic elastic-plastic context, the displacement field in the crack tip region is expressed as:

$$
u(\bar{x}, t)=\sum_{j} a_{j}(t) \cdot u_{j}(\bar{x})
$$

where $u_{j}(\bar{x})$ is a basis of the reference spatial field and $a_{j}(t)$ are the problem temporal parameters. These temporal parameters constitute the freedom degrees of the problem on global scale and, thus, may be applied to the crack propagation criterion.

At first, FEM was employed to calculate displacement fields $\overline{\mathrm{u}}(\overline{\mathrm{x}}, \mathrm{t})^{\mathrm{EF}}$ in the cyclic elastic-plastic context. Then, these calculated fields are treated in order to justify the approach chosen and the problem temporal parameters are calculated.

\section{Methodology}

The displacement field sought is, therefore, assumed to be decomposed into a basis set to the reference site at each point of the crack front, composed of two reference fields: an "elastic" field $\mathrm{u}^{\mathrm{e}}(\mathrm{x})$ and a "plastic" field $\mathrm{u}^{\mathrm{p}}(\mathrm{x})$. This partitioning has the advantage of reducing to two the number of the problem freedom degrees. The two freedom degrees constitute the intensities of the reference fields: stress intensity factor $\widetilde{\mathrm{K}}_{\mathrm{I}}$, pre-factor of the elastic field $\mathrm{u}^{\mathrm{e}}(\mathrm{x})$, and plastic blunting $\rho$, pre-factor of the plastic field $\mathrm{u}^{\mathrm{p}}(\mathrm{x})$ :

$$
\mathrm{u}(\mathrm{x})=\widetilde{\mathrm{K}}_{\mathrm{I}} \cdot \mathrm{u}^{\mathrm{e}}(\mathrm{x})+\rho \cdot \mathrm{u}^{\mathrm{p}}(\mathrm{x})
$$

Numerical solutions may be employed in both fields, but they can also be expressed analytically. In fact, the Karhunen-Loeve decomposition is interesting because it can be performed without having a priori the solution sought, but it is very costly in terms of calculation time. It is simpler, therefore, to provide an analytical expression for the displacement field at the crack tip and then employ the minimum squares method to determine temporal coefficients from the results of a FEM calculation. Furthermore, giving an analytical expression for the reference field entails the interpretation of the results.

It is possible, for instance, to choose Westergaard analytical solution for the reference elastic field, for the stress intensity factor. The analytical expression of the displacement field around a crack tip in Mode I can then be given by:

$$
\mathrm{u}^{\mathrm{e}}(\overrightarrow{\mathrm{x}})=\frac{1}{\mu} \sqrt{\frac{\mathrm{r}}{2 \pi}} \cos \frac{\theta}{2}\left(\frac{\mathrm{x}-1}{2}+\sin ^{2} \frac{\theta}{2}\right)
$$




$$
\mathrm{u}^{\mathrm{p}}(\overrightarrow{\mathrm{x}})=\frac{1}{\mu} \sqrt{\frac{\mathrm{r}}{2 \pi}} \sin \frac{\theta}{2}\left(\frac{\mathrm{x}+1}{2}-\cos ^{2} \frac{\theta}{2}\right)
$$

with: $\mathrm{x}=3-4 \mathrm{v}$ and $\mathrm{r}^{2}=\mathrm{x}^{2}+\mathrm{y}^{2}$

Moreover, the plastic displacement field $\mathrm{u}^{\mathrm{p}}(\mathrm{x})$ retained is the wedge-shaped one, placed in a horizontal direction below the crack plane. Under this assumption, Equations 49 and 50, the normal displacement of surfaces of the crack $\theta=\pi$ is constant and equal to 1 . So the pre-factor $\rho$ of this field can be interpreted as plastic crack blunting or CTOD (crack tip opening displacement).

$$
\begin{gathered}
u_{x}^{p}(\vec{x})=\frac{1}{\pi(x+1)}\left[(x-1) \log r+2 \frac{y^{2}}{r^{2}}\right] \\
u_{y}^{p}(\vec{x})=\frac{1}{\pi(x+1)}[(x+1) \theta-2 \cos \theta \sin \theta]
\end{gathered}
$$

In practice, during post-processing of FE calculations, the numerical solution of a finite element calculation previously done on the same mesh is used for the field $u^{e}(x)$ and Equations 49 and 50 for the field $\mathrm{u}^{\mathrm{p}}(\mathrm{x})$.

\subsubsection{Model Evolution Equation}

The applied approach is concisely as follows. First, a local approximation is employed to calculate in detail, by finite elements, the evolution of stresses and strains in the region of the crack tip due to the material behavior and applied load. Then, one goes from local to global scale by applying the approach under the aforementioned displacement. In general, it is possible to obtain the temporal evolution of plastic blunting, for every finite element calculation. Associated with this development is a model of behavior of the material that will be employed to simulate cyclic plasticity in the crack tip region. Finally, it is necessary to have a crack propagation law that can relate crack propagation speed to plastic blunting speed in order to calculate fatigue life.

\section{Plastic Blunting Law}

Plastic blunting of crack faces is calculated by FEM, supported by a simplified representation of the displacement field in the crack front region. An experimental rounding determination was possible today thanks to novel experimental media employed to measure 
the displacement field, such as image correlation. This experimental technique is more laborious than FEM calculation and enables to characterize the evolution law of $\rho$ only on the surface. Moreover, although this technique has the advantage of not being dependent on the chosen material behavior, it does not allow the separate study of the evolution of model variables in relation to $\rho$ and a, i.e., the crack advances without the occurrence of plastic rounding or plastic rounding occurs without the plastic crack advancing.

This study employed FEM, but it is interesting to use image correlation in future studies at least to validate numerical calculations. Figure 48 shows the evolution of plastic rounding obtained by FEM in relation to intensity factor of the stress applied to an increasing charge-discharge succession. This was achieved by applying the projection defined above. This ensured that the plastic zone in the front crack was confined to the $\mathrm{K}$ dominance region.

The evolution curves of plastic rounding $\rho$ show that after every inversion in loading direction (from tractive to compressive and vice-versa) there is a region $\Delta \mathrm{K}_{\mathrm{I}}^{\infty}$ during which $\rho$ does not vary (Region $\mathrm{C}$ ). As $\rho$ measures plasticity at the crack tip, this region may be defined as the critical cyclic domain of the crack. It is possible to note that this domain can shift throughout solicitation. Hence, it is associated with an internal variable such as linear kinematic hardening, i.e., one that defines the position $\left(\mathrm{K}_{\mathrm{Ixc}}\right)$ of this domain. A second variable is also introduced to define its dimension, which is generally constant.

Likewise, it is possible to note a discontinuity in the evolution curve during tractive loading. This discontinuity appears when the applied load exceeds the maximum load previously reached (D). This rupture in slope corresponds to the loading point beyond which the monotonic plastic zone at the crack tip extends. Point $\mathrm{D}$ is, thus, the limit of the crack monotonic plasticity domain (Region M). The lower limit of the monotonic plastic zone corresponds to the point where surfaces meet. Below this contact point, plastic rounding no longer evolves. It stops evolving even if compression continues, because the crack is closed. Moreover, the proposed projection has no effect now and, therefore, is not performed. Again, two internal variables are brought into the elasticity field, one for position $\mathrm{K}_{\mathrm{Ixm}}$, which is taken as the contact point, and another for its size, which is usually variable (Figure 47). 


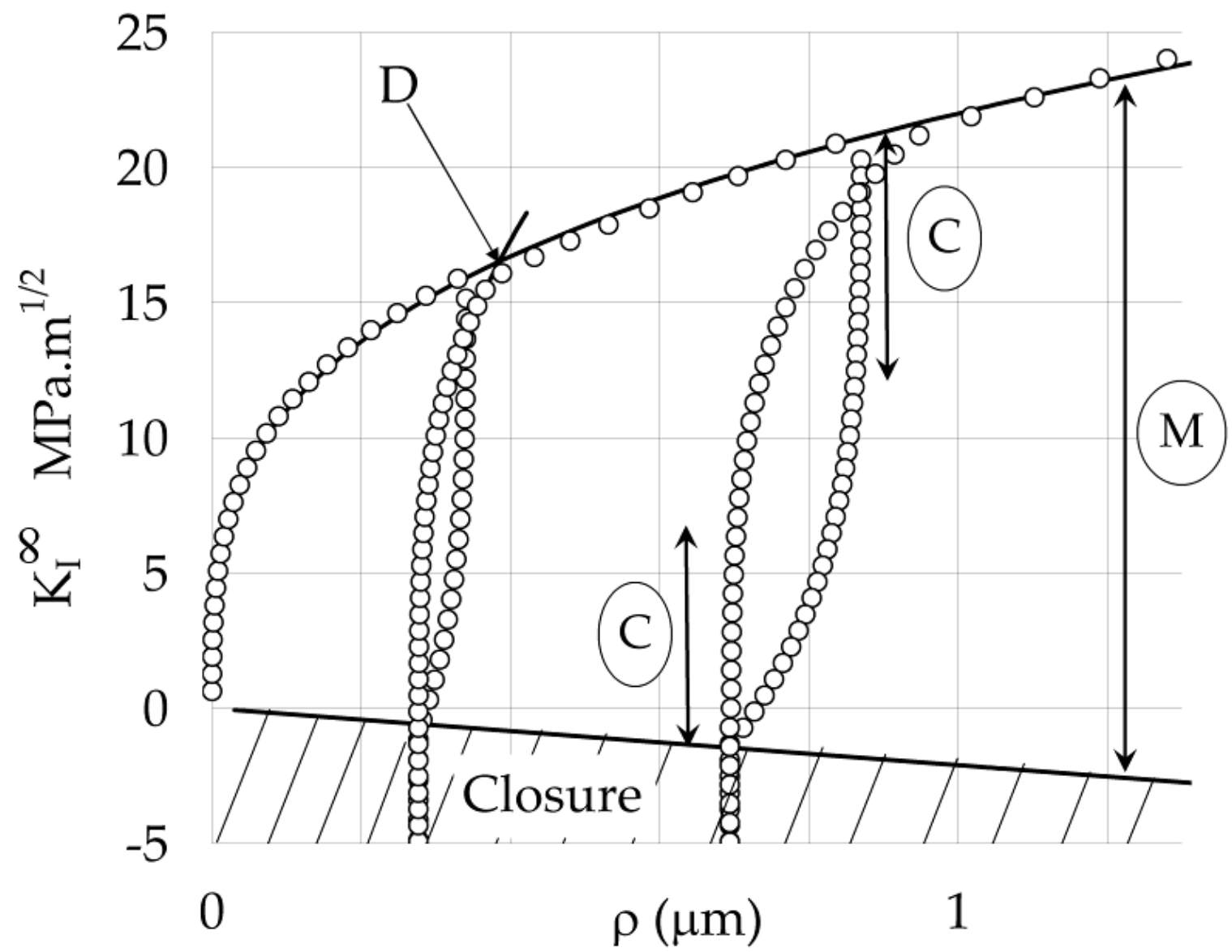

Figure 47. Evolution of plastic rounding in relation to applied stress intensity factor.

The rounding model describes, with the help of a set of analytical equations, the evolution laws of each of these internal variables as well as of $\rho$ in relation to applied load and the current value of these variables. It is important to note that the internal variables $V_{\text {int }}$ may vary as the crack tip region deforms plastically $\partial \mathrm{V}_{\text {int }} / \partial \rho_{\mathrm{I}}$, but also because the tip of the crack moves $\partial \mathrm{V}_{\text {int }} / \partial \mathrm{a}$. To determine these laws of evolution, several calculations are performed from the start of crack propagation. Previous works have established simple evolution laws for each of the variables and defined a strategy to identify parameters of these laws. The main equations of these laws are presented below. In all cases, the following relationship between the nominal stress intensity factor and the thermodynamic force $\varphi$ associated with $\rho$ :

$$
\varphi=A^{2} K^{2} \sin K \quad \quad \operatorname{com} \quad A=\sqrt{\frac{1-v^{2}}{2 E}}
$$

Indeed, as $\mathrm{d} \rho$ and da are related through the crack propagation law, the same rule may be employed to relate the contact point position, known as $\mathrm{K}_{\mathrm{Xm}}$, which is determined by the 
nominal stress intensity factor, to the internal variable $\varphi_{\mathrm{Xm}}$, associated with the center position of the monotonic elasticity domain. In short, the model is written as follows:

- Extension of

$\begin{array}{lll}\text { monotonic plastic } & f_{m}>0 & \frac{d \varphi}{d t}>0\end{array}$

zone:

- Propagation law: $\frac{d a}{d t}=\frac{\alpha}{2} \frac{d \rho}{d t}$

- Plasticity criterion (

$\left.\varphi_{m}<\varphi\right)$ :

$$
f_{m}=\sqrt{\left(\varphi-\varphi_{x m}\right)^{2}}-\varphi_{m}
$$

- Blunting law: $\quad \frac{d \rho}{d t}=\lambda \frac{d f_{m}}{d \varphi}$

- Consistency: $\quad f_{m}=0 \quad \frac{d f_{m}}{d t}=0$

$$
\begin{gathered}
\frac{\partial \varphi_{x m}}{\partial \varrho}=a_{x m} \cdot \sqrt{\varphi_{x m}} \\
\frac{\partial \varphi_{m}}{\partial \varrho}+\frac{\partial \varphi_{x m}}{\partial \varrho}=A^{2} a^{2} \frac{\sqrt{\varphi_{m}+\varphi_{x m}}}{\sqrt{\varphi_{m}+\varphi_{x m}-A b_{m}}}
\end{gathered}
$$

- Evolution equations:

$$
\begin{gathered}
\frac{\partial \varphi_{m}}{\partial a}=p_{a} \cdot \varphi_{m} \\
\frac{\partial \varphi_{x m}}{\partial \varrho}=k_{a} \cdot \varphi_{x m}+k_{b} \cdot \varphi_{m} \\
A^{2}=\frac{(1-v)^{2}}{2 E}
\end{gathered}
$$

- Material parameters:

$$
\left(a_{m}, b_{m}, a_{x m}, p_{a}, k_{a}, k_{b}\right)
$$

- Initial values: $\quad \varphi_{m o}=A^{2} . b^{2} \quad \varphi_{x o}=0$

The material parameters are identified automatically with the help of the minimum squares method and supported by a set of finite elements calculations. The term $\varphi_{\mathrm{xm}}$ represents the point of contact between two crack faces and the elasticity domain dimension associated with the monotonic plastic zone. If the limit $\mathrm{f}_{\mathrm{m}}=0$ is reached, the monotonic plastic zone increases in size.

- Cyclic plasticity:

$$
f_{c}=0
$$

- Propagation law:

$$
\frac{d a}{d t}=\frac{\alpha}{2}\left\langle\frac{d \varrho}{d t}\right\rangle
$$


- Plasticity criterion (

$\left.\varphi_{m}<\varphi\right)$ :

$$
f_{c}=\sqrt{\left(\varphi-\varphi_{x c}\right)^{2}}-\varphi_{c}
$$

- Blunting law: $\quad \frac{d \varrho}{d t}=\lambda \frac{d f_{c}}{d \varphi}$

- Consistency: $\quad f_{c}=0 \quad \frac{d f_{c}}{d t}=0$

$$
\frac{\partial \varphi_{c}}{\partial \varrho}=\frac{\partial \varphi_{c}}{\partial a}=\frac{\partial \varphi_{x c}}{\partial a}=0
$$

- Evolution equations: $\quad \frac{\partial \varphi_{x m}}{\partial \varrho}=A^{2} \frac{a^{2} \cdot \sqrt{\varphi_{x c}+\delta \cdot \varphi_{c}}}{\delta \cdot\left(\sqrt{\varphi_{x c}+\delta \cdot \varphi_{c}}-\frac{\varphi_{c}}{A \cdot b_{c}}\right)-\frac{A \cdot b_{c}}{2}}$

$$
\delta=\sin \frac{d \varrho}{d t}
$$

- Material parameters:

$$
\left(a_{c}, b_{c}\right)
$$

- Evolution of $\varphi_{c}$ and $\varphi_{x c}$

$$
\begin{gathered}
\varphi=\varphi_{c}+\varphi_{x c} \\
\varphi_{x c}=\frac{A^{2} \cdot b_{c}}{2}\left(2 \frac{\sqrt{\varphi}}{A}-b_{c}\right)
\end{gathered}
$$

when monotonic

plasticity is activated:

\section{Implementation Method}

The algorithm to calculate the incremental model is shown in Figure 48, numerical integrations of the plastic rounding law and crack propagation law were done separately in two stages. From the evolution of the applied tension field, the loading variation increment $\mathrm{d} \varphi_{\mathrm{i}}$ is derived for every time increment, the present crack length being known $a_{\mathrm{i}}$. The resolution of the plastic rounding law equations enables the deduction of the resulting plastic rounding increment $d \rho_{\mathrm{i}}$ as well as the evolution of internal variables $d \varphi_{\mathrm{xc}}, \mathrm{d} \varphi_{\mathrm{c}}, \mathrm{d} \varphi_{\mathrm{xm}}$, and $\mathrm{d} \varphi_{\mathrm{m}}$. The new crack length is subsequently determined from the crack propagation law and the internal variables are updated in relation to a. 


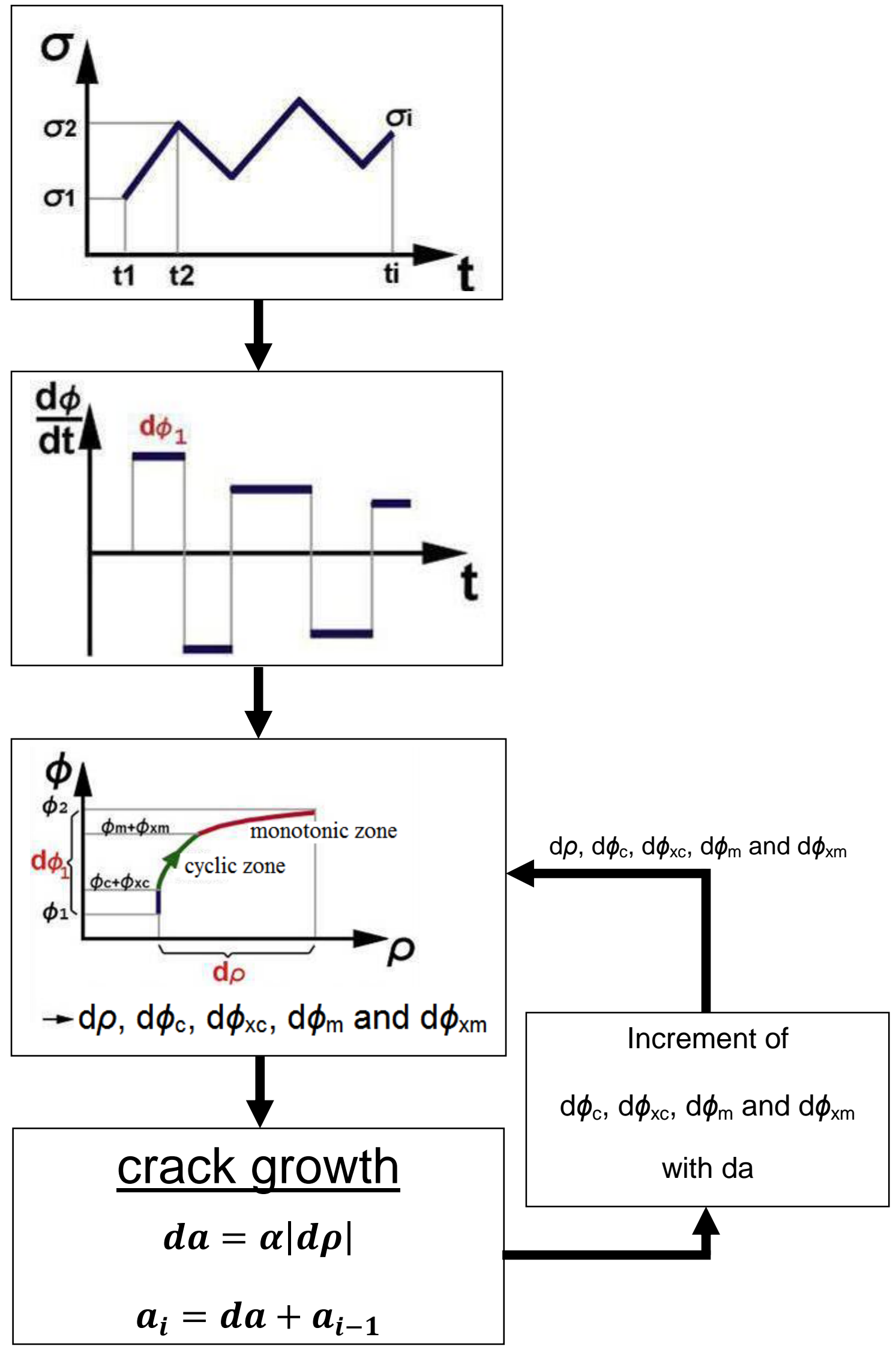

Figure 48: Algorithm of propagation with incremental law. 
The purpose of decoupling the crack propagation law from that of plastic rounding is to facilitate numerical implementation. In addition, it represents a considerable advantage as regards time. This two-stage resolution does not affect the propagation calculation results because internal variables evolve rapidly in relation to plastic rounding (few dozen microns). However, their evolution is much slower with crack growth (few hundred microns). Hence, the scale of a fatigue cycle, the update of internal variables in relation to a can be performed at the end of a calculation increment and not in real time, for crack advancement does not interfere significantly with variables.

Numerical integration of differential equations of the plastic rounding law is done initially by implicit integration, the calculation time required for this type of resolution being in the order of few dozen minutes to simulate one million cycles in two dimensions. Calculation time is the same for variable and constant amplitude loadings since in both cases calculation is done incrementally. Moreover, these calculation times are not prohibitive. However, calculation time increases rapidly when the model is applied to a 3D problem and has to calculate the advance of the crack at various points in the crack front region for every increment. A method of integration by parts validated by making some suppositions with the aim of reducing calculation time is presented in Annex 1.

In short, at the beginning of each calculation increment, the borders of cyclic and monotonic plasticity are known. For a loading increment $d \varphi$, the equations to be integrated depend on the level of applied loading $\varphi_{2}$ and the present state of the crack $\left(\varphi_{1}, \varphi_{\mathrm{m}}, \varphi_{\mathrm{xm}}, \varphi_{\mathrm{cf}}, \varphi_{\mathrm{xcf}}, \varphi_{\mathrm{co}}, \varphi_{\mathrm{xco}}\right)$

- If $\varphi_{1}$ and $\varphi_{2}$ are below the point of contact (Figure 49a), there is no equation to integrate;

- If $\varphi_{1}$ and $\varphi_{2}$ are in the interior of the cyclic plastic zone (Figure 49b), there is no equation to integrate;

- If $\varphi_{1}$ and $\varphi_{2}$ are between the limits of the monotonic and cyclic plastic zones and if it is in the opening phase $d \varphi>0$ (Figure 49c), the equations to be integrated are:

$$
\begin{gathered}
\varrho_{2}=\frac{1}{a_{c f}^{2}}\left[\left(\frac{\sqrt{\varphi_{1}}}{A}-\frac{\varphi_{c o}}{A^{2} b_{c f}}-\frac{b_{c f}}{2}\right)^{2}-\left(\frac{\sqrt{\varphi_{2}}}{A}-\frac{\varphi_{c o}}{A^{2} b_{c f}}-\frac{b_{c f}}{2}\right)^{2}\right]-\varrho_{1} \\
\varphi_{x c o 2}=\varphi_{x c o 1}+d \varphi \\
\varphi_{c o}=c t e
\end{gathered}
$$




$$
\varphi_{c f}=\frac{A^{2} b_{c f}}{2}\left(2 \frac{\sqrt{\varphi_{2}}}{A}-b_{c f}\right)
$$

- If $\varphi_{1}$ and $\varphi_{2}$ are between the limits of monotonic and cyclic plastic zones and if it is in the closing phase $\mathrm{d} \varphi<0$ (Figure $49 \mathrm{~d}$ ), the equations to be integrated are:

$$
\begin{gathered}
\varrho_{2}=\frac{1}{a_{c f}^{2}}\left[\left(\frac{\varphi_{c f}}{A^{2} b_{c f}}-\frac{b_{c f}}{2}-\frac{\sqrt{\varphi_{1}}}{A}\right)^{2}-\left(\frac{\varphi_{c f}}{A^{2} b_{c f}}-\frac{b_{c f}}{2}-\frac{\sqrt{\varphi_{2}}}{A}\right)^{2}\right]-\varrho_{1} \\
\varphi_{x c f 2}=\varphi_{x c f 1}+d \varphi \\
\varphi_{c f}=c t e \\
\varphi_{c o}=\frac{A^{2} b_{c f}}{2}\left(2 \frac{\sqrt{\varphi_{2}}}{A}+b_{c f}\right)
\end{gathered}
$$

- If $\varphi_{2}$ surpasses the monotonic plastic zones in opening phase (Figure 49e), the following equations are activated:

$$
\begin{gathered}
\varrho_{2}=\left(\frac{\frac{\sqrt{\varphi+d \varphi}}{A}-b_{m}}{a_{m}}\right)^{2}-\left(\frac{\frac{\sqrt{\varphi}}{A}-b_{m}}{a_{m}}\right)^{2}+\varrho_{1} \\
\varphi_{x m 2}=A^{2}\left(\frac{\sqrt{\varphi_{x m 1}}}{A}+a_{x m} d \varrho\right)^{2} \\
\varphi_{m 2}=\varphi_{2}-\varphi_{x m 2} \\
\varphi_{c f}=\frac{A^{2} b_{c f}}{2}\left(\frac{2 \sqrt{\varphi_{1}}}{A}+b_{c f}\right)
\end{gathered}
$$




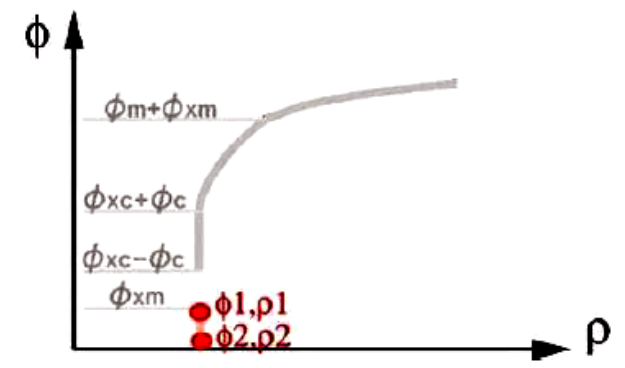

(a)

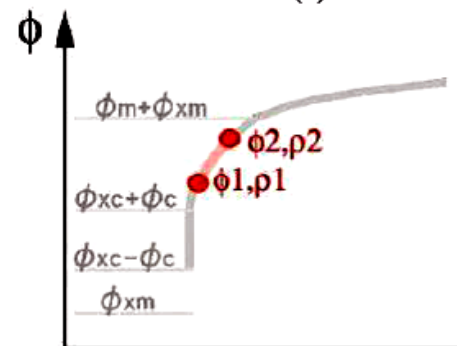

(c)

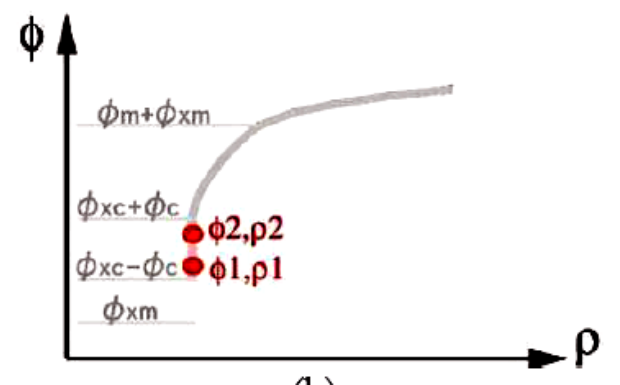

(b)

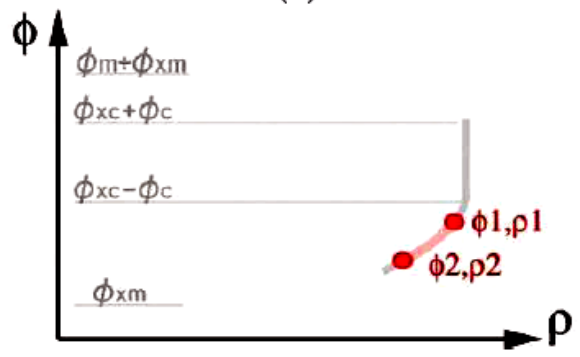

(d)

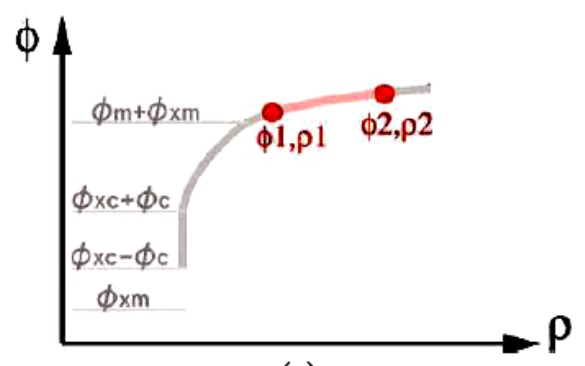

(e)

Figure 49: Evolution of contact point in relation to the plastic rounding law at (a) negative contact level, and (b) positive contact level.

At the end of the increment, the crack length is updated in relation to the crack propagation law. Internal variables dependent on a are updated in the same way.

$$
\begin{gathered}
\varphi_{x m 2}=\left(\varphi_{x m 1}+\varphi_{m 1} \cdot c_{p o s}\right) \cdot e^{k_{a} \cdot d a}-\varphi_{m 2} \cdot c_{p o s} \\
\varphi_{m 2}=\varphi_{m 1} \cdot e^{P_{a} \cdot\left(a_{2}-a_{1}\right)}
\end{gathered}
$$




\subsection{Brief of Chapter 4}

- Numerical simulation is one of the stages to be developed in order to design a component. The component should be analyzed in light of strength criteria, the classic fatigue theory, and the finite element method.

- FEM is suitable to a wide range of problems of boundary values in engineering and this method may be summarized into three stages: (1) pre-processing, (2) solver and (3) postprocessing.

- There are two classic models of material behavior: The first, purely phenomenological, is at a macroscopic scale, i.e., it operates through global variables (e.g., stress, strain, and internal variables); and The second, known as micro-macro, is at a microscopic scale and seeks to predict the macroscopic response by changing scales from physical parameters such as density and movement of discrepancies, nucleation and propagation phenomena, and the distance between precipitates.

- The incremental crack propagation model is composed of two parts: (1) a propagation law that provides the instantaneous speed of creating the cracked area per length of the crack front in relation to the instantaneous speed of plastic rounding of the crack. This first part of the model is identified by using a test of fatigue crack propagation at constant amplitude; and (2) a plastic rounding law that allows the determination of the global speed of plastic deformation in the crack front region in relation to applied load and internal variables of the model. These internal variables define in a comprehensive way the condition of residual stresses and material hardening in the plastic zone. This second part of the model is identified by using finite element calculations and requires identification of the material cyclic elastic-plastic behavior. 


\section{CHAPTER 5: METHODOLOGY}

\subsection{Experimental Procedures}

\subsubsection{Materials}

This study employed an aluminum-silica alloy (A356), whose chemical composition is presented in Table 5. The main characteristics of this alloy are good mechanical strength, high resistance to corrosion, thermal treatability, and weldability. The material was cast in permanent mold (PMC). In addition, material was obtained from the component itself (engine head).

Table 5: Nominal chemical composition of the aluminum-silica alloy under study.

\begin{tabular}{|c|c|c|c|c|c|c|c|c|}
\hline & \multicolumn{9}{|c|}{ ELEMENTO } \\
\cline { 2 - 9 } & $\boldsymbol{S i}$ & $\boldsymbol{M g}$ & $\boldsymbol{F e}$ & $\boldsymbol{C u}$ & $\boldsymbol{Z n}$ & $\boldsymbol{M n}$ & $\boldsymbol{T i}$ & $\boldsymbol{A l}$ \\
\hline \% Peso & $6.5-8.5$ & $0.25-0.45$ & $0.1-0.7$ & $0.1-3.0$ & $0.10-0.8$ & $0.10-0.4$ & 0.10 máx & $86.0-92.0$ \\
\hline
\end{tabular}

\subsubsection{Manufacturing Process}

\section{Permanent Mold Cast}

The permanent cast standardized according to ASTM B108-08 (2008) was employed to obtain two cylindrical strain specimens cast by gravity $(12.5 \mathrm{~mm}$ diameter). The mold design included feeding and distribution channels and heads, which produced specimens with microstructure presenting a very low quantity of defects. The PMC process included degasification, refining, and modification of silica particles. Figure 50 shows the complete cast obtained in this fashion. 


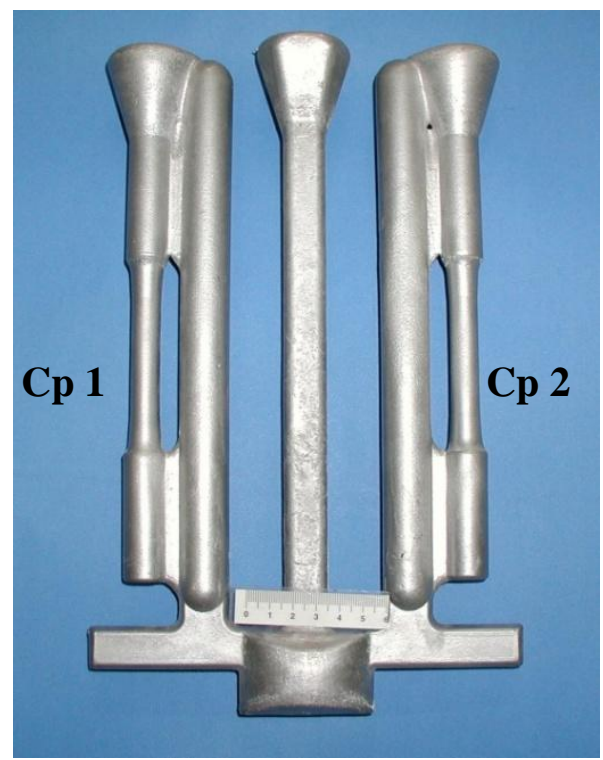

Figure 50: Permanent mold cast produced according to ASTM B108-08 (2008).

\section{Engine Head}

General Motors Brazil supplied ten 1.0 engine heads, from which seven specimens were obtained, totaling 70 specimens (Figure 51).
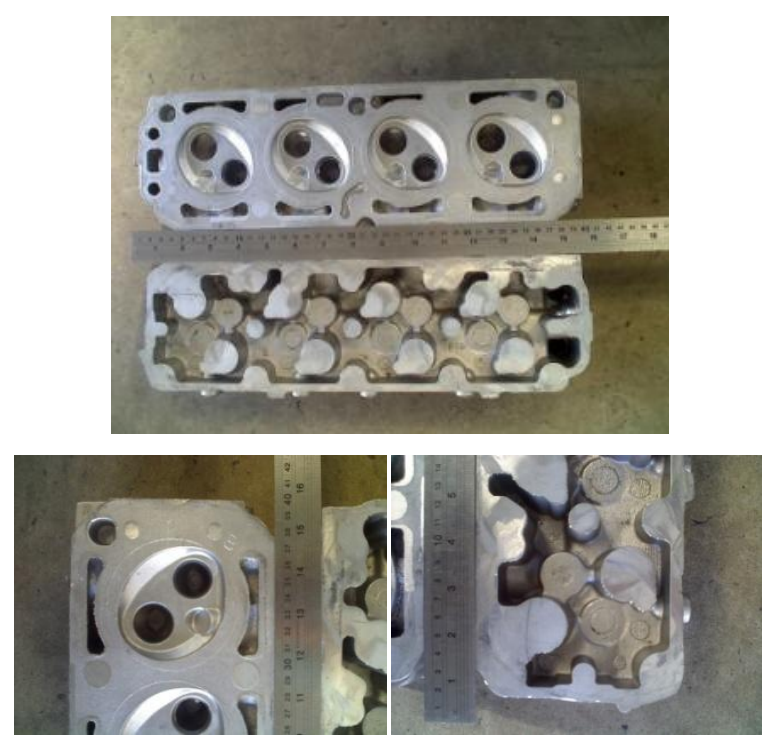

(a)

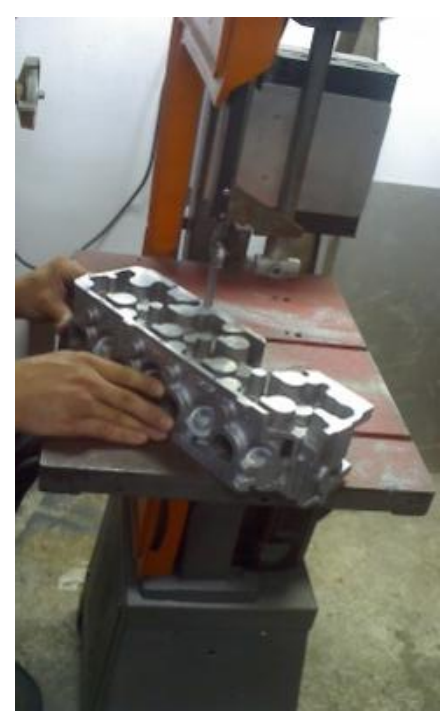

(b)

Figure 51: (a) 1.0 engine head supplied by General Motors Brazil and (b) cutting process employed to obtain specimens.

\subsubsection{Preparation of Test Specimens}


For tensile, isothermal, and anisothermal fatigue tests, specimens were obtained from the central PMC region (Figure 52a) whereas, for relaxation and crack propagation tests under isothermal and anisothermal loading conditions, they were obtained from the component (engine head) (Figure 52b). After machining, the specimens were sanded with 320, 600, and 1200-grit sandpaper and then polished with $10 \mu \mathrm{m}$ chromium oxide down to a mirror finish. Due to the low emissivity coefficient1 of polished aluminum, the usable area of specimens received a coating of matte black paint (heat resistant up to $600^{\circ} \mathrm{C}$ ) prior to testing at elevated temperatures in order to facilitate infrared pyrometer reading.

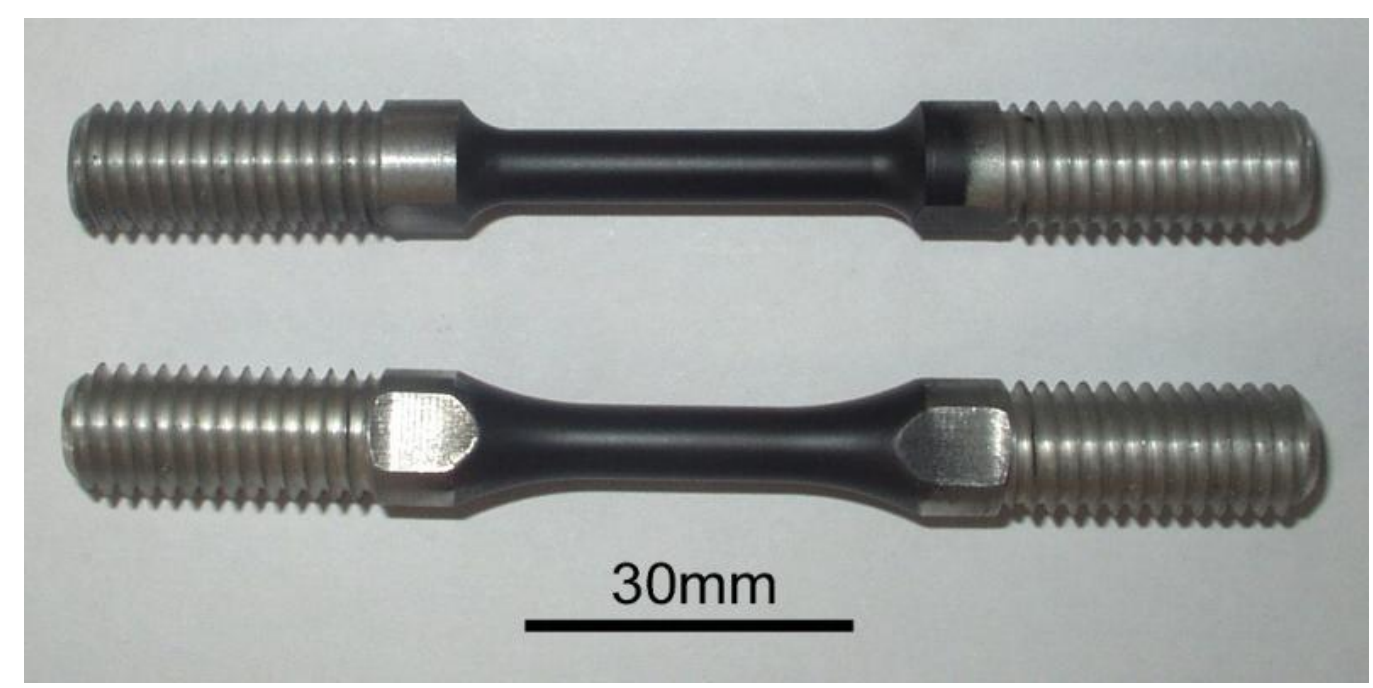

(a)

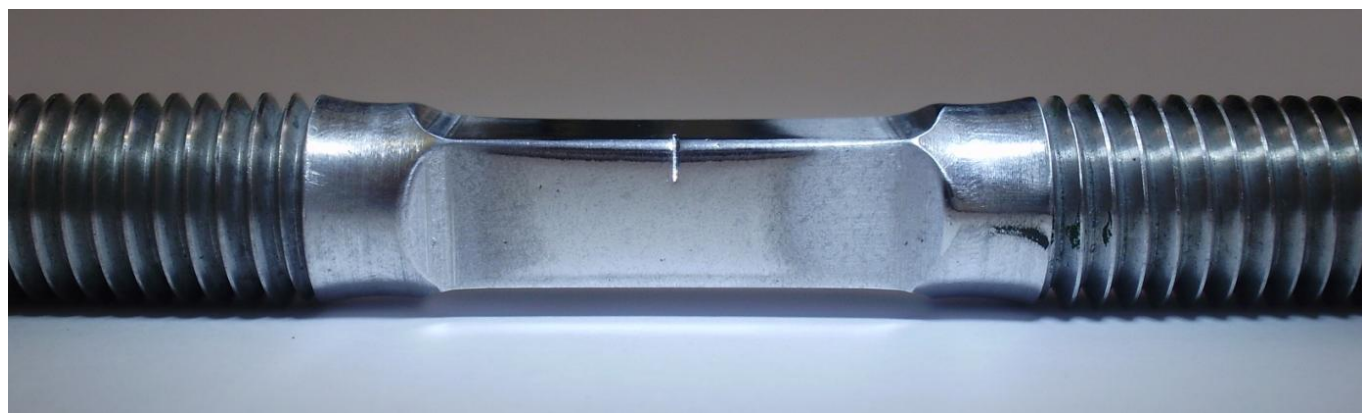

(b)

Figure 52: Machined, polished, and painted specimens.

\subsubsection{Mechanical Test System}

The mechanical testing system was part of LAPROMEC equipment (Laboratory of Mechanical Properties of the Department of Materials, Aeronautical, and Automotive

\footnotetext{
${ }^{1}$ Radiance of a body at a given temperature and radiance of a black body at the same temperature. Radiance or emissivity corresponds to the density of light flow per unit area from a luminous or lit surface.
} 
Engineering) at São Carlos campus of São Paulo University (USP). The testing equipment consisted basically of an induction heating system coupled to an MTS 810 testing machine, in which a microcomputer connected to the system monitored and controlled specimen temperature and deformation during tests through an infrared pyrometer and a ceramic rod extensometer, respectively.

\section{Heating System}

The heating system consisted of an INDUCTOHEAT induction furnace $(7.5 \mathrm{~kW}$ power and $200 \mathrm{kHz}$ frequency), equipped with a CNT110 INCON programmable temperature controller. The furnace had a coupled inductor coil made of copper ( $5 \mathrm{~mm}$ diameter), specially designed to envelope the specimen, heating it uniformly, and allow the extensometer rods to pass in between the coils.

\section{Infrared Pyrometer}

Specimen temperature was measured by means of an infrared pyrometer with laser sight (RAYTEK, Thermalert SX series, D9ETXSLTCF1L2 model) for temperatures ranging from $-18^{\circ} \mathrm{C}$ to $500^{\circ} \mathrm{C}$. The temperature measured by this sensor served as a control parameter for the induction furnace and data acquisition system of the testing program. Fatigue tests above room temperature require precise temperature measurements in the central region of specimens, without physical contact, thereby avoiding problems such as crack initiation due to thermocouples welding.

\section{Testing Machine}

Tensile, isothermal and anisothermal fatigue, crack propagation, and relaxation tests were performed in an MTS $810250 \mathrm{kN}$ servo-hydraulic testing machine, equipped with an MTS Flex Test GT controller. Fatigue and relaxation tests were done under strain control, using an MTS 632.54F14 model extensometer for high temperatures, consisting of two 2-mm diameter ceramic rods attached to the specimen by a coil system. Tensile tests under displacement control also employed this extensometer. Crack propagation tests were conducted under load and displacement control for cyclic and no-dwell loadings. All tests used an MTS 680.01B model system of hydraulic grips for high temperatures and a grip cooling system. Figure 53 presents an overview of the testing system. Figure 54 shows the 
system of grips, infrared pyrometer, and extensometer for fatigue tests. Figure 55 shows the specimen details, induction coil and grip cooling system. The auxiliary grip cooling system for fatigue tests at high temperatures is composed of two coils made with copper pipes, through which chilled water circulates, and two compressed air hoses coupled to the ends of the upper and lower grips.

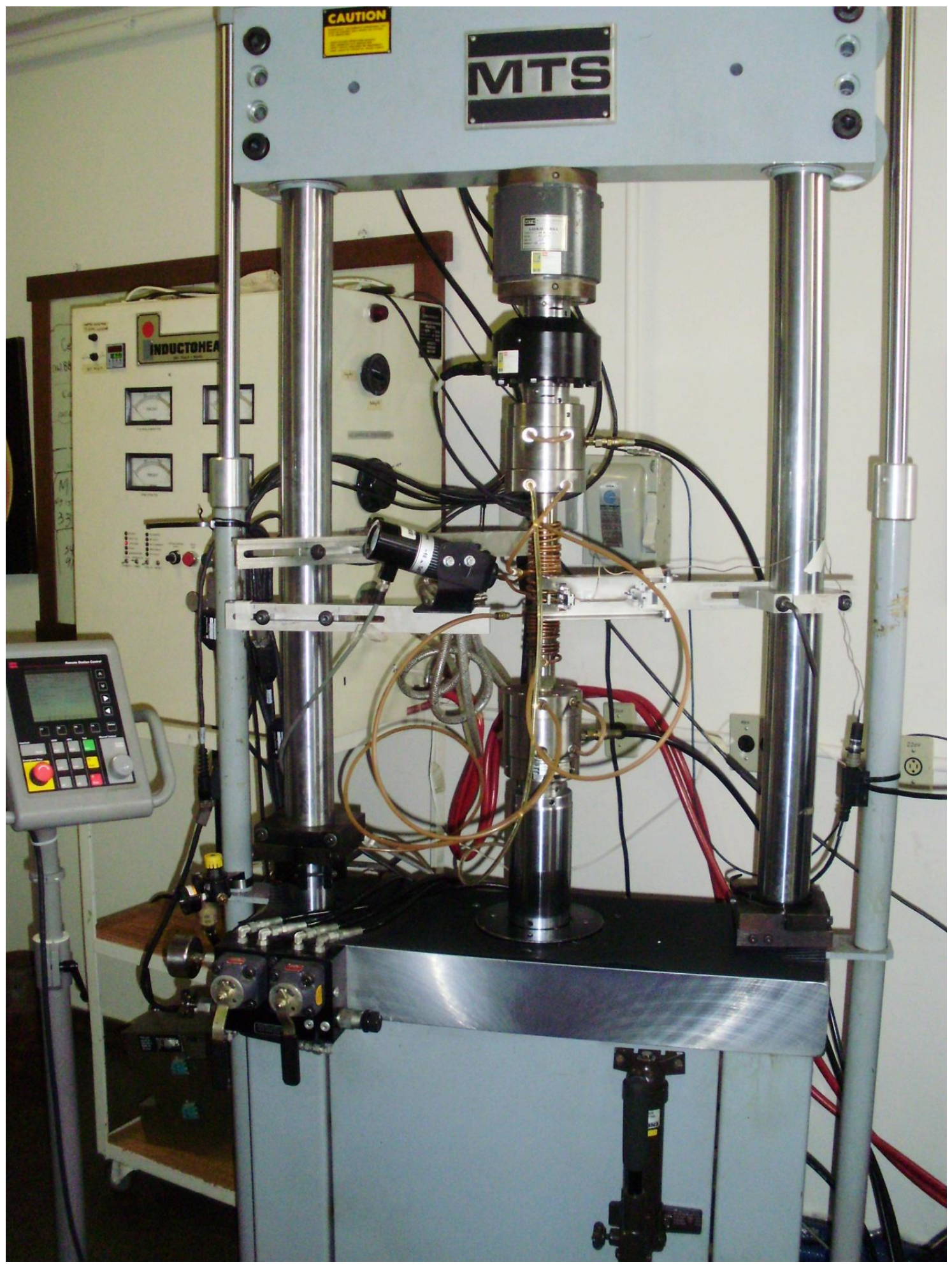

Figure 53: Testing system showing induction furnace and MTS 810 servo-hydraulic equipment. 


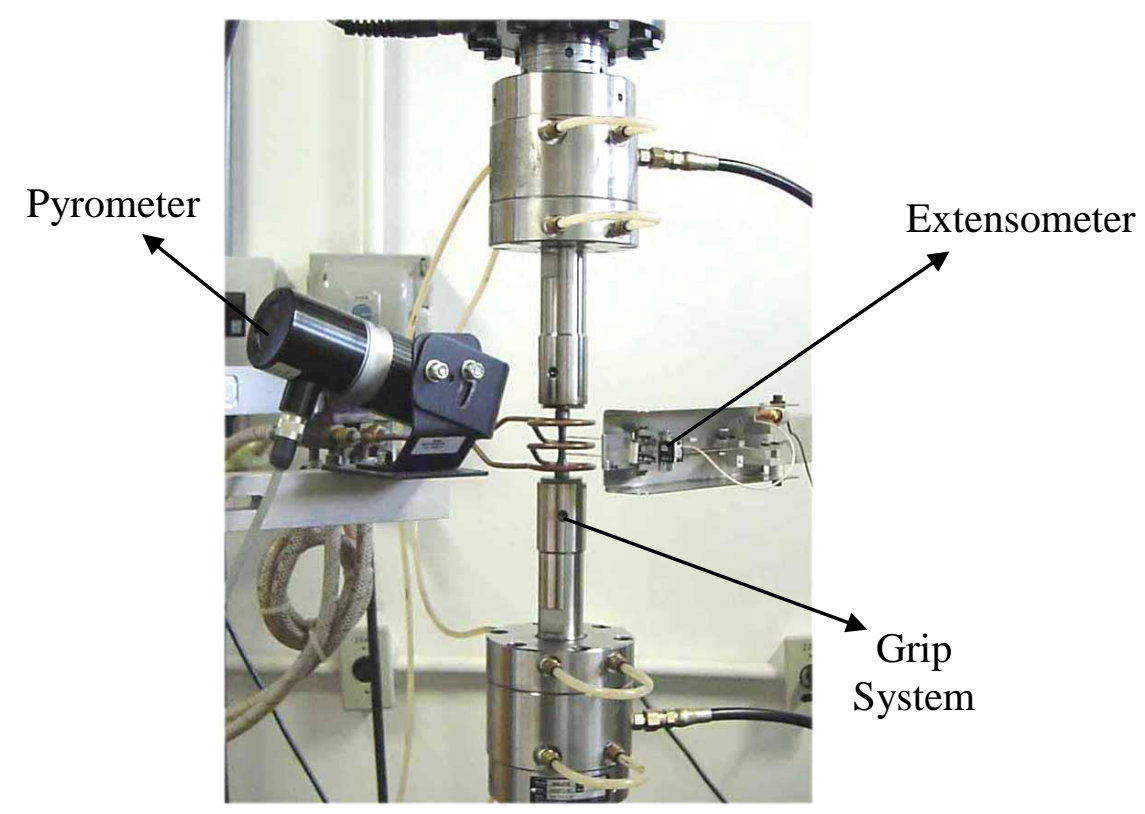

Figure 54: System of grips, infrared pyrometer, and extensometer for fatigue testing..

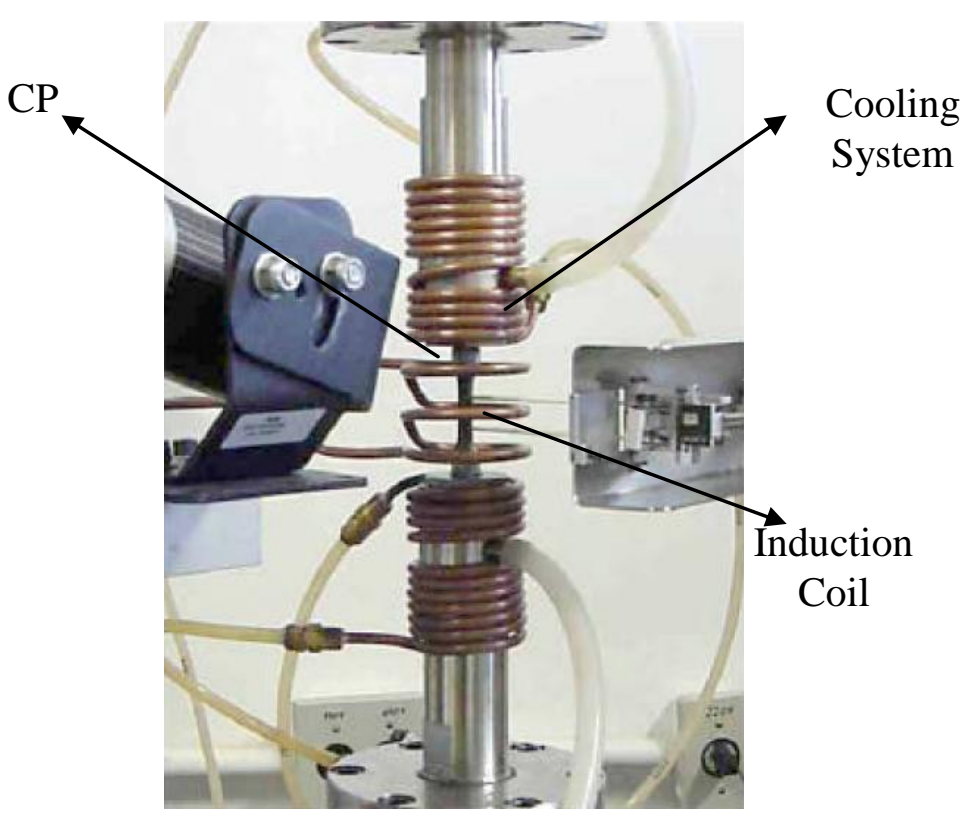

Figure 55: Details of specimen, induction coil, and auxiliary grip cooling system.

\section{Control and Monitoring System}

Each type of mechanical test required a different MultiPurpose TestWare (MPT) software program for the MTS Flex Test GT controller as well as PID adjustment in order to achieve better equipment performance and, consequently, reliable data. Crack propagation tests employed a potential drop method (MDP), in which control was performed through a nano-voltmeter and a DC source connected to the MTS controller. For tests at high 
temperatures, temperature monitoring and control were conducted by a EUROTHERM controller connected to the MTS controller and induction furnace (Figure 56).

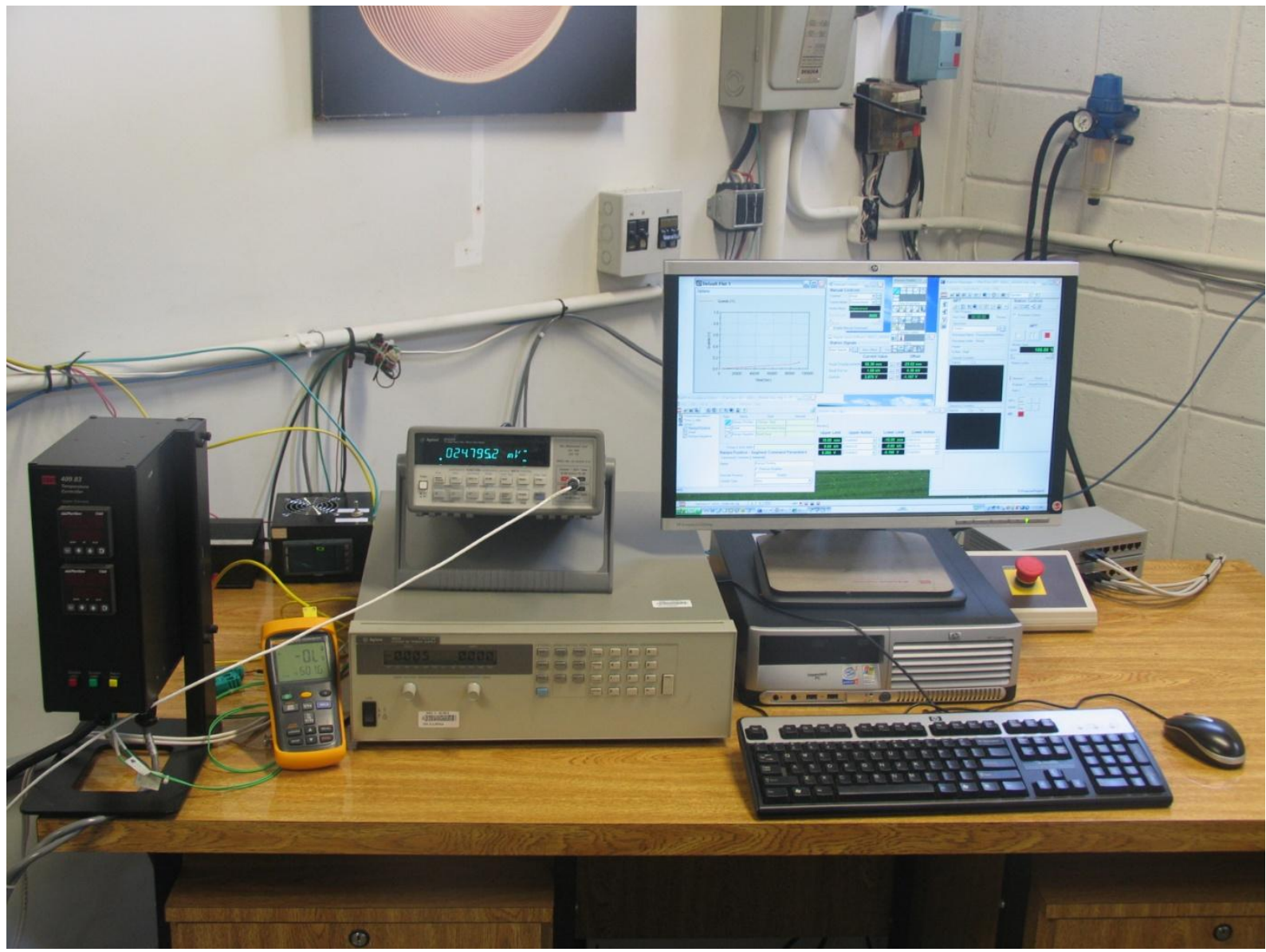

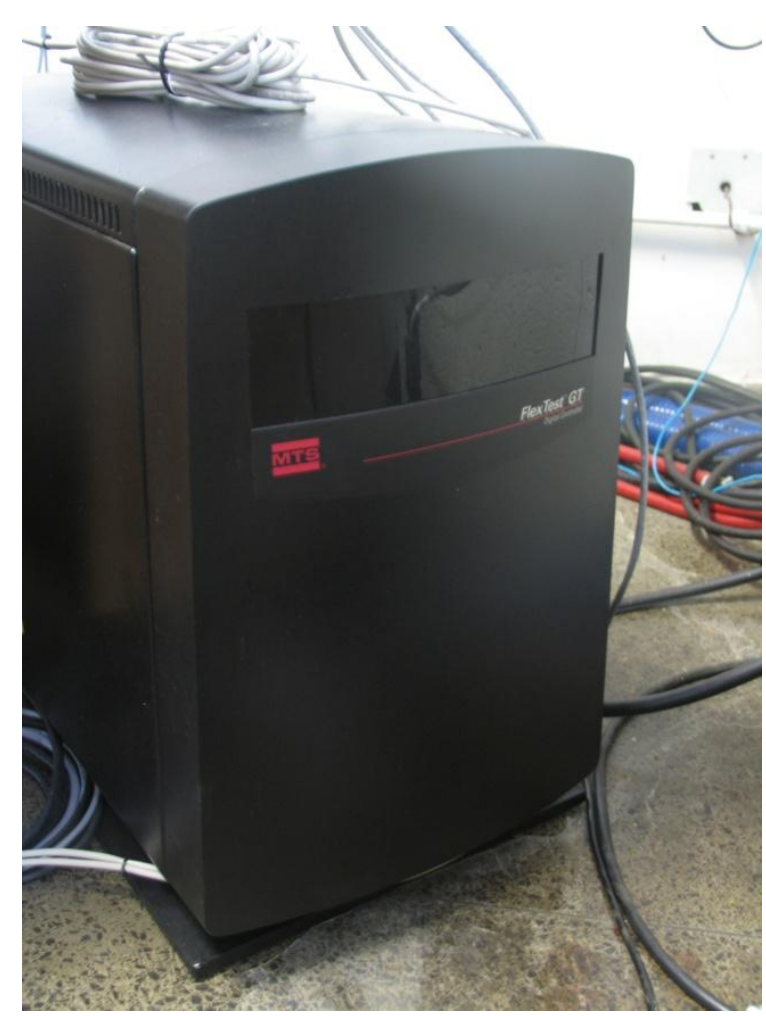

(b) (a)

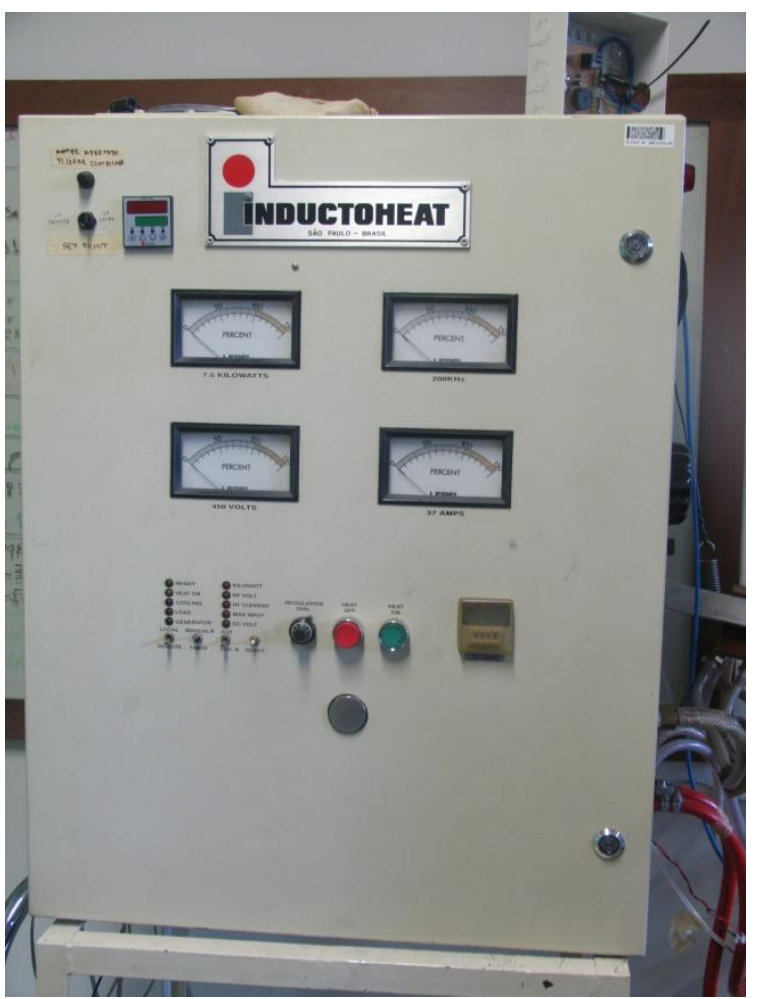

(c)

Figure 56: Anisothermal fatigue testing system. 


\subsubsection{Tensile Tests}

Tensile tests were performed at room temperature, $120^{\circ} \mathrm{C}$, and $280^{\circ} \mathrm{C}$ according to ASTM E21 and ASTM E8/E8M-09-09, under displacement control at $0.003 \mathrm{~mm} / \mathrm{s}$ piston displacement speed, using the MTS-MPT program. Tensile tests were conducted in this work mainly to obtain important properties of the alloy in question, e.g., yield strength and elasticity modulus, which are useful for calculating deformations measured in thermomechanical fatigue tests and to make appropriate parameters available for the finite element model. The tensile test specimen geometry is shown in Figure 57.

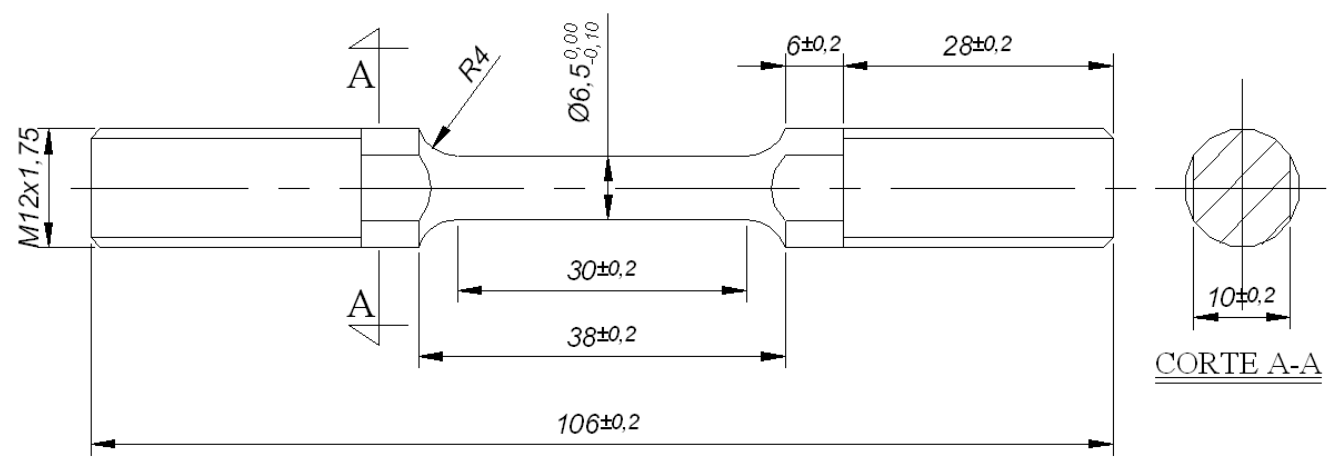

Figure 57: Tensile test specimen for different temperatures.

\subsubsection{Isothermal and Anisothermal Fatigue Testing}

Fatigue tests were performed under controlled strain. All tests were performed at mechanical deformation ratio $R_{\varepsilon}=-1$. The failure criterion used was a $50 \%$ drop of the maximum stress reached during the experiment. Isothermal tests employed $0.1 \mathrm{~Hz}$ frequency in order to come near the maximum frequency used in anisothermal fatigue tests, whose periods are relatively long (about 300s) owing to difficulty in cooling specimens down. However, this type of test is usually conducted over long periods in order to avoid excessive temperature gradients in specimens during heating and cooling. In addition, temperatures in anisothermal tests were imposed by a triangular wave with maximum cycle temperature coinciding with maximum mechanical deformation (in phase) according to initial ASTM E2368-10 guidelines. Isothermal or low-cycle fatigue tests at high temperatures were performed according to ASTM E 606-0 ${ }^{\varepsilon 1}$.

The alloy mechanical behavior was assessed through cyclic hysteresis of inelastic and mechanical strain-stress and progression of some testing parameters in relation to number of 
cycles, e.g., maximum and minimum inelastic strains and stresses as well as total inelastic deformation energy and Ostergren energy (tensile deformation energy of hysteresis) for mechanical strain levels representing overall behavior. Anisothermal fatigue tests were conducted under in-phase conditions, i.e., maximum mechanical deformation coinciding with maximum cycle temperature.

Isothermal and anisothermal fatigue lifetime results were assessed by means of mechanical variation $\left(\Delta \varepsilon_{\mathrm{m}}\right)$, inelastic deformation $\left(\Delta \varepsilon_{\mathrm{in}}\right)$, stress $(\Delta \sigma)$, total deformation energy, and Ostergren energy in relation to number of cycles to fail $\left(\mathrm{N}_{\mathrm{f}}\right)$.

The specimen geometry commonly employed in isothermal and anisothermal fatigue tests is shown in Figure 58.

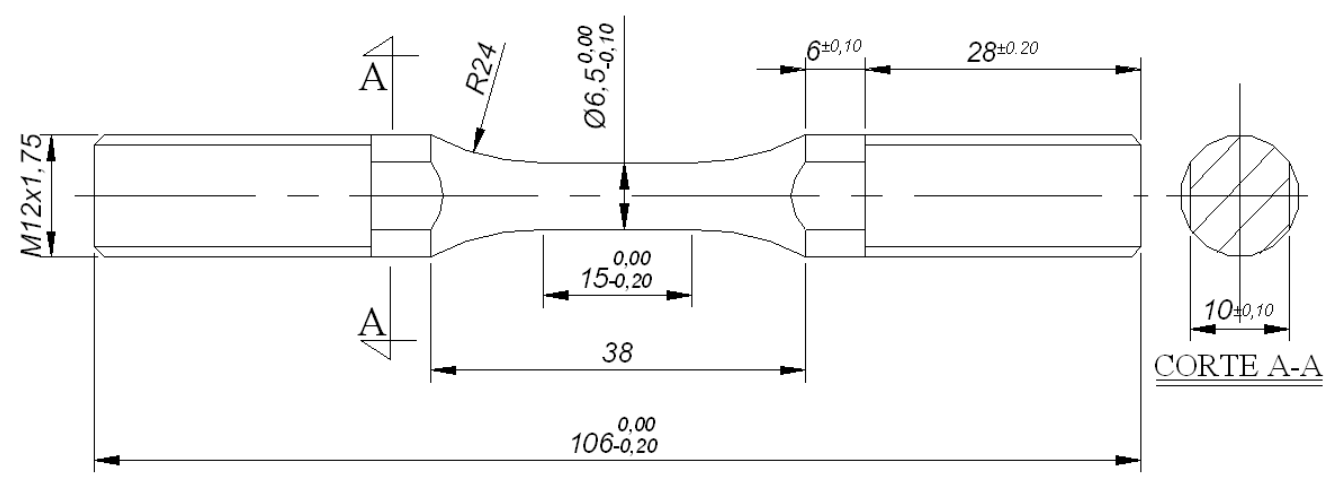

Figure 58: Geometry of fatigue test specimens.

Cyclic hardening curves ( $\sigma$ as a function of $\varepsilon$ ) were determined for different temperatures. The method chosen for obtaining the cyclic hardening curve is the gradual increase in strain applied to the specimen, producing a stable hysteresis for each strain level used (an anisothermal example is given in Figure 59). The effect of strain rate $(\mathrm{d} \varepsilon / \mathrm{dt}$ ) on hysteresis shape was also investigated by varying the test frequency.

Among other factors, the material behavior depends on the gap between $\varepsilon(t)$ and $T(t)$ cycles or, put differently, on the chosen shape of cycle $\varepsilon(T)$. In fact, through a TMF (thermomechanical fatigue) test, it is possible to simulate the behavior of any volume element from a real component as long as the thermo-mechanical load $(\varepsilon(T))$, which the volume element is subjected to during operation, is known. 


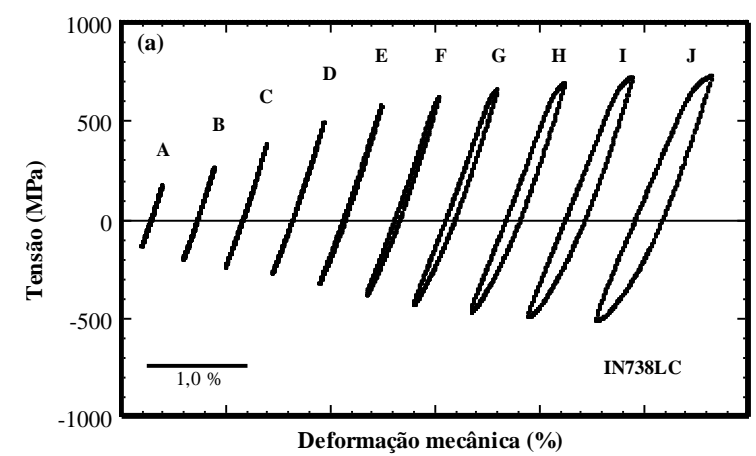

(a)

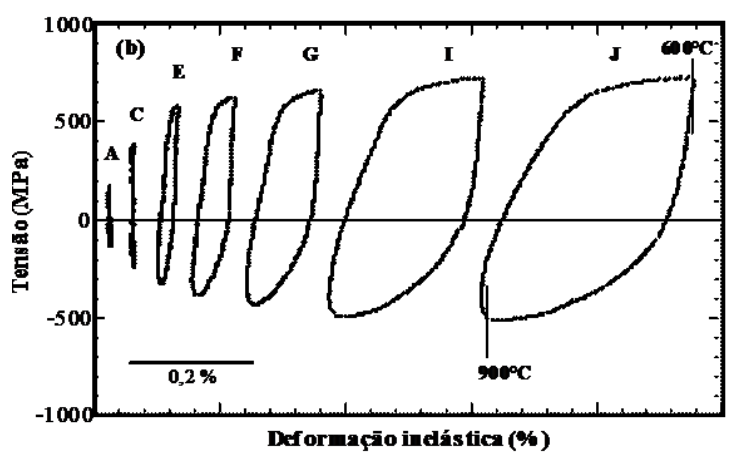

(b)

Figure 59: Anisothermal hystereses obtained between $600^{\circ} \mathrm{C}$ and $900^{\circ} \mathrm{C}$ for IN738LC alloy.

Cycle shape traditionally employed in the study of thermo-mechanical fatigue are inphase, i.e., maximum mechanical deformation coinciding with maximum cycle temperature (Figure 60a), and out-of-phase, minimum mechanical deformation coinciding with maximum cycle temperature (Figure 60a).

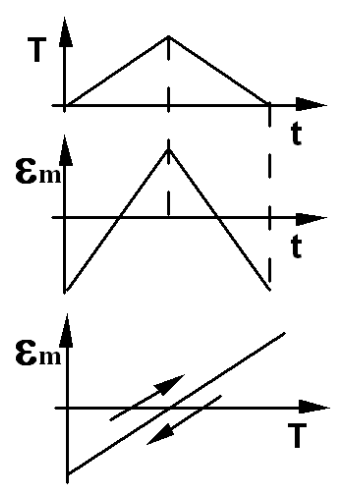

(a) In phase
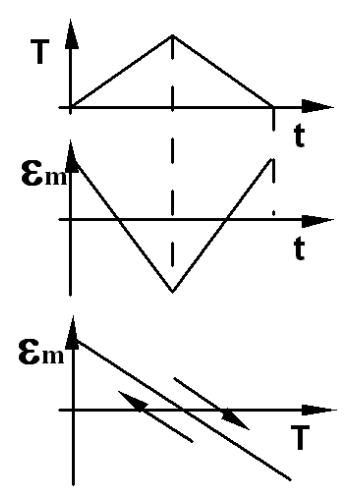

(b) Out of phase
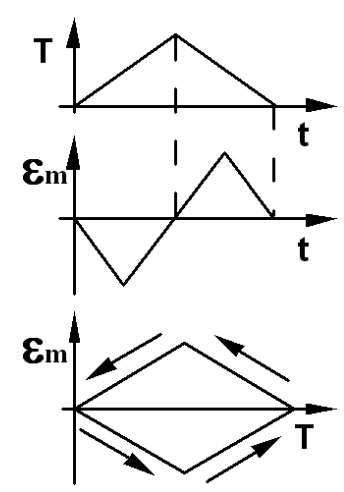

(c) "Diamond"

Figure 60: Cycle formats usually employed in thermo-mechanical fatigue tests.

Thermo-mechanical fatigue testing is also very useful in identifying damage and fracture mechanisms of materials submitted to anisothermal solicitations. At the end of cyclic hardening tests, the specimens were sectioned and metallographically prepared for examination under optical and scanner microscopes. These observations enabled the detection of occasional micro-structural changes due to exposition to test temperatures as well as the identification of sites favoring crack nucleation under isothermal and anisothermal conditions.

\subsubsection{Crack Growth Tests}

Crack propagation tests were conducted at $120^{\circ} \mathrm{C}, 200^{\circ} \mathrm{C}$, and $280^{\circ} \mathrm{C}$ on a corner notch of specimens designed in accordance with the component geometry (Figure 51 and Figure 
52b). The loading rate employed was $R_{\varepsilon}=0.1$ and cracks were monitored by means of the potential drop method. Crack propagation tests under thermo-mechanical loading conditions were attempted during approximately 1 (one) year, but to no avail, despite much effort.

\subsubsection{Micro Structural and Fracture Analysis}

Micro-structural observation of the aluminum alloy was done under an optical microscope. A quantitative image analysis software program (Image Pro Plus) was employed to determine size and morphology of phase $\alpha$, eutectic distribution, and porosity level.

The morphological aspect of fracture surfaces of some specimens tested for isothermal and anisothermal fatigue and crack propagation was analyzed by scanning electron microscopy (SEM).

\subsection{Computational Procedures}

\subsubsection{First Step}

This step comprised the adaptation to a computational modeling software program existing on the market. The chosen program was ABAQUS STANDARD FINITE ELEMENT CODE, due to its simplicity in testing new models and algorithms, e.g., forward EULER, backward EULER, and backward EULER with a radial return. The last one presents the best and fastest results under anisothermal loading conditions, according to Charkaluk (1999).

\subsubsection{Second Step}

It consisted of improving the model of crack propagation developed by Professor Sylvie Pommier in the Laboratoire de Mécanique et Technologie (LMT) at L'École Normale Supérieure de Cachan (ENS-Cachan-France) for loading applied to a real component (engine head), i.e., under anisothermal conditions, given that a component can operate in the presence of cracks as long as they do not reach a critical size conducive to catastrophic failure. 


\subsubsection{Third Step}

This step consisted of a computational implementation of the improved mathematical model. To this end, FORTRAN and PYTHON, whose written code would become part of an ABAQUS subroutine, was employed. Then, the model was evaluated by using test data obtained from strain, fatigue, and crack propagation specimens.

\subsubsection{Fourth Step}

In future studies, this step will comprise the application of the model developed and validated on specimens to real components in operation. To this end, it will be of fundamental importance to map temperatures and stresses to which the component is subject in operation. This step will count on the support of General Motors Brazil and will be conducted experimentally through sensors coupled to the component. 


\subsection{Brief of Chapter 5}

- Thermomechanical fatigue test methodology was developed with successful in the Lab of the University of São Paulo.

- The communication between the equipments purchased, induction furnace, temperature controller and mechanical testing machine was successful.

- The use of the potential drop methodology for measurements of the fatigue crack growth has been improved

- The application of the model of crack propagation developed by Professor Sylvie Pommier in the Laboratoire de Mécanique et Technologie (LMT) at L’École Normale Supérieure de Cachan (ENS-Cachan-France) for isothermal loads applied to a specimen was performed in the Lab of the University of São Paulo with successful.

- Crack propagation tests under thermo-mechanical loading conditions were attempted during approximately 1 (one) year, but to no avail, despite much effort. 


\section{CHAPTER 6: RESULTS AND DISCUTION}

\subsection{Experimental Results}

\subsubsection{Micro-structural and Chemical Analysis}

The results of chemical analysis via optical emission spectrometry by spark is shown in Table 6, the uncertainty value (U) being 2\%. The analysis was performed in an ARL 3400 optical emission spectrometer. ASTM E2055-99 (Standard Practice for Referencing Test Methods for Chemical Analysis of Metals and Related Metals) was used as reference. Before proceeding to the analysis, the spectrometer chamber was cleaned and the spectrometer calibrated using RBC-certified standards. Three (3) surface burnings were performed.

Table 6: Results of chemical analysis through spectrometry (\% in weight).

\begin{tabular}{|c|c|c|c|c|c|c|c|}
\hline Elements & $\mathbf{S i}$ & $\mathbf{F e}$ & $\mathrm{Cu}$ & Mn & Mg & $\mathrm{Cr}$ & $\mathbf{N i}$ \\
\hline Alloy A356 & 8.63 & 0.98 & 3.13 & 0.45 & 0.326 & 0.03 & 0.03 \\
\hline Elements & Zn & $\mathbf{T i}$ & Ca & $\mathbf{P}$ & $\mathbf{P b}$ & Sb & Sn \\
\hline Alloy A356 & 0.66 & 0.027 & ND & 0.002 & 0.06 & ND & 0.02 \\
\hline Elements & $\mathrm{Sr}$ & V & $\mathbf{Z r}$ & B & Cd & Co & Al \\
\hline Alloy A356 & ND & 0.01 & ND & 0.0005 & ND & ND & Base \\
\hline
\end{tabular}

In comparison to nominal values of chemical composition (Table 5), it is possible to observe that $\mathrm{Si}, \mathrm{Fe}, \mathrm{Mn}$, and $\mathrm{Cu}$ weight percentages are slightly above the nominal ones, besides $\mathrm{Mg}$ and $\mathrm{Zn}$, which are near the maximum limit. As previously seen, this can be highly detrimental since iron and copper are the main impurities in $\mathrm{Al}-\mathrm{Si}$ alloys. $\mathrm{Cu}$ weakens these alloys when hot and iron is particularly harmful since it is conducive to formation of acicular platelets, which significantly reduce ductility and fracture toughness (Figure 9). However, when balanced with manganese, iron forms a more compact eutectic phase (A115(Fe, Mn)3Si2, Chinese script-shaped (Figure 61), which is less detrimental to mechanical properties (Crepeau, 1998). Zn is a weakening factor when hot and promotes high shrinkage during cast solidification, giving rise to contraction voids. Mn causes a small reduction in the material ductility. 


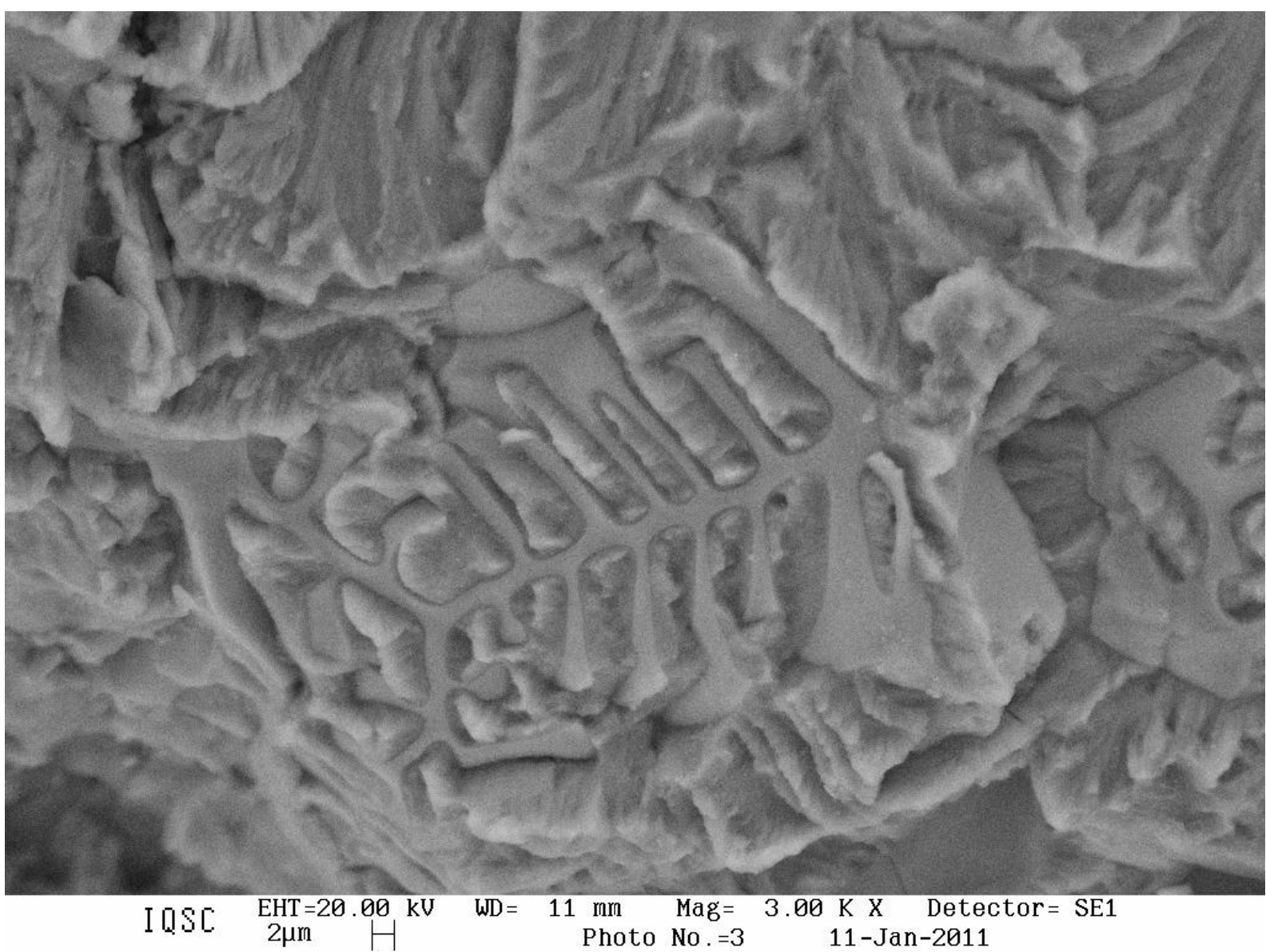

Figure 61: Scanning electron microscopy (SEM) image of fractured surface of a fatigue crack propagation test specimen.

Chemical composition analysis was also performed by EDS (Energy Dispersive System) in EDX LINK ANALYTICAL equipment (Isis System Series 200), with SiLi Pentafet detector, ATW II (Atmosphere Thin Window) ultrathin window, $133 \mathrm{eV}-5.9 \mathrm{keV}$ resolution, coupled to an electron microscope. A Co calibration standard, 20kV electron beam, $25 \mathrm{~mm}$ focal length, $30 \%$ dead time, and 2.82A current were also employed. This analysis was performed on fractured surfaces of fatigue crack propagation specimens from the component (cylinder head) and tested at $120^{\circ} \mathrm{C}, 200^{\circ} \mathrm{C}$, and $280^{\circ} \mathrm{C}$, based on images of the fractured surfaces obtained by scanning electron microscopy (SEM) (Figure 62, Figure 63 e Figure 64).

Firstly, an area of approximately $25 \mathrm{~mm} 2$ was selected to provide an overall chemical composition result. The purpose of this procedure was to establish the occurrence or absence of effects of long test periods at different temperatures on chemical composition through formation of precipitates from synergy between temperature and mechanical deformation. Table 7 shows the lack of homogeneity of the material under study (engine head) due to its manufacturing process (casting). Hence, no conclusion may be drawn. 
Another analysis comprised the selection of a smaller area $(900 \mu \mathrm{m} 2)$ in the region of final fracture and crack propagation under fatigue. Results from this new analysis (Table 7) show brittleness regions with high $\mathrm{Cu}$ and Fe concentrations, which, together with the high level of porosity found (Table 8), cause a decrease in the component mechanical strength.

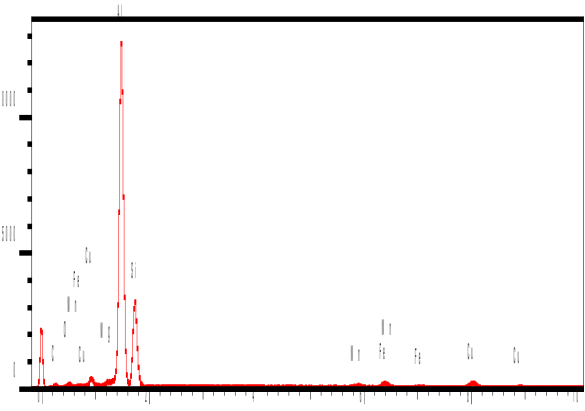

(a)

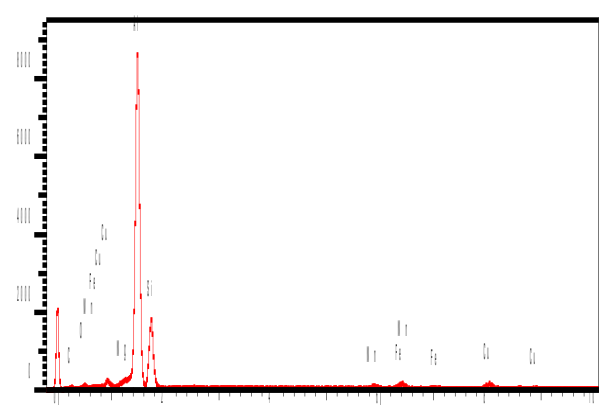

(b)

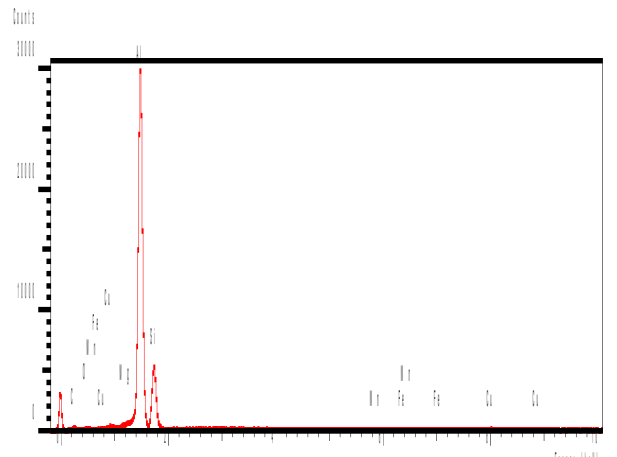

(c)

Figure 62: Analysis of chemical composition via EDX in specimen tested at $120^{\circ} \mathrm{C}$ for areas of (a) $25 \mathrm{~mm}^{2}$ and (b and c) $900 \mu \mathrm{m}^{2}$ in the regions of crack propagation and brittle fracture. 


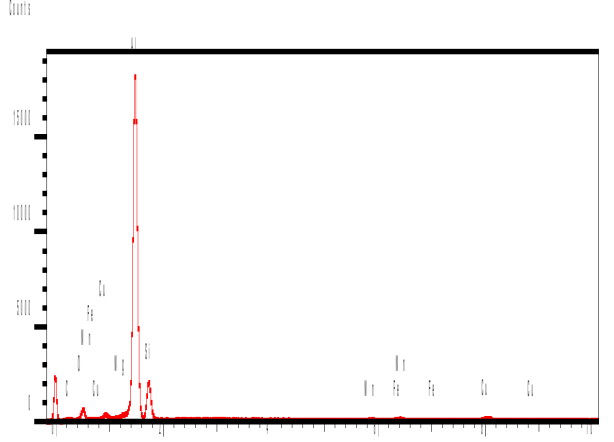

(a)

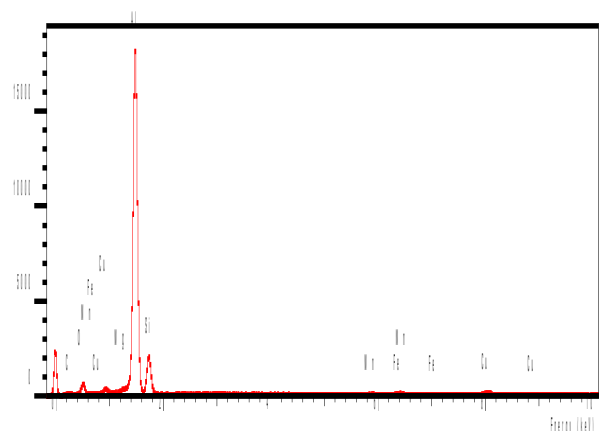

(b)

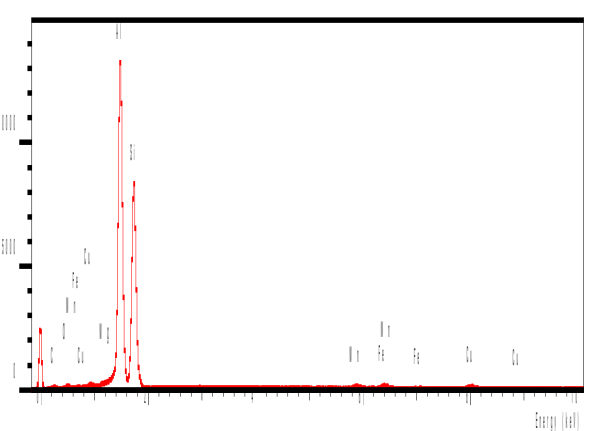

(c)

Figure 63: Analysis of chemical composition via EDX in specimen tested at $120^{\circ} \mathrm{C}$ for areas of (a) $25 \mathrm{~mm}^{2}$ and (b and c) $900 \mu \mathrm{m}^{2}$ in the regions of crack propagation and brittle fracture.

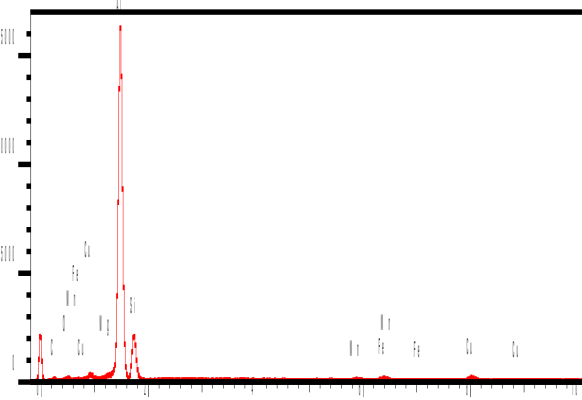

(a) 


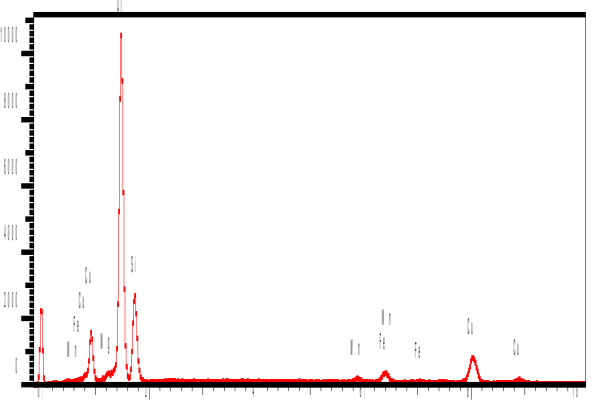

(b)

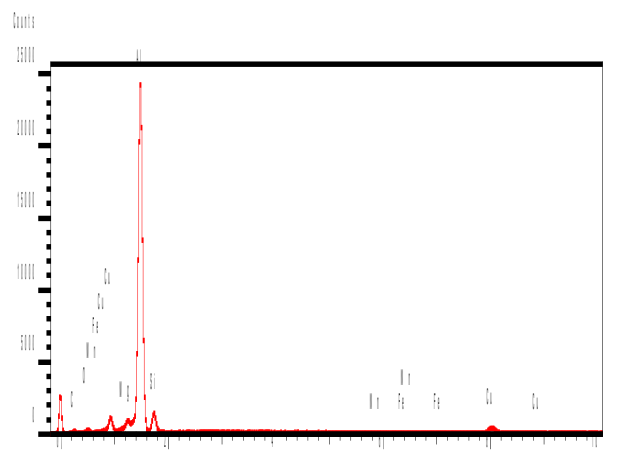

(c)

Figure 64: Analysis of chemical composition via EDX in specimen tested at $280^{\circ} \mathrm{C}$ for areas of (a) $25 \mathrm{~mm}^{2}$ and (b and c) $900 \mu \mathrm{m}^{2}$ in the regions of crack propagation and brittle fracture.

Table 7: Results of chemical analysis via EDX (\% in weight).

\begin{tabular}{|c|c|c|c|c|c|c|c|c|}
\hline & & & \multicolumn{6}{|c|}{ Alloy Elements } \\
\hline $\begin{array}{c}\text { Mechanical } \\
\text { Test }\end{array}$ & Region & Area & Al & $\mathbf{S i}$ & Mg & Mn & $\mathbf{F e}$ & $\mathrm{Cu}$ \\
\hline Temperature & & & & & & & & \\
\hline \multirow{3}{*}{$120^{\circ} \mathrm{C}$} & Total & $25 \mathrm{~mm}^{2}$ & 58.43 & 27.99 & 0.09 & 1.47 & 3.89 & 8.13 \\
\hline & $\mathrm{BF}^{*}$ & $900 \mu \mathrm{m}^{2}$ & 61.27 & 24.08 & 0.12 & 2.05 & 4.41 & 8.07 \\
\hline & $\mathrm{CP} * *$ & $900 \mu \mathrm{m}^{2}$ & 69.72 & 29.23 & 0.33 & 0.03 & 0.01 & 1.4 \\
\hline \multirow{3}{*}{$200^{\circ}$} & Total & $25 \mathrm{~mm}^{2}$ & 73.49 & 18.74 & 0.29 & 0.68 & 1.88 & 5.51 \\
\hline & $\mathrm{BF}^{*}$ & $900 \mu \mathrm{m}^{2}$ & 67.41 & 6.80 & 1.66 & 0.18 & 0.29 & 23.67 \\
\hline & $\mathrm{CP} * *$ & $900 \mu \mathrm{m}^{2}$ & 43.68 & 49.43 & 0.33 & 1.62 & 2.26 & 3.34 \\
\hline \multirow{3}{*}{$280^{\circ} \mathrm{C}$} & Total & $25 \mathrm{~mm}^{2}$ & 69.82 & 19.84 & 0.11 & 1.13 & 2.73 & 6.60 \\
\hline & $\mathrm{BF}^{*}$ & $900 \mu \mathrm{m}^{2}$ & 48.47 & 19.02 & 0.14 & 1.61 & 4.91 & 25.85 \\
\hline & $\mathrm{CP} * *$ & $900 \mu \mathrm{m}^{2}$ & 77.37 & 9.88 & 0.93 & 0.10 & 0.13 & 11.59 \\
\hline
\end{tabular}


Figure 65 shows micrographs of the alloy under investigation. This alloy microstructure is composed of primary $\alpha$ phase, Si-rich eutectic, precipitates $\left(\mathrm{Mg}_{2} \mathrm{Si}, \mathrm{Al}_{2} \mathrm{Cu}\right)$, and inter-metallic components $\left(\mathrm{Al}_{5} \mathrm{FeSi}, \mathrm{Al}_{8} \mathrm{Mg}_{3} \mathrm{FeSi}_{6}, \mathrm{Al}_{5} \mathrm{Mg}_{8} \mathrm{Cu}_{2} \mathrm{Si}_{6}\right)$. The micro-structural aspect of the alloy shows a well-defined dendrite structure with secondary dendrite arm spacing (SDAS) of approximately $25 \mu \mathrm{m}$, in addition to a high porosity level (Figure 65a and b, dotted-dashed arrow) and also shows considerable modification of silicon particles (Figure $65 \mathrm{~b}$, full arrow). It may be observed, in accordance with ASM (2004), the presence of $\mathrm{FeMg}_{3} \mathrm{Si}_{6} \mathrm{Al}_{8}$-type (Chinese-writing type) (Figure 65b dashed arrow) and $\mathrm{Fe}_{2} \mathrm{Si}_{2} \mathrm{Al}_{9}$-type (blade type) precipitates (Figure 65b, dotted arrow).

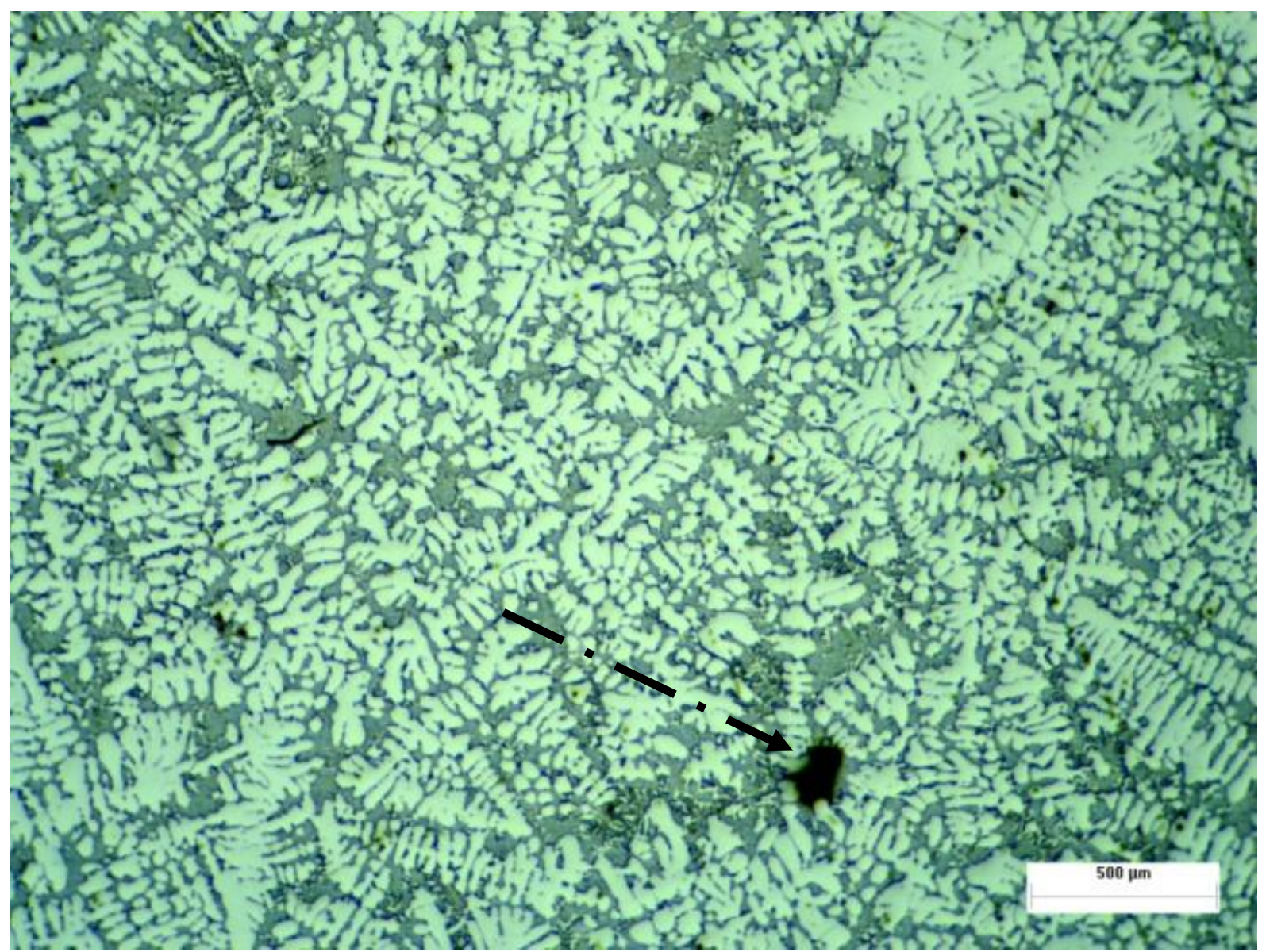

(a) 


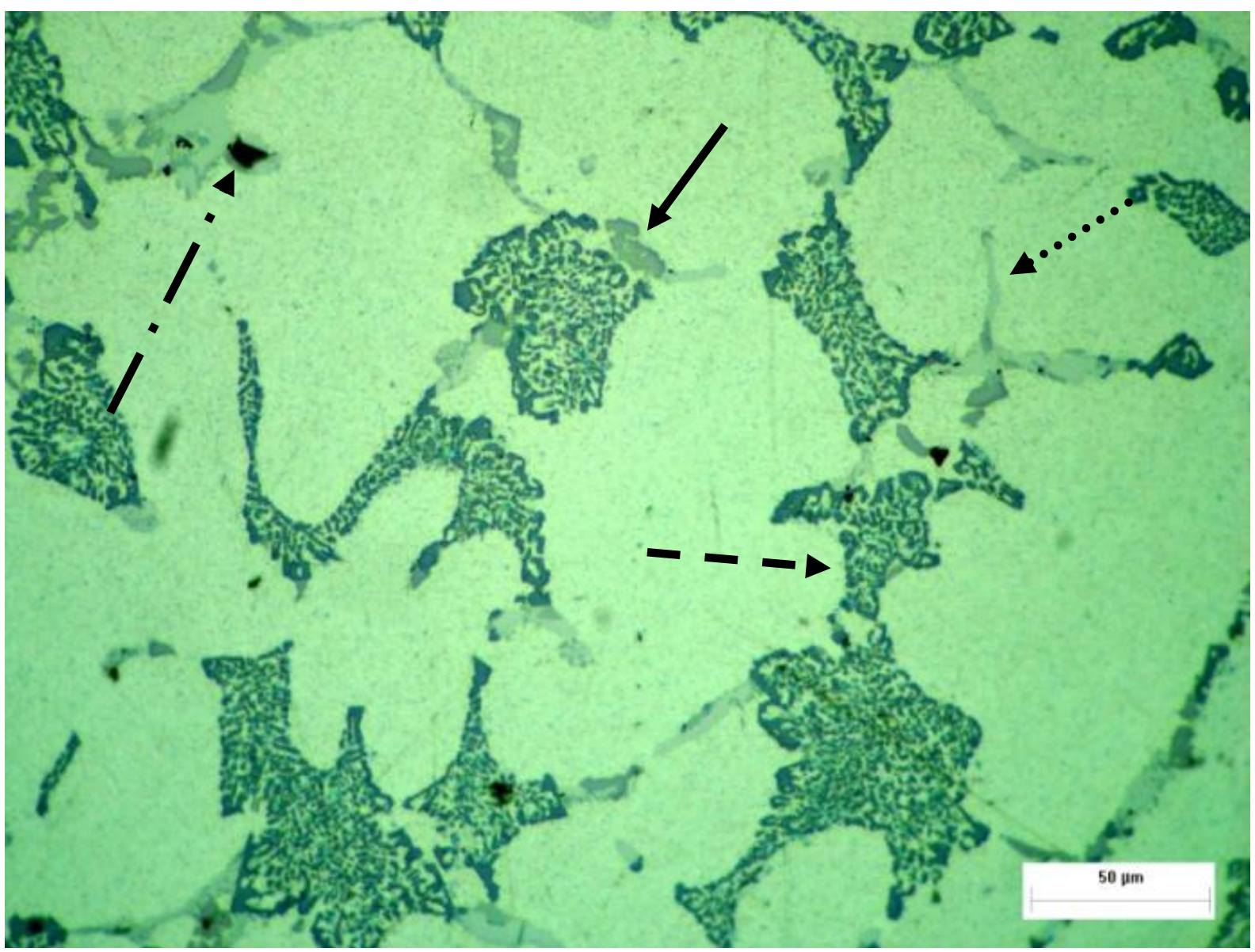

(b)

Figure 65: Optical micrographs of the alloy showing (a) well refined dendrite microstructure and (b) coalesced silica particles that constitute the inter-dendrite eutectic (dark grey indicated by full arrow) and $\mathrm{Fe}_{2} \mathrm{Si}_{2} \mathrm{Al}_{9}$-type precipitates (dotted arrow).

Table 8 shows the average porosity value per area of the alloy. The material under analysis displayed typical porosity as high as $50 \mu \mathrm{m}$. However, the diameter of some voids reached $500 \mu \mathrm{m}$. The porosity analysis was conducted with the aid of Image Pro Plus software from micrographs randomly taken in transversal and perpendicular directions under an Olympus microscope coupled to an image acquisition system. Figure 66 shows the micrographs used in this analysis as well as the corresponding regions of voids. There may be observed a high porosity level deriving from the casting process mainly due to failed cast degasification. Notwithstanding, despite the significant size of voids, they can hardly be detected through conventional techniques, e.g., ultrasound and X-ray, employed by auto factories, more so in sample analysis.

Table 8: Quantitative analysis of material porosity.

Alloy Average Porosity [Area \%] Standard Deviation [Area \%]

$\begin{array}{lll}\mathrm{A} 356 & 9.49 & 3.10\end{array}$




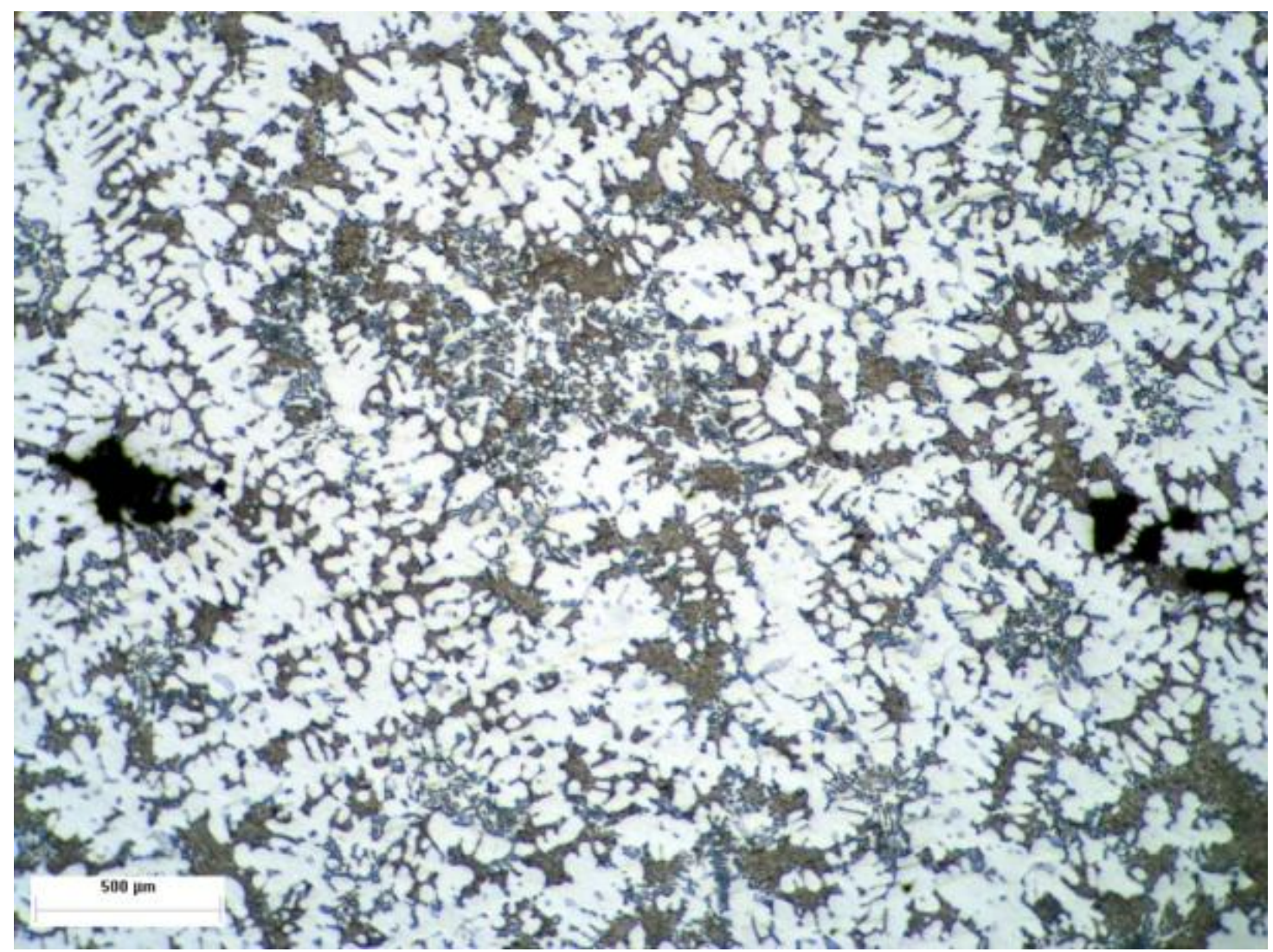

(a)

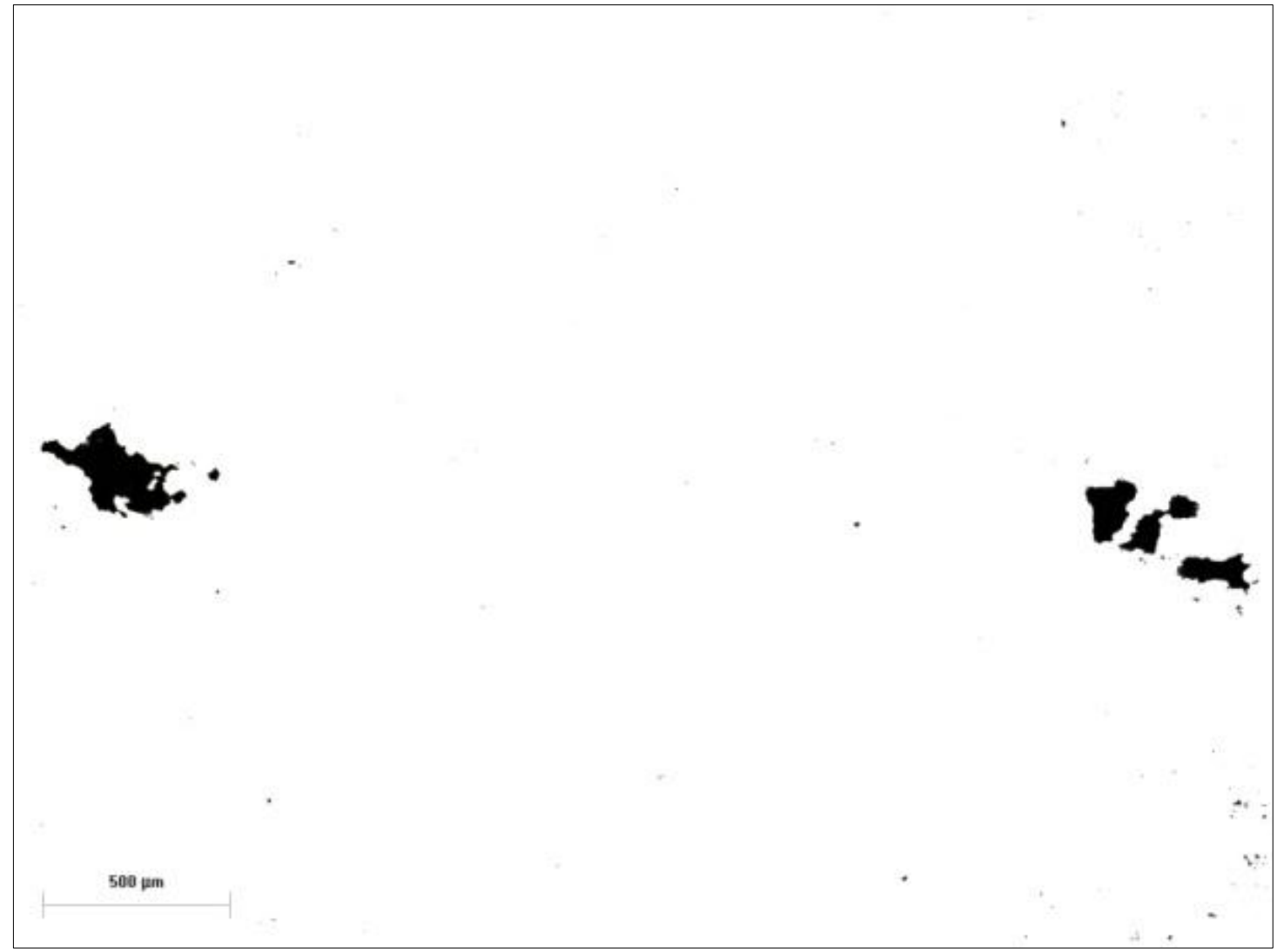

(b) 


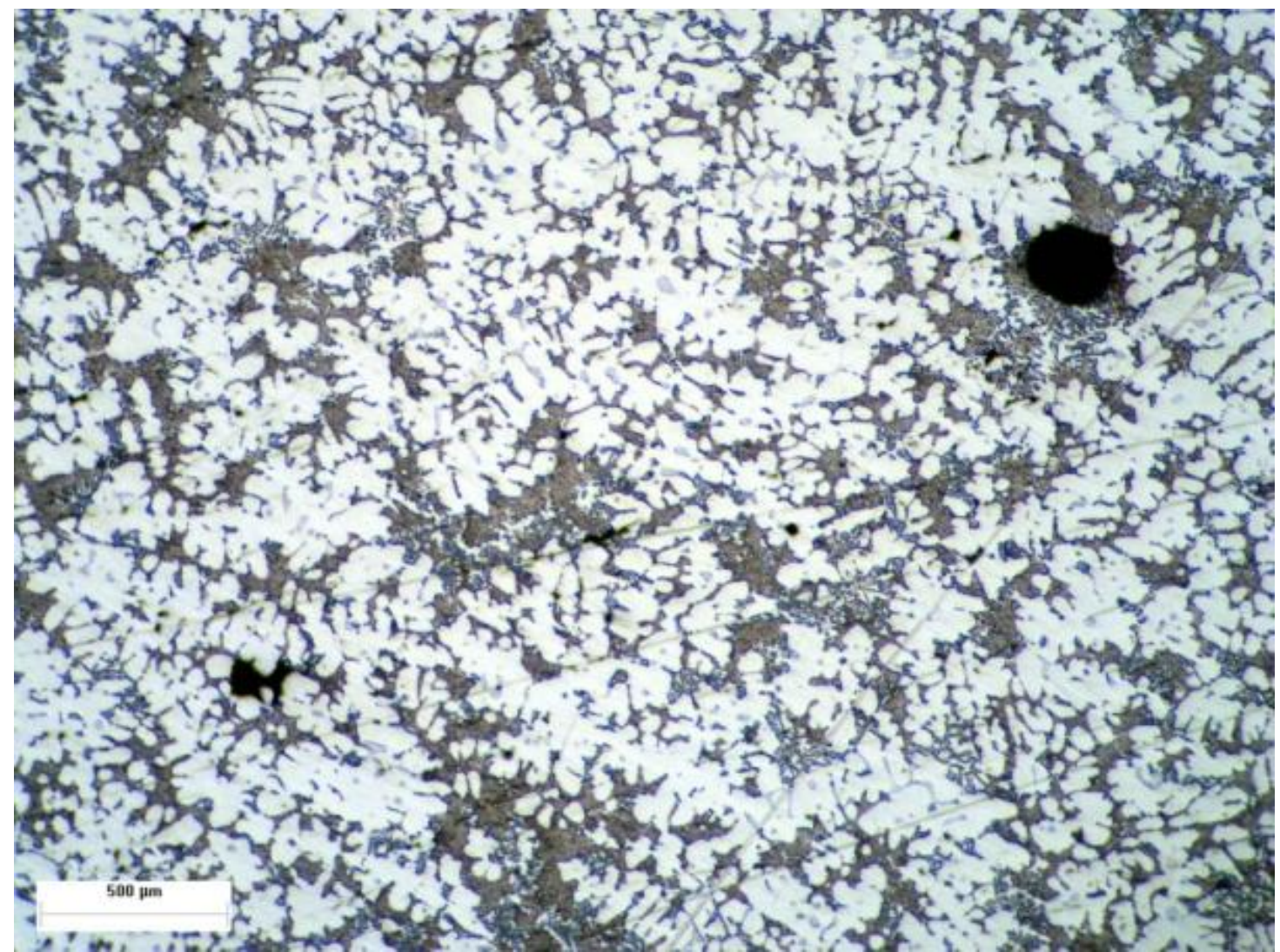

(c)

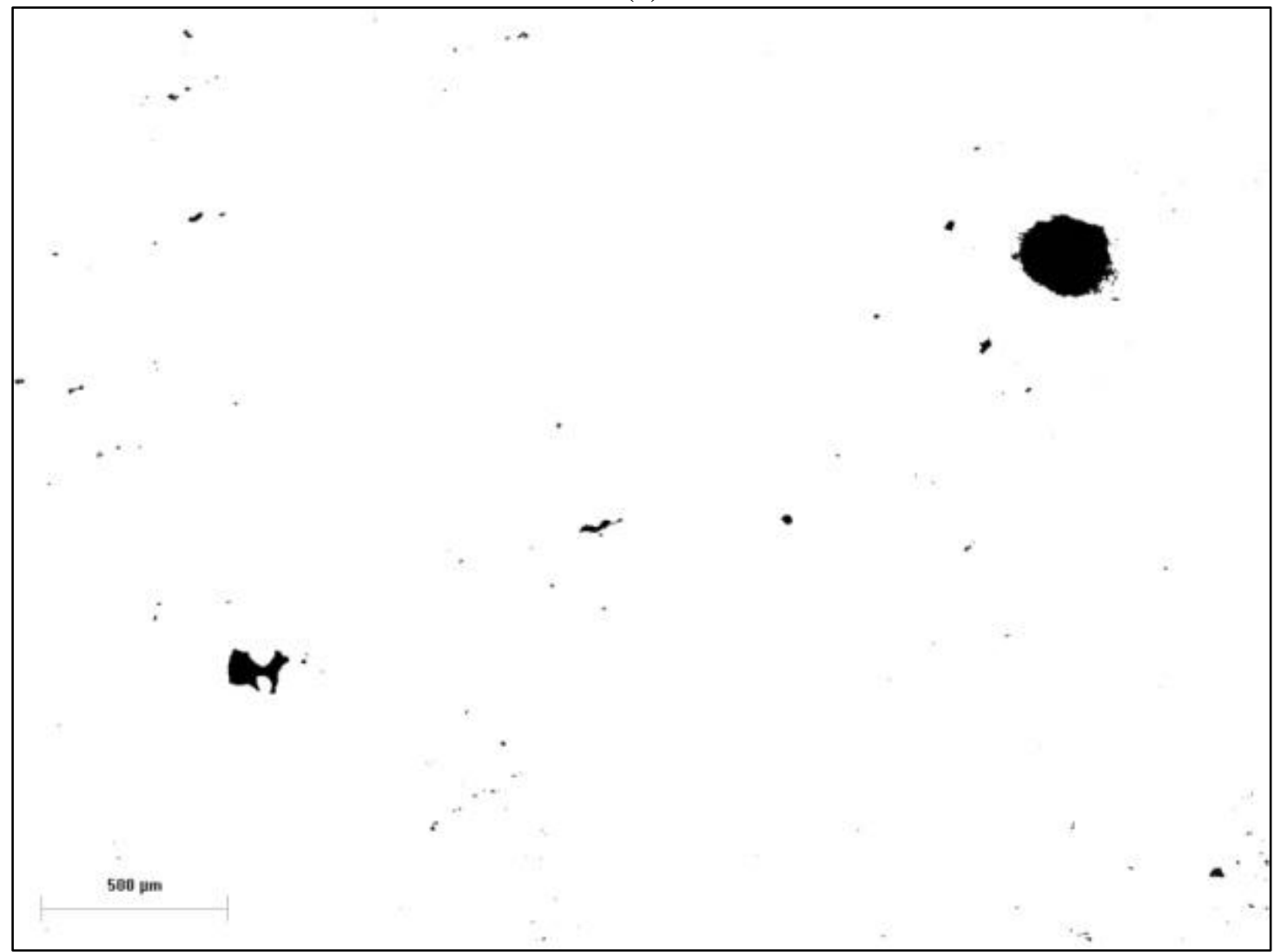

(d) 


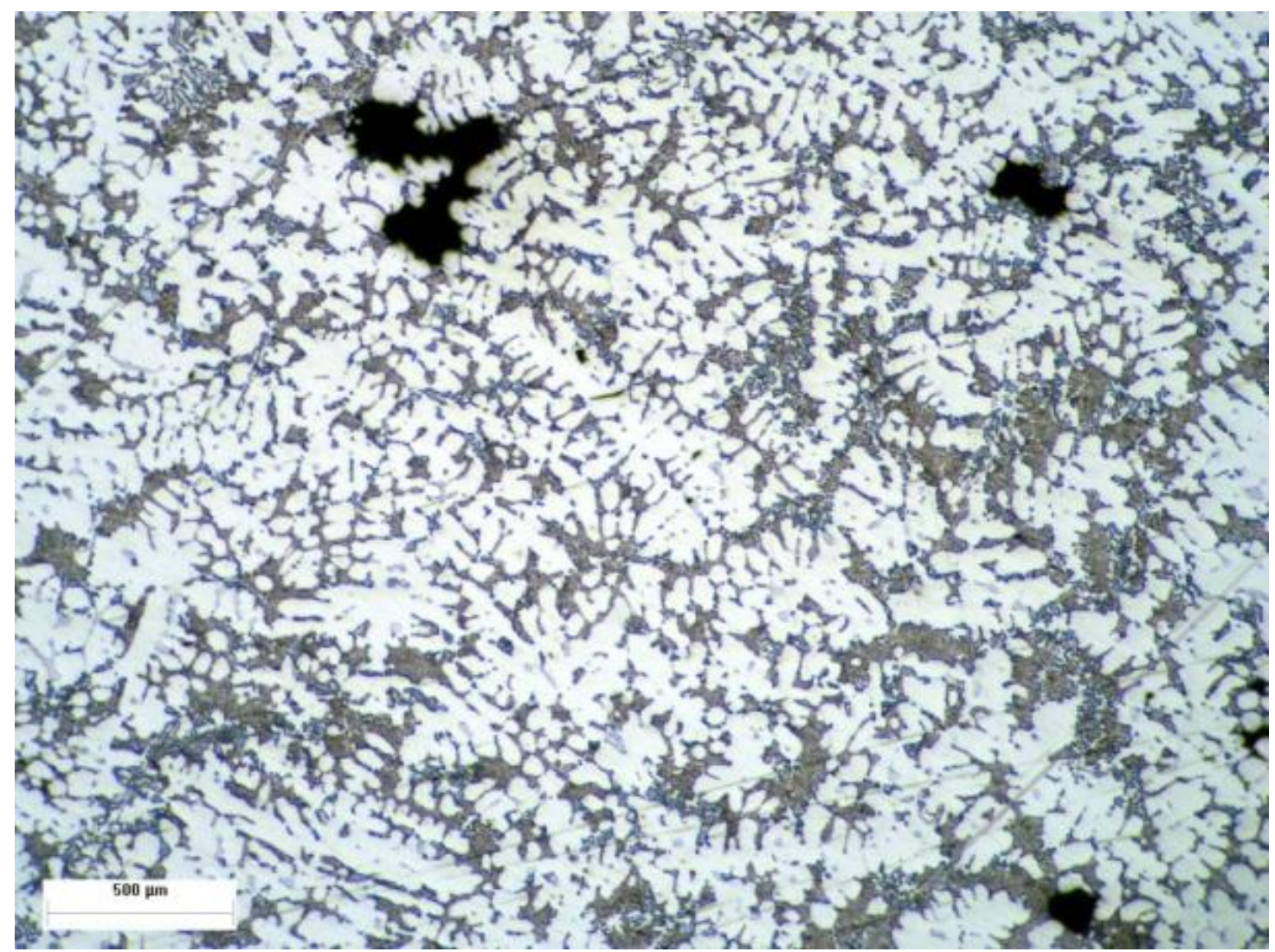

(e)

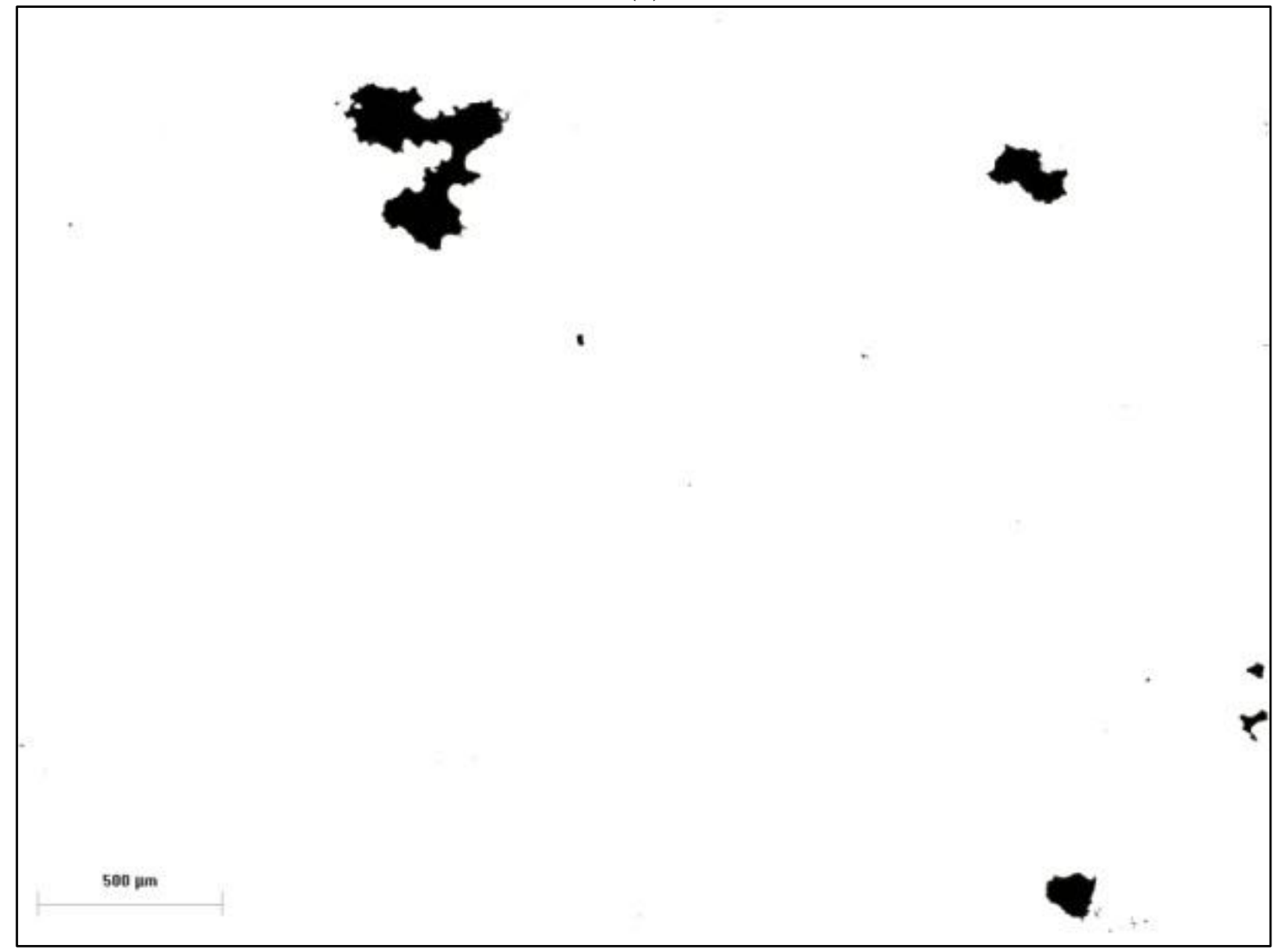

(f) 


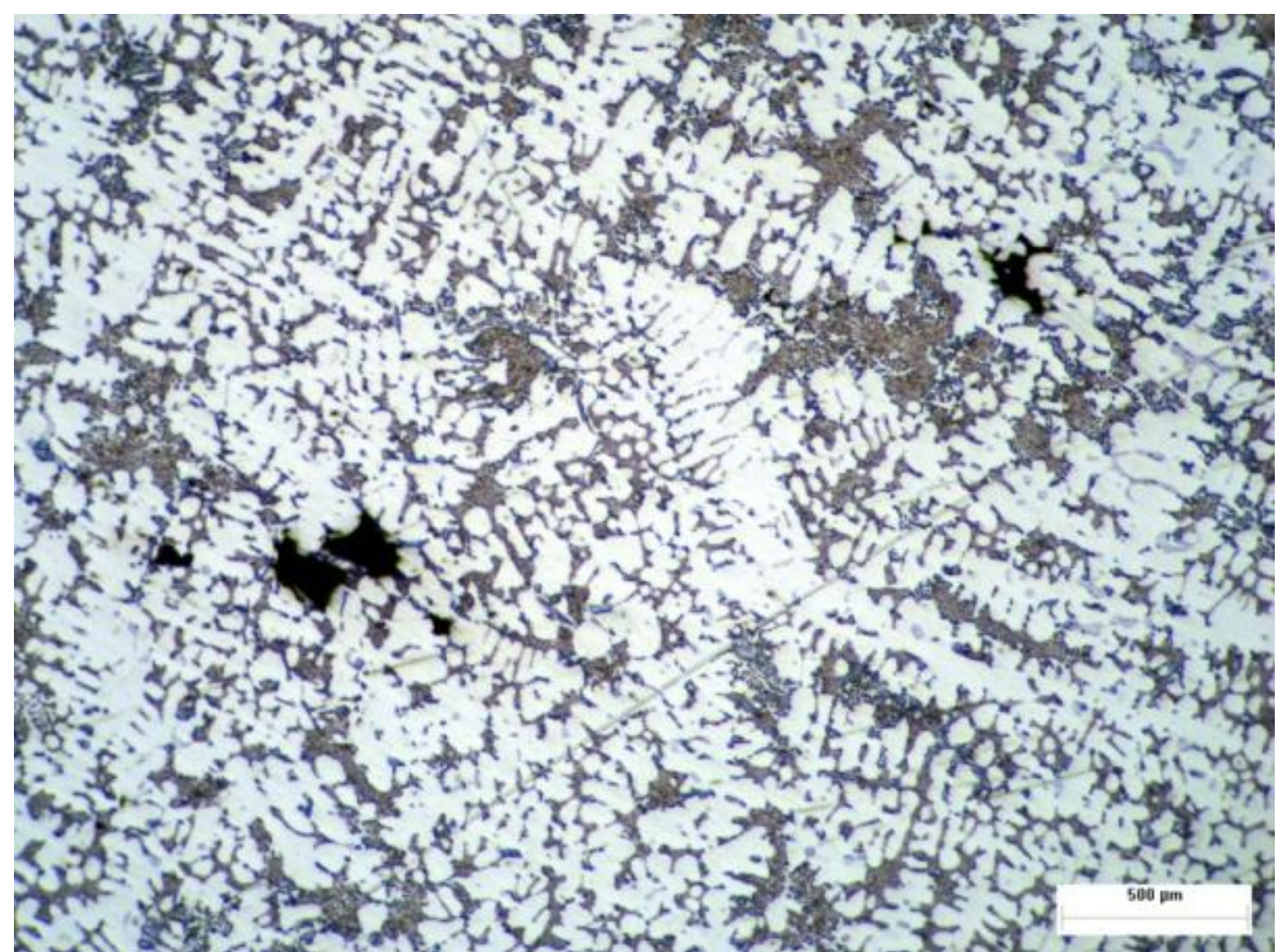

(g)

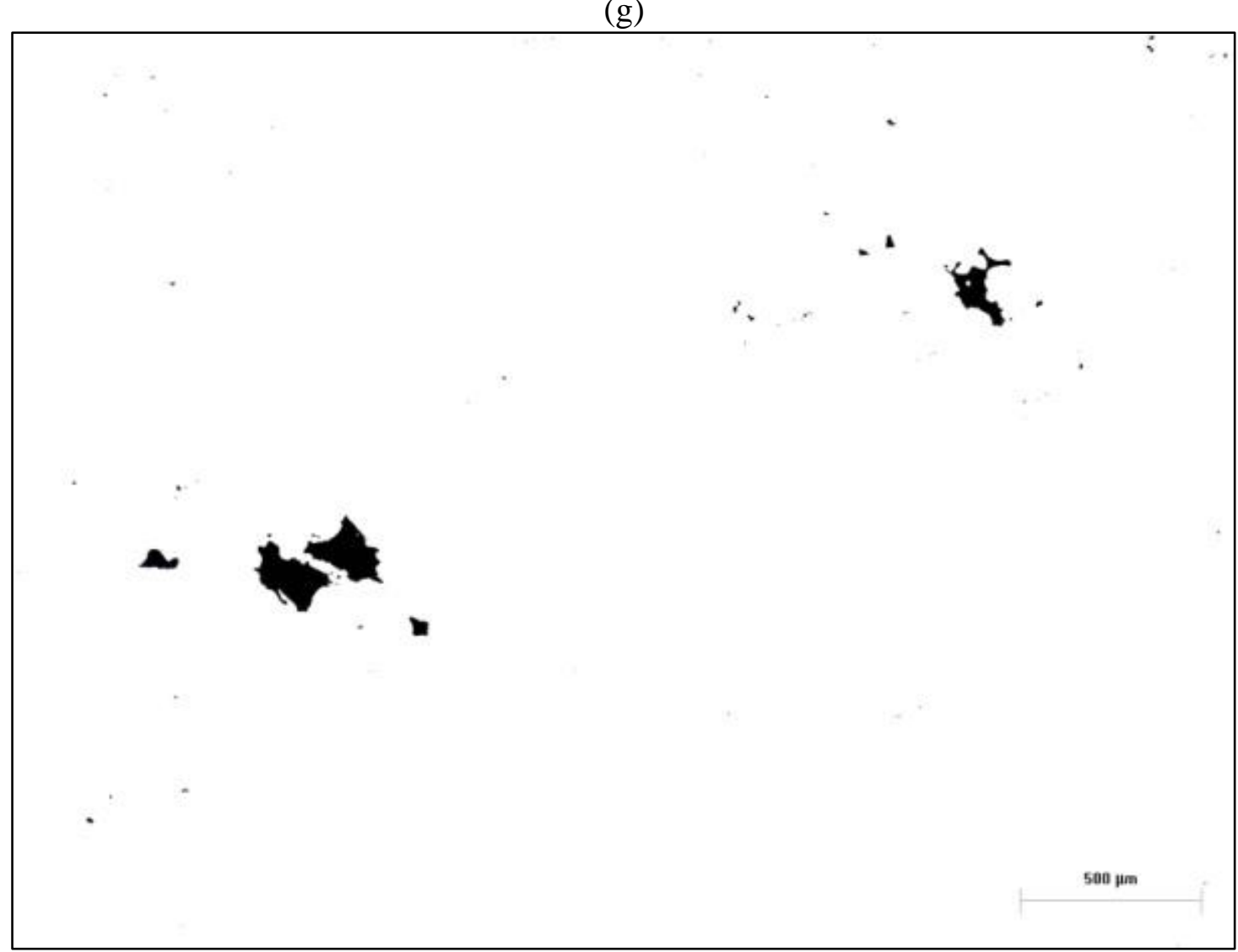

(h) 


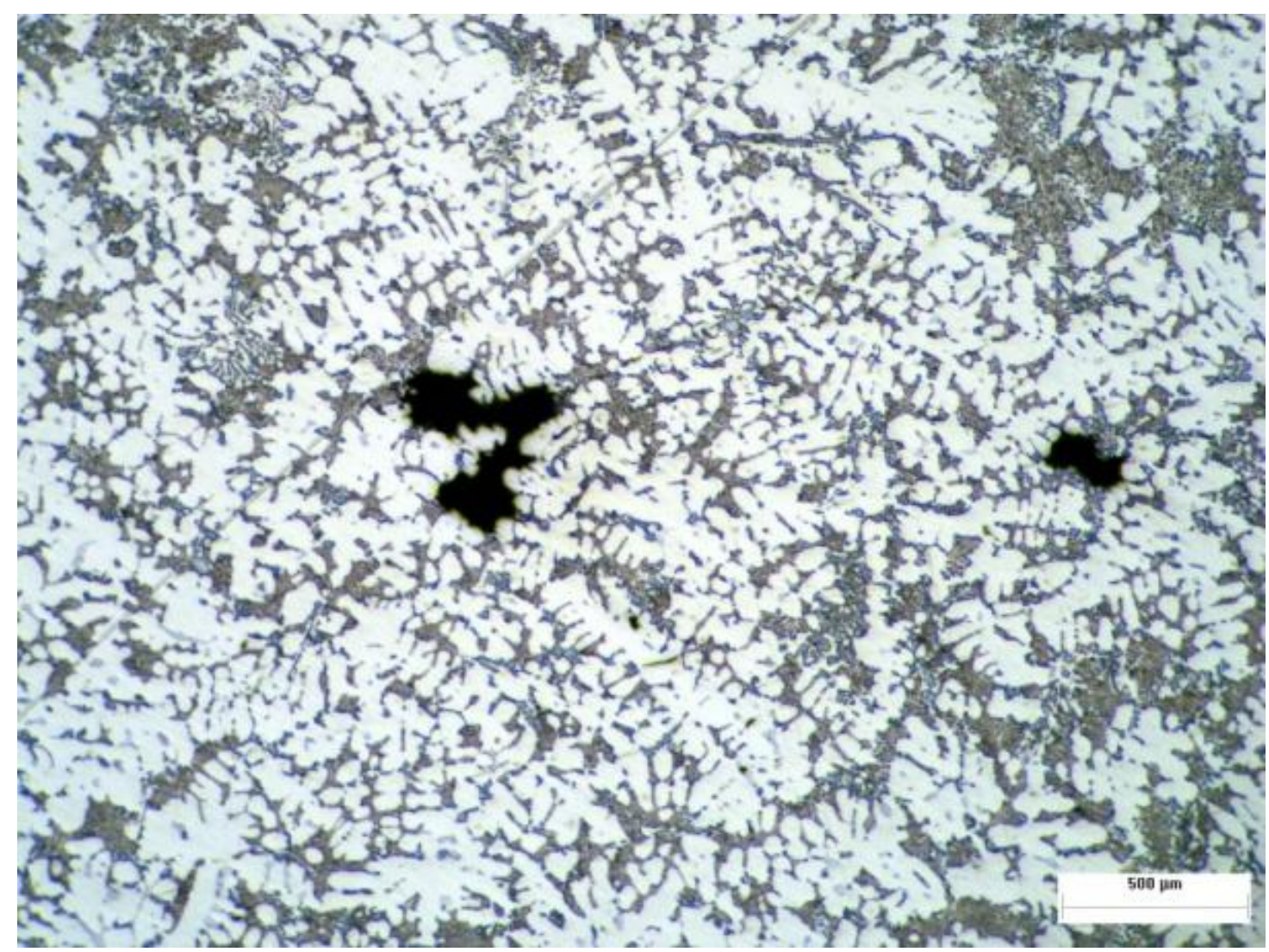

(i)
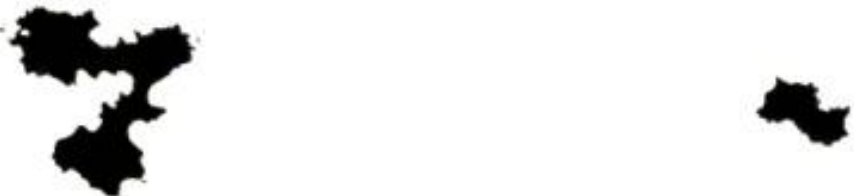

$500 \mu \mathrm{m}$ 


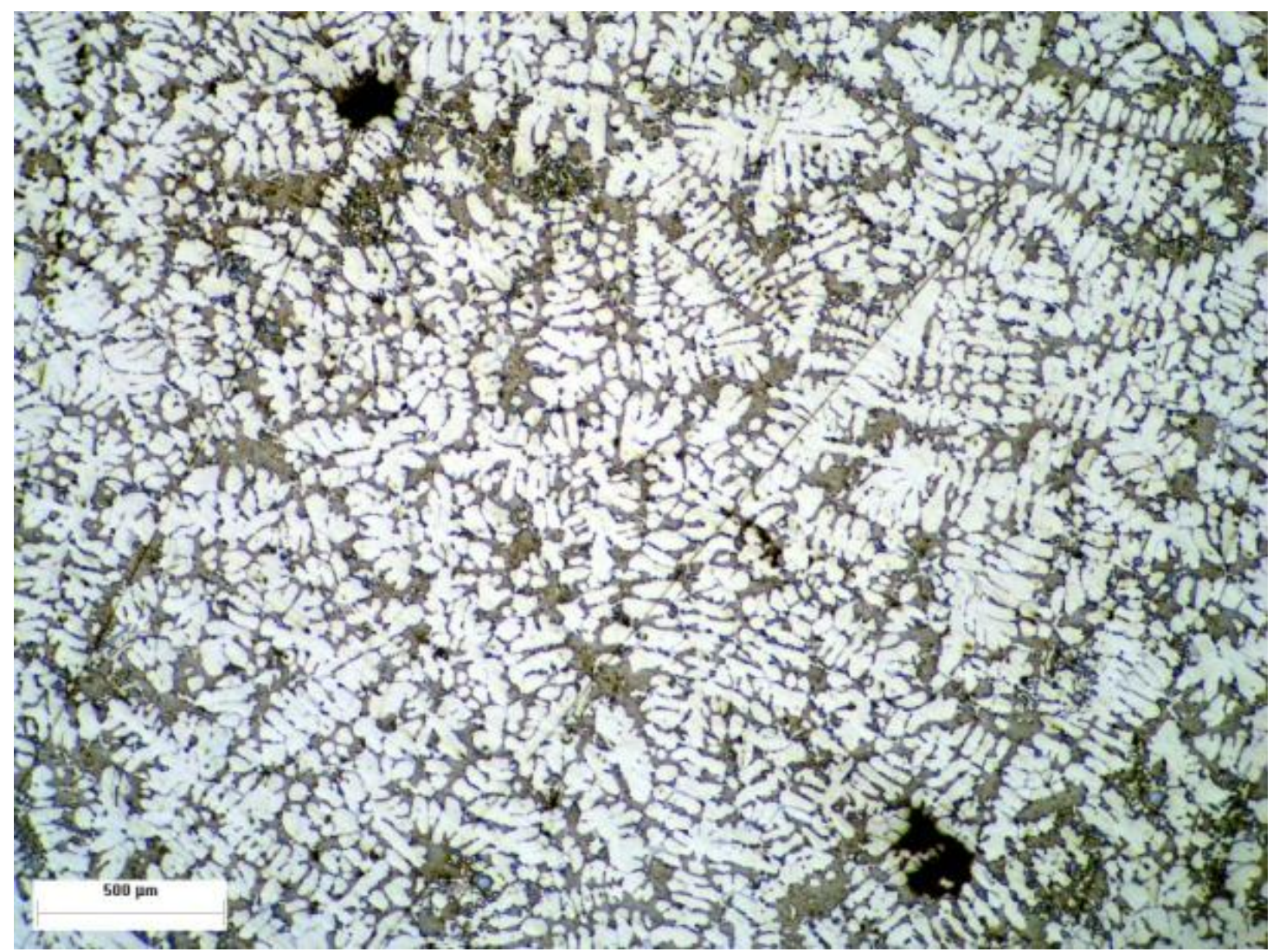

(k)

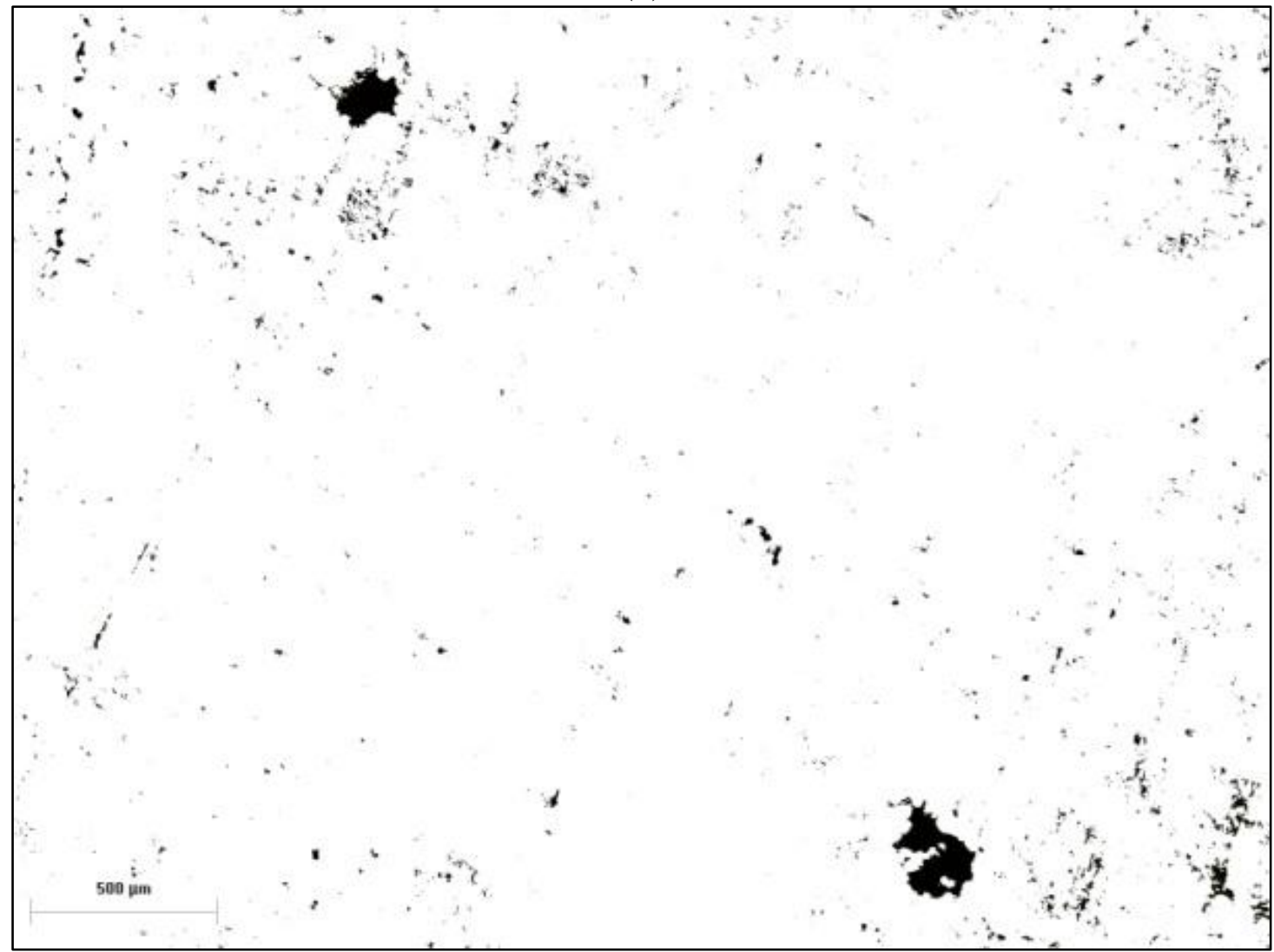

(1)

Figure 66: Metallographic analysis of specimens obtained from engine head transversally (a), (c), and (e) with corresponding images of casting voids (b), (d), and (f); and perpendicularly (g), (i), and (k) with corresponding images of casting voids (h), (j), and (l). 
These voids, shown in detail in Figure 67, constitute stress concentrators, which can nucleate cracks, thus decreasing lifetime in nucleation. From this point of view, component lifetime may be equated to propagation lifetime because the crack-type defect is already present in the material (component).

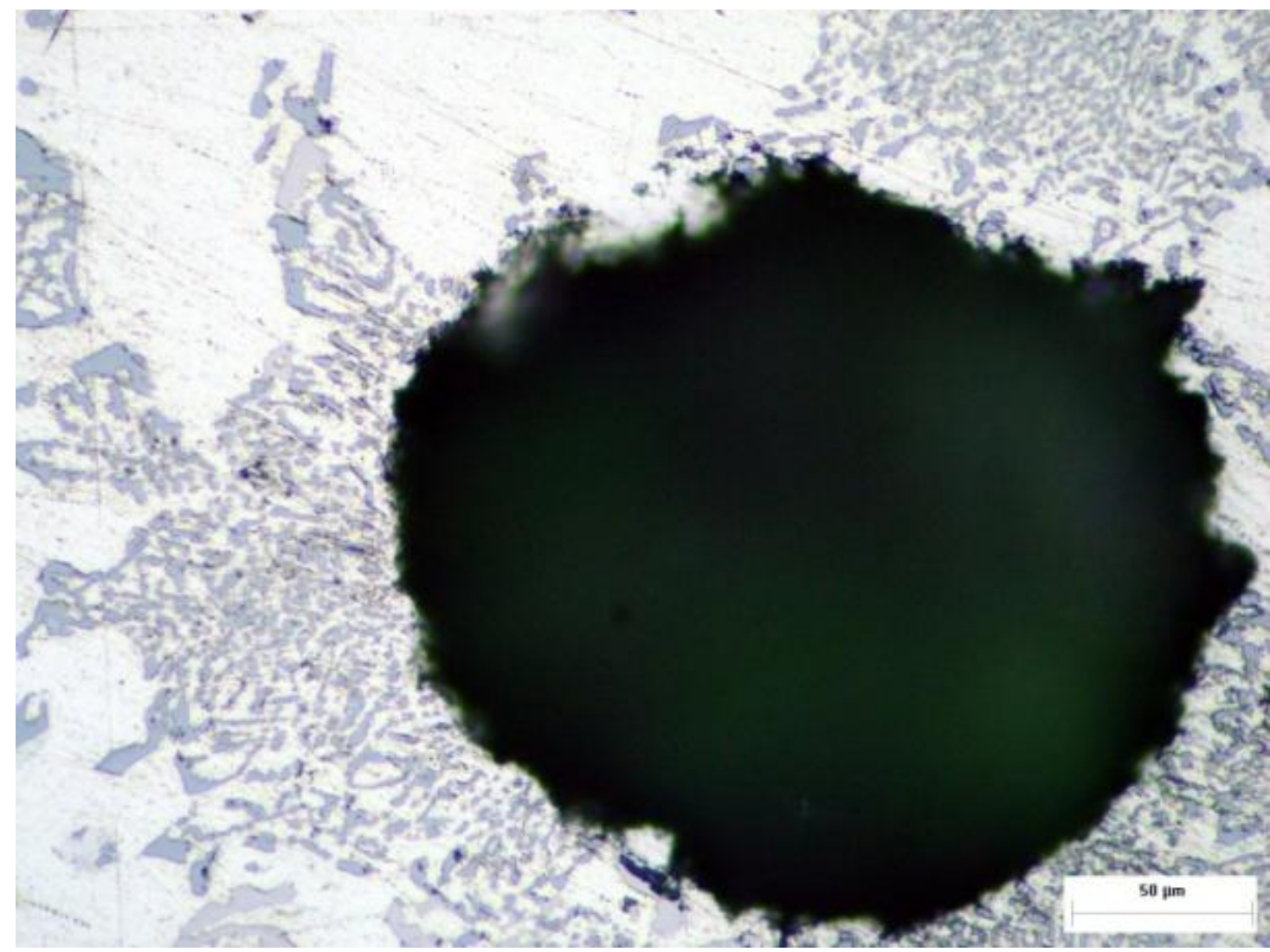

(a)

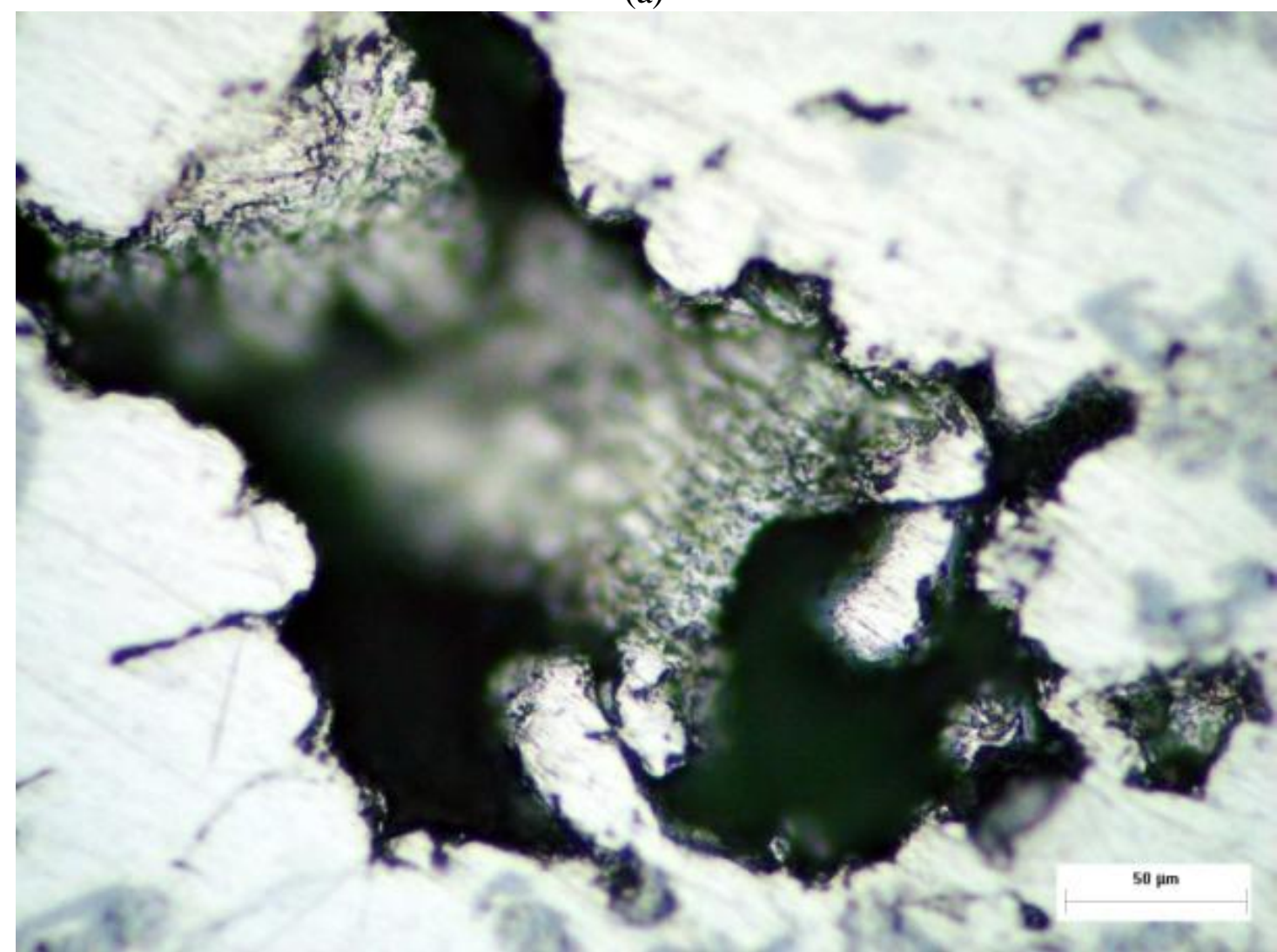


(b)

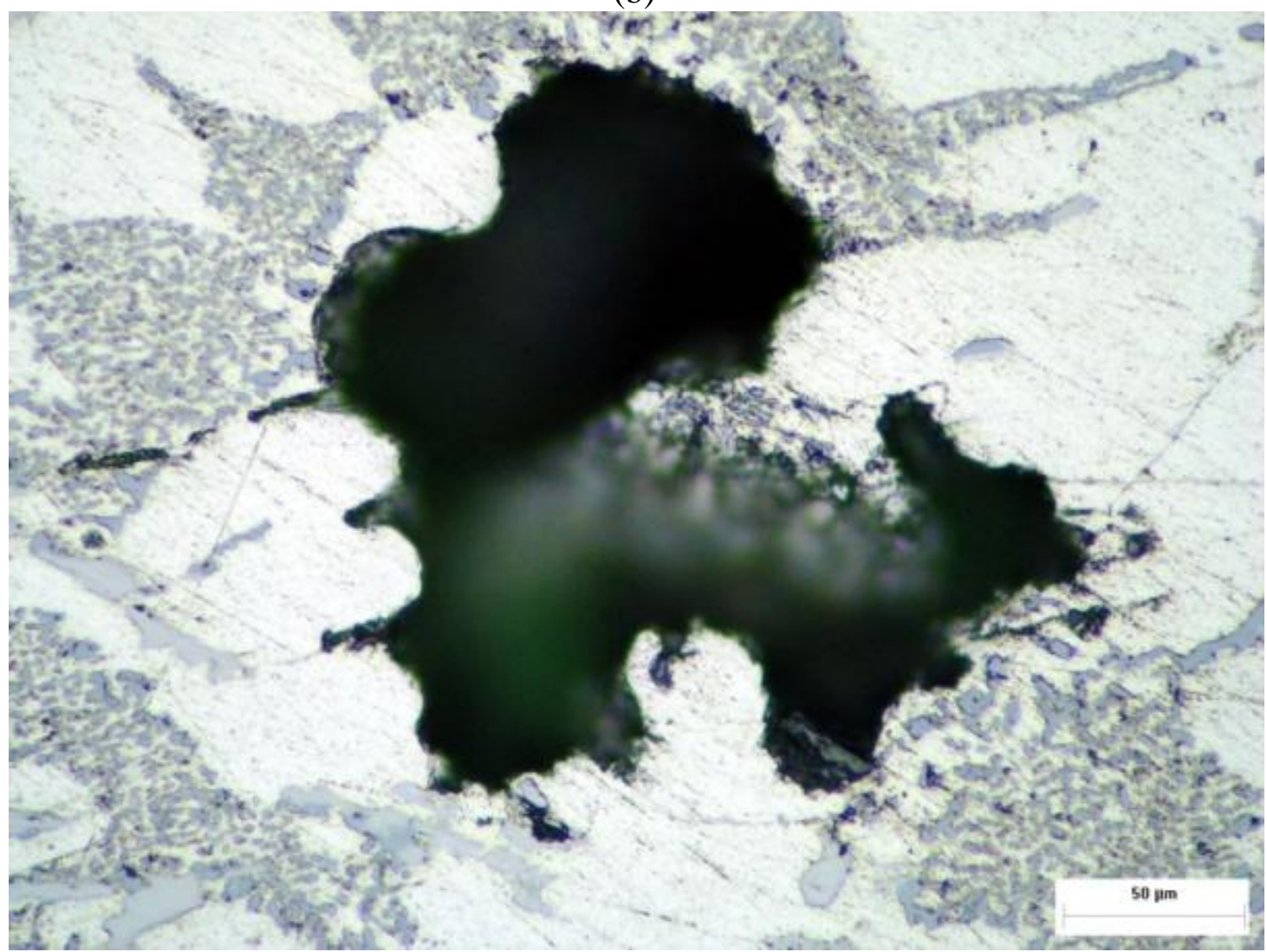

(c)

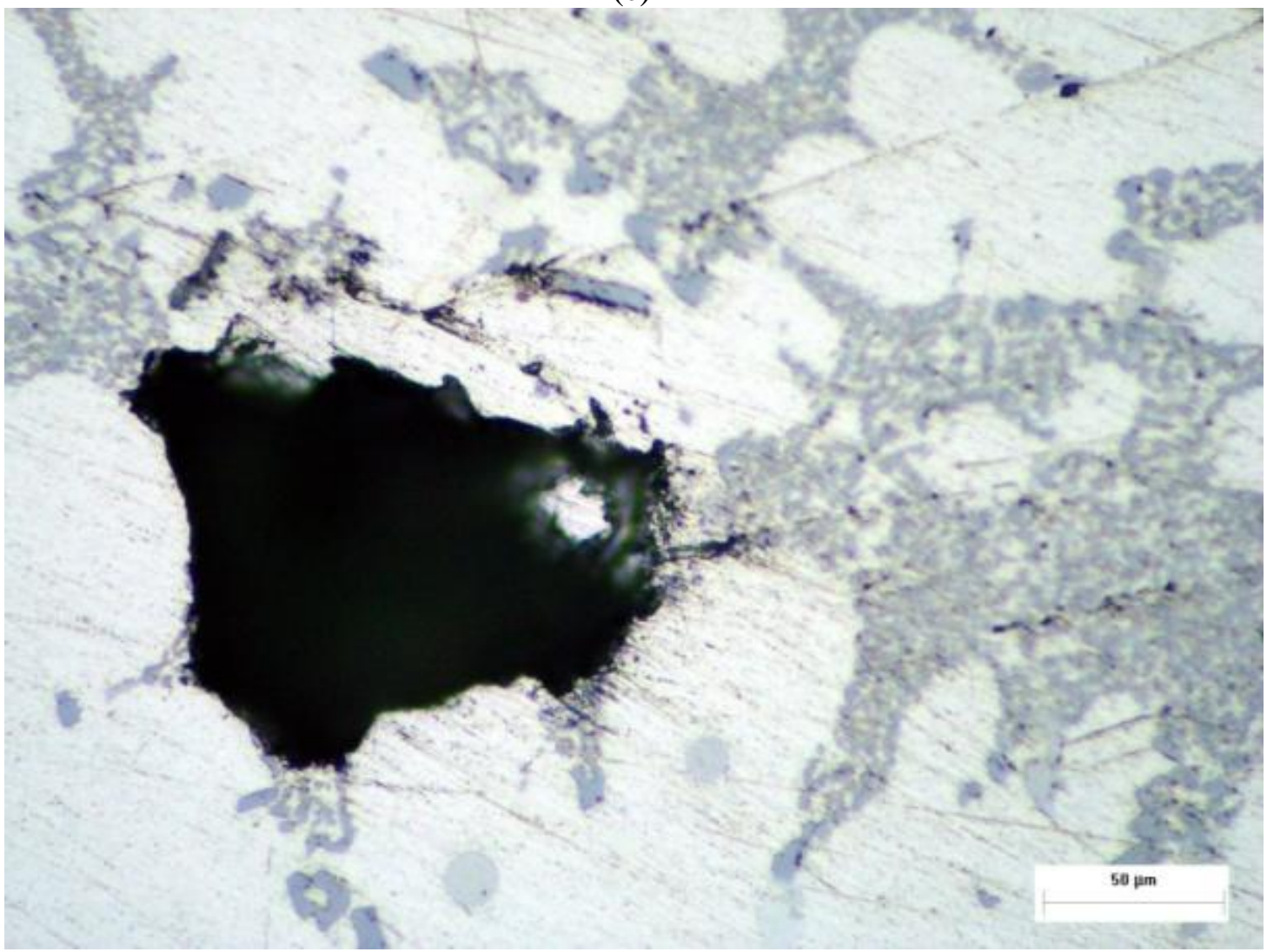

(d)

Figure 67: Detail of casting voids found in specimens obtained from the engine head, transversally ( $a$ and b) and perpendicularly (c and d). 
Figure 68 shows the morphological aspect of fracture surfaces of specimens submitted to crack propagation tests at $120^{\circ} \mathrm{C}$, thermo-mechanical fatigue tests and isothermal fatigue tests at $280^{\circ}$ examined under a scanning electron microscope (SEM). Inter-dendrite porosity and fatigue grooves may be observed in detail on the specimen fracture surface.

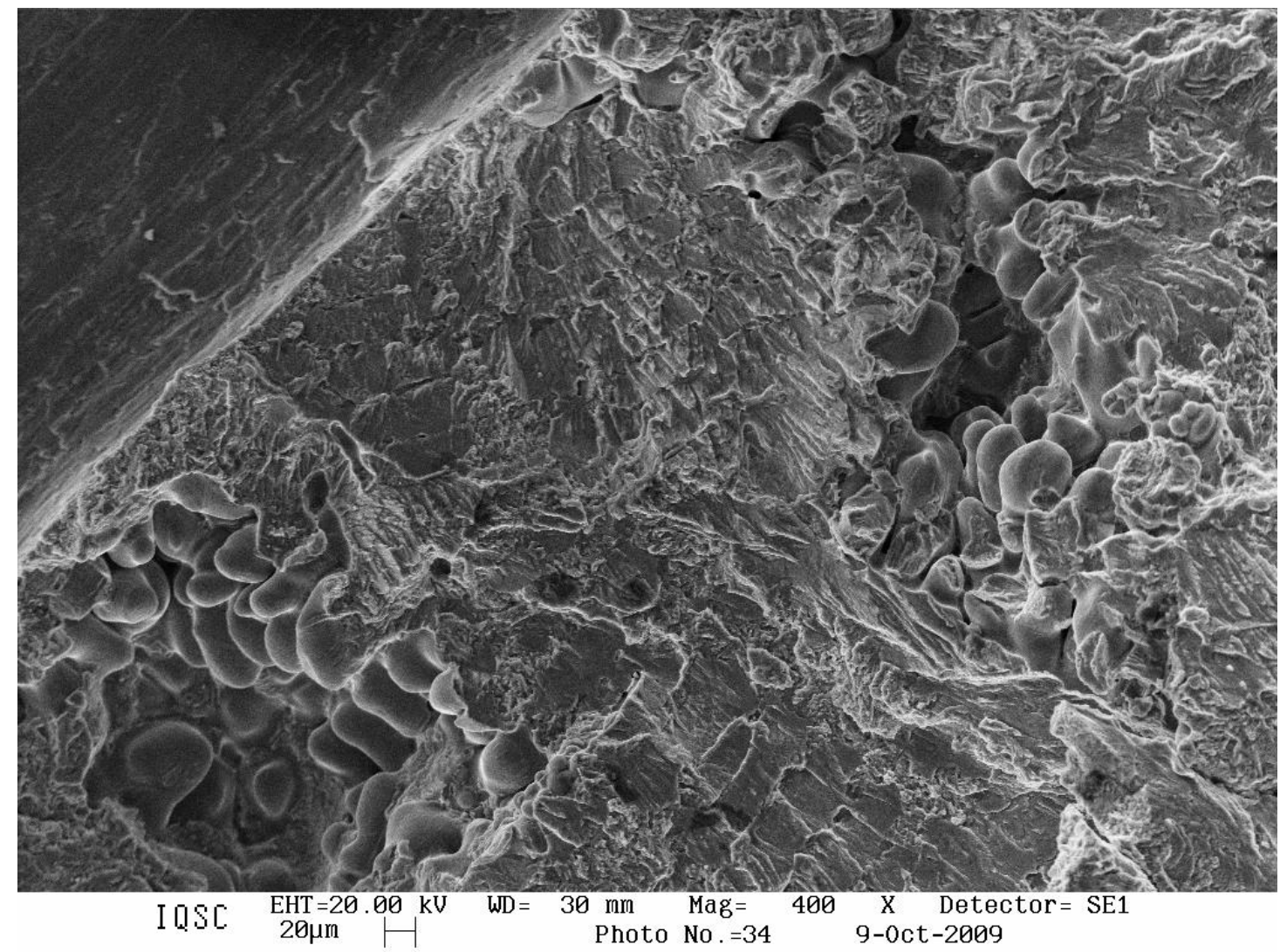

(a)

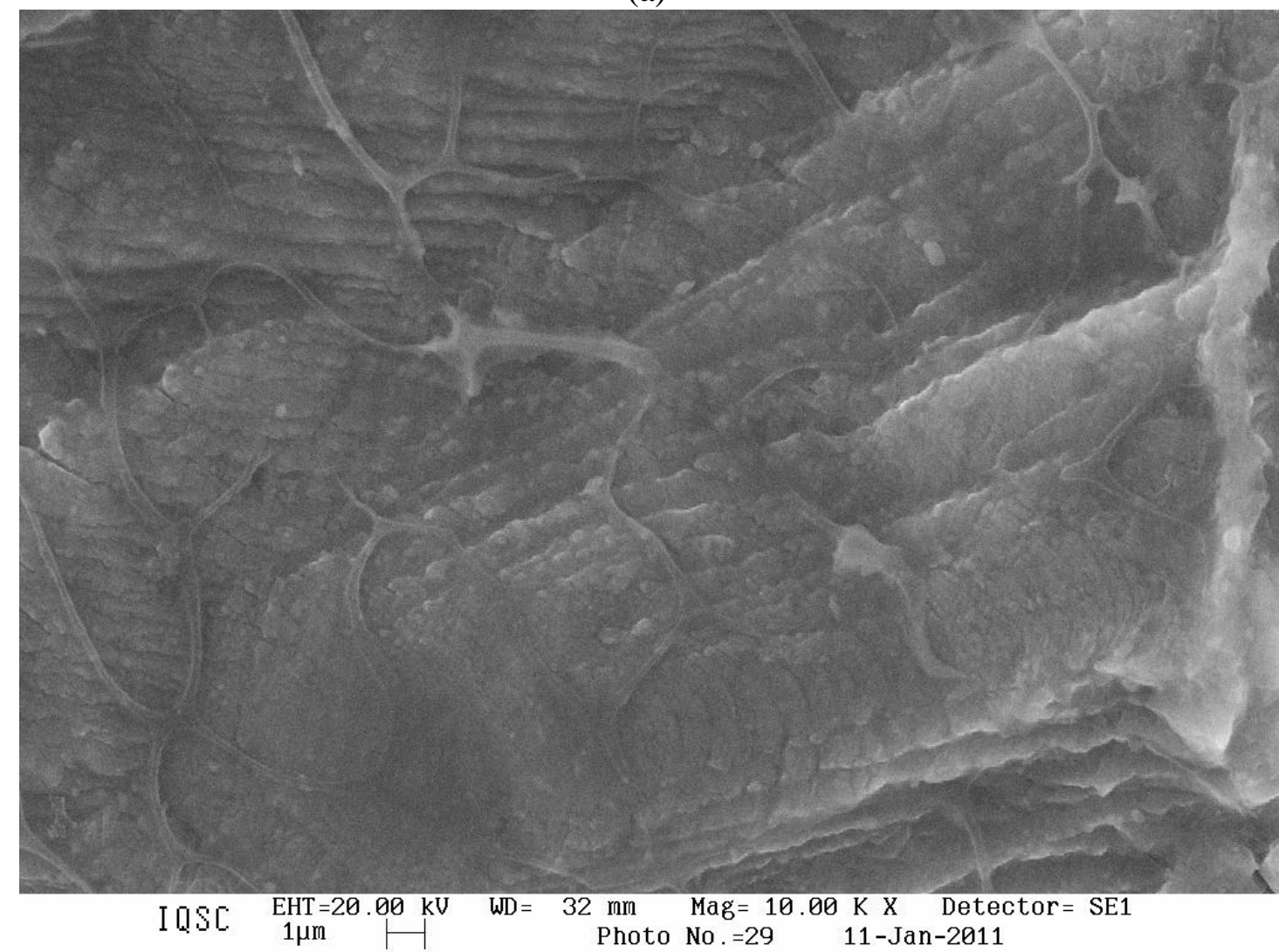


(b)

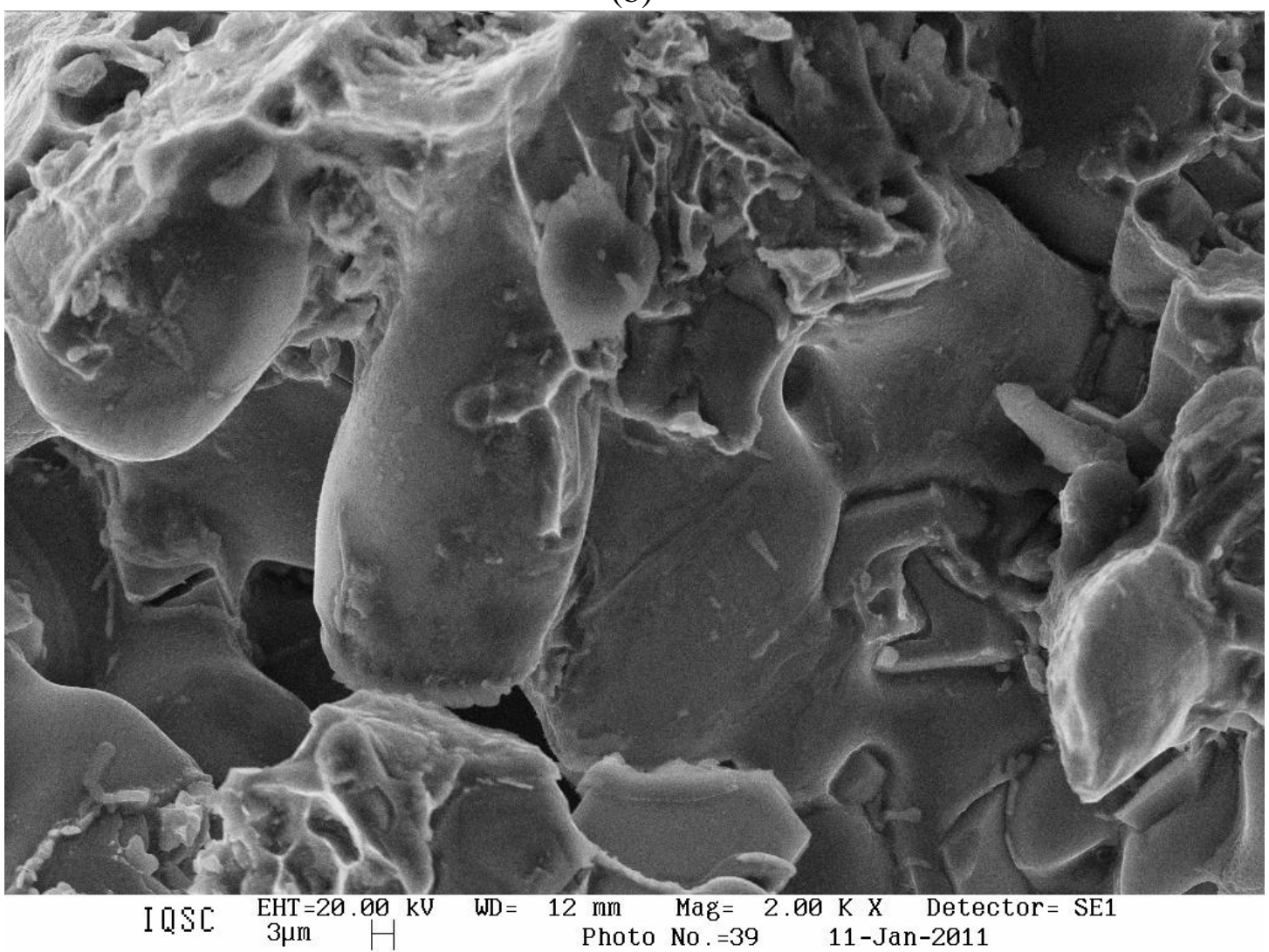

(c)

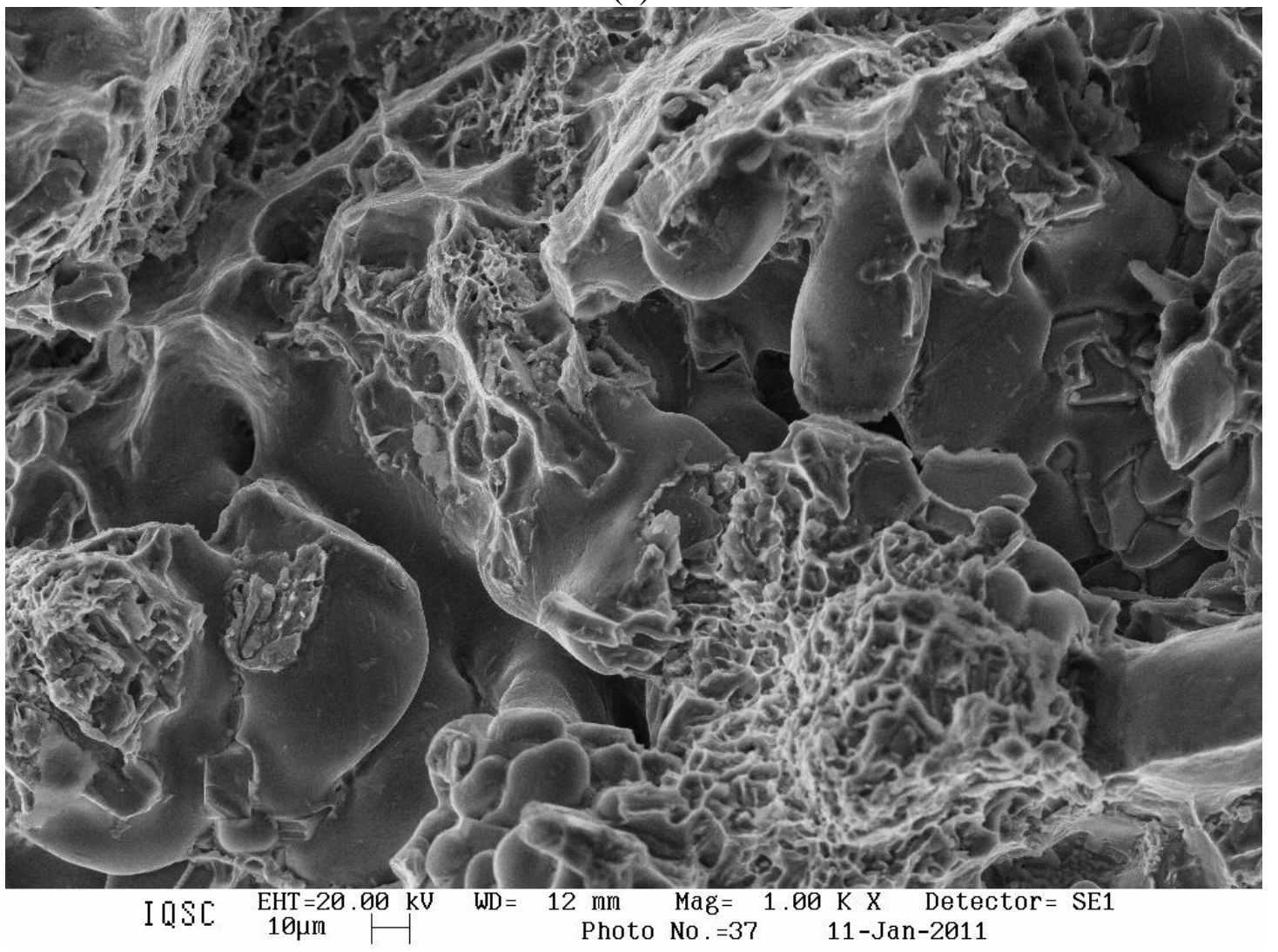

(d)

Figure 68: Fractographic analysis of the specimens submitted to (a) fatigue crack growth test at $120^{\circ} \mathrm{C}$ taken in the region of stable crack propagation and (b) ampliation of the same region, (c) thermomechanical fatigue test and (d) isothermal fatigue test at $280^{\circ} \mathrm{C}$. 
There was observed in all specimens tested the occurrence of several dendrites exposed on the fracture surface, i.e., solidification voids. Fatigue specimens, in general, presented rather irregular surfaces, suggesting the formation of multiple nucleation sites from existing casting defects and indicating that cracks had propagated by connecting theses defects.

From the analyses conducted it is possible to assume that fatigue cracks nucleated from defects (porosity) existing near the specimen surface and that crack propagation mechanisms are similar, a mixture of cleavage and tear grooves at both test temperatures $\left(120^{\circ} \mathrm{C}\right.$ and $\left.280^{\circ} \mathrm{C}\right)$ for the material under investigation.

\subsubsection{Mechanical Tests}

\subsubsection{Tensile Test}

Figure 69 shows average results of tensile tests at $25^{\circ} \mathrm{C}, 120^{\circ} \mathrm{C}$, and $280^{\circ} \mathrm{C}$ and the elasticity modulus $(\mathrm{E})$, the yield stress at $0.2 \%$ strain $\left(\sigma_{\mathrm{y}_{0.2 \%} \%}\right)$, and the rupture stress $\left(\sigma_{\mathrm{U}}\right)$. The test results that were too scattered, probably due to the presence of a large number of casting defects, were discarded.

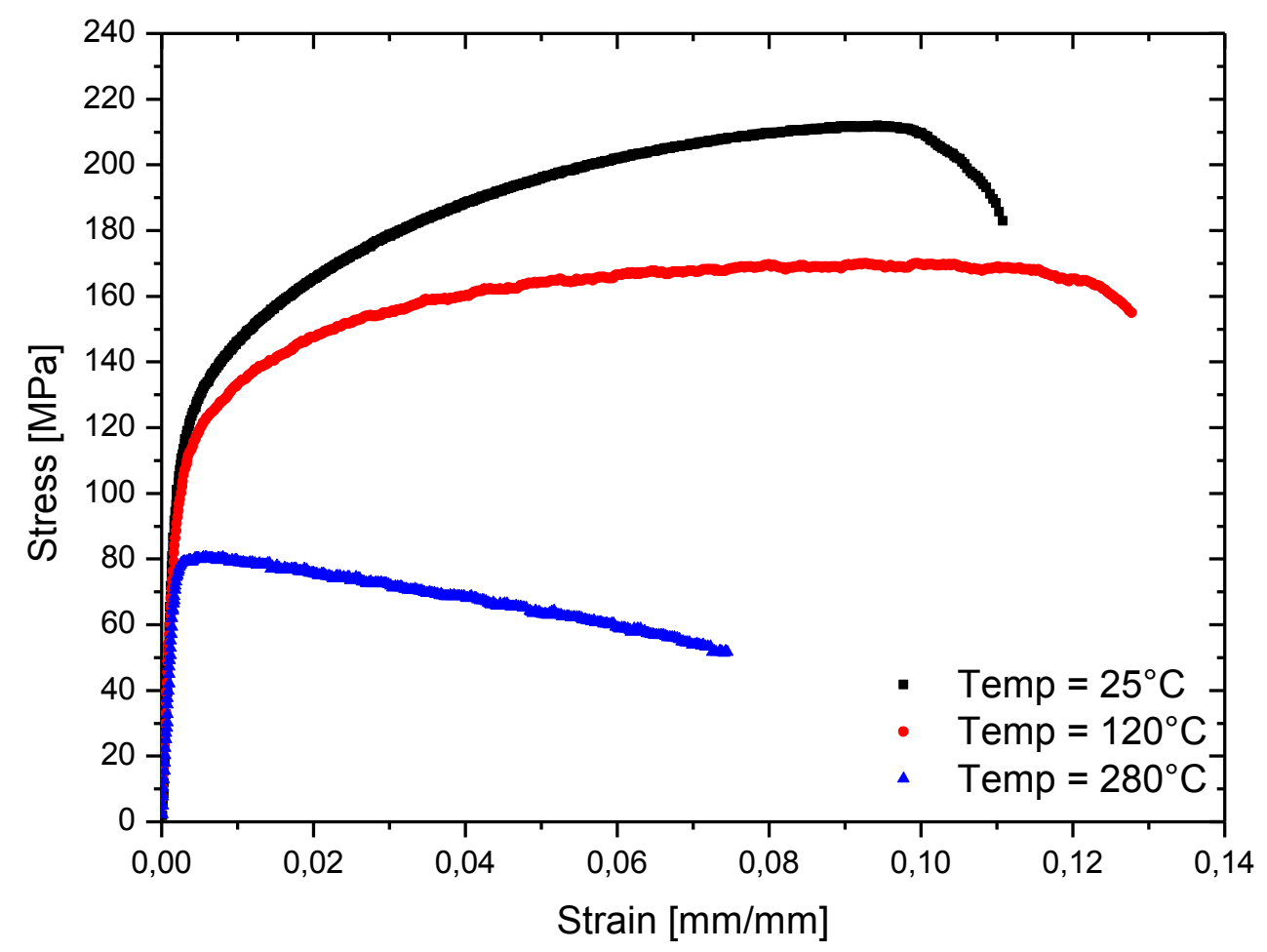

(a) 


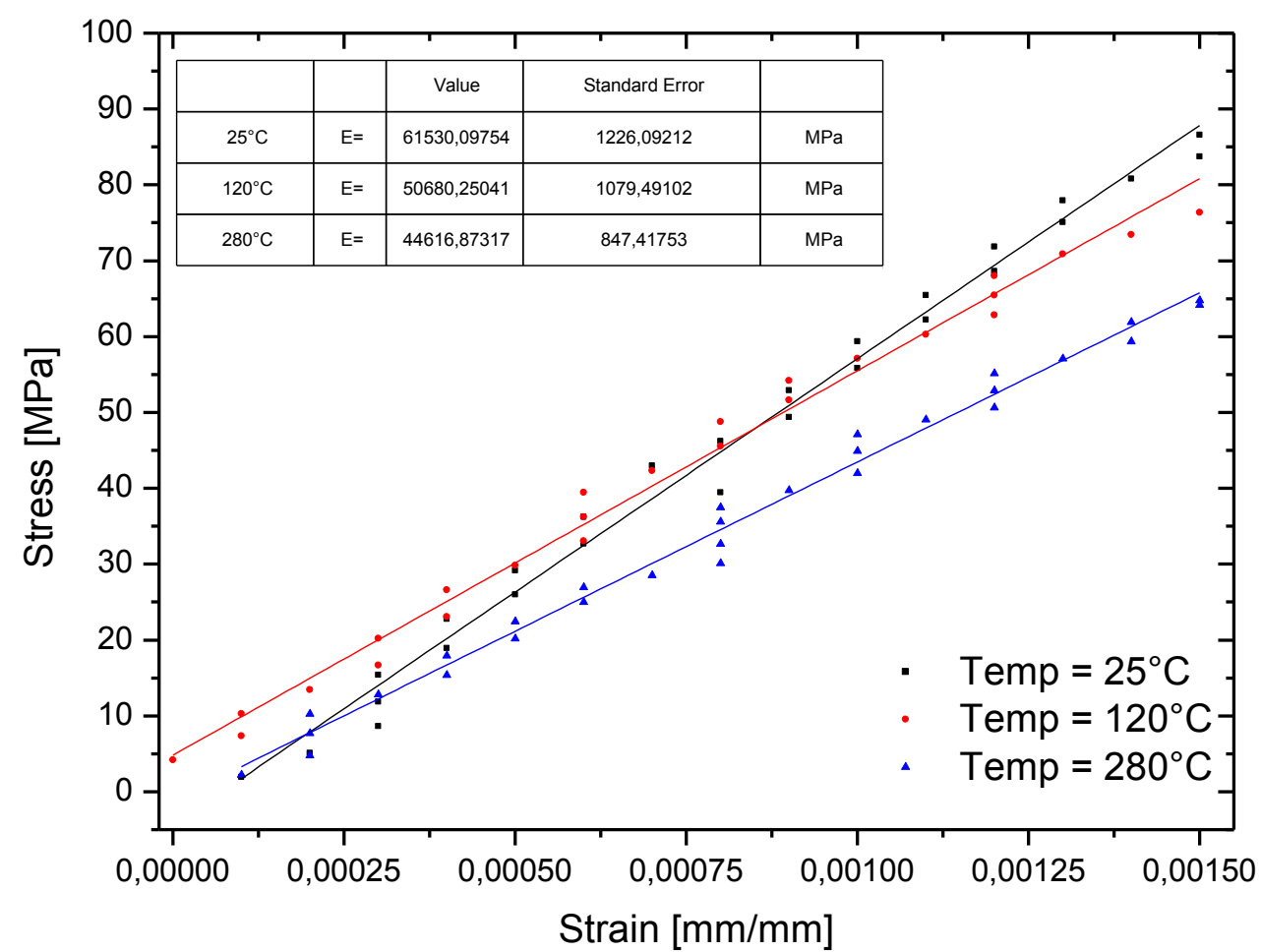

(b)

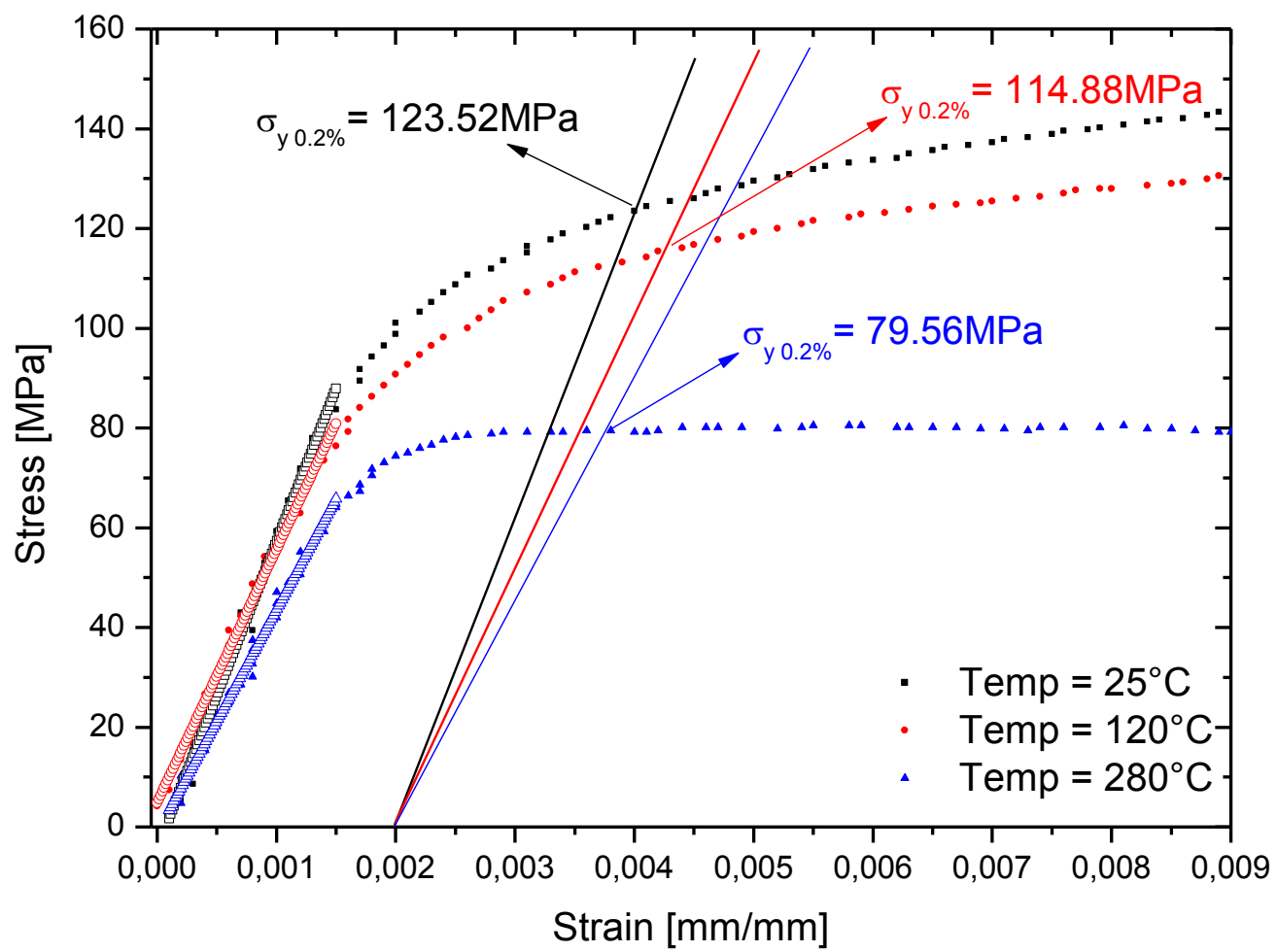

(c) 


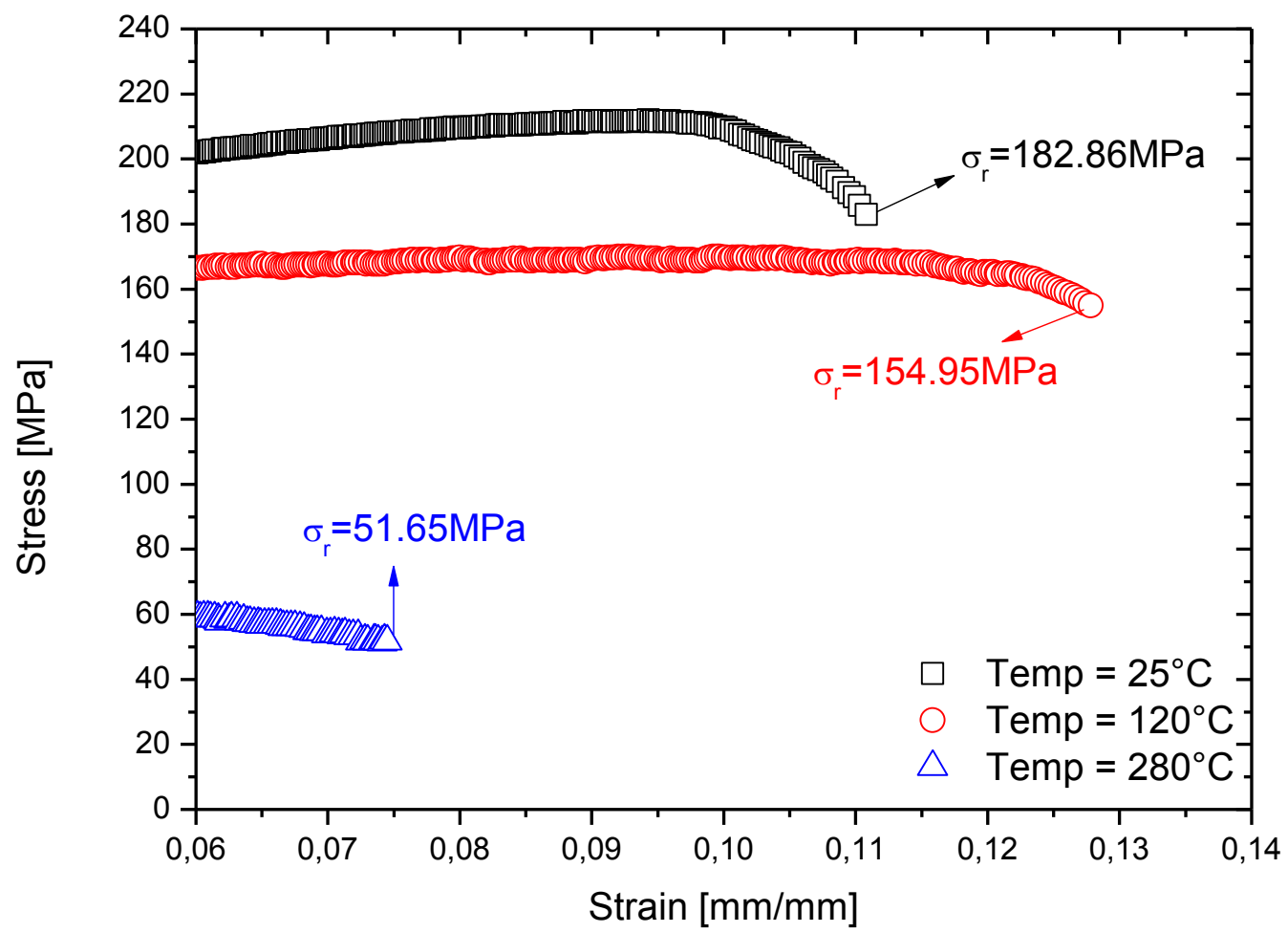

(d)

Figure 69: (a) Results of tensile tests conducted at $25^{\circ} \mathrm{C}, 120^{\circ} \mathrm{C}$, and $280^{\circ} \mathrm{C}$, (b) details of estimation of Young's modulus $(E)$, (c) yield stress at $0.2 \%\left(\sigma_{y_{0.2} \%}\right)$, and $(\mathrm{d})$ rupture stress $\left(\sigma_{r}\right)$.

As is well known, the alloy mechanical strength decreases with increasing temperature. In this study, this fall became pronounced between $120^{\circ} \mathrm{C}$ and $280^{\circ} \mathrm{C}$ when creep mechanisms start acting. In addition to the temperature effect, the presence of regions with high $\mathrm{Cu}$ concentrations may have also contributed to it, since this element is known to have an embrittling effect at high temperatures.

Figure 70 shows the elasticity modulus versus temperature curve.

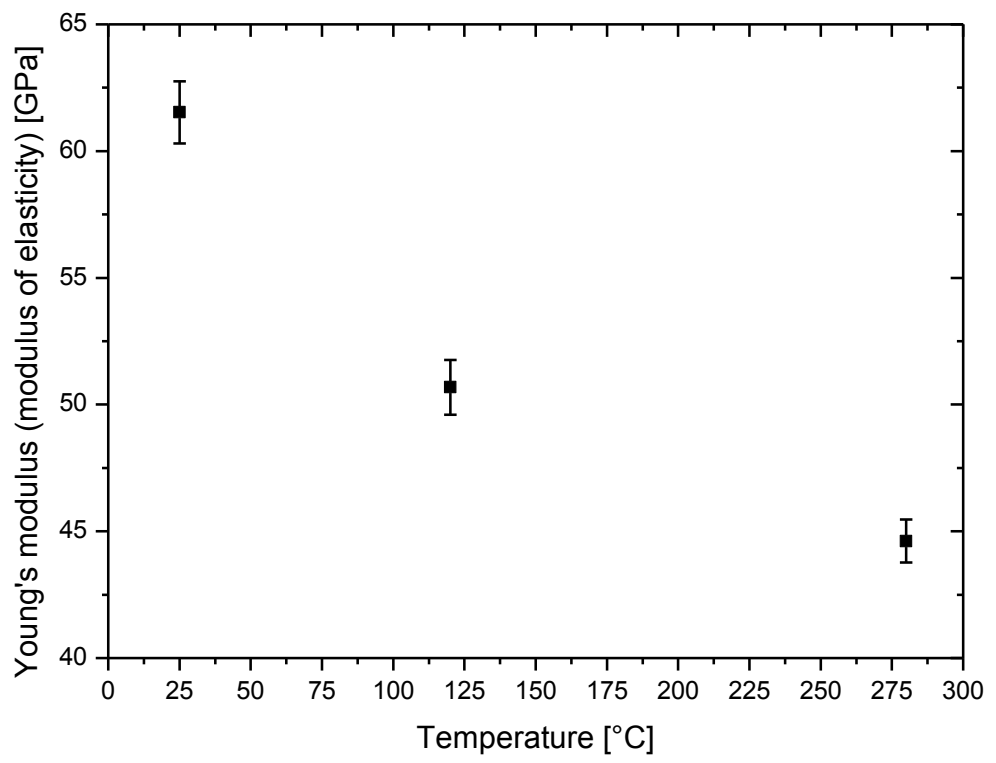

Figure 70: Elasticity modulus vs. temperature for permanent mold cast A356 alloy. 


\subsubsection{Isothermal Fatigue}

Figure 71 shows curves of stress amplitude as a function of the number of reversals to failure obtained from isothermal fatigue tests at $120^{\circ} \mathrm{C}$ and $280^{\circ} \mathrm{C}$, the latter being approximately 0.4 of the melting temperature. The same lifetime indicates a sharp drop of approximately 3 (three) times $\left(\Delta \sigma_{120^{\circ} \mathrm{C}} / \Delta \sigma_{280^{\circ} \mathrm{C}} \cong 3\right)$ in stress amplitude with increasing temperature. Specimens whose results were not consistent with overall behavior were analyzed and discarded if found to be defective.

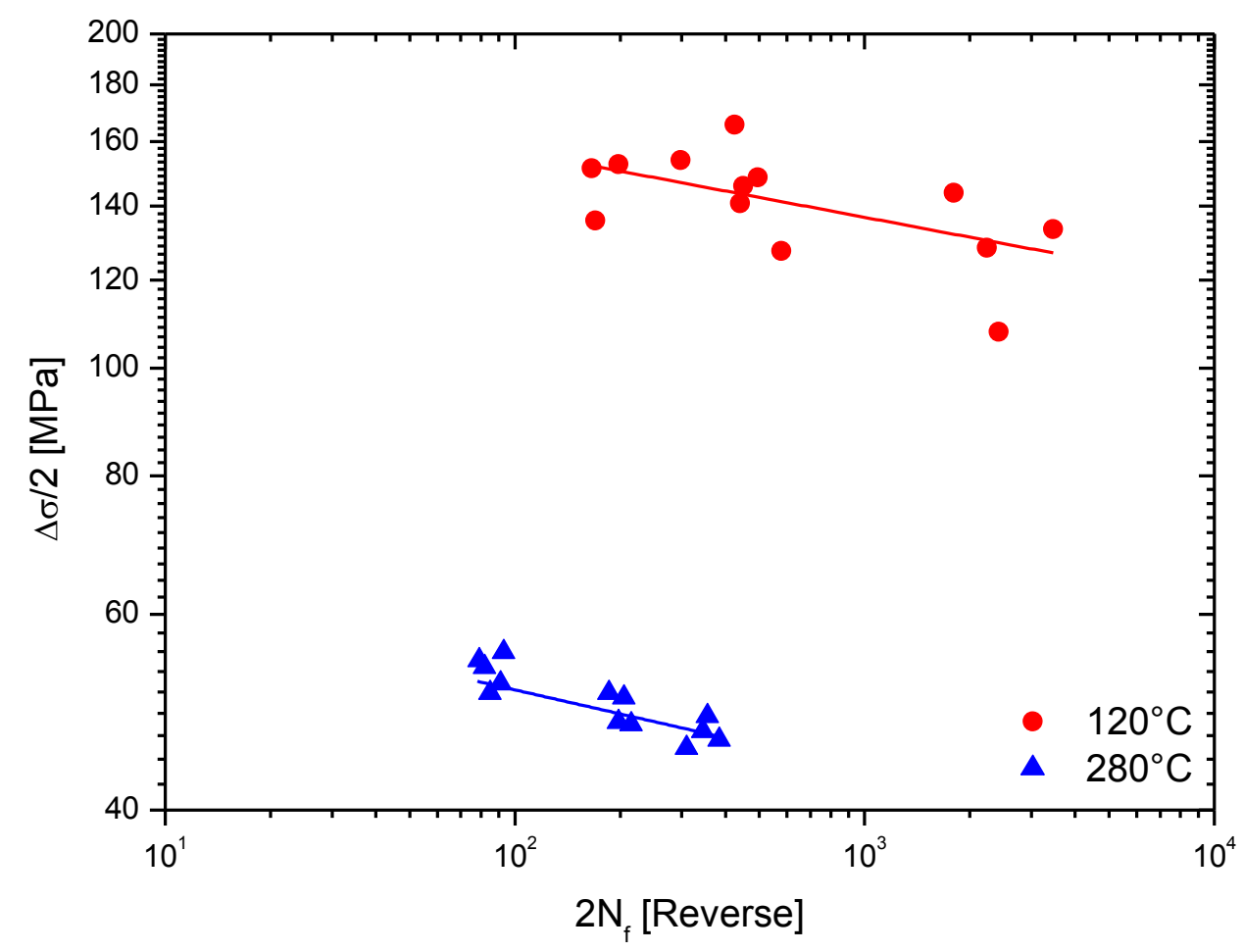

Figure 71: Curve of stress amplitude as a function of the number of reversals to fail for permanent mold cast alloy at $120^{\circ} \mathrm{C}$ and $280^{\circ} \mathrm{C}$.

Figure 72 shows curves of strain amplitude as a function of number of reversals to fail obtained from isothermal fatigue tests at $120^{\circ} \mathrm{C}$ and $280^{\circ} \mathrm{C}$. It may be observed that for low strain amplitude values this influence decreases as higher strain levels are applied. 


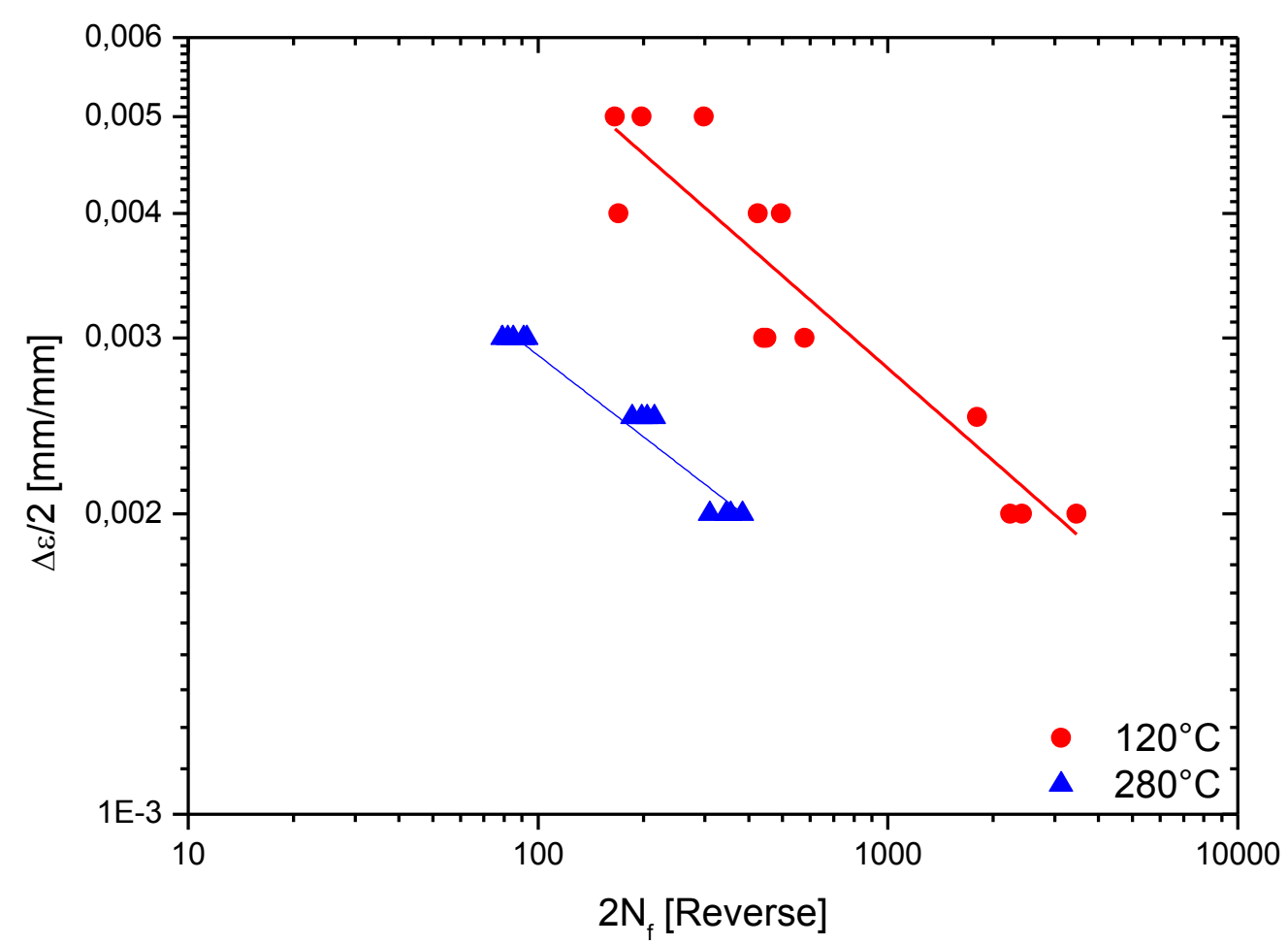

Figure 72: Curve of strain amplitude as a function of the number of reversals to fail for permanent mold cast alloy at $120^{\circ} \mathrm{C}$ and $280^{\circ} \mathrm{C}$.

Analysis of temperature influence on this material fatigue behavior indicates that lifetime decreases with increasing temperature. Reduced fatigue lifetime at high temperatures is due to different mechanisms fostering crack nucleation under these conditions (Suresh, 1998), i.e.:

i. Cyclic slip-induced cracks;

ii. Grain boundary cavitation;

iii. Grain boundary sliding and wedge crack development;

iv. Void nucleation and growth from inclusions and precipitates; and

v. Oxidation and corrosion.

Figure 73 shows stress versus plastic deformation hysteresis curves obtained from isothermal fatigue tests at $120^{\circ} \mathrm{C}$ for total mechanical deformation of (a) $0.3 \%$ and (b) $0.5 \%$. Figure 74 shows stress versus plastic deformation hysteresis curves obtained from isothermal fatigue tests at $280^{\circ} \mathrm{C}$ for $0.3 \%$ total mechanical deformation. Two distinct behaviors may be observed. The material hardens cyclically at $120^{\circ} \mathrm{C}$ and softens cyclically at $280^{\circ} \mathrm{C}$. As is well known, high temperatures have the effect of promoting atom rearrangement in the crystal structures, i.e., atoms shift around in order to reach a more perfect and undeformed arrangement, thus facilitating the movement of dislocations. In general, materials whose 
quotient $\sigma_{\mathrm{e}} / \sigma_{\mathrm{r}}>0.8$ usually undergo cyclic softening, whereas materials whose quotient $\sigma_{\mathrm{e}} / \sigma_{\mathrm{r}}<0.8$ undergo cyclic hardening.

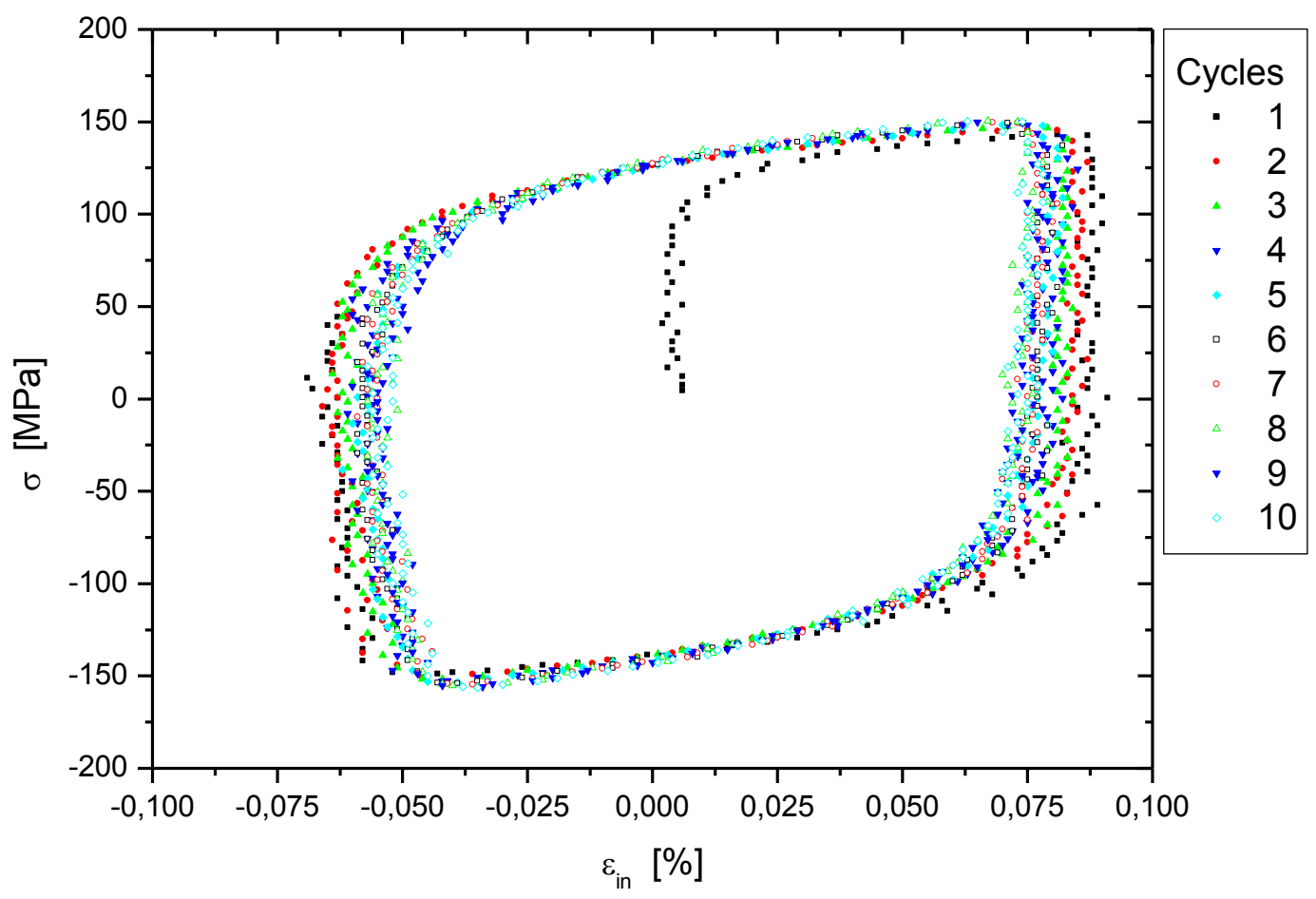

(a)

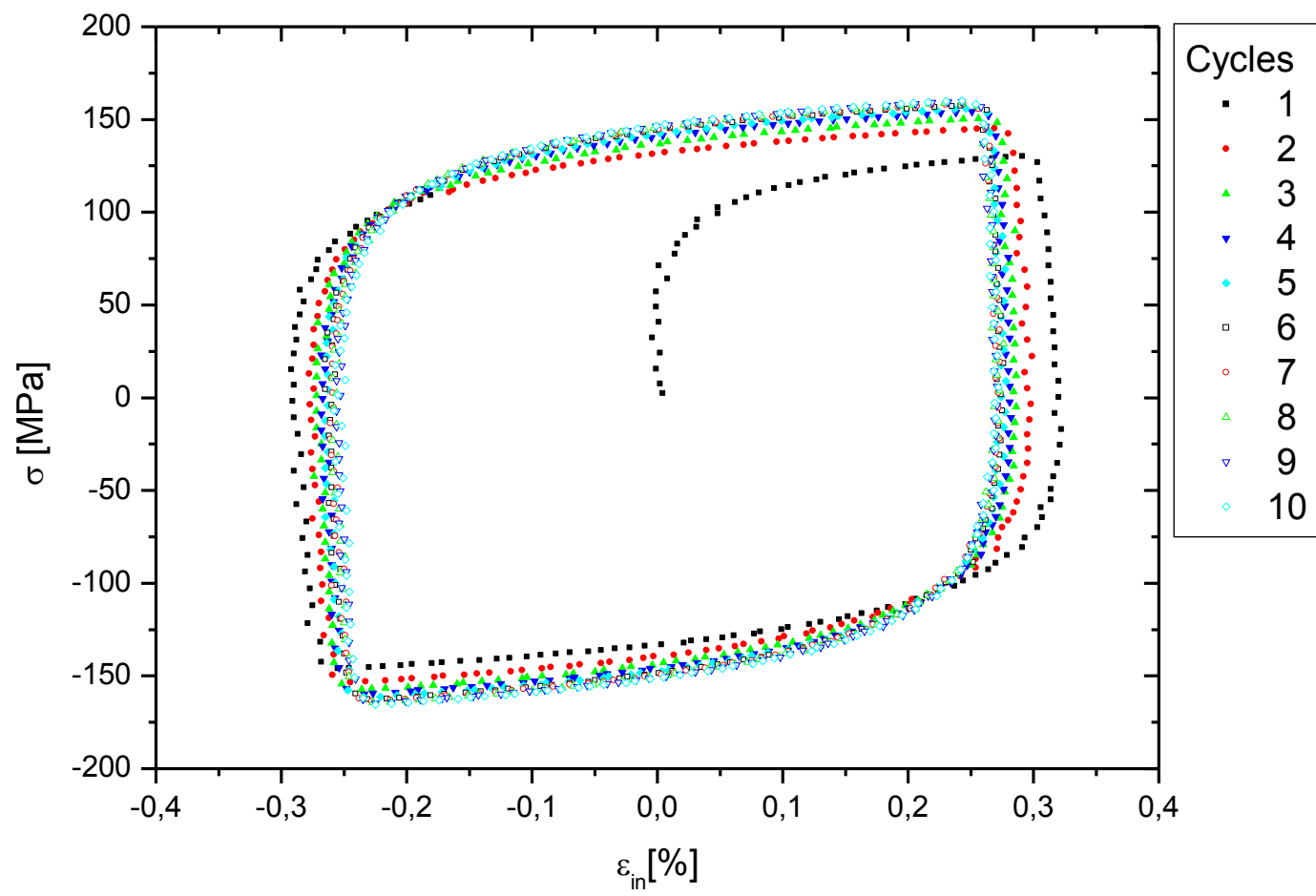

(b)

Figure 73: Hysteresis curve for isothermal fatigue tests at $120^{\circ} \mathrm{C}$ and total mechanical deformation of (a) $0.3 \%$ and (b) $0.5 \%$. 


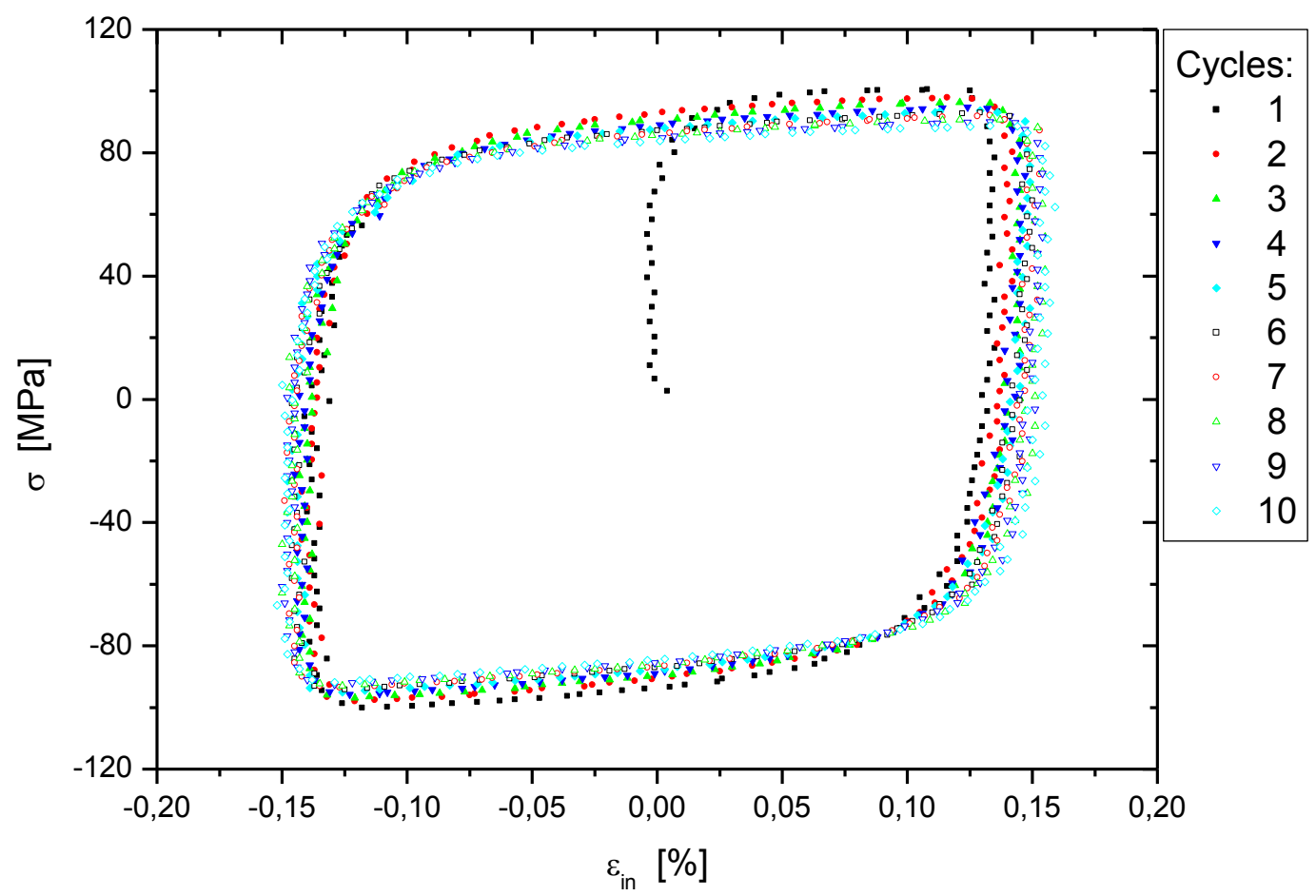

Figure 74: Hysteresis curve for isothermal fatigue tests at $280^{\circ} \mathrm{C}$ and $0.3 \%$ total mechanical deformation.

It is also possible to note that the material displays distinct behaviors under tensile and compressive loading conditions at both temperatures $\left(120^{\circ} \mathrm{C}\right.$ and $\left.280^{\circ} \mathrm{C}\right)$. This difference in behavior is more pronounced at low temperatures. At high temperatures, this difference is subtler, since it can promote a decrease in the strength needed for deformation enabling microstructure recovery and re-crystallization processes, thereby decreasing the density of dislocations.

\subsubsection{Thermomechanical Fatigue (TMF)}

Figure 75 presents the curves of (a) mechanical deformation amplitude and (b) stress amplitude as a function of the number of reversals to failure, corresponding to in-phase anisothermal tests between $120^{\circ} \mathrm{C}$ and $280^{\circ} \mathrm{C}$ starting at the average temperature of $200^{\circ} \mathrm{C}$. As in the case of isothermal fatigue tests, specimens whose results were contrary to overall behavior were analyzed and discarded if found to be defective. 


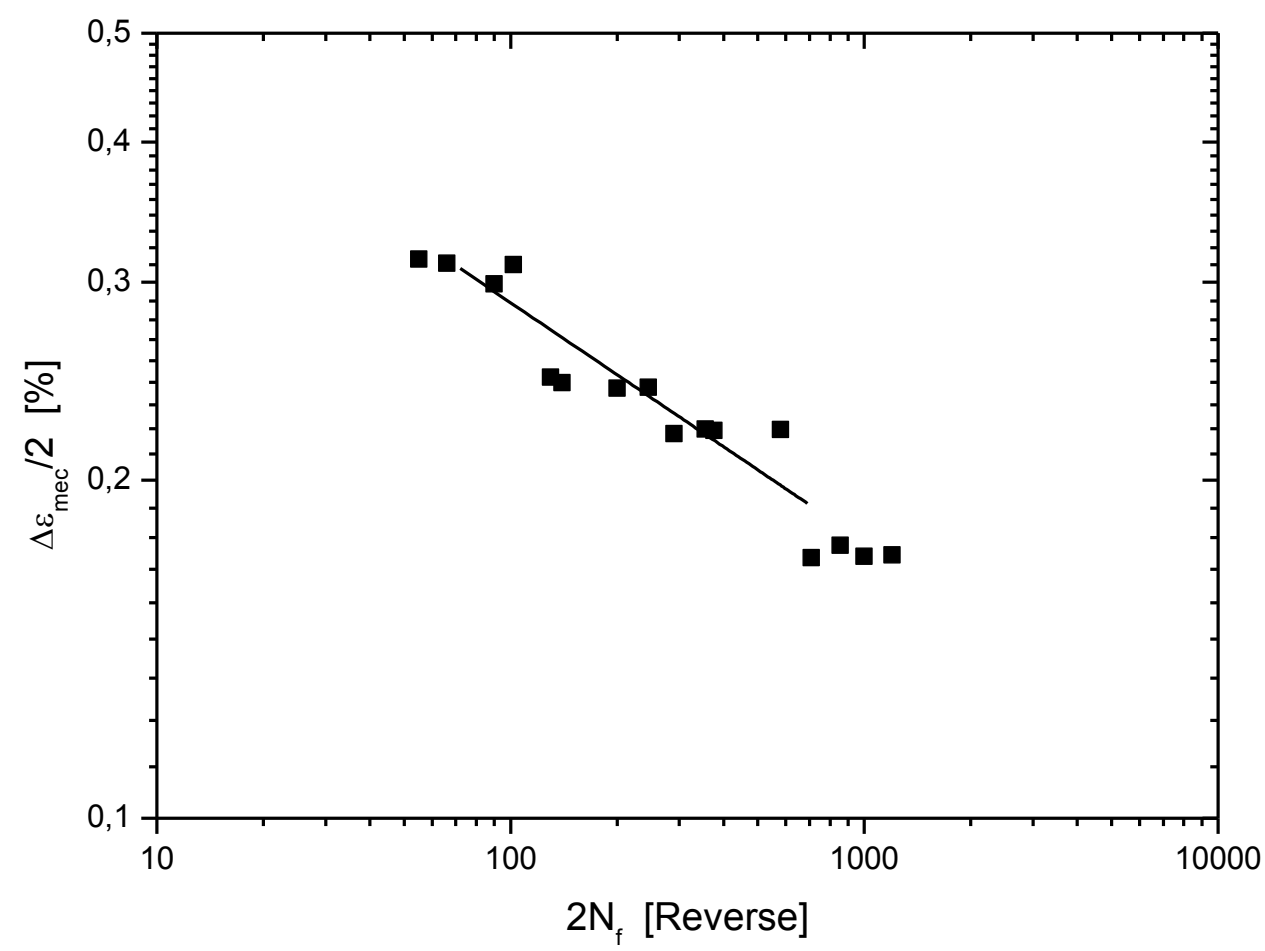

(a)

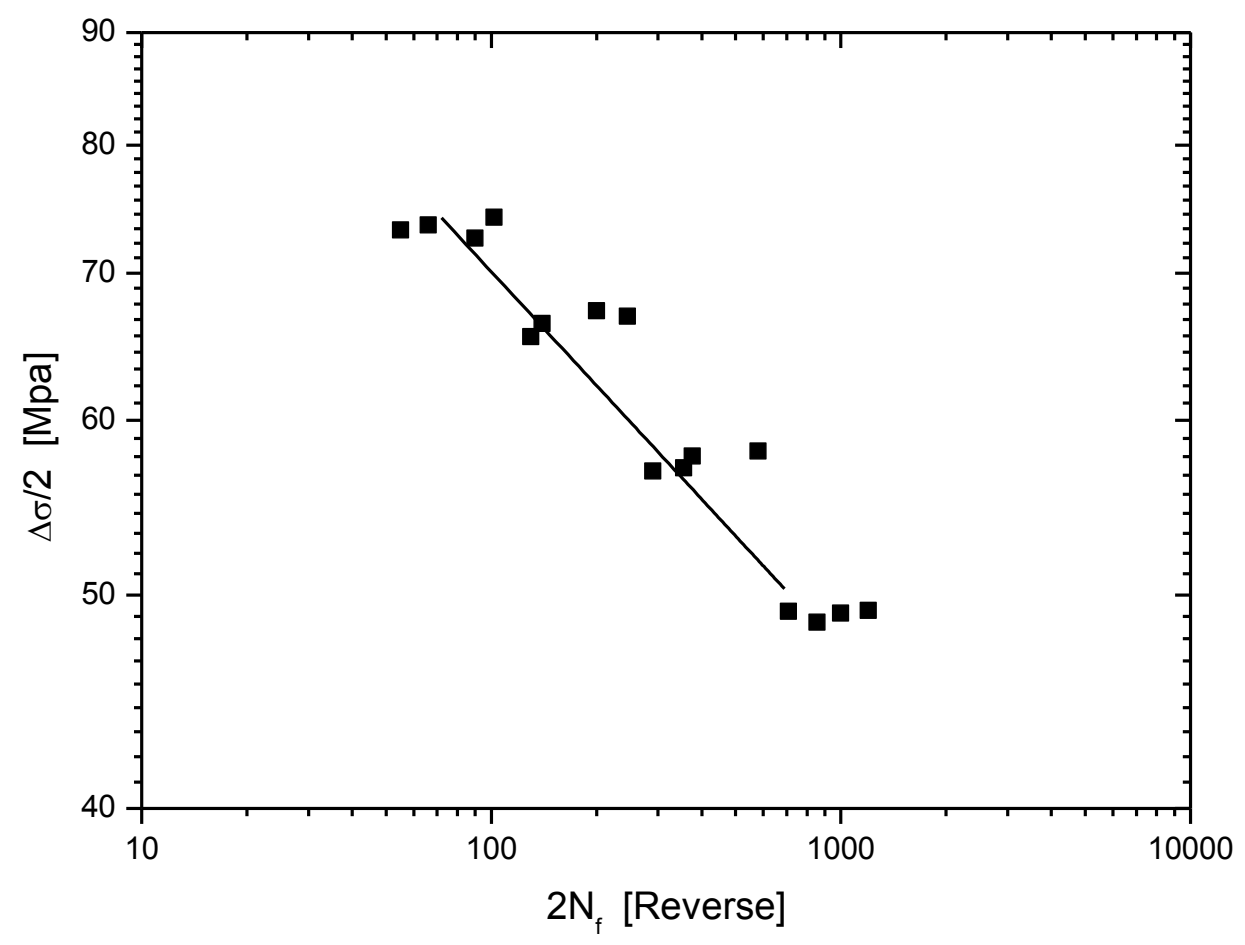

(b)

Figure 75: Curves of (a) mechanical deformation amplitude and (b) stress as a function of the number of reversals to failure. 
Figure 76 shows curves of (a) mechanical deformation amplitude and (b) stress amplitude as a function of the number of reversals to fail as well as hysteresis for (c) $0.3 \%$ total mechanical deformation obtained from IF and TMF tests in order to analyze the material behavior in these different types of tests. It may be observed that, in general, isothermal tests cannot represent thermo-mechanical behavior. An exception may be made, due to the high cost of anisothermal tests, when isothermal tests are conducted under the most critical anisothermal conditions.

Micro-structural refinement and porosity level are closely related in cast alloys because a large part of voids, especially those that are large and irregular, originate in interdendrite regions as a result of local dendrite contractions of microscopic magnitude. Hence, the coarser the microstructure, the greater is contraction in inter-dendrite regions and the larger are the voids. On the other hand, these contractions are minimized in a refined microstructure. It is generally known that there is a higher tendency for crack nucleation in pores with diameters larger than $50 \mu \mathrm{m}$. As the porosity level of the material under study is high (Table 8), fatigue lifetime is believed to have been damaged.

Despite conducting isothermal fatigue tests at a low frequency as compared to that of anisothermal tests, it becomes high. The period of exposition to high temperatures promotes the occurrence of other effects, e.g., relaxation and creep, which explain the anisothermal hysteresis behavior seen in Figure 76(c) and shows that these phenomena interact with fatigue due to extended test periods at high temperatures.

Figure 76(a) indicates that the material behavior under thermo-mechanical fatigue, considering in-phase mechanical and thermal loadings, is similar to the isothermal one at $280^{\circ} \mathrm{C}$, i.e., at maximum test temperature. Thus, it is should be observed that the greatest damage in anisothermal tests occurs when maximum stress is applied at the highest temperature. Figure 76(c) shows that the thermo-mechanical hysteresis is similar to the isothermal one at $280^{\circ} \mathrm{C}$, differences owing to micro-deformation mechanisms at high temperatures, e.g., creep and relaxation. This is because the thermo-mechanical loading cycle is about $0.003 \mathrm{~Hz}$, much lower than the isotherm one $(0.1 \mathrm{~Hz})$. Therefore, in this case, isothermal tests at maximum temperature could offer a good estimate of deformation behavior during cyclic loading, provided that costs and experimental difficulties of thermo-mechanical fatigue tests are taken into account. 


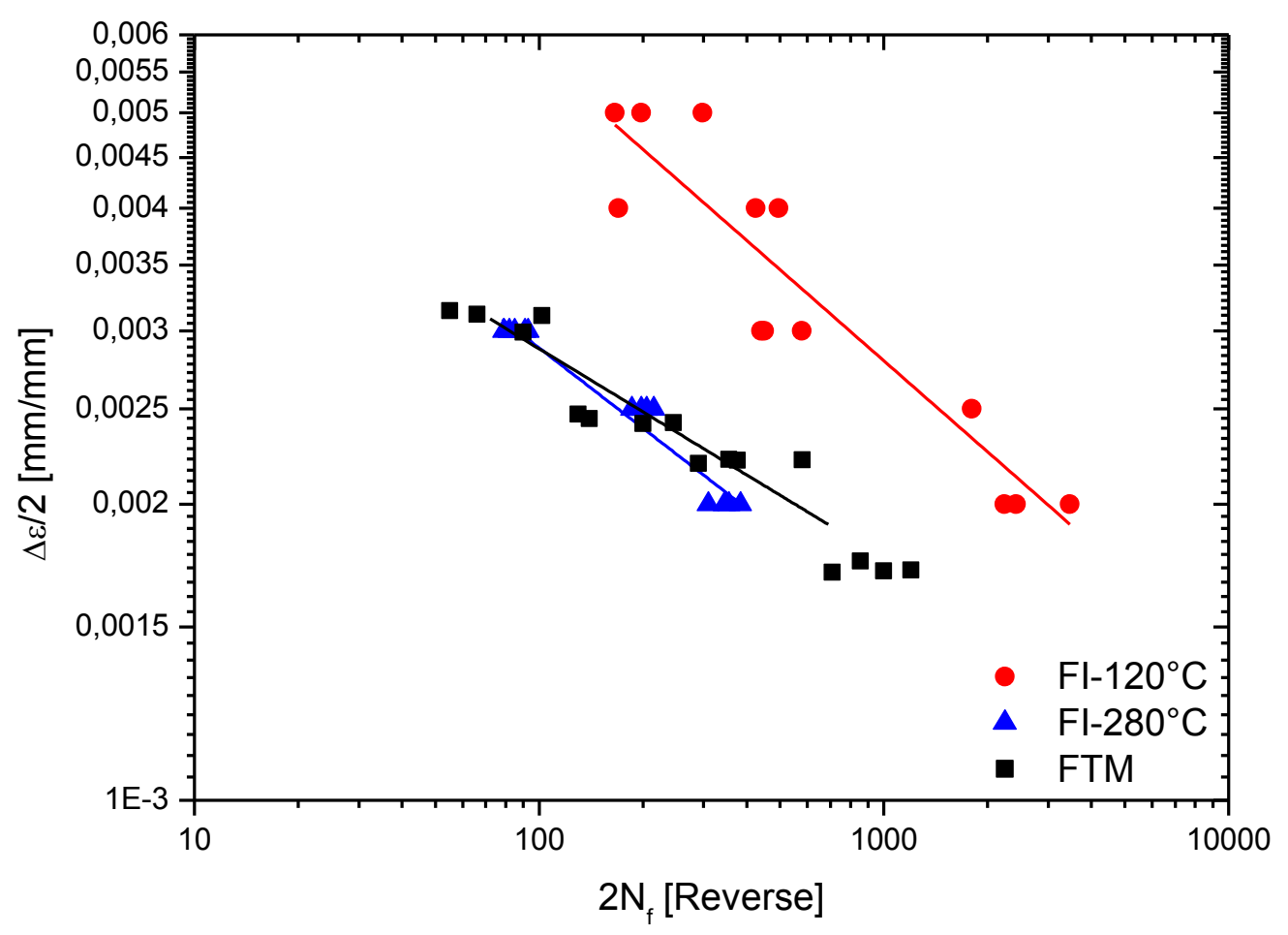

(a)

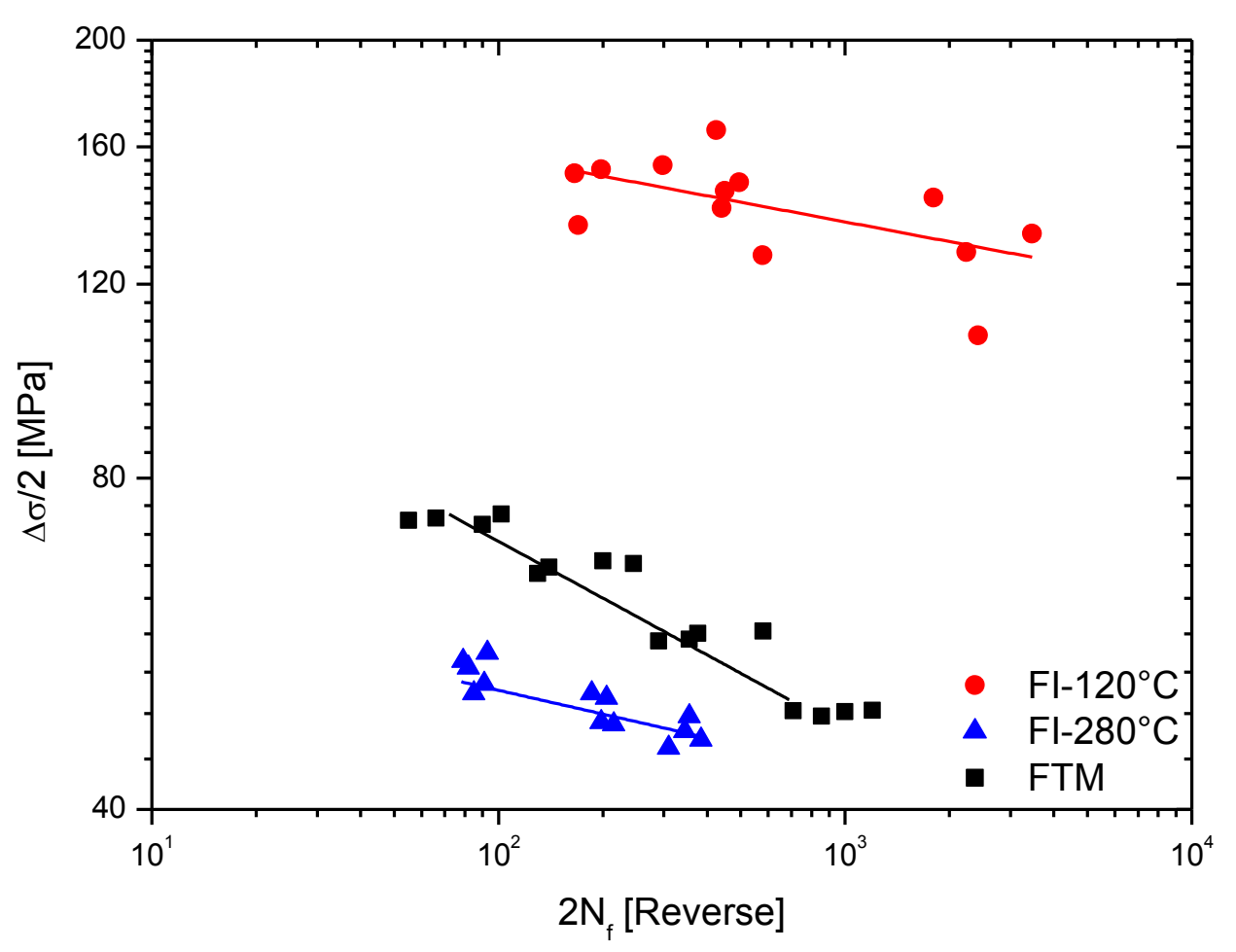

(b) 


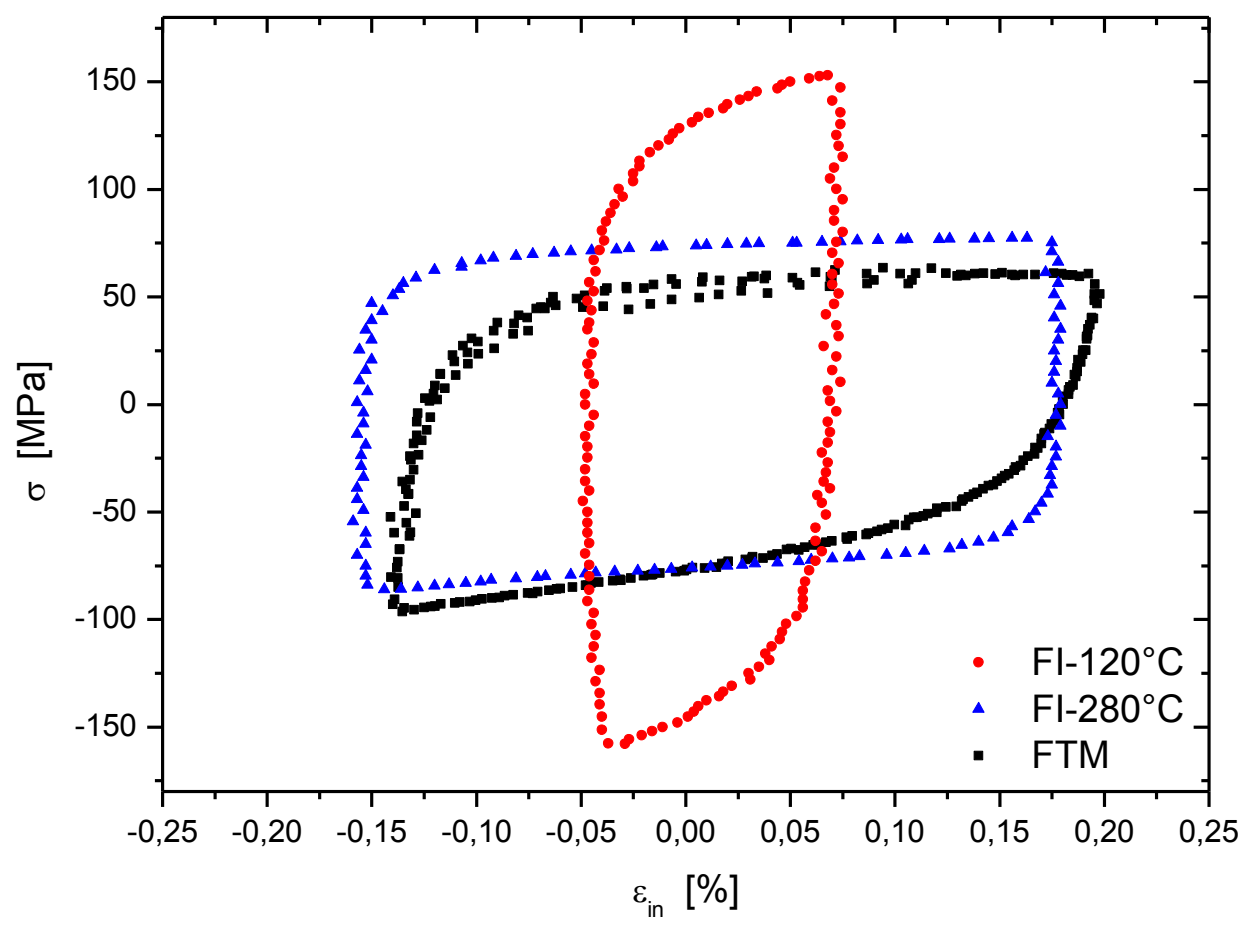

(c)

Figure 76: Comparison between graphs of (a) mechanical deformation amplitude in relation to the number of reversals to fail, (b) stress amplitude as a function of the number of reversals to fail, and (c) hysteresis related to isothermal and anisothermal tests for $0.3 \%$ total mechanical deformation.

In general, in both isothermal and anisothermal tests, cracks began to propagate in regions of inter-dendrite voids near the surface. Crack propagation exhibited similar behavior in both cases. In some instances, intrusion and extrusion mechanisms operated in the beginning of tests, causing cracks to nucleate and propagate through the ductile matrix in a trans-granular mode. Subsequently, because of higher tensile stresses, cracks tended to propagate through the eutectic, which is more brittle.

\subsubsection{Relaxation}

In order to better identify the viscous behavior shown in the hystereses of the thermomechanical fatigue tests, relaxation tests were conducted at $80^{\circ} \mathrm{C}, 100^{\circ} \mathrm{C}, 120^{\circ} \mathrm{C}, 140^{\circ} \mathrm{C}$, $180^{\circ} \mathrm{C}, 240^{\circ} \mathrm{C}$, and $280^{\circ} \mathrm{C}$ for total deformation levels of $0.1 \%, 0.3 \%, 0.5 \%, 0.7 \%$, and $0.9 \%$. The tests were performed by controlling deformation, using the extensometer employed in fatigue tests, and adjusting test parameters to obtain reliable results (Figure 77). The conformity of the elasticity modulus values obtained from tensile tests to those obtained from 
hysteresis loops that did not reach plastic regime was also verified. Figure 78 shows test results. The hysteresis for $240^{\circ} \mathrm{C}$ was suppressed because during this test the hydraulic grip holding the specimen in place lost pressure, altering the curve.

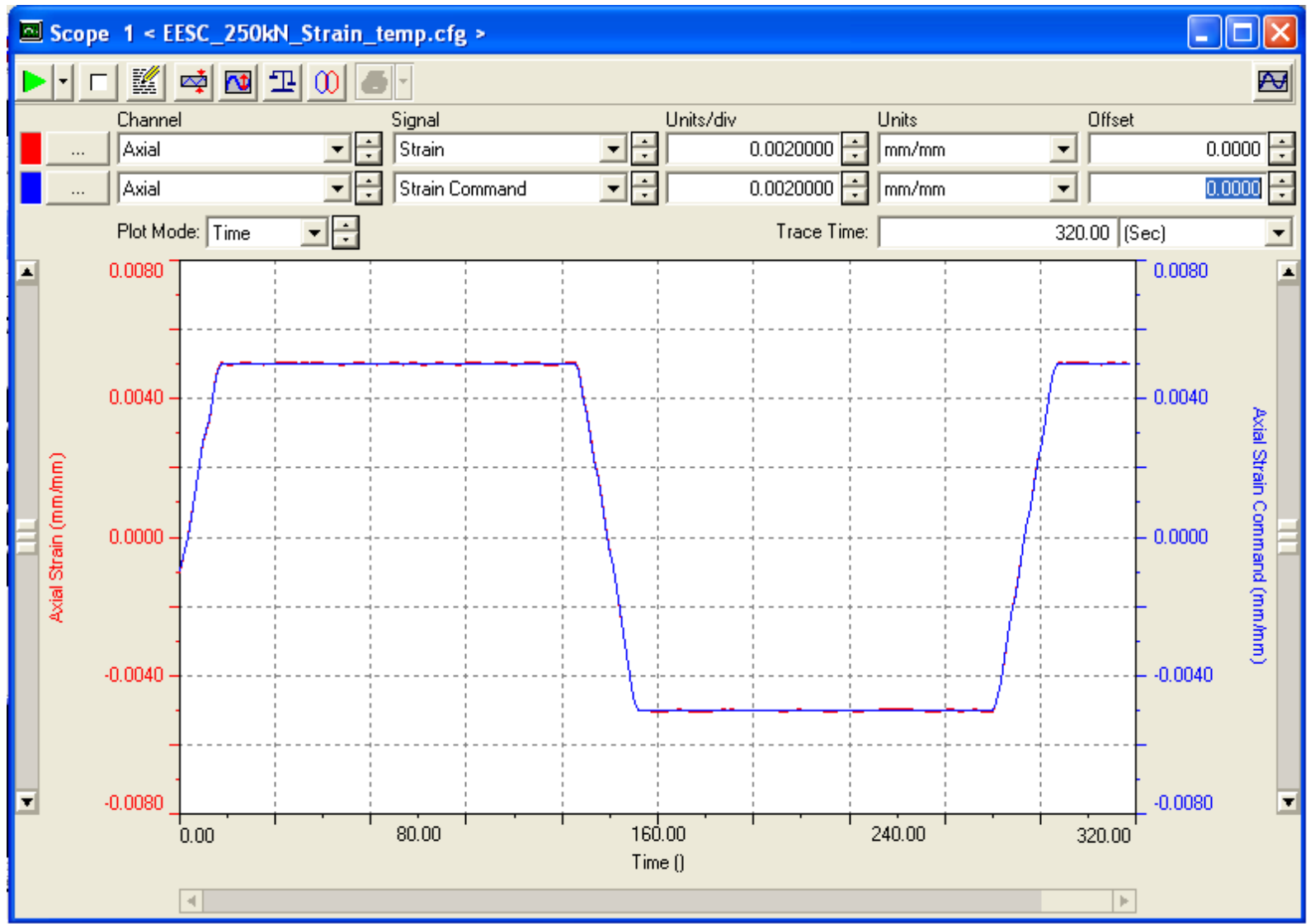

Figure 77: Chart of signal of perfectly adjusted command and response.

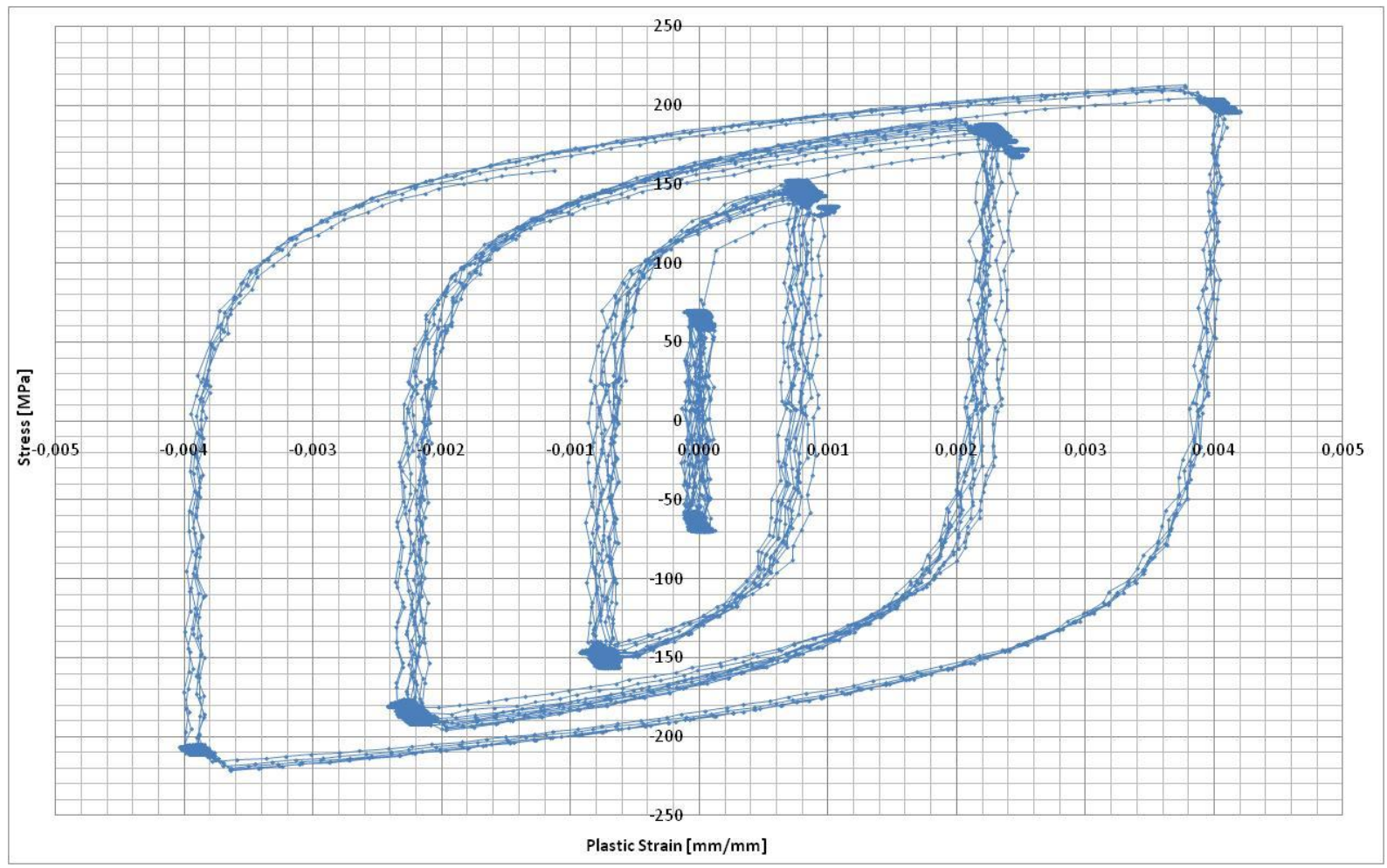

(a) 


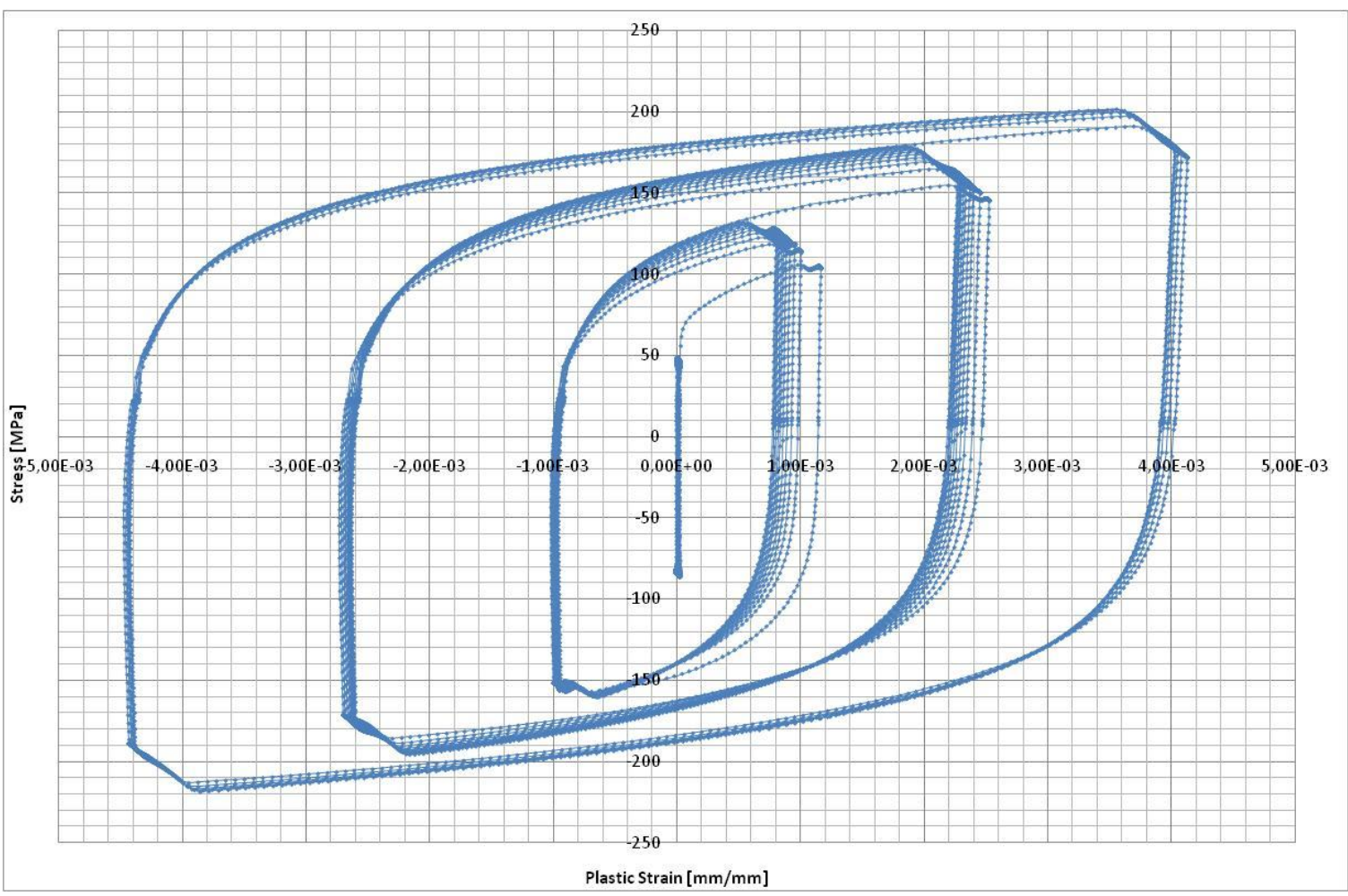

(b)

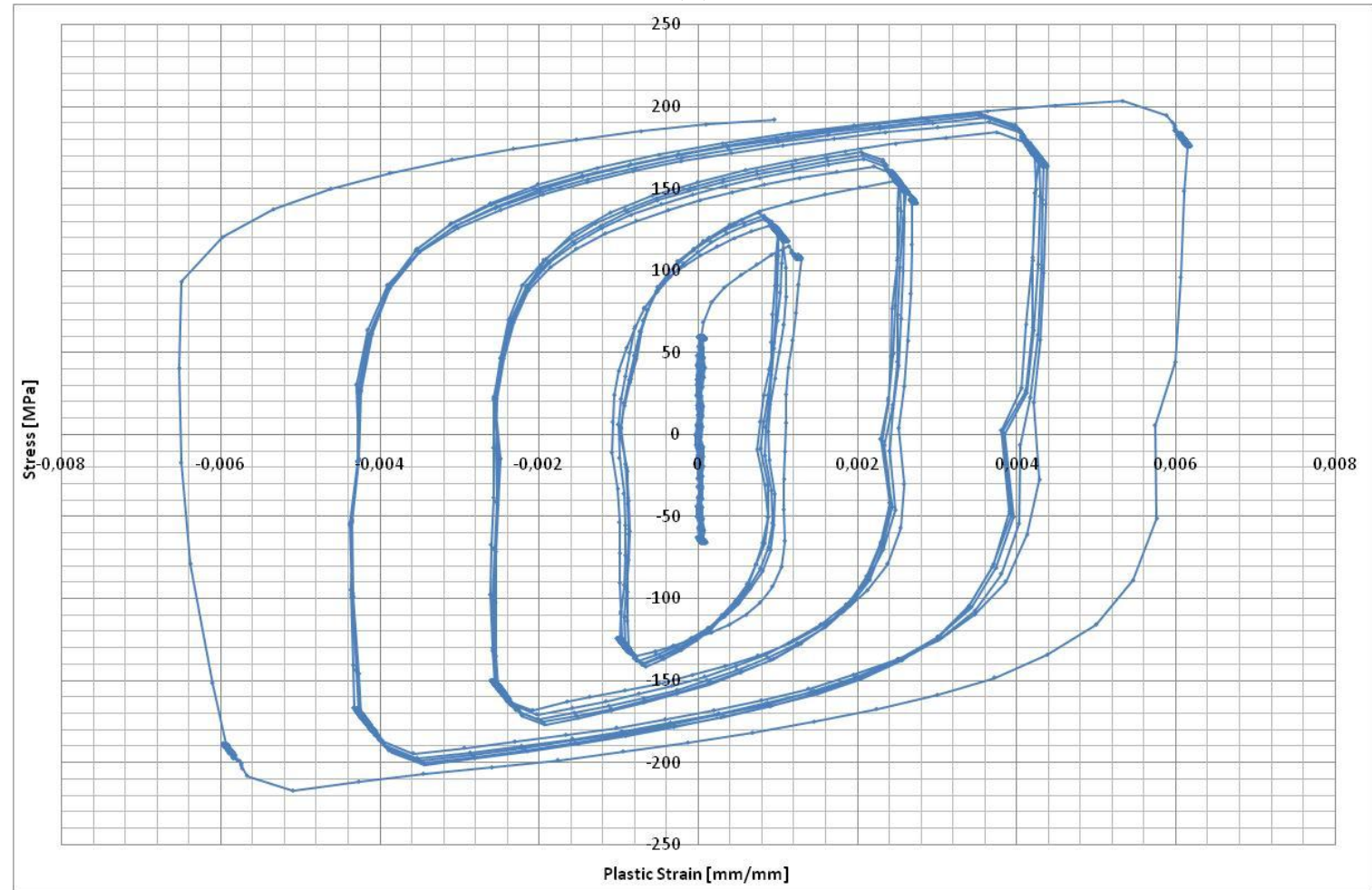

(c) 


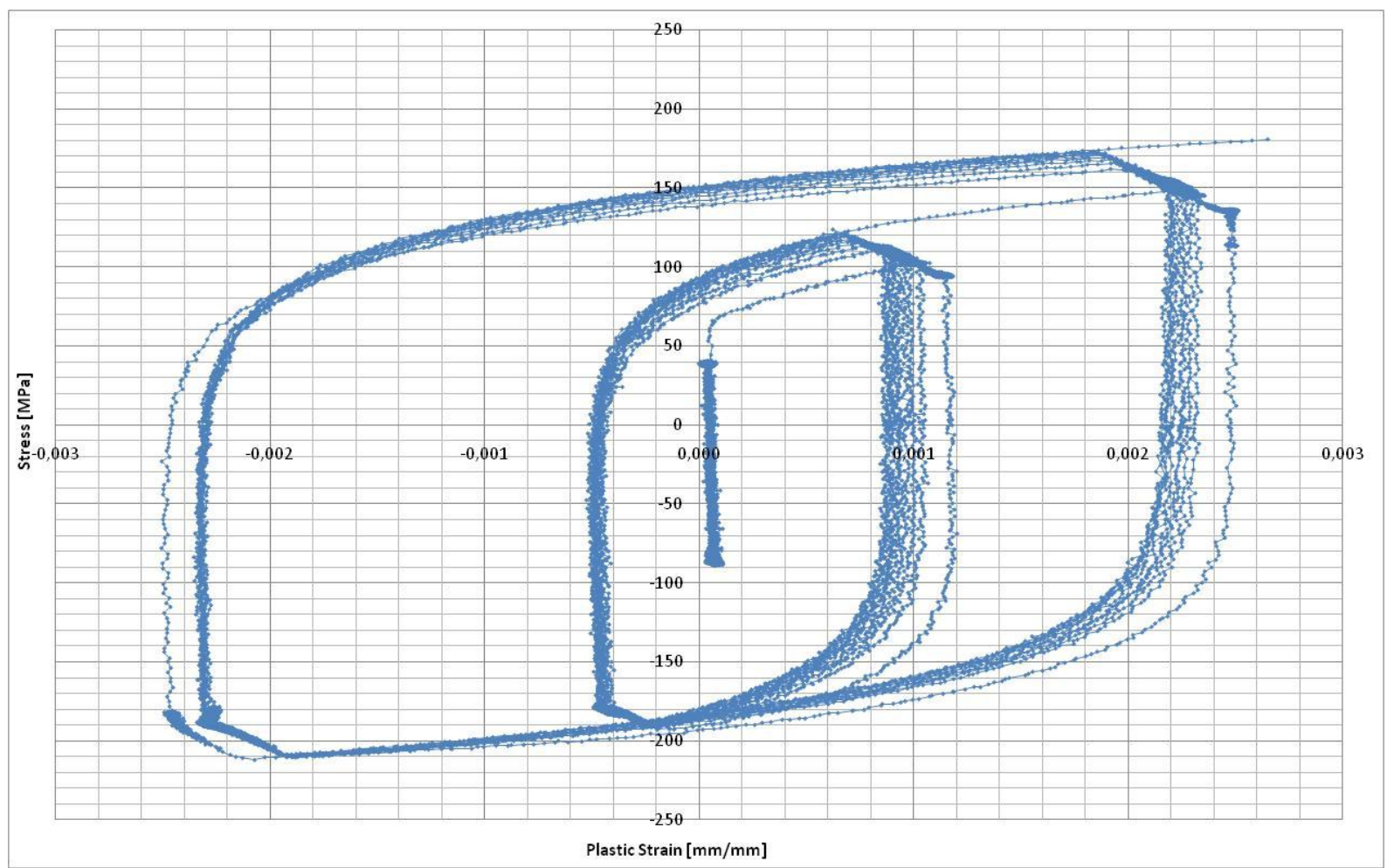

(d)

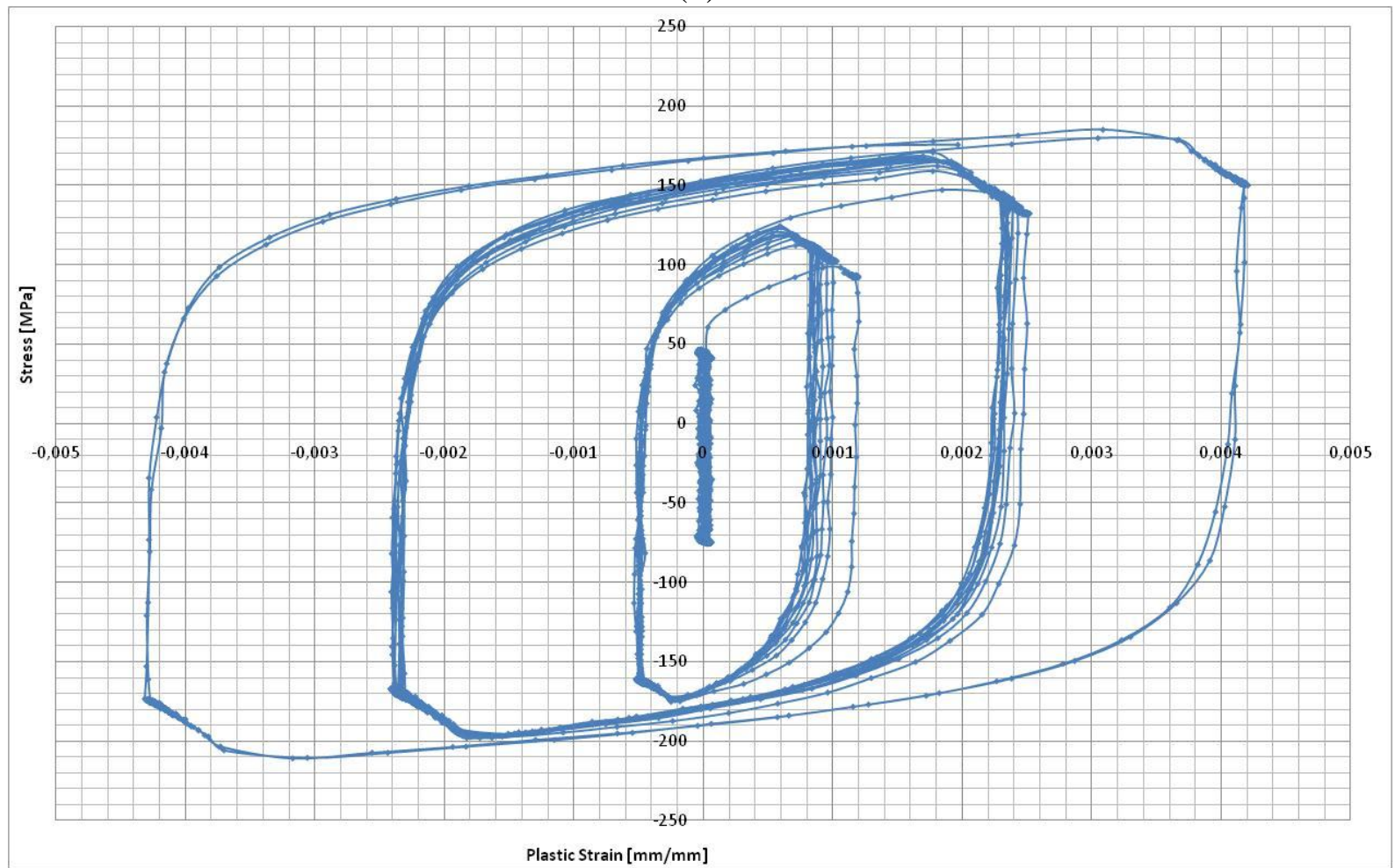

(e) 


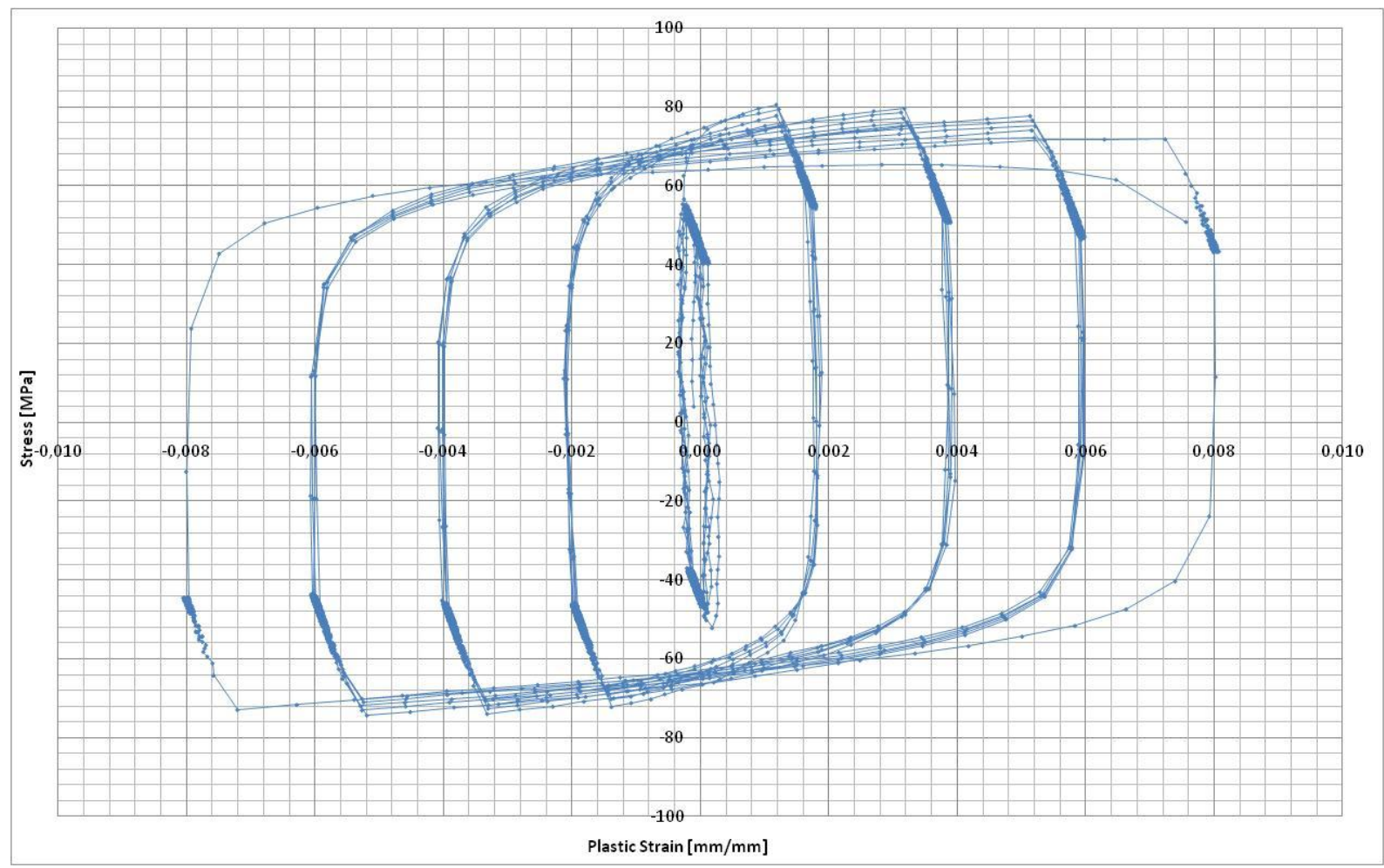

(f)

Figure 78: Relaxation test hystereses for total deformations of $0.1 \%, 0.3 \%, 0.5 \%, 0.7 \%$, and $0.9 \%$ at (a) $80^{\circ} \mathrm{C}$, (b) $100^{\circ} \mathrm{C}$, (c) $120^{\circ} \mathrm{C}$, (d) $140^{\circ} \mathrm{C}$, (e) $180^{\circ} \mathrm{C}$, and (f) $280^{\circ} \mathrm{C}$.

Relaxation tests as well as hysteresis of isothermal fatigue tests indicate two distinct behaviors, the temperature of $240^{\circ} \mathrm{C}$ being a threshold. At lower temperatures, the material hardens cyclically whereas it softens cyclically at higher temperatures. High temperatures have the effect of promoting atom rearrangement in the crystal structure, i.e., atoms shift positions to reach a more perfect and undeformed arrangement, thus facilitating the movement of dislocations. The stress vs. plastic deformation curves shown in Figure 78 also indicate that although the test was performed under controlled strain, i.e., total strain was kept constant throughout the test, there was an increase in the amount of plastic deformation and consequently a decrease in elastic deformation so that total deformation remained constant. Therefore, this increase in plastic deformation corresponded to creep deformation.

Figure 79 shows some stress vs. time curves for a constant deformation level of $0.5 \%$ obtained at $100^{\circ} \mathrm{C}, 180^{\circ} \mathrm{C}$, and $280^{\circ} \mathrm{C}$. It is possible to note that the higher the test temperature $(\mathrm{T})$, the lower is the maximum stress $\left(\sigma_{\max }\right)$ required for this level of deformation. In general, the higher the temperature $(T)$, the higher is the stress variation $(\Delta \sigma)$ at a constant deformation level. When this level of stability is extended, i.e., the period in which deformation remains constant, stress keeps falling. It is important to remark that, since determining a threshold stress was not the purpose of this study, the maximum test period was five minutes, long enough to determine the material behavior and obtain viscosity parameters from the curves. 


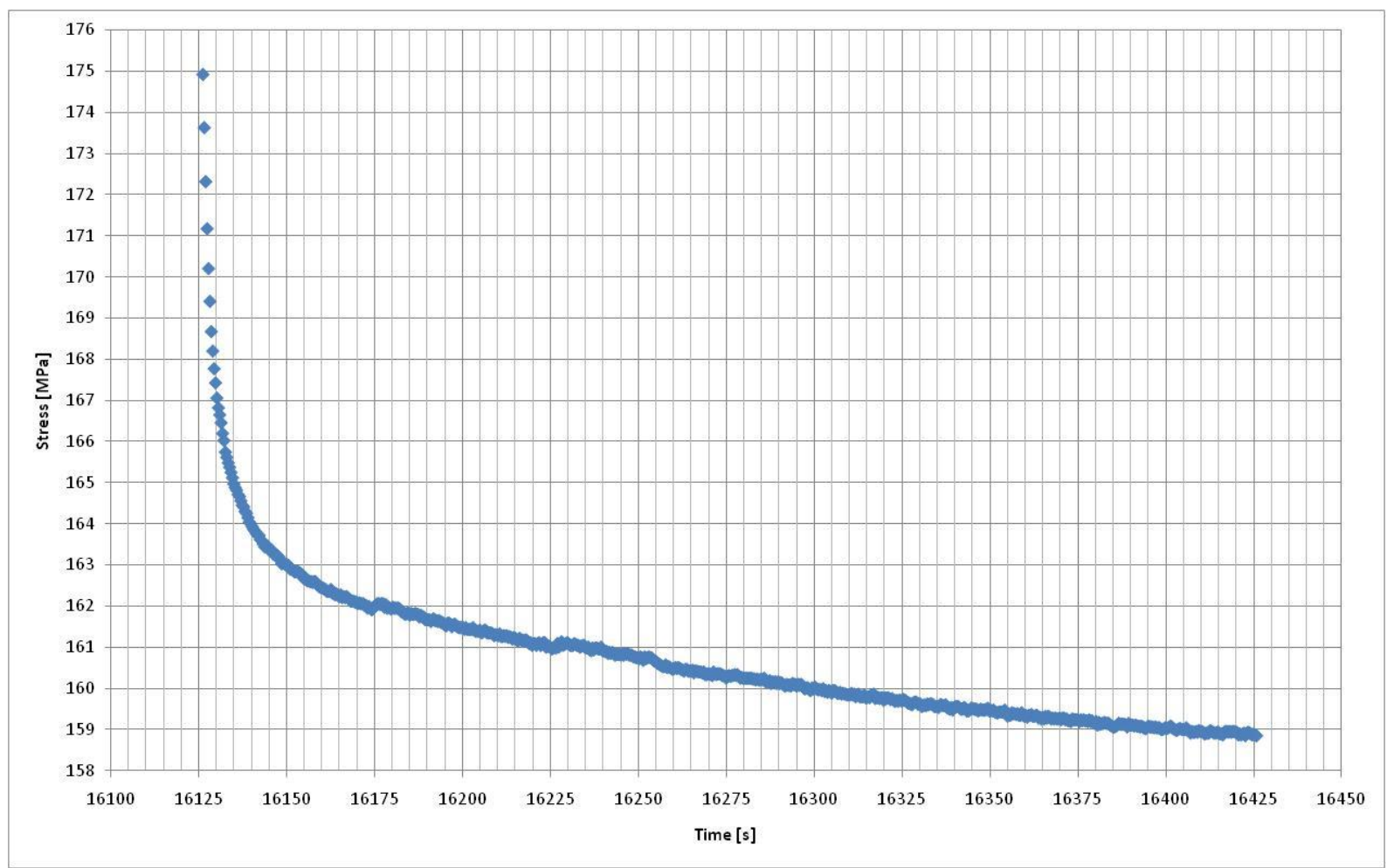

(a)

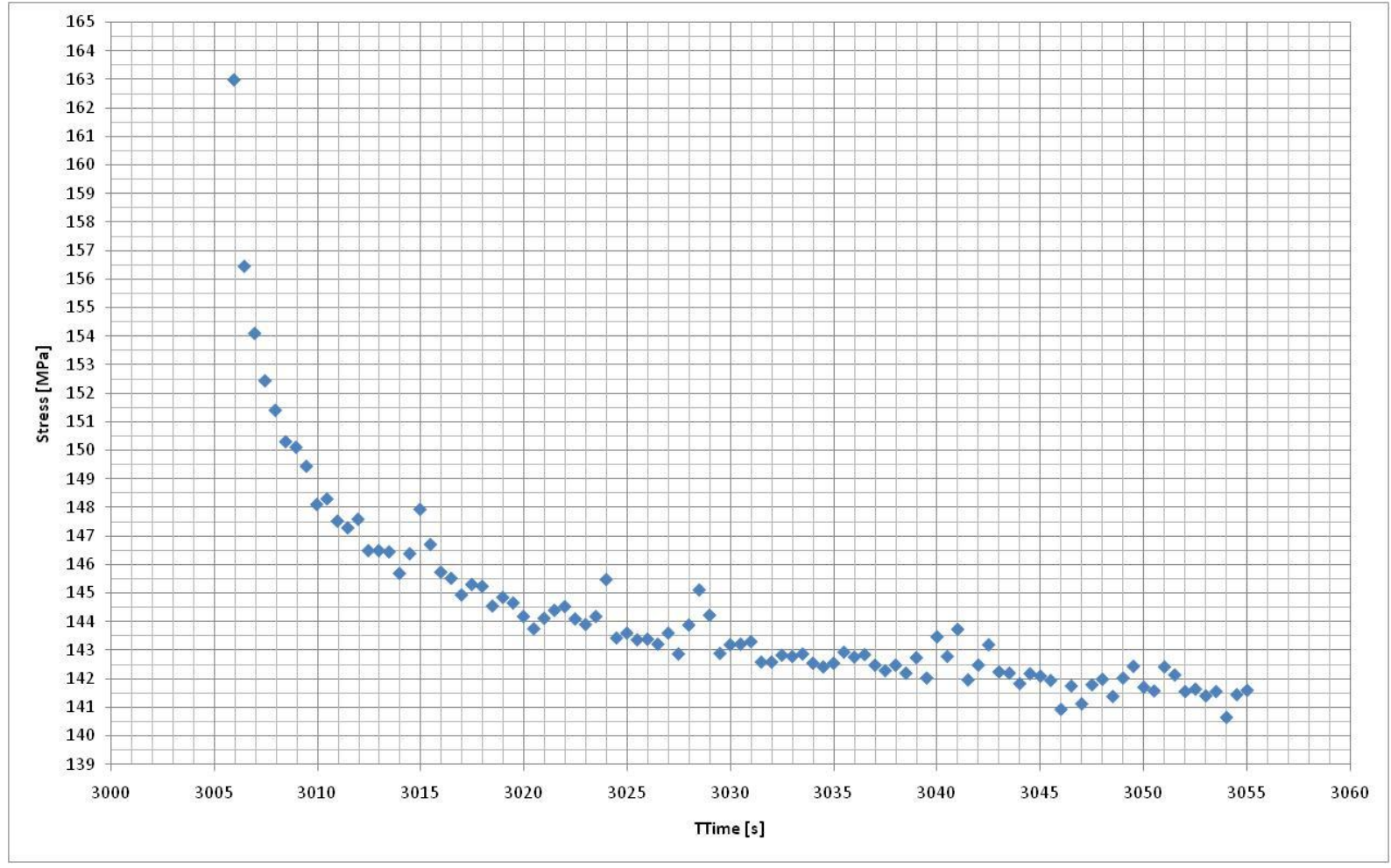

(b) 


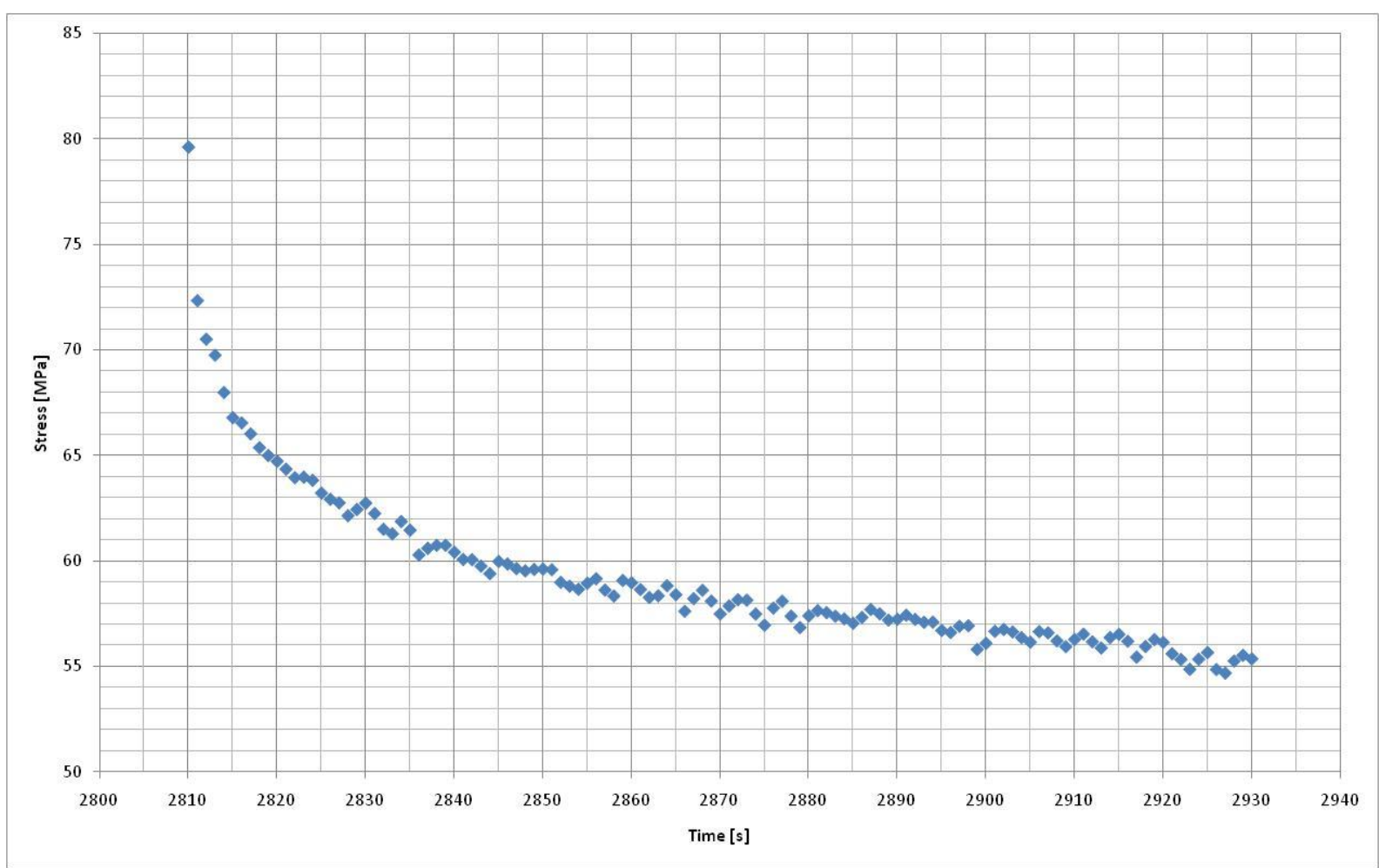

(c)

Figure 79: Stress vs. time curves for a constant deformation level of $0.5 \%$ at $100^{\circ} \mathrm{C}, 180^{\circ} \mathrm{C}$, and $280^{\circ} \mathrm{C}$.

\subsubsection{Fatigue Crack Growth}

Fatigue crack propagation tests were performed with specimens from the component (cylinder head), with special geometry as shown in Figure 52b. The tests were conducted at $75^{\circ} \mathrm{C}, 120^{\circ} \mathrm{C}, 200^{\circ} \mathrm{C}$, and $280^{\circ} \mathrm{C}$, and loadings at $\mathrm{R}=0.1$ produced a sinusoidal wave shape, with $10 \mathrm{~Hz}$ frequency, or a trapezoidal wave shape, consisting of a loading ramp and an unloading ramp lasting $10 \mathrm{~s}$ each and a 180s constant loading level, totaling a $0.005 \mathrm{~Hz}$ frequency. Loading values were estimated for each temperature so as not to plasticize the region ahead of the crack. Several methods for measuring the progress of the crack were tried, i.e., visual method, image correlation method (Correli), and acetate replica method, but to no avail, probably due to the cast material microstructure and the impossibility of polishing the surface as much as required. Then, the potential drop method was used and the relationship between values obtained through it and crack growth was obtained according to ASTM E1457 $07^{\varepsilon 1}$. Figure 80 shows the fracture surface of a specimen tested for fatigue crack propagation, criteria employed to define the points $a_{0}$ and $a_{f}$, and calibration curves. The time used for data acquisition was approximately 1 (one) point per second. These data were filtered to 
eliminate noise due to numerous factors such as power fluctuations, vibrations caused by hydraulic system of mechanical testing machine, small temperature fluctuations, and so on.

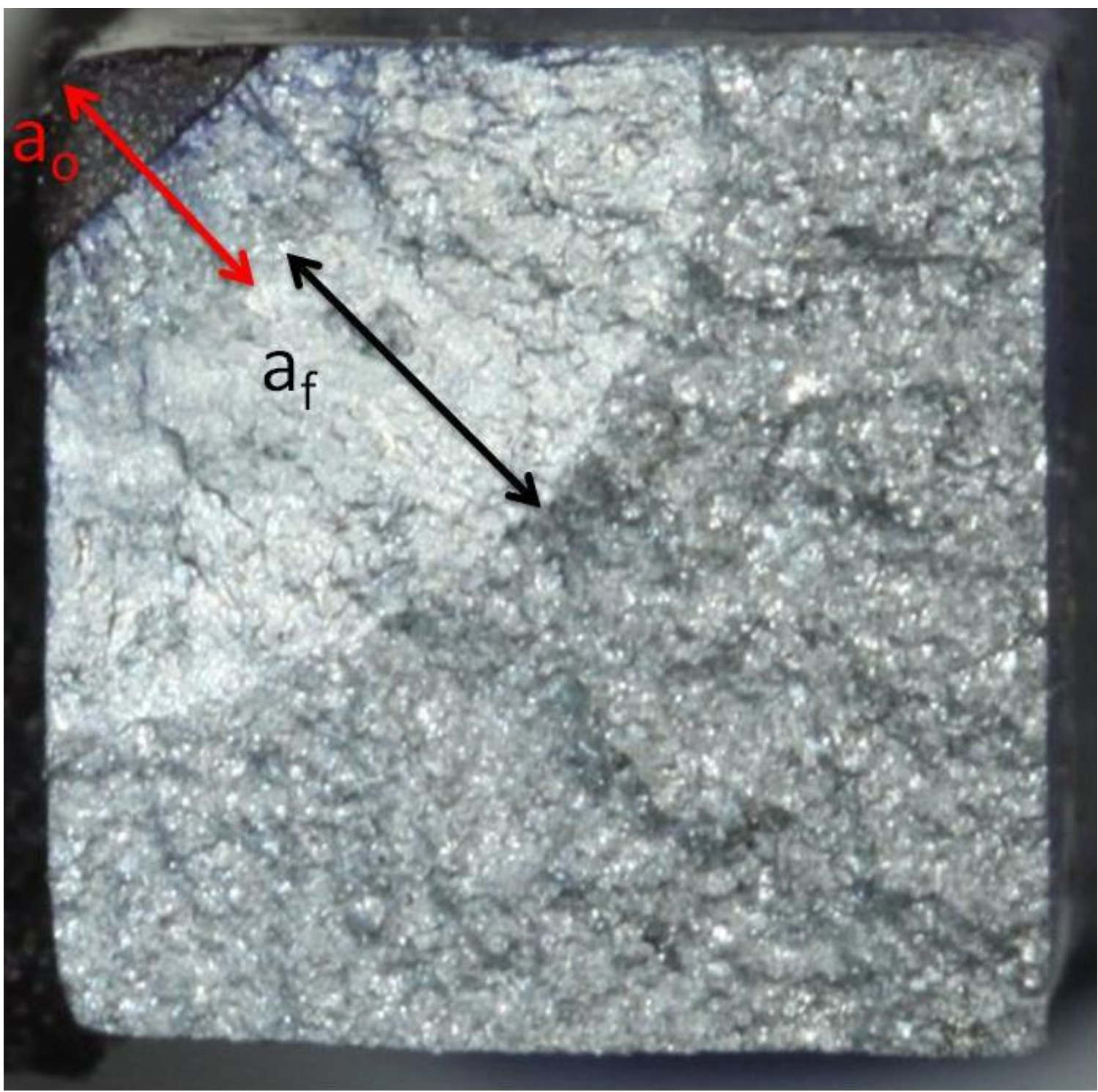

(a)

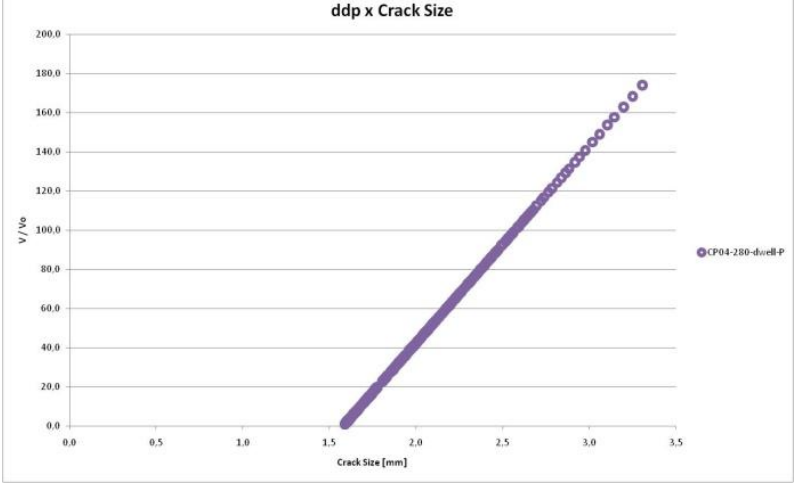

(b)

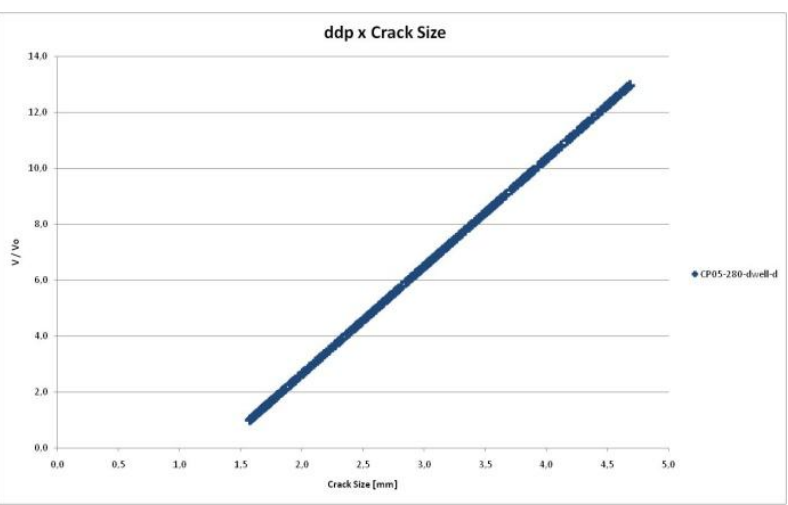

(c)

Figure 80: Fracture surface of a specimen tested for fatigue crack propagation and (a) criteria employed to define the points $a_{0}$ and $a_{f}$, and (b) and (c) calibration curves. 
Figure 81 presents the results of the fatigue crack propagation test at $75^{\circ} \mathrm{C}$ under sinusoidal wave loadings. It would be expected to have values around $20 \mathrm{MPa} \sqrt{\mathrm{m}}$ for the limit stress intensity factor $\left(\Delta \mathrm{K}_{\mathrm{Th}}\right)$, usually found for aluminum alloys. However, values for the material under study (A356 gravity cast aluminum alloy) were well below expected, given the observed quantity of porosity and microstructural heterogeneity.

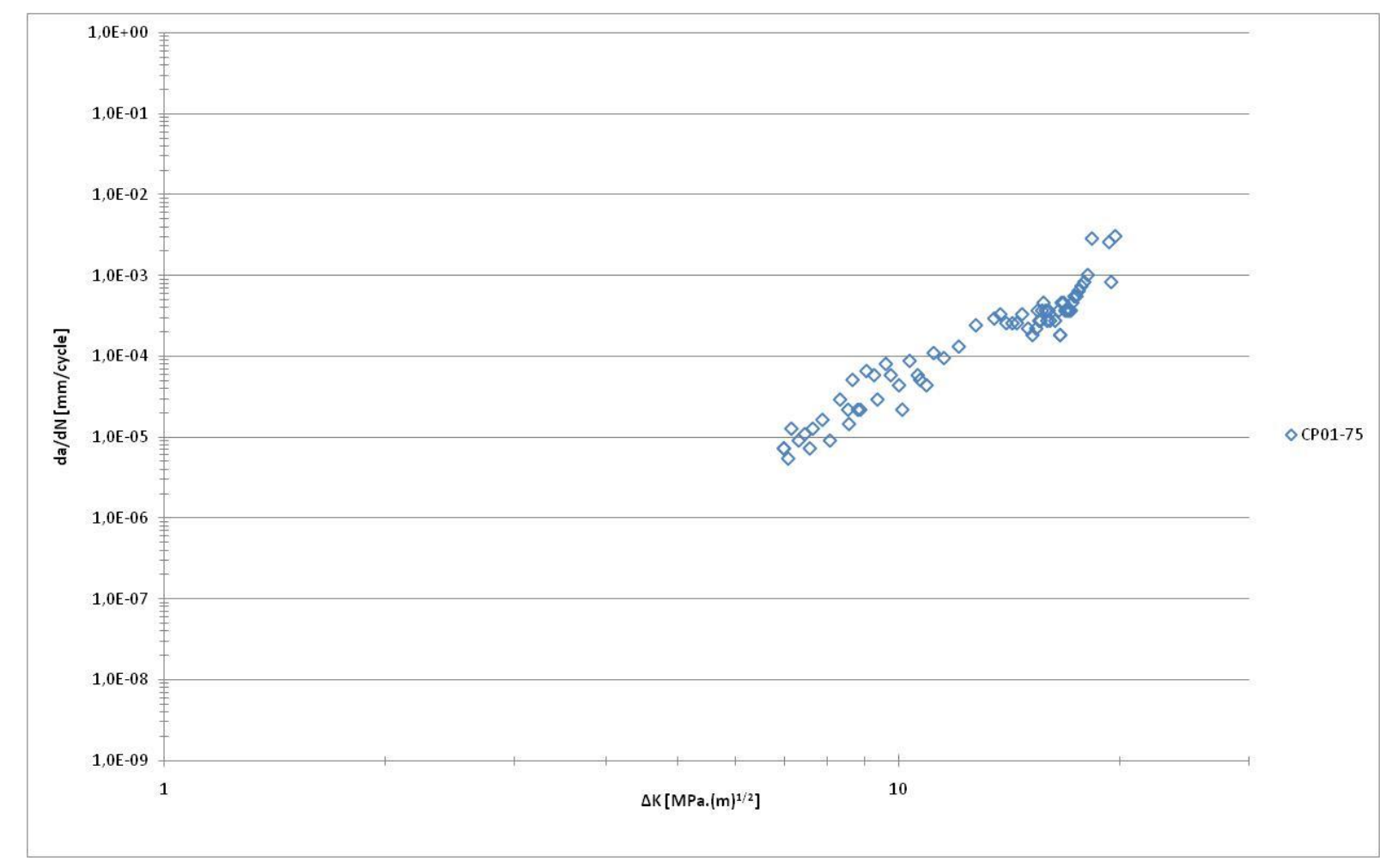

Figure 81: Graph of crack propagation $(\mathrm{da} / \mathrm{dN})$ as a function of stress intensity factor $(\Delta \mathrm{K})$ at $75^{\circ} \mathrm{C}$ under sinusoidal wave loading.

Figure 82 shows results of fatigue crack propagation tests at $120^{\circ} \mathrm{C}$ under sinusoidal wave loading (CP01 and CP02) and trapezoidal wave loading (CP3). It may be observed that for the same level $(\Delta \mathrm{K})$ and sinusoidal wave loading there was a significant decrease in stress intensity as compared to that obtained at $75^{\circ} \mathrm{C}$, which indicates the influence of temperature on crack development and, as a result, formation of a plastic region ahead of it. Trapezoidal wave loading under load control was more detrimental than sinusoidal wave loading, showing an increase in crack growth rate in the order of 100 (one hundred) times. This may be explained by the occurrence of other phenomena, which could not be observed previously due to loading mode, interacting during the test and aggravating it. Indeed, trapezoidal wave loadings with plateau time of the order of 3 minutes allow the occurrence of phenomena such as oxidation, creep, and relaxation, because the material is under maximum loading for a longer period. 


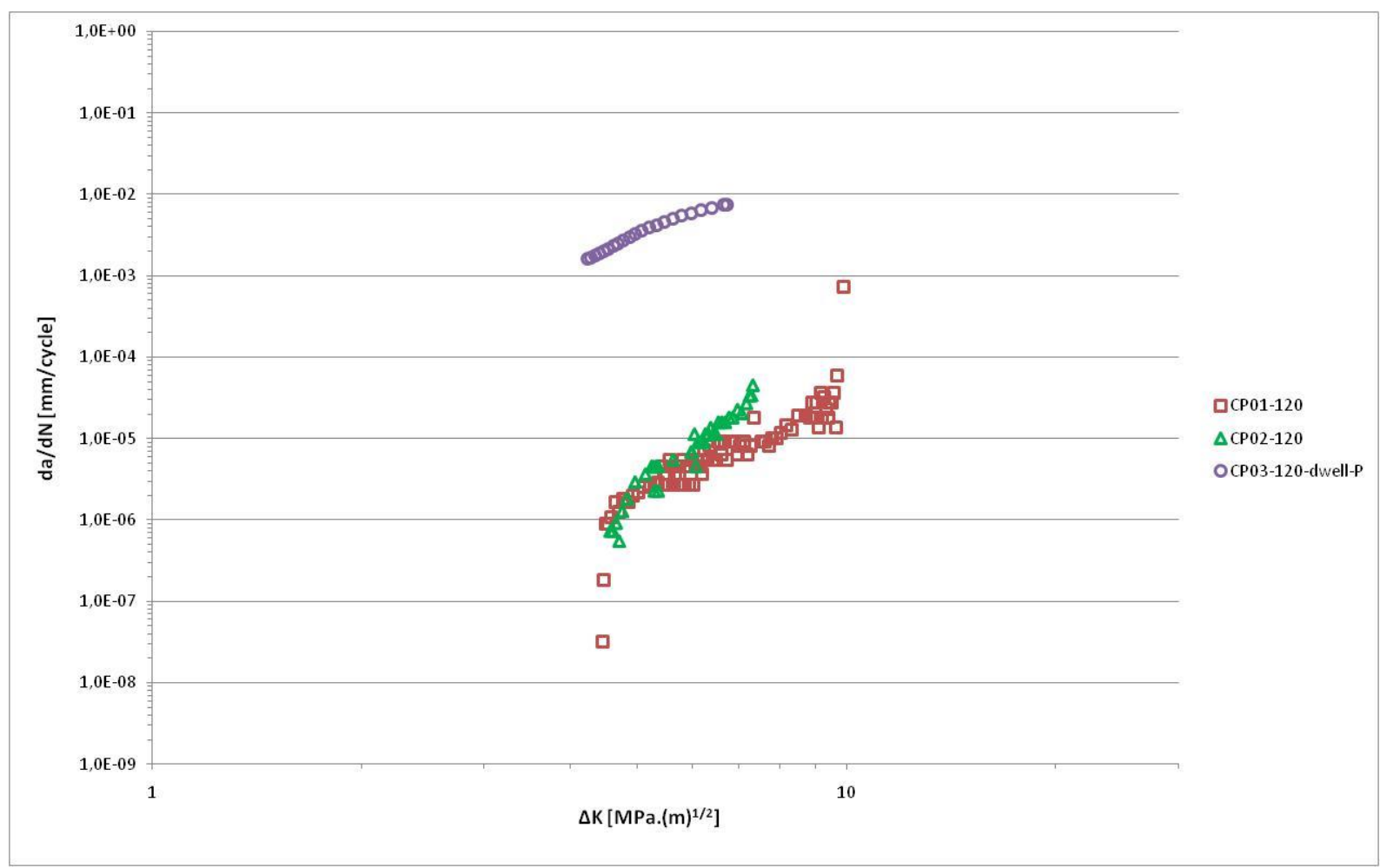

Figure 82: Graph of crack propagation $(\mathbf{d a} / \mathbf{d N})$ as a function of stress intensity factor variation $(\Delta \mathbf{K})$ at $120^{\circ} \mathrm{C}$ and under sinusoidal and trapezoidal wave loadings.

Test results of fatigue crack propagation at $200^{\circ} \mathrm{C}$ in Figure 83 show a slight decrease in stress intensity as compared to tests performed at $75^{\circ} \mathrm{C}$ and $120^{\circ} \mathrm{C}$. However, crack propagation rates remain practically constant in cyclic sinusoidal wave loading tests. There is also a small spread in results (CP04), which may be attributed to lack of homogeneity of the material in question as well as its high porosity level. These behaviors are intensified by increasing the test temperature. 


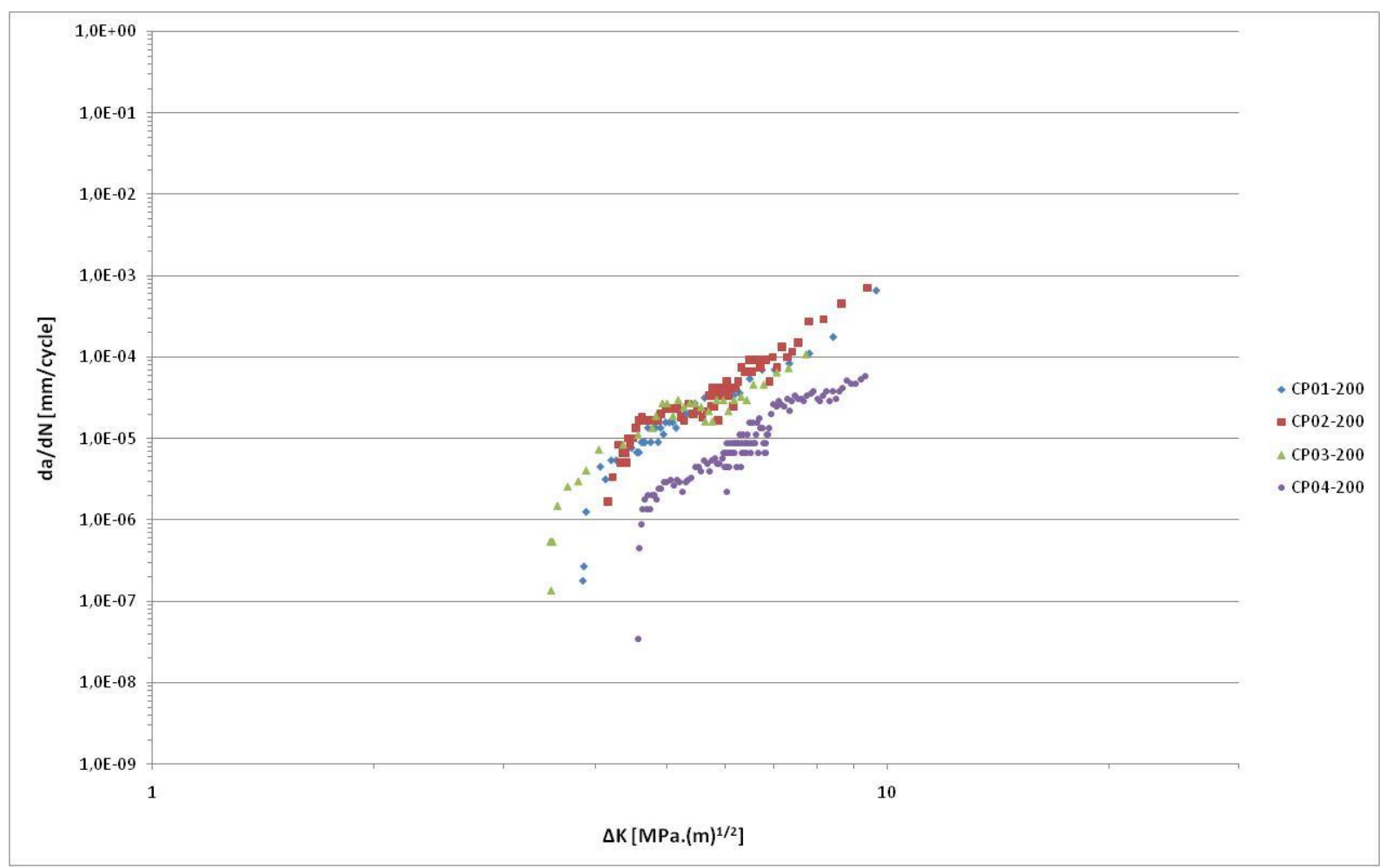

Figure 83: Graph of crack propagation $(\mathrm{da} / \mathrm{dN})$ as a function of stress intensity factor variation $(\Delta \mathrm{K})$ at $200^{\circ} \mathrm{C}$ and under sinusoidal and trapezoidal wave loadings.

Figure 84 presents the test results of fatigue crack propagation at $280^{\circ} \mathrm{C}$ under sinusoidal wave loading (CP01, CP02, and CP03) and trapezoidal wave loading in view of: (1) load control (CP04) and (2) constant deformation control (CP05). Again, it was observed that the propagation rate increased with increasing temperature. The spread of results for tests under sinusoidal wave loading (CP01, CP02 and CP03) increased slightly, indicating a deleterious effect of temperature, which increases further the influence of lack of homogeneity and high porosity of the material under study on its mechanical properties.

When the loading mode was changed to trapezoidal under load control (CP04), the crack propagation rate increased significantly as compared to the same kind of loading conducted at $120^{\circ} \mathrm{C}$. As compared to tests conducted under sinusoidal wave loading at the same temperature $\left(280^{\circ} \mathrm{C}\right)$, trapezoidal wave loading was more detrimental, indicating an increase in crack growth of the order of 100 (one hundred) times. This increase was identical to that observed at $120^{\circ} \mathrm{C}$ for trapezoidal wave loading in relation to sinusoidal wave loading. 


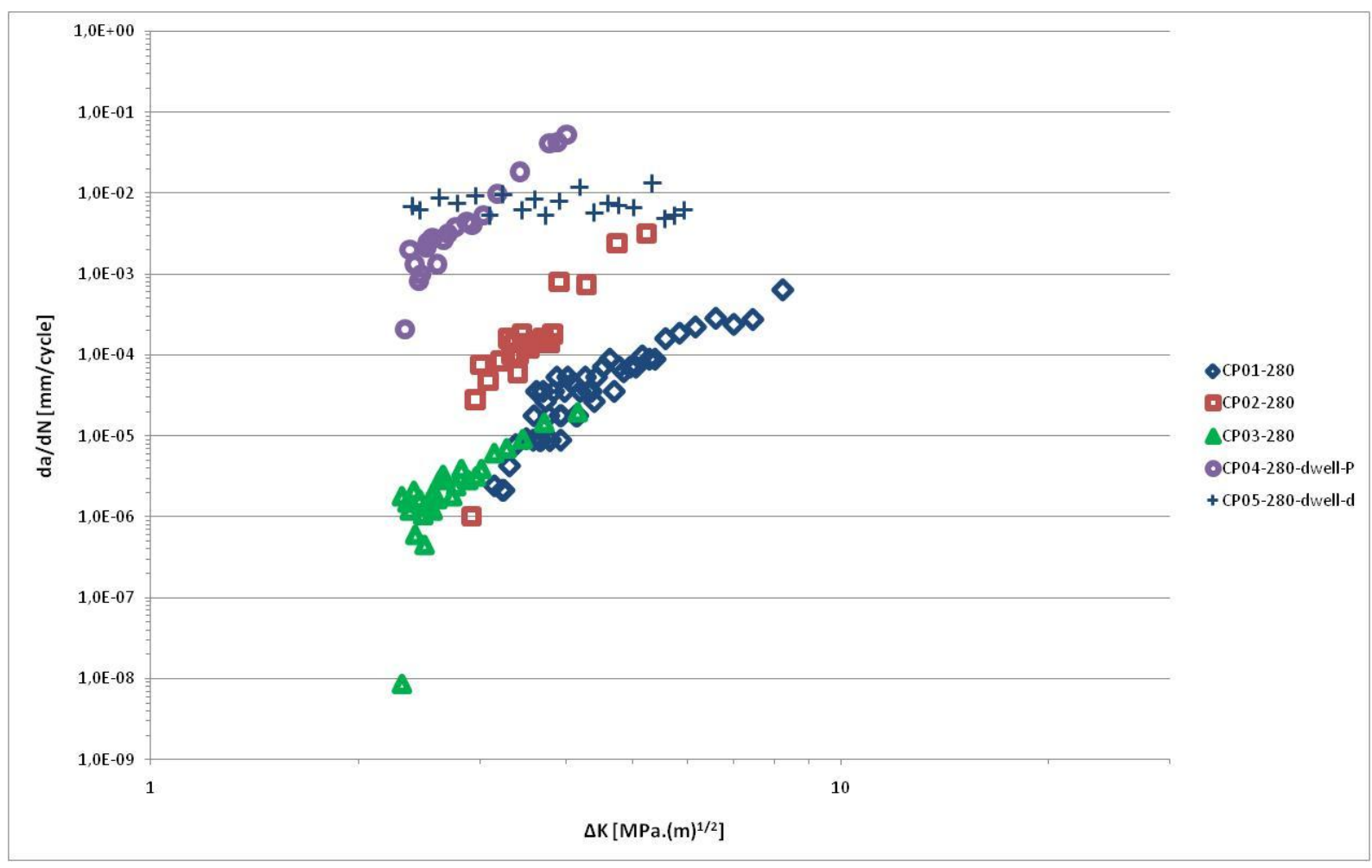

Figure 84: Graph of crack propagation $(\mathrm{da} / \mathrm{dN})$ as a function of stress intensity factor variation $(\Delta \mathrm{K})$ at $280^{\circ} \mathrm{C}$ and under sinusoidal and trapezoidal wave loadings.

In the crack growth test under trapezoidal wave loading and displacement control (CP05), it may be observed that the crack growth rate remains constant, unlike the test conducted under load control. This is because during the plateau, when control is performed by piston displacement (LVDT), the material undergoes relaxation, i.e., a decrease in applied load and, consequently, in stress intensity. This phenomenon is the opposite of creep, observed when the test is performed under load control ( $\mathrm{CP} 04)$, maintained constant during the plateau, where there is specimen elongation. Figure 85 illustrates these two phenomena. During a cycle of a crack propagation test under trapezoidal wave loading and load control during the plateau, the material underwent slight elongation (Figure 85a), whereas under displacement control, the material experienced a release in applied load (Figure 85b), suggesting the occurrence of creep and relaxation, respectively. 


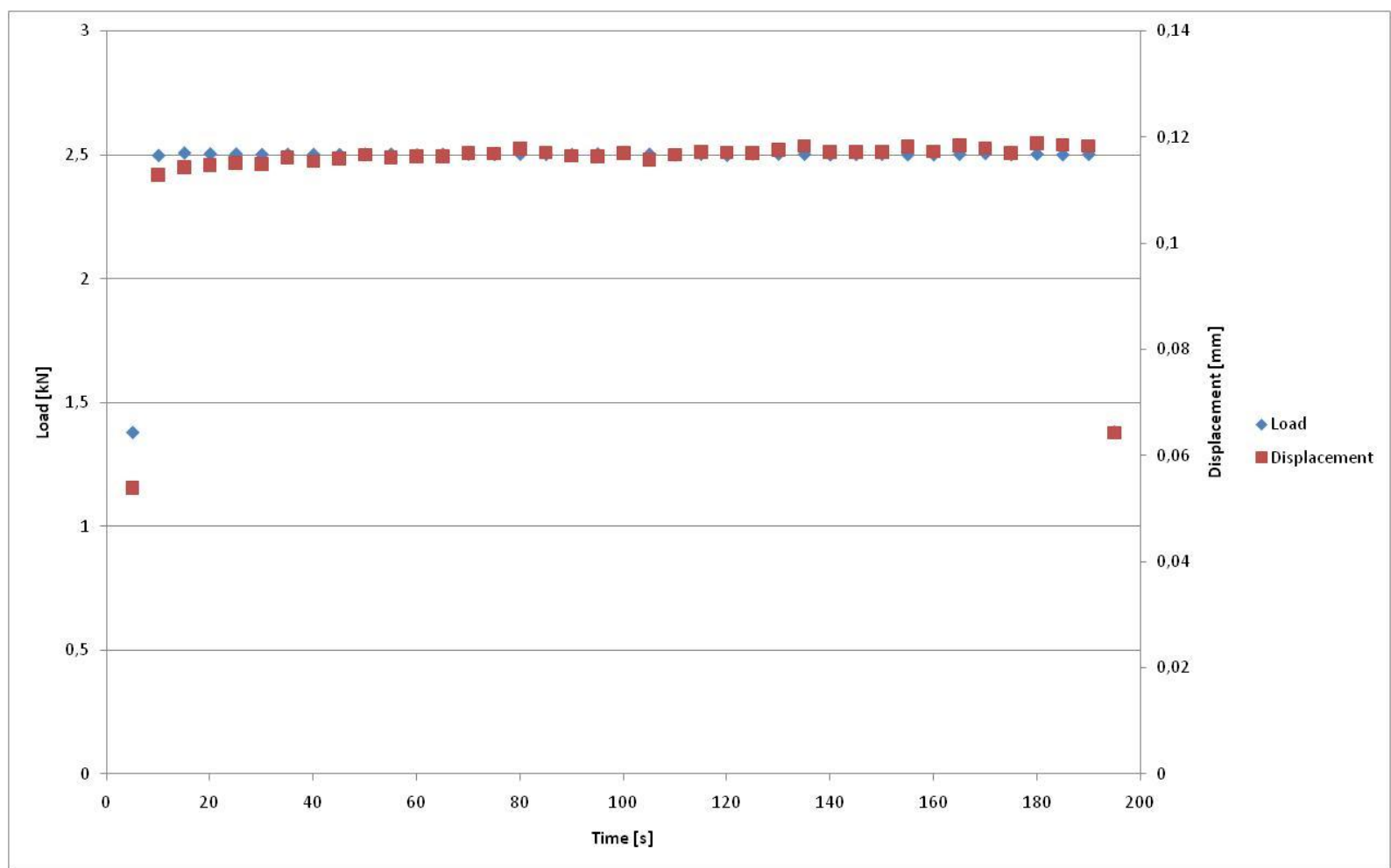

(a)

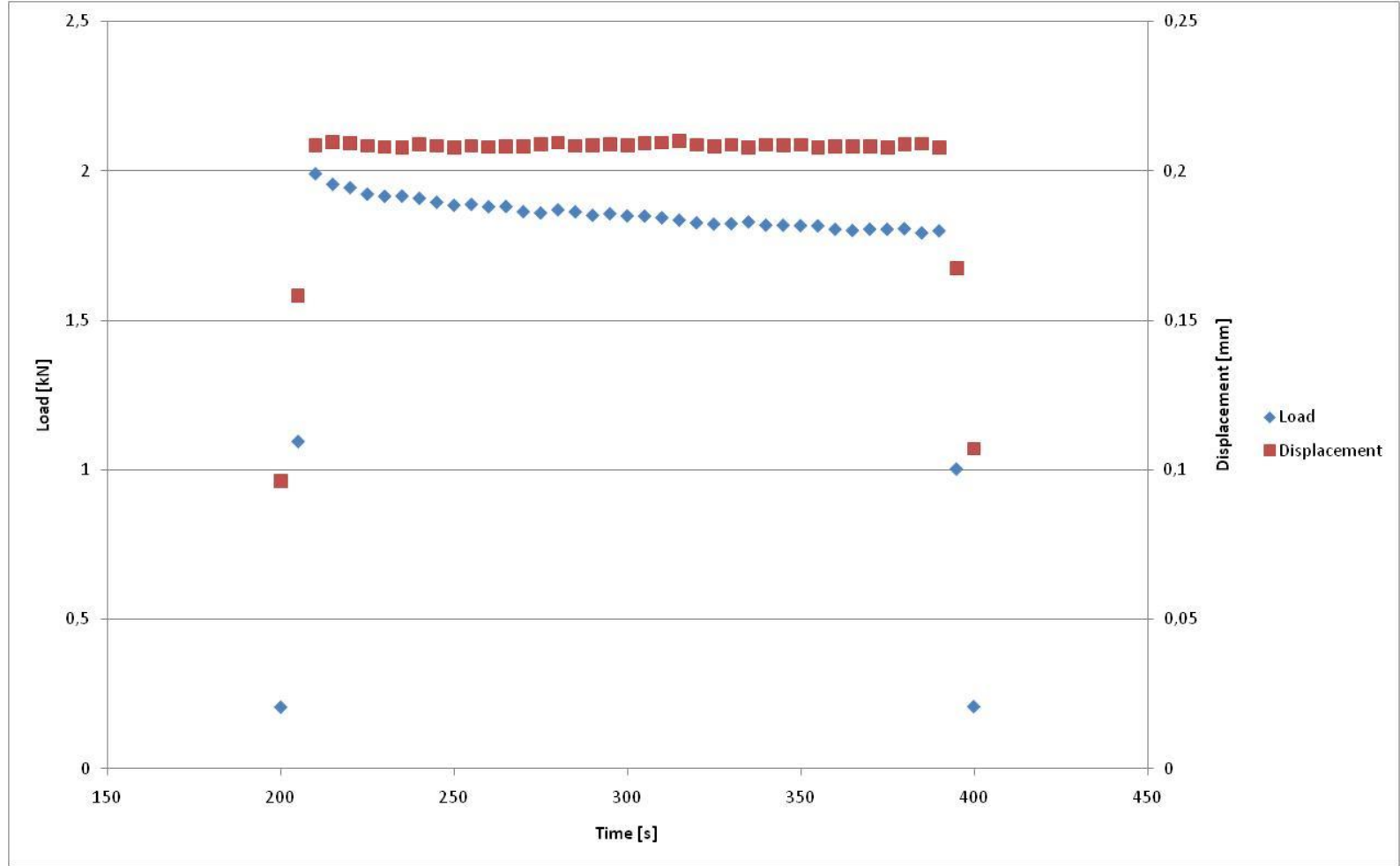

(b)

Figure 85: Comparative graph of displacement and strength in relation to time for a cycle, corresponding to crack growth tests under trapezoidal wave loading and (a) constant load and (b) constant displacement.

Figure 86 presents crack length (a) vs. number of cycles (N) graphs from fatigue crack propagation tests under trapezoidal wave loading and load control (Figure 86a) and 
displacement control (Figure 86b). It may be noticed that in load-control tests, the derivative $(\mathrm{da} / \mathrm{dN})$ is positive and increases as $\mathrm{N}$ rises, generating a linear crack growth rate, beginning at a lower limit in which crack propagation is virtually imperceptible up to a higher limit, close to specimen fracture, in which cracks propagate in an erratic fashion. On the other hand, in controlled-displacement tests, $\mathrm{da} / \mathrm{dN}$ is constant, producing constant crack growth rate throughout the test. This occurs because during long plateau periods, in which displacement is constant, applied stress undergoes relaxation.

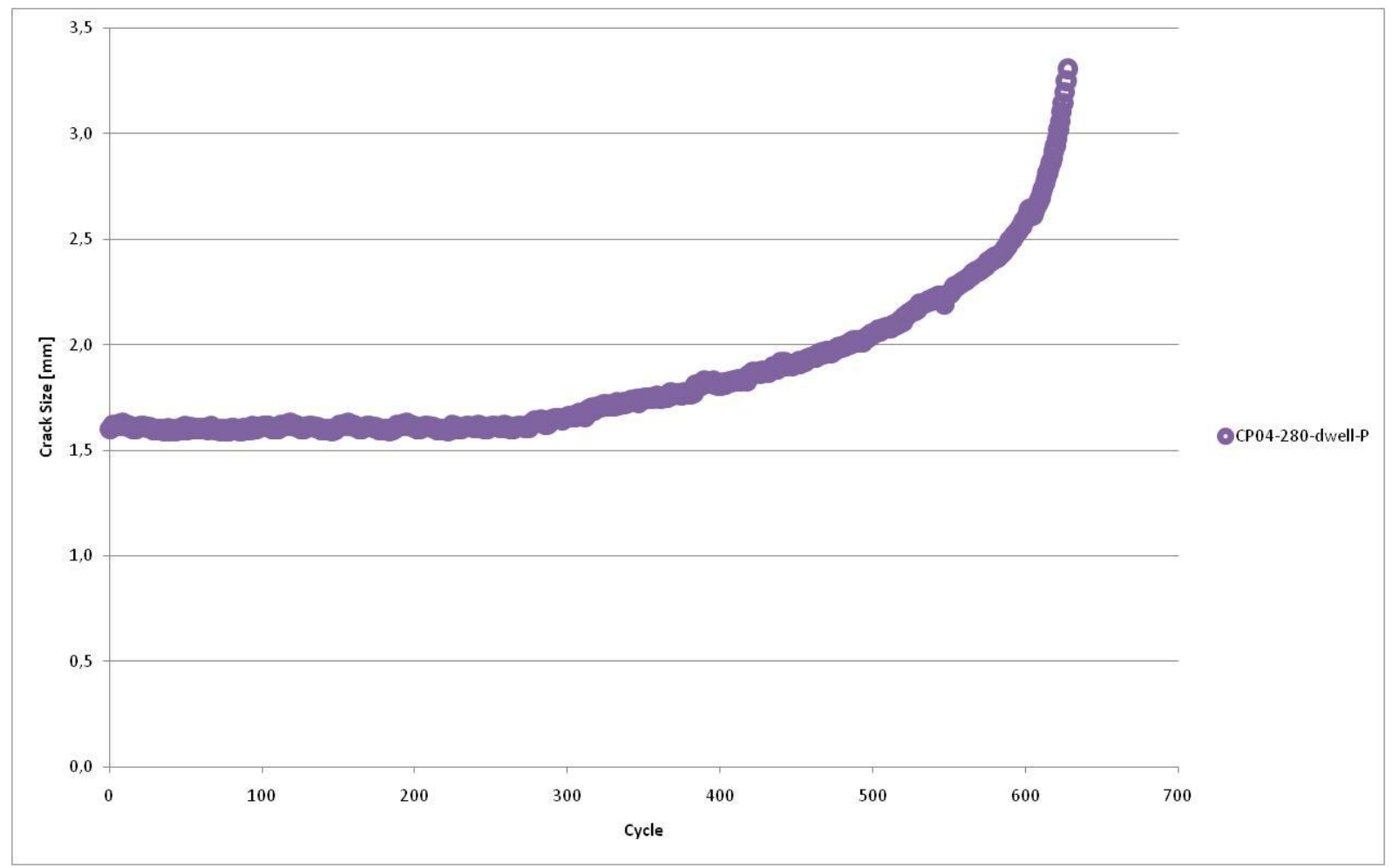

(a) 


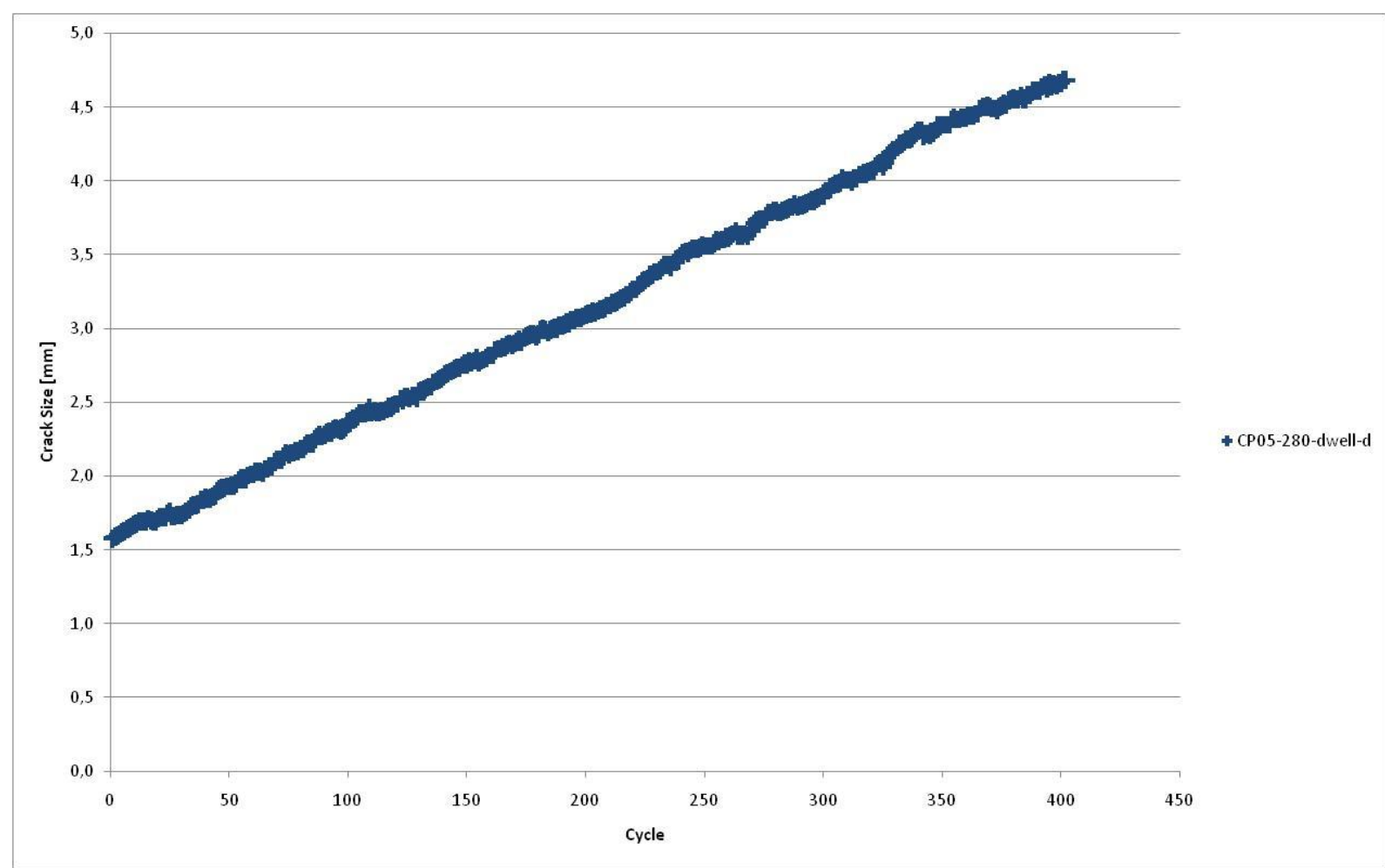

(b)

Figure 86: Graph of crack length as a function of the number of cycles for crack propagation tests under trapezoidal wave loading and (a) load control and (b) displacement control.

Figure 87 shows a comparative graph of crack propagation tests conducted at $75^{\circ} \mathrm{C}$, $120^{\circ} \mathrm{C}, 200^{\circ} \mathrm{C}$, and $280^{\circ} \mathrm{C}$ under sinusoidal wave and dwell loadings and load and displacement control. An Increase in test temperature causes mechanical strength to decrease, ordinarily observed in metallic materials and widely reported in the literature. This fact explains the decrease in the stress intensity shown in Figure 87, which is a function of applied load and, in turn, should decrease as temperature rises so as not to create a plastic zone capable of preventing cracks from growing. There may also be observed a general tendency for increased spread of results as temperature rises, which can be attributed to the influence of temperature on the material micro-structure, exacerbating its flaws, e.g., porosity and lack of homogeneity, that have an impact on its mechanical properties. In addition, as aforementioned, it is possible to note that dwell loadings are more detrimental in that they give rise to other phenomena, which interact during tests due to long plateau periods, causing crack growth rates to rise. 


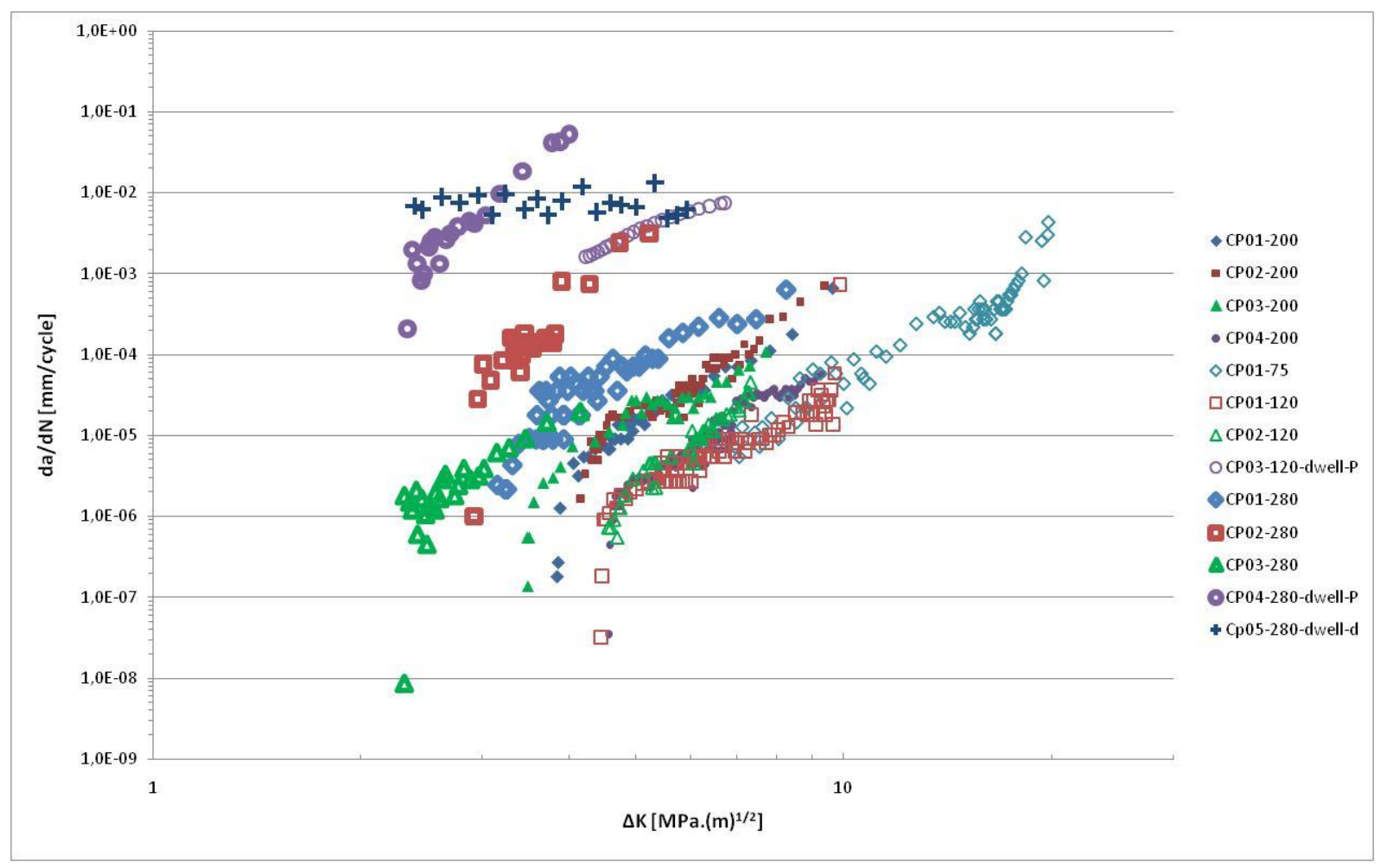

Figure 87: Comparative graph of crack propagation $(\mathrm{da} / \mathrm{dN})$ as a function of stress intensity factor variation $(\Delta \mathrm{K})$ at $75^{\circ} \mathrm{C}, 120^{\circ} \mathrm{C}, 200^{\circ} \mathrm{C}$, and $280^{\circ} \mathrm{C}$ and under sinusoidal wave and dwell loadings.

Figure 88 presents the comparative graph of displacement in relation to time for fatigue crack propagation tests at $120^{\circ} \mathrm{C}$ and $280^{\circ} \mathrm{C}$ under trapezoidal wave loading and load control. Piston displacement analysis (LVDT) of the mechanical testing machine (MTS) was conducted, but not of deformation measured by extensometer because of difficulties in coupling it to specimens. This type of analysis can be performed with no detriment to result reliability because specimen stiffness is very small as compared to that of MTS. In order to plot this graph, points corresponding to loading and unloading ramps were suppressed; only plateau points were maintained so as to render it more clear and objective. 


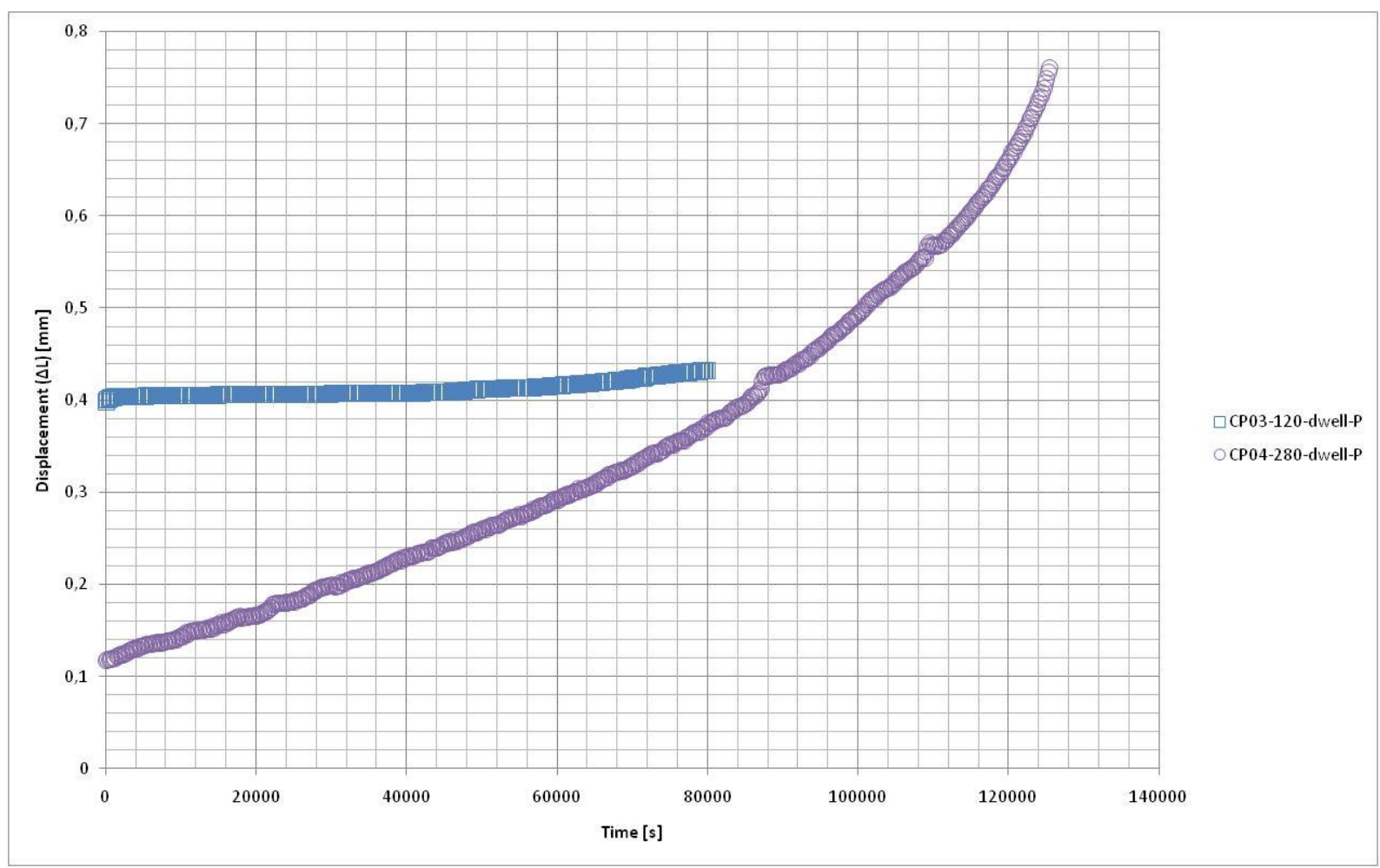

Figure 88: Displacement vs. time graph for a crack propagation test at $120^{\circ} \mathrm{C}$ under dwell loading.

Figure 88 shows the interaction of a phenomenon, distinct from mere fatigue crack propagation, at $280^{\circ} \mathrm{C}$ due to specimen elongation during the test conducted under load control, i.e., kept constant throughout the plateau. This phenomenon, known as creep, interacts during long plateau periods, causing the material to "flow". Therefore, cracks propagate due to interaction between fatigue and creep, i.e., not only due to fatigue as in the case of sinusoidal wave loading. Creep may influence both crack initiation and growth because creep damage is manifested by cavity nucleation in grain boundaries due to coalescing voids, stacking dislocations, and sliding grain boundaries. The same can happen to second-phase particles. Subsequent growth of these cavities is conducive to grain-boundary cracking and inter-granular fracture. Under static loading, failure is established by catastrophic fracture when cavities coalesce. On the other hand, at $120^{\circ} \mathrm{C}$, it is not possible to draw any conclusions about this type of interaction, visible at $280^{\circ} \mathrm{C}$, because the small magnitude of displacement can be attributed to compliance, i.e., to the opening of the crack "mouth" as it propagates through the material.

The fracture surface analyses were performed using a SEM microscope. Unfortunately, this equipment does not allow observation of details of plastic deformation micromechanisms that may took place at high temperature. Therefore, it was used a deformation map generally obtained for $\mathrm{Al}$ and presented in Roesler et al (2007) to guide the 
discussion. From the Figures presented here it was not possible to clearly define the acting micromechanisms, however significant changing may be observed when the tests were conducted under strain or load control and changing in temperature.

Figure 91(a) and (b) present the fracture surface analysis from a specimen submitted to fatigue at $120^{\circ} \mathrm{C}$ and dwell cycles in load control. From Figure 91 (a) it is seen the notch, the regions of stable (fatigue precrack and fatigue at high temperature) and final fracture after soaking in liquid nitrogen and submitted to static loading. In the region of stable crack growth, it was observed that the micromechanism was by fatigue mainly, with formation of striations. Besides, it was also observed cast defects on the crack growth path, which may acts as internal crack nucleation sites (very rough surface). Even considering the large dwell time, there was not clear signal of crack propagation by creep due to the testing temperature being only $120^{\circ} \mathrm{C}$. Analogously to the deformation at low temperature, creep deformation can also occur by dislocation movement in metal. But there is one crucial difference: if an edge dislocation encounters an obstacle, a precipitate for example, it needs a certain minimal stress to overcome the obstacle at low temperatures; if an edge dislocation encounters an obstacle at higher temperature, the dislocation can evade the obstacle by adding or emitting vacancies (Figure 89) and then the dislocation can leave its original slip plane. However, from the graphic presented in Roesler et al (Figure 90), for this temperature two micromechanisms may cause plastic deformation, one time independent called dislocation glide and other by creep denominated of dislocation creep. At low external stresses and at low temperature, the material deforms elastically. At higher temperatures, diffusion creep starts, being stronger at small stresses then dislocation creep because of its lower creep exponent. Because of the lower activation energy for grain boundary diffusion, this mechanism is more important than bulk diffusion at low temperature. Since the creep exponent is the same in both cases, the tow regions are separated by a vertical line. The final fracture performed under static load and after soaking in liquid nitrogen, shows a mixture of ductile and brittle fracture (Figure 91c). Figure 91(c) and (d) shows SEM analysis from a specimen tested by fatigue at $120^{\circ} \mathrm{C}$, sinusoidal wave shape in load control $(10 \mathrm{~Hz}$ frequency). In the region of stable crack growth, it was observed formation of striations indicated by dashed arrows. Also observed the difference between the fracture surfaces of dell and sinusoidal wave shape. 


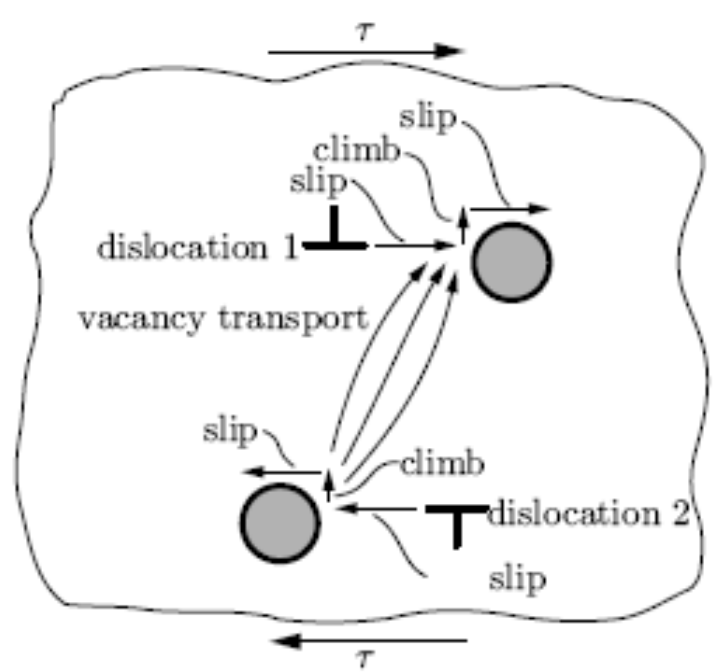

Figure 89: Shows the example of tow edge dislocations pinned at two obstacles. Dislocation 1 has to absorb vacancies to climb and dislocation 2 needs to emit them. Pile-up of dislocation at obstacles and vacancy diffusion. Dislocation 1 is a vacancy sink, dislocation 2 a vacancy source. (Roesler 2007)

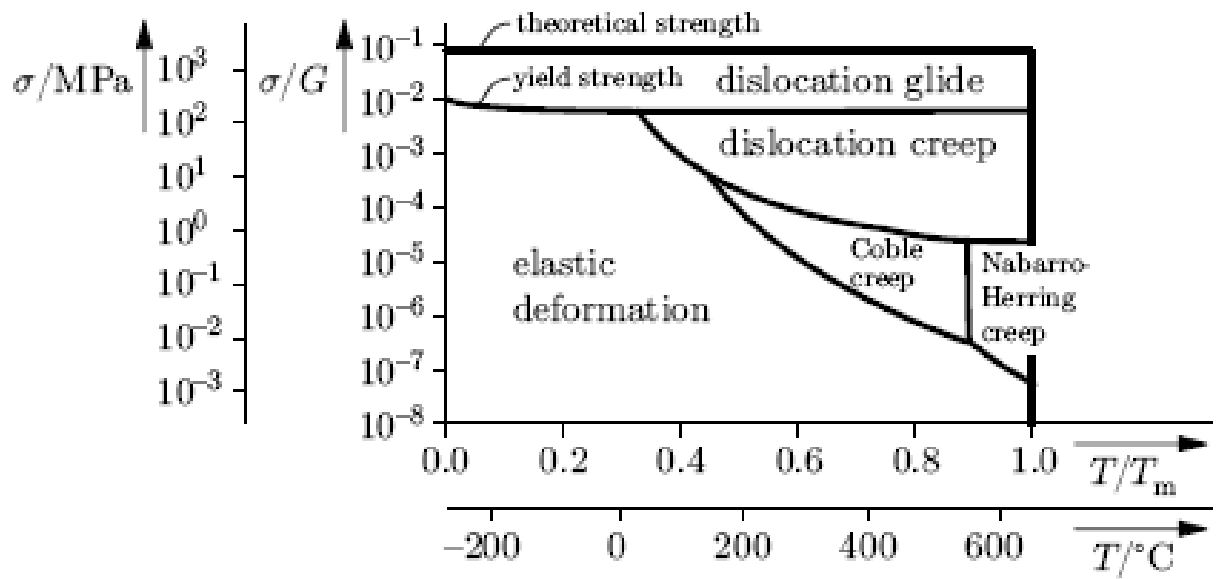

Figure 90: Deformation mechanism maps. The grain size is $32 \mu \mathrm{m}$ in both cases. (Roesler 2007) 


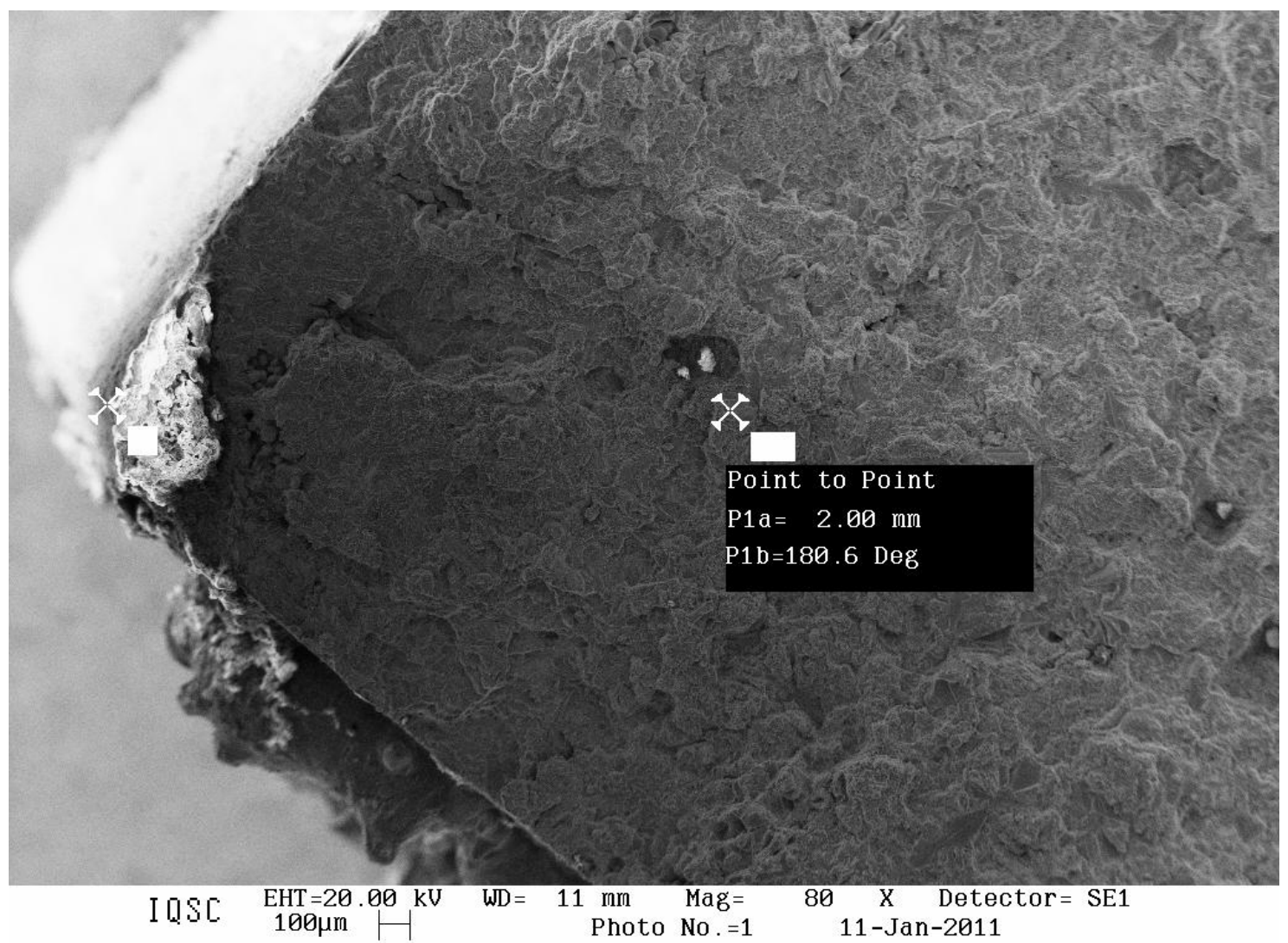

(a)

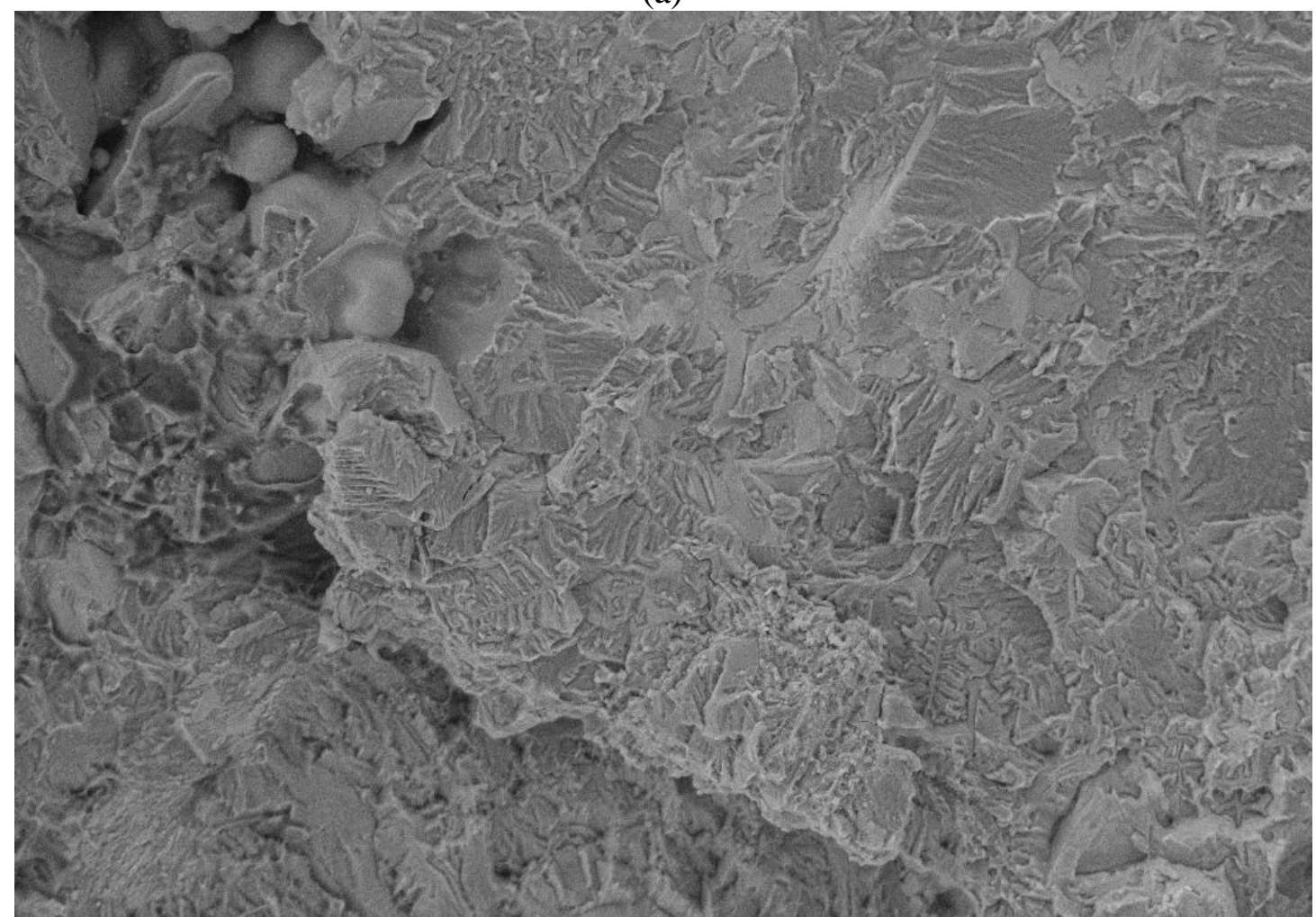

I DSC EHT $=20.00 \mathrm{kV}$ WD $=11 \mathrm{~mm} \quad \mathrm{Mag}=\quad 500 \quad \mathrm{X}$ Dete

(b) 


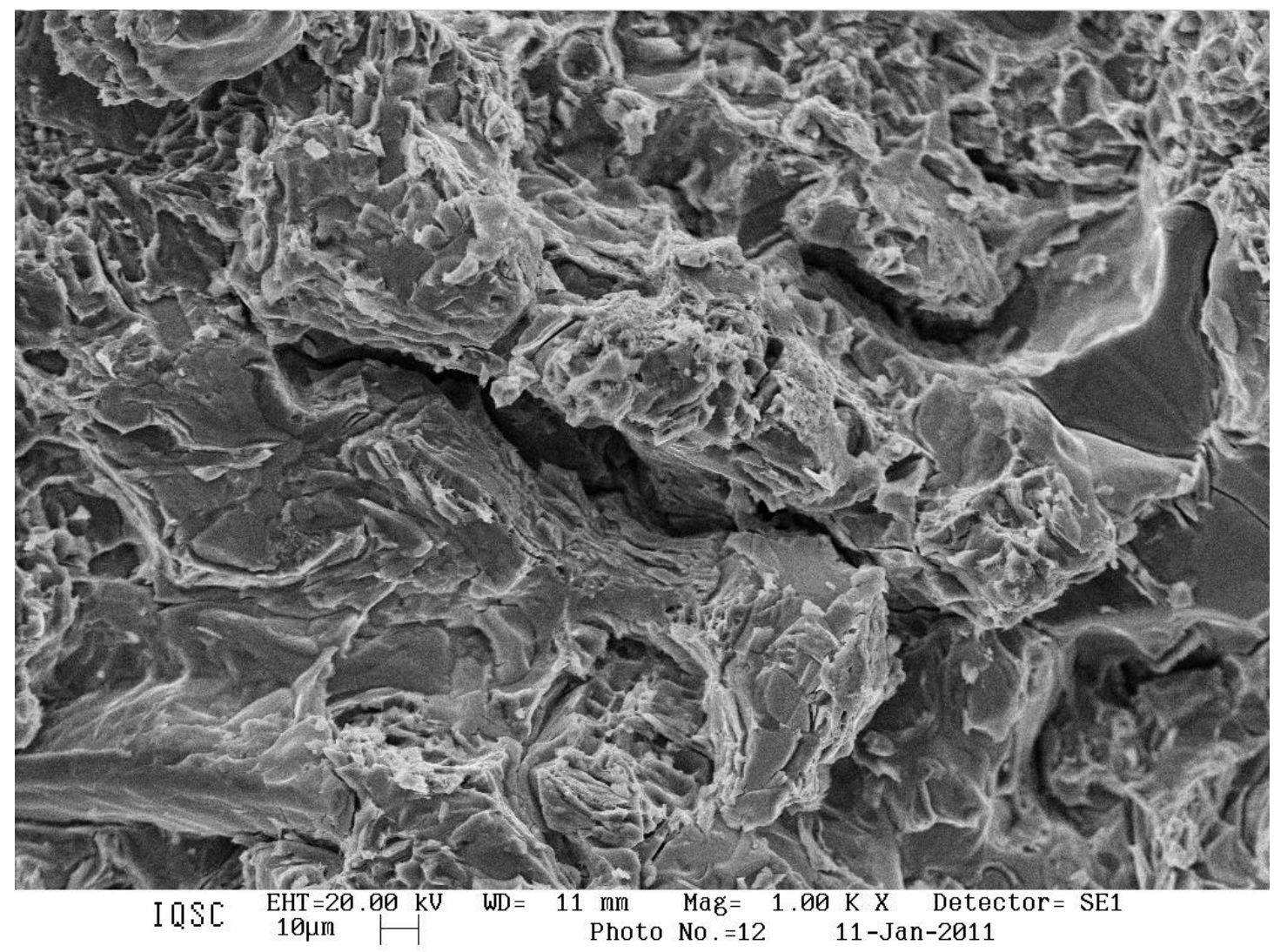

(c)

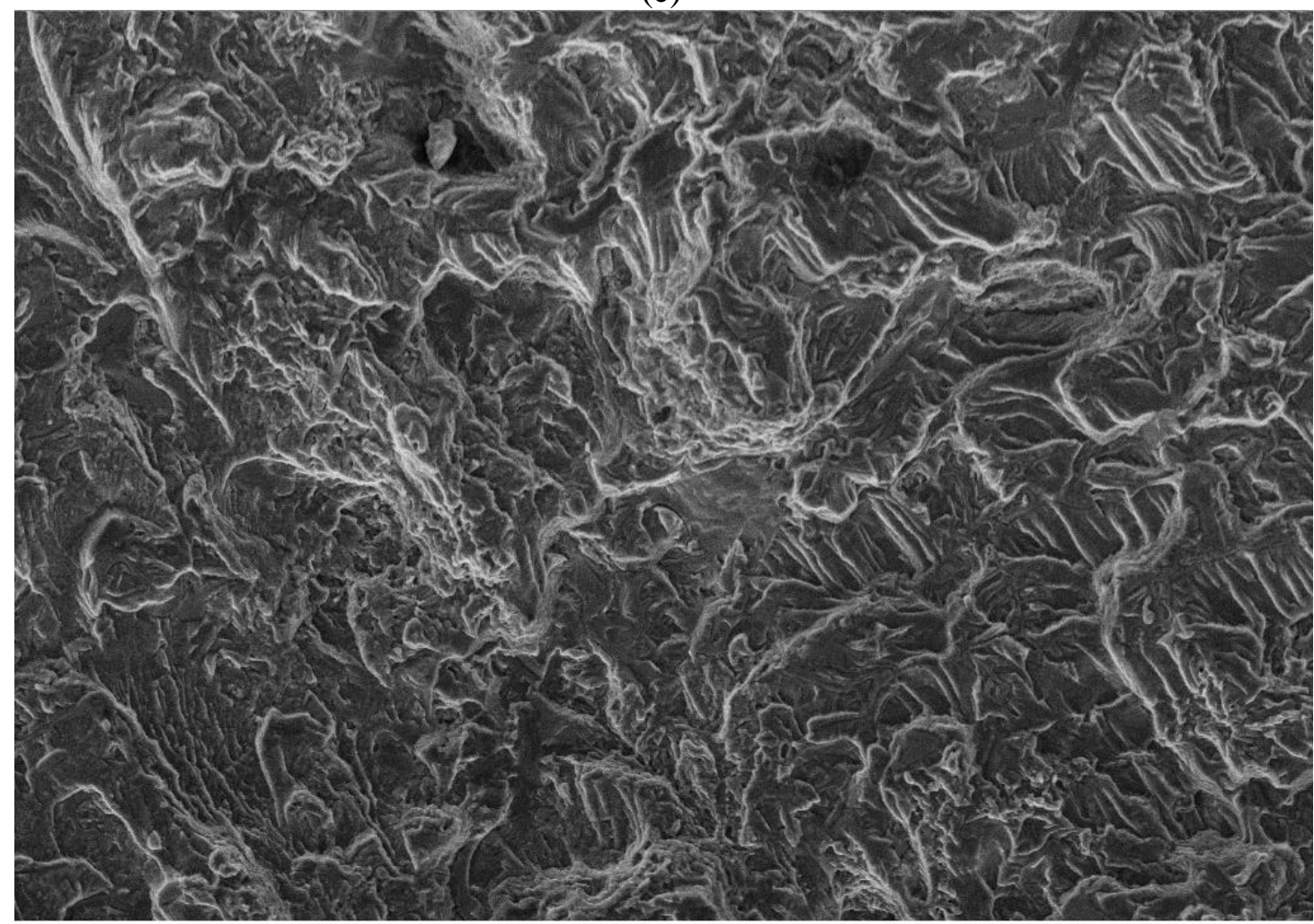

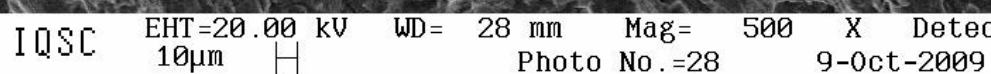

(d) 


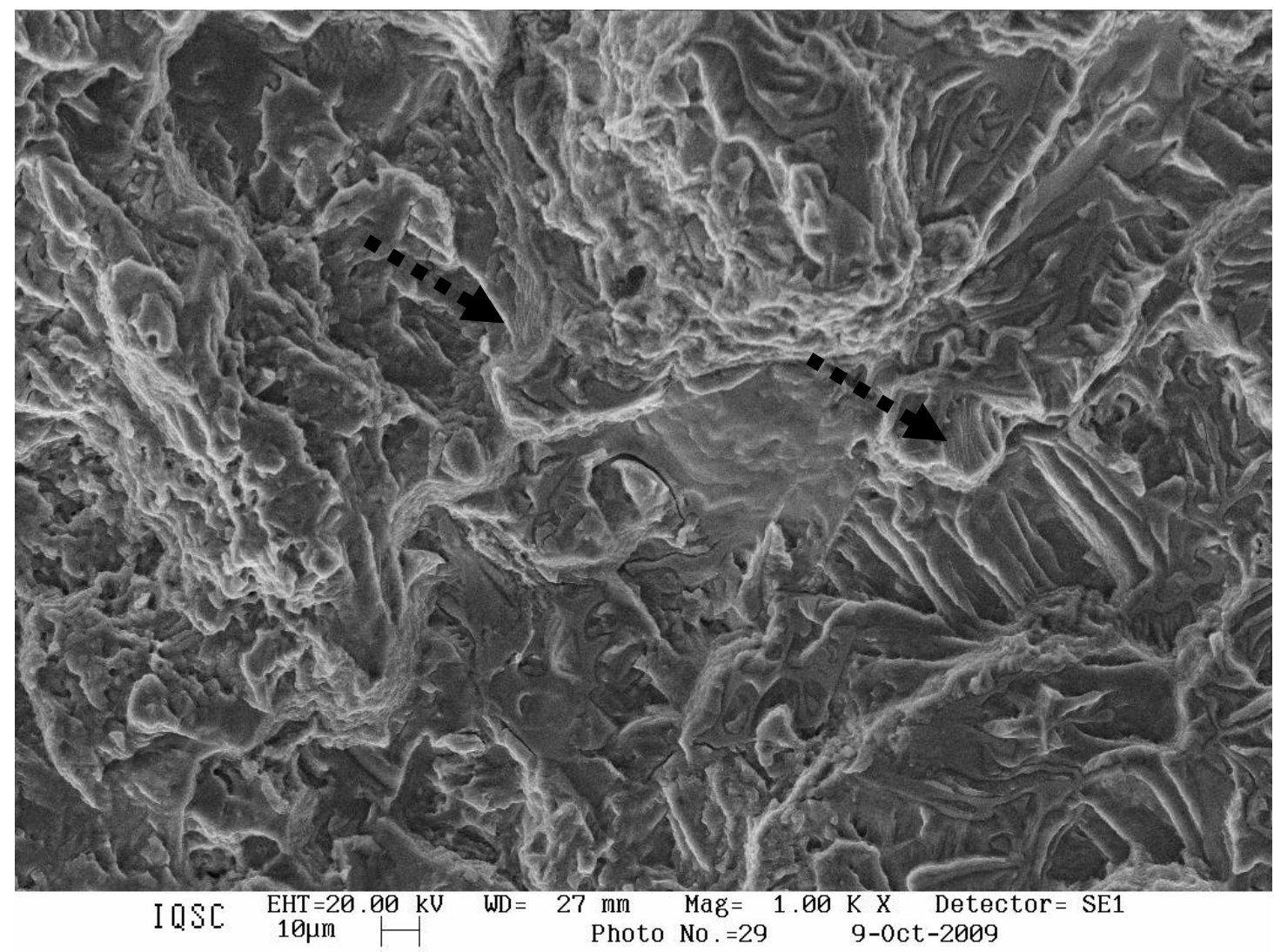

(e)

Figure 91: SEM analysis from a specimen tested by fatigue at $120^{\circ} \mathrm{C}$, dwell cycle in load control: (a) notch and stable crack growth region, (b) detail of the stable crack growth region at $120^{\circ} \mathrm{C}$ and (c) final fracture; (d) is SEM analysis from a specimen tested by fatigue at $120^{\circ} \mathrm{C}$, sinusoidal wave shape in load control $(10 \mathrm{~Hz}$ frequency) and (e) detail of (d).

Figure 92 presents the fracture surface analyses from a specimen submitted to fatigue crack growth at $200^{\circ} \mathrm{C}$ and sinusoidal wave shape $(10 \mathrm{~Hz}$ frequency). In this case, fatigue striations were easily identified on fracture surface, this may be due to the higher temperature than one and the alloy presented a higher ductility than the specimens at $120^{\circ} \mathrm{C}$ and for similar $\Delta \mathrm{K}$, the fatigue crack growth rate was higher than at $120^{\circ} \mathrm{C}$. However, the high frequency did not allow the creep micromechanism being clearly active, and crack propagation was mainly by fatigue. Again, it is possible to see many cast defects on the fracture surface. 


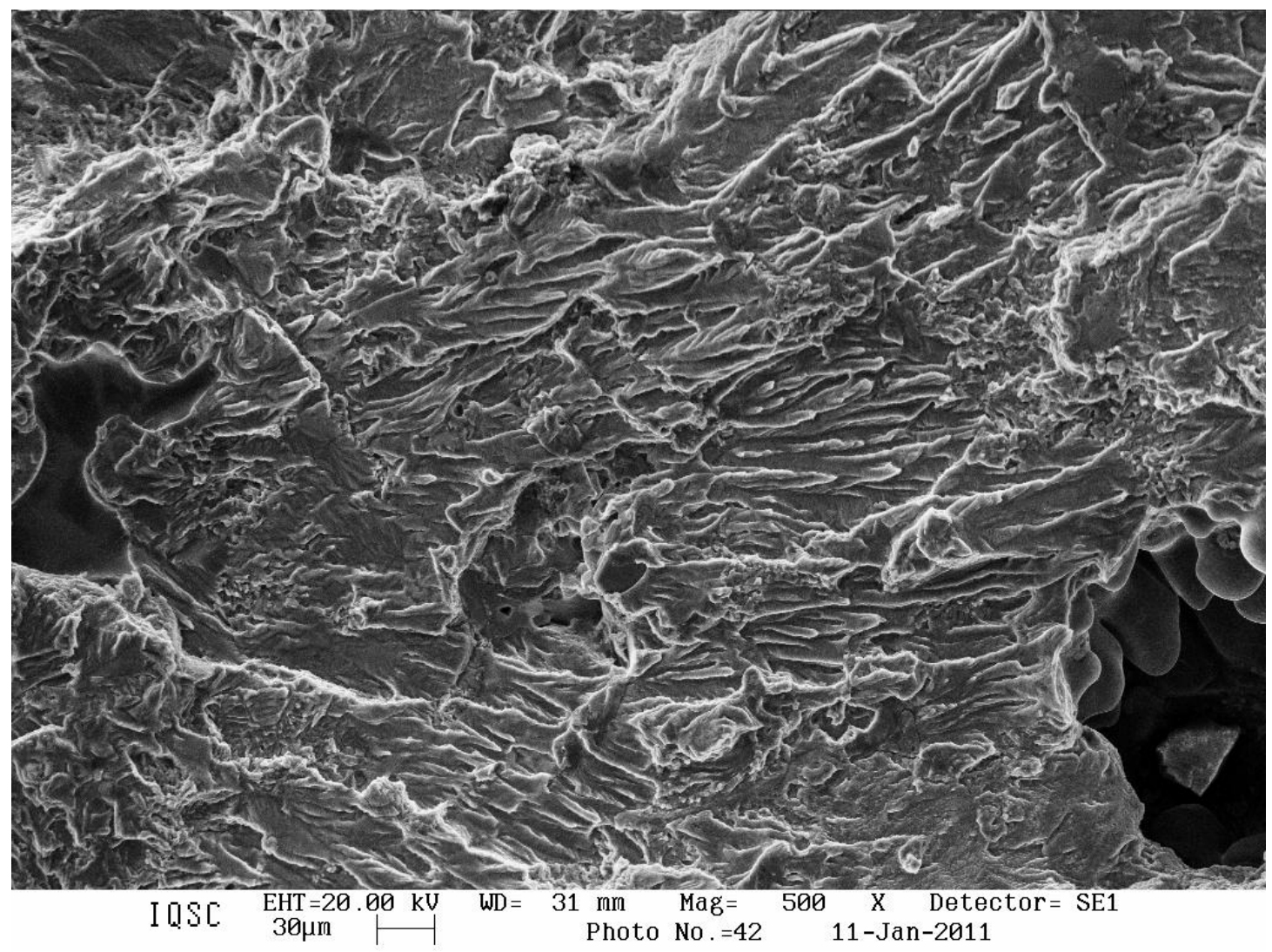

(a)

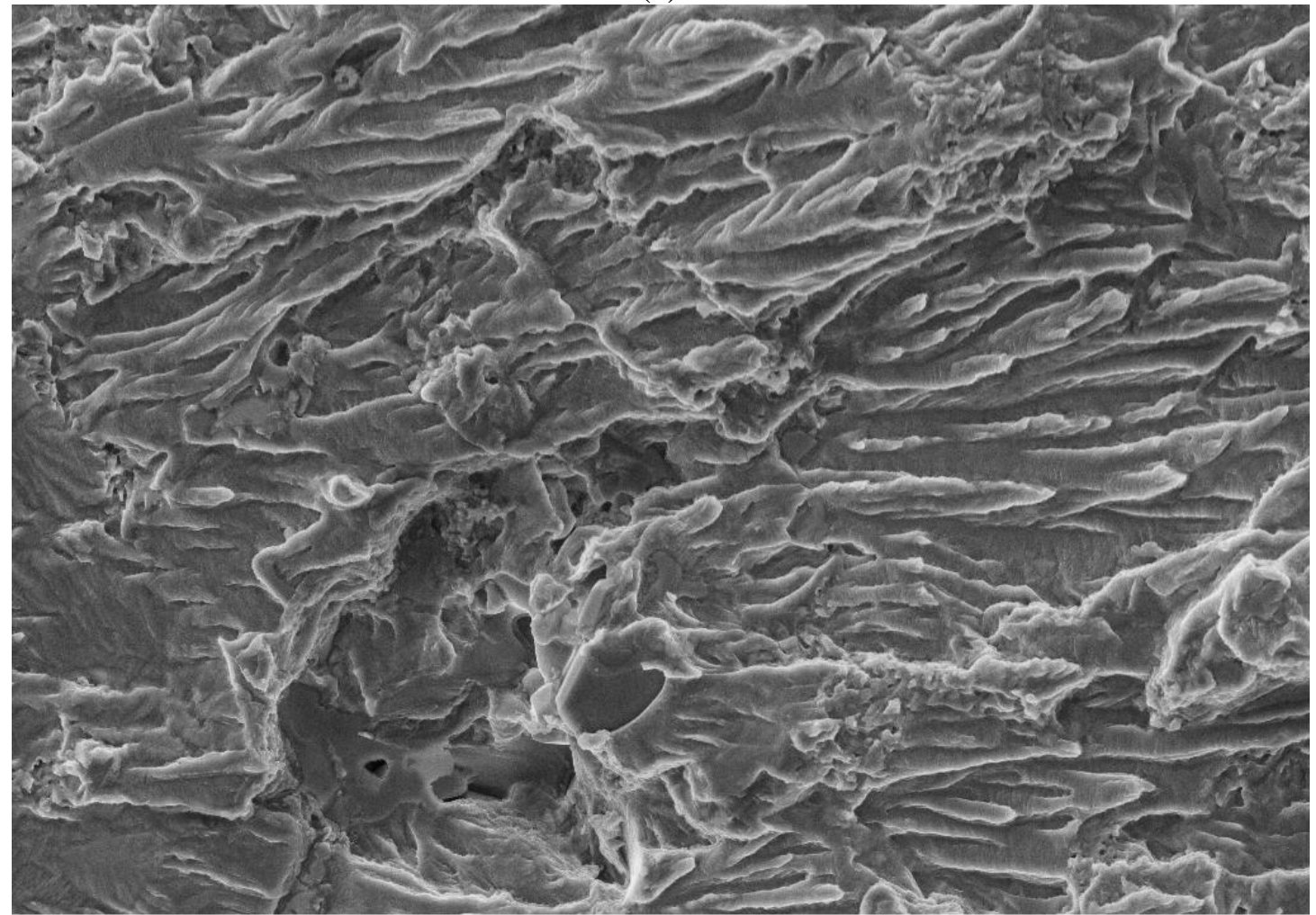

I DSC EHT $=20.00 \mathrm{kV}$
$10 \mu \mathrm{mD}=31 \mathrm{~mm} \quad \mathrm{Mag}=1.00 \mathrm{~K} \mathrm{X}$ Detec
Photo No. $=43$

(b) 


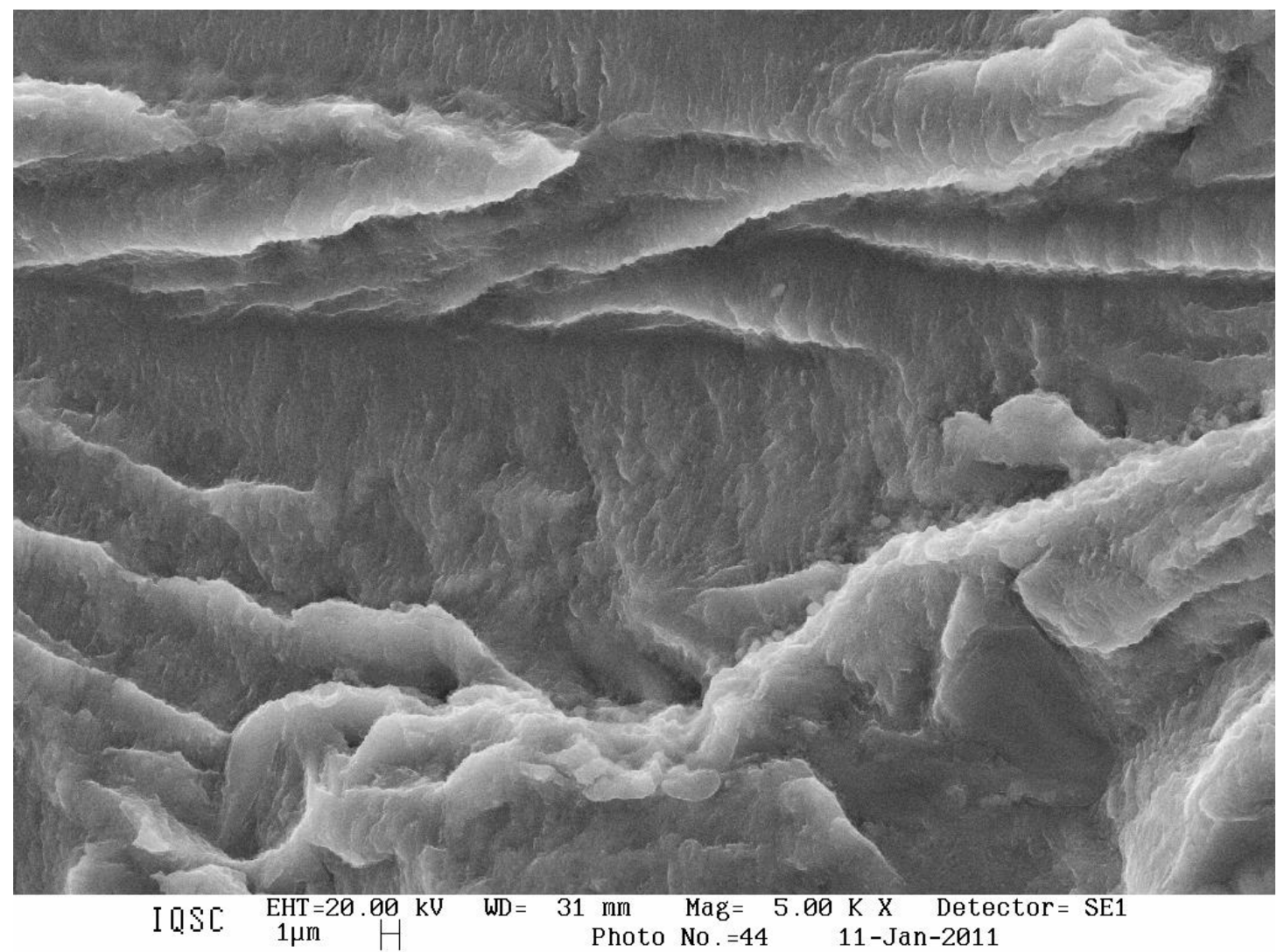

(c)

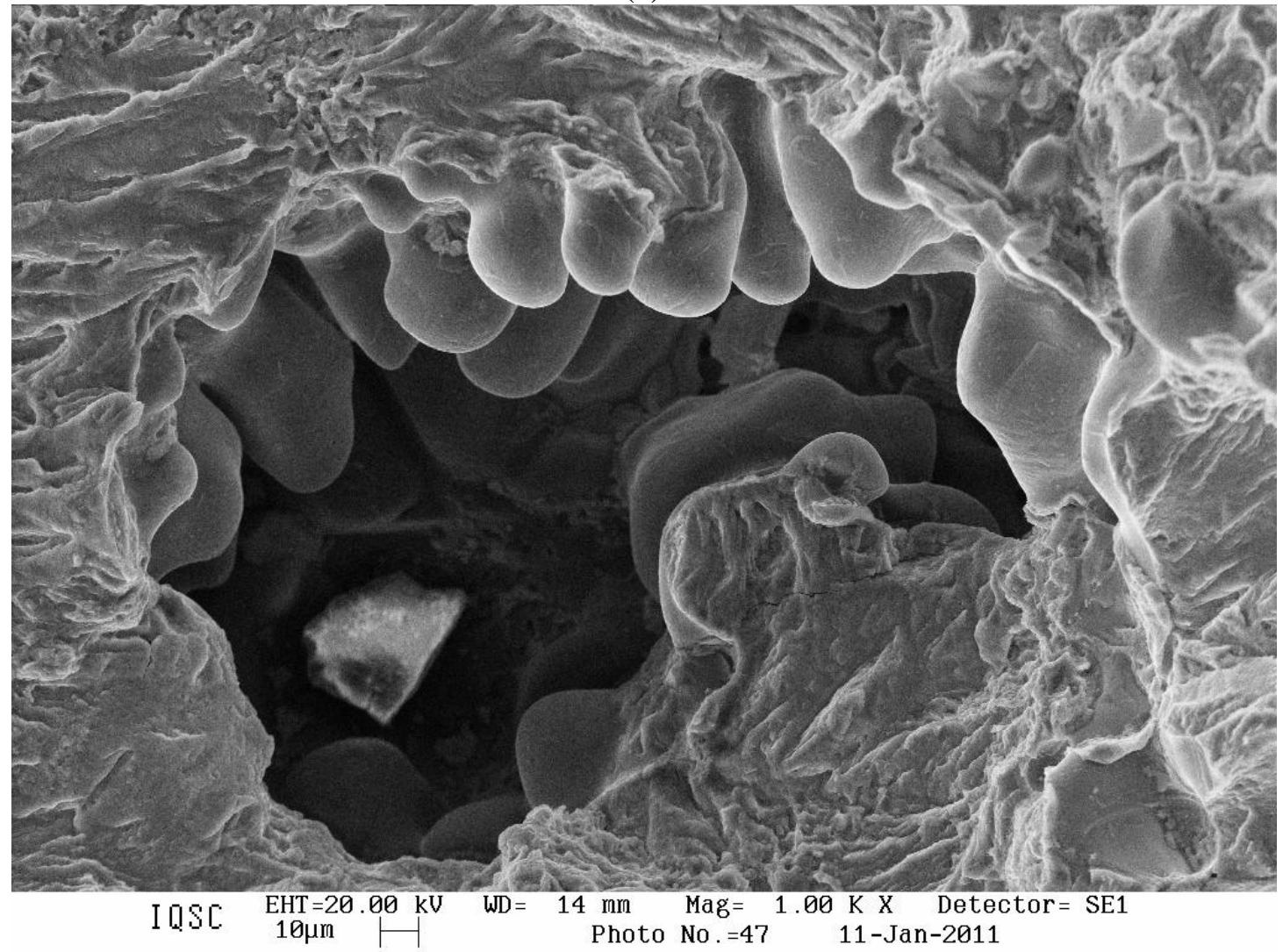

(d)

Figure 92: SEM analysis from a specimen fatigue tested at $200^{\circ} \mathrm{C}$, sinusoidal wave shape, 10 $\mathrm{Hz}$ frequency): (a) region of stable crack growth, (b) detail of (a), (c) fatigue striations and (d) cast defect on fracture surface. 
Figure 93 present the fracture surface analysis from a specimen submitted to fatigue at $280^{\circ} \mathrm{C}$ and dwell cycles in load control. From Figure 93 (a) the regions of fatigue precrack and fatigue at high temperature. As seen in Figure 93 (b), (c) and (d) crack propagation was mainly due to creep, with dimples being formed extensively at the crack front during the dwell period. The crack starts to growth at relatively low $\Delta \mathrm{K}$, therefore, in this case, diffusion creep may be acting. As the crack growth, $\Delta \mathrm{K}$ increases and the cracks accelerates very rapidly.

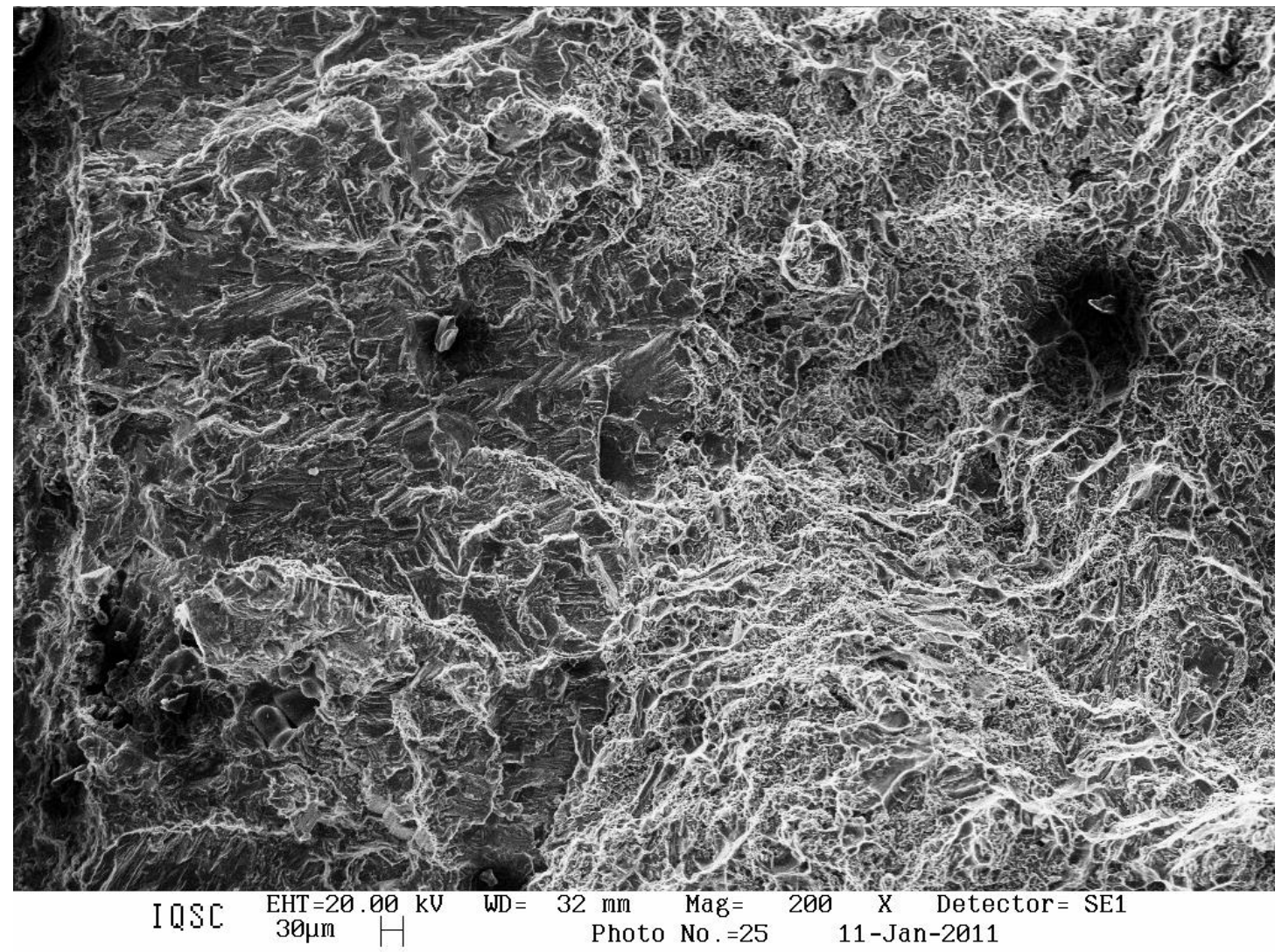

(a) 


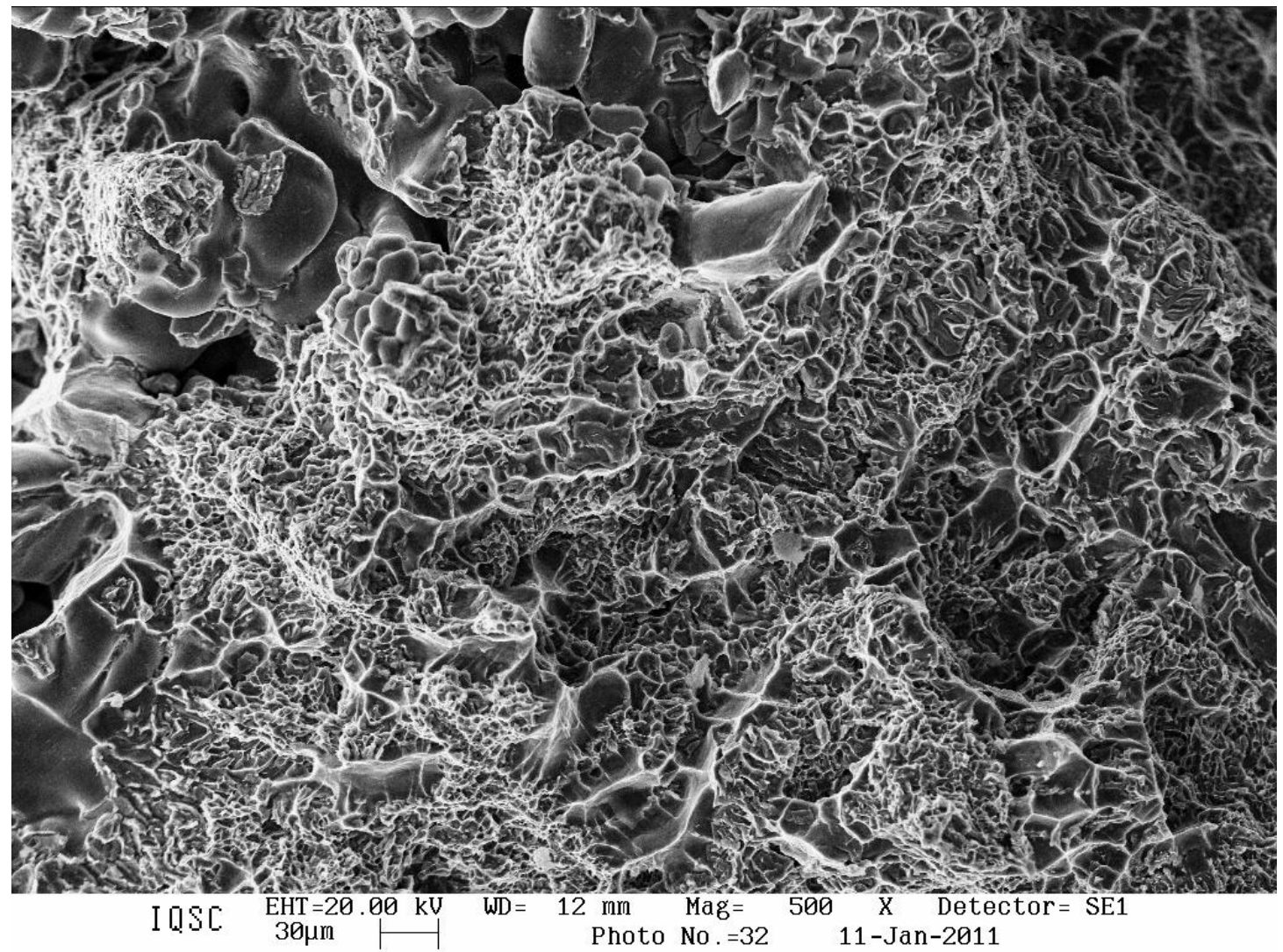

(b)

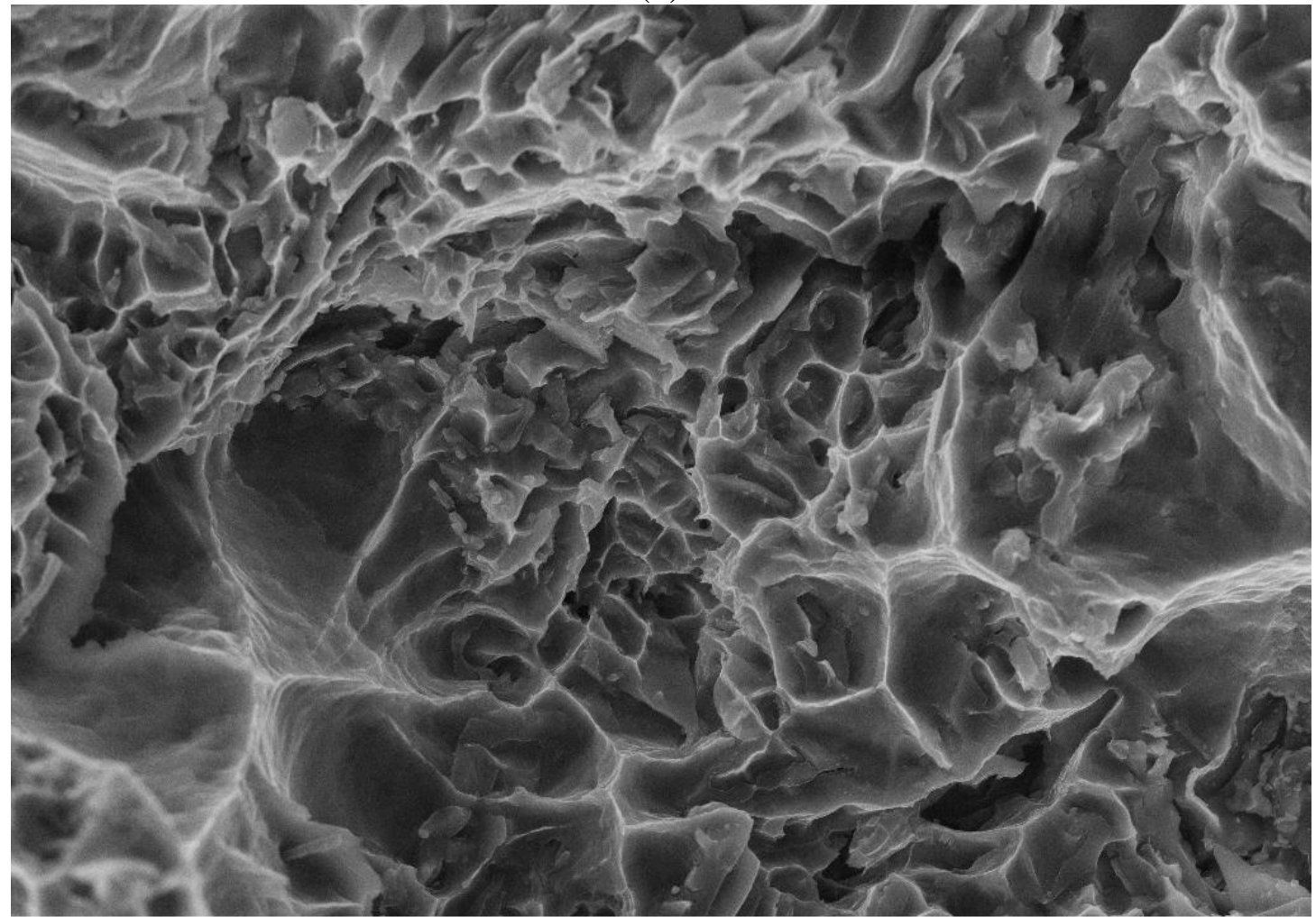

$\mathrm{I} D \mathrm{OSC}$
$2 \mu \mathrm{m}=20.00 \mathrm{mV}$

(c) 


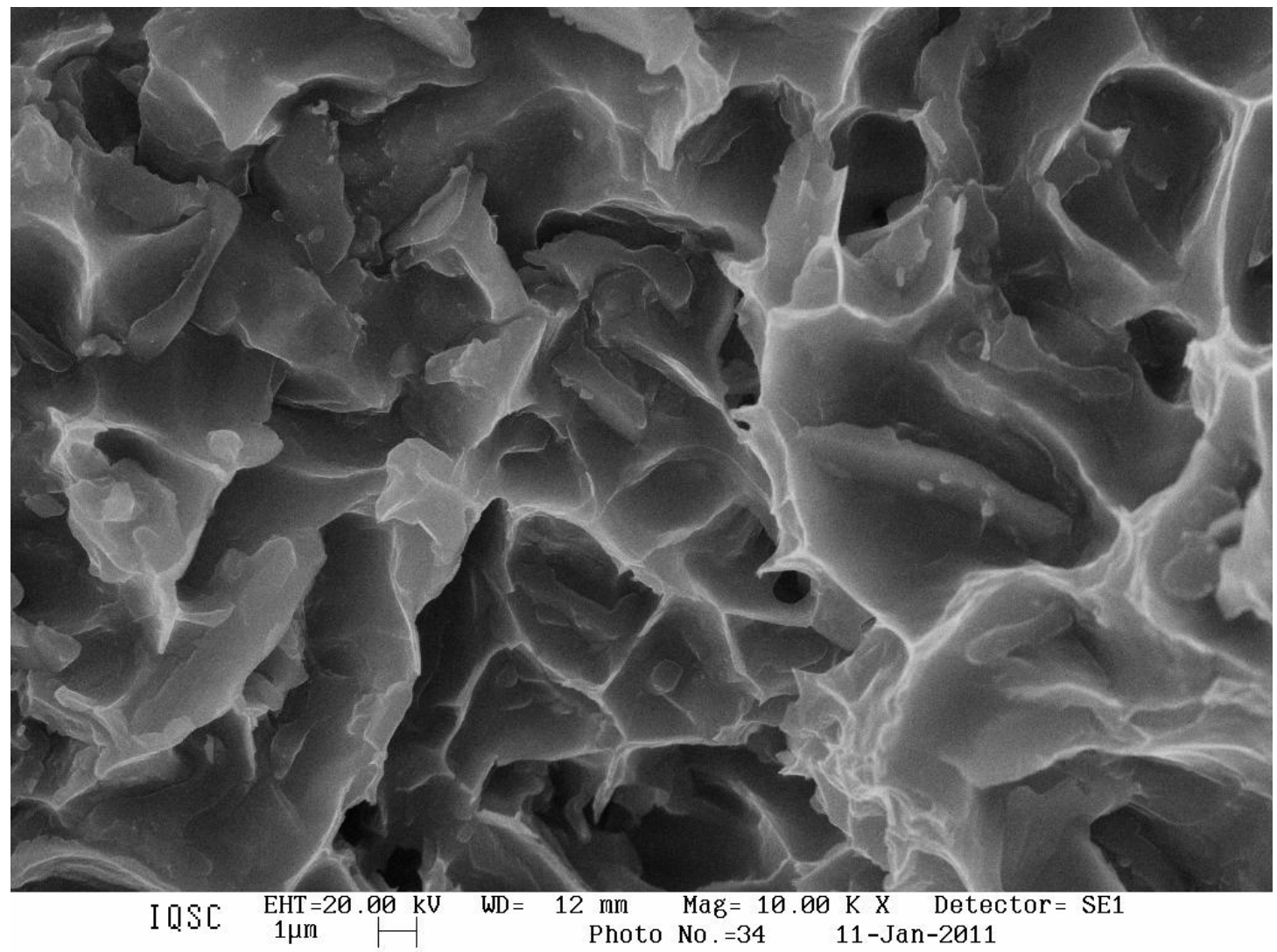

(d)

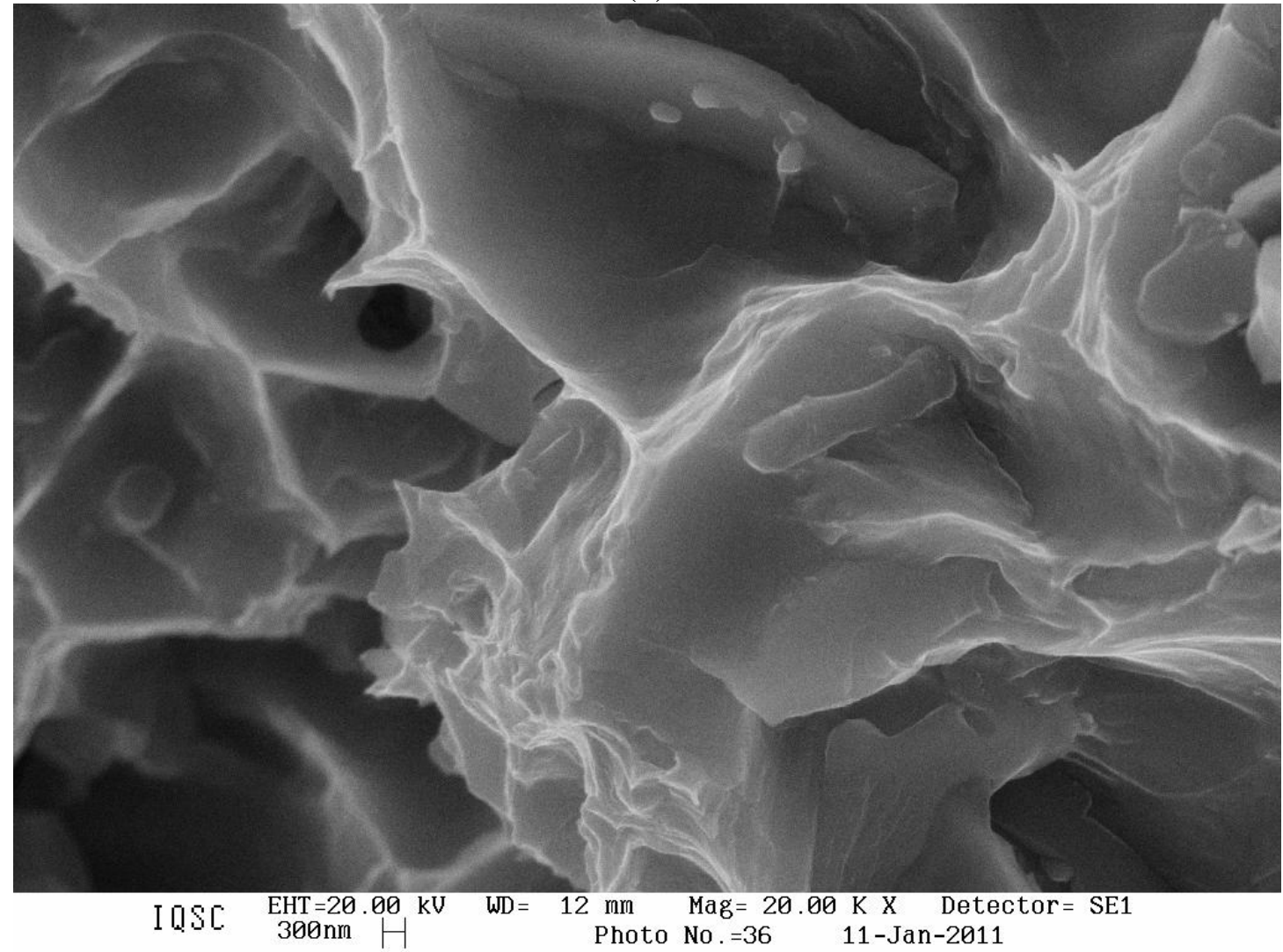

(e)

Figure 93: SEM analysis from a specimen fatigue tested at $280^{\circ} \mathrm{C}$, dwell cycle in load control: (a) notch and stable crack growth region, (b), (c), (d) and (e) detail of the stable crack growth region at $280^{\circ} \mathrm{C}$. 
The fatigue crack propagation at $280^{\circ} \mathrm{C}$ and dwell cycles in displacement control, Figure 94, presented a different fatigue behavior than that in load control. Figure 94(a) presents the regions of fatigue pre-crack and fatigue at high temperature. In this case, stress relaxation takes place at the crack front and $\Delta K$ decreases rapidly as a result, the crack propagation rate is nearly constant. The fracture surface shows a mixture of dimples and features of mechanical fatigue (similar to the features of the pre-crack region). In Figure 94 (b), (c), (d) and (e) shows amplify of the region of fatigue crack growth.

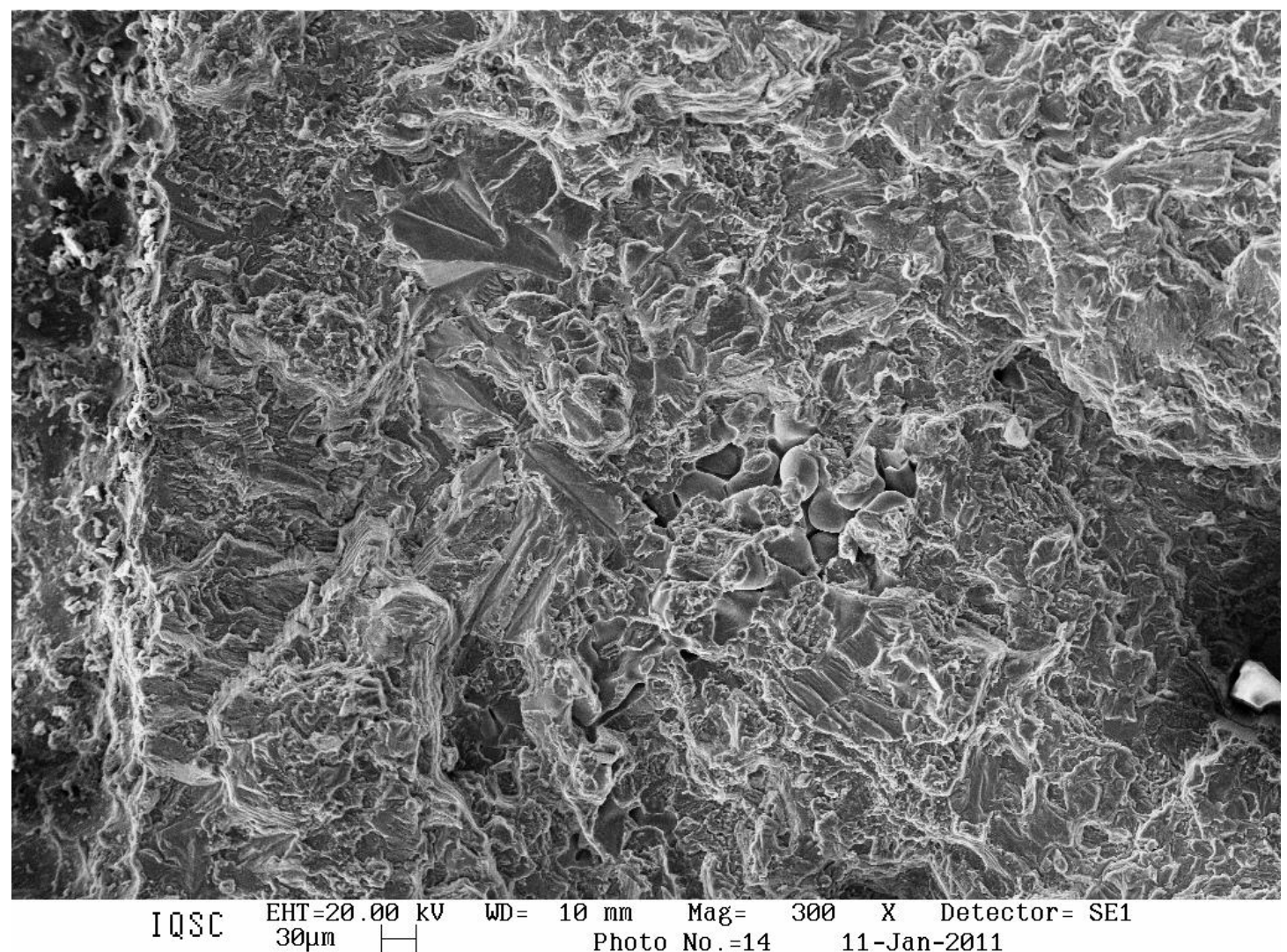

(a) 


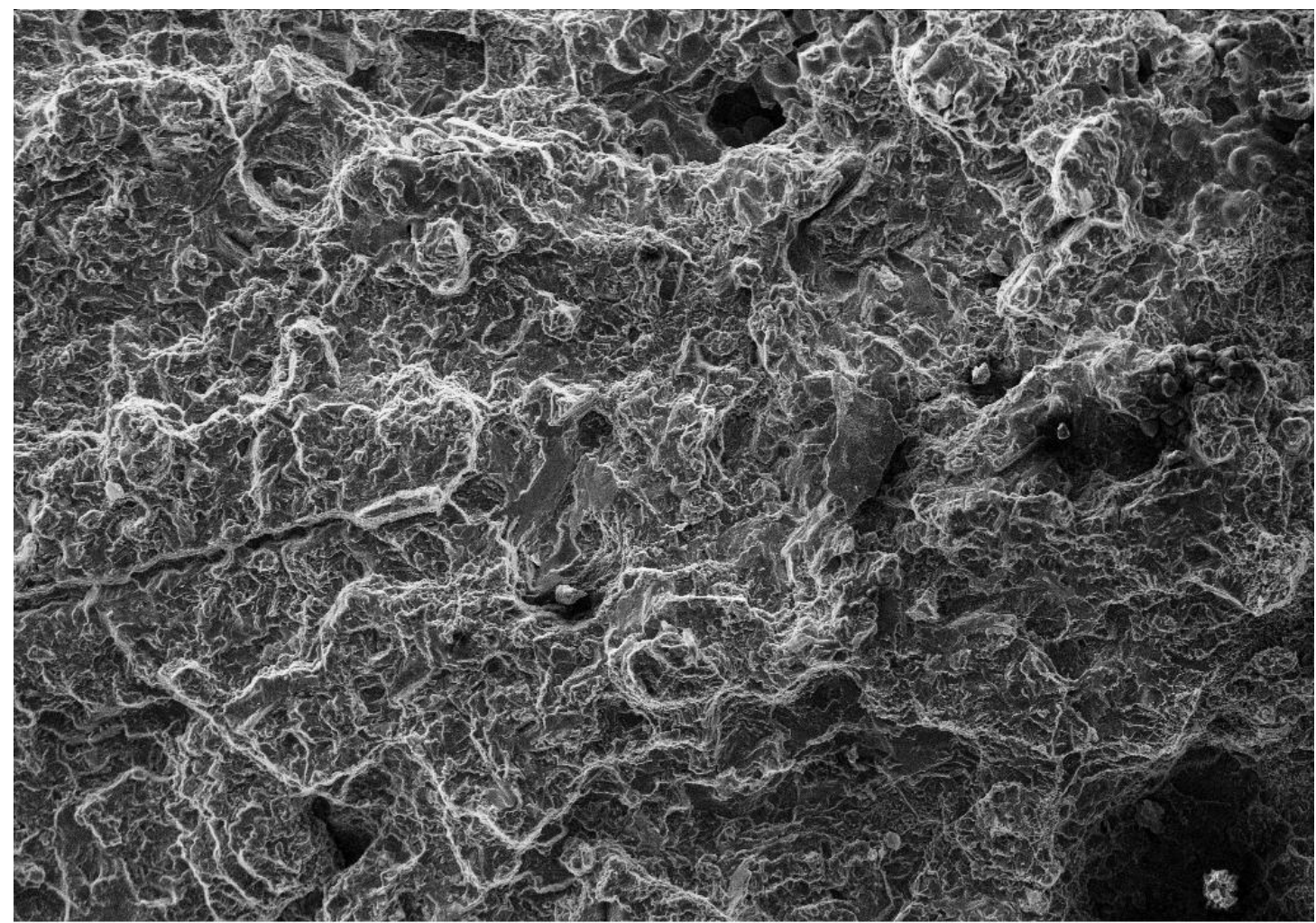

$\mathrm{IDSC}$
$\mathrm{EHT}=20.00 \mathrm{kV}$
$\mathrm{H}$

(b)

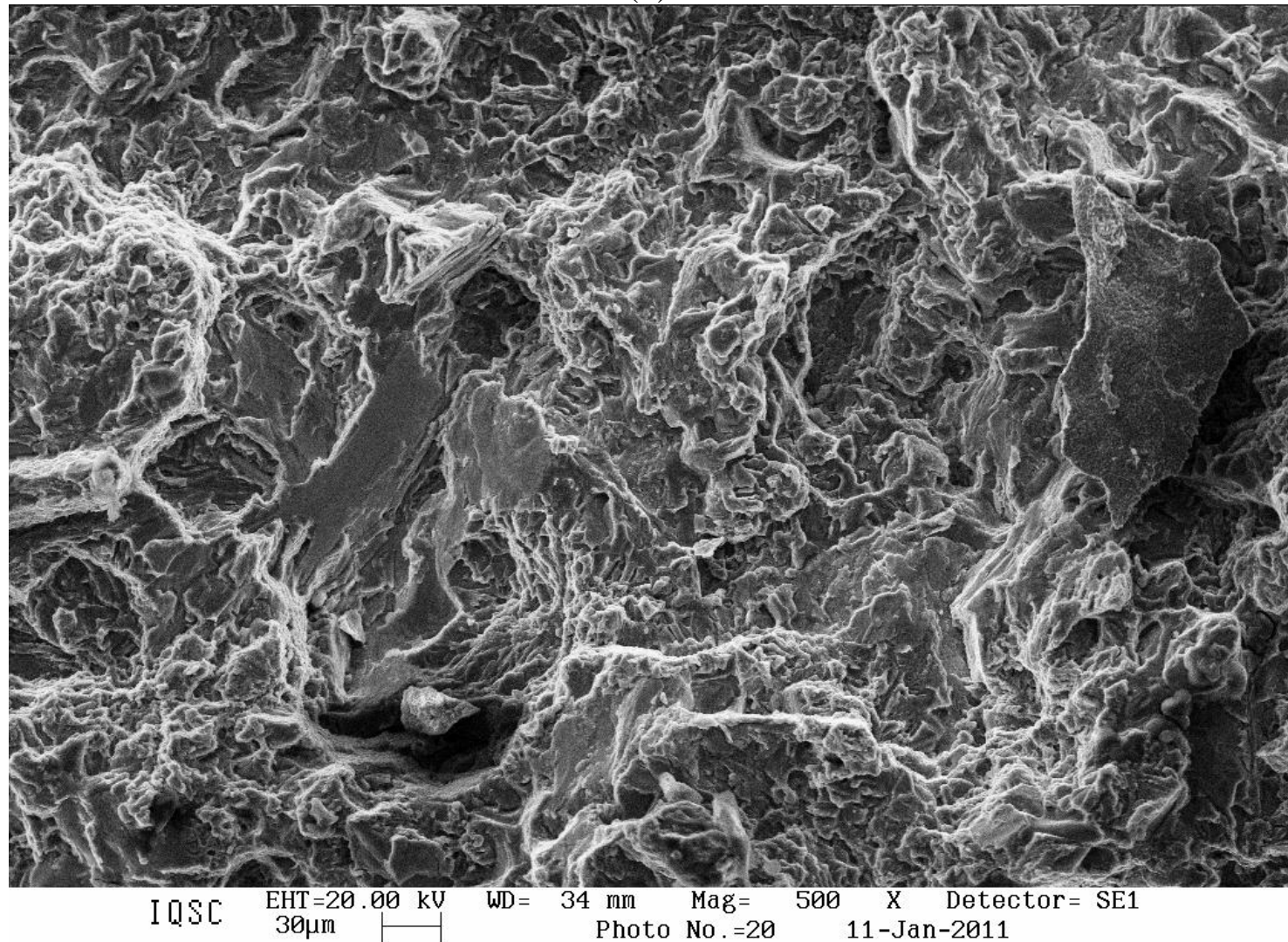

(c) 


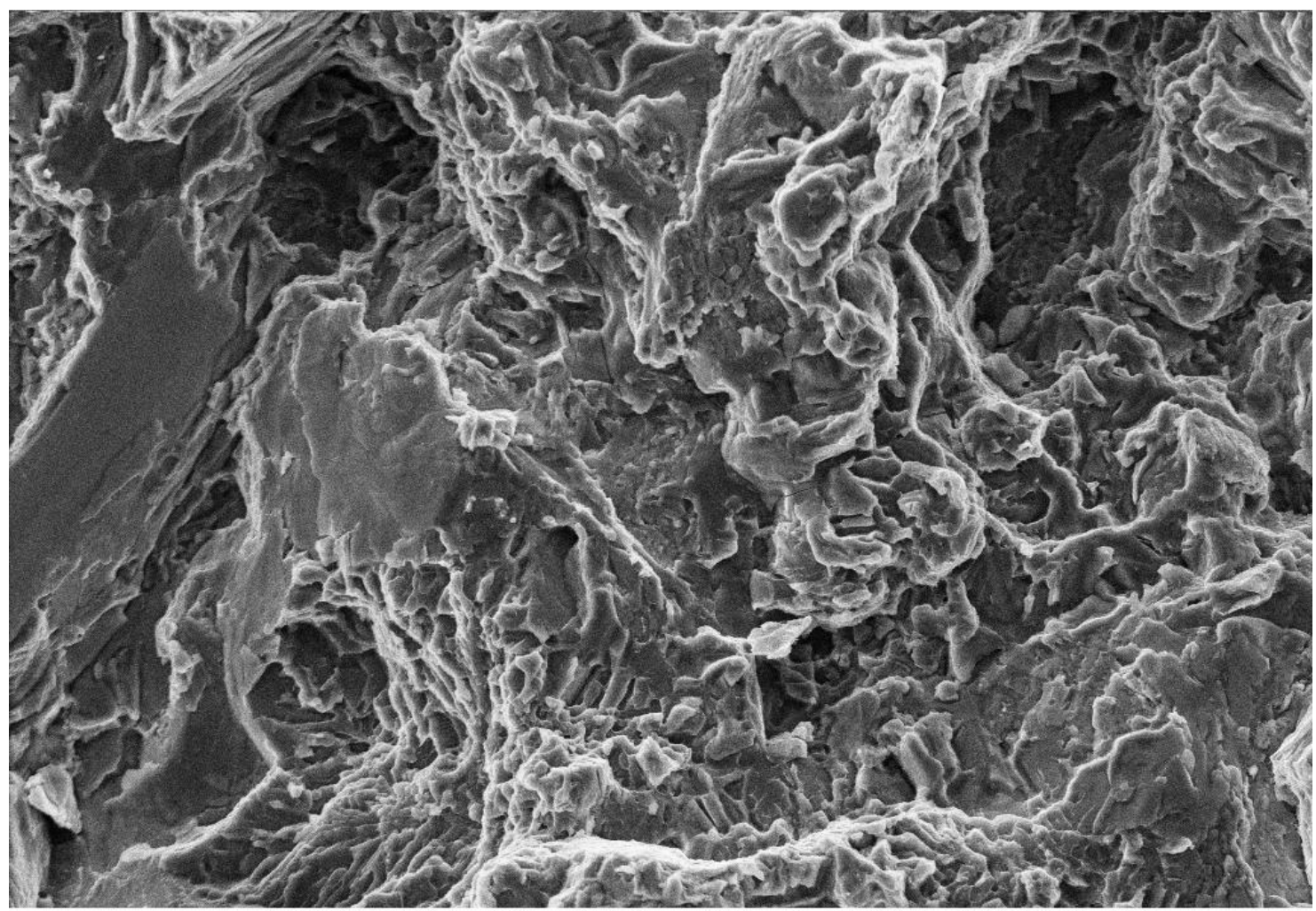

I DSC EHT $=20.00 \mathrm{kV} \quad \mathrm{WD}=34 \mathrm{~mm} \quad \mathrm{Mag}=1.00 \mathrm{~K} \mathrm{X}$ Detector $=$ SE1 Photo No.=21 11-Jan-2011

(d)

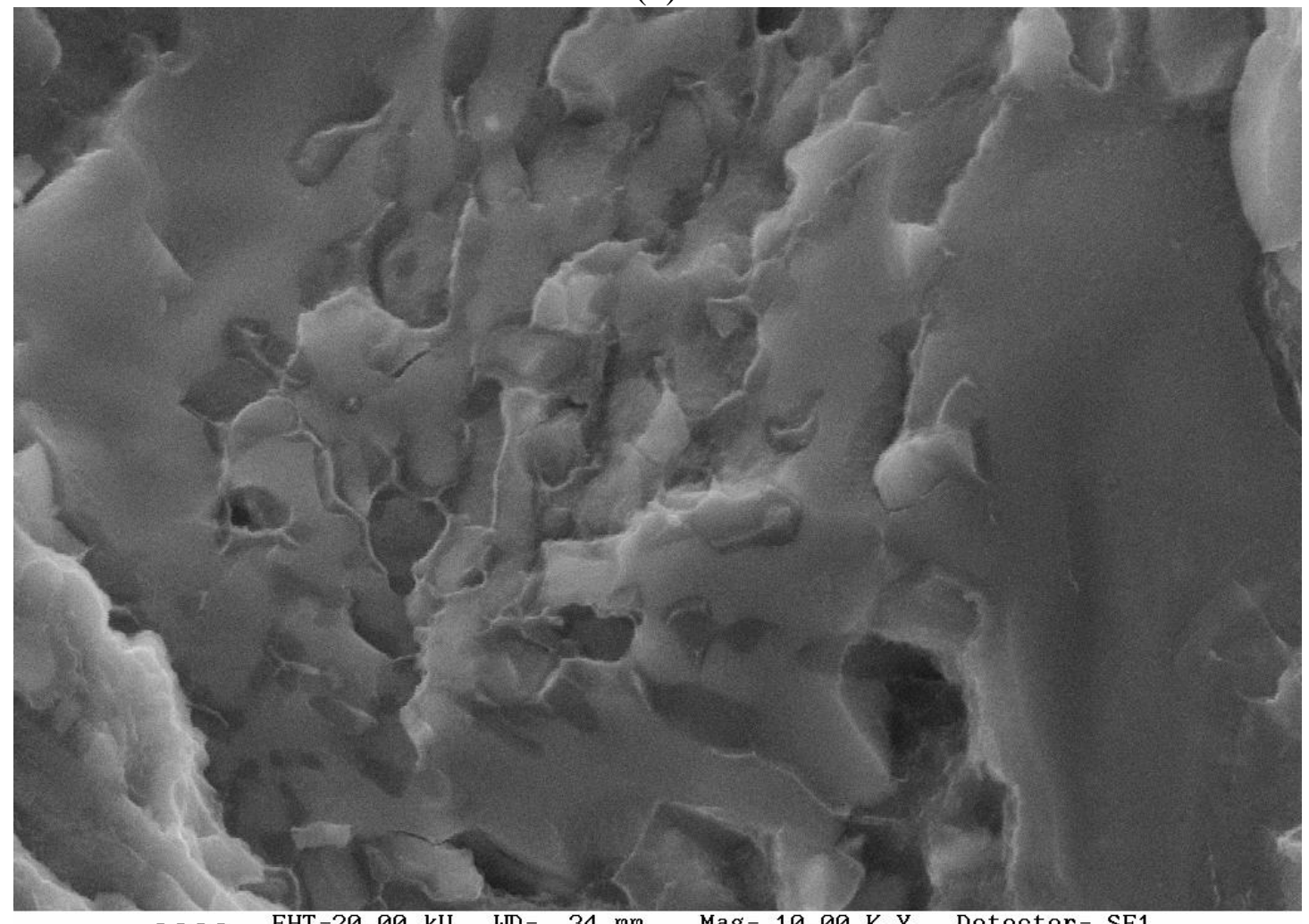

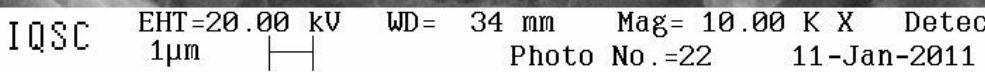

(e) 


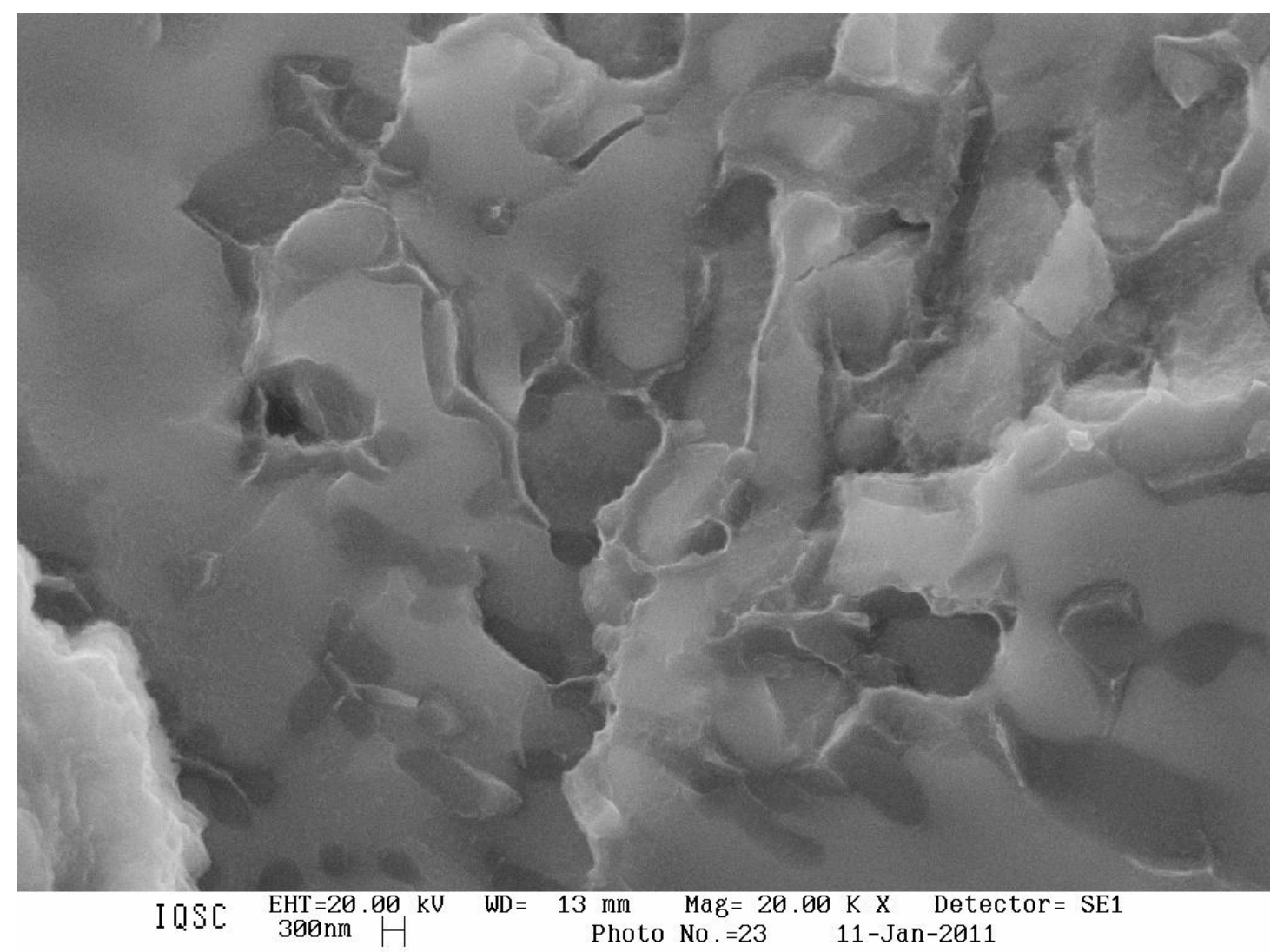

(f)

Figure 94: SEM analysis from a specimen fatigue tested at $280^{\circ} \mathrm{C}$, dwell cycle in displacement control: (a) notch and stable crack growth region, (b), (c), (d) and (e) detail of the stable crack growth region at $280^{\circ} \mathrm{C}$.

Tentative crack propagation tests were conducted under in-phase and out-of-phase anisothermal loadings, but with no valid results. Among the various problems encountered during this study, the most relevant are inadequacy of available and tested methods for measuring crack progress, long duration of tests (approximately one month each), and lack of homogeneity and high porosity of the material under study.

With respect to measuring crack growth, as stated earlier, this study opted for the potential-drop method. This decision was made after trying other methods and finding them to be inefficient. However, despite being the most suitable method among those available, the potential drop method is inadequate when some parameters, such as long testing time and the effects of thermal expansion produced by temperature variation intensified by in-phase mechanical loading, come into play. This occurs because small cracks appear in the region where the specimen is joined, by means of spot welding, with the wires employed to pass electric current, causing them to detach from the specimen after a few hundred cycles. In order to solve this problem, other methods of joining as well as other types of wire were tried. However, due to the specimen geometry, these other methods also proved inadequate. 
Moreover, the potential-drop method proved inadequate when thermal loading is done via induction coils, necessary in order to have plausible heating and cooling times. The electric current passing through the coils induces Foucault current in the wires used by the potential drop, generating large oscillation (noise) in measured values.

The long testing times (approximately one month each) were shown to be inadequate in that several external factors come into play, e.g., climate, energy drop, temperature variation throughout the day, and may even bring about unexpected test breaks, which can generate specimen overloading and, thus, invalidate the test.

As regards the material under study, its lack of homogeneity and high porosity can produce secondary cracking beyond the notch, causing the specimen to rupture outside the measurement region. In addition, due to long loading times (e.g., approximately ten minutes per cycle), this peculiarity of the material microstructure is aggravated by the synergy between high temperature and maximum load (in-phase anisothermal case), giving rise to other phenomena, e.g. creep, which interact with the propagation of fatigue cracks.

Figure 95a shows the notch as well as the pre-crack (indicated by red arrows) of the specimen used in the fatigue crack propagation test at room temperature. Figure 95b shows the specimen after a few weeks of test under in-phase anisothermal loading. The loading direction is shown by black arrows. As may be noticed, the fatigue pre-crack front has undergone significant rounding, indicating how the specimen crept. It is also possible to observe cavity nucleation at grain boundaries due to coalescing vacancies, stacking dislocations, and sliding grain boundaries. This may happen with second phase particles as well. Subsequent growth of these cavities leads to grain boundary cracking, which produces micro-voids, as indicated by blue arrows along the loading line.

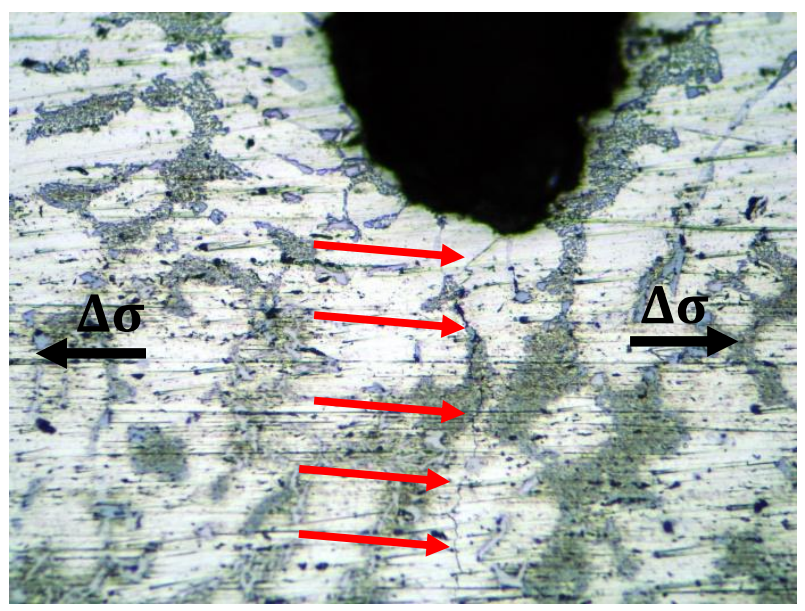

(a)

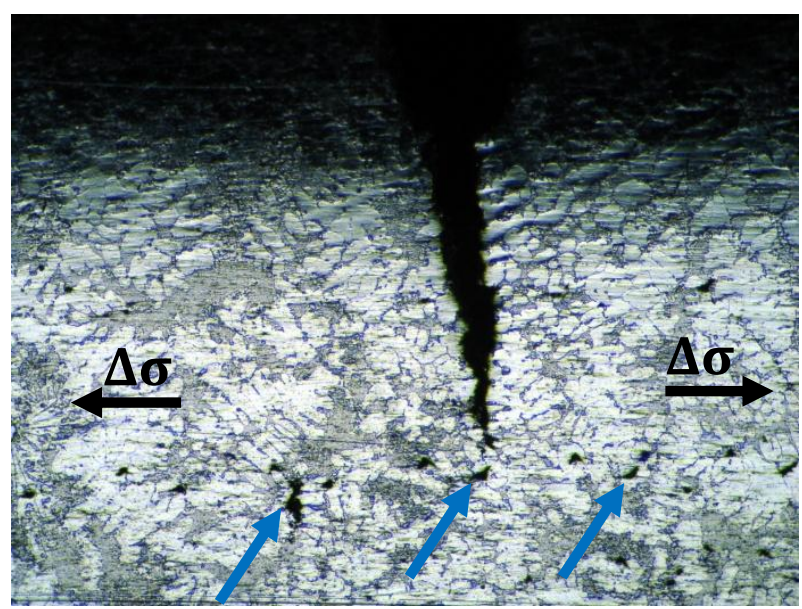

(b)

Figure 95: Photograph of specimen used for fatigue crack propagation testing showing (a) the region of the notch and pre-crack at room temperature and (b) tentative tests under 
anisothermal loading.

\subsection{Computational Results}

\subsubsection{Monotonic Loading (Tensile Test)}

A finite element analysis was carried out to model the material mechanical behavior. After trying several software programs available on the market, ABAQUS STANDARD FINITE ELEMENT CODE was chosen because of its accepting new models and algorithms as subroutine.

The study began with an elastic model of material behavior for the modeling of a specimen of geometry similar to those tested experimentally for stress and fatigue. Data for the material properties measured in these tests were entered at the software pre-processing phase. The same was done with loading and boundary conditions. The load vs. strain curves obtained experimentally provided the 0.2 creep limit in accordance with ASTM-E8/2009. The strain measured by the extensometer was converted into displacement, since the initial specimen length and length of the area measured by the extensometer are known, i.e., $\mathrm{L}_{0}=$ $106 \mathrm{~mm}$ and $\mathrm{L}_{0}=12 \mathrm{~mm}$, respectively. This displacement was applied to the model, which provided the load required. In the resolution phase of the "solver" algorithm, a finite element (FE) series of the software was tested, which indicated that C3D8R ( $\mathrm{C}=$ continuous stress/displacement, 3D = tri-dimensional, $\mathrm{R}=$ reduced integration) was the most suitable element to start this study. Results regarding displacement, strain, and Von Mises stress were obtained in the post-processing phase (Figure 96). 
194

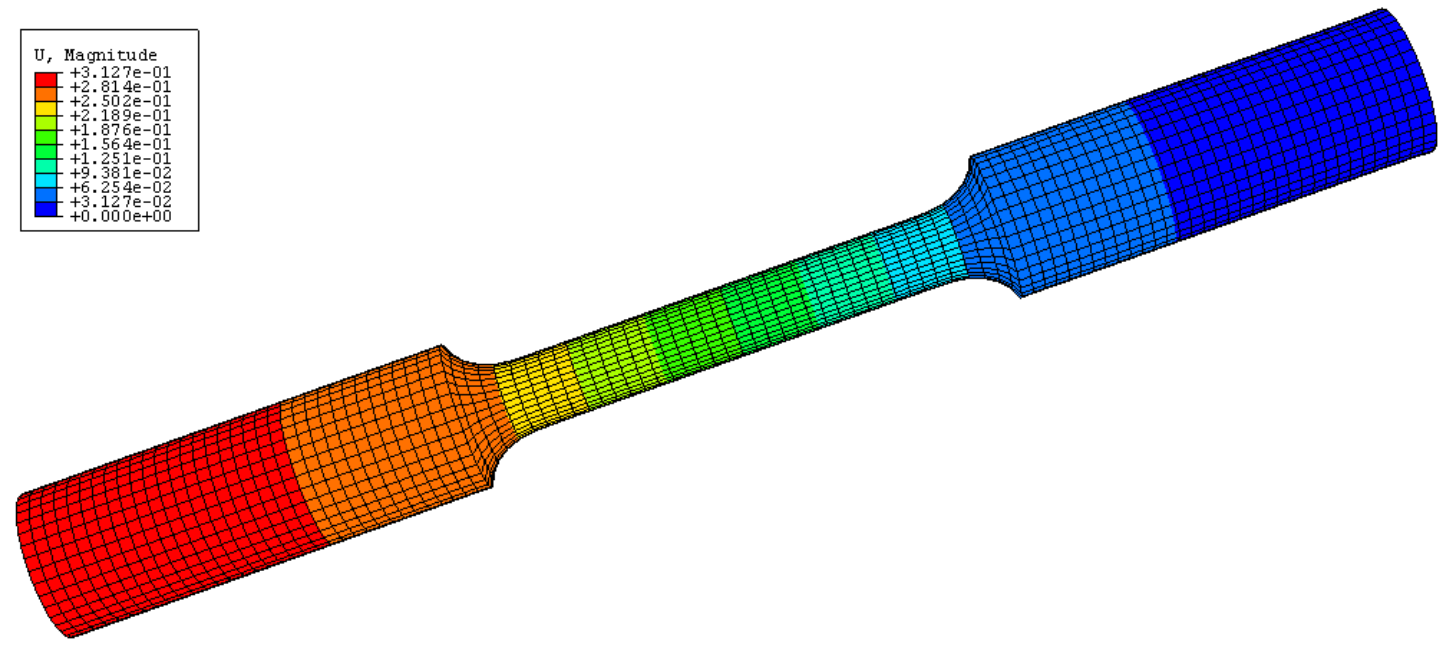

(a)

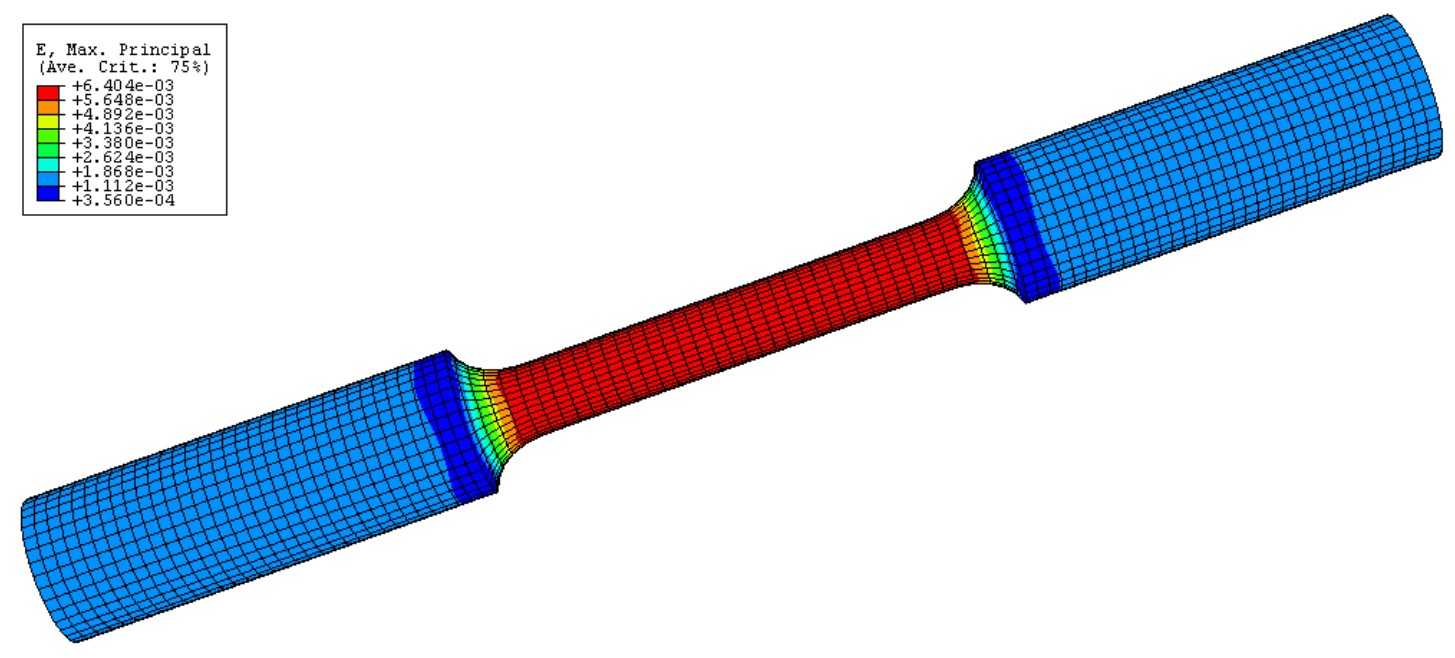

(b)

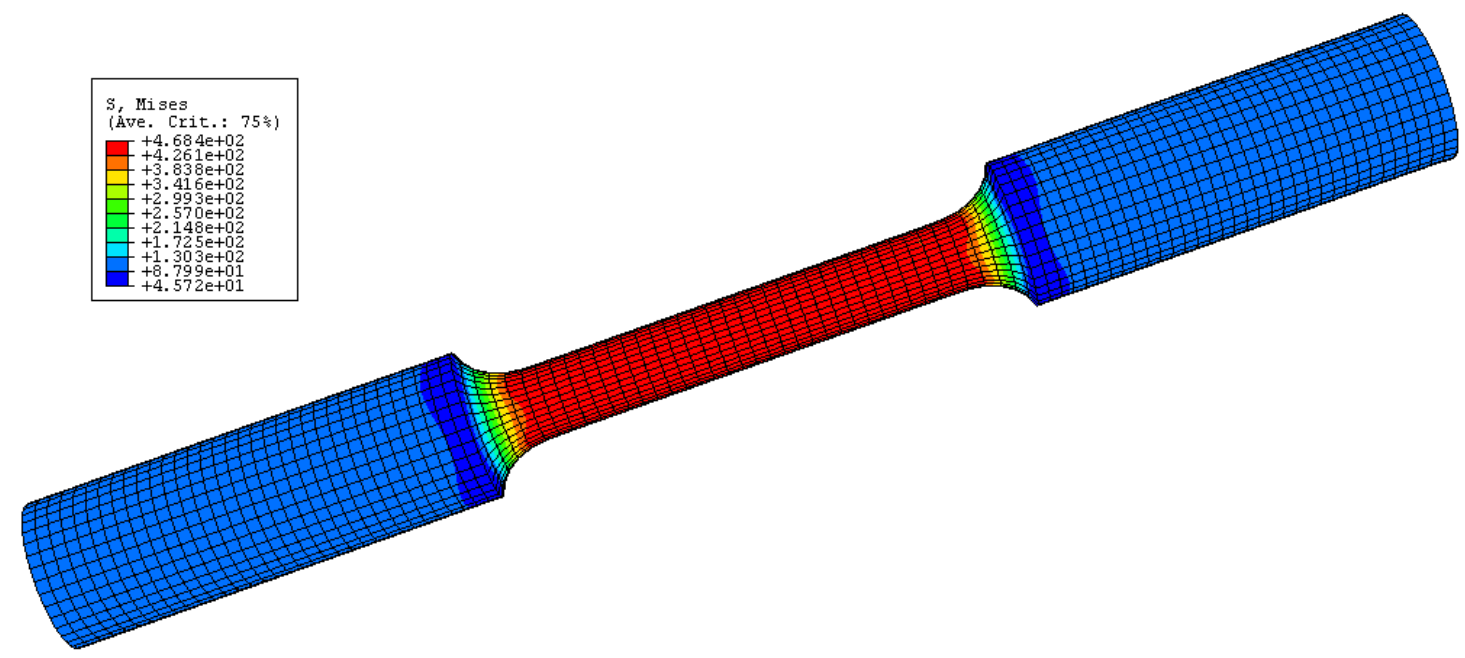

(c)

Figure 96: Modeling results of strain specimen at room temperature in terms of (a) displacement, (b) deformation, and (c) Yon Miss stress. 
It is possible to observe that the useful region of the specimen is the one subjected to the greatest efforts. Therefore, modeling was carried out only on this roughly $12 \mathrm{~mm}$-long region, which corresponds exactly to the region being measured by the extensometer, thus obtaining more accurate results than previous ones (Figure 97).

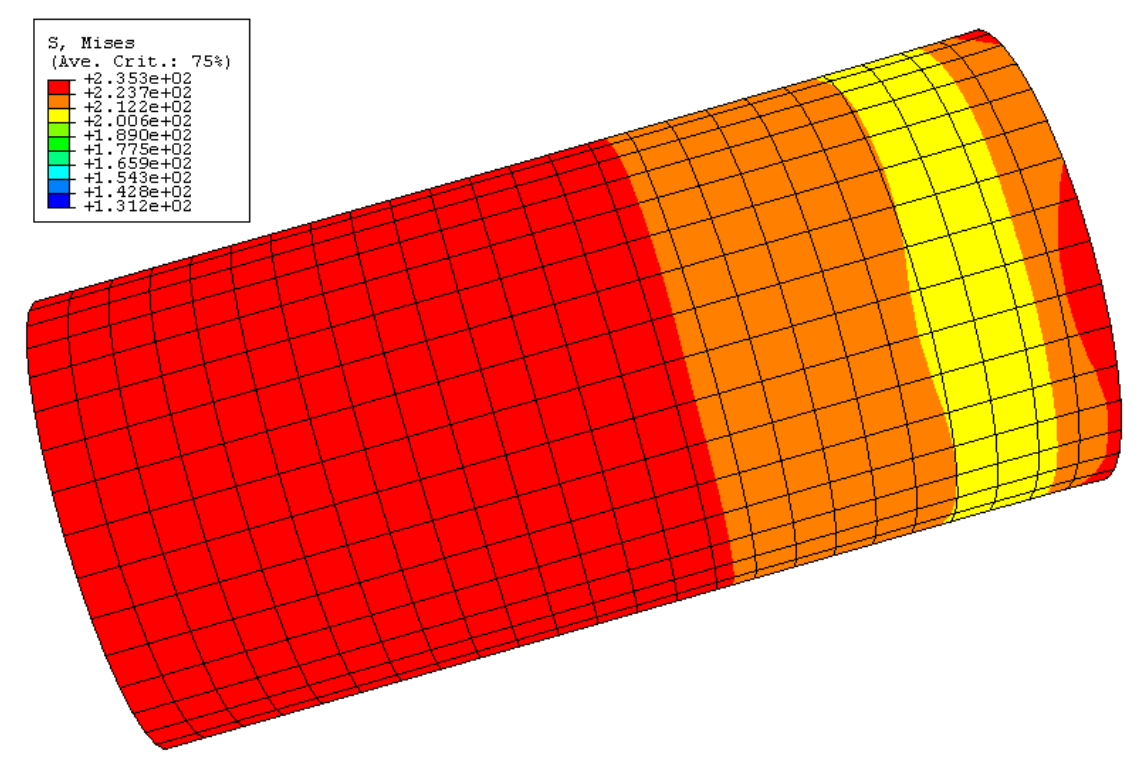

Figure 97: Von Mises stress results of modeling on useful region of strain specimen at room temperature.

As might be expected, if the deformation measured by the extensometer is applied to a small region of the specimen with smaller cross-section than those of the remainder regions, in the whole specimen $\mathrm{L}_{0}=106 \mathrm{~mm}$, it will be subject to higher stresses than under real-life conditions and, thus, erroneous.

In order to compare experimental and computational results, loading vs. deformation curves were plotted for the elastic region at $25^{\circ} \mathrm{C}, 120^{\circ} \mathrm{C}$, and $280^{\circ} \mathrm{C}$ (Figure 98, Figure 99 and Figure 100). 


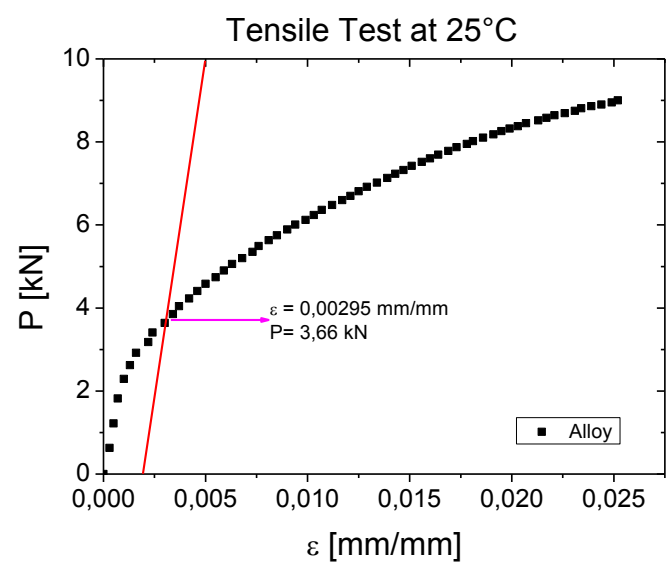

(a)

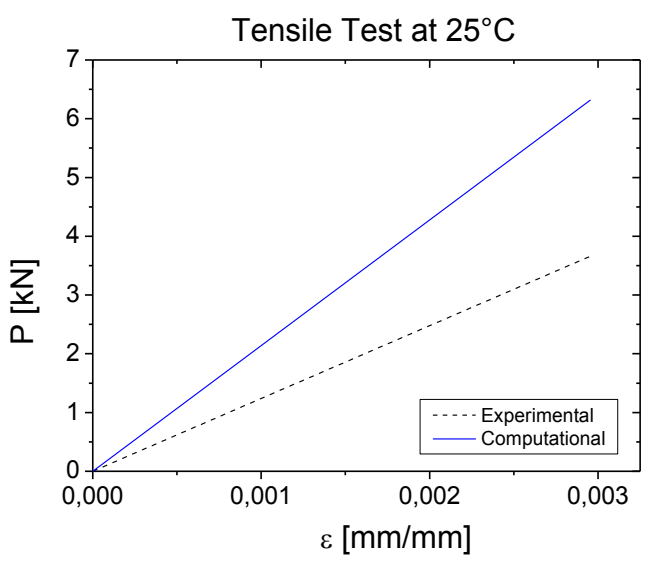

(b)

Figure 98: Tensile tests at $25^{\circ} \mathrm{C}$ : (a) loading as a function of total strain and 0.2 yield point; (b) comparison between experimental and computational curves for elastic region.

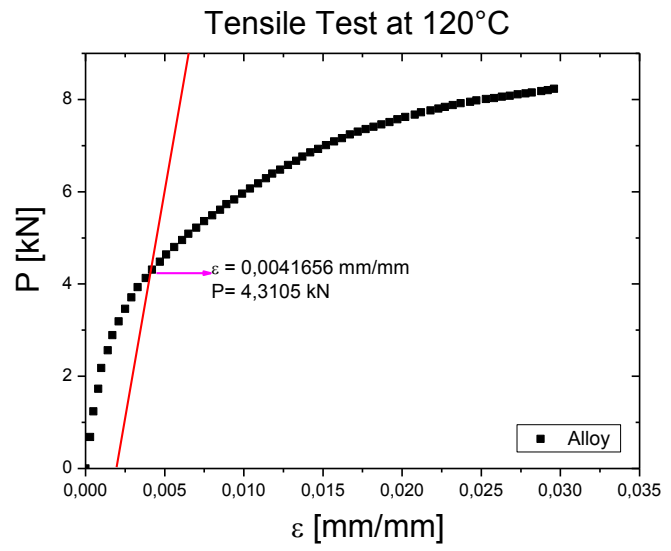

(a)

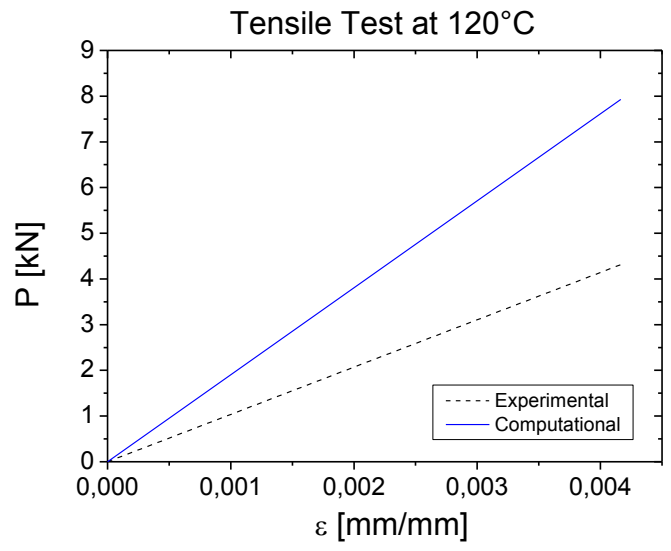

(b)

Figure 99: Tensile tests at $120^{\circ} \mathrm{C}$ : (a) loading as a function of total strain and 0.2 yield point; (b) comparison between experimental and computational curves for elastic region.

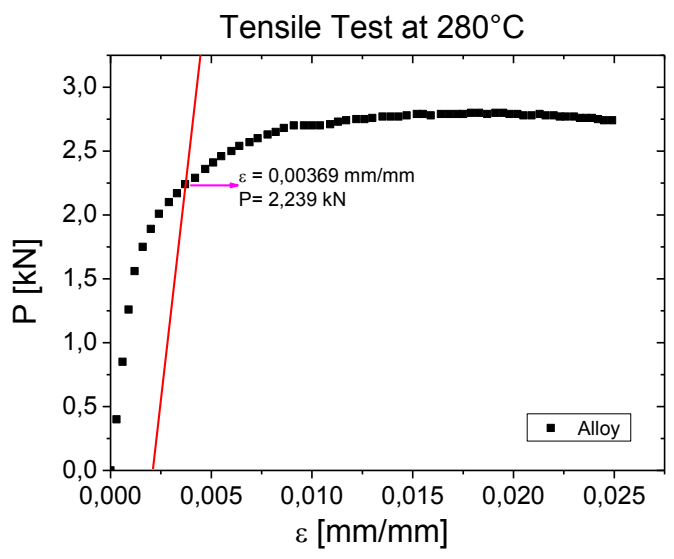

(a)

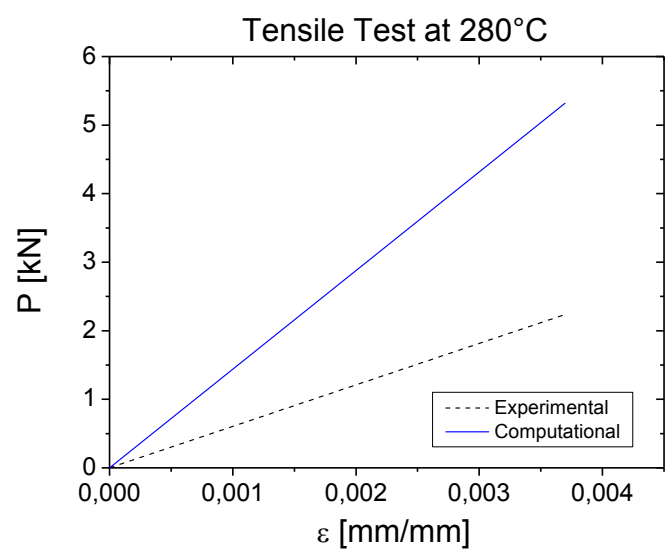

(b)

Figure 100: Tensile tests at $280^{\circ} \mathrm{C}$ : (a) loading as a function of total strain and 0.2 yield point; 
(b) comparison between experimental and computational curves for elastic region.

It is possible to see a great difference between experimental and computational results. This is due to the fact that the adopted creep limit, in accordance with ASTM E8/2009, is not suitable for the type of response provided by the material under study. There is a non-linear region where plastic deformation occurs that is not being properly considered. This region may be under the influence of the material porosity.

When only the linear segment of the curve was taken into account and the creep limit was taken at its end, it was possible to obtain computational results closer to experimental ones, as shown in Figure 101, Figure 102 and Figure 103.

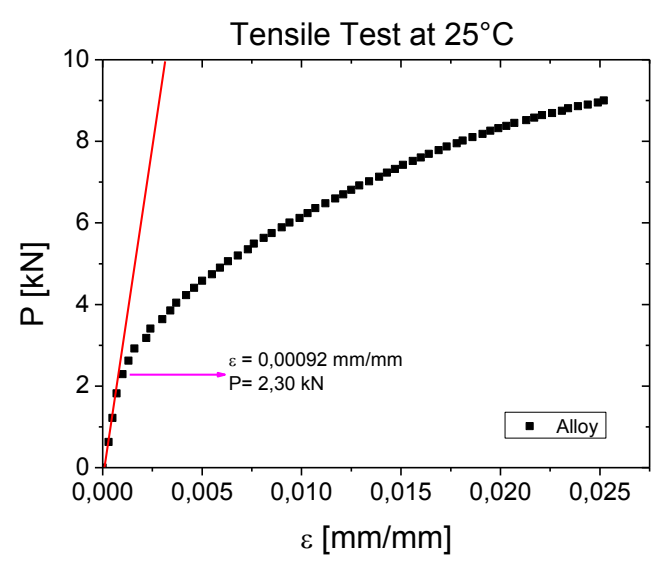

(a)

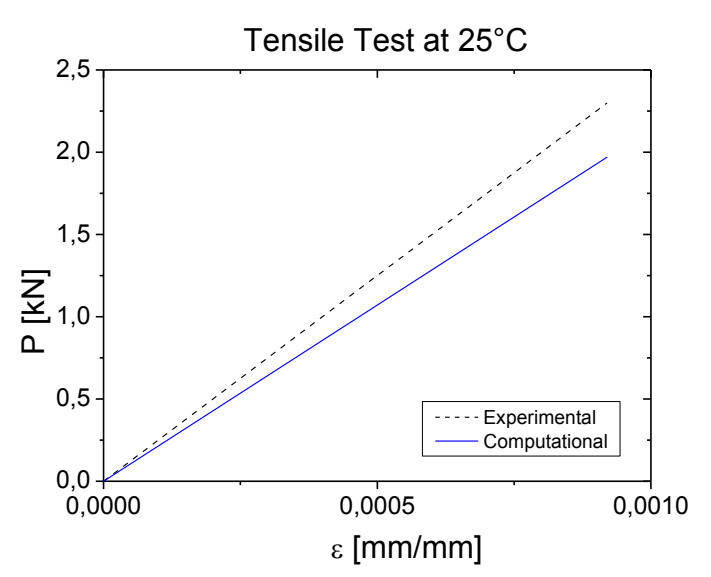

(b)

Figure 101: Tensile tests at $25^{\circ} \mathrm{C}$ : (a) loading as a function of total strain and yield point taken at end of tangent to elastic region of curve; (b) comparison between experimental and computational curves for elastic region.

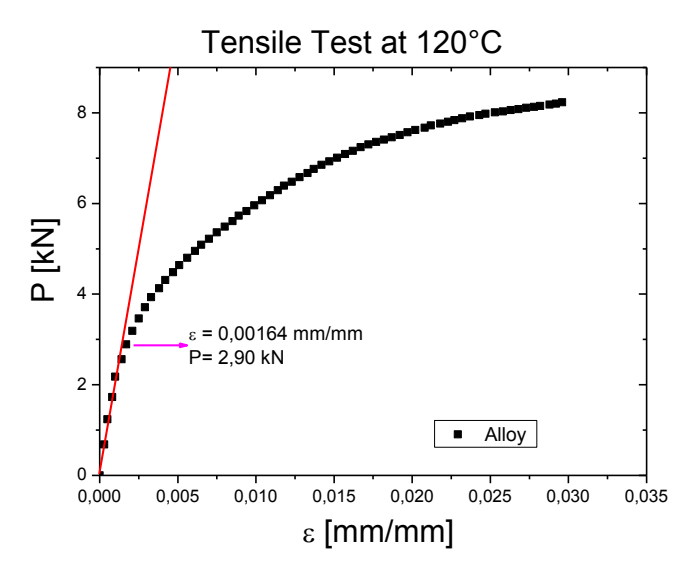

(a)

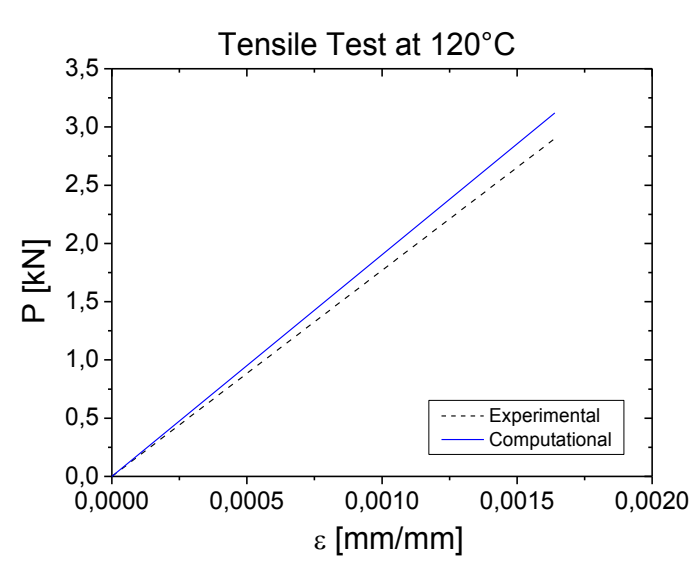

(b)

Figure 102: Tensile tests at $120^{\circ} \mathrm{C}$ : (a) loading as a function of total strain and yield point taken at end of tangent to elastic region of curve; (b) comparison between experimental and 
computational curves for elastic region.

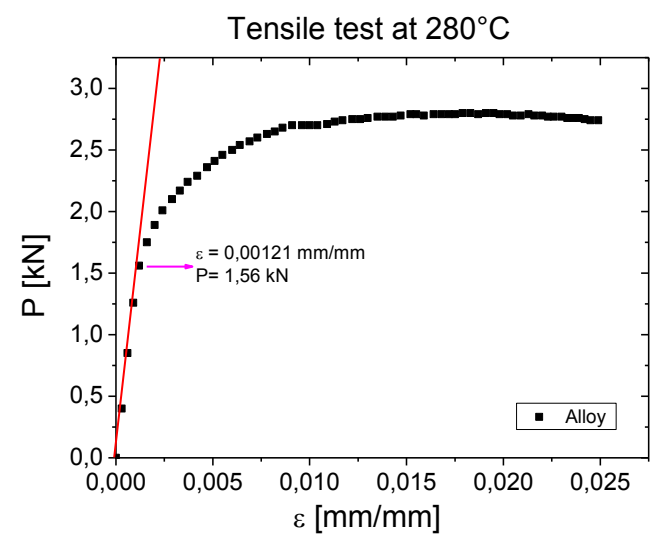

(a)

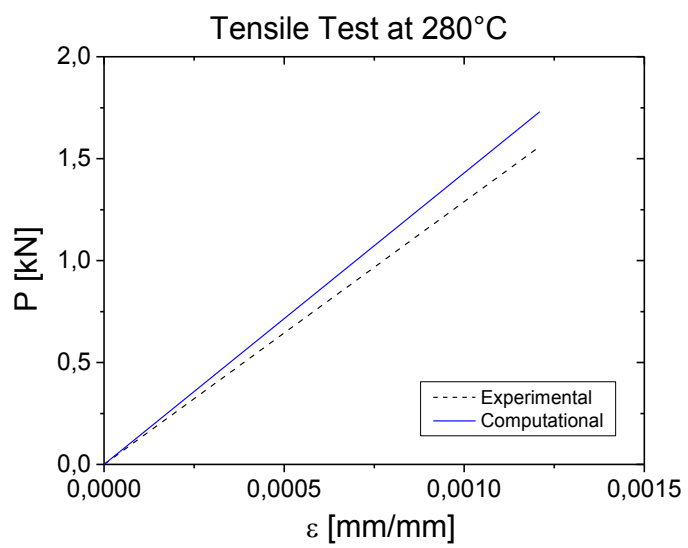

(b)

Figure 103: Tensile tests at $280^{\circ} \mathrm{C}$ : (a) loading as a function of total strain and yield point taken at end of tangent to elastic region of curve; (b) comparison between experimental and computational curves for elastic region.

Figure 104 indicates a difference between experimental and computational curves, especially in the plastic region, which can be attributed to specimen necking and consequent difficulty in turning engineering data into real data to be supplied to the model.

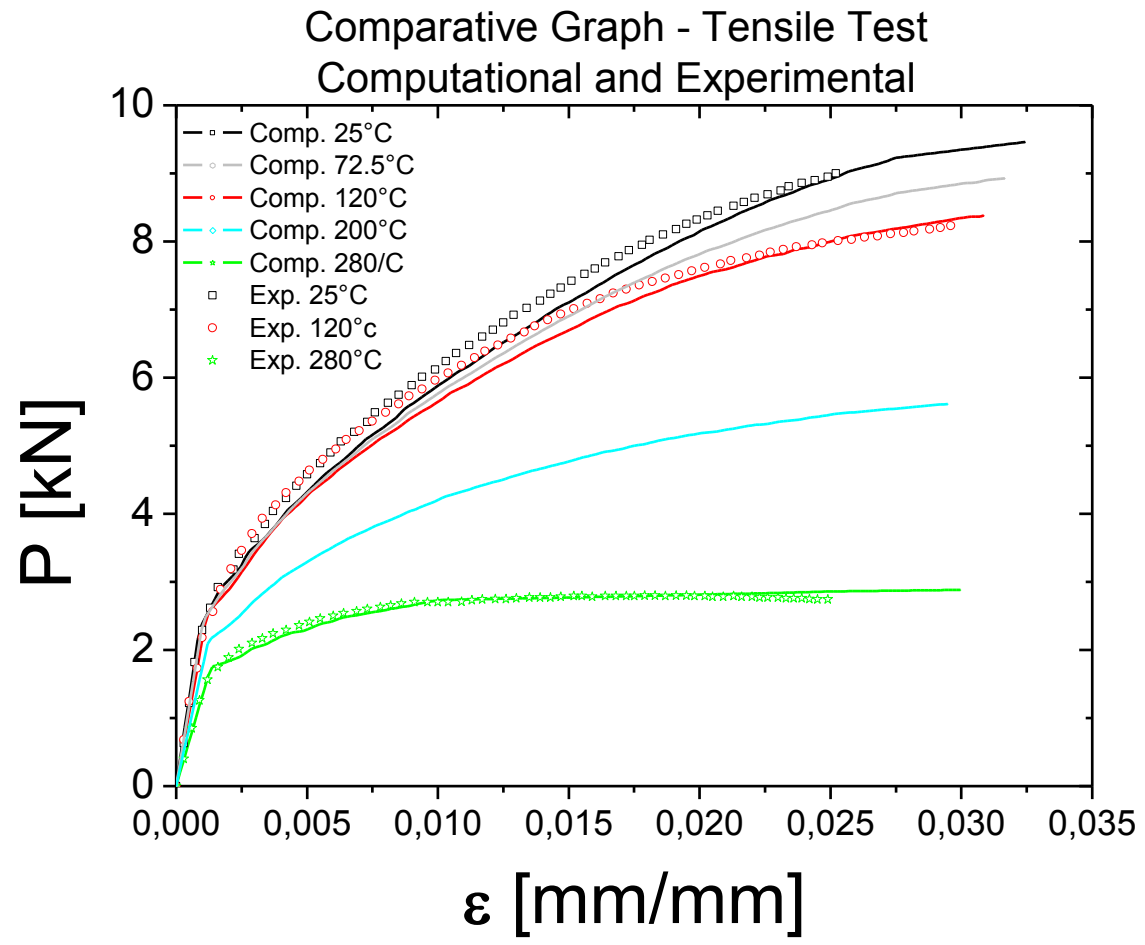

Figure 104: Comparison between computational and experimental tensile test curves.

The data obtained so far indicate that the porosity of the material under study seems to influence strongly its mechanical response. This is because mechanical properties are assumed 
homogeneous throughout the specimen. However, they can vary from region to region, signaling the need to adapt the material model, taking into account its microstructure heterogeneity, so that modeling results are as close to reality as possible. Furthermore, when applying the model to the real component, MAGMA software simulation should be employed to provide data of physical and mechanical properties, thereby ensuring that its mechanical and physical behavior as a whole is taken into consideration. This indicates the need to adapt a material model so that modeling results are as close to reality as possible.

\subsubsection{Cyclic Loading (Fatigue Test)}

\subsubsection{Identification of Material Behavior Law Parameters}

Since the material cyclic behavior is the only way into the incremental model of crack propagation under fatigue (Pommier S., Risbet M., 2005), the correct identification of the material behavior law and its parameters is of fundamental importance to describe plasticity in the region immediately ahead of the crack tip and all its related phenomena. The identification of the material behavior was done by means of isothermal fatigue and relaxation tests described in Sections 6.1.2.2 e 6.1.2.4.

Finite Element Modeling of this behavior will then allow the identification of the eight material parameters of cracked structure behavior. In this case Chaboche law is used with combined nonlinear kinematic hardening $(X)$ and nonlinear isotropic hardening $(R)$. Kinematic hardening evolution is given by:

$$
d X=\frac{2}{3} \cdot C \cdot d \varepsilon_{p}-\gamma \cdot X \cdot d p \operatorname{com} X_{t-0}=X_{0}
$$

whereas isotropic evolution is given by:

$$
d R=b(q-R) d p \text { com } R_{t=0}=R_{0}
$$

where $p$ is accumulated plastic deformation given by: 


$$
p=\int_{t=0}^{t} \sqrt{\frac{2}{3} \dot{\varepsilon}_{p}(\tau): \dot{\varepsilon}_{p}(\tau)} d \tau
$$

and the viscous component is given by the Norton-Hoff law:

$$
\dot{\varepsilon}_{v}=A \cdot \sigma_{v}^{n}
$$

and therefore, the total strain is given by:

$$
\varepsilon=\varepsilon^{e l}+(1-f) \varepsilon^{p l}+f \varepsilon^{v}
$$

A Finite Element simulation was conducted by ABAQUS to identify the material behavior law parameters. The dimensions correspond to the useful region of the specimen employed in the fatigue test. The axisymmetric model was used, with calculations performed under plane strain (Figure 105).

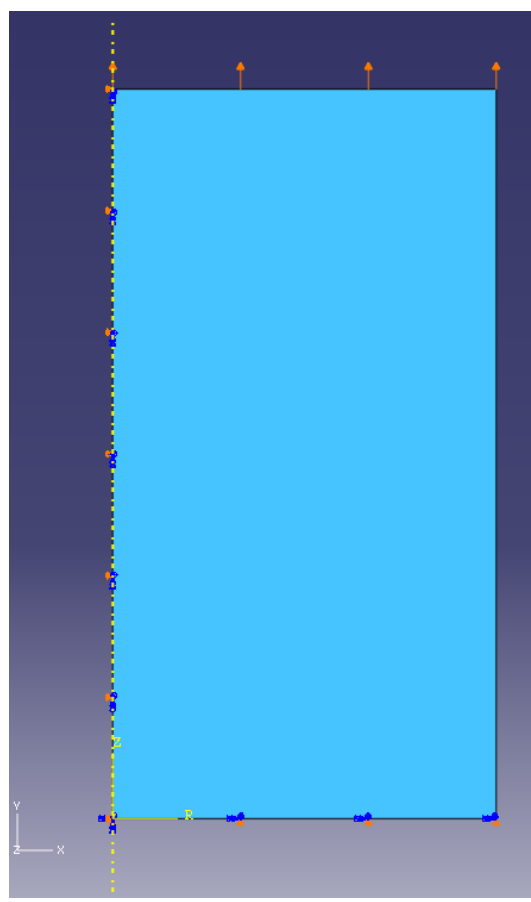

(a)

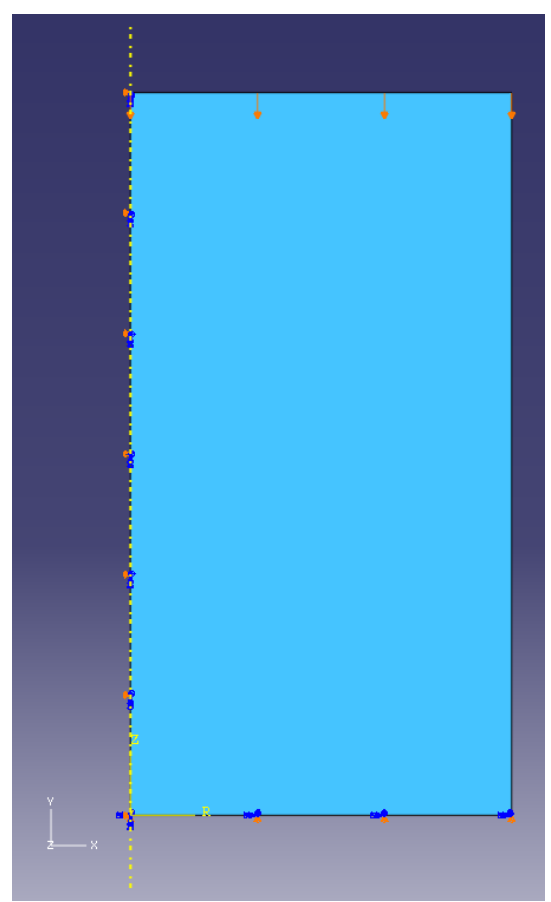

(b)

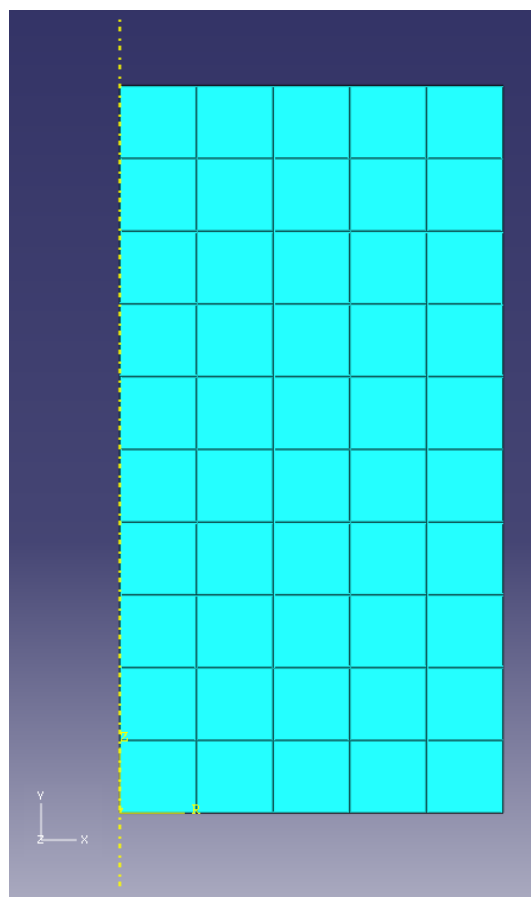

(c)

Figure 105: Axisymmetric model employed to identify the behavior law parameters of the material in question: (a) and (b) boundary conditions; and (c) Finite Element mesh

Table 9 shows the behavior parameters identified for the material under study. Comparison between the experimental hysteresis and that obtained computationally under 
isothermal and thermo-mechanical loading conditions (Figure 106, Figure 107 and Figure 108) shows that the chosen parameters satisfactorily agree with experimental results. It was also noted that when viscous behavior comes into play (Figure 109), discrepancies between the model and experimental results are more pronounced. This point shows the difficulty in modeling the material behavior when two or more phenomena interact.

Table 9: Behavior Law parameters of material under study.

\begin{tabular}{|c|c|c|c|c|c|c|c|c|c|c|c|}
\hline \multirow{3}{*}{ 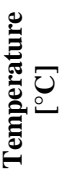 } & \multicolumn{11}{|c|}{ Parameters } \\
\hline & \multicolumn{2}{|c|}{ Elastic } & \multicolumn{5}{|c|}{ Plastic } & \multicolumn{4}{|c|}{ Viscous } \\
\hline & E(MPa) & $\mathbf{v}$ & $\mathbf{R}_{0}$ & $\mathbf{C}$ & $\gamma$ & $\mathbf{Q}$ & b & $\mathbf{A}$ & $\mathbf{n}$ & $\mathbf{m}$ & $\mathbf{f}$ \\
\hline 120 & 63.400 & 0.33 & 93 & 130.000 & 2.500 & 20 & 9 & $3.2 \mathrm{E}-07$ & 3.889 & 0.0 & 0.05 \\
\hline 140 & 62.100 & 0.3325 & 88.9 & 111.517 & 2.500 & -5.73 & 9 & $6.0 \mathrm{E}-08$ & 3.889 & 0.0 & 0.05 \\
\hline 180 & 59.500 & 0.3375 & 82.2 & 85.462 & 2.500 & -24.4 & 9 & $2.85 \mathrm{E}-08$ & 3.889 & 0.0 & 0.05 \\
\hline 200 & 58.200 & 0.34 & 79.4 & 76.089 & 2.500 & -27.8 & 9 & $1.20 \mathrm{E}-08$ & 3.889 & 0.0 & 0.05 \\
\hline 240 & 55.600 & 0.345 & 74.7 & 61.975 & 2.500 & -30.9 & 9 & $8.80 \mathrm{E}-09$ & 3.889 & 0.0 & 0.05 \\
\hline 280 & 53.000 & 0.35 & 71 & 55.000 & 2.500 & -32 & 9 & $5.60 \mathrm{E}-09$ & 3.889 & 0.0 & 0.05 \\
\hline
\end{tabular}

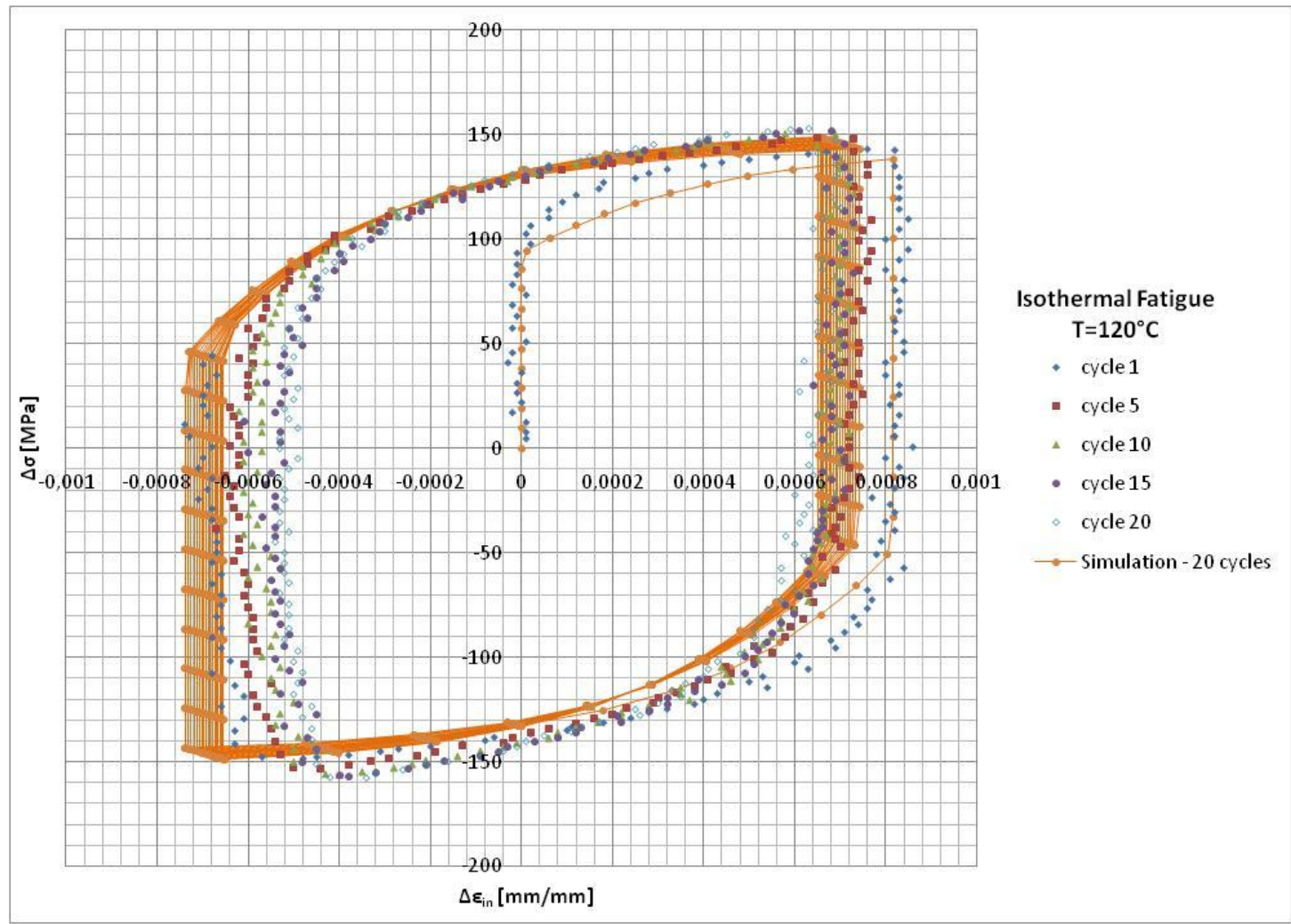

Figure 106: Experimental hysteresis curves for isothermal fatigue tests at $120^{\circ} \mathrm{C}$ and total mechanical deformation of $0.3 \%$ and its corresponding computational hysteresis curve from ABAQUS. 


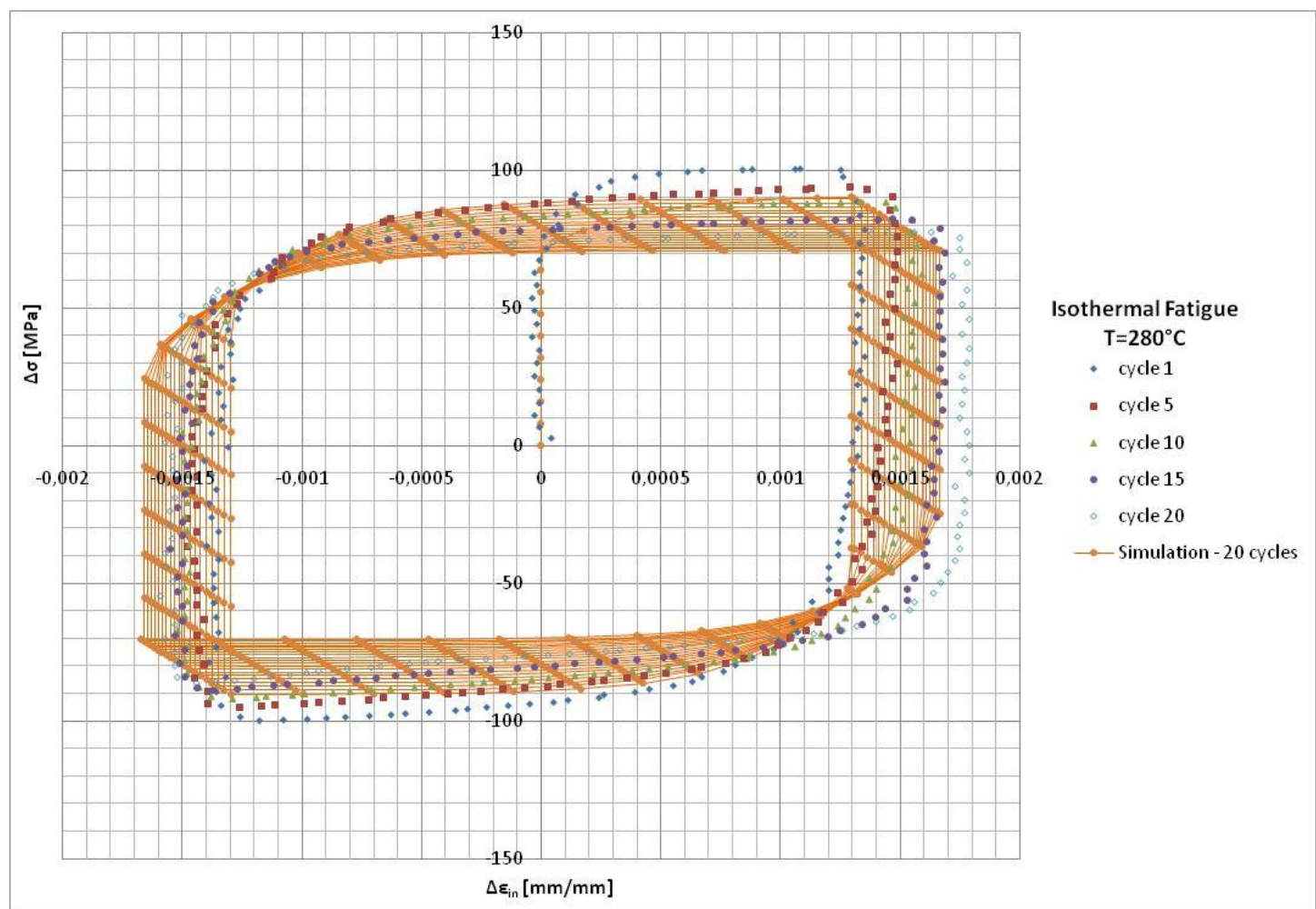

Figure 107: Experimental hysteresis curves for isothermal fatigue tests at $280^{\circ} \mathrm{C}$ and total mechanical deformation of $0.3 \%$ and its corresponding computational hysteresis curve from ABAQUS.

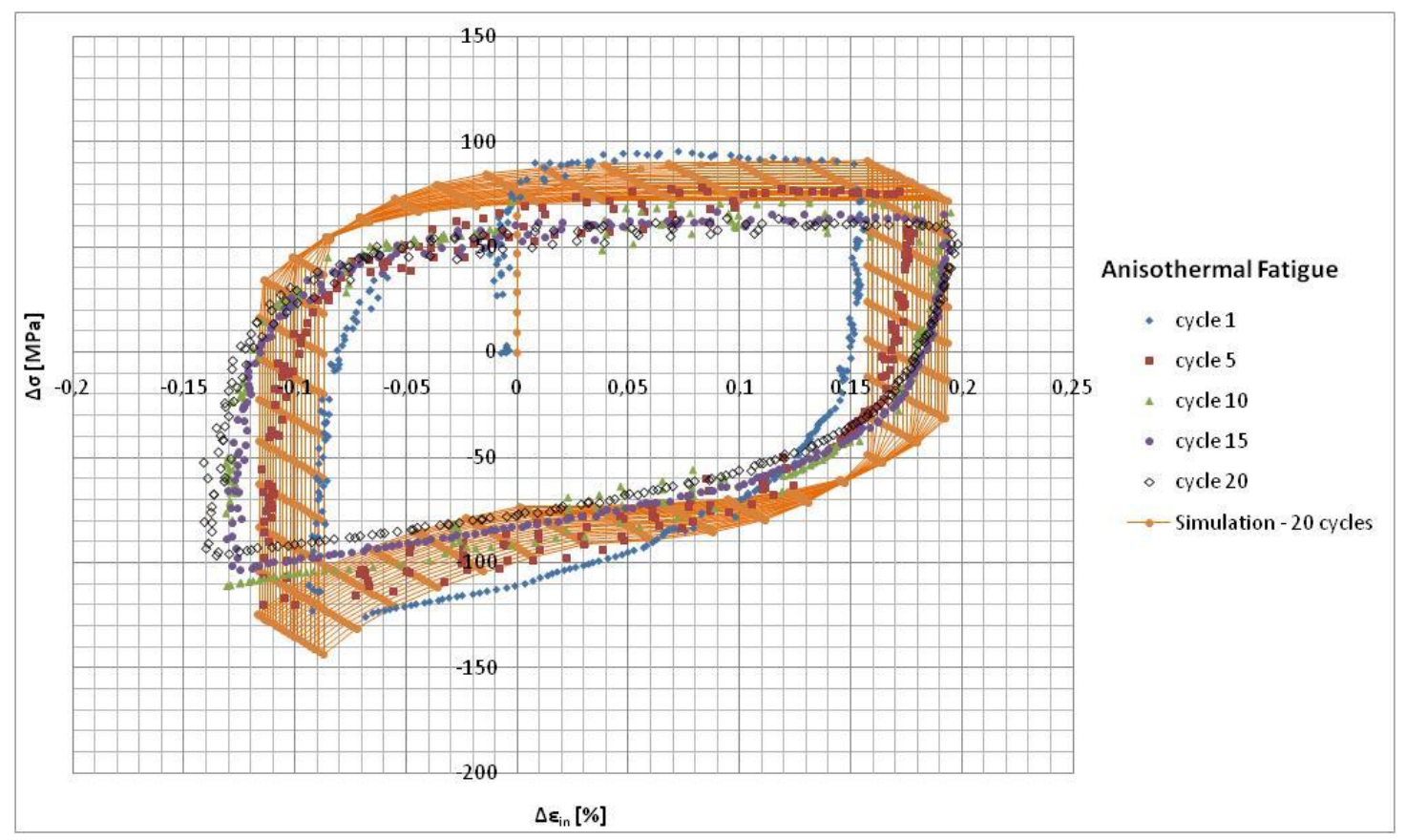

Figure 108: Experimental hysteresis curves for anisothermal fatigue tests between 120 and $280^{\circ} \mathrm{C}$ and total mechanical deformation of $0.3 \%$ and its corresponding computational hysteresis curve from ABAQUS. 


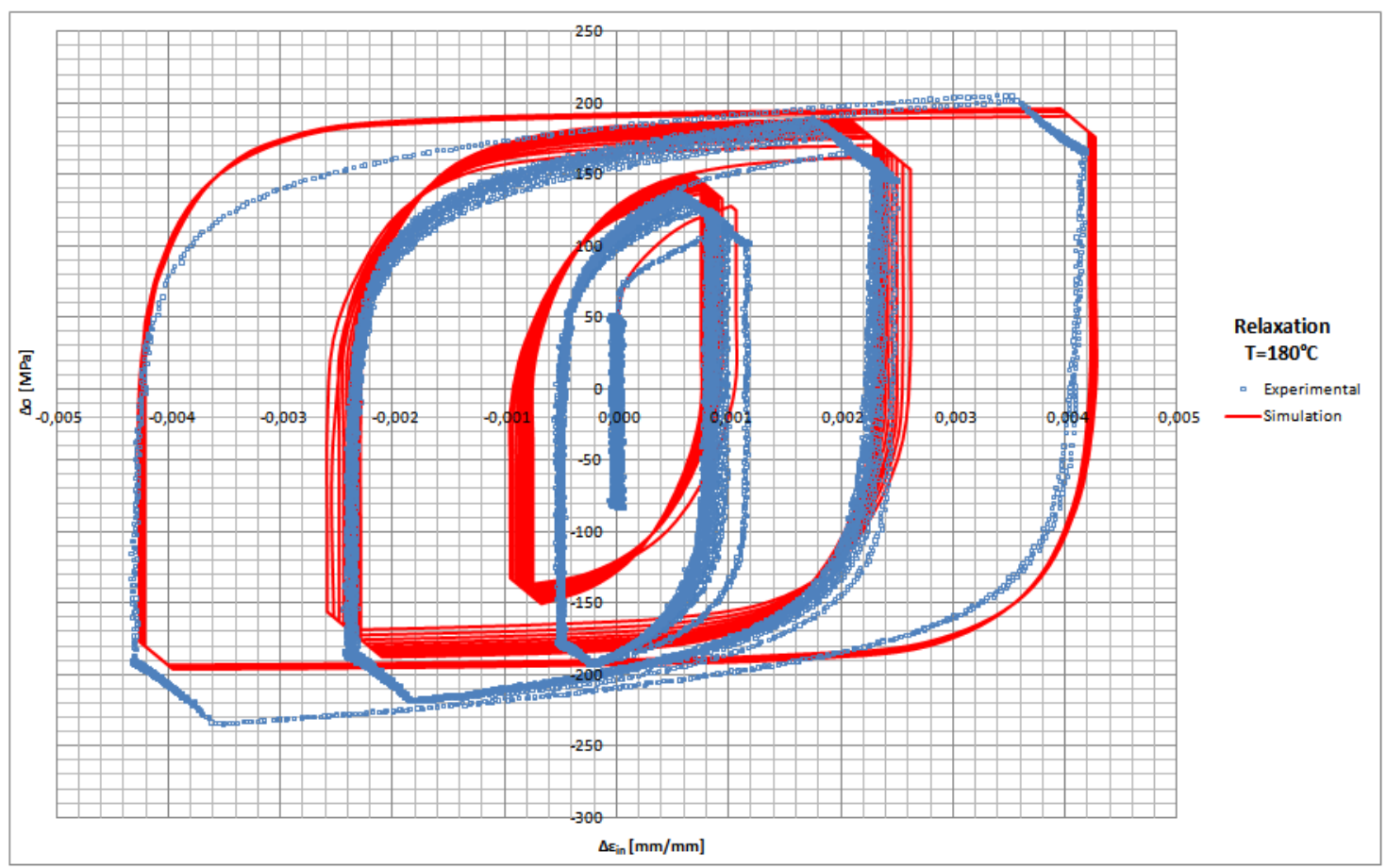

Figure 109: Relaxation test hystereses for total deformations of $0.1 \%, 0.3 \%, 0.5 \%$ and $0.7 \%$ at $180^{\circ} \mathrm{C}$ and its corresponding computational hysteresis curve from ABAQUS.

The relationship between the material behavior parameters and temperature may be obtained by the following equations:

$$
\begin{aligned}
& \frac{C}{\gamma}=\frac{C_{0}}{\gamma_{0}} \cdot e^{(-E / k \cdot T)} \\
& R=R_{0} \cdot e^{(-E / k \cdot T)} \\
& Q=Q_{0} \cdot e^{(-E / k \cdot T)} \\
& A=A_{0} \cdot e^{(-E / k \cdot T)}
\end{aligned}
$$

where $\mathrm{E}=$ Activation energy, $\mathrm{k}=$ Boltzmann constant, $\mathrm{T}=$ Temperature

\subsubsection{Identification of Plastic Blunting Law Parameters}

Since the behavior of the A356 aluminum engine cylinder head is known, the plastic blunting law parameters can be identified. This is done by a Finite Element (FE) calculation on a cracked plate. The plate dimensions are $200 \times 200 \mathrm{~mm}$ and a central crack of length 
2. $a=20 \mathrm{~mm}$. Calculations are performed under plane strain using the behavior identified for the material in question. The plate is subjected to 30 loading and unloading cycles in order to achieve a stabilized cycle for the cyclic part (Figure 110).

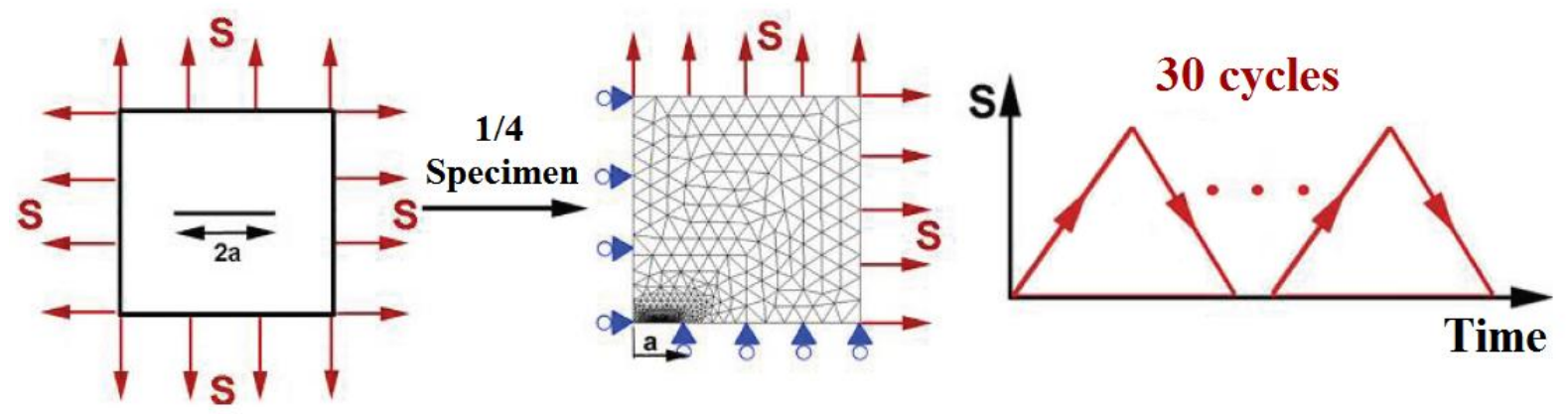

Figure 110: Finite Element calculation performed to identify plastic blunting law parameters.

The identification of parameters $\mathrm{a}_{\mathrm{m}}, \mathrm{b}_{\mathrm{m}}, \mathrm{a}_{\mathrm{cf}}$, and $\mathrm{b}_{\mathrm{cf}}$ is accomplished by plotting the evolution of plastic blunting as a function of the stress intensity factor applied (Figure 111). The identification of $\mathrm{a}_{\mathrm{m}}$ and $\mathrm{b}_{\mathrm{m}}$ is done during the first loading cycle and of $\mathrm{a}_{\mathrm{cf}}$ and $\mathrm{b}_{\mathrm{cf}}$ during the last unloading cycle. The parameters are then calculated by the smallest square method.

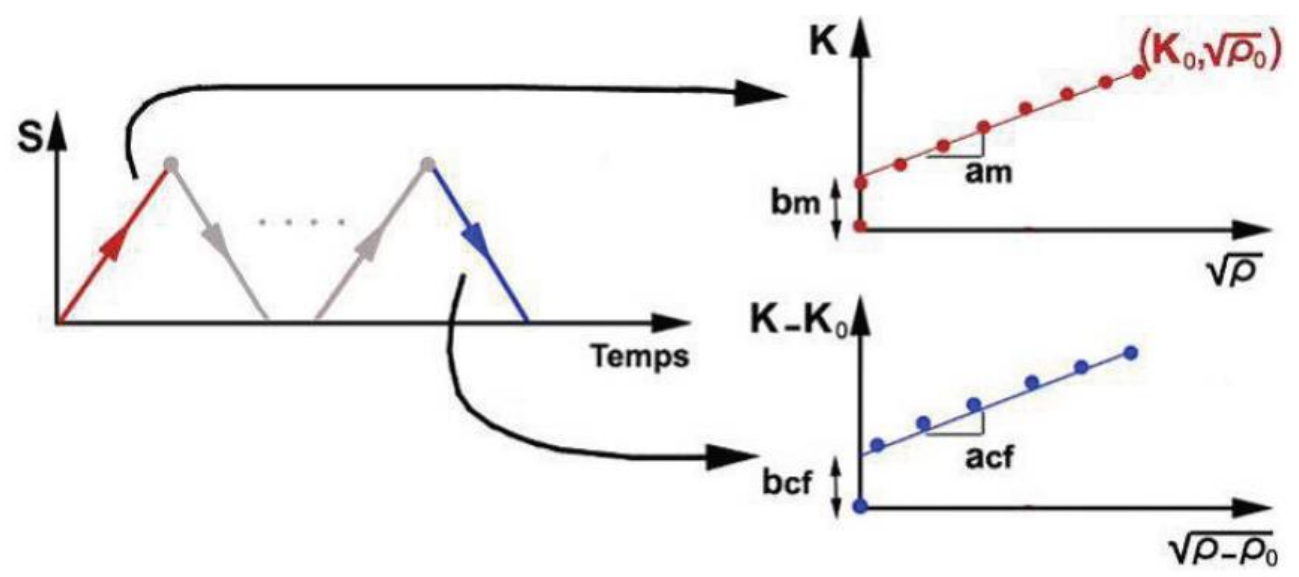

Figure 111: Identification of parameters $a_{m}, b_{m}, a_{c f}$, and $b_{c f}$.

The identification of parameter $\mathrm{a}_{\mathrm{xm}}$ is done by plotting the evolution of contact point $\mathrm{K}_{\mathrm{xm}}$ in the first loading cycle as a function of plastic blunting. The evolution of parameter $\mathrm{K}_{\mathrm{xm}}$ as function of plastic blunting is linear and described by the following equation:

$$
K_{x m}=a_{x m} \cdot \varrho+b_{x m}
$$

Via the smallest square method, it is possible to determine the angular coefficient of the line $\mathrm{a}_{\mathrm{xm}}$, i.e., the slope of curve $\mathrm{K}_{\mathrm{xm}} \mathrm{x} \varrho$ (Figure 112). The identification of $\mathrm{p}_{\mathrm{a}}$ is done by 
plotting the evolution of corresponding plastic strain as a function of distance $r$ from the crack tip during loading. Just the first curve points are employed in the calculation. Parameters $\mathrm{k}_{\mathrm{a}}$ and $\mathrm{k}_{\mathrm{b}}$ are estimated from the contact point evolution as a function of crack length.

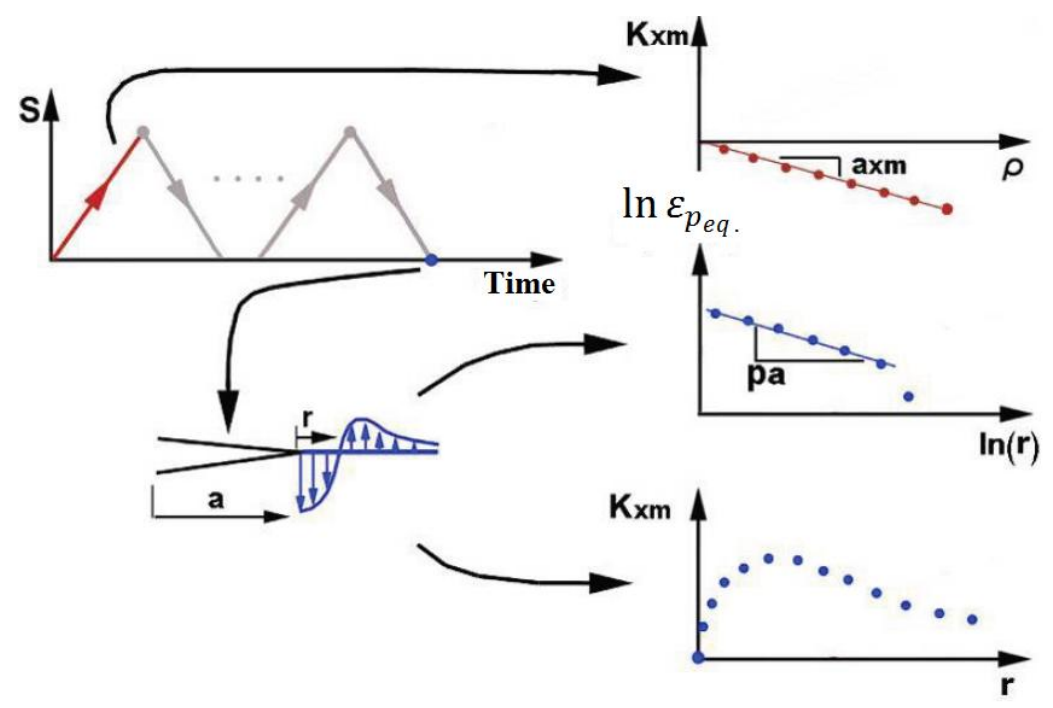

Figure 112: Identification of parameters $a_{x m}, p_{a}, k_{a}$, and $k_{b}$.

Finite Element calculations and procedures to identify plastic blunting law parameters were carried out by ABAQUS, which took a couple of hours. The parameters obtained for the material under study, i.e., A356 aluminum alloy used in the manufacture of automotive engine cylinder heads, are shown in Table 10. Obtaining the parameters for the plastic blunting law depends on choosing the adequate material mechanical behavior model as well as its parameters.

Table 10: Plastic blunting law parameters.

\begin{tabular}{|c|c|c|c|c|c|c|c|c|}
\hline$\tau$ & \multicolumn{8}{|c|}{ Parmeters } \\
\hline 胥 & $a_{m}$ & $b_{m}$ & $a_{c f}$ & $b_{c f}$ & $a_{x m}$ & $p_{a}$ & $k_{a}$ & $k_{b}$ \\
\hline$\stackrel{\Xi}{\varrho}$ & $M P a \sqrt{m} / \sqrt{\mu m}$ & $M P a \sqrt{m}$ & $M P a \sqrt{m} / \sqrt{\mu m}$ & $M P a \sqrt{m}$ & $M P a \sqrt{m} / \mu m$ & $M P a \sqrt{m} / \mu m$ & $M P a \sqrt{m} / \mu m$ & $M P a \sqrt{m} / \mu m$ \\
\hline 120 & 8.37 & 1.37 & 23.2 & 0.85 & -0.85 & -0.001 & -0.32 & 0,023 \\
\hline 200 & 6.37 & 1.46 & 15.1 & 1.99 & $-0,64$ & -0.001 & -0.09 & 0.009 \\
\hline 280 & 4.32 & 1.57 & 9.8 & 2.89 & $-0,52$ & -0.001 & -0.01 & 0.001 \\
\hline
\end{tabular}

\subsubsection{Identification of Crack Propagation Law Parameter}


Once the parameters of the material behavior law and plastic blunting are determined, it is time to determine the fatigue crack propagation law and its only tuning parameter $(\alpha)$ :

$$
\frac{d a}{d t}=\frac{\alpha}{2} \cdot\left|\frac{d \varrho}{d t}\right|
$$

The identification of the fatigue crack propagation law parameter $\alpha$ is done by performing tests under controlled loading and constant amplitude. These tests were carried out by a mechanical testing machine (Figure 53) on specimens with corner cracks (Figure 52b) and loading rate $R=0.1$ (shown in Section 6.1.2.5). Figure 113 presents the fatigue crack propagation curve at $120^{\circ} \mathrm{C}$ plotted in a Paris diagram.

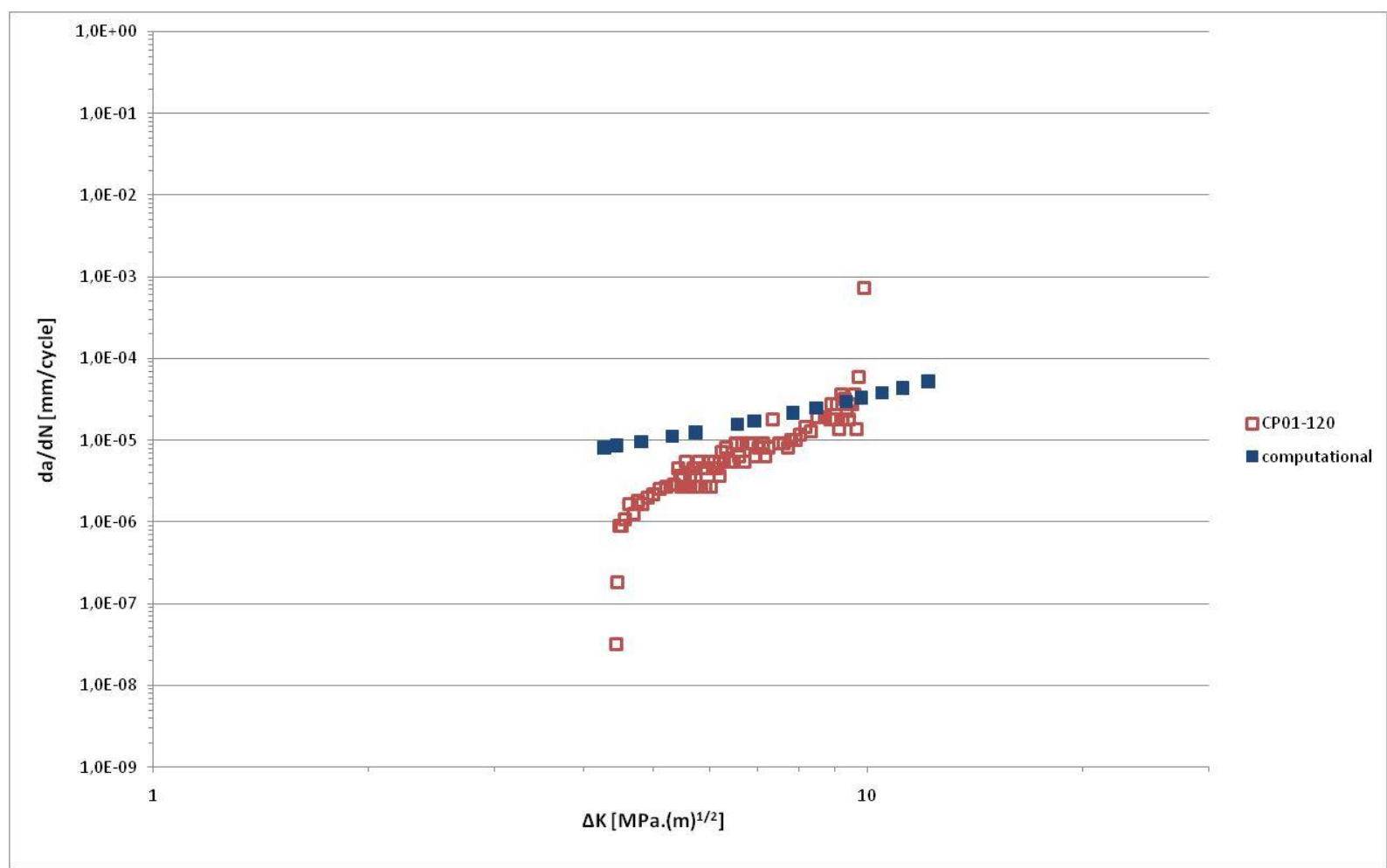

Figure 113: Graph of crack propagation $(\mathrm{da} / \mathrm{dN})$ as a function of stress intensity factor variation $(\Delta \mathrm{K})$ at $120^{\circ} \mathrm{C}$ and under sinusoidal wave loadings at frequency of $10 \mathrm{~Hz}$.

Parameter $\alpha$ exerts influence only on the Paris curve level at constant amplitude and very little on its slope. In fact, the crack propagation rate is directly proportional to the plastic blunting rate. Thus, when $\alpha$ is altered, just the position of the propagation curve in the Paris diagram changes whereas its slope remains constant. The Paris curve slope derives directly from the plastic blunting law. Thus, accurate identification of the material behavior law parameters and, consequently, blunting law parameters is fundamental for the Paris exponent $m$ to be represented correctly. Therefore, in order to identify $\alpha$, an initial simulation is 
performed by adopting its value as equal to 1 (one). Its exact value is then deducted from the relationship between the simulated rate and experimental rate for a same level of $K_{\max }$. 


\section{CONCLUSIONS}

The material studied in this work is an $\mathrm{A} 356 \mathrm{Al}$ alloy, used to produce engine cylinder heads for the automotive industry by die casting process. The chemical analysis has shown that some chemical elements were a slight difference from the nominal values ( $\mathrm{Fe}$ and $\mathrm{Si})$, as well as the EDX analysis, from specific areas, showed that the cast presented some lack of homogeneity.

From microstructural analisys it was observed a quite coarse dentrite microstructure in a eutectic matrix, with a mean SDAS of $25 \mu \mathrm{m}$, intemetallic precipitates and porosities (mean diameter size of $50 \mu \mathrm{m}$ and maximum size of $500 \mu \mathrm{m}$ ).

As expected, the tensile properties were strongly affected by testing temperature (RT till $280{ }^{\circ} \mathrm{C}$ ), with a quite sensitive drop of $\mathrm{E}$ (63 to $\left.45 \mathrm{GPa}\right), \sigma_{\mathrm{y}}(124$ to $80 \mathrm{MPa})$ and $\sigma_{\mathrm{u}}(212$ to $81 \mathrm{MPa}$ ) as the temperature was raised.

Considering the isothermal fatigue tests at 120 and $280{ }^{\circ} \mathrm{C}$, under strain control, it was observed that as the temperature raised from 120 to $280{ }^{\circ} \mathrm{C}$, life dropped markedly (approximately 10 times). This was due to the material has became less resitant to plastic deformation and defects were intensified. From the stabilized hysteresis loop it was possible to observe the difference in the mentioned ductility, as well as, that at $120{ }^{\circ} \mathrm{C}$, during testing, the A356 Al alloy "cyclic hardens" while at 280 'C "cyclically softens".

From the themomechanical in-phase tests, with temperature varying from (120 to 280 ${ }^{\circ} \mathrm{C}$ ), it was possible to observe that life is quite similar to the isothermal fatigue test at $280{ }^{\circ} \mathrm{C}$. In this case, the more sensitive damage caused the in-phase mechanical and thermal cycle take place at the highest temperature. In time, both hysteresis are quite similar in shape, with some differences due to the fact that the thermomechanical cycle is slower $(0.003 \mathrm{~Hz})$ than the isothermal cycle $(0.1 \mathrm{~Hz})$, allowing some stress relaxation (reducing the maximum stress level).

Relaxation tests indicated two distinct behaviors, with the temperature of $240^{\circ} \mathrm{C}$ being a threshold. At lower temperatures, the material hardens cyclically whereas it softens cyclically at higher temperatures. This behavior is similar to the one observed during the isothermal fatigue tests. As the tests are carried out in strain control, during the dwell the stress is relaxed, reducing the elastic component of strain and at same time increasing the plastic component. It was observed that as the test temperature is increased, the amount of stress relaxed increases. 
From the fatigue crack growth results, it was observed that temperature and wave shape has a strong influence on the crack growth rate as well as on the stress intensity threshold. Considering sinusoidal wave shape $(10 \mathrm{~Hz})$, as the temperature increased the $\Delta \mathrm{K}_{\mathrm{th}}$ decreased and the crack propagation rate increased (for a constant $\Delta \mathrm{K}$ ). However, the rate as $\mathrm{da} / \mathrm{dN}$ change with temperature is quite similar, as an indicative that the micromechanism of crack growth has not changed due to the high frequency used, and it was due only to loss of mechanical strength.

Considering a constant temperature $\left(120\right.$ and $\left.280^{\circ} \mathrm{C}\right)$ and stress controlled test, changing the wave shape from sinusoidal to $3 \mathrm{~min}$ dwell at maximum load, the crack propagation rate has increased of approximately 100 times, showing a strong contribution of creep on the crack propagation process, with the amount of creep contribution dependent on temperature, it being higher as higher is the temperature.

Considering a constant temperature $\left(280^{\circ} \mathrm{C}\right)$, strain controlled test and the 3 min dwell at maximum load, it was observed that the rate of da/dN with $\Delta \mathrm{K}$ become almost constant, due to stress relaxation.

It has noticed that is quite difficult to obtain, for the casting material studied in this work, the mechanical behavior parameters, since there is a noticiable discrepancy amount the specimens tested. However, it was shown for the examples presented here, that the model was able to reproduce, with a reasonable approximation, the stress - strain relationship at different temperatures, for the isothermal and anysothermal cases. 


\section{REFERENCES}

AMERICAN SOCIETY FOR METALS - ASM (1997), ASM Handbook, Volume 19: Fatigue and Fracture ASM International, ISBN: 0871703858, Pages: 1057.

AMERICAN SOCIETY FOR METALS - ASM (2000), Metals Handbook Volume 8: Mechanical Testing and Evaluation Publisher: ASM International; 10 edition (September 2000), English, ISBN: 0871703890, 10th Edition, 2235 pages. ASM Handbook

AMERICAN SOCIETY FOR METALS - ASM (2002), ASM Handbook Volume 11: Failure Analysis and Prevention 10 edition, English, ISBN: 0871707047, 2909 pages AMERICAN SOCIETY FOR METALS - ASM (2004), ASM Handbook, Volume 9: Metallography And Microstructures ASM International, ISBN: 0871707063, Pages: 1184.

AMERICAN SOCIETY FOR METALS (1979). Metals Handbook - Properties and Selection: Nonferrous Alloys and Pure Metals. 9ed. Ohio, v.2.

AMERICAN SOCIETY FOR METALS (1988). Semisolid Metal Casting and Forging. In: Metals Handbook - Castings. 9ed. Ohio, v.15, p327-338.

AMERICAN SOCIETY FOR TESTING AND MATERIALS - ASTM B108/B108M-08 (2008). Standard specification for aluminum-alloy permanent mold castings. West Conshohocken, ASTM.

AMERICAN SOCIETY FOR TESTING AND MATERIALS - ASTM E 466-07 (2007).

Standard Practice for Conducting Force Controlled Constant Amplitude Axial Fatigue Tests of Metallic Materials. Philadelphia, v.03.01.2007.

AMERICAN SOCIETY FOR TESTING AND MATERIALS - ASTM E3-01(2007) ${ }^{\varepsilon 1}$.

Standard Practice for Preparation of Metallographic Specimens. Philadelphia, v.03.01.

AMERICAN SOCIETY FOR TESTING AND MATERIALS - ASTM E468-90(2004) ${ }^{\varepsilon 1}$ .Standard Practice for Presentation of Constant Amplitude Fatigue Test Results for Metallic Materials. Philadelphia, v.03.01, 2004.

AMERICAN SOCIETY FOR TESTING AND MATERIALS - ASTM E606-04 ${ }^{\varepsilon 1}$ (2004).

Standard Practice for Strain-Controlled Fatigue Testing. Philadelphia, v.03.01, 2004. 
AMERICAN SOCIETY FOR TESTING AND MATERIALS - ASTM E739-10 (2010). Standard Practice for Statistical Analysis of Linear or Linearized Stress-Life (S-N) and Srain-Life ( $\varepsilon-N)$ Fatigue Data. Philadelphia, v.03.01, 2010.

AMERICAN SOCIETY FOR TESTING AND METERIALS - ASTM E10-10 (2010). Standard test method for Brinell hardness of metallic materials. West Conshohocken, ASTM.

AMERICAN SOCIETY FOR TESTING AND METERIALS - ASTM E2055-99 (1999) Standard Practice for Referencing Test Methods for Chemical Analysis of Metals and Related Materials.

AMERICAN SOCIETY FOR TESTING AND METERIALS - ASTM E21-09 (2009). Standard test methods for elevated temperature tension tests of metallic materials. West Conshohocken, ASTM.

AMERICAN SOCIETY FOR TESTING AND METERIALS - ASTM E2368 - 10 (2010). Standard Practice for Strain Controlled Thermomechanical Fatigue Testing. West Conshohocken, ASTM.

AMERICAN SOCIETY FOR TESTING AND METERIALS - ASTM E606-04 ${ }^{\varepsilon 1}$ (2004). Standard recommended practice for constant-amplitude low-cycle fatigue tests. West Conshohocken, ASTM.

AMERICAN SOCIETY FOR TESTING AND METERIALS - ASTM E8/E8M-09 (2009). Standard test methods of tension testing of metallic materials. West Conshohocken, ASTM.

ANSON J.P., GRUZLESKI J.E. (1999). The quantitative discrimination between shrinkage and gas microporosity in cast aluminium alloys using spatial data analysis. Mater. Char., vol. 43, pp 319-335.

ARGYRIS, J. H. e KELSEY, S. (1964), "Energy Theorems and Structural Analysis", Butterworth, Londres (colletion of papers published in Aircraft Engineering in 1954 and 1955).

ASSAN, A. E. (2003). Método dos Elementos Finitos: Primeiros Passos. 2a ed. Campinas,SP:Editora da Unicamp.

BAIK, S., RAJ, R. (1982). Metallurgical Transactions A (Physical Metallurgy and Materials Science), vol.13A, N.7, p.1207-1214.

BAMBERGER, M.; MINKOFF, I.; STUPEL, M.M. (1986). Some observations on dendritic arm spacing in Al-Si-Mg and Al-Cu alloy chill castings. Journal of Materials Science, v.21, n.8, p.2781-2786. 
BARRALIS J.; MAEDER G. (1997). Précis de métallurgie: élaboration, structurespropriétés, normalisation. AFNOR-Nathan, Paris.

BATTE, A.D. (1983). Creep-fatigue life predictions. In: Fatigue at High Temperature. Skelton, R.P. ed. Elsevier, London.

BEUMLER, H.; HAMMERSTAD, A.; WIETING, B.; DASGUPTA, R. (1988). Analysis of modified 319 aluminum alloy. AFS Transactions, v.96, p.1-12.

BHAT, S. P.; LAIRD, C. (1979a). High temperature cyclic deformation of precipitation hardened alloy-I. Partially coherent precipitates, Acta Metalurgica, vol.27, n.12, p. 1861-1871.

BHAT, S. P.; LAIRD, C. (1979b). High temperature cyclic deformation of precipitation hardened alloy-II. Fully coherent precipitates, Acta Metalurgica, vol.27, n.12, p. 1873-1883.

BOYLAN, J. (1997). Semi-solid formed aluminum. Advanced Materials \& Processes. v.152, n.4, October, p.27-28.

BROWN, S.B.; FLEMINGS, M.C. (1993). Net-shape forming via semi-solid processing. Advanced Materials \& Processes. v.143, n.1, January, p.36-40.

BUFFIÈRE J.Y., SAVELli S., JOUNEAU P.H., MAIRE E., FOUGÈRES R. (2001). Experimental study of porosity and its relation to fatigue mechanisms of model AlSi7-Mg0.3 cast Al alloys. Mater. Sci. Eng. A, vol. 316, pp 115-126.

BYCZYNSKI, G.E.; KIERKUS, W.; NORTHWOOD, D.O.; PENROD, D.; SOKOLOWSKI, J.H. (1996). The effect of quench rate and mechanical properties of 319 aluminum alloy castings. Materials Science Forum, v.217- 222, p.783-788.

CÁCERES, C.H.; DJURDJEVIC, M.B.; STOCKWELL, T.J.; SOKOLOWSKI, J.H. (1999). The effect of $\mathrm{Cu}$ content on the level of microporosity in Al-Si-Cu-Mg casting alloys. Scripta Materialia, v. 40, n.5, p.631-637, February.

CÁCERES, C.H.; SELLING, B.H. (1996). Casting defects and tensile properties of an Al-SiMg alloy. Materials Science and Engineering A, v.220A, p.109-116.

CAI, C., LIAW, P. K., YE, M., YU, J. 1999, Recent Developments in the Thermomechanical Fatigue Life Prediction of Superalloys, JOM-e, V. 51 (4), p. 1 - 19.

CAMPBELL, J. (1991). Castings. Ed. Butterworth-Heinemann, Oxford.

CHABOCHE, J. L. (1997). La Fatigue des Materiaux et des structures, Hermes, p. 617-644.

CHARKALUK, E.; BIGNONNET, A.; CONSTANTINESCU, A.; DANG VAN, K. (2002). Fatigue design of structures under thermomechanical loadings, Fatigue Fracture Engineering Material Structure, vol. 25, p. 1199-1206. 
CHARKALUK, E.; VERGER, L.; CONSTANTINESCU, A.; LEDERER G.; AND STOLZ, C.. (1999). Lois de comportement viscoplastiques anisothermes pour calculs cycliques sur structures, Acte du 4eme colloque national en calcul des structures, Ed. by D. Guedra-Degoerges, Pl. Ladeveze and M. Raous, Teknea, p. 575-580.

CHOI, J.C.; PARK, H.J. (1998). Microstructural characteristics of aluminum 2024 by cold working in the SIMA process. Journal of Materials Processing Technology. v.82, p.107-116.

CHOI, J.C.; PARK, H.J.; KIM, B.M. (1999). The influence of induction heating on the microstructure of A356 for semi-solid forging. Journal of Materials Processing Technology. v.87, p.46-52.

CHOI, J.C.; PARK, H.J.; LEE, B.M. (1998). Finite element analysis of compression holding step in semi-solid forging and experimental confirmation. Journal of Materials Processing Technology. v.80-81, p.450-457.

CLOUGH, R. W. (1980), “The Finite Element Method after twenty-five years: A personal view. Computers \& Structural, vol. 12, pp. 361-370.

COFFIN Jr., L.F. (1954). A study of the effects of cyclic thermal stresses on a ductile metal, Transactions of the ASME, ${ }^{\circ}$ 53-A76, p. $931-949$.

COFFIN, L. F. (1973). Fatigue at high temperatures. In: Fatigue at elevated temperatures, Especial Technical Publication, 520, Philadelphia, ASTM, p.5 - 36.

CONSTANTINESCU, A.; CHARKALUK, E.; LEDERER G.; VERGER, L. (2004). A computational approach to thermomechanical fatigue, International Journal of fatigue, vol. 26, p. 805-818.

COUPER M.J., NEESON A.E., GRIFFITHS J.R. (1990). Castings defects and the fatigue life of an aluminum casting alloy. Fatigue Fract. Eng. Mater. Struct., vol. 13, pp 213227.

COURANT, R. (1943), "Variational Methods for the Solution of Problems of Equilibrium and Vibrations", Bull Am Math Soc,49,1-23.

CREPEAU P.N., ANTOLOVITCH S.D., WORDEN J.A. (1992). FATIGUE CRACK GROWTH IN AN AL-10.7SI-2.2CU-1.2MG CASTING ALLOY. PROCEEDINGS OF THE 22ND SYMPOSIUM ON FRACTURE MECHANICS, PHILADELPHIA, ASTM (USA)., PP 707-726.

CREPEAU, P.N. (1998). Os efeitos da adição de ferro em ligas de alumínio-silício. Fundição e Serviços, v.5, n.68. 
DABAYEH, A.A.; BERUBE, A.J.; TOPER, T.H. (1998). An experimental study of the effect of a flaw at a notch root on the fatigue life of cast Al 319. International Journal of Fatigue, v.20, n.7, p.517-530.

DABAYEH, A.A.; XU, R.X.; DU, B.P.; TOPER, T.H. (1996). Fatigue of cast aluminium alloy under constant and variable-amplitude loading. International Journal of Fatigue, v.18, n.2, p.95-104.

DIETER, G. E., Metalurgia Mecânica, Guanabara Dois, Segunda edição, Rio de Janeiro - RJ, 1981.

DIGHE, M.D.; GOKHALE, A.M. (1997). Relationship between microstructural extremum and fracture path in a cast Al-Si-Mg alloy. Scripta Materialia, v.37, n.9, p.14351440.

DOGLIONE, R.; DOUZIECH, J.L.; BERDIN, C.; FRANÇOIS, D. (1996). Microstructure and damage mechanisms in A356- T6 alloy. Materials Science Forum, v.217-222, p.1435-1440.

DRIVER, J. H. (1971). The effect of boundary precipitates on the high temperature fatigue strength of austenitic stainless steels, Metal Science, V. 5, p. 47 - 50.

DUQUETTE, D. J. (1979). Enviromental effects I - general fatigue resistance and crack nucleation in metals and alloys. In: Fatigue and Microstructure, Metals Park, ASM, p.335- 369 .

ENGLER-PINTO JR., C.C. \& SPINELLI, D. (2002). Cyclcic behavior of the CM247LC-DS superalloy at high temperatures. In HIGH TEMPERATURE FATIGUE. Proceedings of CAMP2002, Bad Lippspringe. p.2-13.

ENGLER-PINTO JR., C.C.; MEYER-OLBERSLEBEN, F.; RÉZAÏ-ARIA, F. (1995). Thermo-mechanical fatigue behaviour of SRR99, in "International Symposium on Fatigue under Thermal and Mechanical Loading", Petten, The Netherlands, May $22-24$.

ENGLER-PINTO JR., C.C.; RÉZAÏ-ARIA, F.; ILSCHNER, B. (1994). Comportamento em fadiga termo-mecânica de superligas à base de níquel, in "11 CBECIMAT Congresso Brasileiro de Engenharia e Ciência dos Materiais", Águas de São Pedro, SP, Brasil, 11-14 de dezembro de 1994, p. 167-169.

ES-SOUNI, M. (2001). Creep behavior and creep microstructures of a high-temperature titanium alloy Ti-5.8-Al-4.0Sn-3.5Zr-0.7Nb-0.35Si-0.06C (Timetal 834) Part I. primary and steady-state creep. Materials Characterization, Vol.46, p.365-379. 
EVANS W.J., JONES H.V., SPITTLE J.A., BROWN S.G.R. (1994). Defect sensitive fatigue in a cast aluminium alloy. Proceedings of the 10th conference on the Strength of Materials, Sendai, Japan. The Japan Inst. of Metals, Strengh of Materials, pp 501504.

FALCÃO, C.A.J. (2002). Estudo do comportamento da fadiga de baixo ciclo em altas temperaturas do aço inoxidável AISI 420. Tese (doutorado). EESC - USP, São Carlos.

FLAIG, B.; LANG, K.H.; LOHE, D.; MACHERAUCH, E. (1995). Thermomechanical fatigue of the cast aluminum alloy GK-AlSi10Mg, in "Symposium of fatigue under Thermal and Mechanical Loadings", Bressers, J. and Rèmy, L. ed. European Commission, Petten.

FLEMINGS, M.C. (1991). Behavior of metal alloys in the semisolid state. Metallurgical Transactions A. v.22A, p.957-981.

FREITAS, E. R.; FERRANTE, M.; SILVA, V. F.; BOSE FILHO, W.; SPINELLI, D. (2004). Thixoextrusion of an A356 alloy: microstructural studies and high temperature fatigue behaviour, Journal of Material Processing and Techonology, vol. 155-156, p. 1629-1633.

FREITAS, E.R. (2001). Identificação dos parâmetros microestruturais e de processo que controlam a conformação de ligas Al-Cu no estado semi-sólido. São Carlos. Tese de Doutorado. Universidade Federal de São Carlos.

FUCHS H. O., STEPHENS R. I., FATEMI A., STEPHENS R. R., (2001), Metal Fatigue in Engineering, Second edition, Wiley-Interscience, p. 472.

FUJINO, S., TAIRA, S. (1980). Effect of thermal cycle on low cycle fatigue of steels and grain boundary characteristics. In: Mechanical behavior of materials, Vol.II, p.4958.

FUJITA, F. E. (1963). Oxidation and dislocation mechanisms in fatigue crack formation. Fracture of Solids. New York, Interscience, p.657-670.

GANDIN C.A., BRÉCHET Y., RAPPAZ M., CANOVA G., ASHBY M., SCHERCLIFF H. (2002). Modelling of solidification and heat treatment for the prediction of yield stress of cast alloys. Acta Mater., vol. 50, pp 901-927.

GANIEV, I.N.; VAKHOBOV, A.V. (1983). Effect of the composition and microalloying with strontium on the structure and properties of Al-Si-Mg alloys. Metal Science and Heat Treatment, v.25, n.7-8, p.623-625.

GLENNY, E. (1961). Thermal fatigue, Metallurgical reviews, vol. 6, nº 24, p. 387-465. 
GLENNY, E.; NORTHWOOD, J.E.; SHAW, S.K.W.; TAYLOR, T.A. (1958-59).A technique for thermal-shock and thermal-fatigue testing based on the use of fluidized solids, Journal of the institute of metals, vol. 87.

GOLOS, K. \& ELLYIN, F. (1988). A total strain energy density theory for cumulative fatigue damage. Transactions of the ASME, v.110. February,p.36-41.

GUEDOU, J.Y.; HONNORAT, Y. (1992). Thermomechanical fatigue on turboengine superalloys, in "International Conference on Low-Cycle Fatigue and Elasto-Plastic Behaviour of Materials 3", Berlin, 7-11 Sept., p. 198-203.

GUNDLACH, R.B.; ROSS, B.; HETKE, A.; VALTIERRA, S.; MOJICA, J.F. (1994). Thermal fatigue resistance of hypoeutectic aluminum-silicon casting alloys, AFS Transactions, vol. 102, p. 205-223.

HALES, R. S. (1983). Fatigue testing methods at elevated temperatures. In: Fatigue at high temperature. Skelton, R.P. ed. Elsevier, London.

HALFORD, G.R.; HIRSCHBERG, M.H.; MANSON, S.S. (1973). Temperature effects on the strain range portioning approach for creep-fatigue analysis. In: Fatigue at elevated Temperature, Especial Technical Publication 520, p.658-667. Philadelphia, ASTM.

HAMAM R., POMMIER S. (2007), « Variable amplitude fatigue crack growth, experimental results and modelling », Int Jal of Fatigue, Vol 29, Num 9-11, Pages 1634- 1646, 2007

HETKE, A.; GUNDLACH, R.B. (1994). Thermal fatigue resistance of hypoeutectic aluminum-silicon casting alloys, AFS Transactions, vol. 102, p. 367-380.

HETNARSKI, R. B. (1991), "Mechanics and Mathematical Methods - Thermal Stress II", North-Holland, Oxford, 2nd Series, V. 2.

HOPKINS, S.W. (1976). Low-Cycle Thermal Mechanical Fatigue Testing, in "Thermal Fatigue of Materials and Components, ASTM STP 612", D.A. Spera and D.F. Mowbray (eds.), American Society for Testing and Materials, p. 157-169.

HOSKIN G.A., PROVAN J.W., GRUZLESKI J.E (1988). The in-situ fatigue testing of a cast aluminium-silicon alloy. Theor. Appl. Fract. Mech., vol. 10, pp 27-41.

HRENNIKOFF, A. (1941), "Solution of Problems in Elasticity by the Framework Method", J. Appl. Mech. 8, p. 169-175.

HUEBNER, K.H. (1994).The finite element method for engineers. New York, John Wiley \& Sons.

INGUANTI P.C. (1985). Overcoming Material Boundaries. Proceedings of the 17th SAMPE technical conference, SAMPE, Covina, CA, pp 61. 
JIANG, H.; BOWEN, P.; KNOTT, J.F. (1999). Fatigue performance of cast aluninium alloy Al-7Si-Mg with surface defects. Journal of Material Science, v.34, p.811-820.

KANG, C.G.; CHOI, J.S. (1998). Effect of gate shape and forging temperature on the injection forging process of semi-solid aluminum material. Journal of Materials Processing Technology. v.73, p.251-263.

KANG, C.G.; CHOI, J.S.; KANG, D.W. (1998). A filling analysis of the forging process of semi-solid aluminum materials considering solidification phenomena. Journal of Materials Processing Technology. v.73, p.289-302.

KANG, C.G.; CHOI, J.S; KIM, K.H. (1999). The effect of strain rate on macroscopic behavior in the compression forming of semi-solid aluminum alloy. Journal of Materials Processing Technology. v.88 p.159-168.

KANG, C.G.; JUNG, H.K. (1999). Finite element analysis with deformation behavior modeling of globular microstructure in forming process of semi-solid materials. International Journal of Mechanical Sciences. v.41, p.1423-1445.

KANG, C.G.; KIM, Y.D.; LEE, S.W. (1997). A coupled solidification analysis of materials and cooling roller in direct rolling process. Journal of Materials Processing Technology. v.66, p.277-286.

KANG, C.G.; YOON, J.H. (1997). A finite-element analysis on the upsetting process of semisolid aluminum material. Journal of Materials Processing Technology. v.66, , p.7684.

KANG, C.G.; YOON, J.H.; SEO,Y.H. (1997). The upsetting behavior of semi-solid aluminum material fabricated by mechanical stirring process. Journal of Materials Processing Technology. v.66, p.30-38.

KAPRANOS, P.; ROBERT, M.H. (1998). Aplicações industriais do processamento de metais no estado semi-sólido (PPS): o estado da arte. Metalurgia e Materiais. v.54, n.475, p.194-197.

KARAYAKA, M.; SEHITOGLU, H. (1991). Thermomechanical fatigue of particulatereiniforced aluminum 2xxx-T4, Metallurgical Transactions A, vol. 22A, p. 697-707.

KICHENIN, J. (1992). Comportement thermomecanique du polyethylene - application aux structures gazieres, PhD Thesis, Ecole Polytechnique, France.

KIM, H.J.; KOBAYASHI, T.; ITO, T. (1997). Effects of Fe and $\mathrm{Ca}$ on usual and impact fatigue characteristics of AC2B-T6 aluminum casting alloy. Materials Science Forum, v.217-222, p.1395-1400.

KIRKWOOD, D.H. (1994). Semisolid metal processing. International Materials Reviews. v.39, n.5, p.173-189. 
KOÇ, M.; VAZQUEZ, V.; WITULSKI, T.; ALTAN, T. (1996). Application of the finite element method to predict material flow and defects in the semi-solid forging of A356 aluminum alloys. Journal of Materials Processing Technology. v.56, p.106-112.

KUMAI, S.; HU, J.; HIGO, Y.; NUNOMURA, S. (1996). Effects of dendrite cell size and particle distribution on the near-threshold fatigue crack growth behaviour of cast AlSi composites. Acta Materialia, v.44, n.6, p.2249-2257.

LAIRD C. (1967). The influence of metallurgical structure on the mechanisms of fatigue crack propagation. ASTM STP 415, pp. 131-168, 1967.

LANGDON, T. G. (2002). Creep at Low Stresses: An Evaluation of Diffusion Creep and Harper __Dorn Creep as Viable Creep Mechanisms. Metallurgical and Materials Transactions. Vol. 33A. p. 249-259.

LEE, S.L.; TAN, Y.H.; YIE, S.N.; LIN, J.C. (1998). Effect of pre-aging on precipitation hardening in Al-Si-Mg alloys. Scandinavian Journal of Metallurgy, v.27, n.3, p.112115.

LIU G., ZHANG G.J., DING X.D., SUN J., CHEN K.H. (2003). Modeling the strengthening response to aging process of heat-treatable aluminum alloys containing plate/disc- or rod/needle-shaped precipitates. Mater. Sci. Eng. A, vol. 344, pp 113-124.

LOUÉ, W.R.; SUÉRY, M. (1995). Microstructural evolution during partial remelting of AlSi7Mg alloys. Materials Science \& Engineering A. v.A203, p.1-13.

MADELAINE-DUPUICH, O.; STOLARZ, J. (1996). Fatigue of eutectic Al-Si alloys. Materials Science Forum, v.217-222, p.1343-1348.

MAIER, H. J., SMITH, T. J. e SEHITOGLU, H., VDI Berichte NR 1472, 1999, P. 409-422.

MAJUMDAR, S., MAIYA, P. S. (1980). Mechanistic model for time-dependent fatigue. Journal Engineering Materials Technology, p.159.

MALAVAZI, J. (2005). Processo de fundição sob pressão. Apostila de fundição. SENAI, Osasco-SP.

MALLET, O.; ENGLER-PINTO JR., C.C.; RÉZAÏ-ARIA, F.; SKELTON, R.P.; NIKBIN, K.; WEBSTER, G.A. (1995). Influence of material stress-strain characteristics on thermomechanical fatigue analysis of IN100 superalloy, Materials at High Temperatures, vol. 13, $\mathrm{n}^{\mathrm{o}}$ 1, p. 47-54.

MALPERTU, J.L.; RÉMY, L. (1988). Thermomechanical Fatigue Behavior of a Superalloy, in "Low Cycle Fatigue, ASTM STP 942", Philadelphia, H.D. Solomon et al. (eds.), American Society for Testing and Materials, p. 657-671. 
MASAHIRO J. (2004). Fatigue damage and crack growth under variable amplitude loading with reference to the counting methods of stress-strain ranges. International Journal of Fatigue, vol. 27 pp. 1006-1015, 2004.

MASSINON D.,CONSTANTIN V. (2002). Formation métallurgie et défauts de fonderie. Montupet, Chateauroux.

MCCLINTOCK F.A. (1967). Discussion to C. Laird's paper « The influence of metallurgical microstructure on the mechanisms of fatigue crack propagation ». Fatigue Crack Propagation, ASTM STP 415, pp. 170-174, 1967.

MCDOWELL, D. L., GALL, K., HORSTEMEYER, M. F. and Fan, J., Engineering Fracture Mechanics 70, 2003, p. $49-80$.

MCHENRY, D. (1943), “A Lattice Analogy for the Solution of Plane Stress Problems”, J. Inst. Civil Eng. 21 (2).

MEDEIROS, S.C. (1999). Failure analysis of an aluminum casting. Advanced Materials \& Processes, v.155, n.4, p.42, April.

MEYER-OLBERSLEBEN, F.; ENGLER-PINTO JR, C.C.; RÉZAÏ-ARIA, F.(1995). On thermal fatigue of nickel-based superalloys, in "Thermo-Mechanical Fatigue Behavior of Materials, ASTM STP 1263", Philadelphia, vol. 2, M.J. Verrilli and M.G. Castelli (eds.), American Society for Testing and Materials.

MILAN, M., MALUF, O., SPINELLI, D., BOSE FILHO, W. W.(2004). “Metais - Uma visão objetiva”, São Carlos, Brasil.

MIN, B. K., RAJ, R.(1979). A mechanism for intergranular fracture during high temperature fatigue. In: Fatigue mechanisms, STP 675, Philadelphia. ASTM, p.569-591, 1979.

MOURA BRANCO, CARLOS A. G. (1985). "Mecânica dos Materisi”, Lisboa: Fundação Calouste Gulbenkian.

MURALI S., ARVIND T.S., RAMAN K.S., MURPHY K.S. (1997). Fatigue properties of sand cast, stir cast and extruded Al-7Si-0.3Mg alloy with trace additions of Be and Mn. Mater. Trans., vol. 38, pp 28-36.

MURALI, S.; RAMAN, K.S.; MURTHY, K.S.S. (1996). Al-7Si-0.3Mg cast alloy: formation and crystal structure of $\beta$-FeSiAls and (Be-Fe)-BeSiFe2Als phases. Materials Science Forum, v.217-222, p.207-212.

NEDBAL I., SIEGEL J. AND HUNZ J. (1989). Relation between striation spacing and fatigue crack growth rate in A1-Alloy Sheets. Advanced in Fracture Research, Pergamon Press, pp. 3483-3491, 1989.

NEU, R. \& SEHITOGLU, H. (1989a). Thermo-mechanical fatigue, oxidation and creep: Part I: Experiments. Metallurgical Transactions A. v.20A, p.1755-1767. 
NEU, R. \& SEHITOGLU, H. (1989b). Thermo-mechanical fatigue, oxidation and creep: Part II: Life prediction. Metallurgical Transactions A. v.20A,p.1769-1783.

NOGOWIZIN, B. (2003). Druckgusslegierungen und ihre Eigenschaften. Druckgess-Praxis, $\mathrm{n}^{\circ} 4, \mathrm{p} 161-168$.

ODEGARD J.A., PEDERSEN K. (1994). Fatigue properties of an A356 (AlSi7Mg) aluminium alloy for automotive applications-fatigue life prediction. SAE technical paper, Society of Automotive Engineers, Warrendale PA, vol. report SAE-940811, pp 25-32.

OLlER, S.; SALOMÓN, O.; OÑATE, E. (2005). A continuum mechanics model for mechanical fatigue analysis, Computational Materials Science, vol. 32, p. 175-195.

OSWALT, K.J.; MISRA, M.S. (1981). Dendrite Arm Spacing (DAS): A nondestructive test to evaluate tensile properties of premium alloy (Al-Si-Mg) castings. AFS International Cast Metals Journal, v.6, n.1, p.26-40.

PELLOUX R.M.N. (1969). Mechanisms of formation of ductile fatigue striation. ASM 62, pp. 281-285, 1969.

PLUMTREE A., SCHAFER S. (1986). The behaviour of short fatigue cracks. K.J. Miller, E.R. de los Rios (Eds.), Mechanical Engineering Publications, London.

POMMIER S., RISBET M. (2005), « Time derivative equations for fatigue crack growth in metals », Int Jal of Fracture, Vol 131, Num 1, Pages 79-106, 2005

POWELL G.W. (1994). The fractography of casting alloys. Mater. Char., vol. 33, pp 275293.

RÉMY, L. (1987). Recent developments in thermal fatigue, in "MECAMAT, International Conference on High Temperature Fracture Mechanisms and Mechanics", Dourdan, France, 13-14-15 October, p. iv/1-iv/19.

RIEDEL HERMANN. (1987). Fracture at high temperatures. Berlin; New York: SpringerVerlag.

ROESLER J., HARDERS H., BAEKER M. (2007). Mechanical Behavior of Engineering Materials. German edition published by Teubner Verlag Wiesbaden. XVI, 536p, 302 illus, Hardcover. ISBN: 978-3-540-73446-8.

ROVIRA, M.M.; LANCINI, B.C.; ROBERT, M.H. (1999). Thixo-forming of Al-Cu alloys. Journal of Materials Processing Technology. v.92-93, p.42-49.

SARABANDA, J.V.L. (1991). Estudo de fadiga sob controle de deformação de duas ligas de alumínio empregadas em pistões de motores diesel. Dissertação de Mestrado, Escola Politécnica - USP, São Paulo 
SAVELLI S., BUFFI'ERE J.Y., FOUG'ERES R. (2000). Pore characterization in a model cast aluminium alloy and its quantitative relation to fatigue life studied by synchrotron Xray microtomography. International Conference on Aluminium Alloys, Charlottesville, Virginia USA. Mater. Sci. Forum, vol. 331, pp 197-202.

SECO, F. J.; IRISARRI, A. M. (2001). Creep failure mechanisms of a Ti-6Al-4V thick plate. p. 741-750.

SEHITOGLU, H. (1995). Thermo-mechanical deformation of engineering alloys and components - experiments and modelling, in NATO-ASI. Mechanical Behaviour of Materials at High Temperature, Sesimbra, Portugal, September 12-22.

SEHITOGLU, H. (1996). Thermal and thermomechanical fatigue of structural alloys, ASM Handbook - Fatigue and Fracture, Ohio, v. 9.

SEHITOGLU, H. ; KARAYAKA, M. (1992). Prediction of thermomechanical fatigue lives in metal matrix composites, Metallurgical Transactions A, vol. 23A, p. 2029-2038.

SENIW M.E., FINE M.E., CHEN E.Y., MESHII M., GRAY J. (1997). Relation of defect size and location to fatigue failure in Al alloy A356 cast specimens. Proceedings of the conference on high cycle fatigue of structural materials, TMS, Warrendale, PA. AIME, pp 371-379.

SERMAGE, J. P.; LEMAITRE, J.; DESMORAT, R. (2000). Multiaxial creep-fatigue under anisothermal conditions, Fatigue Fracture Engineering Material Structure, vol. 23, p. 241-252.

SHI, H. J.; ROBIN, C.; PLUVINAGE, G. (1993). Thermal-mechanical fatigue lifetime prediction of an austenitic stainless steel, Advances in Fatigue Lifetime Predictive Techniques: vol. 2, ASTM STP1211, ASTM, p. 105-116.

SHI, H-J.; KORN, C.; PLUVINAGE, G., (1998). High Temperature Isothermal and Thermomechanical Fatigue on a Molybdenum-Based Alloy, Materials Science and Engineering, A247, P. 180 - 186, 1998.

SIMO, J.C.; HUGHES, T.J.R. (1998). Computational Inelasticity, Springer.

SKALLERUD B., IVELAND T., H“ARKEGARD G. (1993). Fatigue life assessment of aluminum alloys with casting defects. Eng. Fracture Mech., vol. 44, pp 857-874.

SKALLERUD B., LARSEN P.K. (1989). A uniaxial cyclic plasticity model including transient material behavior. Fatigue Eng. Mater. Struct., vol. 12, pp 611-625.

SKELTON, R.P. (1983). Crack initiation and growth in simple metal components during thermal cicling. In: Fatigue at High Temperature, SKELTON, R.P., Elsevier, London. 
SKELTON, R.P. (1994). The determination of hysteresis loops in thermo-mechanical fatigue using isothermal stress data. Fatigue Fracture Engineering Structure, v.17, n.4, p.479-496.

SMITH, T.J.; MAIER, H.J.; SEHITOGLU, H.; FLEURY, E.; ALLISON, J. (1999). Modeling high-temperature stress-strain behavior of cast aluminum alloy, Metallurgical Materials. Transactions, vol. 30A, p. 133-146.

SONSINO, C.M.; ZIESE, J. (1993). Fatigue strength and applications of cast aluminium alloy with different degrees of porosity. International Journal of Fatigue, v.15, n.2, p.75-84.

SOUZA, S. G. S; FREDERICO, G. A. HERING; ANDRÉ, R. TASSIN. (1993). Fundição sob pressão: é possível produzir peças em várias ligas. FS - Fundição e Serviço, ano III, $\mathrm{n}^{\circ}$ 19, Abril/Maio, p 12-18. Aranda editora técnica LTDA.

SOUZA, S.A. (1982). Ensaios Mecânicos de Materiais Metálicos: Fundamentos Teóricos e Práticos. $5^{\mathrm{a}}$ ed. São Paulo: Edgar Blucher.

SPENCER, D.B.; MEHRABIAN, R.; FLEMINGS, M.C. (1972). Rheological behavior of Sn15 pct $\mathrm{Pb}$ in the crystallization. Metallurgical Transactions. v.3, p.1925-1932.

SRINIVASAN, V.S., VALSAN, M., RAO, B. S., MANNAN, S.L., RAJ, B. (2003), Low Cycle Fatigue and Creep-Fatigue Interaction Behavior of $316 L(N)$ Stainless and Life Prediction by Artificial Neural Network Approach, International Journal of Fatigue, V. 25 , p. $1327-1338$.

STANZL-TSCHEGG, S.E.; MAYER, H.R.; BESTE, A.; KROLL, S. (1995). Fatigue and fatigue crack propagation in AlSi7Mg cast alloys under in-service loading conditions. International Journal of Fatigue, v.17, n.2, p.145-155.

SURESH, S, Fatigue of Materials, Cambridge University Press, Cambridge, 1998.

THOMAS, J. J.; VERGER,L.; BIGNONNET, A.; CHARKALUK, E. (2004). Thermomechanical design in the automotive industry, Fatigue Fracture Engineering Material Structure, vol. 27, p. 887-895.

TODINOV, M.T. (1998). A probabilistic method for predicting fatigue life controlled by defects. Materials Science and Engineering A, v.255A, p.117-123.

TOHRIYAMA, S.; KUMANO, M. (1995). Influence of material and mechanical properties on thermal fatigue life of aluminum castings, Aluminum Applications for Automotive Design, SAE, Warrendale, PA, p. 47-57.

TOMKINS, B. \& WAREING, J. (1977). Elevated Temperature fatigue interactions in engineering materials. Metal Science 11, 414-424. 
TURNER, M. J., CLOUGH, R. W., MARTIN, H. C. e TOPP, L. J. "Stiffness and Deflection Analysis of Complex Structures”, J Aeron Sci, 23(9), 805-823, 1956.

VASUDEVAN, A. K., DOHERT, R. (1987). Grain boundary ductile fracture in precipitation hardened aluminum alloys. Acta Metallurgica, V. 35, p.1193 - 1219.

VELASCO, E.; COLÁS, R.; VALTIERRA, S.; MOJICA, M.J. (1995). A model for thermal fatigue in an aluminum alloy, International Journal. of Fatigue, vol. 17, p. 399-406.

VERDU, C.; CERCUEIL, H.; COMMUNAL, S.; SAINFORT, P.; FOUGÈRES, R. (1996). Microstructural aspects of damage mechanisms of Al-7Si-Mg alloys. Materials Science Forum, v.217-222, p.1449-1454.

VORREN, O.; EVENSEN, J.E.; PEDERSEN, T.B. (1984). Microstructural effects on the fatigue properties of a cast Al7SiMg alloy, AFS Transactions, vol. 92, p. 459-466.

WANG, Q. G., APELIAN, D. and LADOS, D. A., Journal of Light Metals 1, 2001, p. 73-97.

WANG, Q.G.; CÁCERES, C.H. (1997). The fracture mode in Al-Si-Mg casting alloys. Materials Science and Engineering A, v.241A, p.72-82.

WAREING, J. (1983). Mechanisms of high temperature fatigue and creep-fatigue failure in engineering materials. In: Fatigue at high temperature, Skelton, R.P. ed. Elsevier, London.

WELLS, C. H. (1979). High-temperature fatigue. In: Fatigue and microstructure. Metals Park, ASM, p.307 - 331.

WICKBERG, A.; GUSTAFSON, G.; LARSON, L.E. (1984). Microstructure and mechanical properties of AlSi $(\mathrm{Mg})$ casting alloys. SAE - Transactions, vol. 93, p. 728-735.

WIGANT C.C., STEPHENS R.I. (1987). Low cycle fatigue of A356-T6 cast aluminum alloy. Society of Automotive Engineers, Warrendale PA, vol. Report No 870096.

YU, Y.B.; SONG, P.Y.; KIM, S.S.; LEE, J.H. (1999). Possibility of improving tensile strength of semi-solid processed A356 alloy by a post heat treatment at an extremely high temperature. Scripta Materialia. v.41, n.7, p.767-771.

ZHANG, D.L. (1996). Precipitation of excess silicon during heat treatment of cast Al7wt\%Si-0.4wt\%Mg alloy. Materials Science Forum, v.217-222, p.771-776. 